THE SEISMIC ATTENUATION STRUCTURE OF THE EAST PACIFIC RISE

by

WILLIAM SAM DOUGLAS WILCOCK

B.A., University of Cambridge (1985)

M.Sc., Imperial College, University of London (1986)

SUBMITTED IN PARTIAL FULFILLMENT

OF THE REQUIREMENTS FOR THE DEGREE OF

DOCTOR OF PHILOSOPHY

at the

MASSACHUSETTS INSTITUTE OF TECHNOLOGY

and the

WOODS HOLE OCEANOGRAPHIC INSTITUTION

June, 1992

(c) William S. D. Wilcock, 1992

The author heareby grants to MIT and WHOI permission to reproduce and distribute copies of this thesis document in whole or in part.

Signature of Author

Joint Program in Oceanography

Massachusetts Institute of Technology /

Woods Hole Oceanographic Institution

$\curvearrowright \curvearrowright \cap$ Kebruary, 1992

Certified by

Sean C. Solomon, Thesis Co-Supervisor

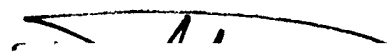

Certified by

G. M. Purdy, Thesis Co-Supervisor

Accepted by

M. K. McNutt

Chair, Joint Committee for Marine Geology and Geophysics

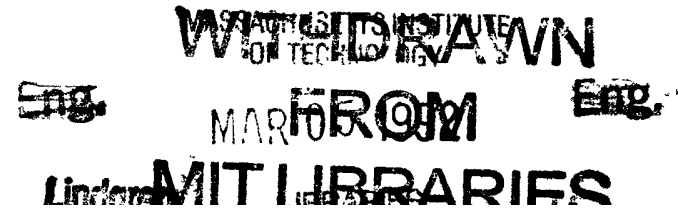




\title{
THE SEISMIC ATTENUATION STRUCTURE OF THE EAST PACIFIC RISE
}

\author{
by \\ William Sam Douglas Wilcock \\ submitted to the Department of Earth, Atmospheric, and Planetary Sciences, \\ Massachusetts Institute of Technology \\ and \\ the Department of Geology and Geophysics, \\ Woods Hole Oceanographic Institution \\ on February 27, 1992, in partial fulfillment of the requirements for the degree of Doctor \\ of Philosophy
}

\begin{abstract}
Studies of seismic propagation through oceanic crust have contributed enormously to our understanding of the generation and evolution of oceanic crust. However, such work has largely been confined to the seismic velocity structure. In this thesis we present results from a study of seismic attenuation using a data set collected for three-dimensional tomographic imaging of a fast-spreading ridge. The experiment location at $9^{\circ} 30^{\prime} \mathrm{N}$ on the East Pacific Rise is the site of a strong mid-crustal seismic reflector which has been inferred to be the roof of a small axial magma chamber at about $1.6 \mathrm{~km}$ depth.
\end{abstract}

A spectral method is used to estimate $t^{*}$, a measure of the integrated attenuation along a wave path. Such a method assumes that the dominant frequency-dependent component of propagation is intrinsic attenuation. A logarithmic parameterization is then used to invert ${ }^{*}$ measurements for $Q^{-1}$ structure assuming that the velocity structure is given from earlier studies. To evaluate the method of $Q$ tomography a full-waveform finitedifference technique which does not include attenuation is used to calculate solutions for seismic propagation through a two-dimensional velocity model. The results show a complex pattern of seismic propagation in the vicinity of the axial magma chamber. The first arrival always passes above the magma chamber. However, for paths of significant length that cross the rise axis the amplitude of this arrival is very small, and the first phase with significant amplitude is a diffraction below the magma chamber. High-amplitude Moho turning and PP arrivals may also be important secondary arrivals. Synthetic inversions show the importance of selecting time windows for power spectral estimation which are dominated by a single phase and of using wave paths which closely corresponds to that of the selected phase.

A comparison of the finite difference solutions and the predictions of the a twodimensional, exact ray-tracing algorithm with record sections obtained during the tomography experiment significantly improves our understanding of seismic propagation across the East Pacific Rise. The results enable an objective choice of the position and length of the time window for $t^{*}$ estimation. Moreover, additional constraints are incorporated into an approximate three-dimensional ray-tracing algorithm used in the inversion so that the wave paths more closely correspond to those of the desired phase. The full data set to be inverted comprises about $3500 t^{*}$ estimates and includes crustal paths which do not cross the rise axis, diffractions above and below the axial magma 
chamber, and Moho-turning phases. Wave paths for the Moho-turning phases cross the rise axis at a wide range of lower crustal depths.

The $\mathrm{Q}^{-1}$ models resulting from two-dimensional and three-dimensional tomographic inversions show that the attenuation of seismic waves on the East Pacific Rise is dominated by two regions of low Q; one in the upper $1 \mathrm{~km}$ of crust, and one at depths greater than about $2 \mathrm{~km}$ below the rise axis. While the data do not resolve the details of vertical variations in near-surface $Q^{-1}$, the results show a substantial variation in shallow attenuation within $0.05 \mathrm{My}$ of the rise axis. On-axis, $Q$ values averaged over the upper 1 $\mathrm{km}$ are about 100 , while off-axis the average value rapidly decreases to about 30 .

Measurements of the seismic velocity suggest that the thickness of the surficial highporosity extrusive layer increases substantially off-axis. If such thickening is entirely responsible for the observed change in near-surface attenuation then $\mathrm{Q}$ within the extrusive layer must be much less than 20 . Alternatively, in situ changes in porosity may also contribute to the observed increase in attenuation. Since significant tectonic activity is apparently restricted to locations well off-axis we suggest that such variations in porosity may result from hydrothermal activity. Regions of hydrothermal downwelling located off-axis will be subject to cooling and thermally-induced cracking while upwelling regions on-axis may be accompanied by rapid infilling of existing pores by hydrothermal deposits.

Estimates of $t *$ for all phases propagating below the magma chamber are markedly higher than those for other phases, resulting in $\mathrm{Q}^{-1}$ models which include a region of low $\mathrm{Q}$ extending from 2 to $7 \mathrm{~km}$ depth below the rise axis. The lowest $\mathrm{Q}$ values resolved are about 25-30 both immediately below the magma chamber and within the lower crust. While there is some evidence for a small decrease in attenuation with depth in the lower crust, axial $Q$ values at depths ranging from less than 2.5 to $6 \mathrm{~km}$ are relatively constant, always lying below 50 . Laboratory measurements at seismic frequencies suggest that $Q$ values of 25-50 require only very small fractions of partial melt. The attenuation observations thus place constraints on the dimensions of the axial magma chamber and strongly suggest that the thickness of the region containing more than a few percent of partial melt is no more than $1 \mathrm{~km}$.

Thesis Co-Supervisors: Dr. Sean C. Solomon

Professor of Geophysics, MIT

Dr. G. M. Purdy

Senior Scientist, WHOI 


\section{ACKNOWLEDGEMENTS}

I would like to express my deep gratitude to my advisors Sean Solomon and Mike Purdy for their excellent support and guidance throughout my tenure as a graduate student. I have also benefitted from the opportunity to work closely with Doug Toomey and Marty Dougherty on aspects of this thesis. In addition many other people have contributed to this work. The excellent quality of the seismic data is testimony to the technical expertise of Jim Broda, Carlton Grant, Don Koelsch, Ken Peal, Beecher Wooding, Tim Barash, Walter Cook, Paul Selkirk, and the captain and crew of the R/V Thomas Washington. Don Koelsch patiently tutored me on the workings of the WHOI ocean bottom hydrophones. I acknowledge useful discussions with Gail Christeson, John Collins, Bob Detrick, Henry Dick, Brian Evans, Kathy Gillis, Steve Holbrook, Tom Jordan, Sang Mook Lee, Jian Lin, Randy Jacobson, Randy Mackie, Ted Madden, Bill Rodi, Peter Shaw, and Anne Sheehan. Dave Krowitz deserves thanks for running the MIT computers so smoothly, while David DuBois assisted with many aspects of my work at WHOI. Outside the confines of my thesis I have enjoyed the opportunity to work with with Jack Whitehead, while the geodynamics courses and field trips organized by Henry Dick have greatly stimulated my interest in mid-ocean ridge processes. Administratively my time in the Joint Program has been greatly simplified by Libby Kurten, Marie Sénat, Katherine Ware, and Faith Hampshire. The work in this thesis was supported by the Office of Naval Research under grant N00014-89-J-1257, while the original data collection was supported by the National Science Foundation under grants OCE-8615797 and OCE-86158920. 


\section{TABLE OF CONTENTS}

$\begin{array}{ll}\text { ABSTRACT } & 3\end{array}$

ACKNOWLEDGEMENTS

TABLE OF CONTENTS

CHAPTER 1

PRINCIPLES OF ATTENUATION TOMOGRAPHY

Introduction 11

Seismic Attenuation 12

A Spectral Method for Attenuation Tomography 14

Tomography Experiment 16

Power Spectrum Estimation 18

Source-Receiver Corrections and $t^{*}$ Estimates 22

A Method of Q Tomography 25

Table $\quad 30$

Figure Captions 31

Figures 33

\section{CHAPTER 2}

A COMPARISON OF THE ATTENUATION STRUCTURE OF 0.35 AND 0.00-MY-OLD CRUST

Introduction 43

The Off-Axis Profiles 43

Velocity Structure $\quad 43$

Western Profile $\quad 44$

Eastern Profile $\quad 45$

t* Estimation $\quad 45$

$\mathrm{Q}^{-1}$ Inversions $\quad 48$

Axial Profile $\quad 50$

Velocity Structure $\quad 51$

t* Estimation $\quad 52$

$\mathrm{Q}^{-1}$ Inversion $\quad 53$

Summary $\quad 54$

Figure Captions $\quad 55$ 
Figures

CHAPTER 3

A FINITE DIFFERENCE EVALUATION OF THE SPECTRAL METHOD OF ATTENUATION TOMOGRAPHY

$\begin{array}{ll}\text { Introduction } & 83\end{array}$

Finite-Difference Model Configurations $\quad 84$

$\begin{array}{ll}\text { Finite Difference Solutions } & 87\end{array}$

Delay-Time Tomography and Wave Paths $\quad 89$

$\begin{array}{ll}\text { Synthetic Inversions for } Q^{-1} & 91\end{array}$

Velocity Model Errors $\quad 91$

Multi-Pathing $\quad 92$

High Surface Smoothing Weights 93

The Effects of Deterministic Scattering 94

$\begin{array}{ll}\text { Summary } & 96\end{array}$

Figure Captions $\quad 97$

$\begin{array}{lr}\text { Figures } & 102\end{array}$

\section{CHAPTER 4}

TWO AND THREE-DIMENSIONAL INVERSIONS FOR THE Q ${ }^{-1}$ STRUCTURE OF THE EAST PACIFIC RISE

Introduction

123

$\begin{array}{lc}\text { Velocity Models } & 123\end{array}$

Receivers 20 km Off-Axis $\quad 125$

Record Sections and Wave Paths 125

$t^{*}$ Estimates 129

Receivers 9 km Off-Axis 132

Record Sections and Wave Paths 133

$t^{*}$ Estimates $\quad 135$

$\begin{array}{ll}\text { Receivers on the Rise Axis } & 136\end{array}$

Biases to the AOBH t* data 137

$\begin{array}{ll}\text { Inversions for } Q^{-1} & 140\end{array}$

$\begin{array}{ll}\text { Station Corrections } & 140\end{array}$

A Two-Dimensional Inversion for a Symmetric $Q^{-1}$ Structure $\quad 141$

$\begin{array}{ll}\text { Inversions for Upper Crustal Structure } & 144\end{array}$

$\begin{array}{ll}\text { Inversions for Shallow Structure } & 148\end{array}$ 
An Inversion of $t^{*}$ Values for Paths Between the Two Outer Refraction Lines

An Inversion of the Moho-Turning Phase 150

Summary

152

Tables 154

Figure Captions

156

Figures

\section{CHAPTER 5}

AN INTERPRETATION OF THE $Q^{-1}$ STRUCTURE

Introduction

The Near-Surface Q Structure

The Axial Low-Q Region

Laboratory Determinations of $Q$ at High Temperatures in Mafic Rocks

Constraints on the Temperature and Melt Concentration Beneath the Axial Magma Chamber

Conclusions

Figure Captions

Figures

APPENDIX A

THE INSTRUMENT RESPONSES

Introduction 247

Analog Ocean Bottom Hydrophone 247

Introduction $\quad 247$

The Low-Frequency Low-Gain Channel 248

Response Tests 250

Testing Configuration 250

Sine Wave Tests 252

Square Wave Tests 255

Uncertainties 257

Reproducibility 259

Calibration Pulses and Absolute Amplitudes 260

Conclusions 263 
Digital Ocean Bottom Hydrophone 263

Introduction 263

Theoretical Response 264

Calibration Tests 266

Conclusions 268

Digital Ocean Bottom Seismometer 268

Tables $\quad 270$

Figure Captions 279

Figures 284

APPENDIX B

THE SPECTRAL ACCURACY OF THE FINITE-DIFFERENCE SOLUTIONS

Numerical Stability

311

Comparisons of Finite-Difference and Reflectivity Solutions

313

Figure Captions

Figures

REFERENCES 


\section{ChAPTER 1}

\section{PRINCIPles OF ATTENUATION TOMOGRAPHY}

\section{INTRODUCTION}

Because seismic wave propagation is affected by the composition, temperature, and porosity of the medium, and by the presence of partial melt, measurements of the seismic properties of oceanic lithosphere have played an important role in developing and refining our understanding of processes by which oceanic crust and upper mantle are generated and evolve. To this end, numerous measurements have been made of seismic velocities. In contrast, while workers have occasionally noted the effects of seismic attenuation on wave amplitudes [e.g., Orcutt et al., 1976; Reid et al., 1977; Spudich and Orcutt, 1980a; Vera et al., 1990], comparatively few studies have been devoted to measuring the attenuative properties of young oceanic crust [Lewis and Jung, 1989; Jacobson and Lewis, 1990; Wepfer and Christensen, 1990, 1991]. In January 1988 a tomographic experiment (Figure 1.1) was conducted at $9^{\circ} 30^{\prime} \mathrm{N}$ on the East Pacific Rise (EPR). A three-dimensional delay time tomographic image of the P-wave velocity structure (Figure 1.2) was obtained by Toomey et al. [1990a]. This model is complemented by a two-dimensional structure (Figure 1.3) derived by interpolating between the results of several expanding spread profiles [Vera et al., 1990]. Near the seafloor, the models are characterized by increased velocities along the rise axis. In contrast, at $2 \mathrm{~km}$ depth beneath the rise axis, the inferred location of a small axial magma body [Herron et al., 1978, 1980; Hale et al., 1982; Detrick et al., 1987; Mutter et al.; 1988; Kent et al., 1990; Vera et al., 1990], anomalously low velocities are observed. Significant along-axis variations in the magnitude of the low velocity anomaly in the delay time tomographic model are interpreted in terms of a thermally segmented rise axis [Toomey et al., 1990a]. This thesis is concerned with the measurement of seismic attenuation using the tomographic data set. The primary objective is to obtain and interpret a model of crustal P-wave anelasticity that is comparable in scale and resolution to the P-wave velocity models [Vera et al., 1990; Toomey et al., 1990a].

The remainder of Chapter 1 is divided into six sections which provide the background to the techniques used in this thesis. The first is a brief overview of the definition of $Q$ and its importance in seismology. The second reviews the theory and 
assumptions of the spectral method of attenuation tomography. The third describes the configuration of the EPR tomography experiment. The next two sections discuss the methods by which spectral measurements are used to obtain estimates of $t^{*}$, an integrated measure of attenuation along a wave path. The final section discusses how estimates of $t^{*}$ are inverted to obtain spatial models of crustal anelasticity parameterized in terms of the reciprocal of the quality factor $\mathrm{Q}$.

The following three chapters discuss specific aspects of this study. Chapter 2 deals with the study of three rise-parallel seismic refraction lines located along the rise axis and $20 \mathrm{~km}$ to the east and west. Chapter 3 discusses the use of a fullwaveform finite-difference method, applied to a two-dimensional velocity model of the EPR, to evaluate the attenuation tomography method. Chapter 4 describes attempts to invert the full $t^{*}$ data set to obtain two and three-dimensional images of crustal attenuation. The final chapter, Chapter 5 attempts to interpret the results of this study and discusses how they contribute to our understanding of the generation and early evolution of oceanic crust along the EPR.

\section{SEISMIC ATTENUATION}

The dissipation of energy (by internal heating) within an anelastic material under periodic stress is described in terms of the quality factor $\mathrm{Q}$. $\mathrm{Q}$ is a measure of the fractional energy lost per cycle and is most unambiguously defined [ $O^{\prime}$ Connell and Budiansky, 1978]:

$$
Q(f)=\frac{4 \pi \bar{V}(f)}{\Delta \phi}
$$

where $f$ is the frequency, $\bar{V}$ is the average stored energy per cycle, $\Delta \phi$ is the energy dissipated per cycle. For a material that can be modelled as a network of linear springs and dashpots this definition is equal to the ratio of the magnitude of the real and complex components of the appropriate modulus [O'Connell and Budiansky, 1978]. If $Q \gg>1$, equation (1.1) leads straightforwardly to an expression for the amplitude decay due to anelasticity [Aki and Richards, 1981] in a homogeneous medium

$$
\frac{A(t)}{A(0)}=\exp \left[\frac{-\pi f t}{Q}\right]
$$

where $\mathrm{A}$ is the amplitude of a harmonic wave at time t. Attenuation is of importance to seismologists not only because of its effect upon seismic amplitudes but also 
because causality requires that it be accompanied by dispersion [e.g., Futterman, 1962; Azimi et al., 1968; Liu et al., 1976].

Seismic observations of $\mathrm{Q}$ range from below $10^{-3} \mathrm{~Hz}$ to over $10^{2} \mathrm{~Hz}$ while laboratory measurements are often obtained at substantially higher frequencies. The degree of attenuation of $\mathrm{P}$ and $\mathrm{S}$ waves is frequently observed to be strongly correlated, with levels of $S$ wave attenuation markedly higher. Assuming that all dissipation occurs in shear rather than compression results in a ratio $\mathrm{Q}_{\mathrm{P}} / \mathrm{Q}_{\mathrm{S}}=\mathbf{2 . 2 5}$ for a Poisson solid [Anderson, 1967], a result in good agreement with many observations [e.g., Cormier, 1982]. Early workers concluded that $\mathrm{Q}$ was substantially independent of frequency [Knopoff, 1964, Anderson, 1967]. However, when observations are made over a large bandwidth there is strong evidence for a frequency dependent $\mathrm{Q}$ at certain frequencies both within the mantle [e.g., Gutenberg, 1958; Kurita, 1968; Archambeau et al., 1969; Solomon, 1972; Sipkin and Jordan, 1979] and the lithosphere [Frantti, 1965; Aki and Chouet, 1975; Aki, 1980; Jacobson, 1987]. The mantle observations can be fit with an absorption band model [Lundquist and Cormier, 1980; Anderson and Given 1982], which specifies a depthdependent frequency interval within which $Q$ is only weakly dependent upon frequency $(\mathrm{Q} \sim \mathrm{f} 0.15)$ but outside which $\mathrm{Q}$ rapidly increases. The lithospheric data are more difficult to model because of the inability to distinguish intrinsic attenuation from the effects of scattering [e.g., Cormier, 1980] which may be important at shallow depths [e.g., Toksöz et al., 1988]. However, when studies are confined to narrow frequency bands it is still usual to assume a constant $Q$.

Many mechanisms may contribute to attenuation [Jackson and Anderson, 1970], and not all processes are well understood. At seismic frequencies dissipation may occur at grain boundaries, at lattice defects, along microcracks, and through fluid flow. Many mechanisms satisfy the properties of a standard linear solid and are characterized by a relaxation time which determines the frequency of maximum $\mathrm{Q}^{-1}$, below and above which $\mathrm{Q}^{-1}$ decreases linearly with the frequency and the reciprocal of the frequency, respectively [Zener, 1948; Jackson and Anderson, 1970]. A broad band of near constant $Q$ can result from the combined effects of a number of different mechanisms with different relaxation times [Liu et al., 1976] or from a single mechanism with a distribution of relaxation times [Kanamori and Anderson, 1977].

Regional attenuation studies in both continental regions [e.g., Asada and Takano, 1963; Sutton et al., 1967; Sacks, 1969; Molnar and Oliver, 1969; Solomon and Toksöz, 1970] and along mid-ocean ridges [Molnar and Oliver, 1969; Solomon 
1973; Sheehan and Solomon, 1991] suggest that volcanic regions are characterized by high levels of attenuation. High-temperature laboratory experiments at both atmospheric [Woirgard and Gueguen, 1978; Berckhemer et al., 1979, 1982; Sacks and Murase, 1983; Kampfmann and Berckhemer, 1985] and elevated pressures [Sato et al., 1988, 1989; Jackson et al., 1992] confirm that such observations are a manifestation of elevated temperatures and possibly partial melt. A large number of local studies of attenuation have also been conducted in volcanically and hydrothermally active areas. Many of the early studies [e.g., Matumoto, 1971; Einarsson, 1978; Latter, 1981; Ryall and Ryall, 1981; Sanders, 1984; Kobayashi et al., 1986; Sanders et al., 1988] employed qualitative estimates of attenuation to locate highly attenuating regions. More recently workers have employed tomographic techniques [Young and Ward, 1980; Hashida and Shimazaki, 1987; Evans and Zucca, 1988; Hashida et al., 1988; Ho-Liu et al., 1988, 1990; Clawson et al., 1989;

Scherbaum and Wyss, 1990] to image low- $\mathrm{Q}$ volumes. However, there is a marked absence of such studies near mid-ocean ridges.

Indeed, while there have been several studies of attenuation in deep sea sediments [e.g., Hamilton, 1976, Mitchell and Focke, 1980; Stoll and Houtz, 1983; Jacobson et al., 1981, 1984; Jacobson, 1987], observations of attenuation near midocean ridges have been limited. From a comparison of the amplitudes of microearthquakes recorded on ocean-bottom instruments, Reid et al. [1977] inferred the presence of a narrow zone of high attenuation coincident with the ridge crest at $21^{\circ} \mathrm{N}$ on the East Pacific Rise. Direct measurements of $\mathrm{Q}$ in the upper $600 \mathrm{~m}$ of 0.4 My old crust near the Juan de Fuca Ridge [Jacobson and Lewis, 1990] show values of 20-50, in good agreement with ultrasonic laboratory measurements of $\mathrm{Q}$ in oceanic basalts [Wepfer and Christensen, 1990]. A model of oceanic Q structure based on laboratory measurements of ophiolite samples at $1 \mathrm{MHz}$ [Wepfer and Christensen, 1991] includes $Q$ values of about 50 near the surface and in the gabbros which form the lower crust. Reflectivity models of wave amplitudes on expanding spread profiles at $9^{\circ} 30^{\prime} \mathrm{N}$ on the EPR [Vera et al., 1990] include $Q$ values beneath 100 near the seafloor and in the vicinity of a 1.6-km-deep magma lens under the rise axis.

\section{A SPECTRAL METHOD FOR ATTENUATION TOMOGRAPHY}

There are a number of different methods to measure the attenuation of seismic body waves [e.g., Cormier, 1980; Tonn, 1989]. The methods can be divided into two categories depending upon whether the measurements are obtained in the time or 
frequency domain. Most time domain methods suffer from the disadvantage that the velocity structure must be accurately known. Simple measurements of peak or averaged amplitude require an accurate correction for wave divergence. More sophisticated waveform modeling techniques require, in addition, reliable calculations of synthetic waveforms. The rise time technique [Gladwin and Stacey, 1974] does not require a good knowledge of the seismic velocity structure, but it may be difficult to implement when waveforms are emergent or have complex shapes or when the signal to noise ratio is low. In contrast, the spectral method does not require a knowledge of the velocity structure, and while certain assumptions are made about the nature of seismic propagation, it can be applied to emergent waveforms with fairly low signal to noise ratios. For this reason we use a spectral technique.

In the time domain a seismic record $\mathrm{x}(\mathrm{t})$ can be described by a convolution

$$
\mathrm{x}(\mathrm{t})=\mathrm{s}(\mathrm{t}) * \mathrm{c}(\mathrm{t}) * \mathrm{i}(\mathrm{t})
$$

where $s$ is the source signature, $c$ is the crustal transfer function, and $i$ the instrument response. Thus, in the frequency domain the power spectrum of the crustal transfer function $\mathrm{C}$ can be obtained from the recorded power spectrum $\mathrm{P}$ by dividing out the source signature $\mathrm{S}$ and the instrument response I

$$
C(f)=\frac{P(f)}{S(f) I(f)}
$$

In many studies either the source signature or instrument response is not sufficiently well known to apply equation (1.4), and a spectral ratio technique [e.g., Teng, 1968] must be employed to obtain estimates of differential attenuation. However, for this study this is not the case and absolute estimates of attenuation are obtainable.

Spectral estimates of attenuation rely on two assumptions. First, it is assumed that by judicious choice of the time interval used for spectral measurements the crustal transfer function can be estimated for a single phase. Second it is assumed that the dominant frequency-dependent component of the crustal transfer function is attenuation, so that for a phase that propagates along a path $\mathbf{s}, \mathrm{C}$ can be described by

$$
C(f, s)=G(s) \exp [-2 \pi f t *(s)]
$$

where $G$ is a frequency-independent term to account for divergence, the exponential term is the attenuation derived from equation (1.2), and $t^{*}$ is defined as an integral of slowness and $\mathrm{Q}^{-1}$ along the wave path 


$$
\mathrm{t}^{*}=\int_{\mathrm{S}} \frac{\mathrm{ds}}{\mathrm{QV}}
$$

An estimate of $t^{*}$ can be obtained by fitting a straight line to a plot of $\ln (\mathrm{C})$ against frequency

$$
\frac{\mathrm{d} \ln [\mathrm{C}(\mathrm{f})]}{\mathrm{df}}=-2 \pi \mathrm{t}^{*}
$$

In practice, non attenuative components of seismic propagation, including scattering, short-path multiples, and focusing, may result in a frequency dependent $\mathrm{G}$. Thus $\mathrm{t}^{*}$ estimates obtained by this method will be measurements of apparent rather than intrinsic attenuation [e.g., Cormier, 1980]. If the velocity structure and wave paths are known, equation (1.6) can be used as a basis for a linear inversion to obtain a $\mathrm{Q}^{-1}$ model from $\mathrm{t}^{*}$ measurements.

\section{TOMOGRAPHY EXPERIMENT}

The three-dimensional tomography experiment [Toomey et al., 1990a] was conducted between $9^{\circ}$ and $10^{\circ} \mathrm{N}$ on the EPR in January 1988. The configuration of the experiment (Figure 1.1) was designed on the basis of synthetic modelling [Toomey, 1987] to determine the P-wave velocity structure within a $16 \times 16 \mathrm{~km}$ area of crust centered upon the rise axis. A total of 15 ocean bottom instruments, comprising 8 Woods Hole Oceanographic Institution (WHOI) analogue ocean bottom hydrophones (AOBHs) [Koelsch and Purdy, 1979], 5 WHOI digital ocean bottom hydrophones (DOBHs) [Koelsch et al., 1982], and 2 Massachusetts Institute of Technology (MIT) ocean bottom seismometers (OBSs) [Mattaboni and Solomon, 1977; Duschenes et al., 1981; Tréhu, 1982], were deployed in a regular pattern at distances of 0,9 , and $20 \mathrm{~km}$ to the east and west of the rise axis. A total of 480 controlled explosive shots were fired in a regular pattern using Global Positioning System navigation. The majority were located within the central $18 \times 16$ $\mathrm{km}$ area of the experiment with a nominal shot spacing of 0.5 or $1 \mathrm{~km}$. In addition three 45-50-km-long rise-parallel shot lines with a 1-km shot spacing were obtained on the rise axis and at $20 \mathrm{~km}$ to either side of the rise axis. With the exception of a few tests and misfires, all the shots were of uniform size $(54.5 \mathrm{~kg})$ and construction and were composed of either $\mathrm{C} 4$ or HBX explosives. A fixed shot depth was maintained by floating the charges.

Considerable effort was devoted to determining the instrument responses; details are presented in Appendix A. Figure 1.4 shows the response of the three 
instruments used in the experiment. Both the DOBH and OBS are digital instruments for which a theoretical response can be calculated. The DOBH has a good response over a broad frequency band $(5-80 \mathrm{~Hz})$. In contrast the bandwidth of the OBS is less $(3-30 \mathrm{~Hz})$, which limits the accuracy of attenuation estimates. Moreover, OBS 1 was clearly poorly coupled to the seafloor during the experiment, making accurate spectral estimates impossible [e.g., Sutton et al., 1981]. The response of the AOBHs had to be measured and shows significant variations between instruments, with the effective bandwidth varying from $5-35 \mathrm{~Hz}$ to $5-60 \mathrm{~Hz}$. Since the calibrations were obtained more than one year after the deployment, it by no means certain that the measured responses were those during the experiment. At ranges less than $10-15 \mathrm{~km}$ the analogue tape drives saturate, and accurate spectral measurements are not possible. AOBH 8 malfunctioned during the experiment and did not record waveforms suitable for spectral analysis.

The source signature for this experiment was measured in a separate source monitoring experiment. A DOBH with $40 \mathrm{~dB}$ of attenuation added to the preamplifier (Appendix A) was moored $1000 \mathrm{~m}$ above the seafloor in $2850 \mathrm{~m}$ of water, and four charges, two composed of $\mathrm{C} 4$ and two of $\mathrm{HBX}$, were detonated directly above the instrument. The recorded pressure signal (Figure 1.5a) is dominated by the primary explosion and the first bubble pulse and their sea-surface reflections. The nominal shot depth was $69 \mathrm{~m}$ for $\mathrm{C} 4$ and $75 \mathrm{~m}$ for HBX shots. However, the timing of the surface reflections suggests that the actual values are $20 \%$ higher. This discrepancy probably results from a systematic error throughout the experiment in measuring the cord used to suspend the charges from the float. A puzzling feature of all four source monitoring shots is that the amplitude of the primary explosion is $10-15 \%$ lower than its surface reflection. Within a homogeneous water column the effects of spherical spreading should produce an amplitude that is about $10 \%$ higher. Moreover the decrease in seismic velocities with depth within the thermocline [Carter, 1980] should decrease the reflected amplitudes further. The only feasible explanation seems to be source directivity. Because this is observed for all test shots and all shots were constructed in an identical manner, this effect was presumably present throughout the tomography experiment.

Figure $1.5 \mathrm{~b}$ shows the recorded pressure signal after applying attenuation using a Azimi's attenuation formula [Azimi et al., 1968; Aki and Richards, 1980] assuming $t^{*}=0.04$, a value similar to the mean $t^{*}$ observed during the experiment. As well as decreasing the relative high frequency content of the waveform, attenuation reduces 
the relative amplitude of the primary pulse and offsets the highest amplitudes to later times. The highest amplitudes occur about $0.2 \mathrm{~s}$ and $0.4 \mathrm{~s}$ after the onset of the waveform. $t^{*}$ estimates obtained from time windows long enough to include the high amplitude portions of the attenuated waveform are likely to be more robust.

Since the bubble pulse time varies slightly between shots and the timing of the surface reflection depends on the take off angle, an attempt was made to deconvolve the instrument response from the source signature. Figures $1.4 \mathrm{c}$ and $\mathrm{d}$ show a source signature model obtained by a constrained frequency domain deconvolution. The time series for the initial explosion is indistinguishable from the impulse response of the DOBH and can be represented by a delta function for the frequency band $5-80 \mathrm{~Hz}$. To model the observed decrease in spectral power with frequency, both the surface reflection of the primary explosion and the first bubble pulse must have a resolvable width. A symmetric bubble pulse with an exponential rise and fall time constant of $\sim 0.005 \mathrm{~s}$ [Helmberger, 1968] fits the data reasonably well. A comparison of the recorded power spectrum corrected for the instrument response and the power spectrum of the deconvolved source signature shows excellent agreement between the location of notches at all but the highest frequencies. The overall decay in power is not fully modelled, though the fit is quite good between 10 and $50 \mathrm{~Hz}$. This discrepancy may be due to a slight underestimation of the first bubble pulse time constant, the failure to include the effects of higher order bubble pulses, or the complexity of underwater explosions [e.g., Holt, 1977].

\section{POWER SPECTRUM ESTIMATION}

There is a considerable body of work devoted to the problem of estimating the power spectra of short time series, the majority of which is located in the electrical engineering literature and is concerned primarily with stationary processes. In this section we will discuss how such methods can be applied to the estimation of power spectra for seismic waveforms.

The direct spectral estimate of the power spectrum $\hat{P}$ of the function $x(t)$ over a time interval 0 to $\mathrm{T}$ is defined as

$$
\widehat{P}(f)=\left|\int_{0}^{T} x(t) w(t) \exp (i 2 \pi f t) d t\right|^{2}
$$

where $w(t)$ is the data window or taper and is normalized according to 


$$
\int_{0}^{T} w^{2}(t) d t=1
$$

It can be shown [e.g., Thomson, 1977] that the expected value of $\widehat{P}$ is a convolution of the true power spectrum $\mathrm{P}$ with the power spectrum of the windowing function $\mathrm{W}$

$$
<\widehat{\mathrm{P}}(\mathrm{f})>=\mathrm{P}(\mathrm{f}) * \mathrm{~W}(\mathrm{f})
$$

Since the window length is finite, $\mathrm{W}$ cannot be a delta function and the estimate is necessarily biased. Examples of two simple windowing functions and their power spectra are shown in Figure 1.6. If $w$ is chosen to be constant (a rectangular or boxcar window), $\hat{\mathrm{P}}$ is the periodogram. In this case the halfwidth of the central lobe of the power spectrum, which determines the spectral resolution, has the minimum achievable value of $1 / \mathrm{T}$. However, the side lobes of the window power spectrum have large amplitudes. In the event that the data do not smoothly approach zero at the window limits, spectral leakage will severely contaminate the spectral estimates and produce large biases. To overcome this problem it is generally necessary to use a tapered window. The cosine squared (Hanning) window (Figure 1.6) is commonly used in seismology but is only one of a large number of tapered windowing functions [Harris, 1978]. Tapered windows result in a decrease in the amplitude of side lobes at the expense of a broader central lobe. Thus the bias from spectral leakage is reduced at the expense of spectral resolution. The optimal choice of windowing function depends on the nature of the signal to be analyzed. If it is necessary to resolve features that are closely spaced in the frequency domain then a narrow central lobe is required, whereas if the spectrum is smooth and includes large power variations then side lobe suppression will be more important.

Since attenuation increases exponentially with frequency, strongly attenuated seismic waveforms might be expected to show large variations in spectral power over a significant frequency band. Moreover, considering solely the effects of attenuation, the spectral power will decrease smoothly with frequency. For these reasons a windowing function exhibiting high side-lobe suppression is preferable for attenuation studies. However, there are two drawbacks to using such windows. First, the low window amplitudes at the start and end of the window discard a significant amount of statistical information on the spectral content of the time series and increase the variance of spectral estimates. Second, since seismic waveforms are non-stationary, the unequal weighting within the window may bias the results in 
a manner that is sensitive to the precise position of the window with respect to the waveform.

This problem may be overcome partially using the method of overlapping windows [Welch, 1967]. A series of equal-length data segments are chosen, each of which overlaps adjacent segments by a significant amount (typically 50\%). Spectral estimates are obtained from each segment after the application of a suitable windowing function, and the normalized sum of these measurements is chosen as the final estimate. By more evenly weighting the time series such methods can produce a significant variance reduction over single-window estimates which have been smoothed by means of a running average to yield the same frequency sampling interval. However, sub-dividing short time series may produce an unsatisfactorily large frequency sampling interval. Moreover, while the weighting of the time series is more even, it is far from uniform, and biases may still remain.

A better method, and the one that is used in this study, is the multiple-window spectral analysis (MWSA) technique [Thomson, 1982; Park et al., 1987a, b; Zhu et al., 1989]. The method is based upon the desire to seek windows which minimize spectral leakage by maximizing the fractional energy $\lambda$ within an estimation bandwidth of halfwidth $\Delta \mathrm{f}$.

$$
\lambda(N, \Delta f)=\frac{\int_{-\Delta f}^{\Delta f}|W(f)|^{2} d f}{\int_{-N / 2 T}^{N / 2 T}|W(f)|^{2} d f}
$$

where $\mathrm{N}$ is the number of samples in the time series. Equation (1.11) may be written in a discrete form [Thomson, 1982; Park et al., 1987a]

$$
\lambda(\mathbf{N}, \Delta \mathrm{f})=\frac{\mathbf{w}^{\mathrm{T}} \mathbf{A} \mathbf{w}}{\mathbf{w}^{\mathrm{T}} \mathbf{w}}
$$

where $\mathbf{w}$ is the windowing function, $\mathbf{w}^{\mathrm{T}}$ denotes the transpose of $\mathbf{w}$, and $\mathbf{A}$ is defined by

$$
A_{k l}=\frac{\sin [2(k-1) \pi T \Delta f]}{\pi(k-1)}
$$

It is straightforward to show [Thomson, 1982; Park et al., 1987a] that the stationary points of the functional are solutions of the eigenvalue problem

$$
\mathbf{A} \mathbf{w}-\lambda(\mathrm{N}, \Delta \mathrm{f}) \mathbf{w}=0
$$


The set of ordered eigenvalues $1>\lambda_{0}>\lambda_{1}>\lambda_{2}>\ldots>\lambda_{\mathrm{N}-2}>\lambda_{\mathrm{N}-1}>0$ have eigenvectors $w^{(k)}(N, \Delta f)$ which are termed discrete prolate spheroidal sequences, or prolate eigentapers. The prolate eigentaper with the quantity $\mathrm{M}=\mathrm{N} \mathrm{T} \Delta \mathrm{f}$ is termed an $\mathrm{M} \pi$ prolate taper. Low-order tapers for the $2 \pi$ and $4 \pi$ prolate sequences and their power spectra are shown in Figure 1.7. The lowest order taper [Thomson, 1977] is similar in shape to other conventional tapers. However, the higher order tapers are oscillatory and exhibit $\mathrm{k}$ zero crossings where $\mathrm{k}$ is the order of the taper. The first 2M-1 tapers have eigenvalues that are close to unity (Table 1.1) and hence are suitable for spectral analysis.

To obtain the multiple-window spectral estimate the discrete Fourier transform of the data is calculated after windowing with each of the first $2 \mathrm{M}-1$ tapers

$$
\mathrm{X}_{\mathrm{k}}(\mathrm{f})=\sum_{\mathrm{t}=0}^{\mathrm{N}-1} \mathrm{w}_{\mathrm{l}}^{(\mathrm{k})} \mathrm{x}_{1} \exp (\mathrm{i} 2 \pi \mathrm{ft})
$$

An estimate of the spectrum is given by

$$
\hat{\mathrm{P}}(\mathrm{f})=\sum_{\mathrm{k}=0}^{\mathrm{M}-1} \frac{\left|\mathrm{X}_{\mathrm{k}}(\mathrm{f})\right|^{2}}{\mathrm{M} \lambda_{\mathrm{k}}}
$$

Figure 1.8 shows the effective window amplitude of such estimates; the amplitude can be seen to be fairly uniform away from the window limits. However, a better estimate may be obtained using an adaptive method [Thomson, 1982]. Such a method recognizes that if the spectrum is highly colored, spectral leakage will be a serious problem at frequencies with low spectral power. Since the leakage characteristics are much better for the lowest order windows, they should be weighted more highly at such frequencies. By making the approximation that $|\mathrm{W}|$ is constant outside the estimation bandwidth, the spectral estimate with the minimum variance can be approximated

$$
\hat{P}(f)=\frac{\sum_{k=0}^{M-1}\left|d_{k}(f) X_{k}(f)\right|^{2}}{\sum_{k=0}^{M-1}\left|d_{k}(f)\right|^{2}}
$$

where $d_{k}(f)$ are frequency dependent weights given by

$$
d_{k}(f)=\frac{\sqrt{\lambda_{k}} P(f)}{\lambda_{k} P(f)+\left(1-\lambda_{k}\right) P(f) \sum_{l=1}^{N-1} x_{l}^{2}}
$$


Since $d_{k}$ depend upon the the spectral power ratio, which is unknown, an iterative procedure is employed to obtain the spectral estimate. $P$ in equation (1.18) is replaced by $\widehat{P}$, and the initial value $\widehat{P}=\left|X_{0}(f)\right|^{2}$ is used. Such a method converges in a few iterations for all time series considered in this study.

\section{SOURCE-RECEIVER CORRECTIONS AND T* ESTIMATES}

Once an estimate of the power spectrum is obtained a correction for the source signature and instrument response must be made as outlined in equation (1.4). However, since MWSA estimates have low frequency resolution, careful consideration must be paid as to how best to calculate this correction. If it is assumed that spectral leakage is negligible, the expected value of an MWSA spectral estimate can be approximated using equation (1.10)

$$
\left\langle\widehat{P}_{i}\right\rangle=\sum_{j=i-M+1}^{i+M-1} P_{j} V_{i-j}^{(i)}
$$

where $V$ is the effective central lobe power distribution

$$
V_{j}^{(i)}=\frac{\sum_{k=0}^{2 M-1}\left|d_{k}\left(f_{i}\right) w_{k}\left(f_{i-j}\right)\right|^{2}}{\sum_{k=0}^{2 M-1}\left|d_{k}\left(f_{i}\right)\right|^{2}}
$$

For all estimates $\mathrm{V}$ will lie between that of the lowest order prolate taper (Figure 1.7) and that resulting from evenly weighting the first $M-1$ tapers (Figure 1.8b). The expected value of an estimate of the power spectrum of the crustal transfer function obtained directly from equation (1.4) is thus

$$
<\widehat{C}_{i}>=\frac{\sum_{j=i-M+1}^{i+M-1} S_{j} C_{j} I_{j} V_{i-j}^{(i)}}{S_{j} I_{j}}
$$

Such an estimate will generally be biased if one or more of S, C, or I are not constant within the estimation bandwidth which runs from $\mathrm{f}_{\mathrm{i}-\mathrm{M}+1}$ to $\mathrm{f}_{\mathrm{i}+\mathrm{M}-1}$. If the product of the source signature and instrument response power spectra are first convolved with the effective MWSA window power spectrum (equation 1.20), then $\langle\hat{\mathrm{C}}\rangle$ may be approximated 


$$
\langle\widehat{C}\rangle=\frac{\sum_{j=i-M+1}^{i+M-1} S_{j} C_{j} I_{j} V_{i-j}^{(i)}}{\sum_{j=i-M+1}^{i+M-1} S_{j} I_{j} V_{i-j}^{(i)}} .
$$

The estimate will be unbiased if $C$ is constant within the estimation bandwidth, but for an attenuated phase this clearly will not be the case. If both $\mathrm{S}$ and I are constant within the estimation bandwidth and $C$ is described correctly by equation (1.5), the systematic bias in $\left\langle\hat{\mathrm{C}}>\right.$ will be a constant factor and the estimate of $\mathrm{t}^{*}$ obtained from the spectral slope according to equation (1.7) will be unaffected. However, when $S$ and $I$ are not constant within the estimation bandwidth, the estimate will generally be biased.

Inspection of Figures 3 and 4 shows that $S$ and I are never constant within any significant bandwidth. The instrument response may be fairly constant within a passband but decreases rapidly outside, particularly at low frequencies. The source signature shows rapid variations at all frequencies. The expected value of a $t^{*}$ estimate $\left\langle\mathrm{t}^{*}\right\rangle$ can be calculated for a given $\mathrm{t}^{*}, \mathrm{M}$, and frequency interval from equations (1.5) and (1.13) together with the correct source signature and instrument responses. Figure 1.9 shows an example of such a calculation for $t^{*}$ estimates made for AOBH 1 over the frequency interval $10-40 \mathrm{~Hz}$ using $2 \pi$ and $4 \pi$ prolate MWSA. For a data window much shorter than $0.3 \mathrm{~s}$, the $4 \pi$ prolate MWSA estimates (Figure 1.9b) are significantly biased towards low values, while for longer windows there is a slight bias toward higher values. Such biases result primarily from the rapid changes in instrument response at low frequencies and may be reduced if the lower frequency bound is increased. However, for highly attenuated waves for which the available bandwidth for $\mathrm{t}^{*}$ estimates is small, such an increase may significantly increase the uncertainty of spectral slope estimates.

Biases may also be reduced for a given time interval by decreasing M. Figure 1.9a shows that for $2 \pi$ prolate MWSA, large biases are limited to window lengths less than $0.15 \mathrm{~s}$. However, decreasing $M$ increases the variance of the spectral estimates and hence $t^{*}$ values, an effect whose importance must be assessed using real data. Moreover, decreasing $M$ may also increase source biases. The source signature (Figure 1.5) displays a series of power spectral notches the location of which will be significantly affected by small errors in the shot depth, take off angle, and bubble pulse time. Low-resolution spectral estimates reduce the importance of such uncertainties since the spectrum is smoothed and the notches are not resolved. 
If the spectral resolution is too high, misplaced notches in the source signature may result in an inaccurate source correction, introducing a potentially large error to $t^{*}$ estimates. Such an effect is investigated in Figure 1.9 by using an incorrect source term in the denominator of equation (1.22). While the resulting errors are clearly larger for the $2 \pi$ prolate window, they are surprisingly small and do not exceed $5 \%$ for this example.

In this work the source-receiver correction is calculated for the $\mathrm{k}^{\text {th }}$ receiver and the $1^{\text {th }}$ shot according to

$$
\widehat{\mathrm{C}}_{\mathrm{i}}=\frac{\widehat{\mathrm{P}}_{\mathrm{i}}^{(\mathrm{MWSA})}}{\mathrm{Y}_{\mathrm{i}}}
$$

where $\mathrm{Y}$ is the source-receiver correction and is calculated for the $\mathrm{k}^{\text {th }}$ receiver and the $1^{\text {th }}$ shot according to

$$
Y_{i}=\sum_{j=i-M+1}^{j=i+M-1} \frac{V_{i-j} I_{j}^{(k)} R_{j}^{(0)} D_{j}^{(l)}}{I_{j}^{(0)} D_{j}^{(0)}}
$$

where $I^{(k)}$ is the response of the $k^{\text {th }}$ instrument, $I^{(0)}$ is the response of the DOBH used in the source monitoring experiment, $R^{(0)}$ is the power spectrum of the recording obtained during the source monitoring experiment (Figure $1.5 \mathrm{a}$ ), $\mathrm{D}(0)$ is the power spectrum of the deconvolved model of the source monitoring source signature (Figure $1.5 \mathrm{~b}$ ), and $\mathrm{D}^{(1)}$ is the model of the $1^{\text {th }}$ shot incorporating estimates of the shot depth, take-off angle, and bubble pulse period. While the estimate of $\mathrm{D}(\mathrm{l})$ may have considerable uncertainties, it can be shown that provided the frequency

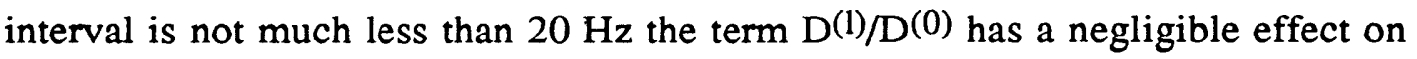
estimates of $t^{*}$.

Estimates of $t^{*}$ are obtained according to equation (1.7) using a least squares straight line fit to a plot of the natural logarithm of crustal transfer power spectrum against frequency. The minimum frequency considered is normally $10 \mathrm{~Hz}$. The maximum frequency is determined by either the upper limit of the instrument response or the frequency at which the power spectrum of a noise sample obtained immediately prior to the waveform is comparable to that of the waveform. An estimate of the uncertainty in $t^{*}$ values can also be obtained from a least squares fit provided that an uncertainty can be ascribed to $\ln (\widehat{\mathrm{C}})$ values. One approach is to obtain such estimates from the noise sample. However, such a method results in unrealistically small estimates of the $t^{*}$ uncertainty since it assumes that $\widehat{C}$ is perfectly described by equation (1.7) except for the presence of noise. A more 
realistic estimate of the $\ln (\widehat{C})$ uncertainty may be obtained from the misfit of the straight line fit

$$
\sigma_{\ln (\mathrm{C})}^{2}=\sum_{\mathrm{i}=1}^{\mathrm{N}} \frac{\left[\ln \left(\mathrm{C}_{\mathrm{i}}\right)-\ln \left(\mathrm{C}_{\mathrm{i}}^{\mathrm{LS}}\right)\right]^{2}}{\mathrm{~N}-2} \mathrm{~g}(\mathrm{M}, \mathrm{N})
$$

where $\mathrm{N}$ is the number of spectral values, $\mathrm{CLS}$ is the spectrum predicted by the least squares fit, and $\mathrm{g}$ is a factor to account for the smoothing effects of the spectral estimator. If it is assumed that all the misfit results from random variations in spectral amplitudes then approximating the effective central lobe power distribution of the MWSA estimate by a boxcar and considering the covariance of adjacent spectral estimates yields an approximate value of $g$

$$
g(M, N)=\frac{N M^{2}-2 \sum_{i=1}^{M-1} i(M-i)}{N M}
$$

which can be approximated $\mathrm{g} \sim 2 \mathrm{M}-1$ when $\mathrm{N}$ is large. However, in practice much of the misfit may arise from spectral uncertainties that are correlated within the estimation bandwidth. In this case equation (1.26) yields too large a value of $g$ and therefore overly pessimistic uncertainties in $t^{*}$.

In this work we use $t^{*}$ uncertainties obtained from equations (1.25) and (1.26), except that for waveforms for which the least squares fit yields a value of $\sigma_{\ln (C)}$ smaller than the average for a particular instrument, an average value for all waveforms recorded by the instrument is used. Such uncertainties are best thought of as relative rather than absolute and can be scaled on the basis of the fit of $\mathrm{Q}^{-1}$ models to obtain an estimate of the absolute, non-systematic uncertainties in $t^{*}$.

\section{A METHOD OF Q TOMOGRAPHY}

Equation (1.6) may be written in a discrete form

$$
\mathrm{t}^{*}=\sum_{\mathrm{k}=1}^{\mathrm{N}} \mathrm{V}\left(\mathbf{x}_{\mathrm{k}}\right)^{-1} \mathrm{Q}\left(\mathbf{x}_{\mathrm{k}}\right)^{-1} \delta \mathrm{S}_{\mathrm{k}}
$$

where the wave path has been split into $\mathrm{N}$ segments of length $\delta \mathrm{S}_{\mathrm{k}}$ with center coordinates $\mathbf{x}_{\mathbf{k}}$. As is the case for the velocity models [Toomey et al., 1990a], $\mathrm{Q}^{-1}$ is parameterized using a nodal representation [Thurber, 1983]. $\mathrm{Q}^{-1}$ values are defined on a Cartesian grid which may be irregular and may be sheared vertically to conform with the seafloor. A smoothly varying model is obtained by linearly interpolating $\mathrm{Q}^{-1}$ values between nodal values at the corners of a grid cell. If the velocity model and 
wave paths derived by delay time tomography or some other means are assumed to be correct, then equation (1.27) yields a linear relationship between a vector $\mathbf{t}$ of $\mathbf{t}^{*}$ values and a vector $\mathbf{q}$ of nodal $\mathrm{Q}^{-1}$ values

$$
\mathbf{t}=\mathbf{G} \mathbf{q}
$$

where $\mathrm{G}_{\mathrm{ij}}$ is the contribution of the $\mathrm{j}^{\text {th }} \mathrm{Q}^{-1}$ node to the $\mathrm{i}^{\text {th }} \mathrm{t}^{*}$ value. There are several methods for directly inverting equation (1.26) to obtain q [e.g., Tarantola, 1987;

Menke, 1989]. Unfortunately, the most straightforward applications of such methods do not include the physically required constraint that $\mathrm{Q}^{-1}$ values be positive. Such constraints may be added to a least squares solution [e.g., Lawson and Hanson, 1974; Menke, 1989], but the algorithm is inefficient when the number of model parameters becomes large. Iterative solutions obtained by the back projection tomography technique [Ho-Liu et al., 1988, 1990] will always yield positive $\mathrm{Q}^{-1}$ values provided all $t^{*}$ estimates are positive. However, back projection tomography does not provide formal error estimates and resolution, though it may be combined with a generalized inverse method to obtain such estimates [Ho-Liu et al., 1989; Trampert et al., 1990].

In this study positivity is achieved at the expense of linearity by parameterizing the model in terms of $\ln \left(\mathrm{Q}^{-1}\right)$. Equation (1.28) may be linearized about a model $\mathbf{q}$

$$
\mathbf{t}=\mathbf{G} \mathbf{q}+\mathbf{A} \delta \mathbf{r}
$$

where $\delta \mathbf{r}$ is the perturbation to the $\ln \left(Q^{-1}\right)$ model $\mathbf{r}$, and $\mathbf{A}$ is related to $\mathbf{G}$ according to

$$
A_{i j}=q_{j} G_{i j}
$$

Such a parameterization has the advantage that it naturally downweights nodes that achieve a high $Q$ value, a reflection of the small contribution such nodes make to $t^{*}$ values. Since, the relationship is no longer linear an iterative solution must be obtained. A least squares approach would seek to minimize the squared data misfit $\chi^{2}$ which is expressed

$$
\chi^{2}=(\mathbf{t}-\mathbf{G} \mathbf{q}-\mathbf{A} \delta \mathbf{r})^{\mathrm{T}} \mathbf{R}_{\mathrm{rr}}^{-1}(\mathbf{t}-\mathbf{G} \mathbf{q}-\mathbf{A} \delta \mathbf{r})
$$

where $\mathbf{R}_{\mathrm{tt}}$ is the data covariance matrix. For uncorrelated $\mathrm{t}^{*}$ estimates $\mathbf{R}_{\mathrm{tt}}$ is related to the uncertainty $\sigma$ in $t^{*}$ by

$$
\left(\mathrm{R}_{\mathrm{tt}}\right)_{\mathrm{ij}}=\delta_{\mathrm{ij}} \sigma_{\mathrm{i}}^{2}
$$

In practice the problem is unlikely to be fully determined, and a direct minimization of equation (1.31) will lead to an unstable solution. It is therefore 
necessary to include a priori constraints in the solution in the form of additional terms in the minimization. In this study two such constraints are included. The first assumes some prior knowledge of the model [Tarantola and Valette, 1982a, b; Tarantola, 1987] expressed in terms of an initial $\ln \left(\mathrm{Q}^{-1}\right)$ model $\mathbf{r}^{(0)}$ and a model covariance $\mathbf{R}_{\mathrm{rr}}$. A solution is sought that minimizes the deviation from the a priori model

$$
v^{2}=\left(\mathbf{r}^{(0)}-\mathbf{r}-\delta \mathbf{r}\right)^{\mathrm{T}} \mathbf{R}_{\mathrm{rr}}^{-1}\left(\mathbf{r}^{(0)}-\mathbf{r}-\delta \mathbf{r}\right)
$$

The second is a smoothing constraint [Tikhonov and Arsenin, 1977] which seeks to minimize the perturbations relative to the $a$ priori model and which can be expressed

$$
\eta^{2}=\left(\mathbf{r}^{(0)}-\mathbf{r}-\delta \mathbf{r}\right)^{\mathrm{T}} \Delta^{\mathrm{T}} \Delta\left(\mathbf{r}^{(0)}-\mathbf{r}-\delta \mathbf{r}\right)
$$

where $\Delta$ is the the model roughness expressed in terms of first differences between adjacent node pairs. For a one-dimensional model with a uniform unit node separation $\Delta$ is simply

$$
\Delta=\left[\begin{array}{ccccccc}
-1 & 1 & & & & & 0 \\
& -1 & 1 & & & & \\
& & \cdots & \ldots & & \\
& & & -1 & 1 & \\
& & & & -1 & 1
\end{array}\right]
$$

In the case that $\mathbf{q}^{(0)}$ is chosen to be constant this smoothing constraint is the smoothest model approach [Tikonov and Arsenin, 1977; Constable et al., 1987]. A similar smoothing constraint may be included in the a priori model constraint by introducing positive off-axis elements into the model covariance matrix [Tarantola and Valette, 1982a] whose magnitude decreases with the node-pair separation according to some functional relationship (a gaussian function is commonly used). However, once off diagonal elements are introduced into $R_{\mathrm{rr}}$, the inverse is no longer trivial to calculate, and so for computational reasons the two constraints are separated.

The full solution is therefore expressed as a combined minimization of

$$
\chi^{2}+a v^{2}+b \eta^{2}
$$

where $\mathrm{a}$ and $\mathrm{b}$ are weights (which may be zero) ascribed to the smoothing constraints. An iterative solution is given by [e.g., Tarantola, 1987]

$$
\begin{gathered}
\mathbf{r}^{\mathrm{k}+1}=\mathbf{r}^{\mathrm{k}}+\delta \mathbf{r} \\
\delta \mathbf{r}=\left[\mathrm{a} \mathbf{R}_{\mathrm{rt}}^{-1}+\mathrm{b} \Delta^{\mathrm{T}} \Delta+\mathbf{A}_{(\mathrm{k})}^{\mathrm{T}} \mathbf{R}_{\mathrm{tt}}^{-1} \mathbf{A}_{(\mathrm{k})}\right]^{-1} \\
\times\left[\left(\mathrm{a} \mathbf{R}_{\mathrm{rr}}^{-1}+\mathrm{b} \Delta^{\mathrm{T}} \Delta\right)\left(\mathbf{r}^{(0)}-\mathbf{r}^{(\mathrm{k})}\right)+\mathbf{A}_{(\mathrm{k})}^{\mathrm{T}} \mathbf{R}_{\mathrm{tt}}^{-1}\left(\mathbf{t}-\mathbf{G} \mathbf{q}^{(\mathbf{k})}\right)\right]
\end{gathered}
$$


Provided the a priori constraints are adequately weighted and $\left|\mathbf{q}^{(0)}\right|$ is not much less than $\left|\mathbf{q}^{(\infty)}\right|$ the scheme is stable and converges upon a solution within a few iterations.

It is important to estimate the uncertainty associated with solutions, a problem that is complicated by the presence of a priori information. If a smoothest model approach is used (i.e., $\mathrm{a}=0$ ), then the solution may be combined with the Backus and Gilbert [1968] approach to obtain estimates of resolution and uncertainty [Rodi, 1989; Yonovsha and Ditmar, 1990]. However, when an a priori model is assumed it is necessary to compare the a posteriori covariance $\mathrm{C}_{\mathrm{qq}}$ with the effective a priori covariance $\mathbf{C}_{\mathrm{qq}}$ [Tarantola, 1987]. A linear approximation to the $a$ posteriori covariance is defined [Tarantola, 1987] by

$$
\mathbf{C}_{\pi^{\prime}}=\left[\mathrm{a} \mathbf{R}_{\pi \mathrm{rr}}^{-1}+\mathrm{b} \Delta^{\mathrm{T}} \Delta+\mathbf{A}_{(\infty)}^{\mathrm{T}} \mathbf{R}_{\mathrm{tt}}^{-1} \mathbf{A}_{(\infty)}\right]^{-1}
$$

while the effective a priori covariance, which includes the smoothing constraint, is

$$
\mathbf{C}_{\mathrm{Ir}}=\left[\mathrm{a} \mathbf{R}_{\mathrm{rr}}^{-1}+\mathrm{b} \Delta^{\mathrm{T}} \Delta\right]^{-1}
$$

If a node is well constrained by the data then the diagonal element of $\mathrm{C}_{\mathrm{rr}}$ corresponding to that node will be much smaller than the equivalent element in $\mathrm{C}_{\mathrm{rr}}$. Alternatively if diagonal elements have similar values, the data have contributed little information to constraining the model at the node. When equating the absolute value of the diagonal elements of $\mathbf{C}_{\mathrm{rr}}$ to model uncertainties it is important to remember that the estimates of $t^{*}$ are best thought of as relative rather than absolute. If the normalized squared data misfit $\overline{\chi^{2}}$, which is given by

$$
\overline{\chi^{2}}=\frac{\chi^{2}}{N}
$$

where $\mathrm{N}$ is the number of $\mathrm{t}^{*}$ observations, differs greatly from unity then the model variances should be scaled by a similar factor. Indeed the fact that the absolute data uncertainties are not known precludes an approach that seeks to find the smoothest model that reduces $\overline{\chi^{2}}$ to unity [Constable et al., 1987].

When considering the variance of the solution it is also necessary to consider how well the model is resolved. This information is contained in the off-diagonal elements of the $a$ posteriori covariance matrix and is probably best examined using the resolution matrix, which is defined [Tarantola, 1987]

$$
\mathbf{R}=\mathbf{I}-\mathbf{C}_{\boldsymbol{\pi}^{\prime}} \mathbf{C}_{\mathbf{r}^{\prime}}^{-1}
$$

The rows of the resolution matrix can be thought of as a linear filter that shows how the estimate of a model parameter is really a weighted sum of model parameters. If 
a row of $\mathbf{R}$ is close to a delta function then the model parameter is well resolved, whereas if there are large off-diagonal elements for adjacent nodes the solution is spatially smoothed. Since it is impractical to examine $\mathbf{R}$ in detail for all model parameters it is frequently convenient to summarize each row in terms of an element of the spread function S [Backus and Gilbert, 1968] which in this study we define

$$
S_{i}^{2}=\sum_{j=1}^{m}\left|\mathbf{x}_{j}-\mathbf{x}_{i}\right|^{2} R_{i j}
$$

where $x_{k}$ is the location of the $k^{\text {th }}$ of $m$ model nodes. A small value of $S_{i}$ indicates that a model parameter is spatially well resolved. A fundamental feature to all inversion techniques is the trade off between the model variance and resolution [e.g., Backus and Gilbert, 1968, 1970; Tarantola, 1987]. An improved spatial resolution results in an increase in model variance, and vice-versa. Moreover, the choice of the solution which optimally balances the model resolution and variance is necessarily a subjective one.

When considering the uncertainties associated with a $\mathrm{Q}^{-1}$ model obtained by the techniques described in this chapter it is important to remember that the formal uncertainties are based on the assumption that the forward solution (equations 1.27 - 1.29) is known exactly. The uncertainties arising from assumptions behind the technique will not be included in the formal model uncertainties and must be investigated separately. 
Table 1.1. Fractional leakage of prolate eigentapers for a 256-point time series.

\begin{tabular}{lcc}
\hline & \multicolumn{2}{c}{$M \pi$ Prolate } \\
\cline { 2 - 3 } & $\mathrm{M}=4$ & $\mathrm{M}=2$ \\
\cline { 2 - 3 }$\lambda_{0}$ & 0.9999999997 & 0.9999428126 \\
$\lambda_{1}$ & 0.9999999725 & 0.9975632086 \\
$\lambda_{2}$ & 0.9999987966 & 0.9594018056 \\
$\lambda_{3}$ & 0.9999676885 & 0.7217684703 \\
$\lambda_{4}$ & 0.9994117491 & 0.2746520408 \\
$\lambda_{5}$ & 0.9925173871 & 0.0430015741 \\
$\lambda_{6}$ & 0.9367030504 & 0.0034756339 \\
$\lambda_{7}$ & 0.6988882538 & 0.0001867680 \\
$\lambda_{8}$ & 0.2993280554 & 0.0000074487 \\
$\lambda_{9}$ & 0.0641890774 & 0.0000002316 \\
& & \\
\hline
\end{tabular}




\section{FIGURE CAPTIONS}

Figure 1.1. Configuration of the East Pacific Rise tomography experiment. Ocean bottom receivers are shown as solid symbols and comprise 8 analog ocean bottom hydrophones (triangles), 5 digital ocean bottom hydrophones (squares), and 2 digital ocean bottom seismometers (circles). Explosive sources are shown as small open circles. Bathymetric contours are spaced at $100 \mathrm{~m}$ and are obtained from the Sea Beam data collected during the experiment [Wilcock et al., 1992].

Figure 1.2. Horizontal cross-sections showing the horizontal perturbations from the average one-dimensional model $(0.25 \mathrm{~km} / \mathrm{s}$ contour interval, bold contours at $0.5-\mathrm{km} / \mathrm{s}$ intervals) through a three-dimensional P-wave velocity structure obtained from an inversion of travel times obtained during the tomography experiment [Toomey et al., 1990a]. Sections are shown at (a) the seafloor and (b) $2 \mathrm{~km}$ depth and show the perturbations from the average vertical structure.

Figure 1.3. Velocity model derived from an interpretation of expanding spread profile and common depth point reflection data obtained at the same location as the tomography experiment [from Vera et al., 1990].

Figure 1.4. Normalized instrument power responses for (a) AOBHs 1 (solid) and 2 (dashed), (b) DOBH 14, and (c) OBS 1.

Figure 1.5. (a) Mid-water DOBH recording of the source signature obtained during the source monitoring experiment. (b) The recorded source signature after applying attenuation using Azimi's attenuation formula [Azimi et al., 1968; Aki and Richards, 1980] assuming $\mathrm{t}^{*}=0.04$. (c) A model of the source signature obtained from a constrained frequencydomain deconvolution of the DOBH instrument response. (d) The normalized power spectra of the recorded source signature corrected for instrument response (solid line) and the source signature model (dash). 
Figure 1.6. (a) Time series and (b) power spectra for a rectangular (boxcar) window (solid) and a cosine squared (Hanning) window (dashed).

Figure 1.7. Lowest order eigentapers and their power spectra for (a) $2 \pi$ prolate and (b) $4 \pi$ prolate spheroidal sequences.

Figure 1.8. (a) Effective squared window amplitudes and (b) power spectra for the $2 \pi$ prolate (dashed) and $4 \pi$ prolate (solid) multiple-window spectral estimate obtained using equation (1.16).

Figure 1.9. Expected $t^{*}$ estimates (solid lines) obtained over a frequency interval of $10-40 \mathrm{~Hz}$ plotted against window length using (a) $2 \pi$ prolate and (b) $4 \pi$ prolate multiple-window spectral analysis. The crustal transfer function is calculated according to equation (1.5), the source signature used is that of Figure 1.4, and the instrument response is that of AOBH 1 (Figure 1.3a). Expected $t^{*}$ values are calculated according to equation (1.7) using a least-squares fit to the crustal transfer function obtained from equation (1.22). The effect of using an incorrect source signature (the source depth is $6 \mathrm{~m}$ shallower and the bubble pulse period $14 \mathrm{msec}$ smaller) in the correction term is also shown (asterisks). 


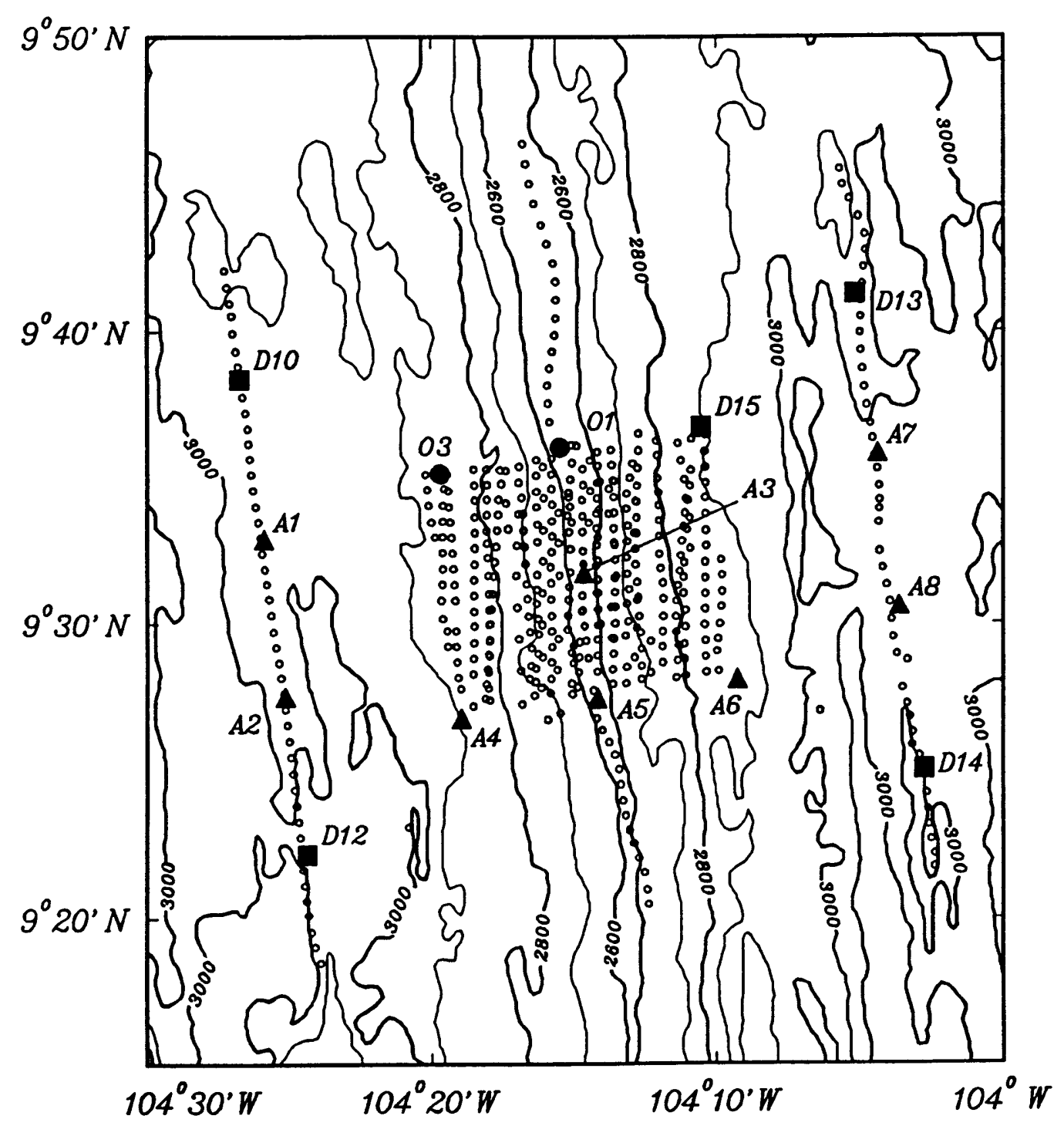

Figure 1.1 
(a)

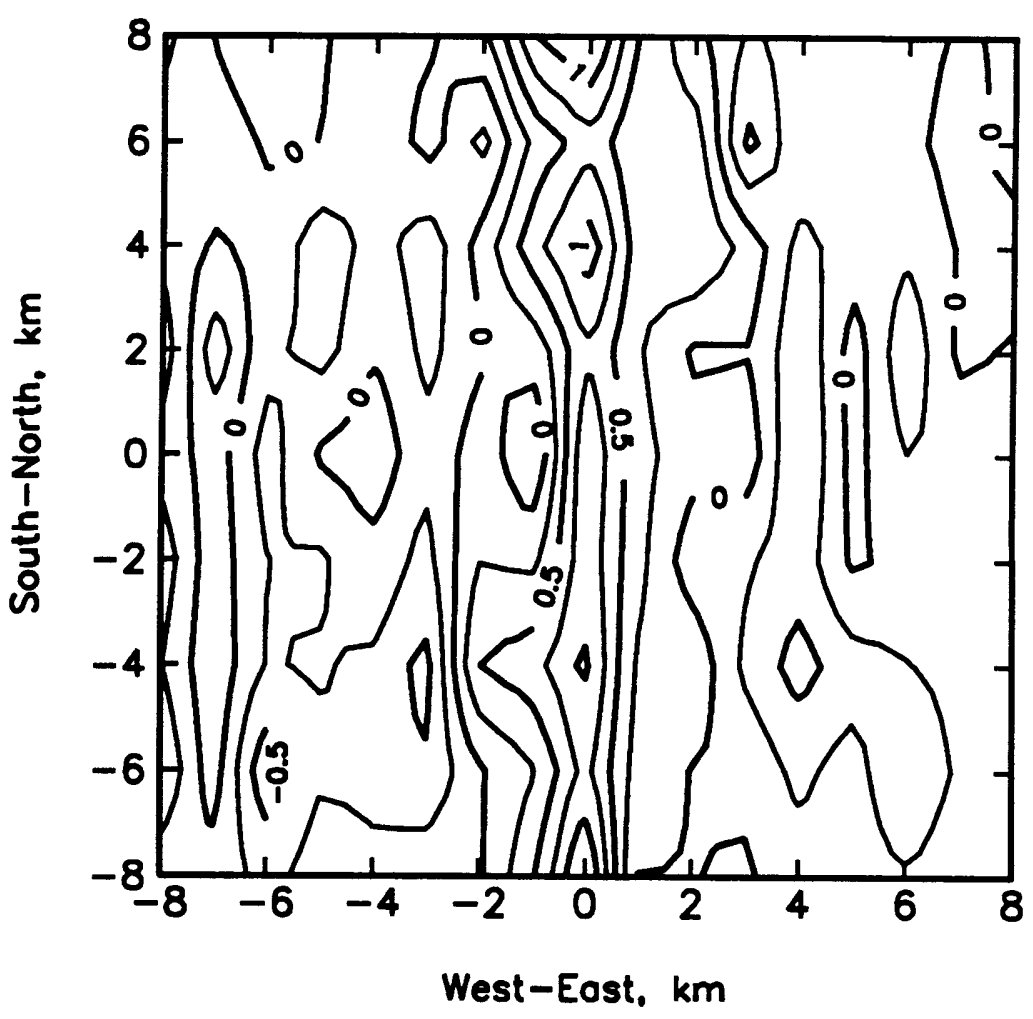

(b)

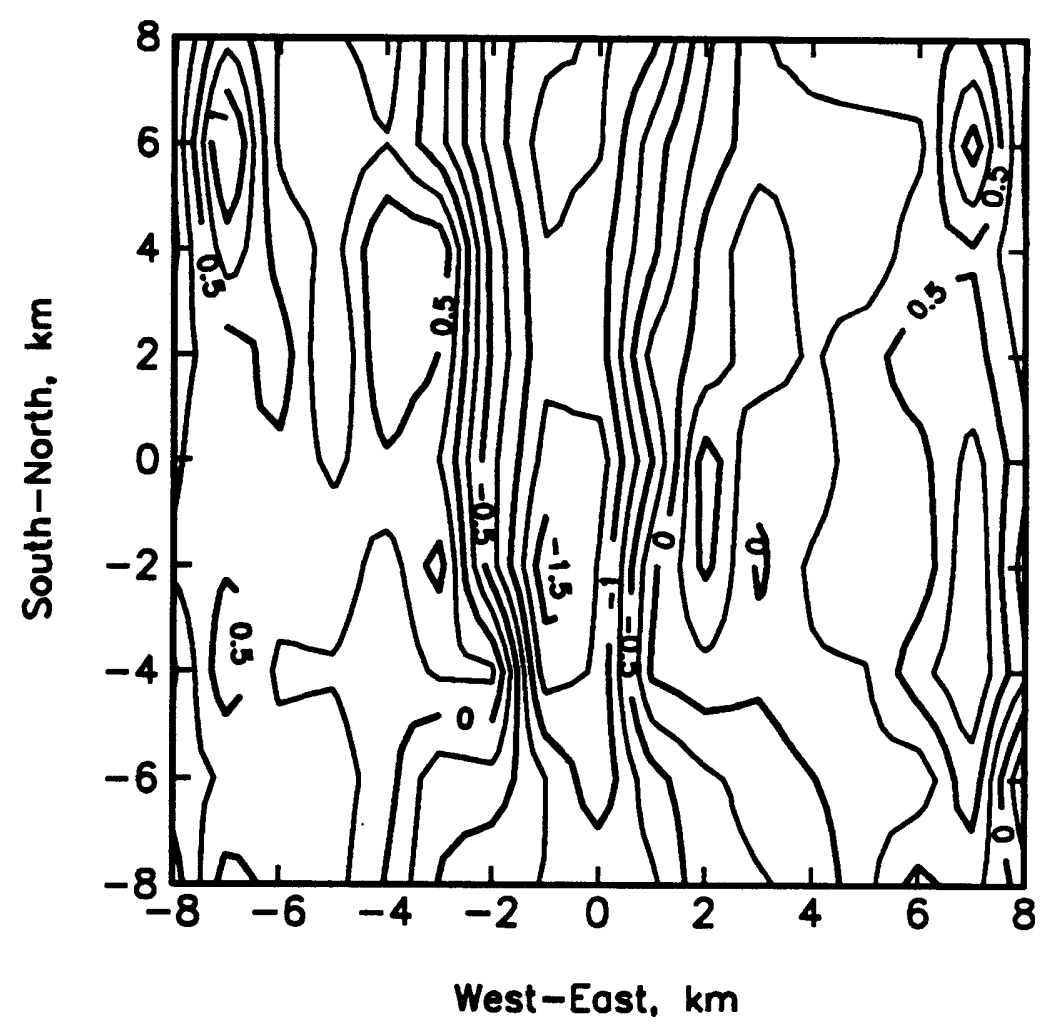

Figure 1.2 


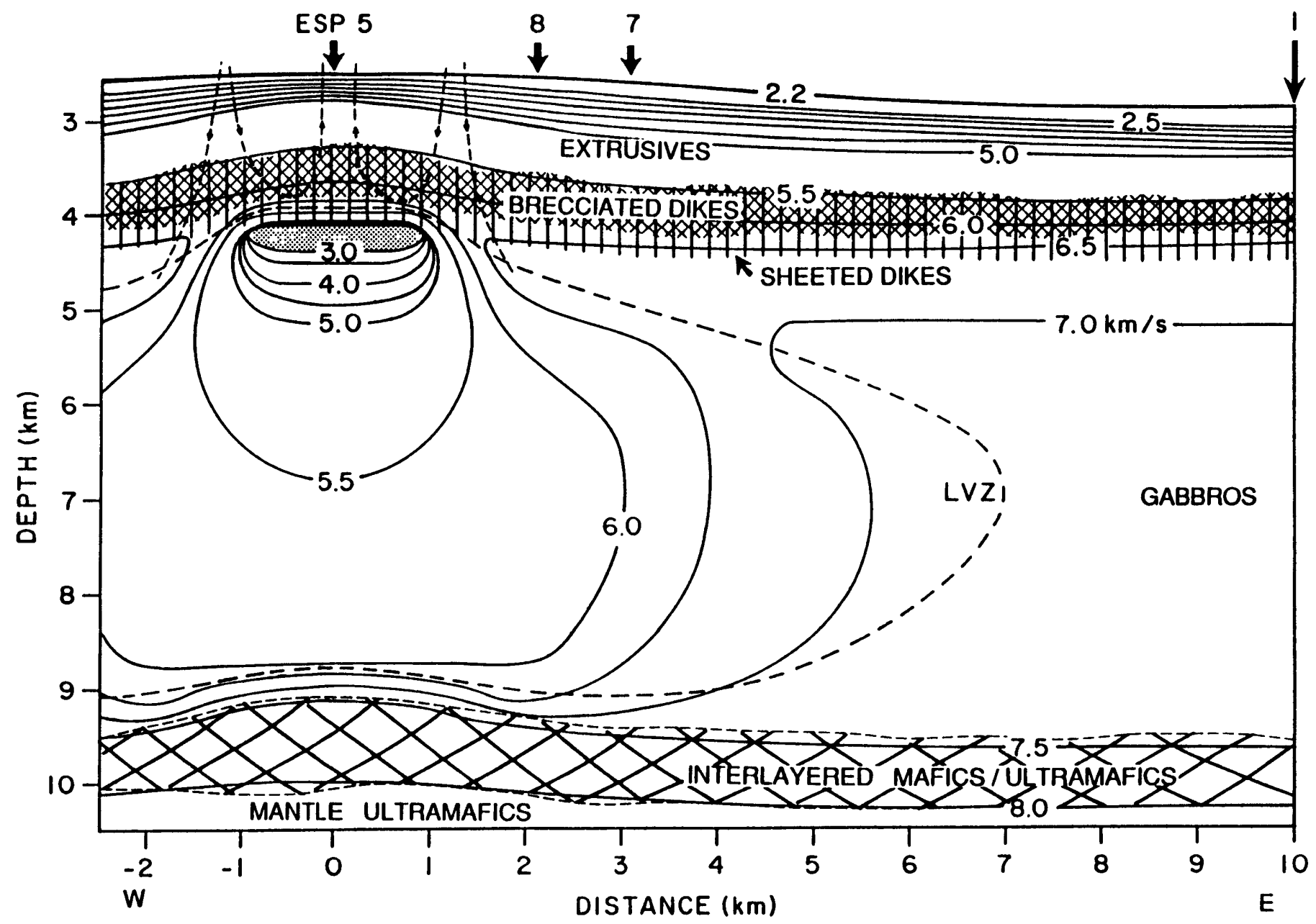




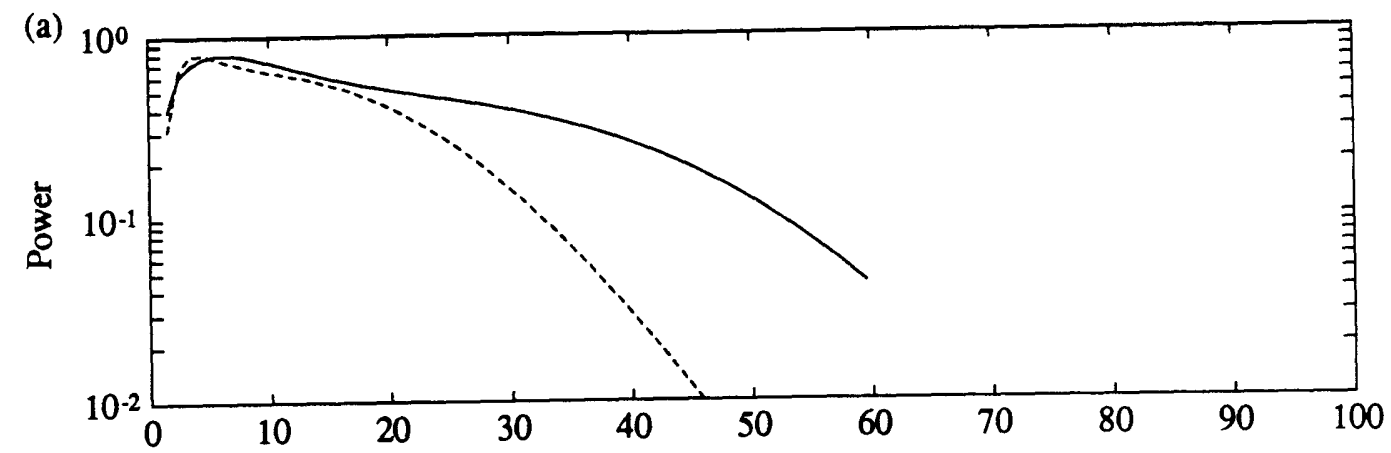

Frequency, $\mathrm{Hz}$
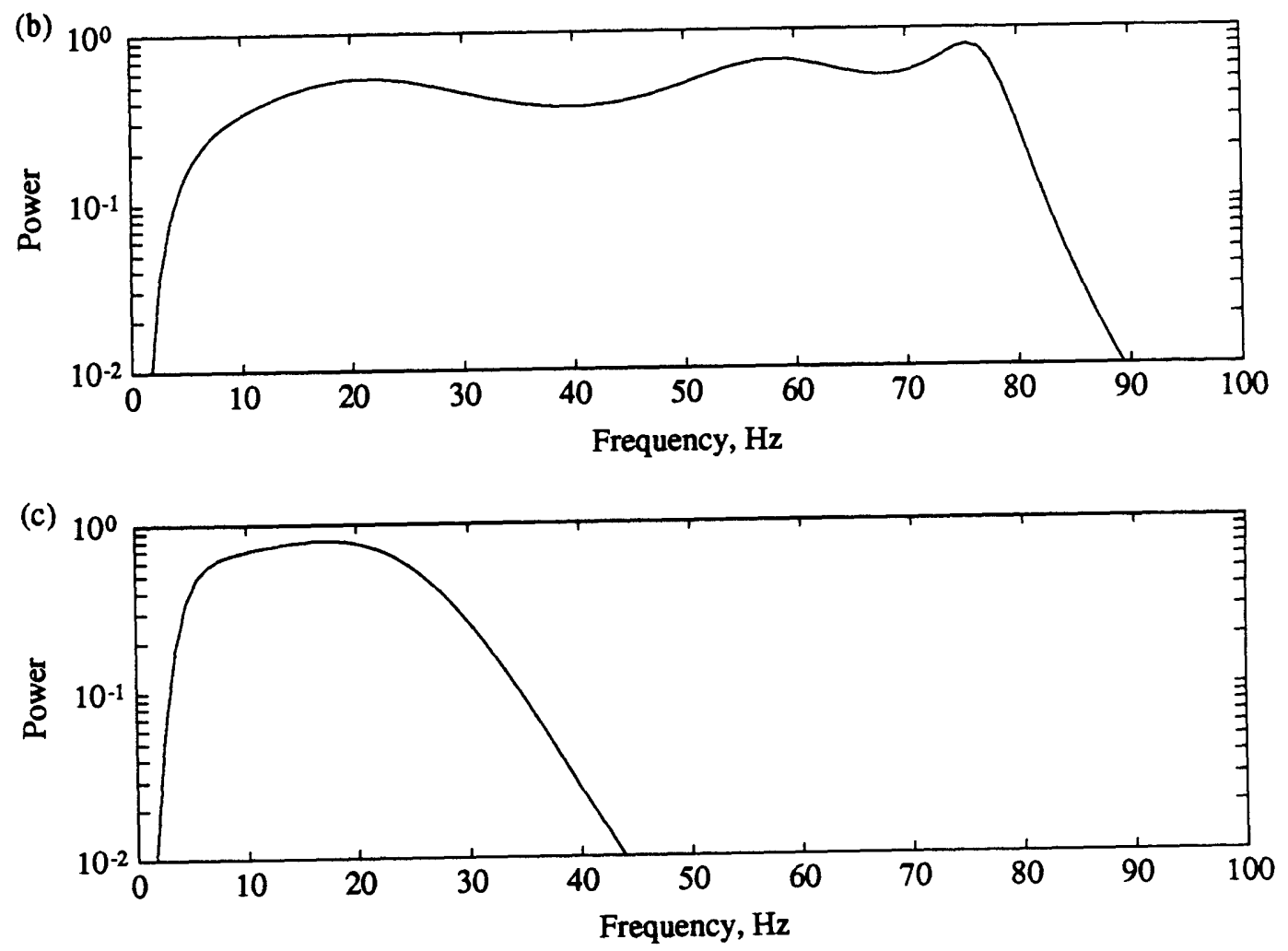

Figure 1.4 

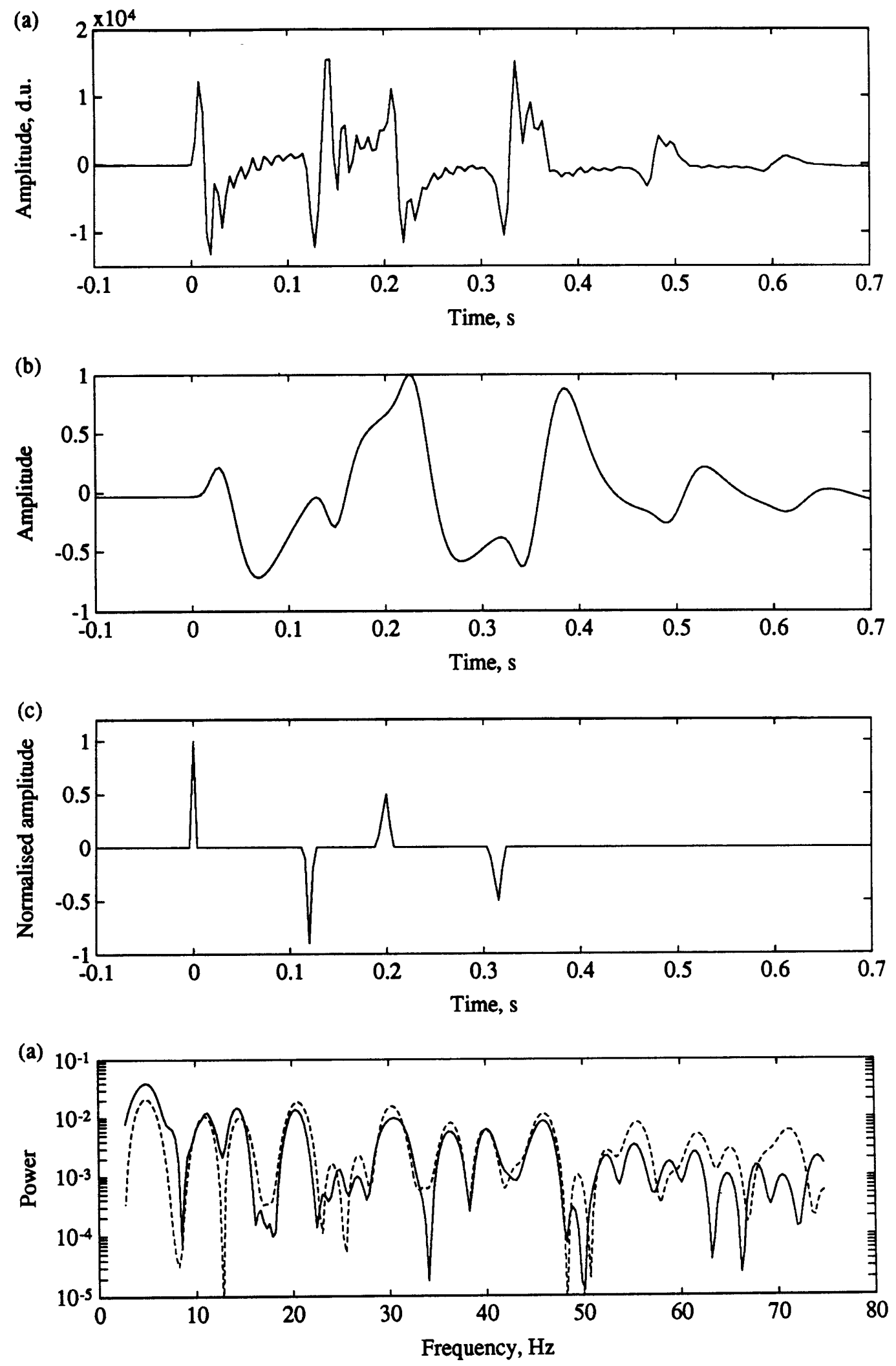

Figure 1.5 

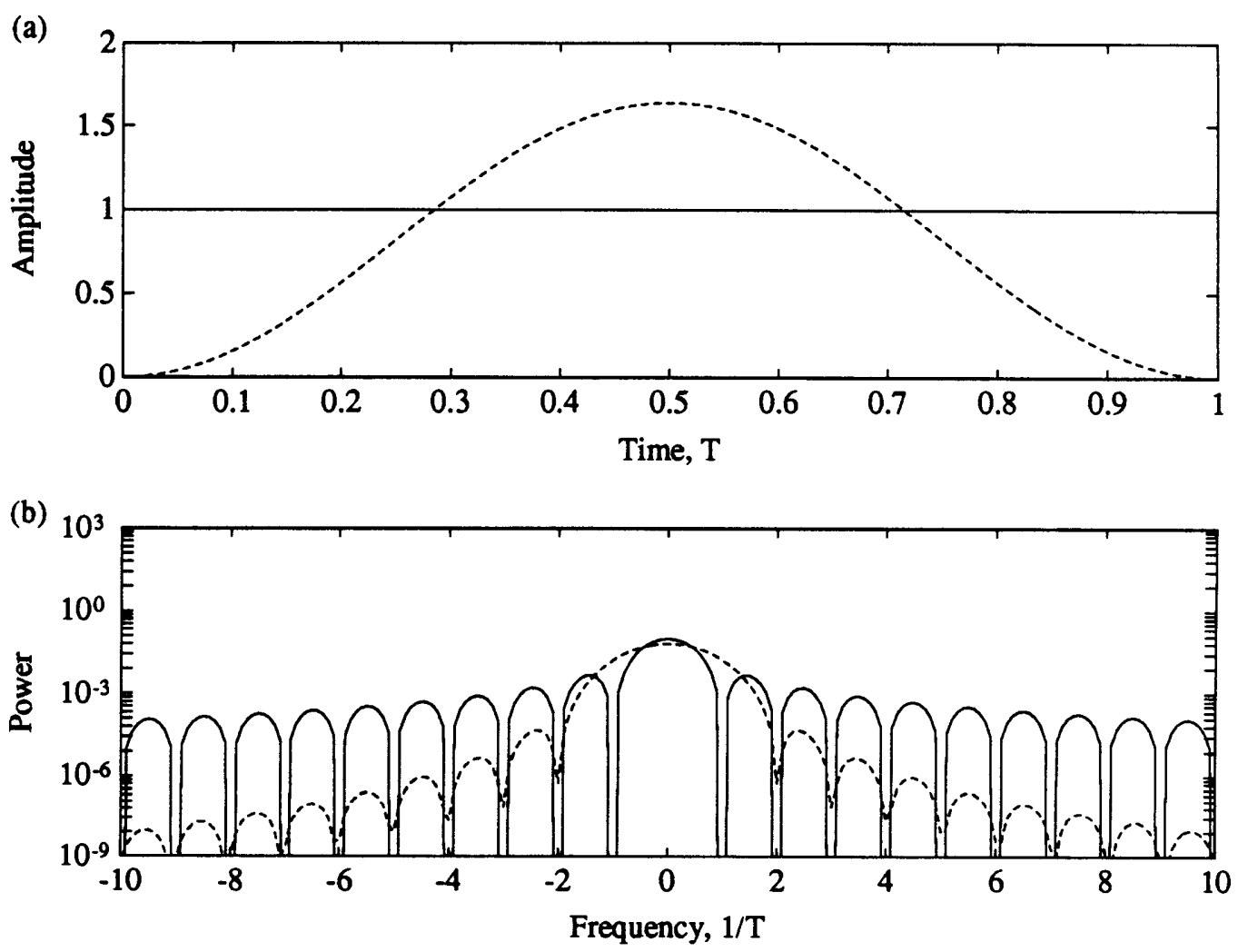

Figure 1.6 

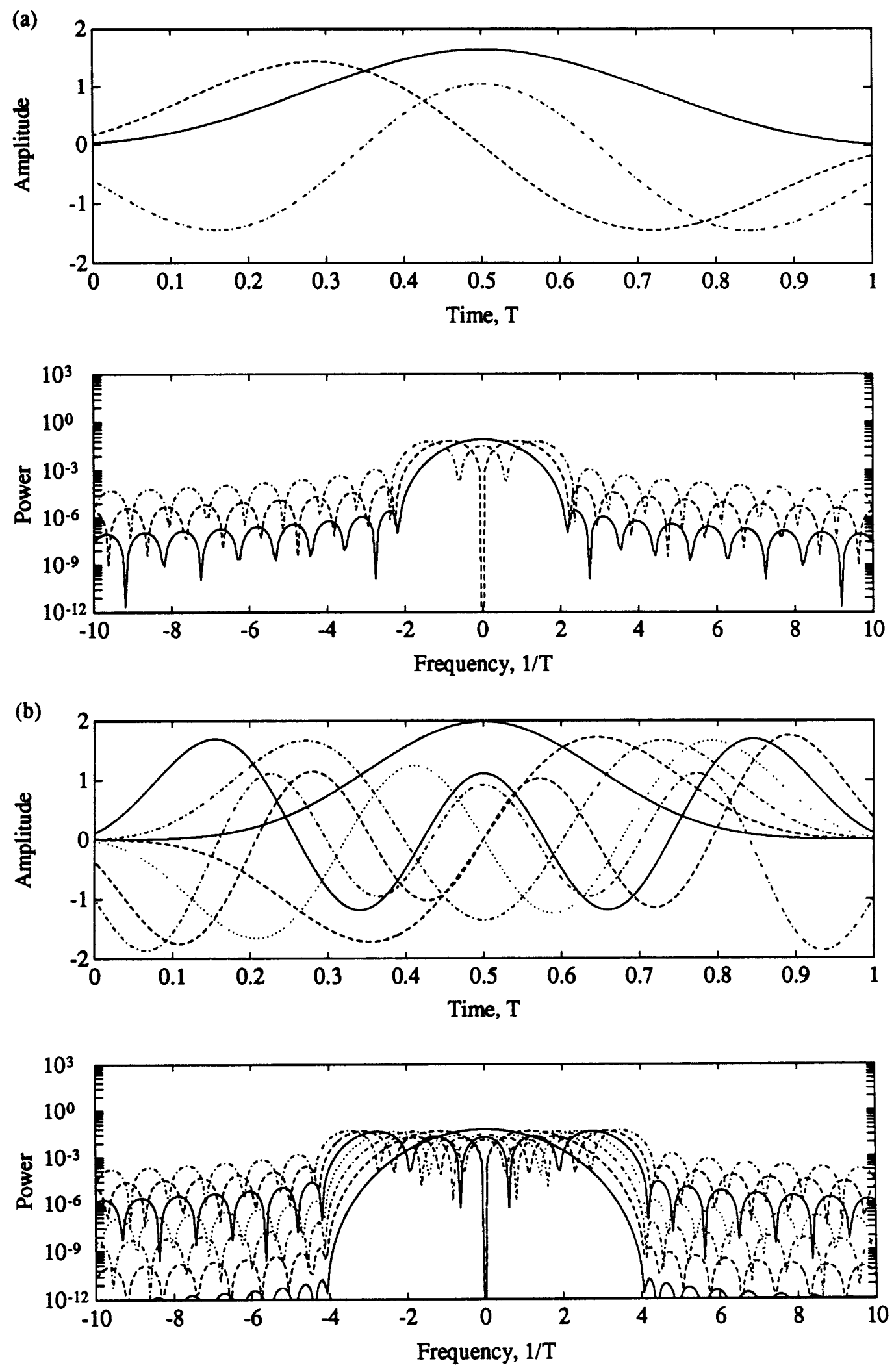

Figure 1.7 

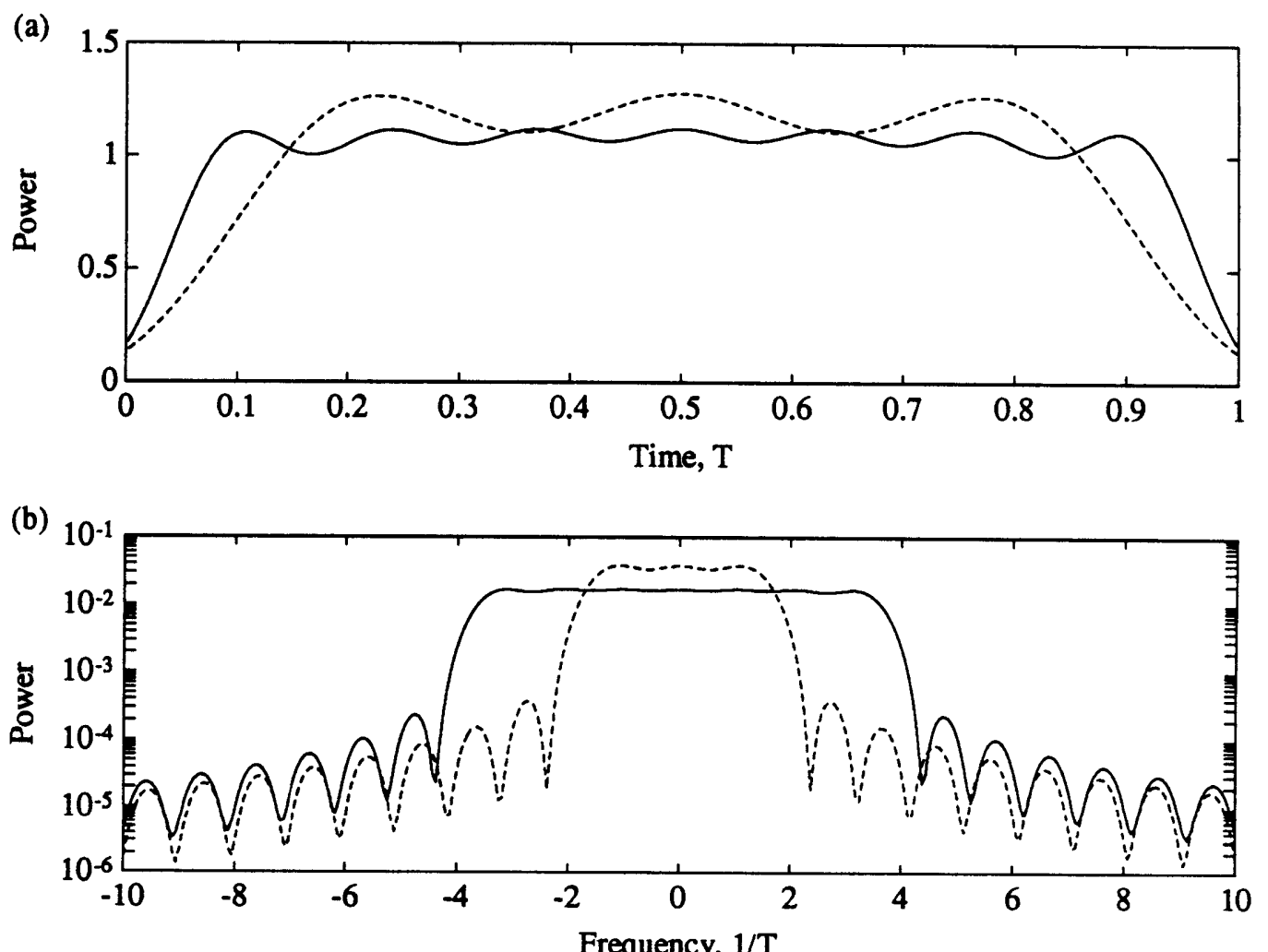

Frequency, 1/T

Figure 1.8 
(a)

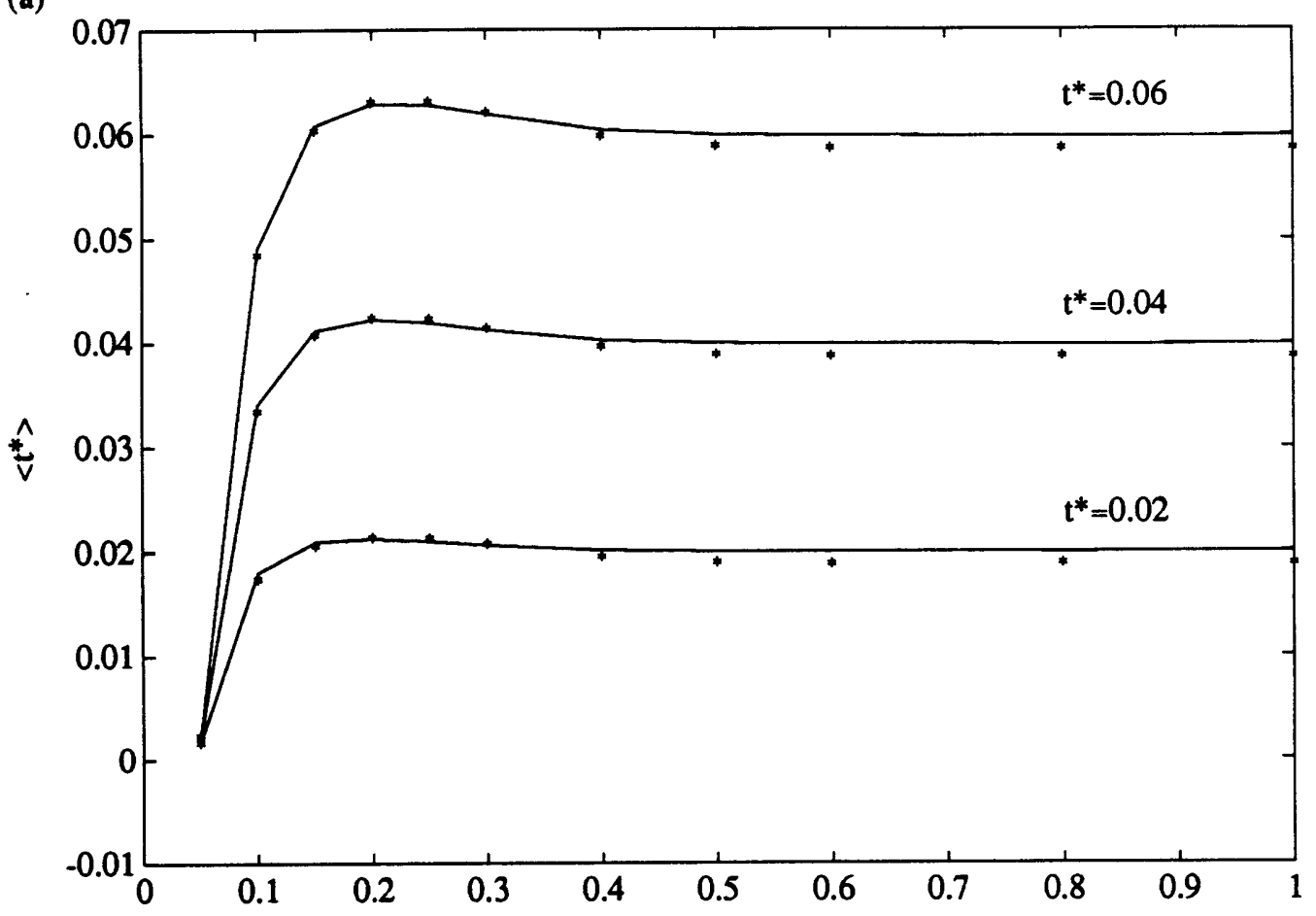

Window length, $s$

(b)

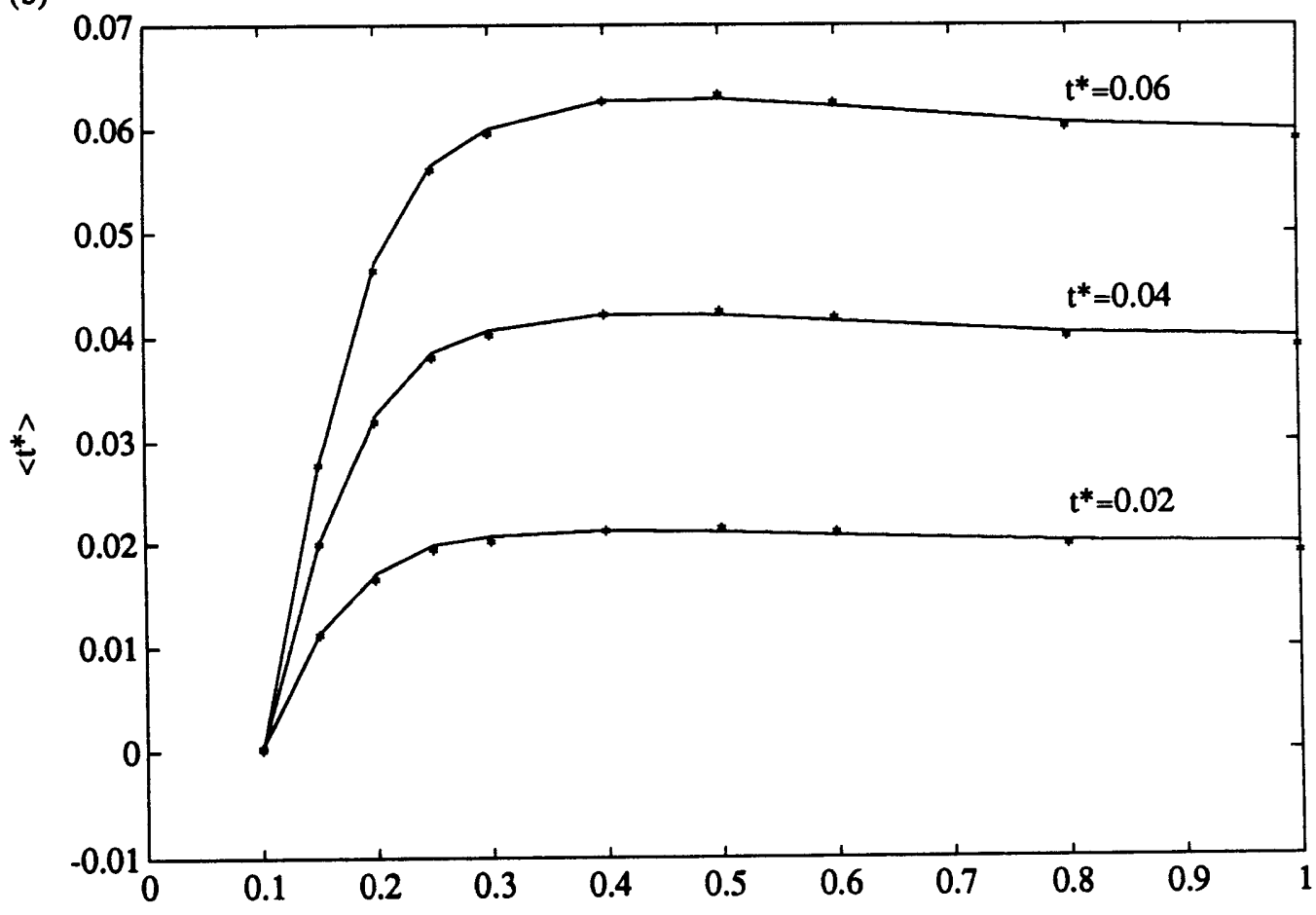

Window length, $s$

Figure 1.9 


\title{
ChaPTER 2
}

\section{A Comparison Of THE ATtenuation StRuCture OF 0.35 AND 0.00-MY-OLD}

\author{
Crust
}

\section{INTRODUCTION}

This chapter is concerned with the analysis of three $45-$ to-50-km-long refraction profiles which form a subset of the tomography experiment (Figure 2.1). The profiles, which are orientated parallel to the EPR and are located on the rise axis and $20 \mathrm{~km}$ to either side, provide a good data set to evaluate the spectral method for estimation of $t^{*}$ and to illustrate the inversion techniques used to obtain $\mathrm{Q}^{-1}$ models. The one-dimensional models of $\mathrm{Q}^{-1}$ obtained in this chapter can be used to constrain inversions of the whole data set presented in Chapter 4. Moreover, since previous measurements of attenuation in young oceanic crust have been limited to one experiment that was confined to the upper $650 \mathrm{~m}$ of $0.4-\mathrm{My}$-old crust [Jacobson and Lewis, 1990], the results contribute to an understanding of the structure and evolution of young oceanic crust which is discussed in Chapter 5.

\section{OFF-AXIS PROFILES}

The two off-axis profiles (Figures 2.1) are located $20 \mathrm{~km}$ to the east and west of the rise axis and have almost identical configurations. Each line comprised four OBHs deployed at $10 \mathrm{~km}$ intervals and about 45 explosive shots spaced at $1 \mathrm{~km}$ intervals. For both lines the inner receivers are $\mathrm{AOBHs}$ while the outer receivers are DOBHs. Since the AOBHs saturate at all but the largest ranges, the attenuation studies are confined to the DOBHs, though arrival times obtained from the AOBH records are used to constrain the velocity structure.

\section{Velocity Structure}

A full study of the velocity structure along the two profiles would require a detailed analysis of travel times and amplitudes for both the explosive shots and the airgun data that were also collected along each line. However, while a velocity model and ray paths are required for attenuation tomography, the resolution of the $\mathrm{Q}^{-}$ 1 models presented in this chapter is not such as to warrant a detailed knowledge of the fine-scale velocity structure. In particular, the inability to obtain spectral $t^{*}$ 
measurements from surface shots at ranges less than about $4 \mathrm{~km}$ due to the presence of the water wave minimizes the necessity for an accurate determination of the shallow velocity structure. For this reason, this study is limited to forward modelling the principal features of the explosive-shot travel time data, the $1-\mathrm{km}$ spacing of which does not permit good resolution of the shallow structure.

The P-wave arrival time data were obtained using an automated picking routine that uses waveform alignment and a simulated annealing formalism [Allen, 1982; Rothman, 1986; Toomey et al., 1990a]. The location of receivers and shots together with the shot origin times were estimated for the tomography experiment using a formal inversion of the water-wave arrival times and navigation data [Creager and Dorman, 1982; Toomey et al., 1990a]. Such a procedure generally yields origin times and shot and receiver locations that are accurate to within $\pm 0.004 \mathrm{~ms}$ and $\pm 15 \mathrm{~m}$ respectively. However, the shots at the margins of the experiment, including shots at the ends of the refraction lines, are not recorded by enough receivers at sufficiently small ranges to permit good locations based on arrival time data alone since there is a strong trade off between range and origin time. Moreover, since the ship was steaming slowly ( 4 knots) during shooting and the charges were in the water about 4 minutes prior to detonation, shipboard estimates of origin time are not sufficiently accurate to constrain the inversion. Therefore, determining accurate locations and origin times for such shots is critically dependent on navigational constraints. The western profile was obtained during a period of high quality GPS satellite coverage and is accurately navigated [Wilcock et al., 1992]. In contrast, there are few navigational constraints on the eastern profile and the range and travel time data obtained for the profile are consequently of lower quality.

Western Profile

After the application of a water path correction [Purdy, 1982], the travel time data to the north and south of each receiver were forward modelled to obtain a series of simple velocity-depth profiles. The starting model for these calculations was based on the off-axis velocity model of Vera et al. [1990]. Figure 2.2 shows the results obtained from shots to the south of DOBH 10 (profile 10S) and to the north of DOBH 12 (profile 12N). The two velocity models are markedly different. Layer 2B $\left(\mathrm{V}_{\mathrm{P}}=5-5.5 \mathrm{~km} / \mathrm{s}\right)$ [Houtz and Ewing, 1976] is not resolved by profile 10S, which indicates velocities approaching $6 \mathrm{~km} / \mathrm{s}$ at $0.5 \mathrm{~km}$ depth. In contrast, profile $12 \mathrm{~N}$ incorporates a $400 \mathrm{~m}$ thick layer with velocities of about $5.4 \mathrm{~km} / \mathrm{s}$, and the velocity does not reach $6 \mathrm{~km} / \mathrm{s}$ until a depth of $1.2 \mathrm{~km}$. In addition, lower crustal (layer 3) 
arrivals have a higher phase velocity for profile $12 \mathrm{~N}(7.15 \mathrm{~km} / \mathrm{s})$ than for profile $10 \mathrm{~S}$ $(7.0 \mathrm{~km} / \mathrm{s})$.

To obtain a two dimensional velocity model the data for all four instruments were forward modelled by ray tracing through a layered two-dimensional velocity structure which includes the bathymetry along the profile [Luetgert, 1988]. The initial one-dimensional model is an average of the models obtained for individual profiles, except that the upper $0.5 \mathrm{~km}$, a portion of the structure which is not resolved by the data, is modelled by a constant gradient. Rather than exhaustively investigating a large range of possible velocity structures, an attempt was made to model the travel time data by perturbing the velocity at the base of the second crustal layer (layer 2B) and by changing the layer thicknesses. The resulting model is shown in Figure 2.3, while the fit to the travel time data is shown in Figure 2.4.

The primary feature of the model is a south-to-north thinning of the upper crust by $0.25-0.5 \mathrm{~km}$ coupled with an increase in velocities at the base of the second model layer (layer 2B). This feature successfully models the difference in the apparent phase velocities between northward and southward propagating lower crustal arrivals (Figure 2.2) without any modification of lower crust velocities. There is some indication that the trend in upper crustal thickness may not persist to the outer limits of the profile, particularly to the north, but since the shot parameters for the outer shots have higher uncertainties, the model is not well resolved in these regions.

\section{Eastern Profile}

Figure 2.5 shows the results of forward modelling travel times for shots to the south of DOBH 13 and to the north of DOBH 14. While the travel time data are of lower quality than that for the western line, there is no indication of lateral heterogeneity on the scale observed for the western profile. Indeed the data are compatible with the laterally invariant velocity model obtained by forward modelling expanded spread profile (ESP) data $10 \mathrm{~km}$ to the east of the rise axis [Vera et al., 1990].

\section{t* Estimation}

Because of the relative uniformity of the crustal structure, the off-axis data provide a good opportunity to evaluate the MWSA $t^{*}$ estimation technique described in the last chapter. Figures 2.6 and 2.7 show an example of the application of this technique to a single waveform using two different data window lengths and both $2 \pi$ 
prolate and $4 \pi$ prolate windows. Figure 2.6 shows estimates obtained from the first $0.6 \mathrm{~s}$ of the waveform and clearly illustrates the advantage obtained from the additional smoothing of the $4 \pi$ prolate MWSA estimate. The $2 \pi$ prolate spectral estimate is characterized by rapid variations in power over the signal band. As a result, the choice of the upper frequency bound for spectral slope estimation is strongly dependent upon the minimum acceptable signal to noise ratio. Moreover, the source-receiver correction contains several pronounced notches which greatly decrease the stability of the correction. The $2 \pi$ prolate spectral estimate of $t^{*}$ for this example varies by almost a factor of 2 depending upon the upper frequency bound chosen for slope estimation. In contrast both the spectral estimate and source-receiver correction for the $4 \pi$ MWSA estimate are relatively smooth. The upper frequency bound for slope estimation can be chosen unambiguously and has little effect on $t^{*}$ values.

In Figure 2.7 the window length is reduced to include only the first $0.3 \mathrm{~s}$ of the waveform. The decreased spectral resolution of the shorter window results in smoother spectra for both the $2 \pi$ and $4 \pi$ prolate tapers. Both of the power spectra show a leveling off at low frequencies, a feature that is particularly apparent for the $4 \pi$ prolate estimate. Since the half-width of the estimation band is $10 \mathrm{~Hz}$ for the $4 \pi$ prolate and $5 \mathrm{~Hz}$ for the $2 \pi$ prolate estimate, this feature apparently results from the inclusion of frequencies lying outside the instrument passband. However, even after applying the source-receiver correction the feature is still apparent. Using a spectral ratio method, a technique which need not be sensitive to the spectral estimation method, Lewis and Jung [1988] observe a decrease in spectral amplitudes at frequencies below $10 \mathrm{~Hz}$ which they attribute to the reflectivity of the upper crust. Since the MWSA technique does not have good spectral resolution and the instrument responses decrease rapidly below $5 \mathrm{~Hz}$, we cannot directly confirm this observation, but we note that it may contribute to the levelling off of power spectra at low frequencies.

Figure 2.8 shows $\mathrm{t}^{*}$ estimates for the DOBHs, derived from $0.3 \mathrm{~s}$ of the waveform using both $2 \pi$ and $4 \pi$ prolate MWSA. In most instances the two estimates are in close agreement and show little or no systematic offset. In the cases where the discrepancies between the two estimates are large, it is invariably the result of a significant difference between the upper frequency bounds used for spectral slope estimation. Since the variation between adjacent $t^{*}$ estimates is 
larger for the $2 \pi$ prolate MWSA estimates, the additional smoothing of the $4 \pi$ prolate MWSA is desirable as it appears not to introduce additional bias.

Figure 2.9 compares $4 \pi$ prolate MWSA t* estimates obtained from 0.3 - and 0.6$s$ data windows. The variation between adjacent values is noticeably larger for the 0.3-s estimates, a reflection of the greater uncertainties accompanying spectral estimates obtained from the shorter window. At small ranges the two estimates are generally in good agreement while at larger ranges the 0.3-s estimates are commonly larger, particularly for DOBH 10. There are two effects that might cause differences between spectral estimates, neither of which completely explains the observed discrepancy. First, the longer window is likely to include a larger proportion of secondary arrivals. These may include reverberations within layer $2 \mathrm{~A}$, PP and higher order phases which include one or more downward reflection from the seafloor, and at larger ranges mantle phases. The high levels of attenuation observed in the shallow crust [Jacobson and Lewis, 1990] should result in an increase in apparent $\mathrm{t}^{*}$ values when secondary phases which propagate longer distances at shallower depths are included in the spectral window. However, since $t^{*}$ values decrease with spectral window length it is reasonable to conclude that such phases do not affect estimates significantly. Figure 2.10 shows record sections for the two DOBHs on the western profile. The maximum source-receiver separation for is about $37 \mathrm{~km}$, a range that is too short to observe primary mantle arrivals [e.g. Spudich and Orcutt, 1980b]. However, PmP (mantle reflections) arrivals with amplitudes that are strongly dependent on the range are observed at ranges greater than about 20-25 km in typical oceanic crust [e.g. Spudich and Orcutt, $1980 \mathrm{~b}]$ and may contribute to the discrepancy.

A second source of the differences in $t^{*}$ values may arise from the source component of the source-receiver correction. When $t^{*}$ is estimated from a window which is as long as the whole source signature, as is essentially the case for the 0.6$s$ estimates, then the application of the source-receiver correction is relatively straightforward. However, if the window is shorter than the source signature then the correction is considerably more problematic. First, the source signature recorded during the source monitoring experiment must be truncated using a taper, a procedure that may in itself introduce a bias to spectral estimates. Second, it is by no means clear at what point to apply the taper. For a 0.3-s data window the surface reflection of the first bubble pulse lies close to the window limit. However, because attenuation and convolution with the instrument response significantly broaden a 
pulse (Figure 1.5b) and data in the last $10 \%$ of the window are downweighted (Figure 1.8), this feature will not contribute significantly to the spectral estimate. Indeed, for the same reasons the first bubble pulse, which occurs at about $0.2 \mathrm{~s}$, may not be fully weighted in a 0.3 -s spectral estimate. If a source-receiver correction derived from the whole source signature is always used, the power spectrum obtained from a shorter window should be richer in high frequencies and hence yield lower $t^{*}$ estimates. In this study we truncate the source signature to account for shorter windows and apply a cosine squared taper over the last $0.1 \mathrm{~s}$. Any remaining bias in $t^{*}$ estimates after such a procedure is difficult to estimate but should not be range dependent.

\section{$Q^{-1}$ Inversions}

In the following $\mathrm{Q}^{-1}$ inversions we chose to use the estimates obtained from 0.3 $\mathrm{s}$ of the waveform to ensure that the effects of secondary arrivals are minimized. Figure 2.11 shows the $t^{*}$ estimates together with the estimates of relative uncertainty obtained following the method outlined in Chapter 1. A striking feature of the data is that to first order $t^{*}$ values are independent of range, which suggests qualitatively that attenuation is concentrated in the upper crust. Moreover, it explains why waveform modelling techniques are frequently successful in matching the relative amplitudes of seismic waveforms with little regard for the effects of attenuation.

$\mathrm{Q}^{-1}$ inversions require knowledge of the velocity structure and ray paths. For the western profile the two-dimensional velocity structure obtained from travel time modelling (Figure 2.3) is used, while for the eastern profile a one-dimensional model obtained by averaging the models for individual instruments (Figure 2.4) is assumed. The ray paths for the western profile [Luetgert, 1988] are shown in Figure 2.12. The largest depth sampled by the rays is about $3.5 \mathrm{~km}$. Since $t^{*}$ estimates are not available at ranges much less than $4 \mathrm{~km}$, very few ray paths turn in the uppermost crust and $\mathrm{Q}^{-1}$ inversions are consequently unlikely to have good resolution at depths less than $1 \mathrm{~km}$.

A useful approach to inverting the $t^{*}$ data is to obtain a vertical $Q^{-1}$ model for each instrument [Constable et al., 1987]. Each model is parameterized by values at nodes spaced at $0.25-\mathrm{km}$ depth intervals, and a solution is sought that simultaneously minimizes the misfit to the data and the roughness of the model (equation 1.36 with $\mathrm{a}=0$ ). Figure 2.13 shows the results for different choices of 
smoothing weight. The effect of using a large smoothing weight is to produce an almost constant $\mathrm{Q}^{-1}$ model which fits the data poorly. As the smoothing is decreased, the model progressively becomes more complex. Initially, there is a rapid decrease in the data misfit, but as the degree of smoothing is decreased further the improvement in fit becomes progressively smaller despite marked increases in the roughness of the model. Constable et al. [1987] suggest choosing a smoothing weight that yields a normalized squared data misfit (equation 1.40) equal to one. However, since the absolute data uncertainties are unknown the choice of the optimal smoothing weight is necessarily subjective. Indeed, the estimates of uncertainty are probably too large, since solutions for two of the four instruments have normalized squared data misfits which are much less than one. In Figure 2.14, the formal uncertainty and spread are plotted as functions of depth for several choices of smoothing weight for DOBH 10. The trade off between resolution and model variance is immediately apparent. The smoothest model $(b=100)$ has a small uncertainty but the resolution is very poor. In contrast the roughest model $(b=0.1)$ has good resolution at shallow depths, but the uncertainties are extremely large and are clearly compatible with a much smoother model. The intermediate solutions ( $b=10$ and 1) probably represent better choices of smoothing weights. Below $1 \mathrm{~km}$ depth, the spread (equation 1.42) progressively increases with depth, indicating a decrease in resolution. This results in part from the logarithmic parameterization and is a reflection of the increase in $Q$ values with depth and the smaller contribution of the lower part of the model to $t^{*}$. The solutions obtained for all instruments show a strikingly similar progressive increase of $Q$ with depth. $Q$ values of $30-50$ are observed within the upper $1 \mathrm{~km}$, while at the base of the model $\mathrm{Q}$ ranges from 500 to over 1000 .

Inspection of the smoothest model results suggests that the data might be alternatively fit using a simple two layer model. In Figure 2.15 the results of such inversions are shown as a function of upper layer thickness. Plots of misfit versus depth show that all four solutions are characterized by two minima which correspond to upper layer thicknesses of $\leq 0.5$ and $1-1.5 \mathrm{~km}$. For upper layers thicker than 1.5 $\mathrm{km}$, the two-layer model fits the data poorly. A simple F-test, which is based on the assumption that the two-layer model configuration is correct, shows that at the $95 \%$ confidence levels it is impossible to discriminate between solutions with upper layer thicknesses of less than $0.5 \mathrm{~km}$ and of $1-1.5 \mathrm{~km}$. However, models with an upper layer thickness less than $0.5 \mathrm{~km}$ require that $\mathrm{Q}$ in the upper layer be less than 10-15, 
a value that is markedly lower than previous measurements on the Juan de Fuca Ridge [Jacobson and Lewis, 1990]. The double minimum observed in the misfit suggests that a three-layer model might produce a better fit to the data, but in view of the limited number of shallow ray paths and the uncertainties in the velocity model, we do not present such solutions. It should be noted that the smoothest model solutions which show a progressive decrease in $\mathrm{Q}$ with depth fit the data better though this may be a result of a larger number of independent model parameters.

A comparison of the predictions of the one-dimensional models with the $t^{*}$ observations (Figure 2.16) shows that there are systematic misfits. One possibility is that these misfits result from lateral inhomogeneities in the $\mathrm{Q}$ structure. Indeed the results of Jacobson and Lewis [1990] show significant changes in upper crustal $\mathrm{Q}$ over distances of the order of $1 \mathrm{~km}$, a distance that corresponds to the separation of adjacent $t^{*}$ observations in this experiment. To investigate whether upper crustal heterogeneities in $\mathrm{Q}$ might account for the misfit to the data, inversions were performed for both the western and eastern lines. Below $0.625 \mathrm{~km}$ depth, the $\mathrm{Q}$ structure was held constant at values determined by a smoothest model inversion $(b=10)$ of the combined data for the two DOBHs on each line, while in the upper $0.625 \mathrm{~km} \mathrm{Q}^{-1}$ was parameterized by nodal values with a $1 \mathrm{~km}$ spacing. The results for the eastern profile (Figure 2.17) show that fluctuations in upper crustal $Q$ can significantly improve the fit to the data. The solutions show a general decrease in upper crustal attenuation of about $20 \%$ from $N$ to $S$ (the western profile shows the opposite). Moreover, the solutions with smaller smoothing weights achieve significant additional misfit reduction by introducing short-wavelength variations in upper crustal $Q$. The solution with $b=1.0$, a smoothing weight below which there is little additional variance reduction, achieves a $50 \%$ variance reduction over the onedimensional model and includes an upper crustal $\mathrm{Q}$ which locally fluctuates by a factor of about 2 , in excellent agreement with variations observed by Jacobson and Lewis [1990]. The range of upper crustal Q (17-88) is greater than range (20-50) observed by Jacobson and Lewis [1990], however, suggesting that the smoothing weight may be slightly too small.

\section{AXIAL Profile}

The 50-km-long axial profile (Figure 2.1) combines selected shots from the central region of densely spaced shots with shot lines extending to the north and 
south. Along most of its length, the line closely follows the crest of the rise axis, though at the southern end shots are offset appreciably to the east. Three instruments, two AOBHs and one OBS, were deployed on the rise axis. The central instrument, $\mathrm{AOBH}$ 3, suffered from stick-slip tape motion (Appendix A) while recording shots from the northern portion of the profile which prohibits accurate spectral estimates of $t^{*}$. Moreover, at ranges greater than $15 \mathrm{~km}$, records for shots to the south of AOBH 3 cannot be considered representative of axial structure, since the shots are displaced to the east of the rise axis, while the magma chamber is offset to the west in this region [Mutter et al., 1988]. This study is therefore limited to the instruments AOBH 5 and OBS 1.

Unfortunately the frequency responses of the AOBH and OBS (Figure 1.4) are not as broadband as the DOBH. In addition, the measured AOBH response has a high level of uncertainty, and $A O B H 5$ saturates at ranges below $10-15 \mathrm{~km}$ (Appendix A), while the OBS response may be affected by poor coupling to the seafloor. Therefore, the $t^{*}$ measurements obtained for the axial profile will have larger uncertainties than those for the off-axis profiles.

\section{Velocity Structure}

The velocity structure along the rise axis has been studied in detail by Vera et al. [1990] using ESP data centered at $9^{\circ} 34^{\prime} \mathrm{N}$, a location that coincides very closely to the center of the tomography experiment. The velocity model obtained is shown in Figure 2.18. The most prominent feature is a thin low velocity lens with $V_{P}=3.0$ $\mathrm{km} / \mathrm{s}$ and a $1.6-\mathrm{km}$-thick lid. The lens, which is interpreted as a magma body, is underlain by generally low velocities $(5.5 \mathrm{~km} / \mathrm{s})$ which extend to the base of the crust. The model also includes $\mathrm{Q}$ values of $80-100$ above the magma chamber. Examination of the most prominent Moho arrival in the explosive portion of the ESP data [Vera et al., 1990, Figure 12] shows that the phase velocity is lower than that predicted by the model. The data would be better modelled by lowering the velocities at the base of the Moho transition zone to about $7.25 \mathrm{~km} / \mathrm{s}$.

Figure 2.19 shows ray paths and travel times [Luetgert, 1988] predicted by a slightly modified version of the the model of Vera et al. [1990] which includes lower mantle velocities (Figure 2.18). Ray theoretical direct arrivals propagating above the magma chamber are limited to ranges less than $9 \mathrm{~km}$. At larger ranges shallow crustal ray paths are modelled by PP, PPP and PPPP phases which include one or more downward reflections from the seafloor, although non-ray-theoretical phases 
may arrive earlier. At ranges greater than $16 \mathrm{~km}$ a Moho-turning arrival is observed; between 16 and $20 \mathrm{~km}$ this arrival is characterized by a high-amplitude turning phase from the Moho transition zone.

Figure 2.20 shows record sections for AOBH 5 and OBS 1 which lend support to the general features of the velocity model. The times of the first arrival are overestimated by the ray-theoretical PP and PPP phases. Variations in amplitudes

of the first arriving phase on AOBH 5 may be explained by triplications in the PP and PPP phases that are offset slightly to higher ranges. The observed arrival times of the Moho phase is in excellent agreement with the model predictions. For AOBH 5 the Moho arrival has high amplitudes between 16 and $28 \mathrm{~km}$, while the Moho arrival recorded by OBS 1 is of lower quality since at larger ranges the peak-to-peak amplitudes are only a few digital units. A higher amplitude phase, delayed $0.75 \mathrm{~s}$ with respect to the Moho arrival and with a similar phase velocity, is also observed on OBS 1. Since this phase is also very prominent on the horizontal channels it may be a $\mathrm{P}$ to $\mathrm{S}$ conversion.

\section{t* Estimation}

The waveforms of the Moho arrivals have a monochromatic appearance that suggests a high degree of attenuation. Indeed, the amplitudes, while larger than those of the first arrival at similar ranges, are small considering the high velocity gradients within the Moho transition zone. $t^{*}$ estimates were obtained for both the shallow crustal and the Moho phases using $4 \pi$ prolate MWSA. A 0.6-s data window was used for the shallow crustal waveforms, though this was shortened for some of the AOBH 5 records to exclude saturated portions of the waveform. To increase the frequency band for $t^{*}$ estimation for the highly attenuated Moho phase, the minimum frequency was lowered to $5 \mathrm{~Hz}$ and data windows of $1.0 \mathrm{~s}$ and $0.75 \mathrm{~s}$ were used for AOBH 5 and OBS 1, respectively. Examples of $t^{*}$ estimates for the Moho phase are shown in Figure 2.21. It is immediately apparent that such estimates must be accompanied by a considerable level of uncertainty. The recorded power spectra have a step-like appearance which is a result of the high level of attenuation coupled with a notch in the source signature just above $10 \mathrm{~Hz}$. While the source-receiver correction results in a relatively good straight line fit for these examples, the $t^{*}$ estimate can be very sensitive to the frequency limits chosen, and the degree of uncertainty is large because of the limited frequency interval available for slope estimation. It is plausible that $t^{*}$ estimates obtained over such small 
frequency intervals may be biased due to frequency dependent focusing within the Moho transition zone. To investigate this phenomenum a number of reflectivity models [Fuchs and Müller, 1971] were calculated for simple velocity profiles incorporating a variety of gradients within the Moho transition zone. While the results cannot be used to quantify the effects of frequency-dependent focusing, since the velocity structure at the Moho is not known, they suggest that the biases are probably only a small fraction of the large $t^{*}$ values measured for this phase.

The $t^{*}$ estimates are shown in Figure 2.22. Shallow crustal phases show relatively low levels of attenuation that increase approximately linearly with range. The Moho phases have very high $t^{*}$ values that are about twice those of the crustal phase at the same range. The $t^{*}$ estimates for AOBH 5 are significantly lower than for OBS 1 . In the case of the shallow crustal phases this is probably primarily a result of uncertainties in the slope of the $\mathrm{AOBH}$ instrument response at high frequencies (Figure 1.4 and Appendix A), an effect that produces in a fixed offset to $t^{*}$ estimates obtained over a given frequency interval. For the Moho $t^{*}$ estimates, the discrepancy may also result from systematic biases in the source-receiver correction, a resonance in the OBS response, the low digital amplitude of the OBS recording, or spectral leakage in the $\mathrm{AOBH}$, a phenomenon resulting from a distorted recording of a monochromatic waveform that includes significant power at the frequencies of higher order harmonics (Appendix A).

\section{$Q^{-1}$ Inversion}

Since the wave paths are not very well known for either the upper crustal or Moho arrivals, the $t^{*}$ data are inverted using a simple two-layer model with the boundary at $1.6 \mathrm{~km}$ depth, the roof of the magma chamber. The results of inversions of $t^{*}$ from AOBH 5 and OBS 1 are shown in Figure 2.23, while the fit to the data is shown in Figure 2.22. Values for $Q$ in the lower layer are $60 \pm 10$ and $40 \pm 10$ for AOBH 5 and OBS 1 , respectively. Since the fit to $t^{*}$ values for the Moho phase is no better than the assumed errors, these uncertainties are probably realistic though they may not include the effect of all biases. The $Q$ value for the upper layer recorded by OBS $1,90 \pm 10$, is in excellent agreement with the value proposed by Vera et al. [1990]. This value is probably more reliable than the value of $200 \pm 40$ for AOBH 5 because of the possible offset in $t^{*}$ values for this instrument. Indeed, applying the instrument $t^{*}$ correction derived for $\mathrm{AOBH} 5$ during the inversion of the 
whole data set (Chapter 4) yields an upper layer $Q$ value close to the value obtained for OBS 1.

\section{SUMMARY}

In this chapter we have applied the spectral technique outlined in Chapter 1 to derive one-dimensional models of $\mathrm{Q}^{-1}$ from refraction profiles located $20 \mathrm{~km}$ off-axis and on the rise axis. Off-axis $t^{*}$ values are relatively invariant with range, an observation which requires that most of the attenuation be concentrated in the upper 1-1.5 km. The $t^{*}$ values require an average $Q$ of 30 in the upper $1 \mathrm{~km}$, in contrast with values in the range 500-1000 at mid-crustal depths. Our results show no evidence for high levels of attenuation in the gabbros which form layer 3, a feature which is included in an ultrasonic model of oceanic $\mathrm{Q}$ structure derived from ophiolite samples [Wepfer and Christensen, 1991]. The structure on the rise axis is markedly different. Near-surface levels of attenuation are markedly lower with average $Q$ values over the upper $1.6 \mathrm{~km}$ determined from OBS 1 of about 100. A Moho-turning phase yields very high $t^{*}$ values and suggests that average crustal $Q$ values beneath the roof of the axial magma chamber at $1.6 \mathrm{~km}$ depth are about 40-60. 


\section{Figure CaPTIONS}

Figure 2.1. Simplified Sea Beam bathymetric map (100-m contour interval, bold contours at 200-m intervals) showing the configuration of the three refraction profiles. Solid symbols show the locations of analogue ocean bottom hydrophones (triangles), digital ocean bottom hydrophones (squares), and an ocean bottom seismometer (circle), while explosive shots are shown as open circles.

Figure 2.2. Results of modeling travel times for the western refraction profile for shots (a) to the south of DOBH 10 and (b) to the north of DOBH 12. Observed travel times are shown as asterisks, while the predictions of the model are shown as a solid line. The increased scatter in travel time residuals observed at large ranges results from greater uncertainties in both the shot parameters and the water path correction as well as from crustal heterogeneities.

Figure 2.3. Two-dimensional velocity model for the western refraction profile obtained by forward modelling of travel times. The contour interval is $0.25 \mathrm{~km} / \mathrm{s}$ with solid contours every $1 \mathrm{~km} / \mathrm{s}$. The velocity model is parameterized at nodes (shown as pluses) which mark the boundaries between model layers [Luetgert, 1988]. The origin of the model coincides with location of the most northerly shot (Figure 2.1). The bathymetry along the profile has been removed from the model.

Figure 2.4. Travel time predictions (solid) and observations (asterisks) plotted against source-receiver range for the two-dimensional velocity model for the western profile that is shown in Figure 2.3.

Figure 2.5. Results of modeling travel times from the eastern profile for (a) DOBH 13 and (b) DOBH 14. The scatter in the travel time data is noticeably larger than for the western profile (Figure 2.2).

Figure 2.6. (a) Example of $2 \pi$ prolate MWSA $t^{*}$ estimation using a 0.7 -s-long window which includes the first $0.6 \mathrm{~s}$ of a waveform recorded by 
DOBH 10. (i) The waveform and the time windows used to obtain spectral estimates for the waveform (dashed) and a noise sample (dotted). (ii) The power spectrum obtained for the waveform (solid) and the noise sample (dashed). A vertical dashed line delineates the frequency interval used for $t^{*}$ estimation. (iii) The source-receiver correction calculated according to equation (1.24) (solid). The sourcereceiver correction obtained without smoothing is also shown for comparison (dotted). (iv) The source and receiver-corrected power spectrum (solid) and the least squares straight line fit used to obtain the $t^{*}$ estimate (dashed).

(b) As for (a) except $4 \pi$ prolate MWSA is used.

Figure 2.7. As for Figure 6 except the MWSA t* estimates are obtained from 0.4$\mathrm{s}$-long time windows which include the first $0.3 \mathrm{~s}$ of the wavefrom.

Figure 2.8. A comparison of DOBH $\mathrm{t}^{*}$ estimates obtained from the first $0.3 \mathrm{~s}$ of the waveform using both $2 \pi$ prolate (circles and dashed line) and $4 \pi$ prolate (crosses and solid line) MWSA. The origin of the plots corresponds to the most northerly shot of each profile. The receiver location is shown by a dotted line.

Figure 2.9. As for Figure 2.8 but showing $t^{*}$ estimates obtained using $4 \pi$ prolate MWSA for $0.3 \mathrm{~s}$ (crosses and solid line) and $0.6 \mathrm{~s}$ (circles and dashed line) of the waveform.

Figure 2.10. Record sections recorded by (a) DOBH 10 and (b) DOBH 12 for the western refraction profile; amplitudes are scaled linearly with range for display purposes. The apparently lesser high-frequency content of the records from $\mathrm{DOBH} 10$ is a result of the greater low-frequency response of this instrument (Appendix A).

Figure 2.11. DOBH t* estimates and relative uncertainties obtained using $4 \pi$ prolate MWSA of $0.3 \mathrm{~s}$ of the waveform. 
Figure 2.12 Ray paths for DOBH 10 and 12 obtained using the two-dimensional velocity model shown in Figure 2.2 [Luetgert, 1988]. The ray paths have been corrected for bathymetry along the profile.

Figure 2.13. Results of smoothest model inversions of data for each DOBH for one-dimensional vertical $\mathrm{Q}^{-1}$. The left hand plots show the normalized squared data misfit (equation 1.40) as a function of the smoothing weight. The right hand plots show $\mathrm{Q}^{-1}$ profiles obtained for five choices of smoothing weight, $b=10^{4}$ (dashed), 1000 (dotted), 100 (solid), 10 (dot-dashed), and 1 (dashed).

Figure 2.14. Linearized formal uncertainty and spread for the smoothest model inversion of data for DOBH 13 using smoothing weights of (a) $b=100$, (b) $b=10$, (c) $b=1$, and (d) $b=0.1$. The left hand plots show the $Q^{-1}$ profile (solid) together with the formal uncertainty (dashed). The right hand plots show the spread (equation 1.42), which is a measure of the formal spatial resolution. The spread has been normalized to give a value of unity for a 1-km-wide boxcar.

Figure 2.15. Results of two-layer $\mathrm{Q}^{-1}$ inversions for each DOBH. The left hand plots show the normalized squared data misfit (equation 1.40) as a function of upper layer thickness. A dashed line shows $95 \%$ confidence levels predicted by an F-test using the minimum misfit. The left hand plot shows the upper layer (solid) and lower layer (dashed) $\mathrm{Q}^{-1}$ values predicted by the model together with the formal uncertainties (dotted).

Figure 2.16. Comparison of $t^{*}$ observations for the eastern line with the predictions of a two models: a smoothest model $(b=10)$ solution for a laterally invariant $\mathrm{Q}^{-1}$ structure (open circles) and an inversion solution that includes lateral inhomogeneity in the upper crust (asterisks) (Figure $2.17, b=0.1$ ).

Figure 2.17. Results of an inversion for lateral variations in $Q^{-1}$ in the upper 0.625 $\mathrm{km}$ for the eastern refraction profile. (a) Starting $\mathrm{Q}^{-1}$ model obtained 
from a smoothest model $(b=10)$ inversion for vertical $Q^{-1}$ structure of $t^{*}$ estimates for DOBH 10 and 12. (b) A plot of the normalized squared data misfit against smoothing weight for the inversion for upper crustal $Q^{-1}$. (c) The upper crustal $Q^{-1}$ model plotted for four different choices of smoothing weight, $b=100$ (solid), 10 (dashed), 1 (dot-dashed) and 0.1 (solid).

Figure 2.18 The axial P-wave velocity model obtained by Vera et al. [1990] (solid) and the modified version used to calculate ray paths and travel times (dashed).

Figure 2.19 (a) Ray paths and (b) travel times for the slightly modified version of the velocity model of Vera et al. [1990] (Figure 2.18). Direct phases are shown as solid lines, PP phases as dashed lines, and PPP and PPPP phases as dotted lines.

Figure 2.20 Record sections for (a) AOBH 5 and (b) the vertical channel of OBS 1; amplitudes are scaled linearly with range for display purposes. The predicted arrival time of the Moho-turning phase is shown by a solid line.

Figure 2.21 Examples of $t^{*}$ estimates for the Moho-turning phase for (a) AOBH 5 and (b) OBS 1. The plot descriptions are the same as for Figure 2.6.

Figure 2.22 $t^{*}$ estimates and relative uncertainties obtained for the shallow crustal (solid) and Moho (dashed) waveforms. The predictions of the two layer models shown in Figure 2.23 are shown as asterisks.

Figure 2.23 Two-layer $\mathrm{Q}^{-1}$ models obtained from the axial $\mathrm{t}^{*}$ data (solid) and the formal uncertainties (dashed) for (a) AOBH 5 and (b) OBS 1. 


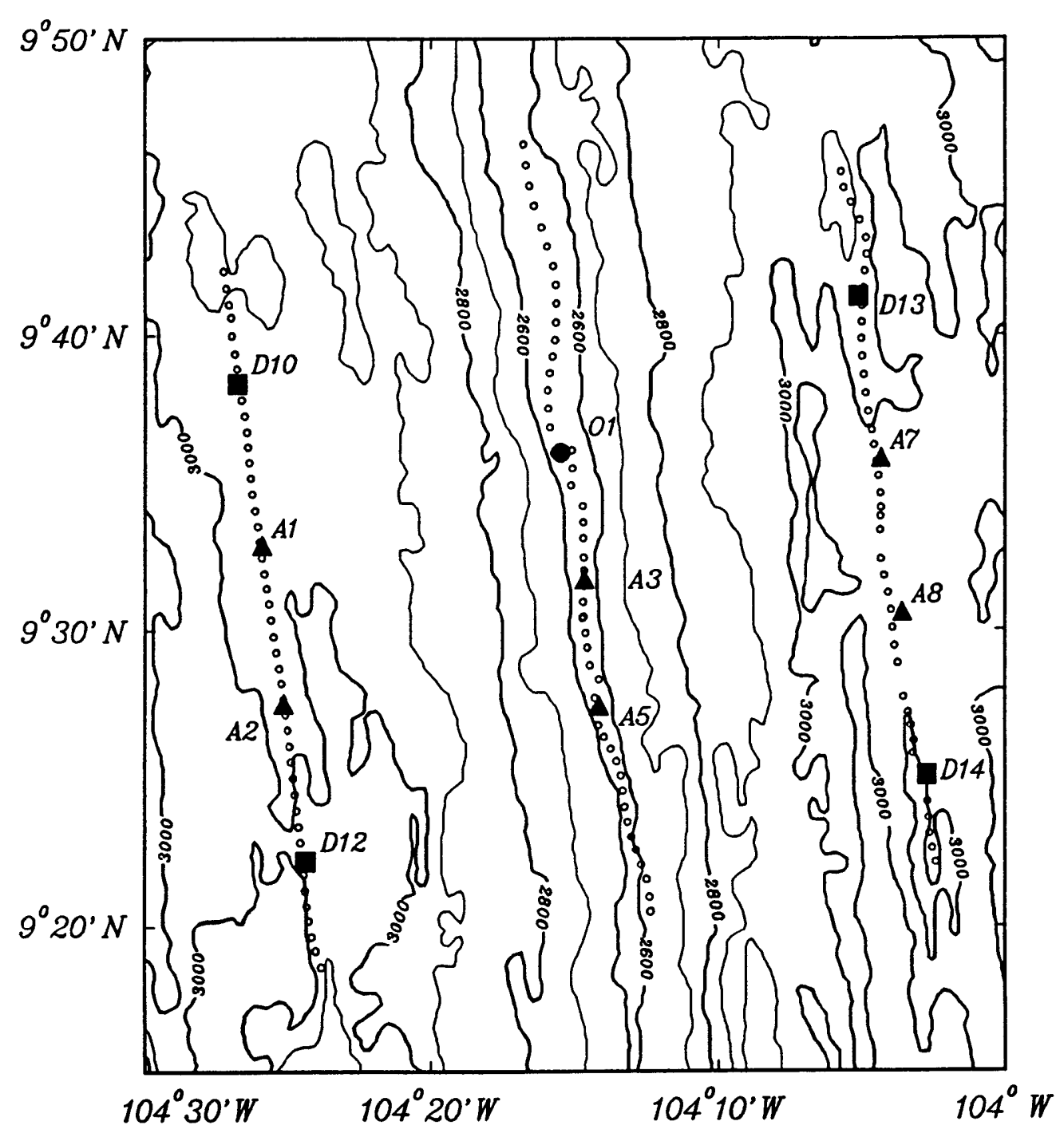

Figure 2.1 
(a)

DOBH 10 South
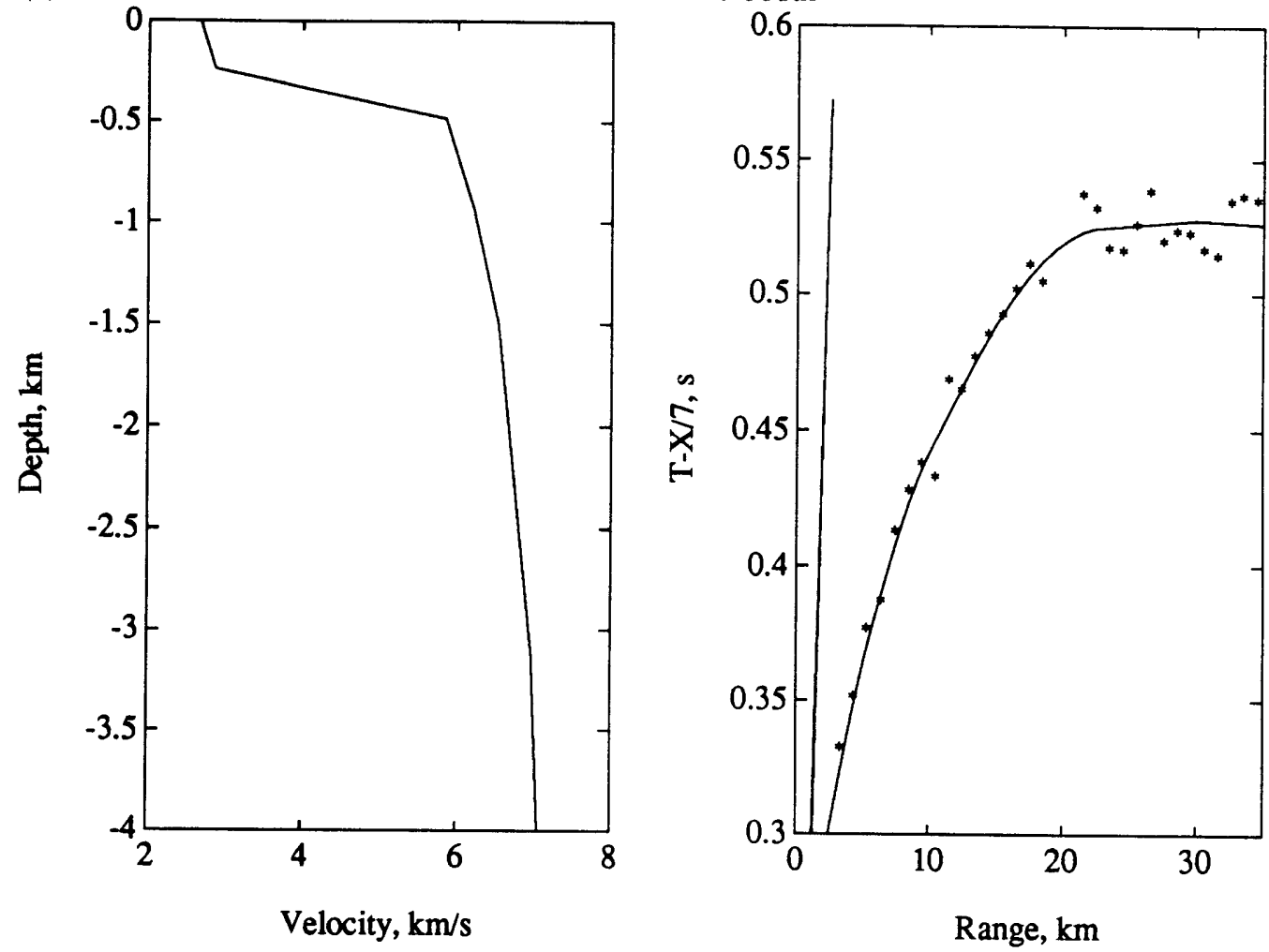

(b)

DOBH 12 North
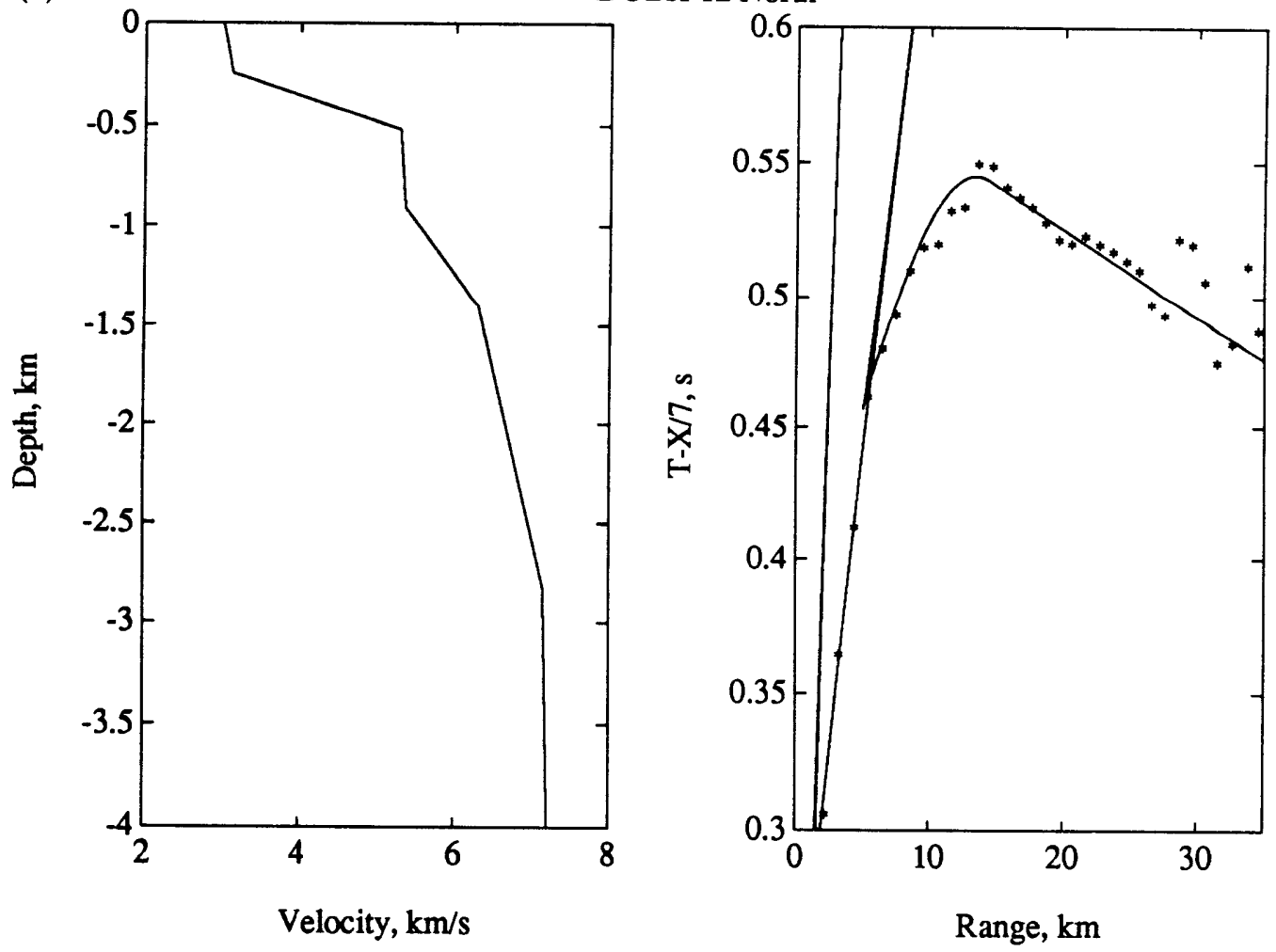

Figure 2.2 


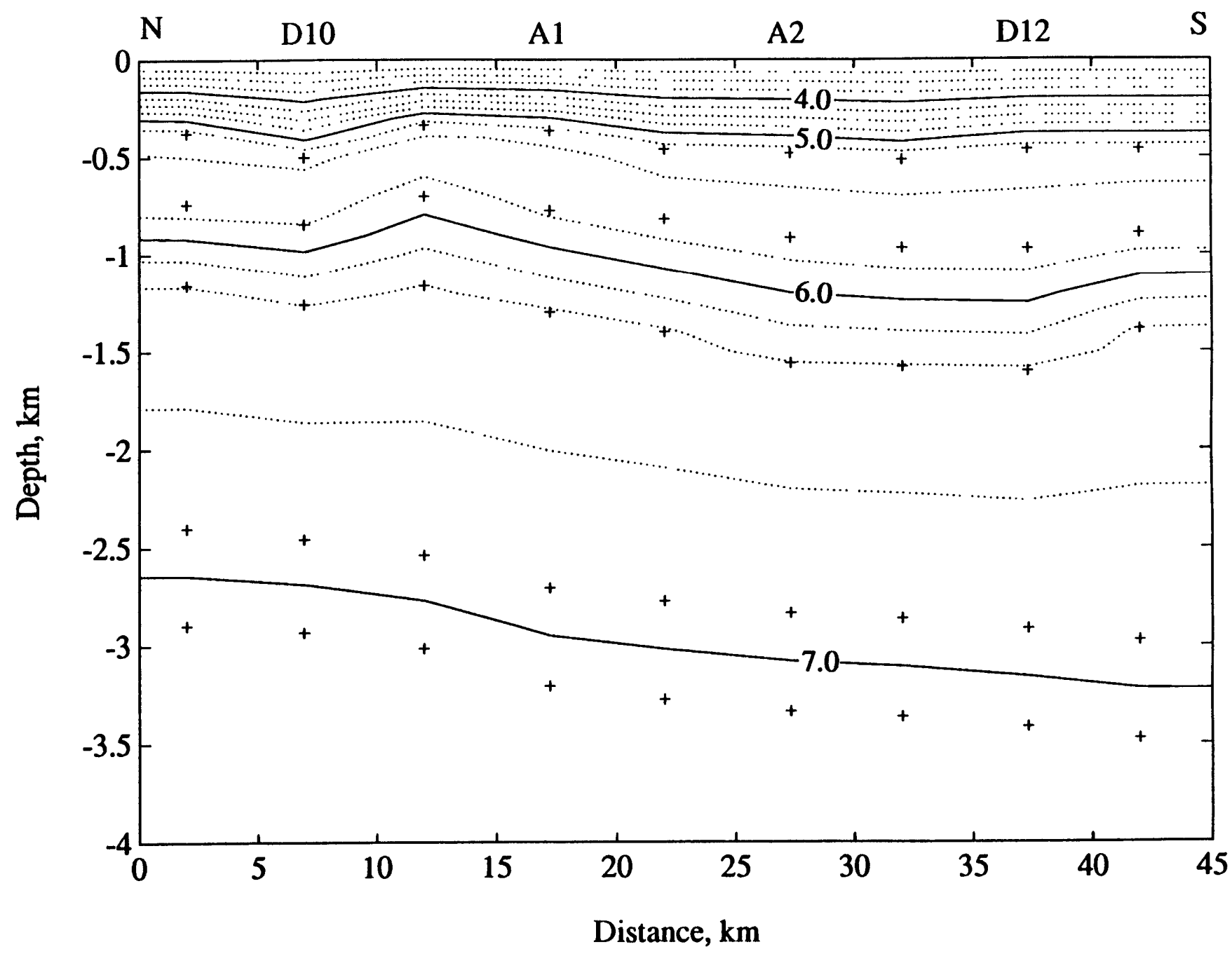



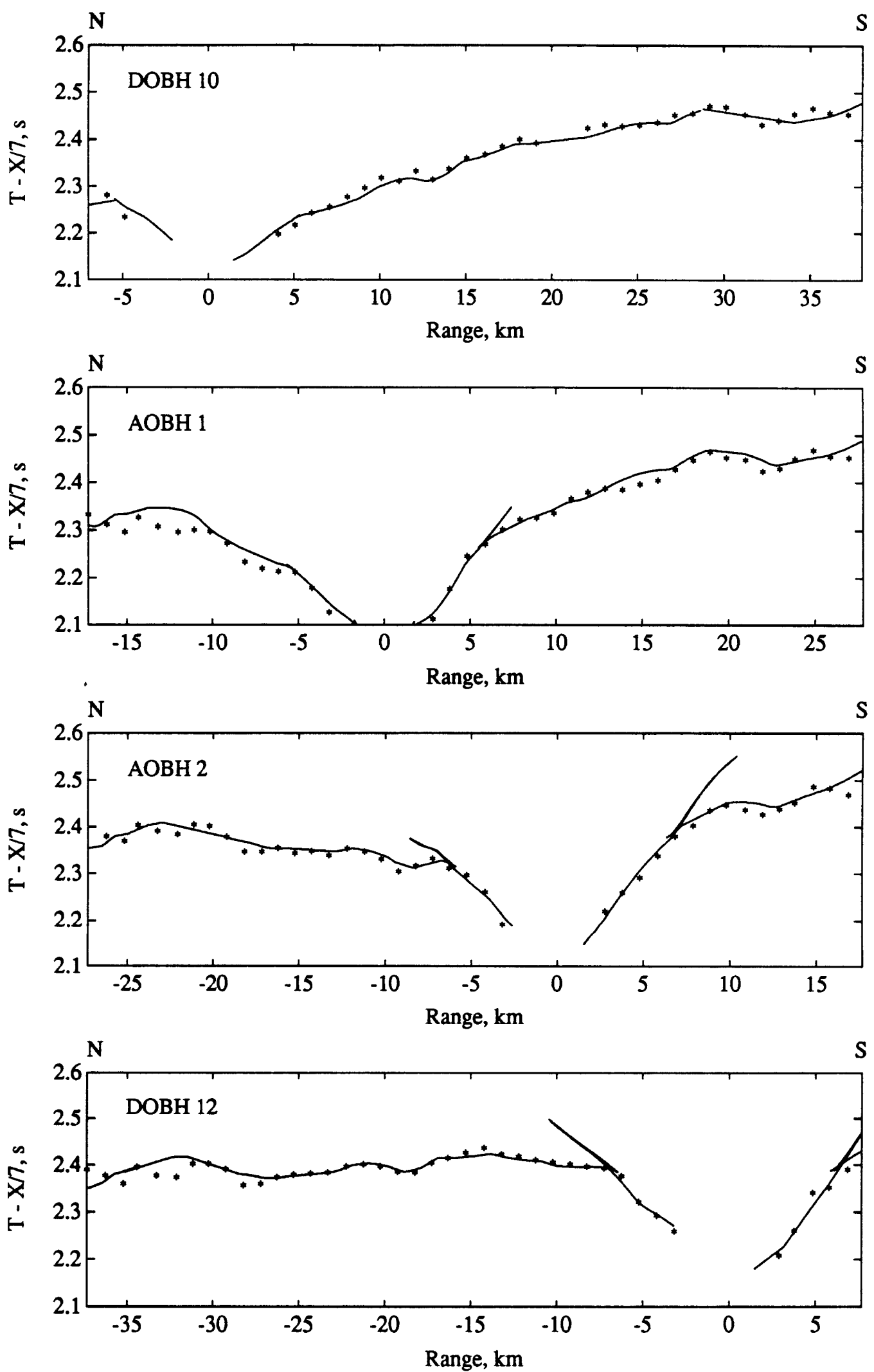

Figure 2.4 
(a)

DOBH 13 South
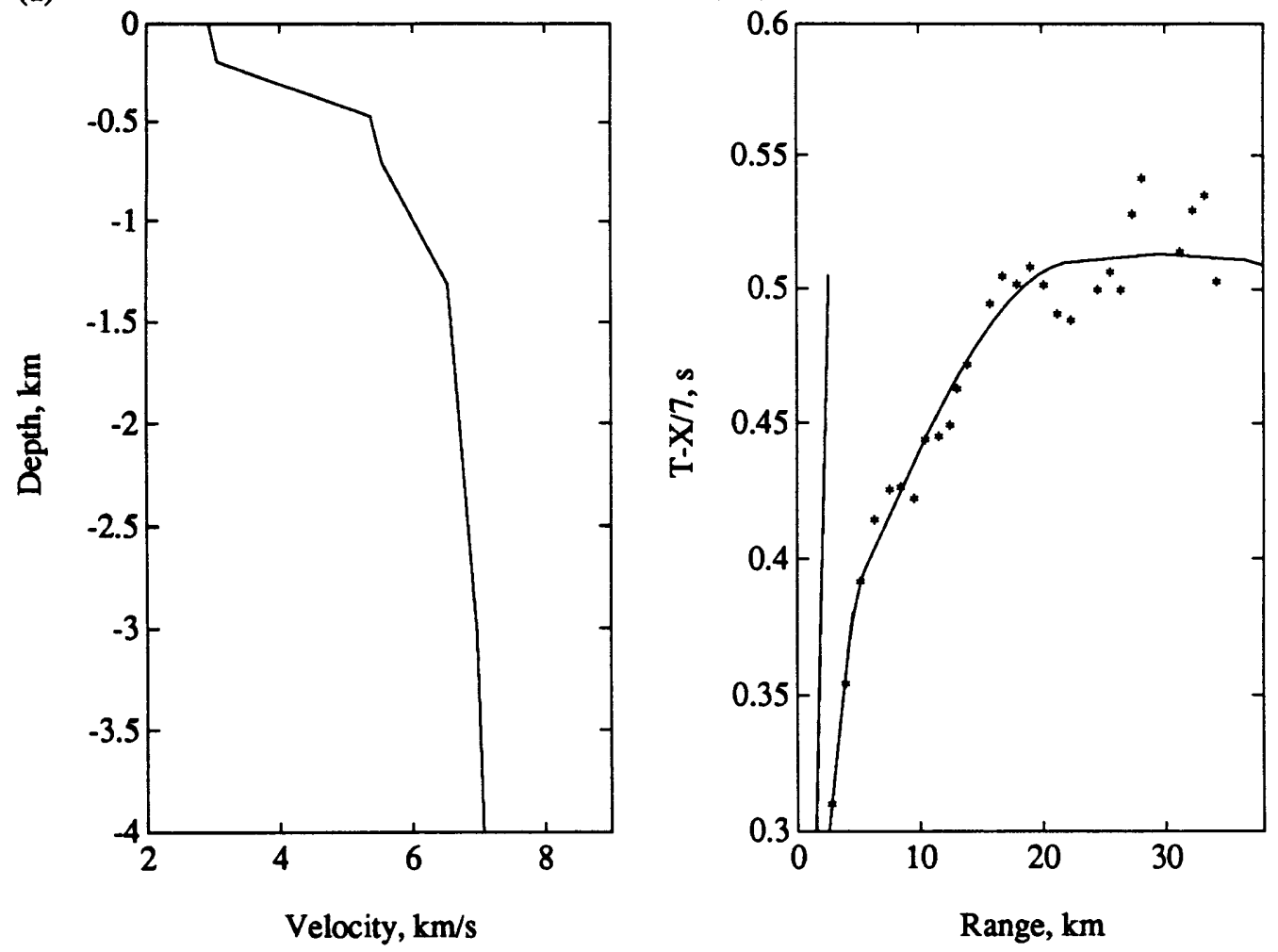

(b)

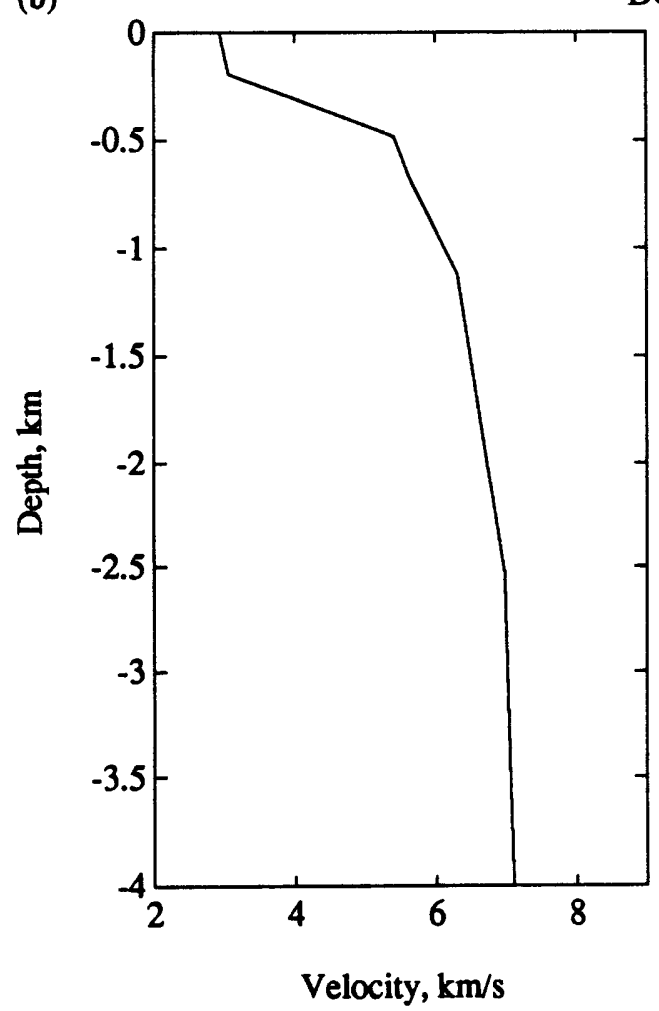

DOBH 14 North

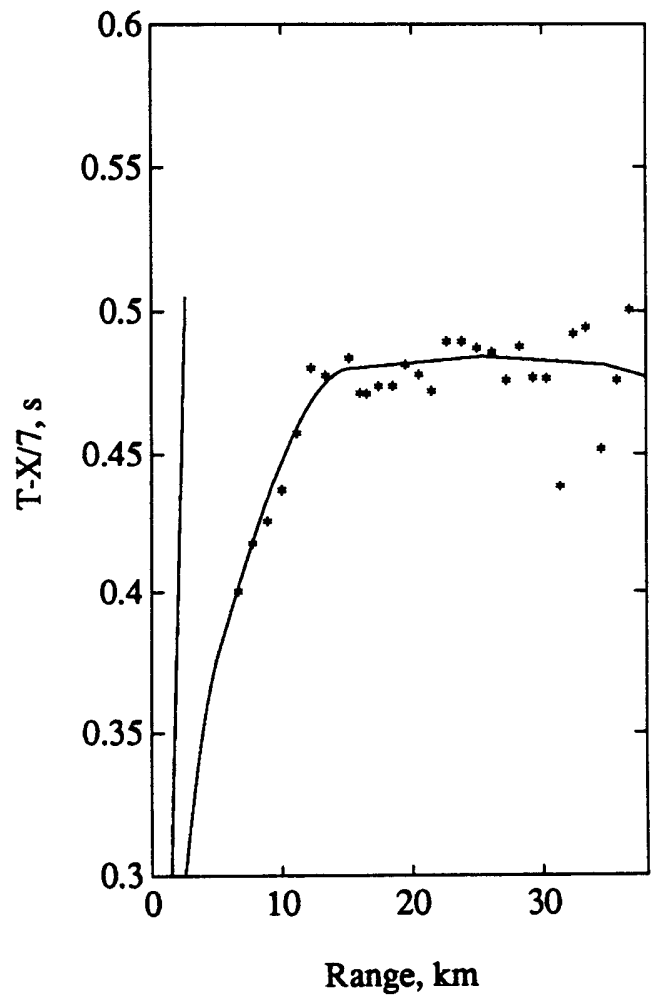

Figure 2.5 
(a)
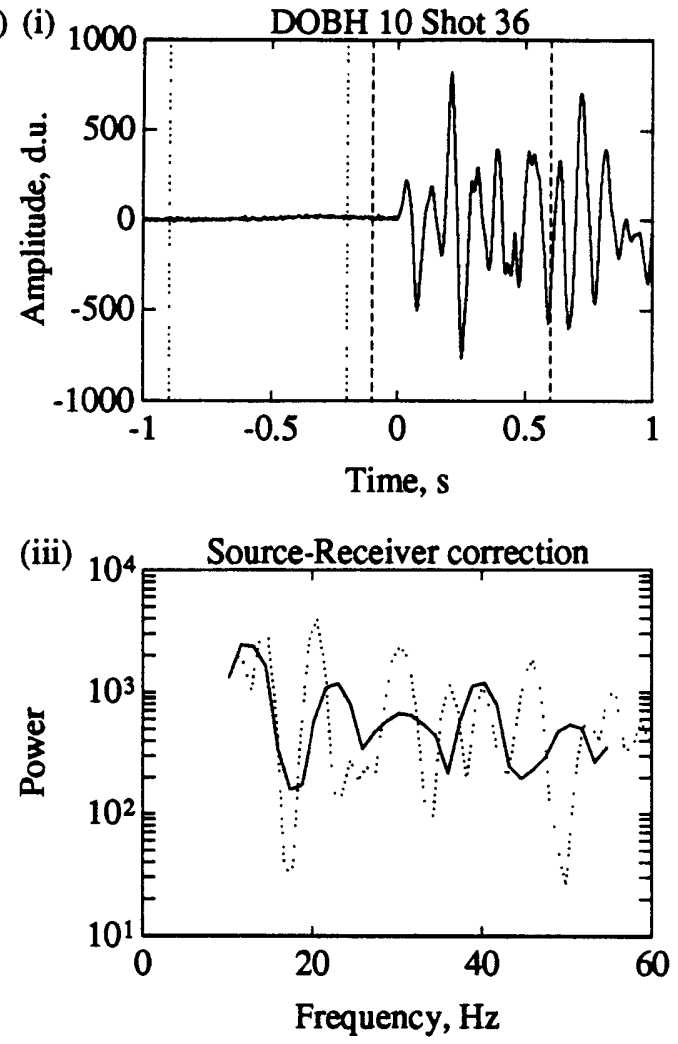

(b) (i)
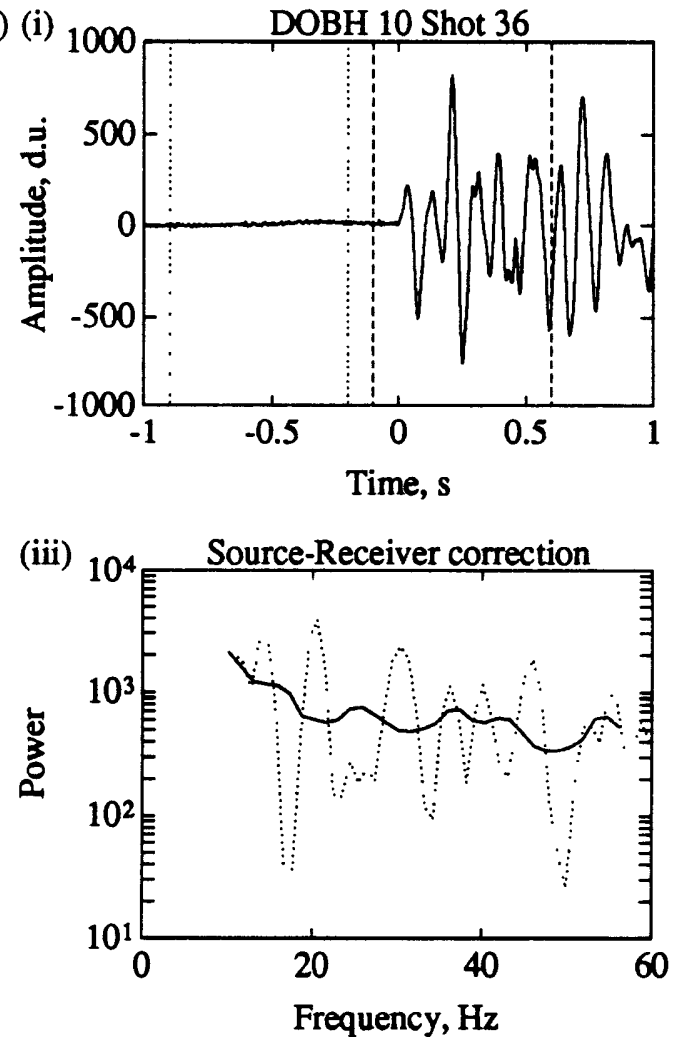
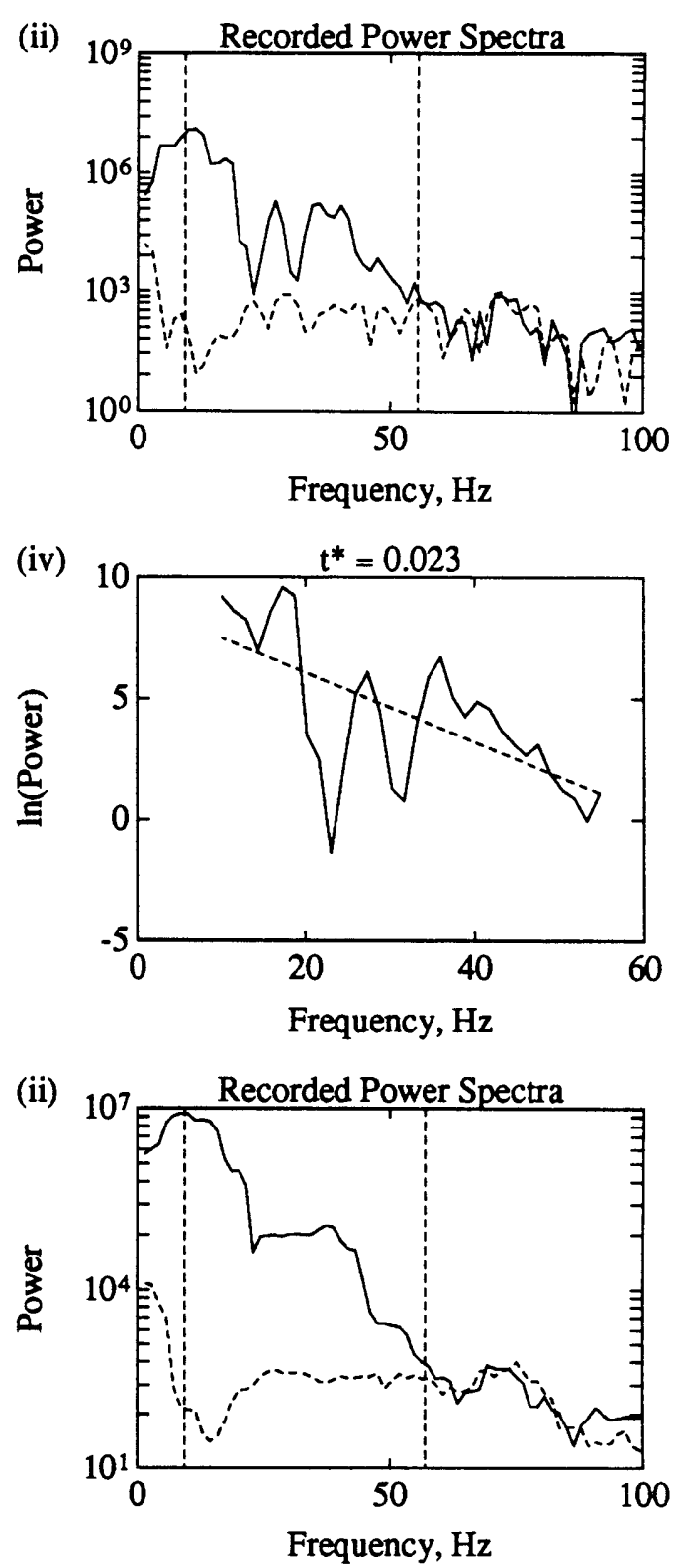

(iv)

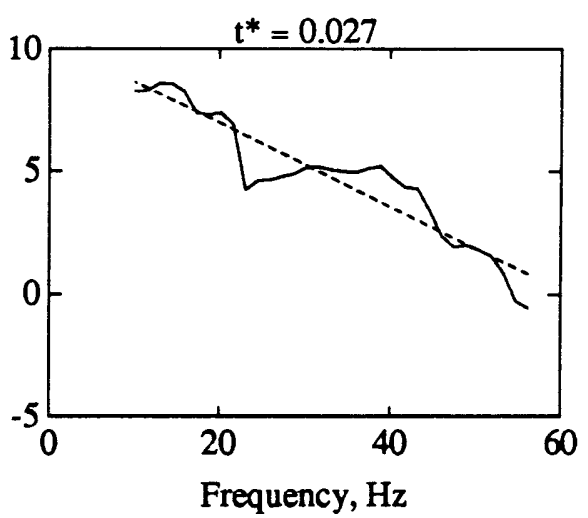

Figure 2.6 
(a) (i) $2000 \quad \mathrm{DOBH} 10$ Shot 30
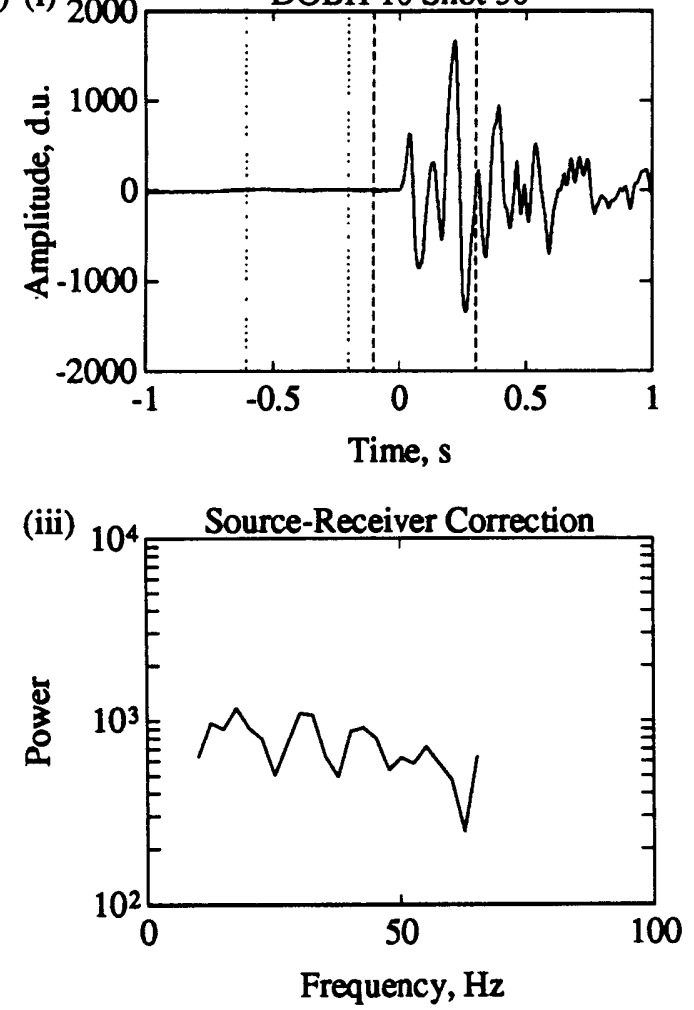

(b)

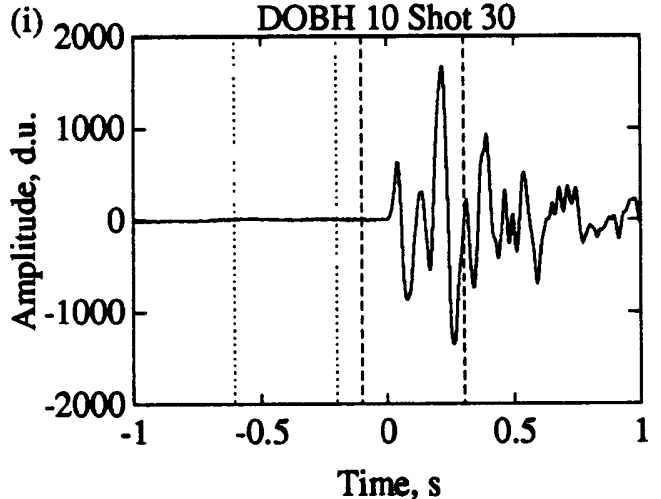

(iii)

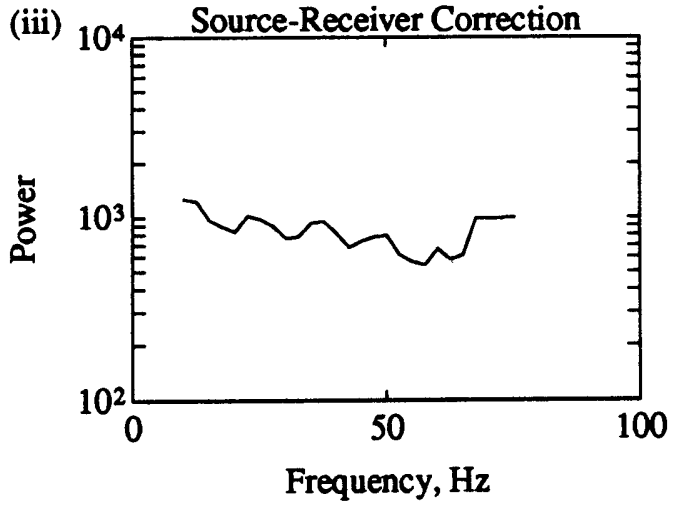

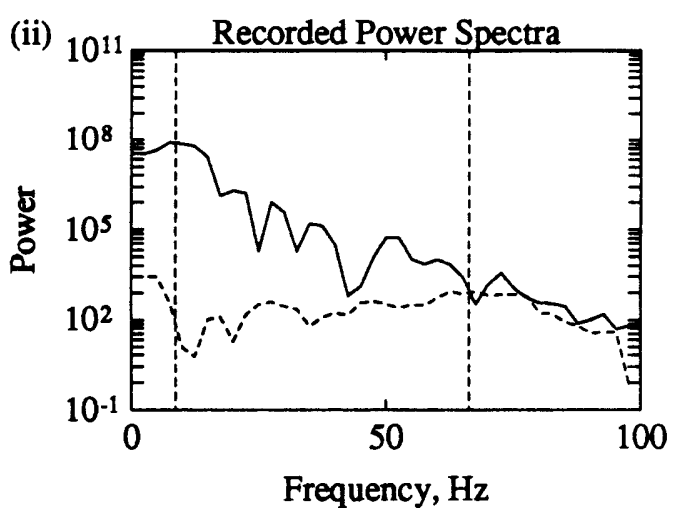
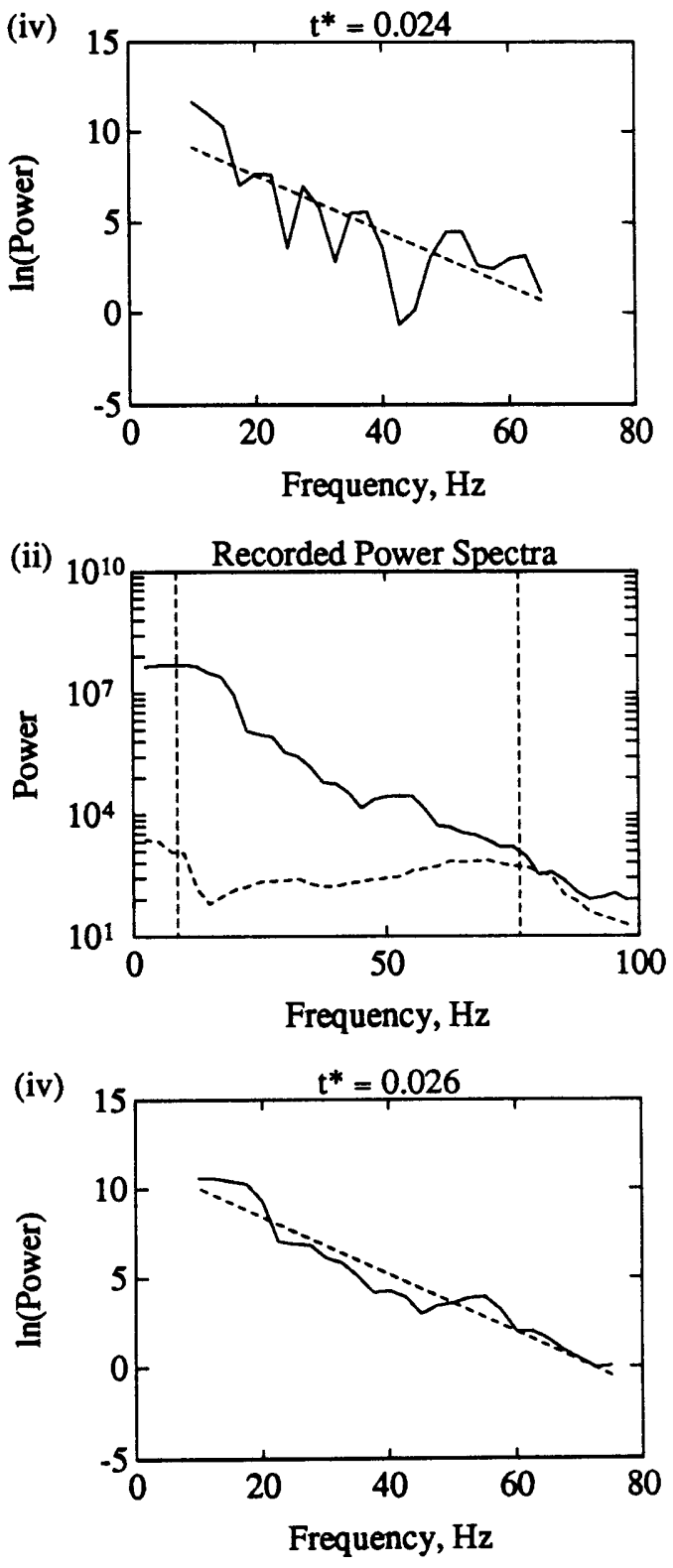

Figure 2.7 

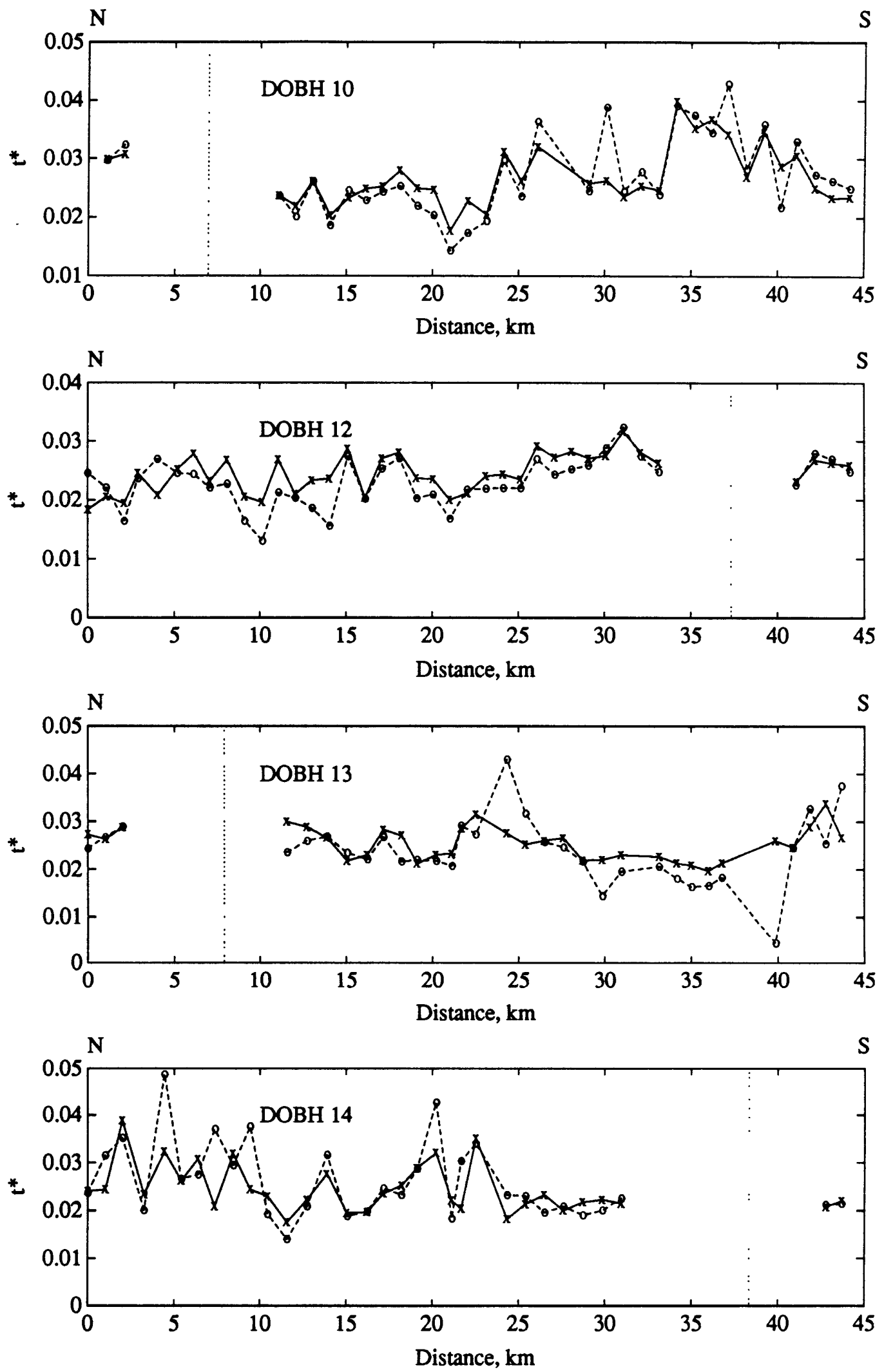

Figure 2.8 

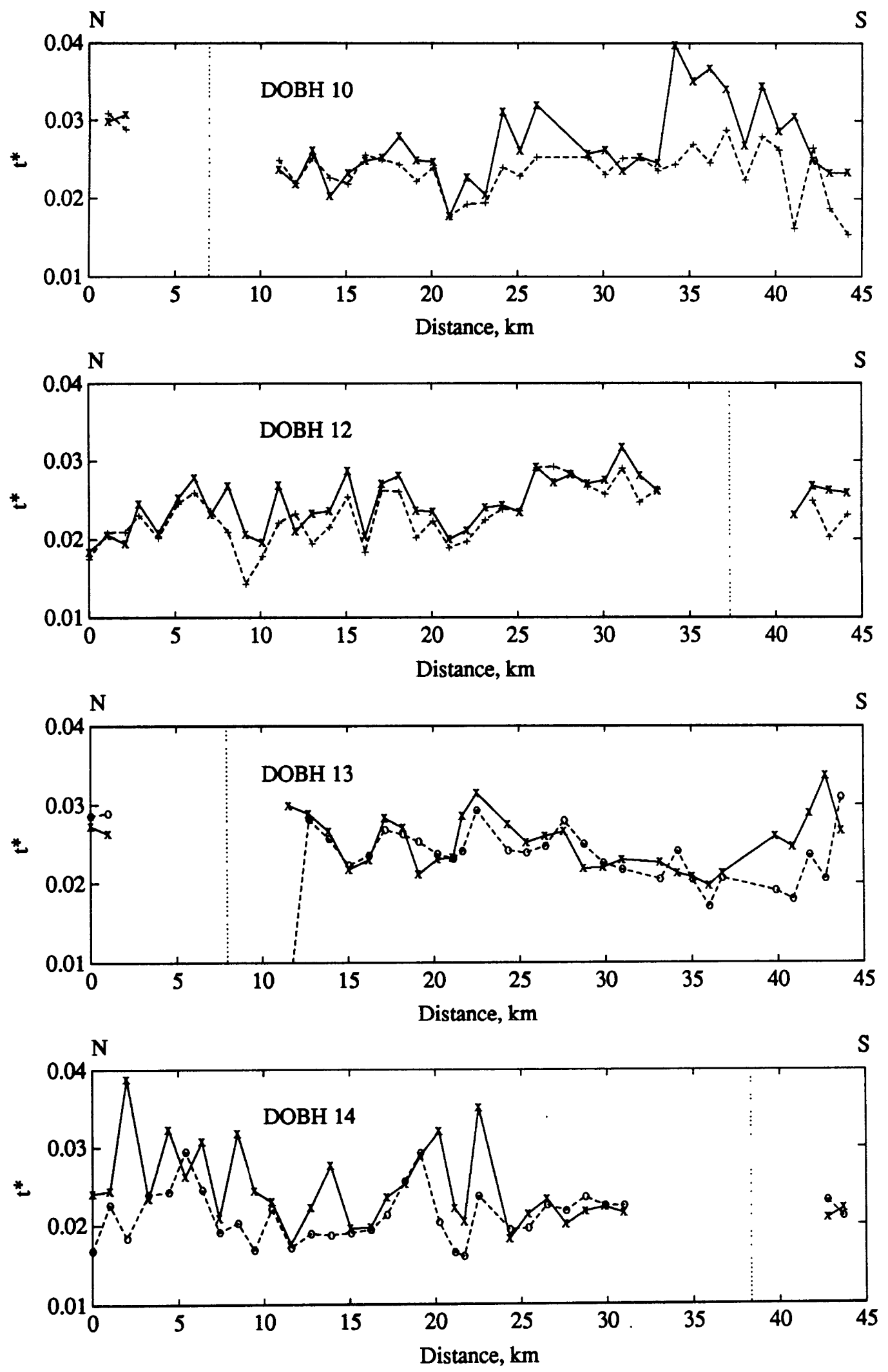

Figure 2.9 
(a) $\mathrm{N}$ DOBH 10 South $\mathrm{S}$
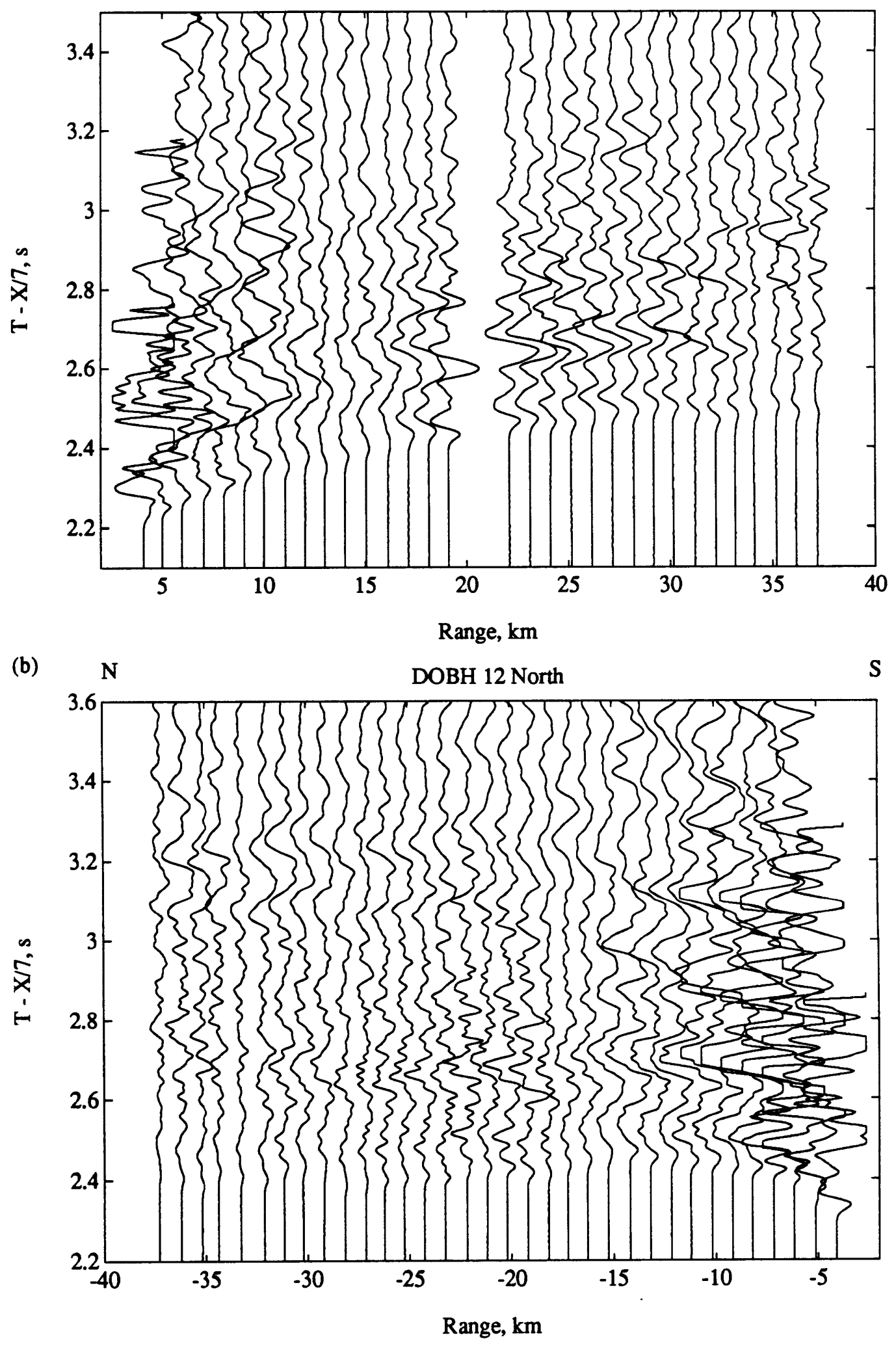

Figure 2.10 

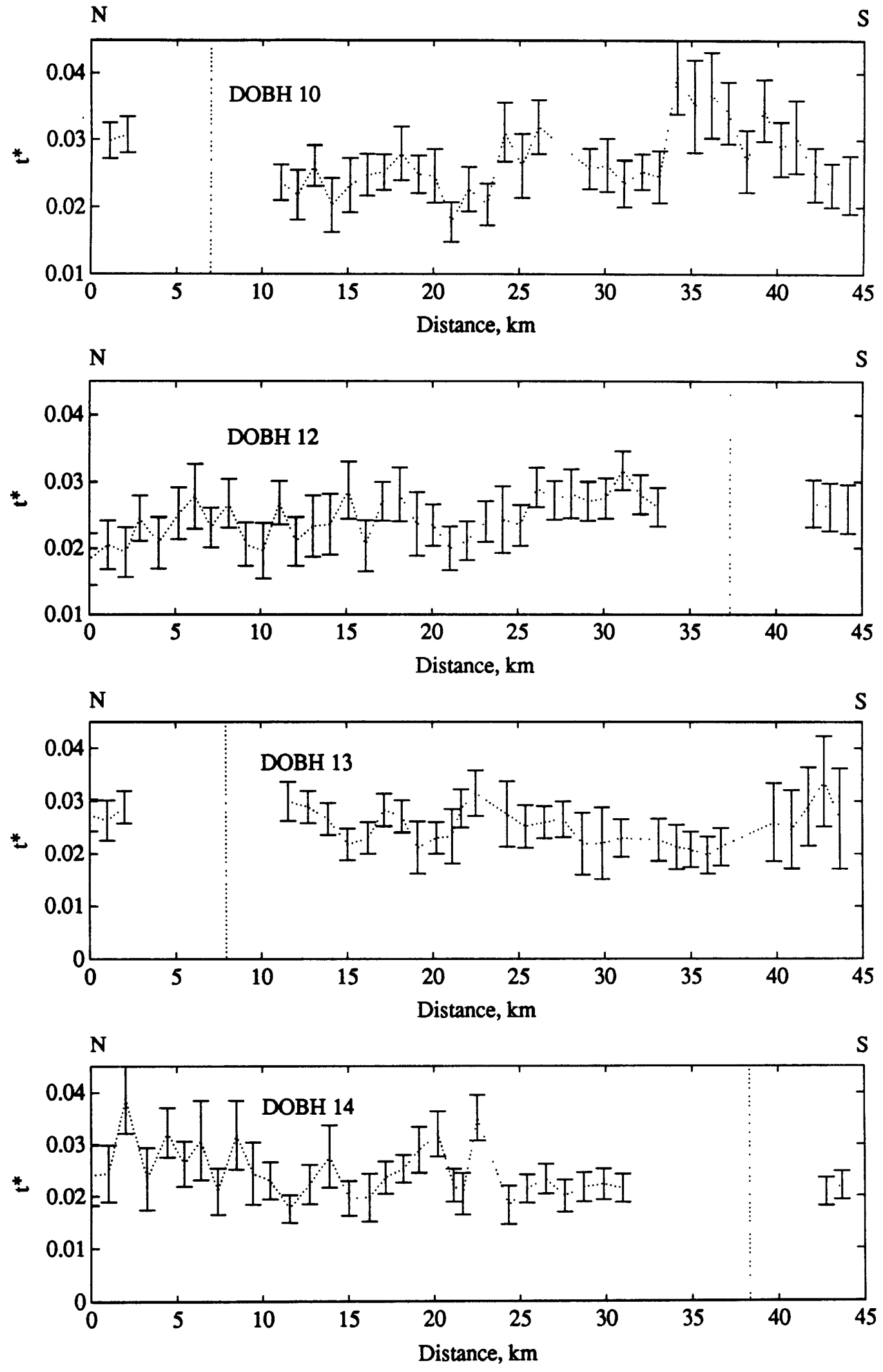

Figure 2.11 


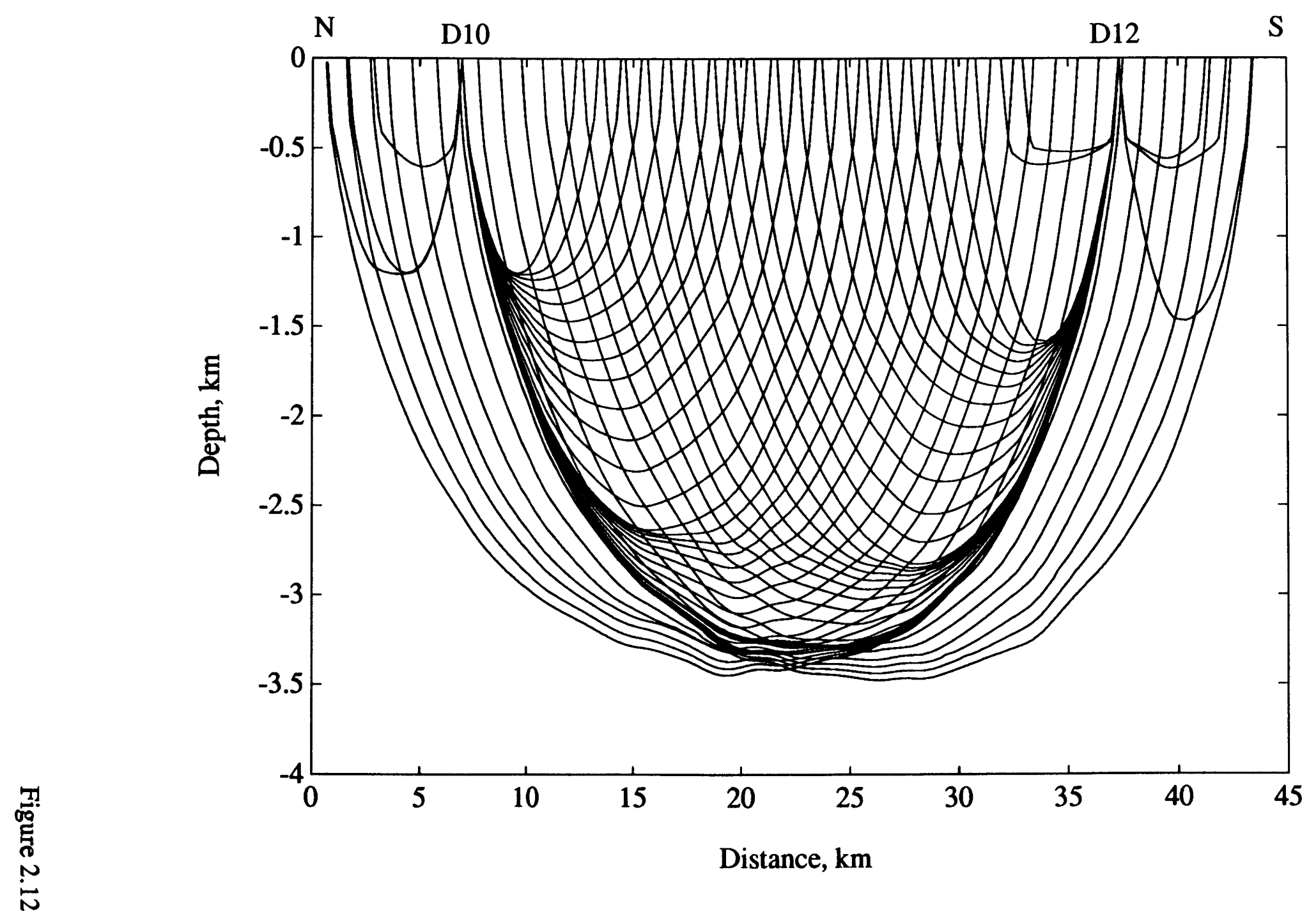



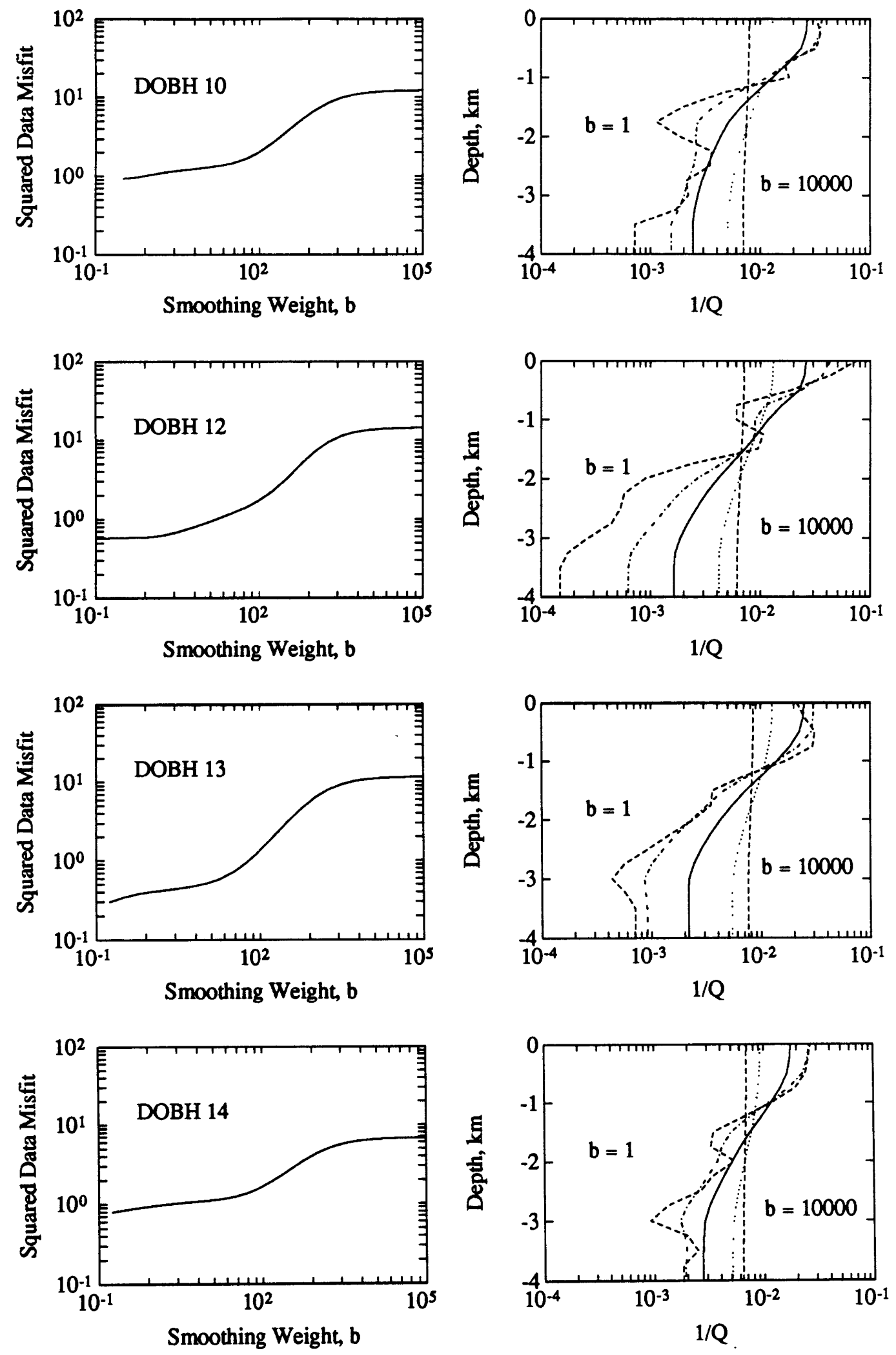

Figure 2.13 

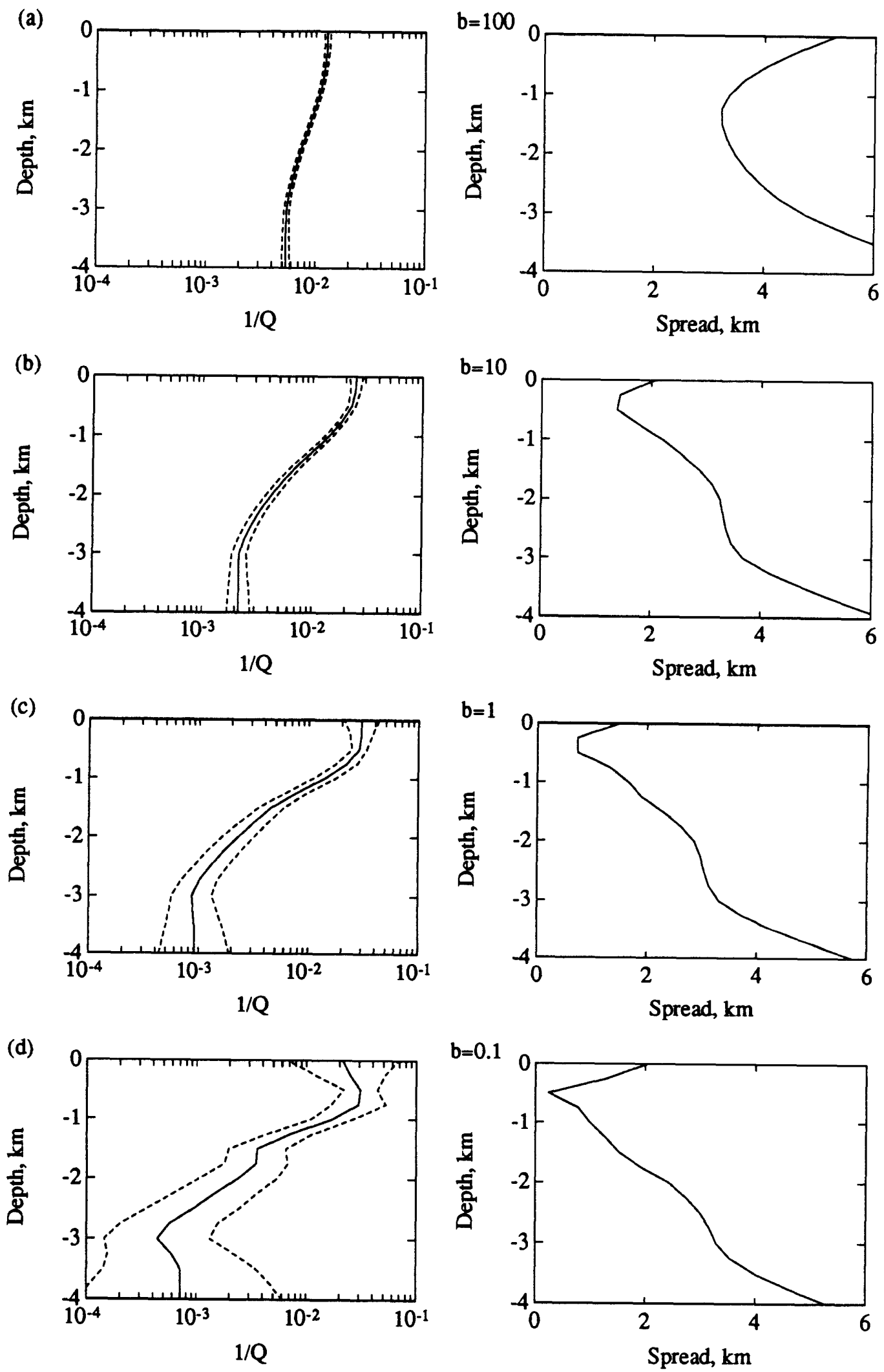

Figure 2.14 

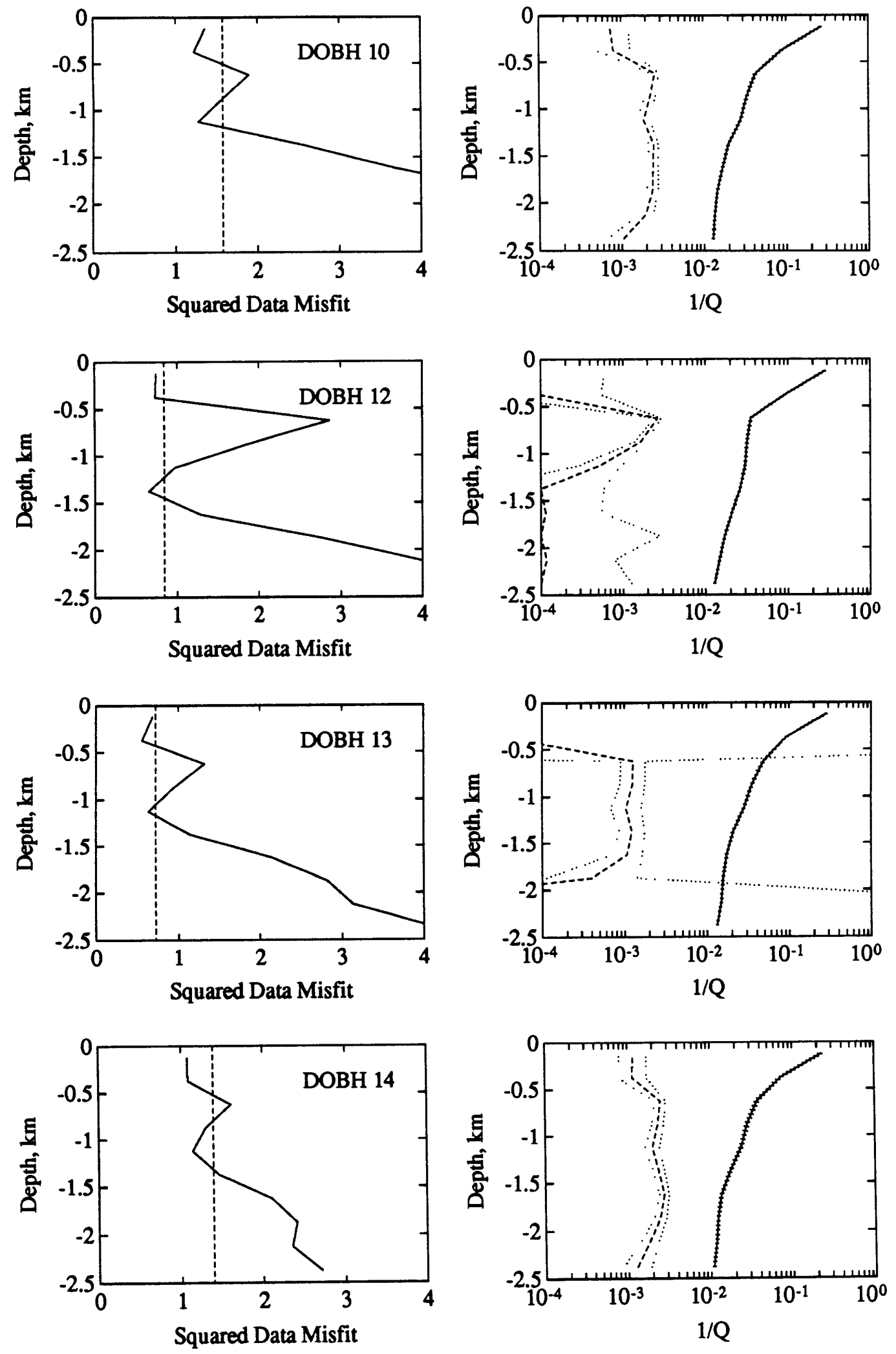

Figure 2.15 


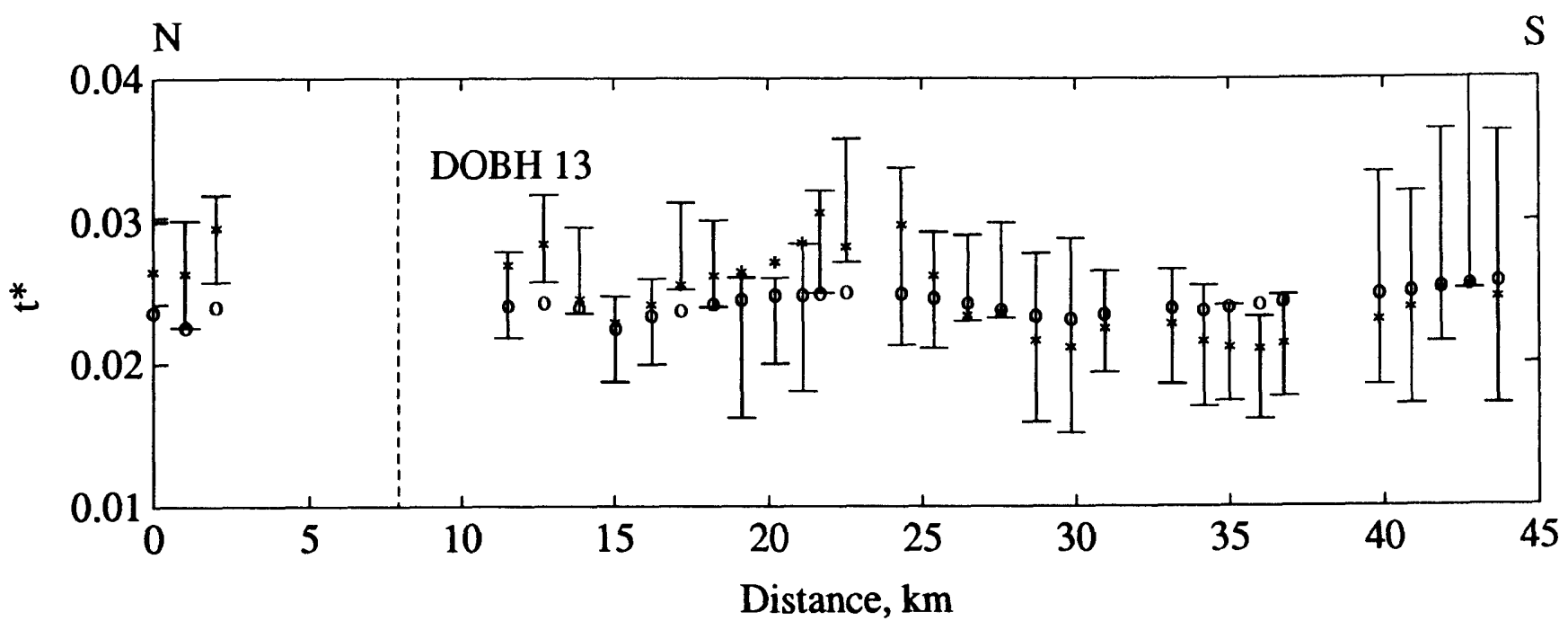

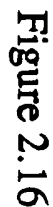

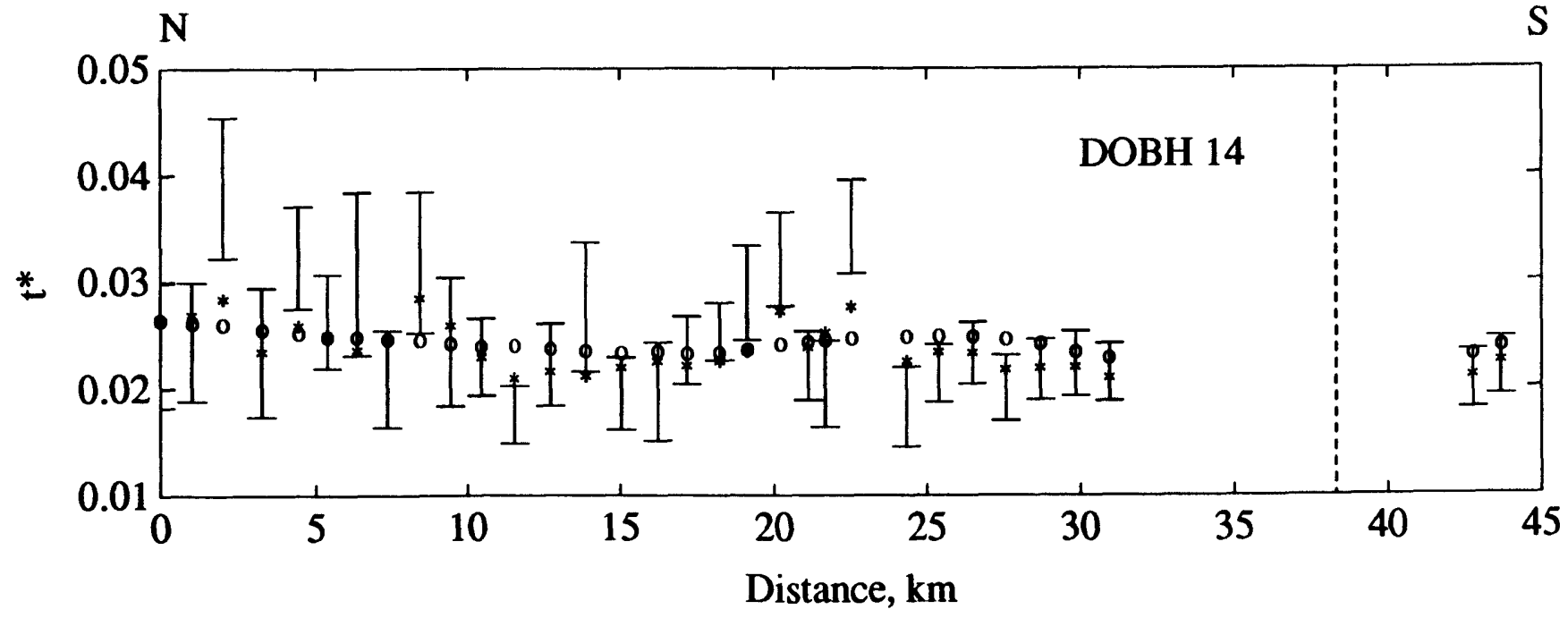



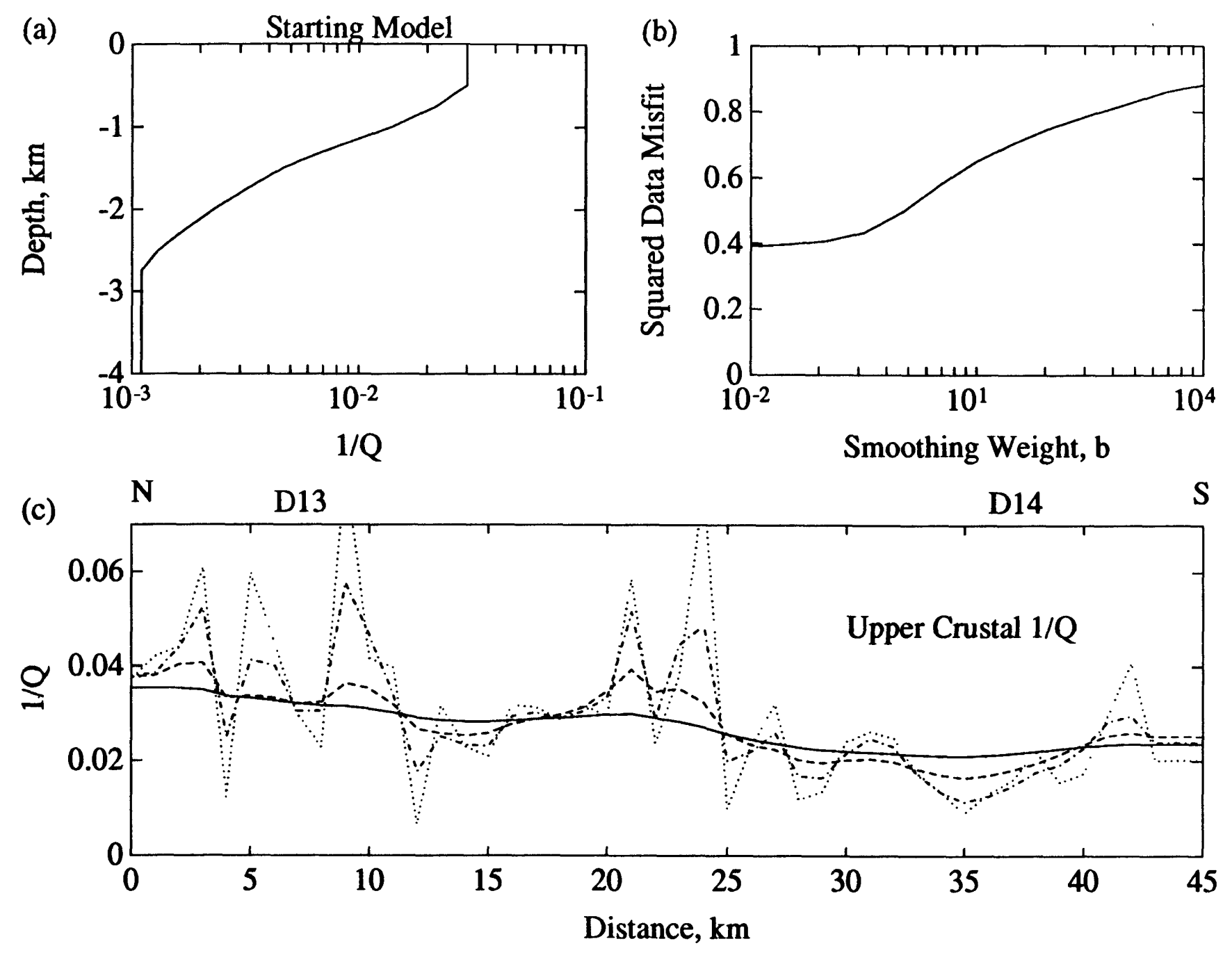


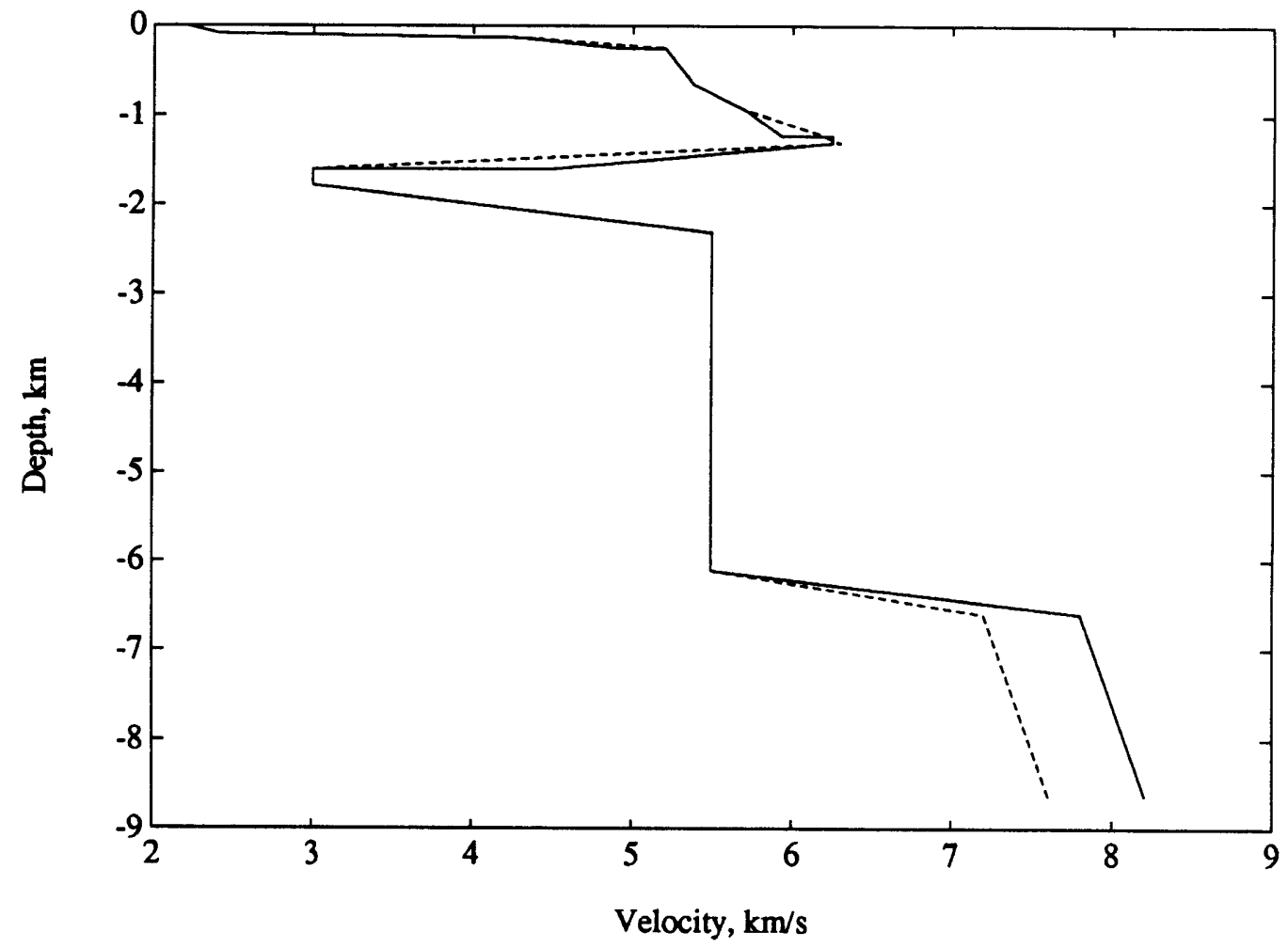

Figure 2.18 

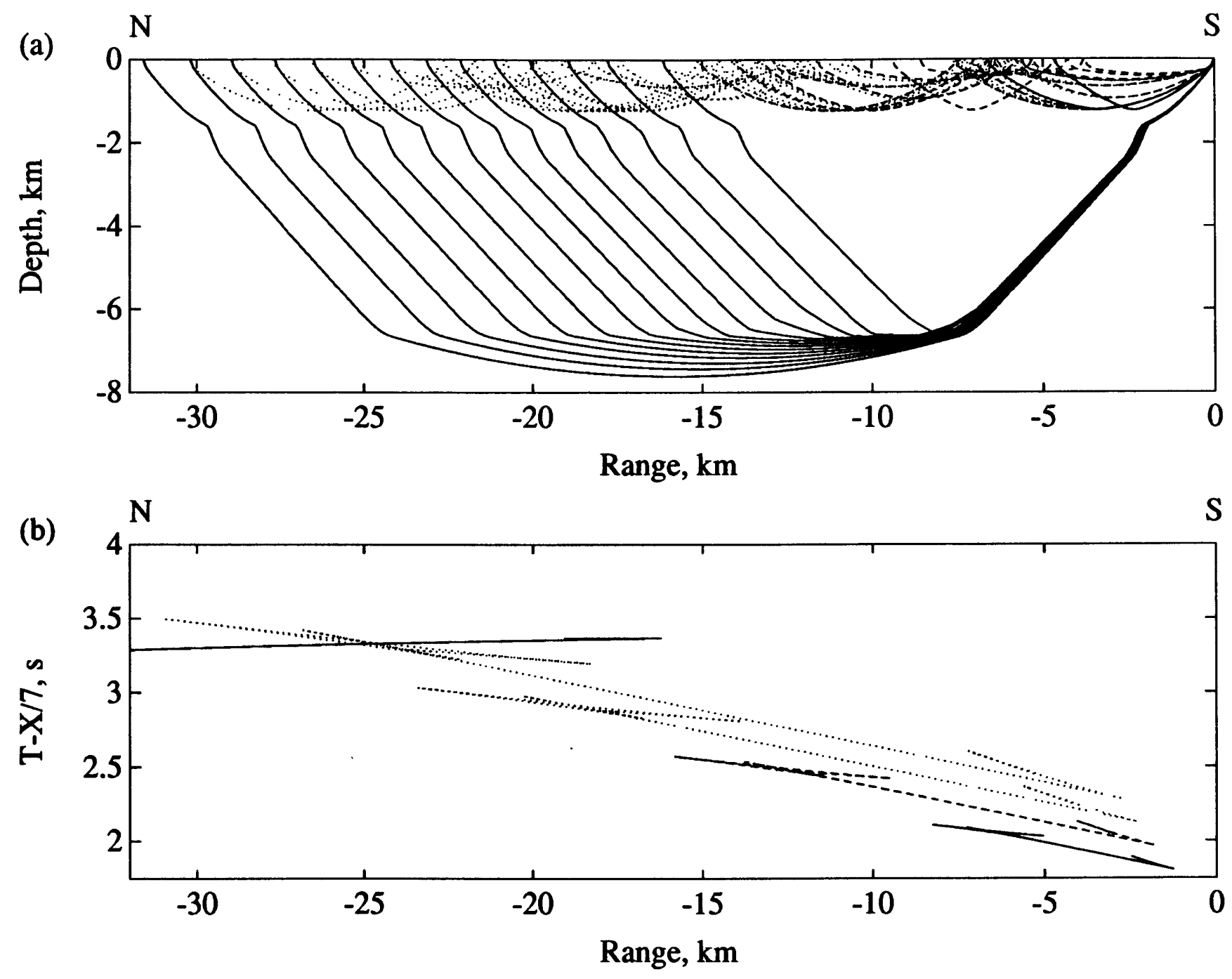
(a)

AOBH 5

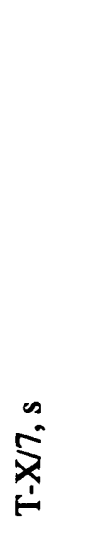

\section{5}

\section{Noms}

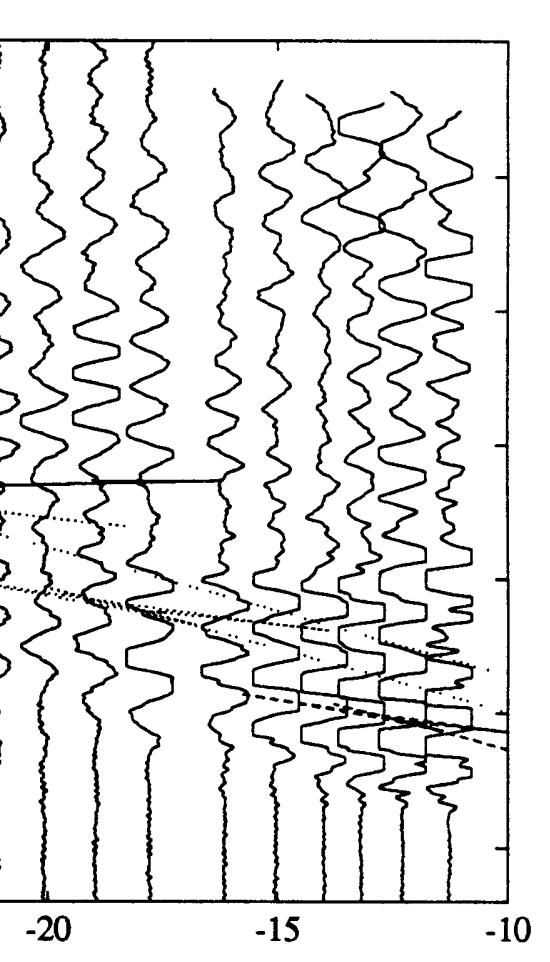

\section{Range, km}
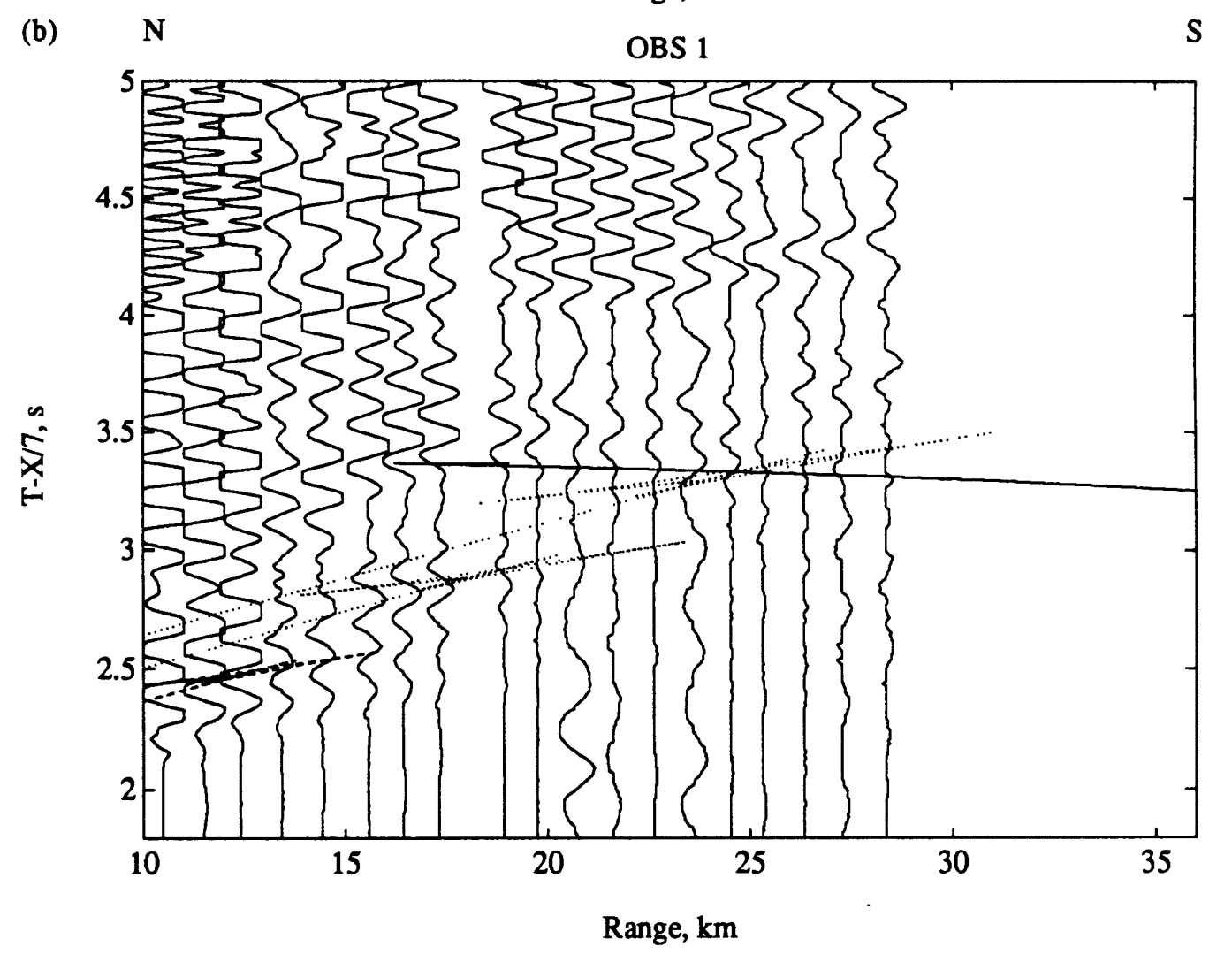

Figure 2.20 
(a) (i)
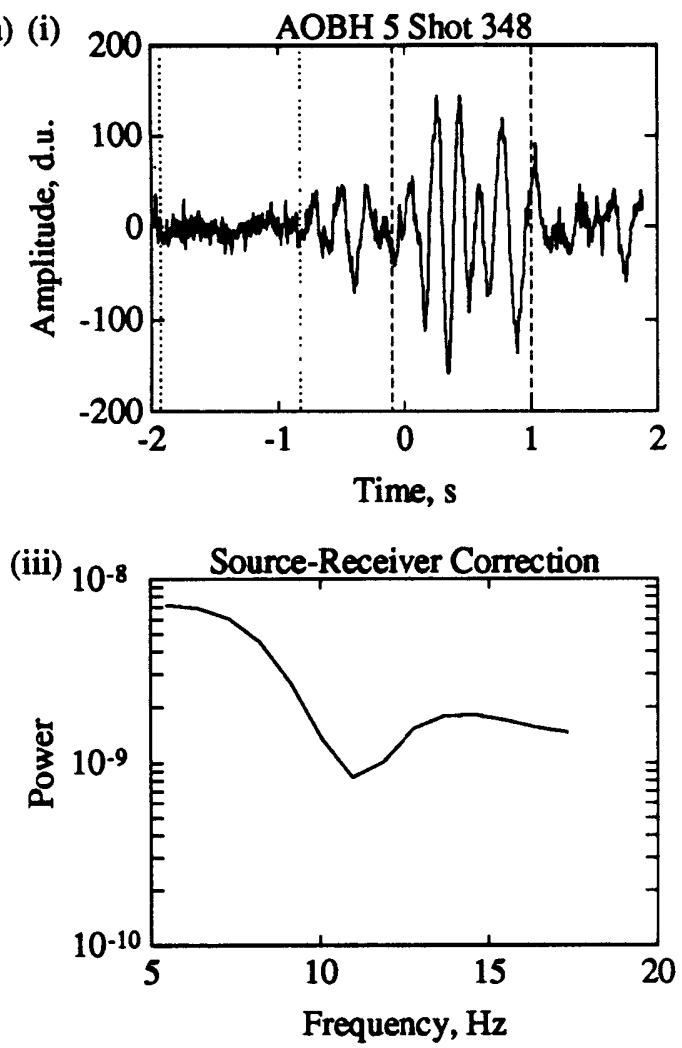

(b) (i)
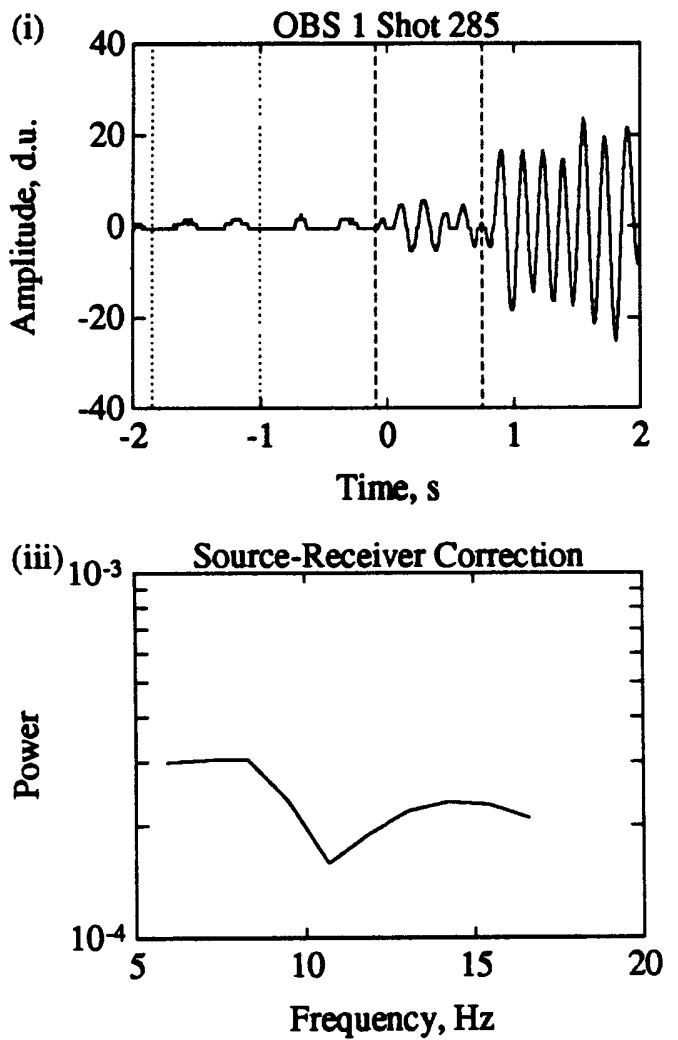
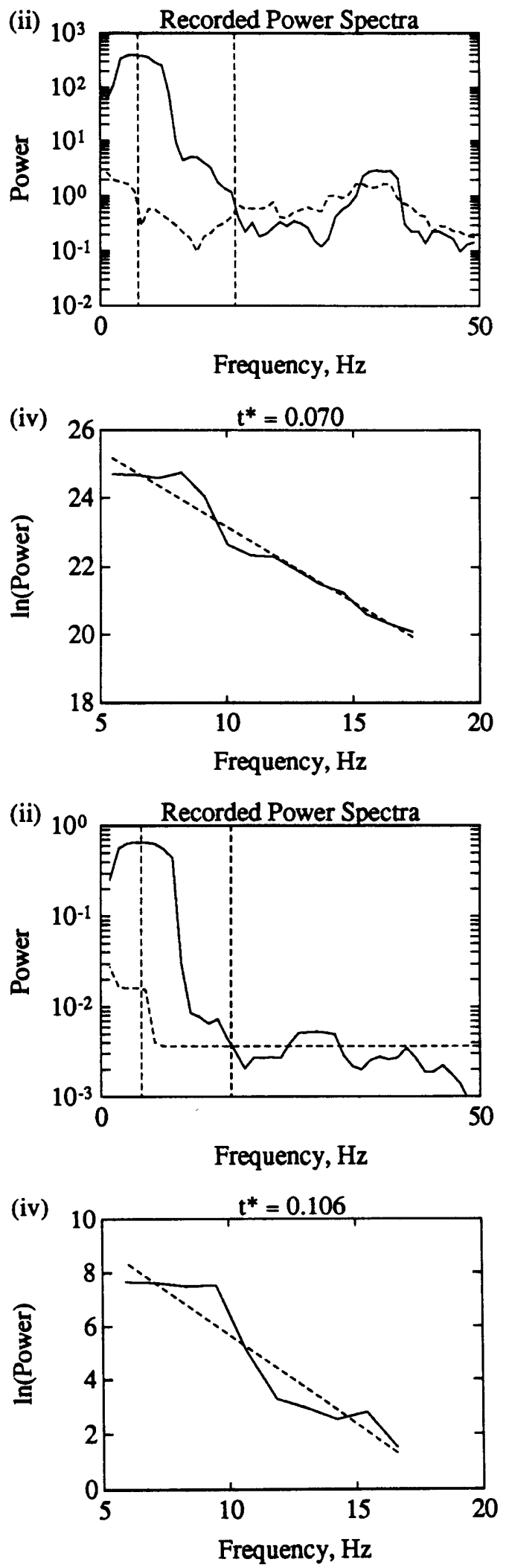

Figure 2.21 

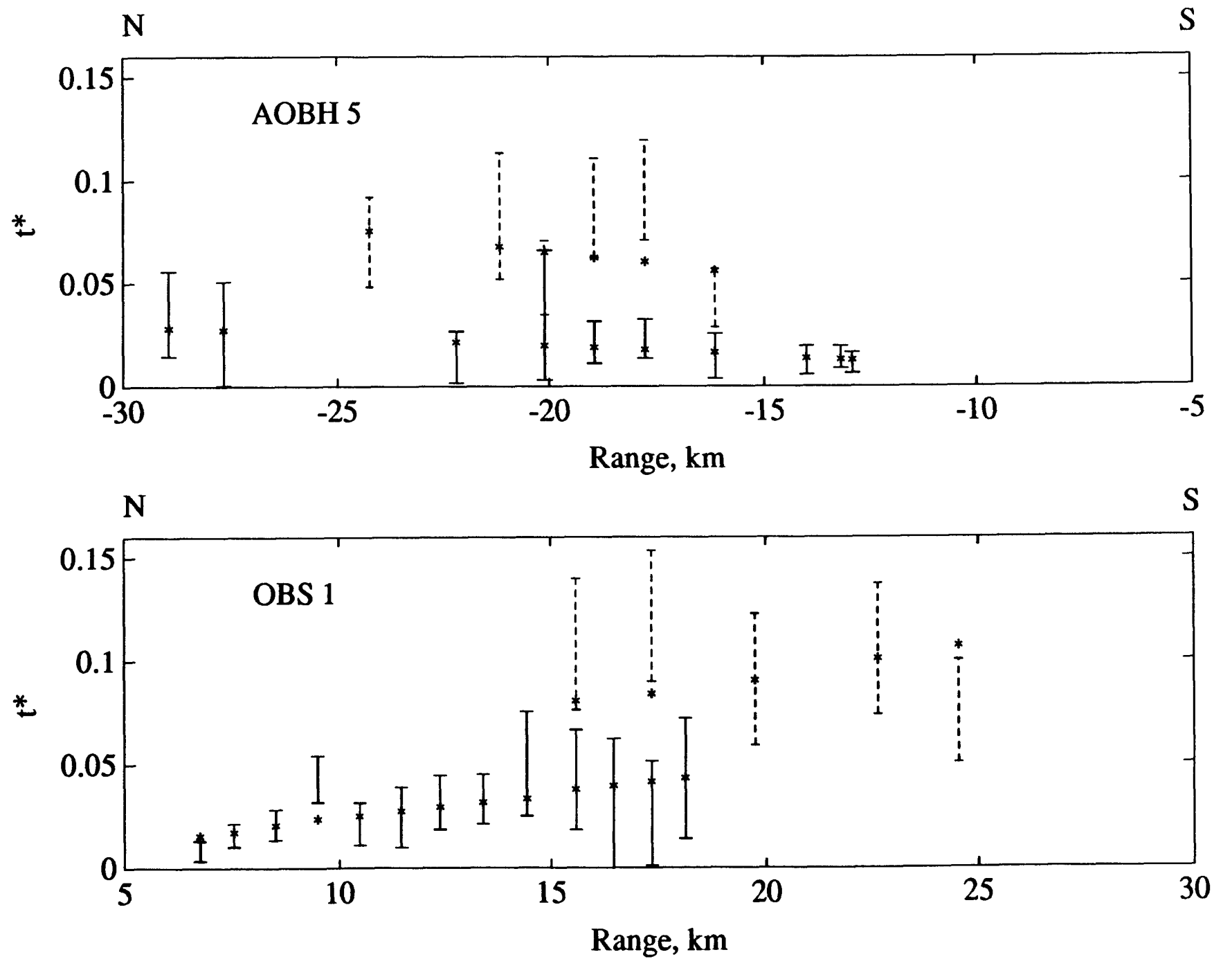

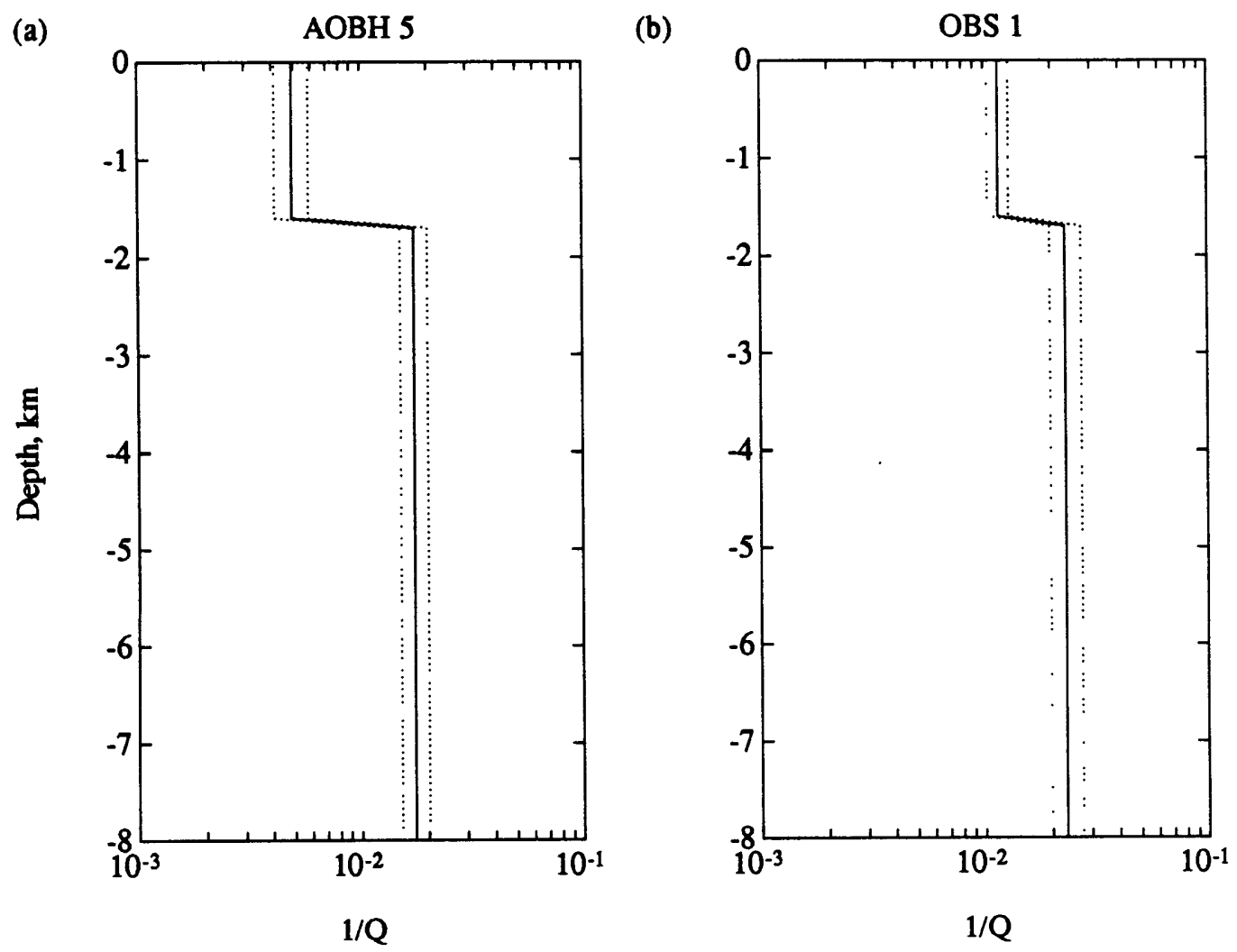

Figure 2.23 


\section{CHAPTER 3}

\section{A Finite-Difference Evaluation of the SPECTRal Method of ATTENUATION TOMOGRAPHY}

\section{INTRODUCTION}

The majority of attenuation studies which have employed tomographic techniques on local scales have been located in continental regions [Young and Ward, 1980; Hashida and Shimazaki, 1987; Evans and Zucca, 1988; Hashida et al., 1988; Ho-Liu et al., 1988; Clawson et al., 1989]. While velocity heterogeneities undoubtedly exist in such regions, the velocity structure in these studies has generally been successfully approximated by simple, laterally-invariant models in which velocity progressively increases with depth. In comparison to continental regions, the upper $10 \mathrm{~km}$ of oceanic lithosphere is characterized by high velocity gradients both in the upper crust and at the Moho. In addition, the velocity structure at $9^{\circ} 30^{\prime} \mathrm{N}$ on the EPR includes high gradients and pronounced lateral inhomogeneities in the vicinity of the axial magma chamber [Vera et al., 1990; Toomey et al., 1990a]. These characteristics raise three serious issues concerning the validity of spectral $t^{*}$ estimates and the reliability of tomographic $Q^{-1}$ models derived in this study.

First, errors in the assumed velocity structure will affect the inversions for $\mathrm{Q}^{-1}$ both directly, by biasing equation (1.6), and through the resulting errors in wave paths. For data sets that are compatible with a laterally invariant velocity structure, detailed velocity models may be obtained by forward modelling both travel times and amplitudes. However, because of the infeasibility of forward modelling data for complex, laterally varying velocity structures, velocity models for two- and threedimensional seismic data sets collected at mid-ocean ridges are generally obtained using delay-time tomographic techniques [Burnett et al., 1989; White and Clowes, 1990; Toomey et al., 1990a; Caress et al., 1992]. Such models have both limited resolution and tend to underestimate the magnitude of anomalies. Moreover, even if the true velocity structure were known, estimating accurate wave paths in the presence of a low-velocity anomaly is a difficult problem since ray-theoretical paths 
do not always exist [Caress et al., 1992] and an approximate ray-tracing technique must be used.

Second, the window used for spectral estimates may include two or more phases which propagate along significantly different paths. Such multi-pathing invalidates the linear relationship between $t^{*}$ and $\mathrm{Q}^{-1}$ (equation 1.6). If the true $\mathrm{t}^{*}$ differs significantly between multiple paths, $\mathrm{Q}^{-1}$ models obtained assuming a single path may provide very distorted images of the true structure, especially if the wave paths do not correspond to those of the highest amplitude arrivals.

Third, the diffraction and focusing of seismic waves in regions containing high velocity gradients may result in a significant non-attenuative, frequency-dependent component of propagation. As a result, the spectral t* estimates will be measure of apparent rather than intrinsic attenuation [e.g., Cormier, 1982], and the resulting $\mathrm{Q}^{-1}$ models may be biased. In particular, frequency-dependent scattering in the vicinity of the axial magma chamber may significantly affect $\mathrm{Q}^{-1}$ estimates in this region.

Previous studies [Burnett et al., 1988; Swift et al., 1990] have demonstrated the feasibility of applying numerical techniques to model seismic propagation across mid-ocean ridges. In this chapter we apply a two-dimensional full-waveform finitedifference technique to address the reliability of the $\mathrm{Q}$ tomography method. We employ a velocity structure based on the cross-sectional model proposed by Vera et al. [1990] for the EPR at $9^{\circ} 30^{\prime} \mathrm{N}$ (Figure 1.3). Solutions obtained for several source locations are used to construct a synthetic data set. An inversion of the travel time data [Thurber, 1983] reproduces the potential errors in the velocity model. The approximate wave paths obtained during the delay-time inversion are compared with estimates of the wave paths derived from the finite-difference solutions. Intrinsic t* values, which may include the contributions of multiple arrivals, are calculated for a plausible $\mathrm{Q}^{-1}$ model. Inversions of these synthetic data for $\mathrm{Q}^{-1}$ are used to assess the potential biasing effects arising from errors in the velocity structure and from multi-pathing. Spectral analysis of the finite-difference waveforms, which do not include the effects of intrinsic attenuation, provides a basis for assessing the contribution of deterministic scattering to $t^{*}$ estimates and to the resulting $\mathrm{Q}^{-1}$ models.

\section{Finite-DifferenCE MOdEL CONFIGURATIONS}

The finite-difference algorithm used in this study is based on the scheme of Virieux [1986] and is described in detail by Dougherty and Stephen [1988] and 
Stephen [1990]. The method utilizes second-order differences on a staggered grid and is stable at liquid-solid interfaces without the incorporation of explicit boundary conditions [Virieux, 1986; Stephen, 1988]. The finite-difference grid (Figure 3.1) comprises a heterogeneous zone sandwiched between thin homogeneous water and solid layers. A source located $2.81 \mathrm{~km}$ above the seafloor is introduced as a boundary condition along the top of the grid, $150-600 \mathrm{~m}$ above the water-solid interface. The upper, lower, and right-hand boundary regions are absorbing layers [Levander, 1985; Cerjan et al., 1985; Stephen, 1990] which prevent reflections of seismic energy from the edges of the model, while the left-hand boundary may include either an absorbing region or alternatively an axis of symmetry if the source is located at the left-hand edge of the grid.

In order to facilitate spectral analysis of the finite-difference waveforms, a source function with a large bandwidth is required. However, accurate finitedifference calculations require a minimum of about ten grid points per wavelength [e.g., Alford et al., 1974; Virieux, 1986]. This criterion, together with the small time steps required for stability [Alford et al., 1974; Virieux, 1986], places strong computational limitations on the maximum frequency of the source. In this study solutions are obtained with a grid spacing that is equal in horizontal and vertical directions, and which has values of $15,7.5$, and $3.75 \mathrm{~m}$, depending upon the physical dimensions of the model. The time steps used for the three grid spacings are 1.5, 0.75 , and $0.375 \mathrm{~ms}$ respectively. The source time series (Figure 3.2 ), which is identical for all models, is constructed by successively applying low and high pass Butterworth filters to a delta function, a procedure that results in a power spectrum that is optimally flat within the passband. For solutions with $15-\mathrm{m}, 7.5-\mathrm{m}$, and $3.75-$ m grids, the half power source bandwidths are $2-12.5 \mathrm{~Hz}, 4-25 \mathrm{~Hz}$, and $8-50 \mathrm{~Hz}$, respectively. At the upper half-power source frequency there are 8 grid points per wavelength within the thin water layer and over 10 grid points per wavelength for both $\mathrm{P}$ and $\mathrm{S}$ waves within the crust. Since the grid spacing is close to or even below the generally accepted minimum value, considerable effort was devoted to confirming the spectral accuracy of the finite-difference $P$ waveforms, the results of which are presented in Appendix B.

The P-wave velocity model used in this study (Figure 3.3) is based on that obtained by Vera et al. [1990] at $9^{\circ} 30^{\prime} \mathrm{N}$. Vera's model, which is symmetric about the rise axis, was obtained by interpolating between several one-dimensional velocity models obtained from expanding-spread-profile data at $0,2,3$ and $10 \mathrm{~km}$ 
distance from the rise axis, taking into account the constraints imposed by reflection profiles. While parts of the model are necessarily subjective, it is preferred for this study over the delay time tomographic velocity model [Toomey et al., 1990a], since it includes many fine-scale features of the velocity structure and the absolute velocities are better constrained. The model also includes S-wave velocities and densities that are required for the finite-difference algorithm. The principal feature of the $\mathrm{P}$-wave velocity structure is a thin, 200-m-thick, 2-km-wide magma lens with $V_{P}$ $=3 \mathrm{~km} / \mathrm{s}$, at a depth of $1.6 \mathrm{~km}$ beneath the rise axis. Beneath the magma lens a broad low-velocity region extends to the base of the crust, while the roof of the magma chamber is characterized by very high velocity gradients which grade into a region of heightened shallow crustal velocities on the rise axis.

The model of Vera et al. [1990] has been modified in a number of ways to meet the requirements of the finite-difference technique. Seafloor topography has been removed from the model since a very fine grid spacing would be required to model its effects accurately [Dougherty and Stephen, 1991]. In order to avoid very low but non-zero $S$ wave velocities, the thin, 50-200 $\mathrm{m}$ thick surface low-velocity layer (layer $2 A$ ) is removed and replaced by a constant gradient from a $P$ wave velocity of $4 \mathrm{~km} / \mathrm{s}$ at the seafloor to $5.4 \mathrm{~km} / \mathrm{s}$ at the base of layer $2 \mathrm{~B}$. To satisfy the stability criterion imposed by the time increment, all velocities exceeding $7 \mathrm{~km} / \mathrm{s}$ are reduced to $7 \mathrm{~km} / \mathrm{s}$. At depths of 5 to $6 \mathrm{~km}$, a linear vertical velocity gradient is assumed between the model value at $5 \mathrm{~km}$ depth and a constant value of $7 \mathrm{~km} / \mathrm{s}$ at $6 \mathrm{~km}$ depth. Thus, the Moho velocity gradient is absent away from the rise axis while beneath the rise axis it is about $1 \mathrm{~km}$ shallower and the velocity increase is reduced in magnitude over that proposed by Vera et al. [1990].

$A V_{S} / V_{P}$ ratio of 0.54 (equivalent to a Poisson's ratio of 0.29 ) is assumed throughout most of the model. In the upper kilometer $\mathrm{V}_{S} / \mathrm{V}_{\mathrm{P}}$ ranges between 0.5 and 0.54 and is calculated according to:

$$
\begin{array}{ll}
\mathrm{V}_{\mathrm{S}} / \mathrm{V}_{\mathrm{P}}=0.54-0.0287\left(5.4-\mathrm{V}_{\mathrm{P}}\right) & \mathrm{V}_{\mathrm{P}}<5.4 \mathrm{~km} / \mathrm{s} \\
\mathrm{V}_{\mathrm{S}} / \mathrm{V}_{\mathrm{P}}=0.54 & \mathrm{~V}_{\mathrm{P}} \geq 5.4 \mathrm{~km} / \mathrm{s}
\end{array}
$$

Within the magma body $V_{S}=0 \mathrm{~km} / \mathrm{s}$ while in the surrounding region $V_{S} / V_{P}$ increases with decreasing $V_{\mathrm{P}}$ :

$$
\begin{array}{ll}
\mathrm{V}_{\mathrm{S}} / \mathrm{V}_{\mathrm{P}}=0.54+0.0422\left(6.0-\mathrm{V}_{\mathrm{P}}\right) & \mathrm{V}_{\mathrm{P}}<6.0 \mathrm{~km} / \mathrm{s} \\
\mathrm{V}_{\mathrm{S}} / \mathrm{V}_{\mathrm{P}}=0.54 & \mathrm{~V}_{\mathrm{P}} \geq 6.0 \mathrm{~km} / \mathrm{s}
\end{array}
$$


While such an increase is primarily incorporated to maintain a minimum value of $\mathrm{V}_{\mathbf{S}}=$ $2 \mathrm{~km} / \mathrm{s}$ it is compatible with the results of Vera et al. [1990] and experimental measurements [Murase and McBirney, 1973].

Following Vera et al. [1990], the density (in $\mathrm{g} / \mathrm{cm}^{3}$ ) throughout most of the model is calculated according to

$$
\rho=0.165 \mathrm{VP}+1.852
$$

However, this relationship predicts unrealistically high densities in the uppermost crust and produces numerical instabilities at the seafloor in the finite-difference algorithm. Therefore, the density relationship of Nafe and Drake [1957]

$$
\rho=0.379 V_{P}+0.252
$$

is used at the seafloor and a linear gradient links the two relationships over the upper $0.5 \mathrm{~km}$. In the vicinity of the magma chamber equation (3.3) yields densities that are too low, and so the minimum density in this region is limited to $2.6 \mathrm{~g} / \mathrm{cm}^{3}$.

\section{FinITE-DiffERENCE SOLUTIONS}

Finite-difference solutions were calculated for sources at distances $x$ of $0,-2,-4$, $-6,-8$, and $-20 \mathrm{~km}$ from the rise axis. Solutions for the $0,2,4,6$, and $8-\mathrm{km}$ sources were obtained using both $7.5-\mathrm{m}$ and $3.75-\mathrm{m}$ grid spacings. The $7.5-\mathrm{m}$-grid solutions extend $8 \mathrm{~km}$ to either side of the rise axis, while solutions using the smaller grid spacing are confined to shorter ranges at which the P-wave phases of interest propagate well within the upper $2.5 \mathrm{~km}$. A $15-\mathrm{m}$ grid was used for the $20-\mathrm{km}$ source solution for which the velocity model extended from $x=-20 \mathrm{~km}$ to $x=10 \mathrm{~km}$. This solution does not have sufficient high frequency content for short-window spectral t $^{*}$ analysis and was obtained primarily to reproduce the geometry of the outer shot lines (Figure 1.1) for the synthetic inversions.

Figure 3.4 shows record sections for the $8-\mathrm{km}$ and $20-\mathrm{km}$ sources, both of which include several compressional phases within $0.5 \mathrm{~s}$ of the first arrival. The first arrival always propagates within the upper crust and passes over the roof of the magma chamber. At ranges that do not greatly exceed that of the rise axis the first energy is a ray-theoretical arrival with a small triplication at $7-9 \mathrm{~km}$ range. At greater ranges this phase is diffracted over the roof of the magma chamber and is characterized by very low amplitudes, particularly for the $20-\mathrm{km}$ source. Indeed in Figure 3.4 this phase is not discernible at the largest ranges. 
The record sections also contain high amplitude PP phases whose paths include a downward reflection from the seafloor as well as higher order phases with more than one such reflection. In the case of the 8- $\mathrm{km}$ source a single branch of the PP travel-time curve extends to over $14 \mathrm{~km}$ range. Additional PP arrivals with smaller ray parameters also exist at ranges of $11-12 \mathrm{~km}$ and $14-16 \mathrm{~km}$, resulting in a marked increase in amplitudes. In the case of the $20-\mathrm{km}$ source the PP phase is not observed at ranges much greater than $20 \mathrm{~km}$ because of the effects of the axial low velocity zone. A strong triplication at $12-15 \mathrm{~km}$ range results in very high PP amplitudes as well as high amplitude PPP and PPPP phases at correspondingly greater ranges. However, the amplitude of downward seafloor reflections in the finite-difference solutions will be appreciably higher than might be expected in a physically realistic model due to the high seafloor crustal velocities. The amplitude of the seafloor reflection depends strongly on the seismic properties at the seafloor, the ray parameter, and the presence of additional near-surface discontinuities. In particular, the amplitude is very sensitive to the S-wave velocity, which is poorly known. For $\mathrm{P}$ - and S-wave velocities at the seafloor of $2.2 \mathrm{~km} / \mathrm{s}$ and $0.95 \mathrm{~km} / \mathrm{s}$, values similar to those obtained by Vera et al. [1990], and a density given by equation (3.4), the true amplitudes of the downward-reflected plane $P$ waves are reduced by a factor of 0.40.7 [Ergin, 1952]. In addition, there will be an further decrease in amplitudes on reflection due to seafloor roughness. The high levels of attenuation in the upper $1 \mathrm{~km}$ (Chapter 2) will also generally reduce the amplitude of PP phases relative to direct phases that propagate at larger depths. However, in the case of the 8-km source, the high amplitude PP phase at $14-16 \mathrm{~km}$ range includes a surface reflection near the rise axis, a region that is characterized by relatively low levels of surface attenuation. Since, the rise axis is also characterized by high levels of attenuation at larger depths this arrival may be important at such ranges.

A diffracted arrival from beneath the magma chamber is also observed (Figure 3.4), which at ranges much greater than that of the rise axis is the first phase with significant amplitude. The propagation of this phase is fairly complex. The high velocity gradients above and below the magma body act as a waveguide that propagates energy in a broad region centered below the magma body. Upon emerging from beneath the magma body the phase is strongly diffracted, producing back-scattered arrivals of appreciable amplitude.

At the largest ranges, a very high amplitude Moho-turning phase is apparent in both sections. Because the Moho transition zone has been elevated $1 \mathrm{~km}$ under the 
rise axis and is not present off-axis, this arrival will be misplaced. In the case of the $8-\mathrm{km}$ source such an arrival would be offset to larger ranges in a more realistic model. A Moho arrival might be expected at such ranges for the $20-\mathrm{km}$ source, but the relative arrival time may be incorrect. The high amplitudes are a consequence of the large velocity increase at the Moho beneath the rise axis, a feature that is also present in the model of Vera et al. [1990].

\section{DELAY-TIME TOMOGRAPHY AND WAVE PATHS}

Since attenuation tomography is dependent upon the assumed velocity structure, the first step in analysis of the finite-difference solutions is to obtain a velocity structure with errors that are comparable to those in the delay time tomographic model obtained at $9^{\circ} 30^{\prime} \mathrm{N}$ [Toomey et al., 1990a]. To achieve this end travel times obtained from the finite-difference solutions were inverted for a velocity model. A geometry that closely mimics that of a cross-section through the tomography experiment is obtained by combining travel times for sources at $\mathrm{x}=0$, \pm 8 , and $\pm 20 \mathrm{~km}$ and receivers at $0.5-\mathrm{km}$ intervals between $x=-8$ and $8 \mathrm{~km}$, though the source and receiver positions are reversed.

The very low amplitudes of the diffracted first arrivals observed in Figure 3.4 raise the important question as to whether such arrivals would be observable in the presence of ambient noise. Figure 3.5 compares typical noise levels observed during the tomography experiment with the amplitude of the first arrival for both the 8-km and $20-\mathrm{km}$ sources. The finite-difference arrivals have been adjusted to account for the difference between two and three-dimensional divergence and to include the effects of attenuation. In the case of the $20-\mathrm{km}$ source the amplitude of the first arrival is well below noise levels, strongly suggesting that this phase will not be observable at such ranges. In the case of the $8-\mathrm{km}$ source the signal amplitude drops slightly below noise levels at the largest ranges. Inspection of the waveforms recorded by receivers at $9 \mathrm{~km}$ range from the rise axis supports this observation. A low-amplitude first arrival is generally observed for interior shots located across the rise axis, though at the largest ranges the automatic picking routine [Toomey et al., 1990a; Allen, 1982; Rothman, 1986] does not always pick this phase. The presence of a first-arriving phase with amplitudes below the detection threshold illustrates a potential pitfall of using minimum time algorithms [Vidale, 1988, 1990; Moser, 1991] to calculate travel times and wave paths. 
In this study, travel times are obtained by picking the first zero in the third derivative of the first cycle whose amplitude exceeds the minimum background noise amplitude shown in Figure 3.5. Following Toomey et al. [1990a] the velocity model is parameterized on a $1-\mathrm{km}$ grid, and the delay times are inverted using the algorithm of Thurber [1983]. The delay-time inversion is able to resolve both the high-velocity anomaly at $0-1 \mathrm{~km}$ depth and the low-velocity anomaly at $2 \mathrm{~km}$ depth. However, there is a strong trade off between the magnitudes of these two anomalies, which are strongly dependent upon the starting model. A one-dimensional starting model yields a large high-velocity anomaly, while starting models which include a portion of the two-dimensional structure have larger low-velocity anomalies. The final travel time residual is almost identical for a wide range of starting models. Clearly, the inclusion of travel times for short rise-parallel paths on the rise axis would significantly improve the resolution of the final model since the velocities in the highvelocity anomaly would be independently constrained. Since such paths are included in the three-dimensional delay-time inversions [Toomey et al., 1990a], we chose a solution (Figure 3.6) that closely reproduces the magnitude of the low-velocity anomaly resolved by the tomography experiment. The inversion underestimates the magnitude of the low-velocity anomaly and includes a number of artifacts off-axis.

In Figure 3.7 ray paths obtained from the delay-time inversion algorithm are compared with those estimated from the finite-difference solutions. The delay time tomographic algorithm [Thurber, 1983] calculates paths using a pseudo-bending technique which attempts to iteritive distort a minimum time path of constant curvature into a ray-theoretical path. The finite-difference wave paths are obtained by combining an exact ray-tracing algorithm with visual identification of wavefronts in the finite-difference snapshots. In the case of the diffraction from beneath the magma chamber it is difficult to estimate objectively the depth of propagation beneath the magma body. Propagation occurs in a fairly broad zone extending from the roof of the magma chamber at about $1.5 \mathrm{~km}$ depth to over $2.5 \mathrm{~km}$ depth. While a number of fairly complex and unstable ray theoretical solutions exist in this region, a simple path was chosen through this region with a depth beneath the rise axis of between 2 and $2.5 \mathrm{~km}$.

At shorter ranges the shapes of the ray paths are in good agreement. However, at the largest ranges, Thurber's algorithm fails to find the minimum time path and places the ray beneath the magma chamber. To some extent this failure is fortuitous since it occurs at ranges where where the amplitude of the first arrival is very small. 
However, the range at which the transition between the two paths occurs is very sensitive to poorly resolved details of the velocity structure and generally coincides neither with the range at which the first arrival becomes unpickable nor the range at which the amplitude of the deeper diffraction exceeds that of the first arrival. Therefore, some paths will be grossly in error for both velocity and $\mathrm{Q}^{-1}$ inversions.

Away from the rise axis, approximate ray paths generally bottom at slightly smaller depths at short ranges and significantly greater depths at large ranges. Beneath the rise axis there are no approximate paths between about 1.5 and $2.5 \mathrm{~km}$ depth, a region that coincides with the center of the low velocity anomaly. The finitedifference paths show a similar though less extensive gap, though as noted earlier the diffraction from below the magma chamber propagates in part within this region.

\section{SYNTHETIC INVERSIONS FOR $Q^{-1}$}

To assess the potential effects of errors in the velocity structure and multipathing, a series of synthetic inversions were conducted using intrinsic $t^{*}$ values derived from a plausible $\mathrm{Q}^{-1}$ model (Figure 3.8). The $\mathrm{Q}^{-1}$ model is based on the velocity model and is compatible with the constraints on the axial and off-axis $Q^{-1}$ structure derived in Chapter 2. For the inversions $Q^{-1}$ is parameterized on a $0.5-\mathrm{km}$ grid, and a smoothest model inversion is performed in which the smoothing operator is a simple first-order difference between all adjacent nodes. Rather than present solutions for a variety of smoothing weights, results are compared for a single constant smoothing weight chosen subjectively by comparing solutions with the true model.

\section{Velocity Model Errors}

Given the path and velocity structure, synthetic $t^{*}$ values can be calculated from the $\mathrm{Q}^{-1}$ model using equation (1.6). In Figure $3.9 \mathrm{t}^{*}$ values obtained assuming the finite-difference velocity model (Figure 3.3 ) and the finite-difference wave path corresponding to the maximum amplitude direct crustal arrival (Figure 3.7) are shown. Values were calculated for sources at $x= \pm 20, \pm 8$ and $0 \mathrm{~km}$, receivers at 0.5 $\mathrm{km}$ intervals between $\mathrm{x}=-8$ and $8 \mathrm{~km}$, and a minimum source-receiver separation of $3.5 \mathrm{~km}$. The principal feature of these data is a sudden increase in $\mathrm{t}^{*}$ values for the off-axis sources at the range where paths switch from passing above to below the magma chamber. Figure 3.10 shows the results of inverting these data assuming 
alternatively the correct velocity structure and wave paths and the results of delay time tomography.

Not surprisingly, the solution obtained using the correct velocity structure and wave paths is a fairly good representation of the true $\mathrm{Q}^{-1}$ model and matches the $t^{*}$ data (Figure 3.9) very well. The primary effect of smoothing is to increase slightly the thickness of the surface low-Q zone and the width of the axial low-Q region and to increase the minimum $Q$ values in these regions. The lowest axial $Q$ values are at $2 \mathrm{~km}$ depth while the axial structure between 1 and $2 \mathrm{~km}$ depth is not wellresolved because of the lack of ray paths in this region.

The solution derived from the delay time tomographic velocity model differs significantly from the first solution. While the principal features of the model are still resolved the lowest axial $\mathrm{Q}$ values are located at a depth of $1.5 \mathrm{~km}$ rather than 2.0 $\mathrm{km}$, and the magnitude of the anomaly is smaller. Off-axis the near-surface $\mathrm{Q}$ structure shows much larger lateral variations and includes very low $Q$ values $2 \mathrm{~km}$ off-axis. These differences are a direct consequence of the misplacement of ray paths beneath the rise axis. In particular $t^{*}$ values obtained from ray paths that diffract below the magma chamber are incorrectly ascribed to shallower ray paths at smaller ranges, while at longer ranges the ray paths turn at too large a depth. As a result the the lowest axial $Q$ values are not fully resolved, and some of the anomaly is incorporated into the shallow structure. Inspection of the $t^{*}$ predictions (Figure 3.9) shows that the model fails to match fully the sudden increase in $t^{*}$ values observed for off-axis sources.

\section{Multi-Pathing}

An intrinsic $t^{*}$ value for a time window which includes more than one phase may be estimated using a straight line fit to a power spectrum $\mathrm{C}$ calculated according to

$$
C(f)=\sum_{i=1}^{N} a_{i}^{2} \exp \left(-f t *_{i}\right)
$$

where $\mathrm{N}$ is the number of phases, and $\mathrm{a}_{\mathrm{i}}$ and $\mathrm{t}^{*}{ }_{\mathrm{i}}$ are the amplitude and intrinsic $\mathrm{t}^{*}$ value (equation 1.6) of individual phases. Such a calculation makes the simplifying assumption that the full spectral content of a phase is concentrated at the arrival time and will therefore tend to overestimate the effect of later arrivals whose onsets just fall within the window. In Figure 3.11 such multiple-phase $t^{*}$ values are shown for arrivals within the first $0.3 \mathrm{~s}$ and $0.6 \mathrm{~s}$ of the waveform. The amplitudes used in the calculation are the peak-to-peak amplitudes observed in the record sections. PP 
amplitude are corrected for a seafloor reflection assuming $\mathrm{V}_{\mathrm{P}}=2.2 \mathrm{~km} / \mathrm{s}, \mathrm{V}_{\mathrm{S}}=0.95$ $\mathrm{km} / \mathrm{s}$, and $\rho=1.2 \mathrm{~g} / \mathrm{cm}^{3}$ in the uppermost crust. The Moho phase is not included in the calculations for the $8-\mathrm{km}$ source since in a realistic model it would be offset to larger ranges.

While there are noticeable differences, both sets of $t^{*}$ values are generally similar. Inspection of the the amplitude and timing of phases (Figure 3.4) and of the the relative squared amplitude of phases contributing to the $t^{*}$ values for a $0.6-\mathrm{s}$ window suggests that this similarity is somewhat coincidental. At larger ranges the 0.6-s-window t* value for the $8-\mathrm{km}$ and $20-\mathrm{km}$ sources are dominated by PP and Moho arrivals, respectively, while the 0.3-s-window values at the same ranges results almost entirely from energy diffracted below the magma chamber. However, since the single path $t^{*}$ values are similar for these phases, the multi-path $t^{*}$ values are little changed. Indeed the inversion of the two data sets (Figure 3.12) yields very similar models. While the similarity of the 0.3 - and $0.6-\mathrm{s}$-window multi-path $\mathrm{t}^{*}$ values is a consequence of the choice of $\mathrm{Q}^{-1}$ model, the results do suggest that the effects of multi-pathing may be fairly subtle. However, if the results of inversions for an unknown $\mathrm{Q}^{-1}$ structure are to be considered robust, it is clearly necessary to exclude high-amplitude arrivals whose propagation path does not correspond to that of the wave paths used in the inversion.

\section{High Surface Smoothing Weights}

Figure 3.13a shows the results of inverting the 0.3-s-window multi-path $t^{*}$ values assuming the delay time tomography results. In marked contrast to previous solutions the lowest axial $\mathrm{Q}$ values at about $2 \mathrm{~km}$ depth are barely resolved. Much of the variation in $\mathrm{t}^{*}$ values resulting from the $2-\mathrm{km}$-deep axial low-Q zone is modelled by variations in the uppermost crustal structure off-axis. Figure $3.13 \mathrm{~b}$ shows the results of an identical inversion except that the smoothing weight in the upper $1 \mathrm{~km}$ has been quadrupled. The resulting model resolves an region of axial low- $Q$ values that extends from $1.5 \mathrm{~km}$ to over $2.5 \mathrm{~km}$ depth. The effect of increasing the near-surface smoothing weight is to limit the lateral variations in the shallow $\mathrm{Q}^{-1}$ structure off-axis, while the relatively high near-surface $Q$ values on-axis are still resolved. As a result the inversion is forced to include an axial low- $Q$ zone though the magnitude of the anomaly is too small and the dimensions reflect the depth distribution of approximate ray paths. 


\section{THE EFFECT OF DETERMINISTIC SCATTERING}

The finite-difference method has been used by several workers to study the effects of stochastic perturbations to simple velocity models [Frankel and Clayton, 1984, 1986; McLaughlin et al., 1986; McLaughlin and Anderson, 1987; Dougherty and Stephen, 1988; Charette, 1991; Toksöz et al., 1991]. Such studies show that stochastic scattering can appreciably alter the spectral content of the first arrival [Frankel and Clayton, 1986; Charette, 1991]. The highest apparent $\mathrm{Q}^{-1}$ values occur for the frequencies given by $\mathrm{ka} \sim 1-2$ where $\mathrm{k}$ is the wave number and a the correlation length of the velocity perturbations. At lower frequencies $(\mathrm{ka} \ll 1) \mathrm{Q}^{-1}$ $\propto \mathrm{k}^{2}$ while at higher frequencies the rate of decrease in $\mathrm{Q}^{-1}$ is strongly dependent upon the assumed relationship between velocity perturbations and density perturbations [Charette, 1991]. Self-similar models which are characterized by a range of correlation lengths can result in approximately constant apparent $\mathrm{Q}^{-1}$ over a broad frequency band [Frankel and Clayton, 1986]. For velocity perturbation with a standard deviation of 10\%, Frankel and Clayton [1986] observe maximum apparent $\mathrm{Q}^{-1}$ values of about 0.01 , while Charette [1991] obtains higher values.

While stochastic scattering may well be important in oceanic crust, particularly at shallow depths [Dougherty and Stephen, 1988], its effect is hard to quantify without a better knowledge of the stochastic properties of the oceanic crust. In this section we use spectral analysis to evaluate the effect of deterministic scattering. Lewis and Jung [1988] show that the reflectivity of high gradients in the upper crust modify the spectral content of waveforms significantly at frequencies below $10 \mathrm{~Hz}$. A similar effect resulting from the very high velocity gradients observed around the magma chamber might alter the spectra of waveforms. The dimensions of the magma lens yield $\mathrm{ka}=1-2$ at frequencies ranging from about 1 to $20 \mathrm{~Hz}$. Spectral $\mathrm{t}^{*}$ estimates for such waveforms are frequently obtained over a fairly narrow frequency band $(10-30 \mathrm{~Hz})$ and may thus be strongly influenced by scattering.

Figure 3.14 shows apparent $t^{*}$ values derived from the finite-difference solutions using an 0.3-s-long time window and $4 \pi$ prolate MWSA. A comparison of finitedifference $t^{*}$ values obtained for one-dimensional on- and off-axis structures with those obtained by the reflectivity method [Fuchs and Müller, 1971] suggest that the results are probably reliable at all but the shortest ranges (Appendix 2). The results show apparent $t^{*}$ values that oscillate rapidly with range and that generally lie between \pm 0.01 . The largest variations are observed at longer ranges, suggesting that deterministic scattering around the magma chamber may influence $t^{*}$ values. 
The $t^{*}$ values obtained cannot be directly equated to those obtained from the tomography experiment, for two reasons. First, the true velocity model in the vicinity of the magma chamber will probably differ significantly from that used for the finite-difference solutions. There are few constraints on the detailed structure beneath the magma body [Vera et al., 1990] or on the thickness of the magma lens [Vera et al., 1990; Kent et al., 1990], while the modelling of diffraction hyperbolae suggest the width of the magma lens may be only $0.8-1.2 \mathrm{~km}$ rather than $2 \mathrm{~km}$ [Kent et al., 1990]. In addition, the delay-time tomography resolves significant asymmetry and along-axis variations in axial structure [Toomey et al., 1990a]. Second, the source-receiver position are reversed between the finite-difference solutions and the experiment, so only a handful of measurements have similar configurations. In Figure 3.15 six finite-difference $t^{*}$ estimates are compared with similarly configured $t^{*}$ estimates obtained for DOBH 15 (see Chapter 4). The results suggest that while deterministic scattering may significantly affect $t^{*}$ it does not dominate the observed trends in $t^{*}$.

Figure 3.16 shows the results of inverting the contributions to $t^{*}$ values from scattering $\mathrm{Q}$ for apparent $\mathrm{Q}^{-1}$ structure using the velocity model and wave paths from delay time tomography. While such an inversion is able to achieve a $60 \%$ variance reduction over a model with $\mathrm{Q}^{-1}=0$ using very small smoothing weights, the variance reduction for smoother solutions is much smaller. Solutions which show variations on a similar scale to those in $\mathrm{Q}^{-1}$ model are characterized by absolute $\mathrm{Q}^{-1}$ values that are less than 0.01 throughout the model.

To investigate the effect of deterministic scattering on an inversion for intrinsic $\mathrm{Q}^{-1}$, inversions were performed using both forward-modelled intrinsic $\mathrm{t}^{*}$ values and the sum of intrinsic $t^{*}$ and the $t^{*}$ estimates from deterministic scattering (Figure 3.17). Since the inversions are confined to sources and receivers at distances $\geq 8$ $\mathrm{km}$ from the rise axis it is necessary to use the finite-difference wave paths to include enough paths below the magma chamber to resolve adequately the axial low$\mathrm{Q}$ region. Including the contribution to $\mathrm{t}^{*}$ from scattering decreases the variance reduction of the best fitting constant- $Q$ model from over $99 \%$ to $80 \%$, since the contribution of scattering to $t^{*}$ is not fully consistent with a smooth $\mathrm{Q}^{-1}$ model. Moreover the final model is characterized by significant lateral variations in the nearsurface $\mathrm{Q}$ structure off-axis and an intensification of the 2-km-deep low- $\mathrm{Q}$ anomaly. This latter effect suggests that deterministic scattering may contribute to low apparent $Q$ values imaged in the vicinity of a low-velocity magma chamber. Whether 
this will be the case for the source-receiver configuration used in the tomography experiment is not clear. To answer this question fully will require a better knowledge of the velocity structure and three-dimensional models of wave propagation.

\section{SUMMARY}

In this chapter we have used a full-waveform finite-difference technique to evaluate the spectral method of $\mathrm{Q}$ tomography. Solutions for seismic propagation through a two-dimensional model similar to that proposed by Vera et al. [1990] for the East Pacific Rise at $9^{\circ} 30^{\prime} \mathrm{N}$ show that propagation near an axial magma chamber is complex and that a number of phases may arrive within $0.5 \mathrm{~s}$ of the onset of the waveform. The first arriving phase always propagates above the magma chamber. However, for paths of significant length that across the rise axis the amplitude of the first arrival is very small, and a diffraction below the magma chamber is the first phase with significant amplitude. A PP phase and a high amplitude Moho-turning phase may be important secondary arrivals.

Synthetic inversions show the importance of using wave paths appropriate to the phase that dominates $t^{*}$ estimates. Care must be taken in selecting the positions and lengths of the time windows used for spectral $t^{*}$ estimates in order to exclude high-amplitude arrivals whose paths do not correspond to that of the desired arrival. Deterministic scattering from the large velocity gradients in the vicinity of the axial magma chamber may contribute to $t^{*}$ estimates. While such affects can produce noticeable changes in $\mathrm{Q}^{-1}$ models, including an intensification of the axial low- $Q$ anomaly, the results suggest that intrinsic attenuation will dominate scattering attenuation. In the following chapter the results of the finite-difference studies are used to constrain the position of approximate wave paths and to select objectively the time windows used for spectral estimation. 


\section{Figure CAPTIONS}

Figure 3.1. Configuration of the finite-difference grid. A heterogeneous region is sandwiched between homogeneous water and solid layers. The grid spacing is equal in the vertical and horizontal directions and the source is introduced as a boundary condition along the top of the grid. The grid is enclosed by 120-point-wide absorbing regions which are not shown, though the left-hand boundary may alternatively be an axis of symmetry. The maximum dimensions of the models and the grid indices are shown for both the $7.5-\mathrm{m}$ and $3.75-\mathrm{m}$ grids (values for the smaller grid spacing are enclosed in parentheses).

Figure 3.2. (a) Source pressure time series used in the finite-difference solutions with a $7.5-\mathrm{m}$ grid. The source is constructed by digitally filtering a spike with a 4-pole high-pass Butterworth filter with a 4-Hz cuttoff and a 8-pole low-pass Butterworth filter with a $25-\mathrm{Hz}$ cutoff. (b) The power spectrum of the source shown in (a). The time series for the $3.75 \mathrm{~m}$ and $15 \mathrm{~m}$ grid solutions are identical but the sample intervals are proportional to the grid spacing.

Figure 3.3. Contours of gridded P-wave velocity for the model used in this study $(0.5-\mathrm{km} / \mathrm{s}$ contour interval), which is based on the model of Vera et al. [1990]. At all but the shallowest depths the gridded model was obtained by interpolating between contours using a minimum curvature algorithm [Briggs, 1974; Swain, 1976]. Such a procedure produces a predominantly smoothly varying velocity model, although in some regions there is a small residual roughness whose amplitude does not exceed $0.025 \mathrm{~km} / \mathrm{s}$ and which produces the jagged contours visible in areas with small velocity gradients. At ranges greater than $8 \mathrm{~km}$ from the rise axis the model is laterally invariant.

Figure 3.4. Record section for the sources at (a) $x=-8 \mathrm{~km}$ and (b) $x=-20 \mathrm{~km}$ showing predicted arrival times for a number. of $P$-wave phases. Predicted times are calculated from estimates of the wave paths obtained by combining an exact ray-tracing algorithm with the visual 
identification of wavefronts in the finite-difference snapshots. For display purposes the seismic records are scaled by the square root of the range to account for cylindrical divergence. The direct $\mathrm{S}$-wave arrival is just visible in (a) at $\mathrm{x}=-1 \mathrm{~km}$ and at a reduced time of $3.2 \mathrm{~s}$ and shows appreciable dispersion (Appendix B).

Figure 3.5. Peak-to-peak amplitudes of the first arriving phase for the model with the sources at (a) $x=-8 \mathrm{~km}$ and (b) $x=-20 \mathrm{~km}$. Amplitudes have been adjusted to include the effects of attenuation assuming $t^{*}=0.045$ $S$ and for the difference between two- and three-dimensional divergence. The range of typical background noise amplitudes observed during the tomography experiment is also shown (dashed lines). Amplitudes are normalized to that of the water wave at $1 \mathrm{~km}$ range.

Figure 3.6. A delay-time tomographic velocity model obtained by inverting travel times obtained from the finite-difference solutions (see text). Both (a) the absolute velocities $(0.5-\mathrm{km} / \mathrm{s}$ contour interval) and (b) the perturbations from the average one-dimensional model $(0.25-\mathrm{km} / \mathrm{s}$ contour interval) are shown. The velocity structure is parameterized using 1-km-spaced nodes. This solution was obtained using a starting model that contains $50 \%$ of the smoothed two-dimensional structure contained in the true velocity model (Figure 3.3).

Figure 3.7. Comparison of approximate wave paths obtained from the travel time inversion (solid lines) and from the finite-difference solutions (dashed and dotted lines) for sources at (a) $x=-20 \mathrm{~km}$ (b) $x=-8 \mathrm{~km}$ and (c) $x$ $=0 \mathrm{~km}$. The delay-time tomography wave paths are calculated using a pseudo-bending technique which iteritively distorts a minimum time path of fixed curvature. The finite-difference wave paths are obtained by combining an exact ray-tracing algorithm with the visual identification of wavefronts in the finite-difference snapshots. The path of the highest amplitude direct crustal arrival is shown as a dashed line while the lower amplitude arrival is shown as a dotted line. As noted in the text, propagation beneath the magma chamber is 
fairly complex and occurs over a fairly large range of depths. The paths in this region represent a visual estimate of the mid-depth of propagation

Figure 3.8. $\quad \mathrm{Q}^{-1}$ model used for the finite-difference inversions $(0.005$ contour interval, solid contours at 0.01 intervals). Away from the rise axis a simple model is assumed which linearly interpolates between values of $0.04,0.012,0.002$, and 0.002 at depths of $0,1,2$, and $6 \mathrm{~km}$ depth. Perturbations to this model are derived from the lateral variations in the velocity structure. The resulting $\mathrm{Q}^{-1}$ model is smoothed using the weighted average within a $1-\mathrm{km}$ square.

Figure 3.9. Synthetic $t^{*}$ values (asterisks) obtained for the $Q^{-1}$ model shown in Figure 3.8 assuming a single finite-difference wave path corresponding to the highest-amplitude direct crustal arrival. Predicted $t^{*}$ values are shown both for an inversion which uses the correct wave paths and velocity model (pluses) and one which uses the delay time tomography results (circles).

Figure 3.10. $\mathrm{Q}^{-1}$ models obtained from inversions of the $\mathrm{t}^{*}$ data shown in Figure 3.9 using (a) the correct velocity structure and wave paths and (b) the delay time tomography results. $t^{*}$ values are used for sources at $x=$ $0, \pm 8$, and $\pm 20 \mathrm{~km}$, receivers at $0.5 \mathrm{~km}$ intervals between $x=-8$ and 8 $\mathrm{km}$, and a minimum source-receiver separation of $3.5 \mathrm{~km}$. The $\mathrm{Q}^{-1}$ models are parameterized on a $0.5-\mathrm{km}$ grid.

Figure 3.11. (a) Multi-path $t^{*}$ values for a source at $x=-20 \mathrm{~km}$. (i) $t^{*}$ values are shown for phases arriving within an 0.3-s window (pluses) and an 0.6$s$ window (circles). (ii) The relative squared amplitudes ( $\mathrm{a}^{2}$ in equation 3.5) or power of the different phases used to calculate $t^{*}$ values for the 0.6-s-long window. Weights are shown for raytheoretical direct arrivals and diffractions above the magma chamber (asterisks), PP arrivals (circles), diffractions beneath the magma chamber (pluses), and a Moho-turning phase (crosses). (b) As for 
(a) except the source is at $\mathrm{x}=-8 \mathrm{~km}$ and the Moho arrival is excluded from the calculations.

Figure 3.12. Results of inverting synthetic multi-path $t^{*}$ values obtained from (a) 0.3-s-long windows and (b) 0.6-s-long windows. The data are inverted assuming the correct velocity structure and the finitedifference wave paths corresponding to the maximum amplitude direct crustal arrival.

Figure 3.13. (a) Results of inverting synthetic multi-path $t^{*}$ values obtained from a 0.3-s-long window using the velocity structure and wave paths obtained from the delay time tomographic inversion. A constant smoothing weight is used throughout the model. (b) As for (a) except the smoothing weight is quadrupled in the upper $1 \mathrm{~km}$.

Figure 3.14. Spectral $t^{*}$ estimates for sources at (a) $x=-8 \mathrm{~km},(\mathrm{~b}) \mathrm{x}=-6 \mathrm{~km}$, (c) $\mathrm{x}$ $=-4 \mathrm{~km},(\mathrm{~d}) \mathrm{x}=-2 \mathrm{~km}$, and (e) $\mathrm{x}=0 \mathrm{~km}$. Estimates are obtained from the finite-difference solutions using $4 \pi$ prolate MWSA and a 0.4-slong window that includes $0.3 \mathrm{~s}$ of the waveform. A least squares line is fitted to the natural logarithm of the source-corrected spectrum over the frequency interval defined by the half power bandwidth of the source. Values are presented for both the $4-25 \mathrm{~Hz}$ (crosses) and the $8-50 \mathrm{~Hz}$ (circles) sources. The source loacation is shown by a dashed line.

Figure 3.15. A comparison of variations in observed $t^{*}$ values estimated from the real data (pluses) with the finite-difference $t^{*}$ values (circles). $t^{*}$ values obtained for the tomography experiment are shown for approximately rise-perpendicular paths recorded by DOBH 15 , located $9 \mathrm{~km}$ off-axis. A constant value of 0.02 has been added to the finitedifference $t^{*}$ values, which are shown for a receiver located $8 \mathrm{~km}$ offaxis. In both cases $t^{*}$ values have been estimated from $0.3 \mathrm{~s}$ of the waveform using $4 \pi$ prolate MWSA. 
Figure 3.16. Results of inverting the contribution to $t^{*}$ from scattering as predicted by the finite difference models (Figure 3.14) for apparent $Q^{-1}$ structure using the delay time tomographic velocity model and wave paths. To avoid negative $\mathrm{Q}^{-1}$ in the inversion, $\mathrm{t}^{*}$ values resulting from a constant $\mathrm{Q}^{-1}$ of 0.02 is added to all data prior to the inversion and then subtracted from the model before displaying the results. $t^{*}$ estimates are used for sources at $x=0, \pm 2, \pm 4, \pm 6$, and $\pm 8 \mathrm{~km}$, receivers at 0.5 $\mathrm{km}$ intervals between $\mathrm{x}=-8$ and $8 \mathrm{~km}$, and a minimum source-receiver separation of $3.5 \mathrm{~km}$. Where available, $\mathrm{t}^{*}$ estimates are from the frequency interval $8-50 \mathrm{~Hz}$, while elsewhere they are for the frequency interval 4-25 Hz (Figure 3.14). (a) A plot of variance versus smoothing weight for the inversion. (b)-(d) The apparent $\mathrm{Q}^{-1}$ models obtained for smoothing weights $b=0.001$ (contour interval reduced to 0.001 ), $b=0.01$ (contour interval reduced to 0.0025 ), and $b=0.1$, respectively.

Figure 3.17 (a) Results of inverting forward-modelled intrinsic $t^{*}$ values for the same source-receiver configuration as used in Figure 3.16. The wave paths and velocity model assumed correspond to those of the highest amplitude direct crustal path. (b) As for (a) except the $t^{*}$ contributions from deterministic scattering (Figure 3.14) are added to the intrinsic $\mathrm{t}^{*}$ values. 


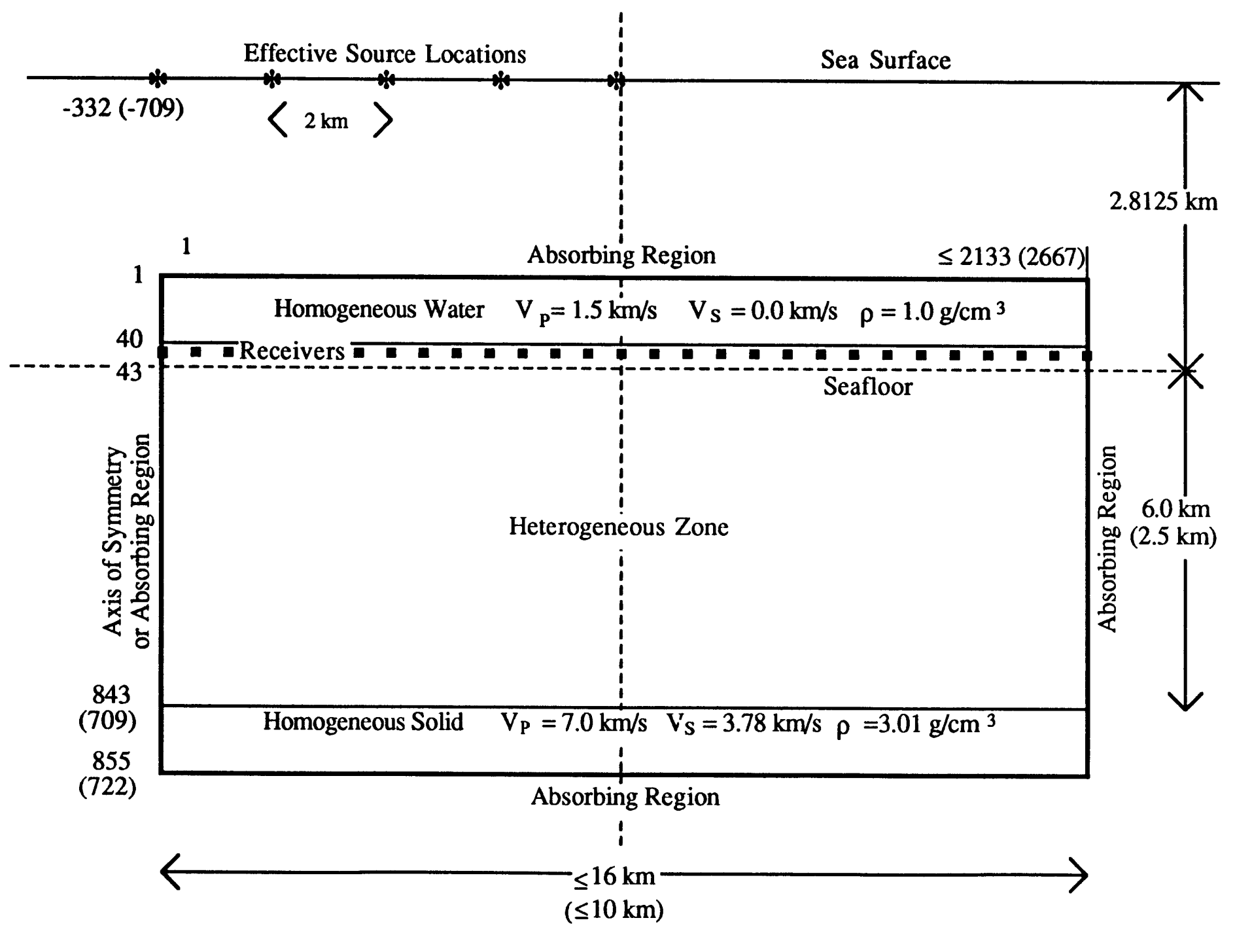



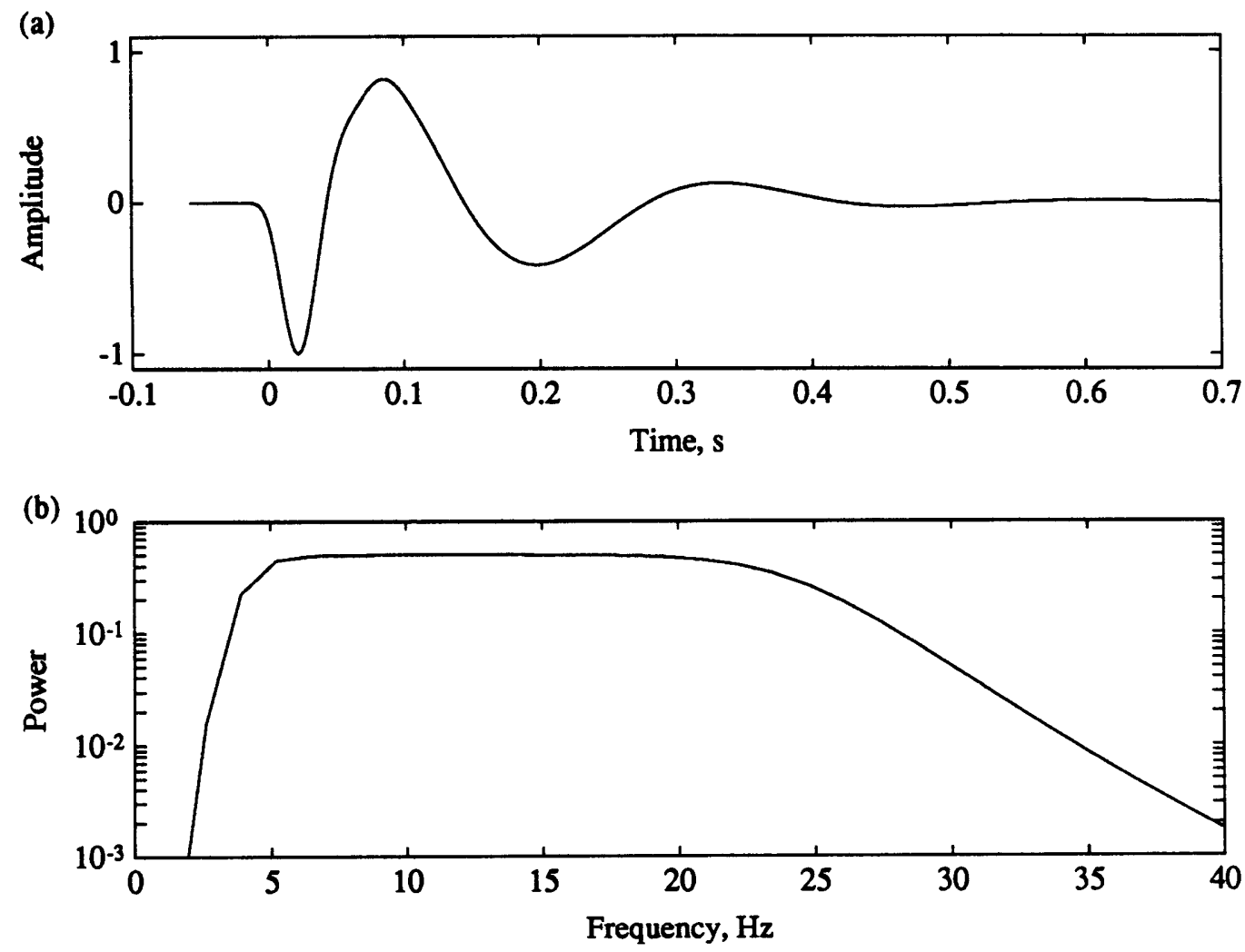

Figure 3.2 


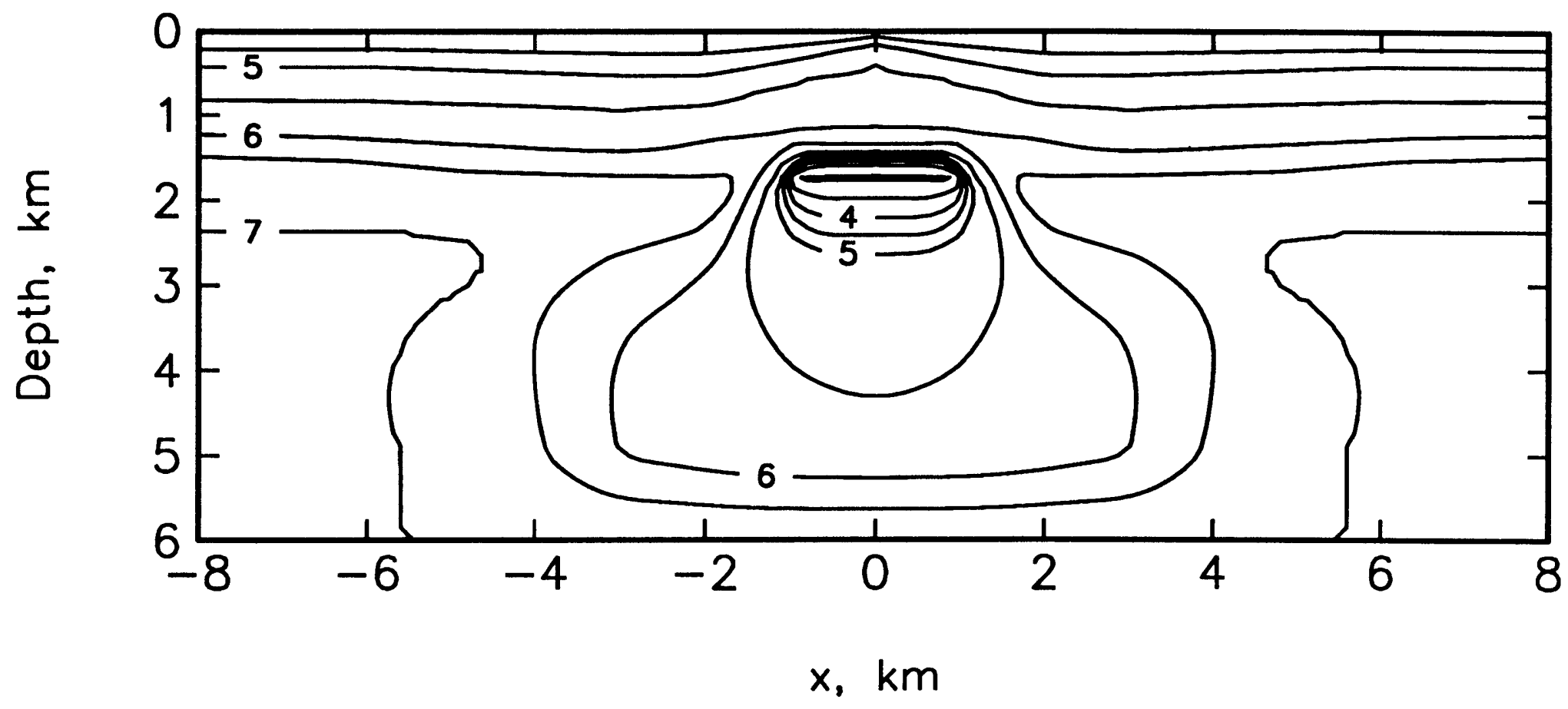




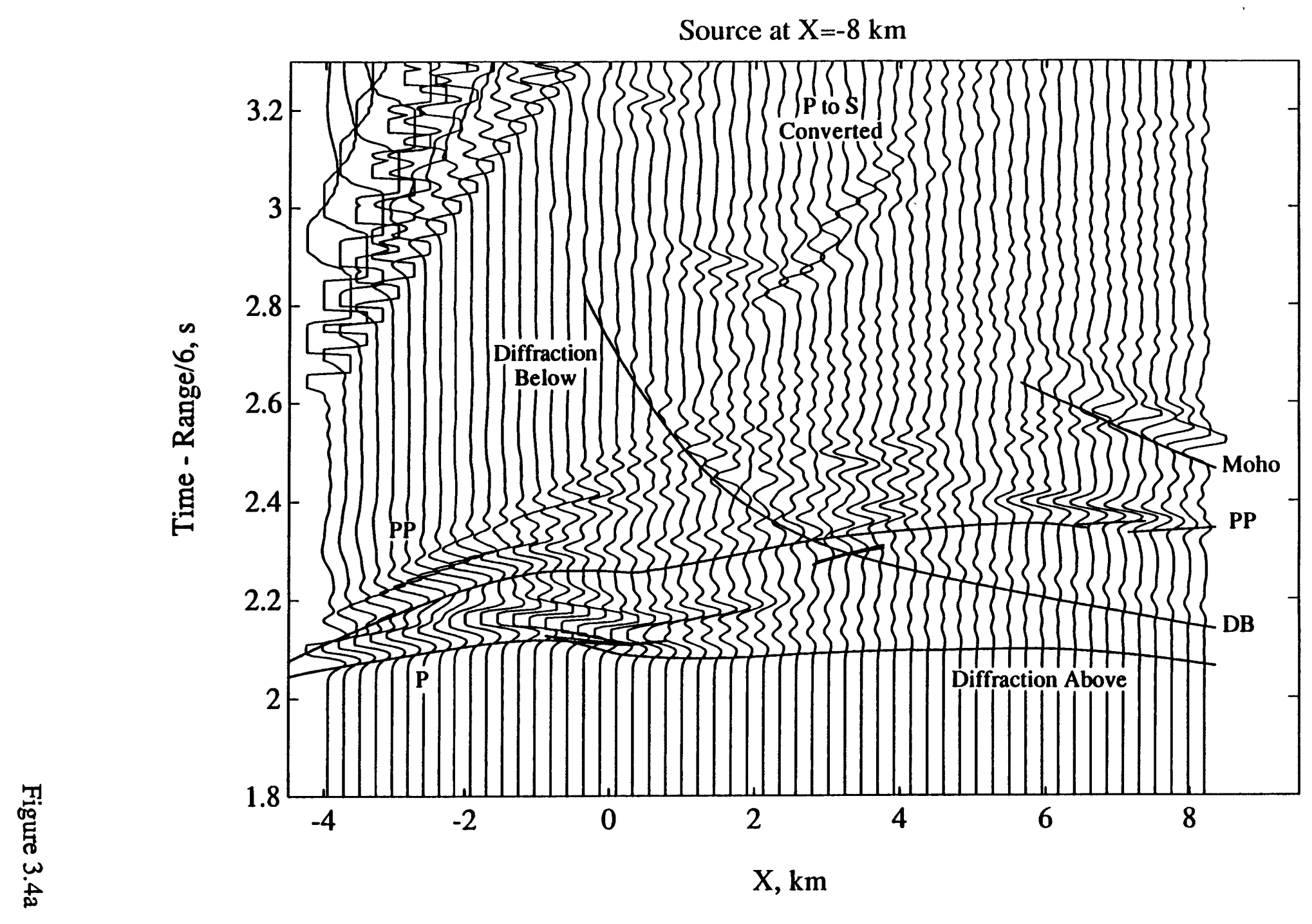

옹 


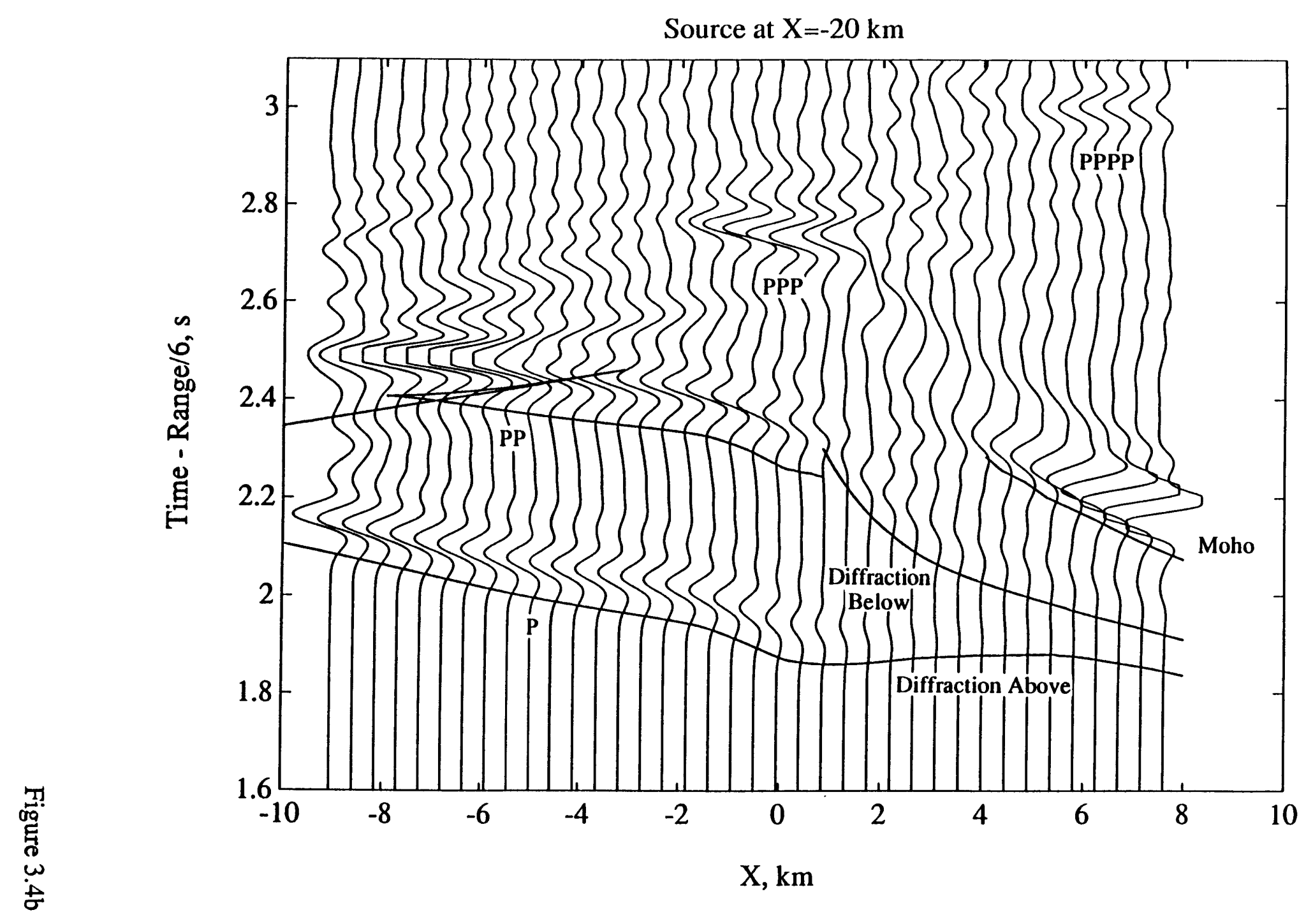


(a)

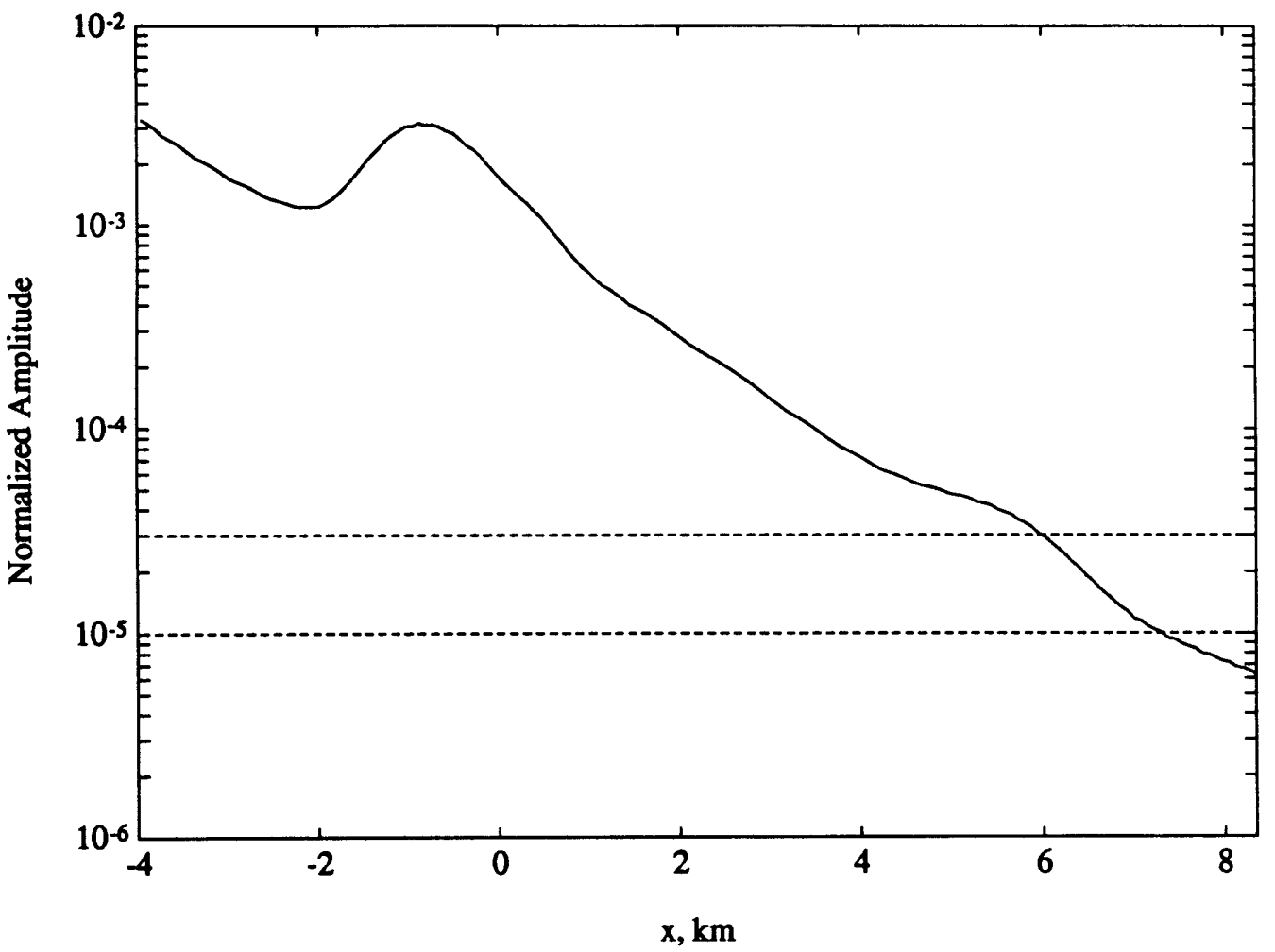

(b)

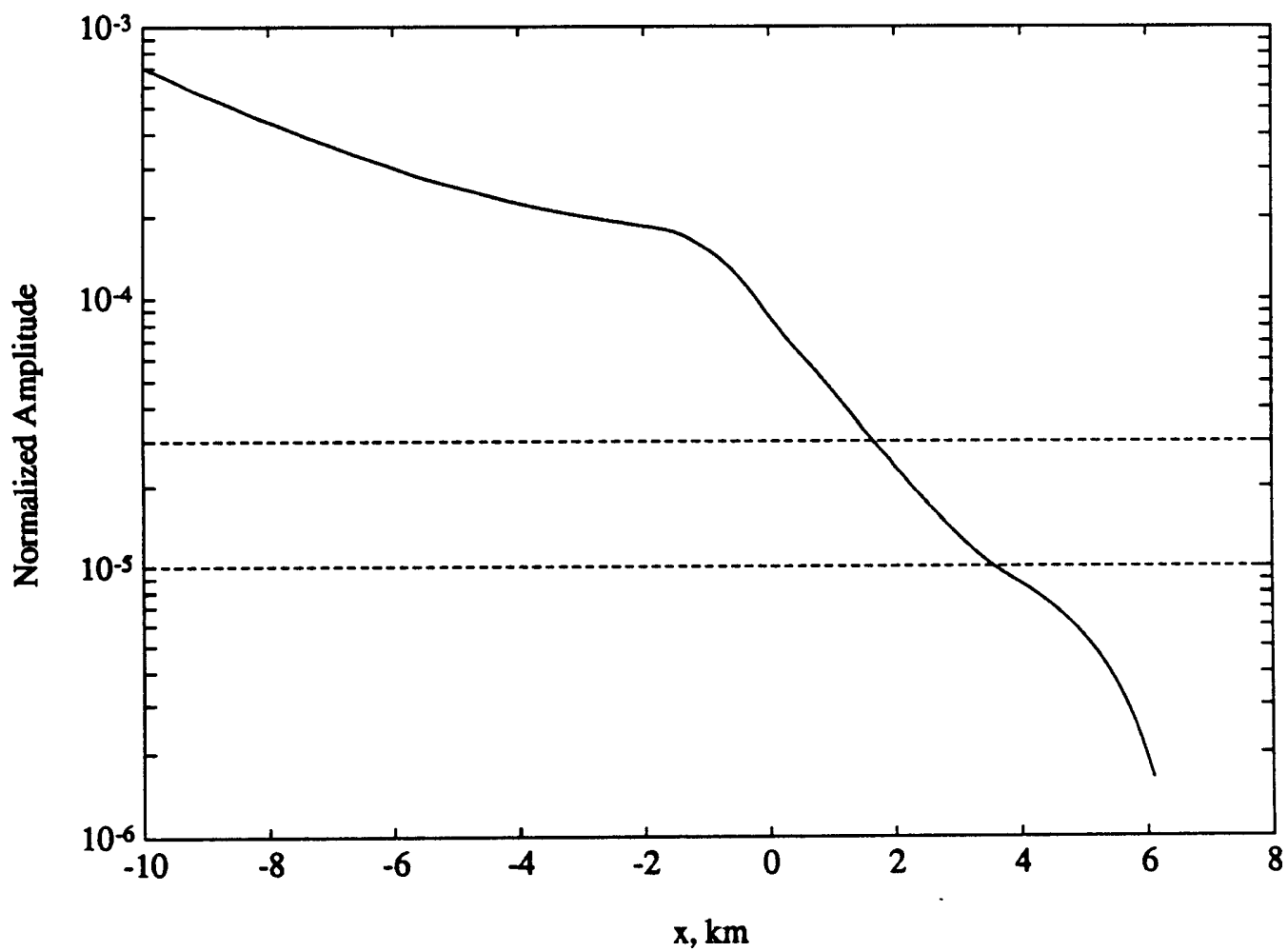

Figure 3.5 
(a)

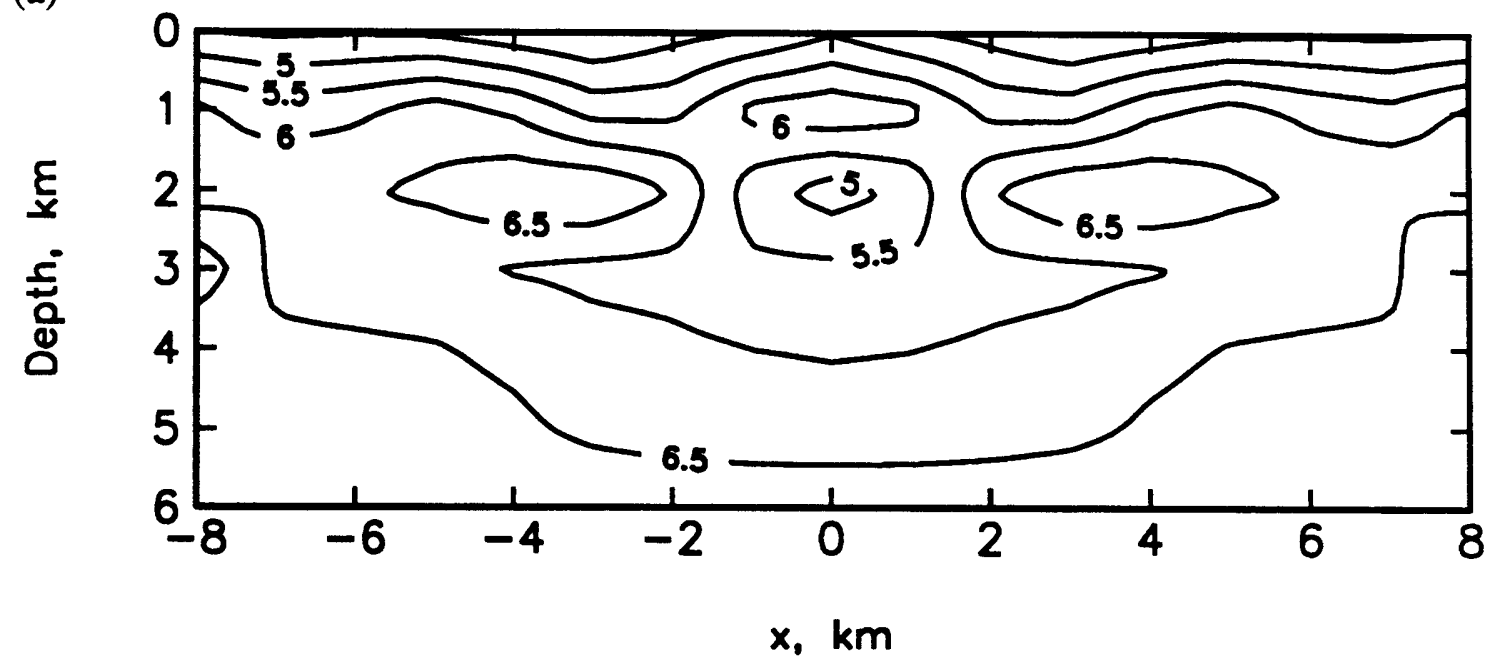

(b)

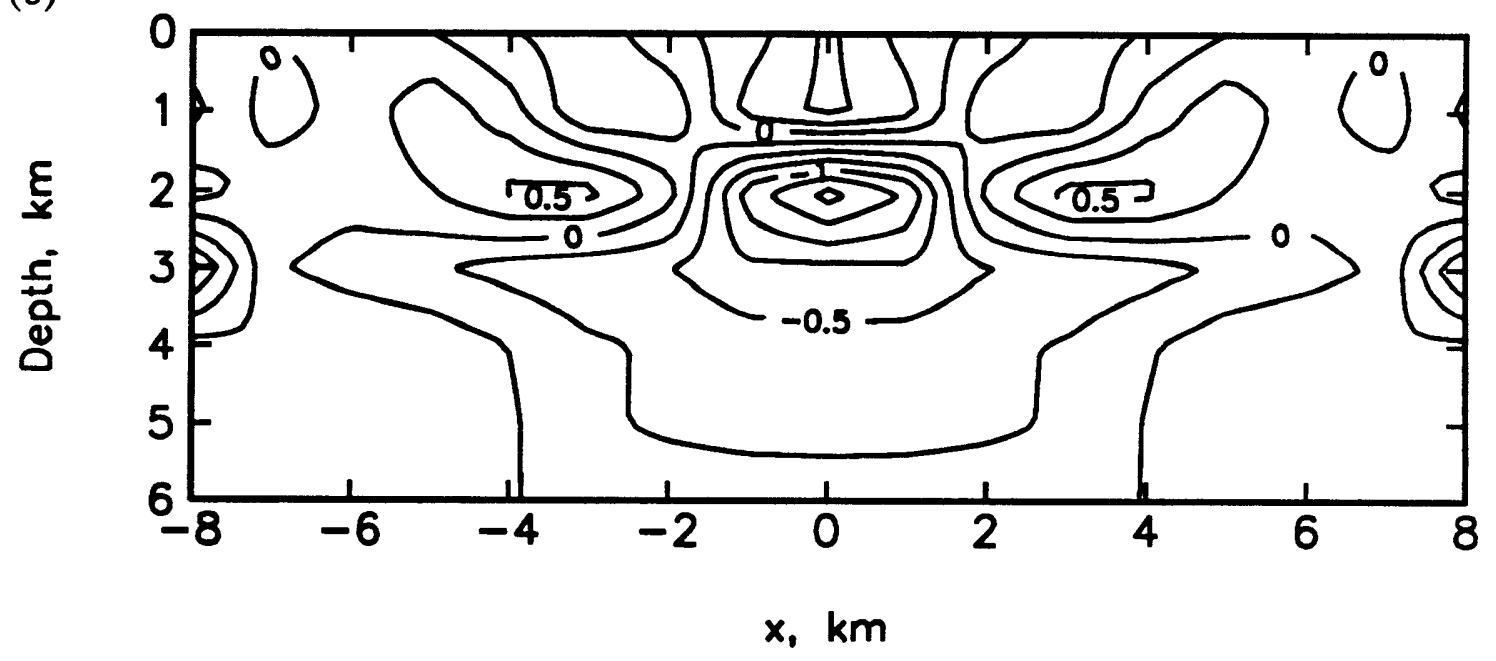

Figure 3.6 


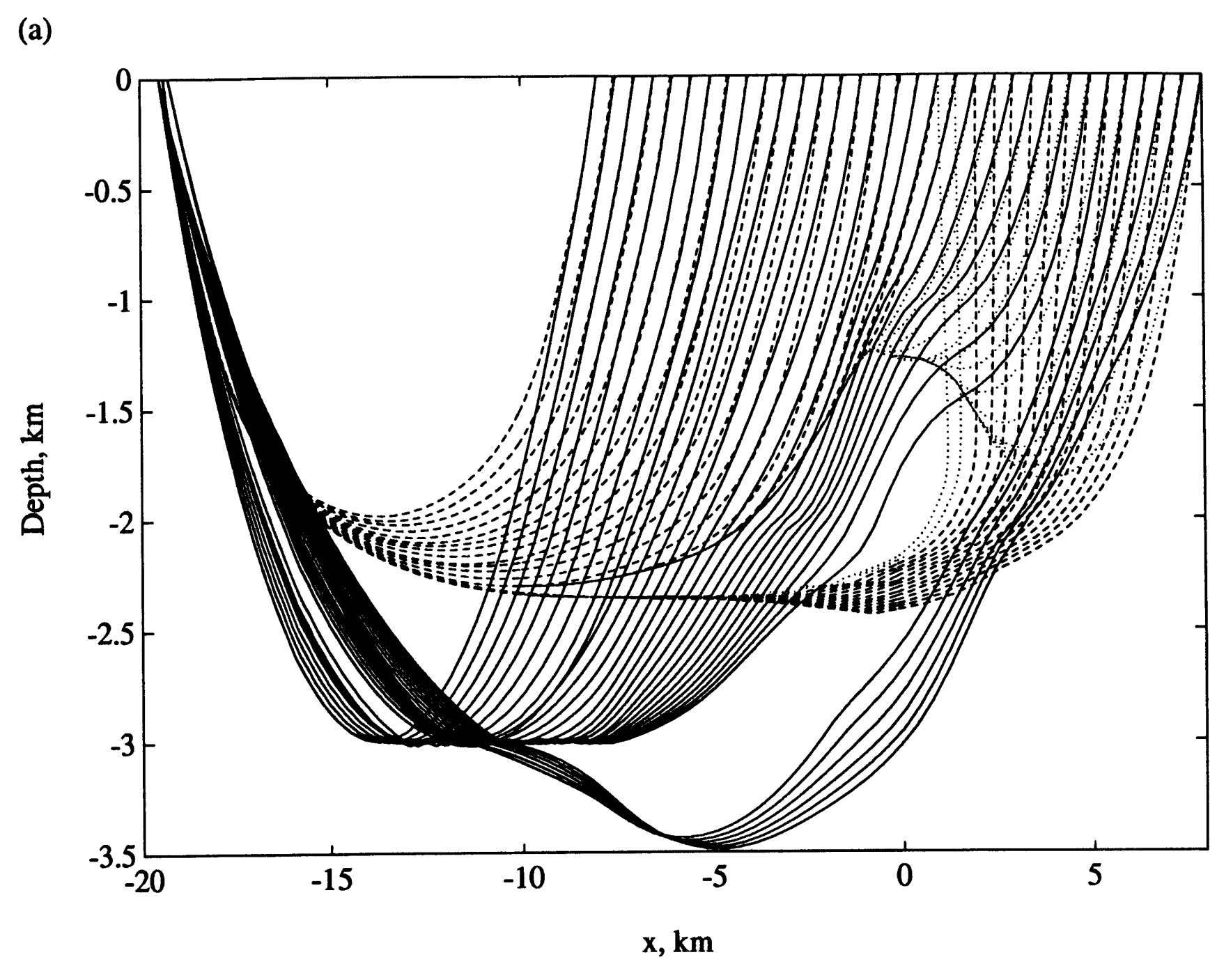

管 
(b)

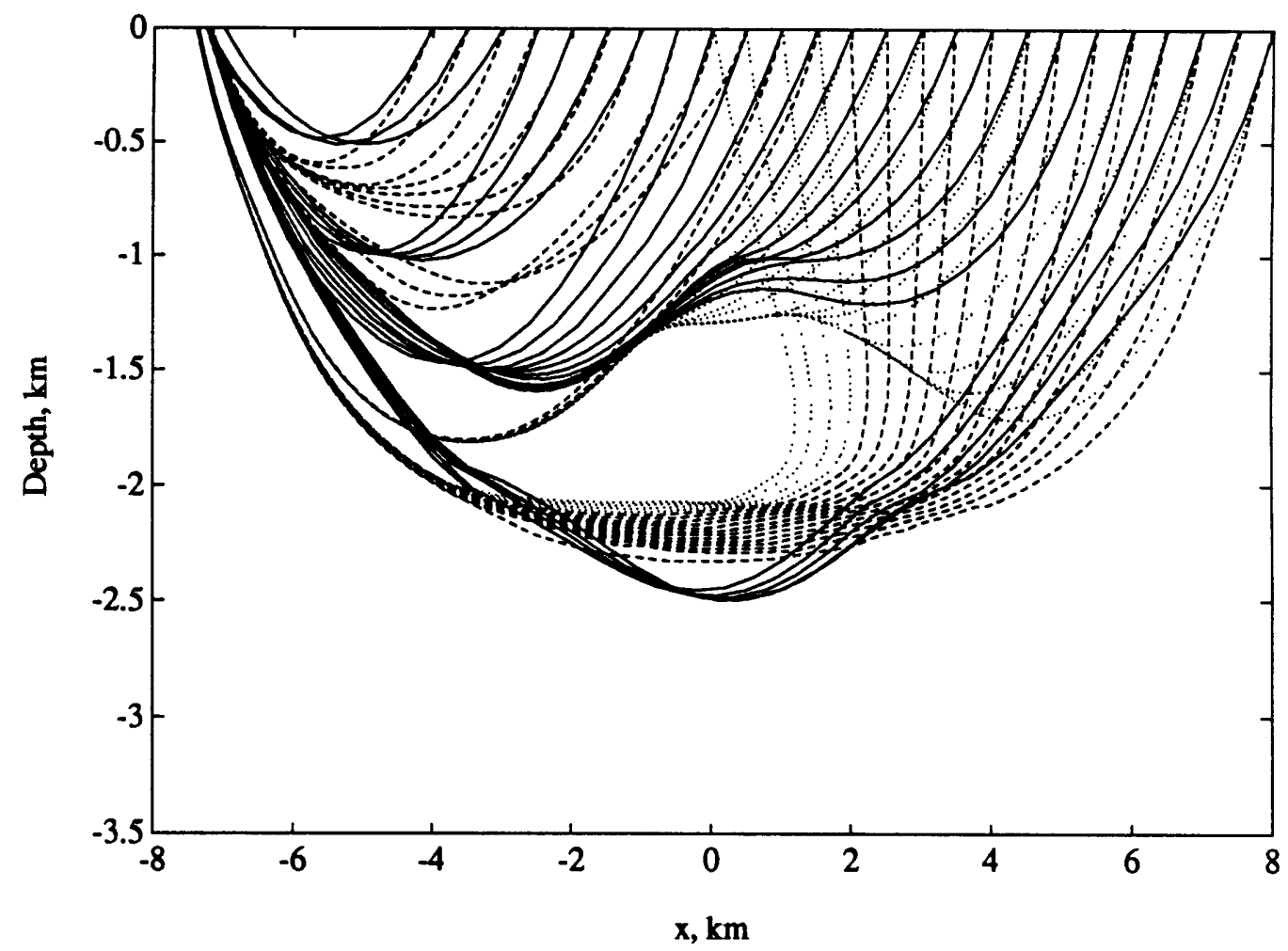

(c)

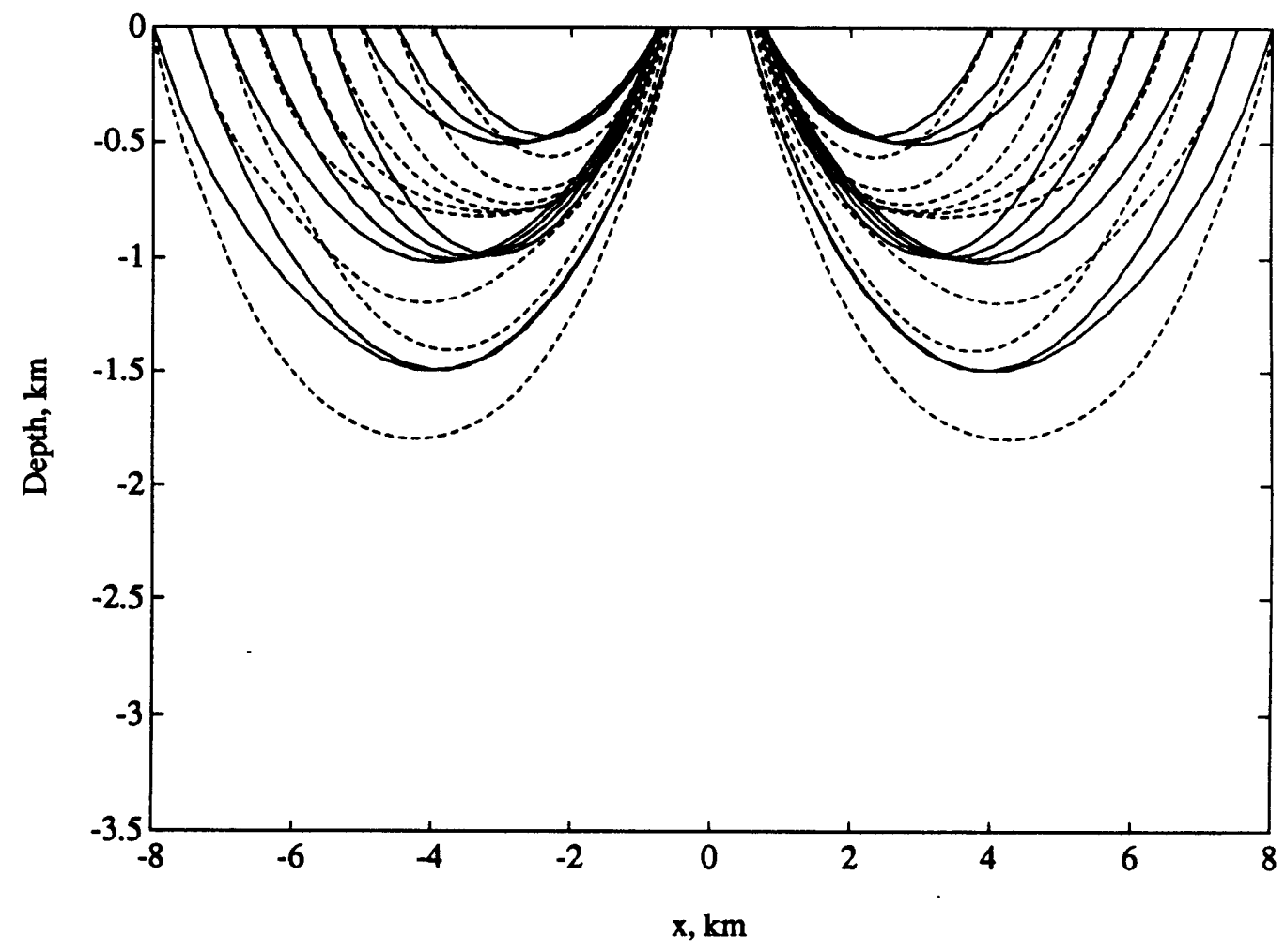

Figure 3.7 cont. 


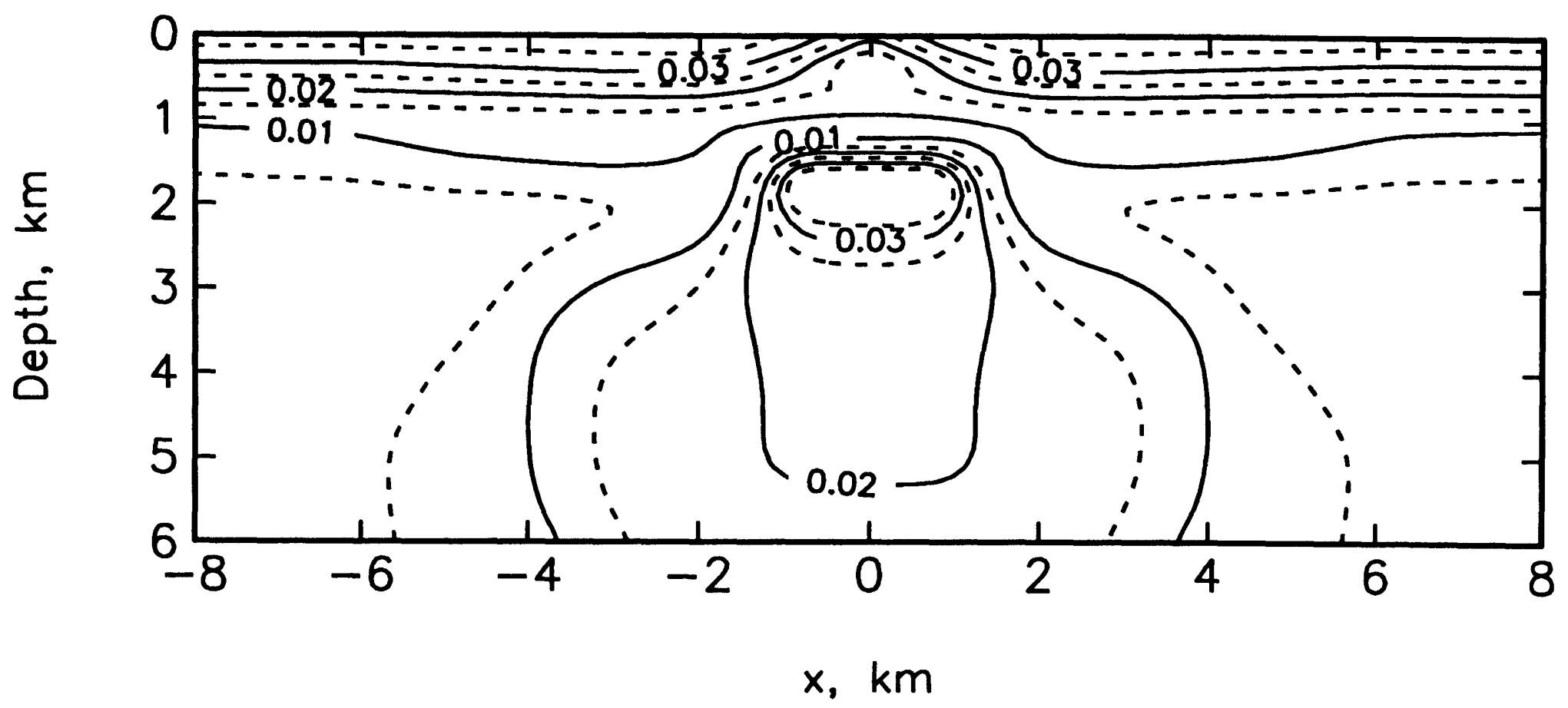


(a)

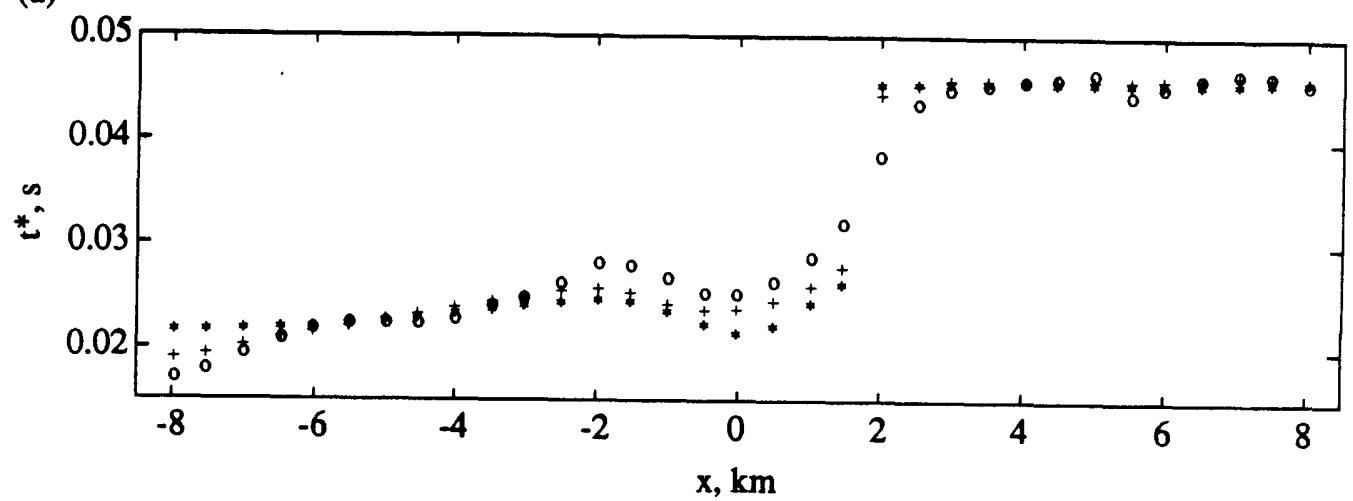

(b)
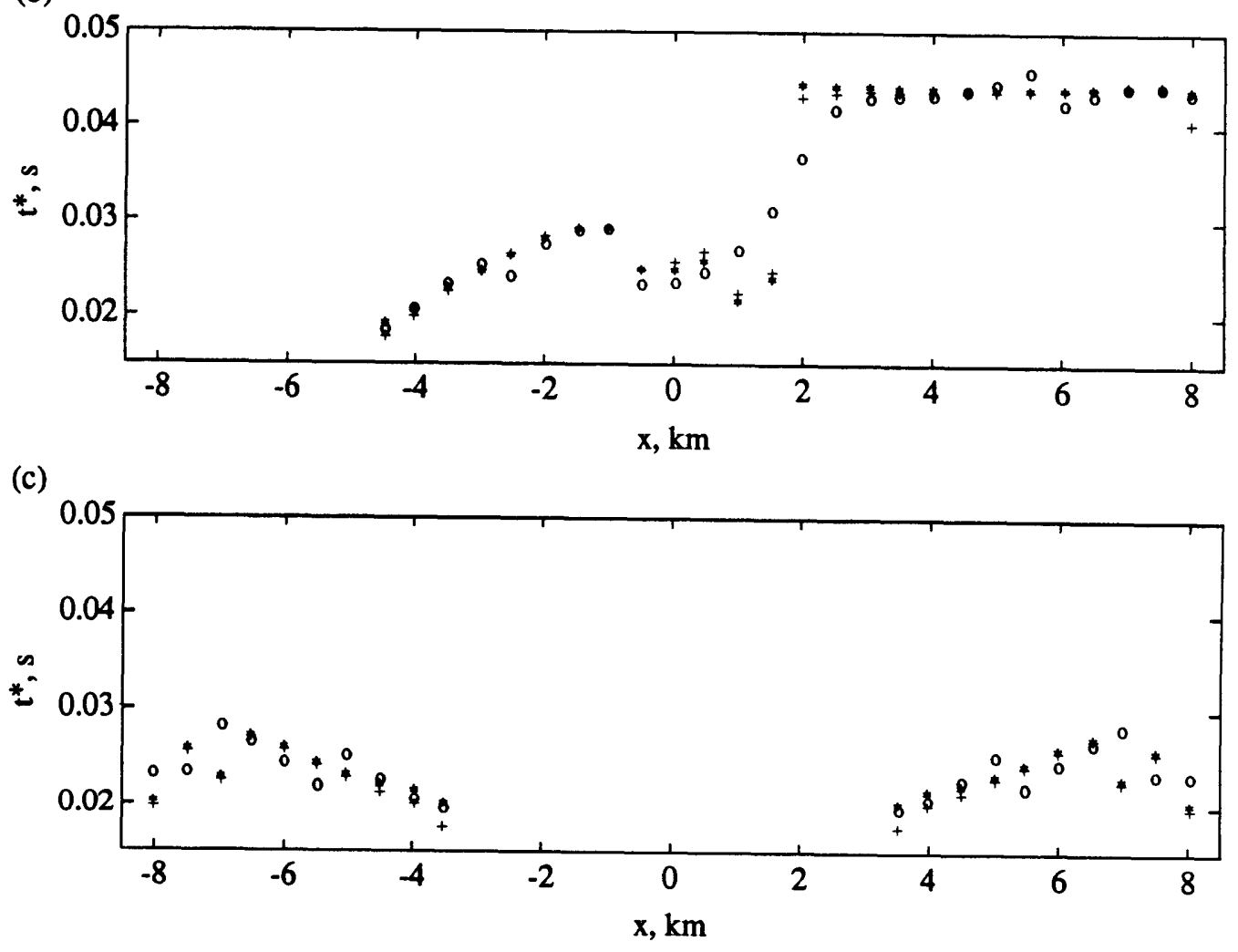

Figure 3.9 
(a)

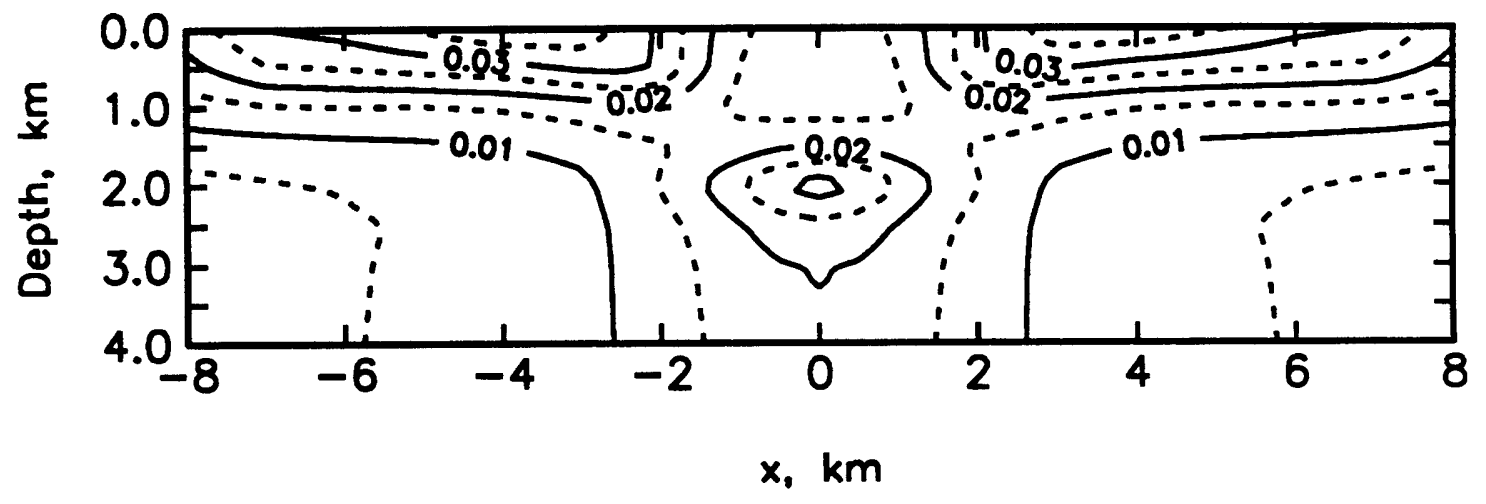

(b)

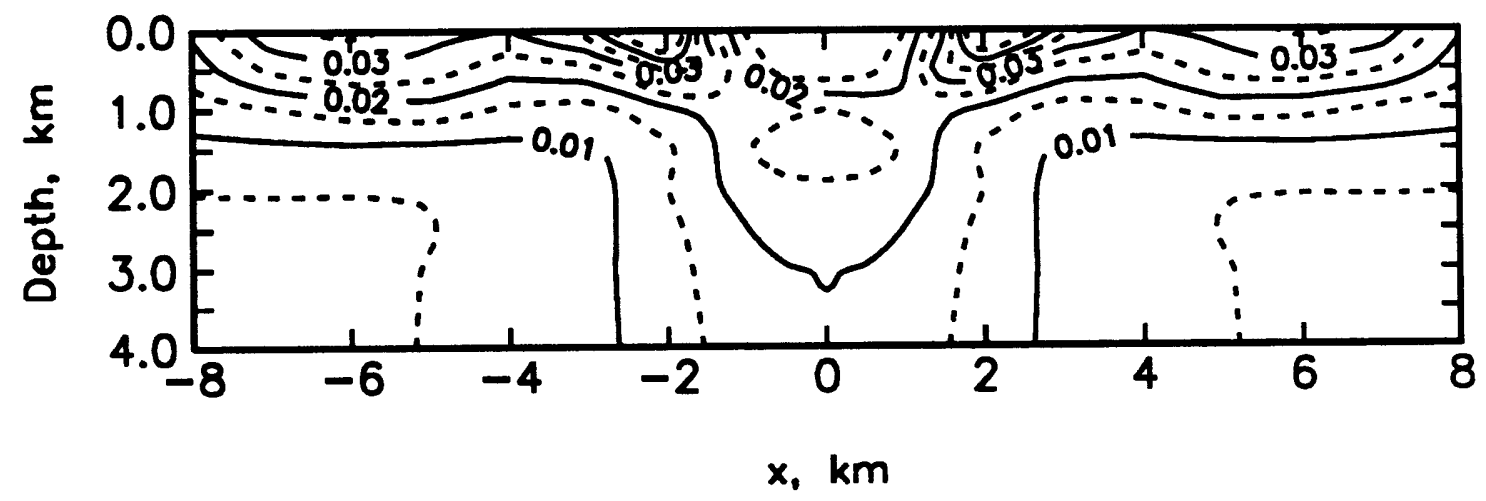

Figure 3.10 
(a) (i)
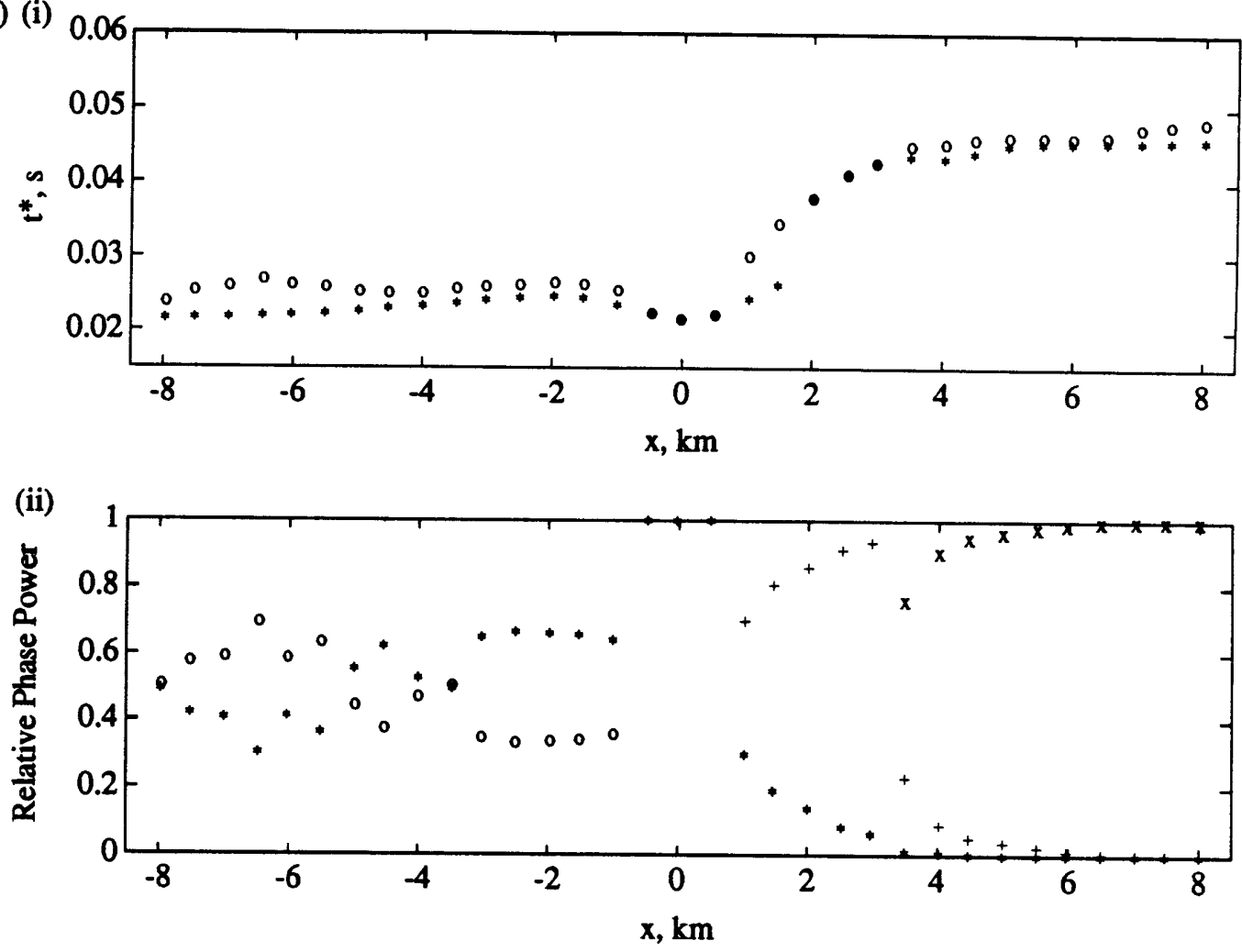

(b) (i)
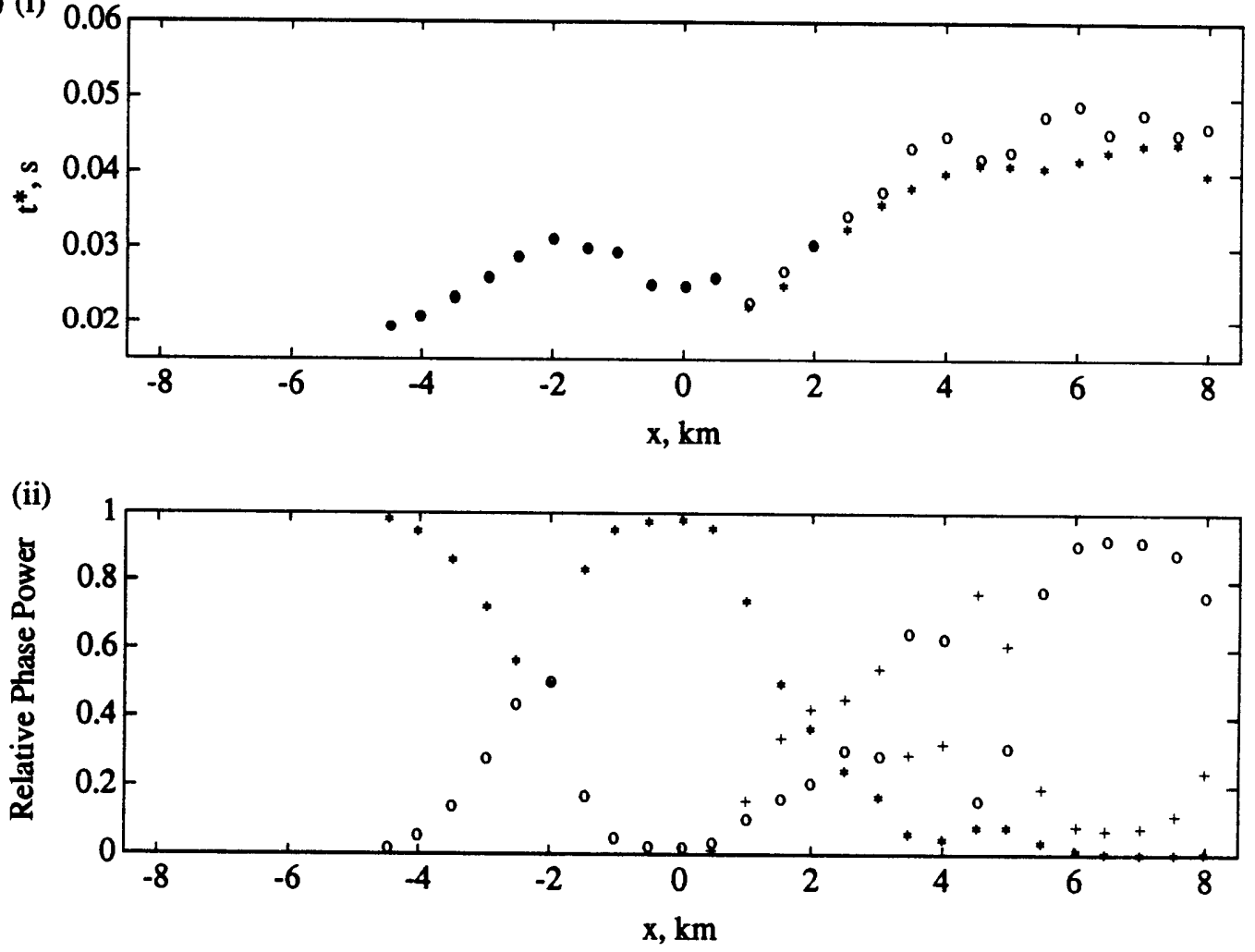

Figure 3.11 
(a)

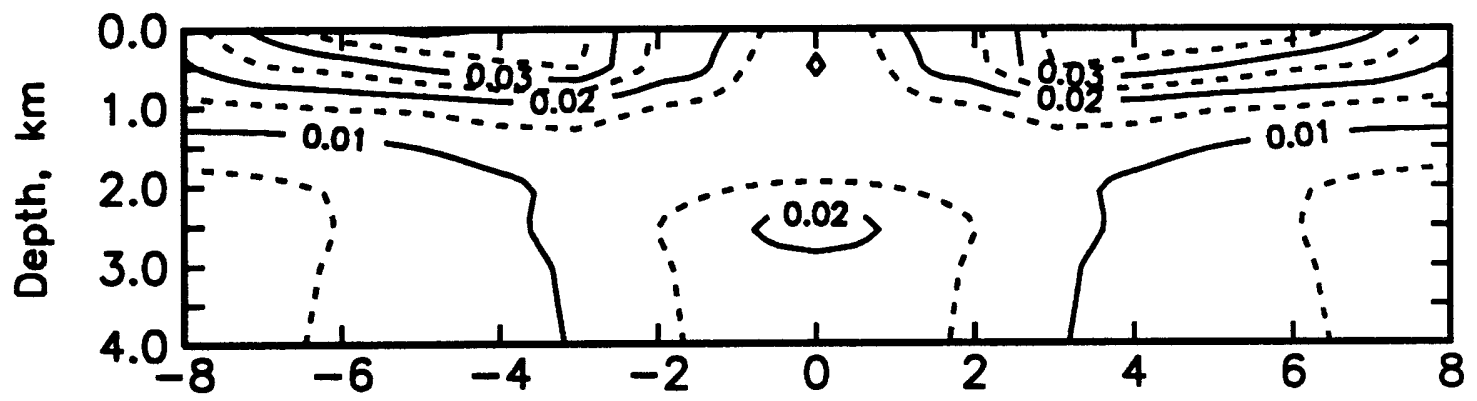

$x, \mathrm{~km}$

(b)

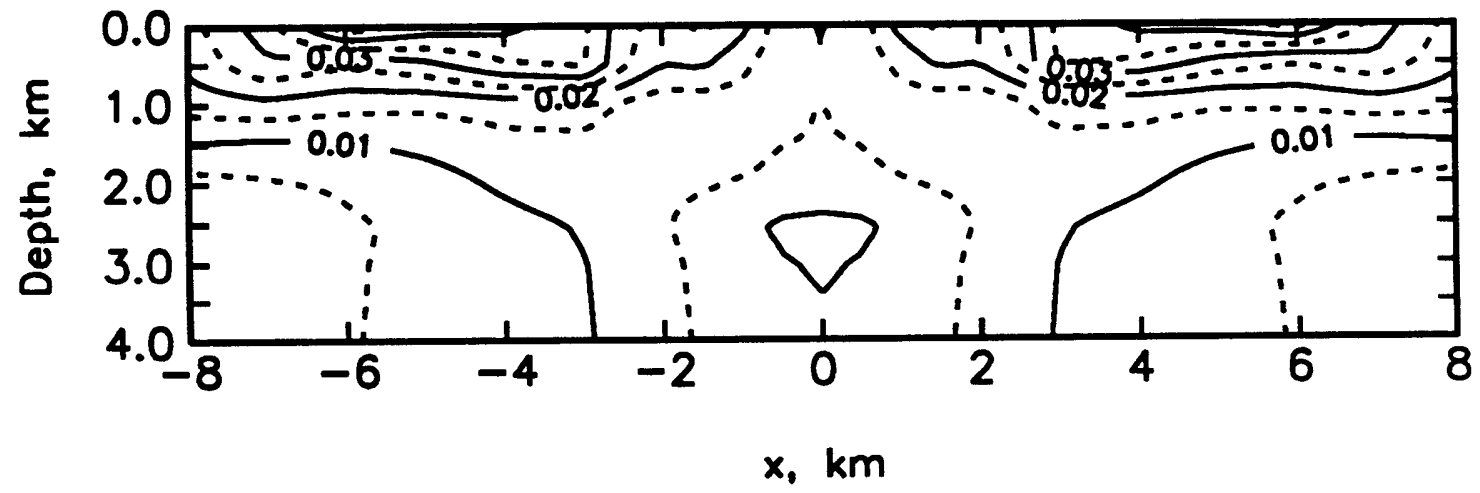

Figure 3.12 
(a)

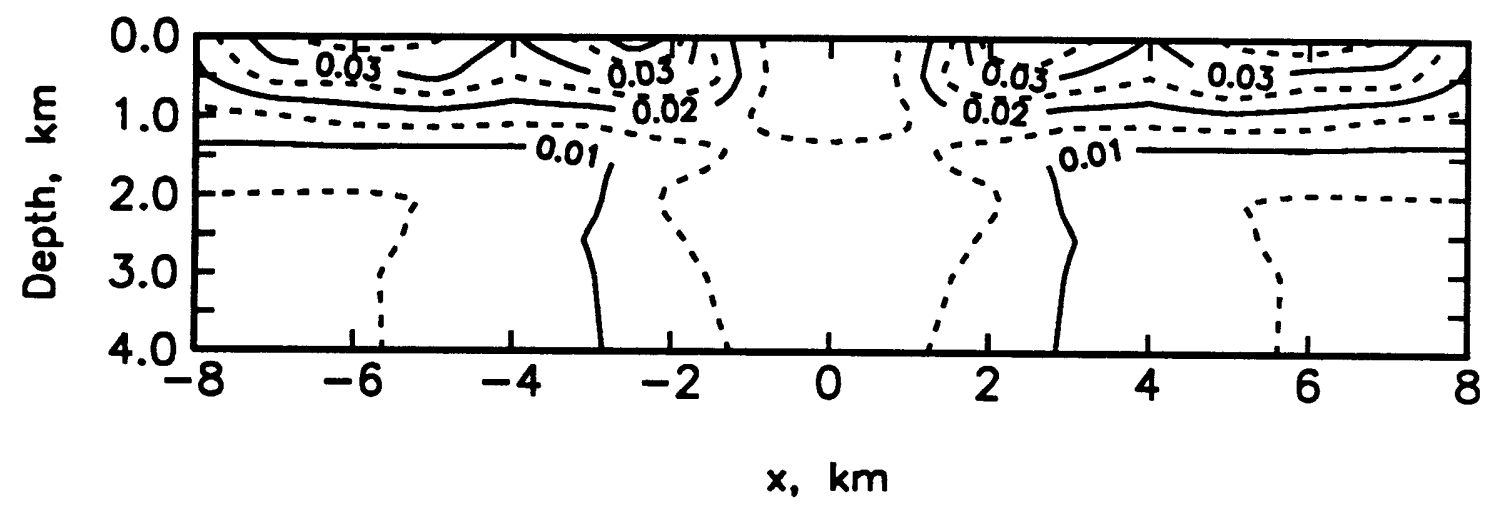

(b)

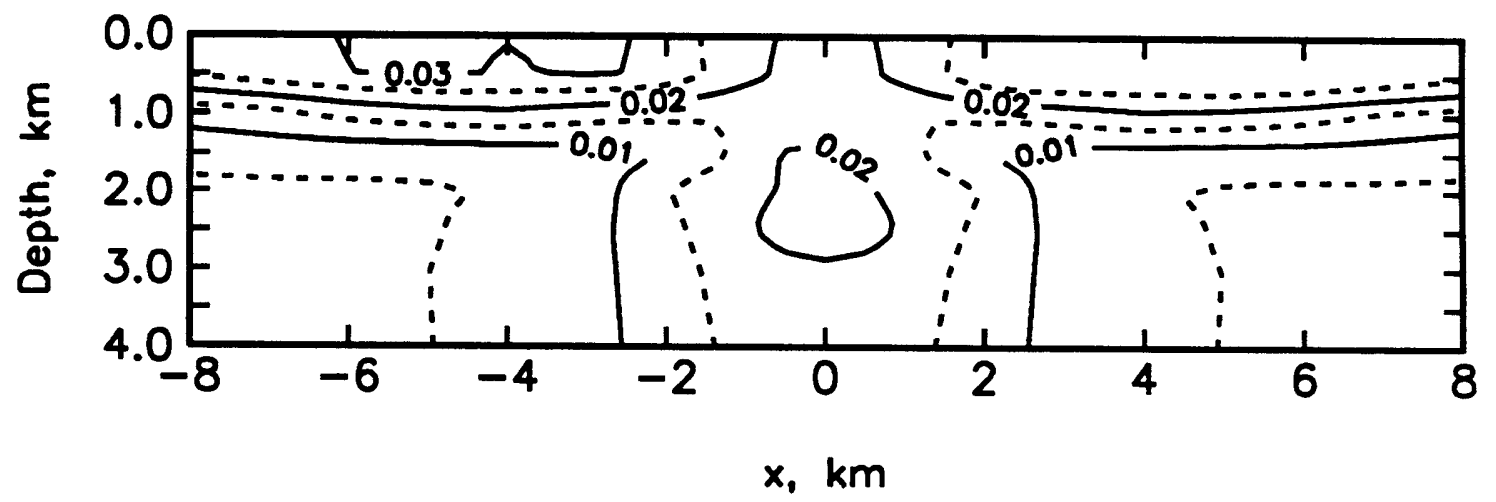

Figure 3.13 

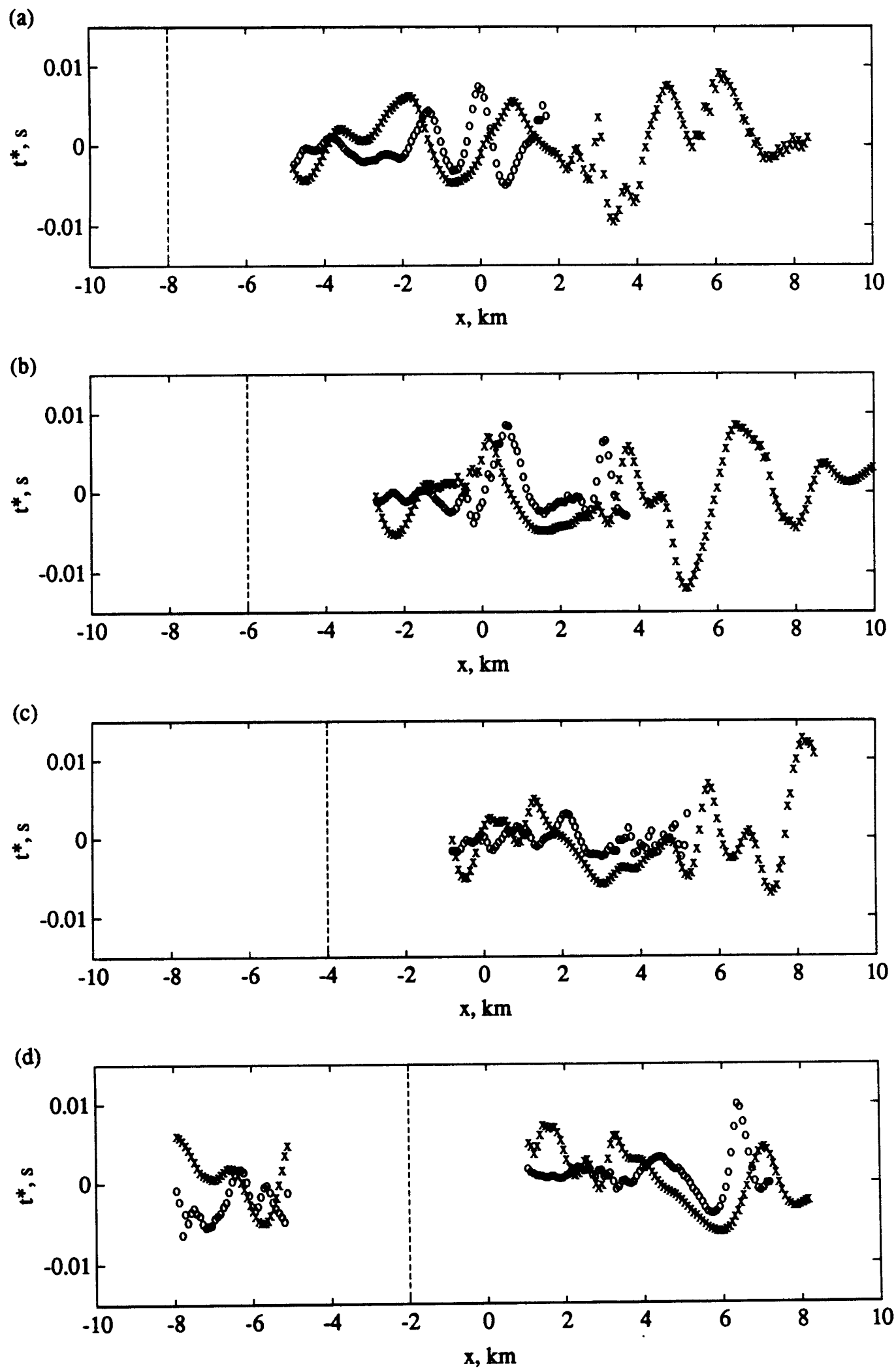

Figure 3.14 


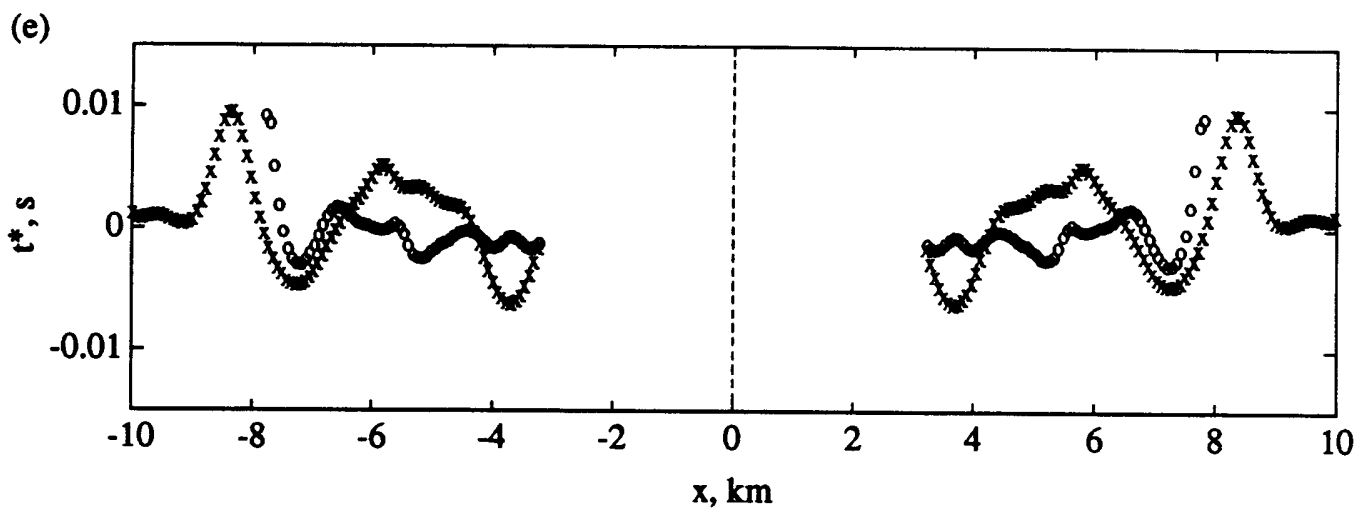

Figure 3.14 cont. 


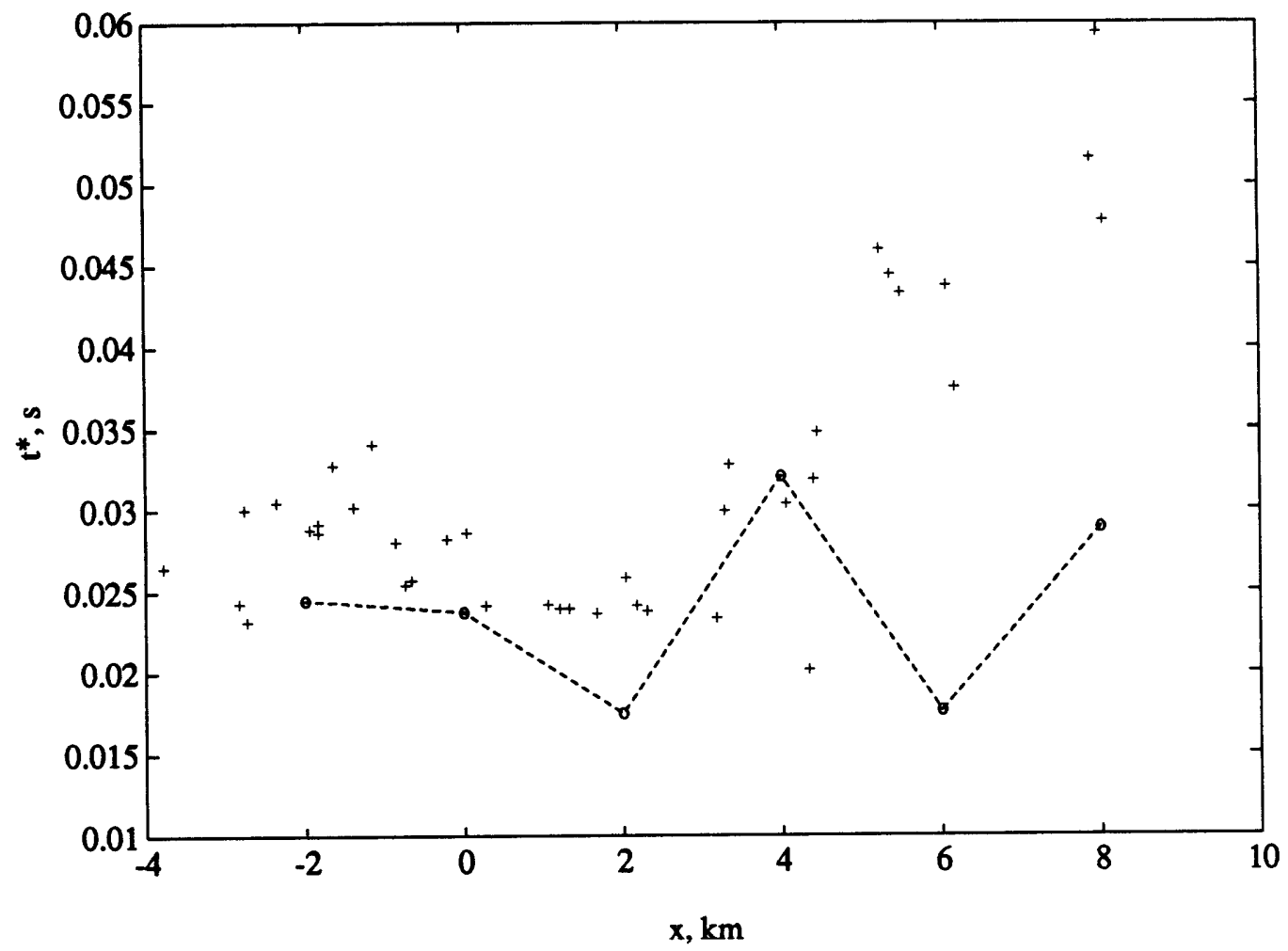

Figure 3.15 


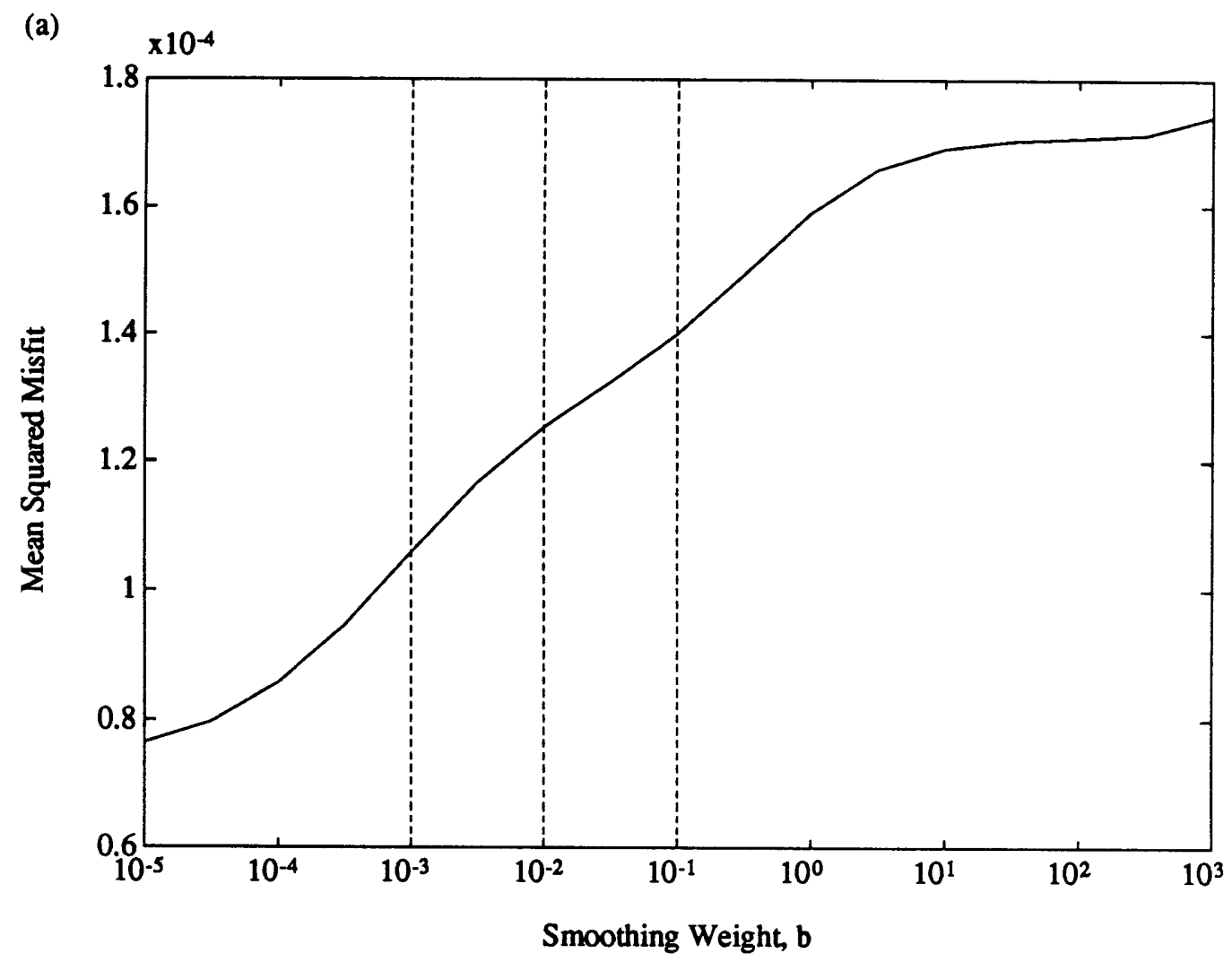

Figure 3.16a 
(b)

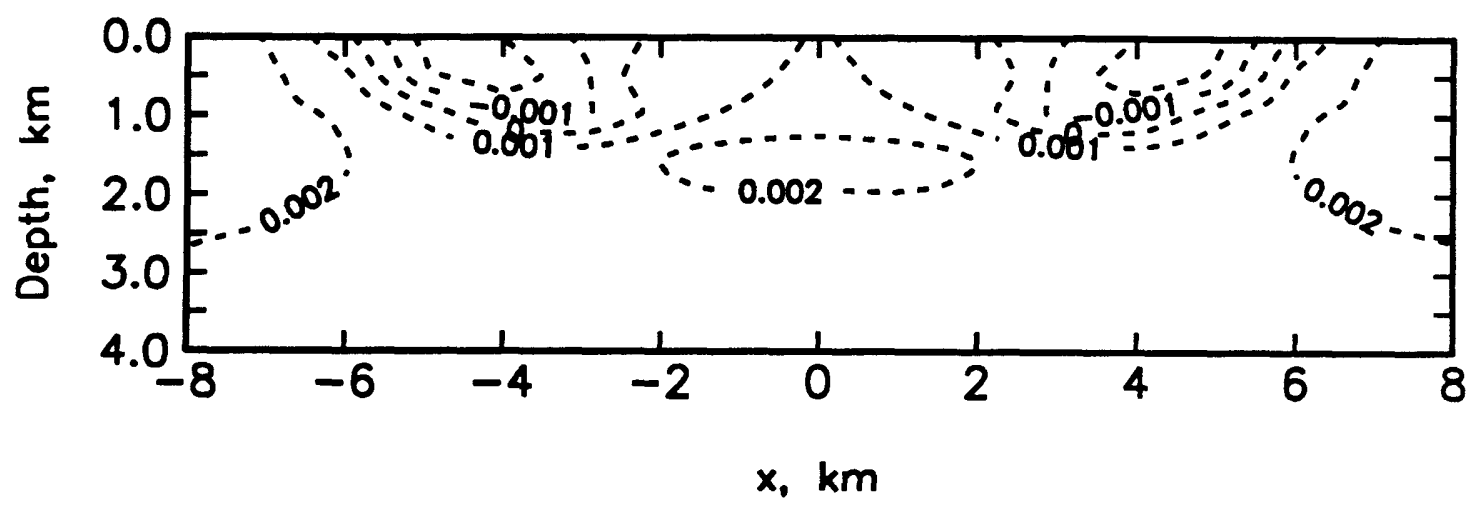

(c)

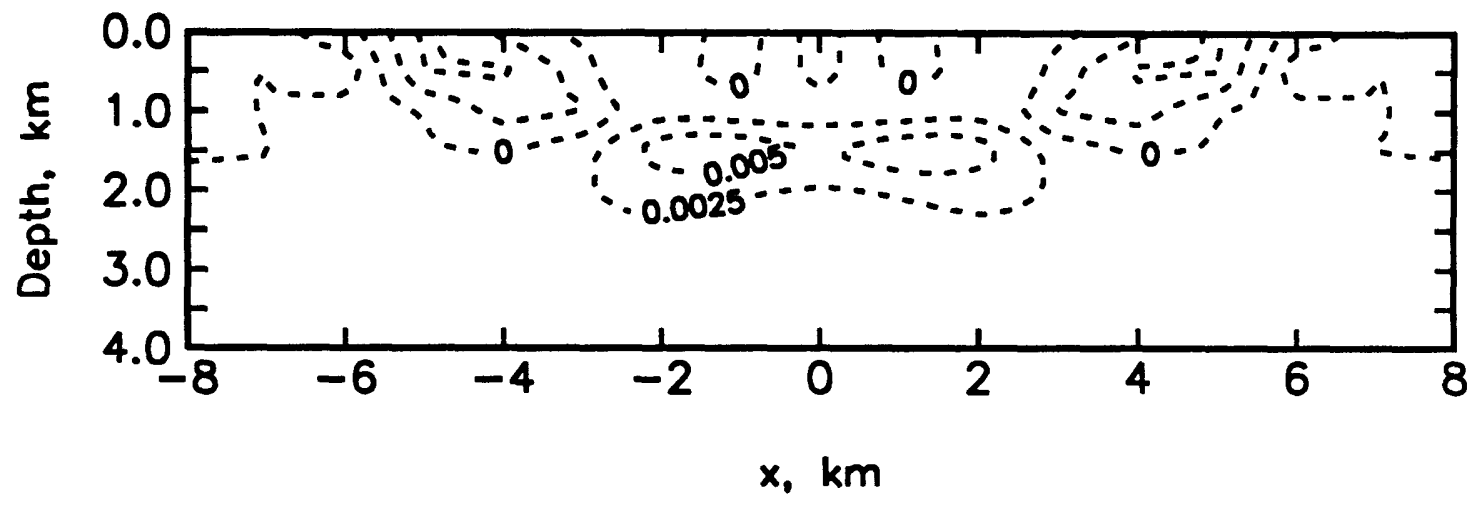

(d)

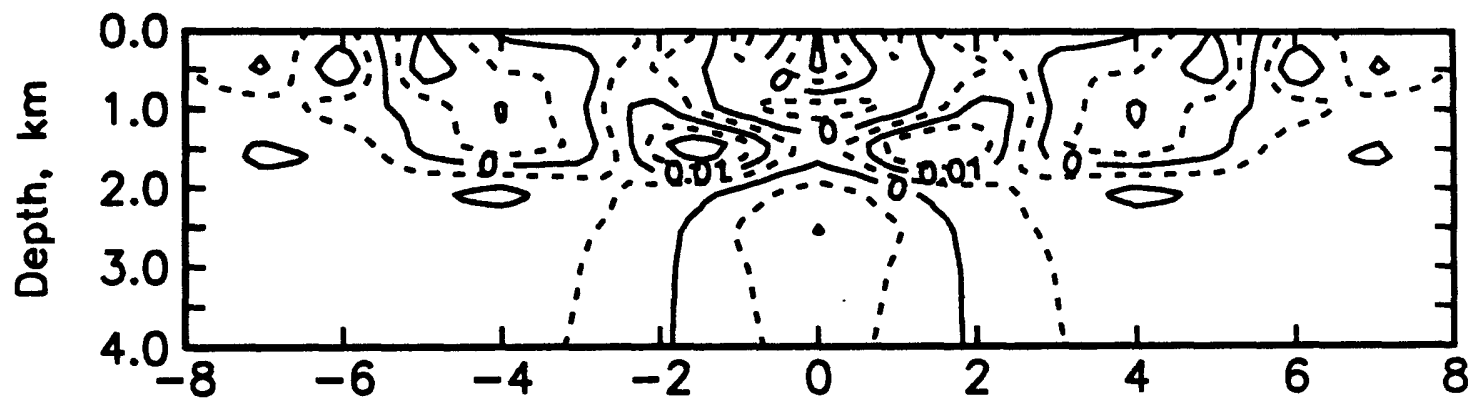

$x, \mathrm{~km}$

Figure 3.16 cont. 
(a)

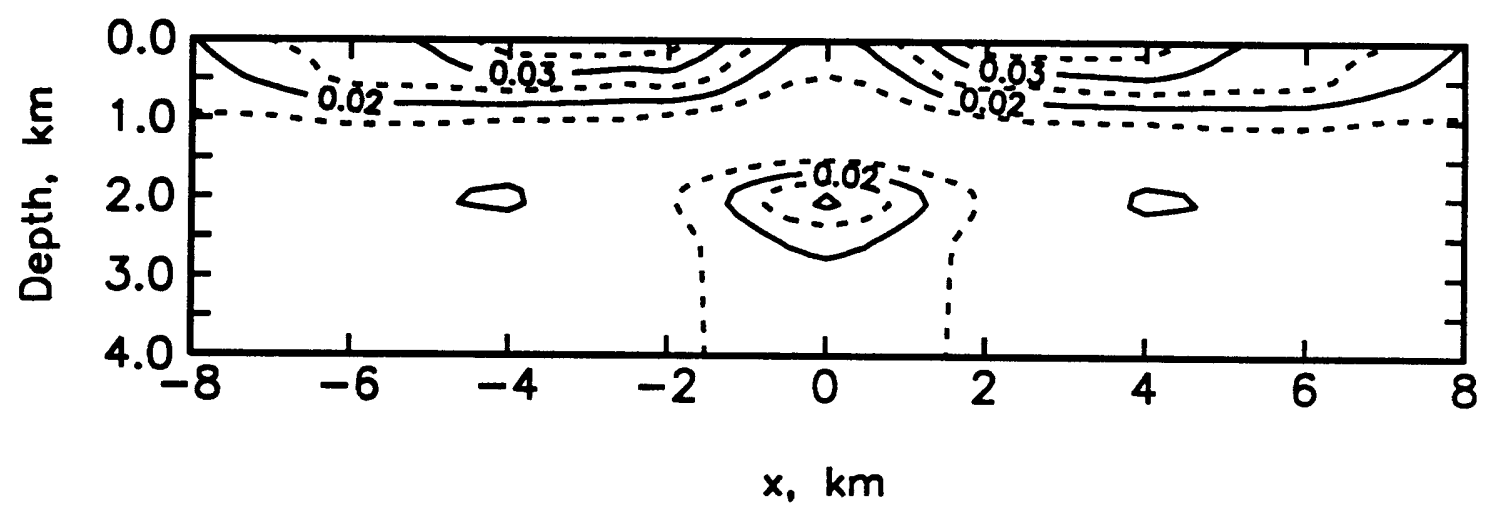

(b)

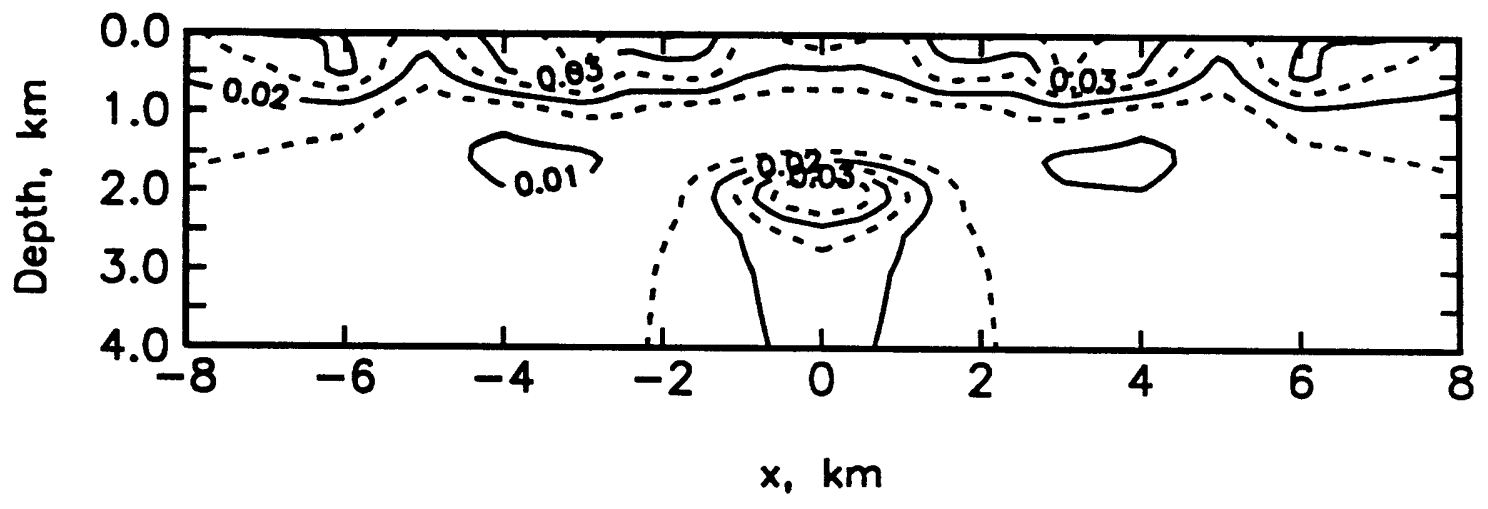

Figure 3.17 


\section{Chapter 4}

\section{TWO AND THREE-DIMENSIONAL INVERSIONS FOR THE $\mathbf{Q}^{-1}$ STRUCTURE OF THE EAST PACIFIC RISE}

\section{INTRODUCTION}

In this chapter we present the results of attempts to invert the full tomography data set for the compressional wave $Q^{-1}$ structure across the the EPR at $9^{\circ} 30^{\prime} \mathrm{N}$. In the last chapter we demonstrated the importance of ensuring that $t^{*}$ estimates are obtained from a time window dominated by a single phase and of correctly determining the appropriate wave paths. Therefore, the first half of this chapter is devoted to a systematic description of the early-arriving compressional phases observed on seismic records and an evaluation of the quality of $\mathrm{t}^{*}$ estimates and approximate wave paths. In the second half of this chapter, the inversion techniques described in Chapter 1 are used to obtain a series of $Q^{-1}$ models. The primary features of the models are shown to be qualitatively apparent from inspection of the $\mathrm{t}^{*}$ and wave path data.

\section{VELOCITY MODELS}

As discussed in previous chapters, the method of $\mathrm{Q}^{-1}$ tomography requires a good knowledge of the velocity structure. While the $t^{*}$ estimates are assumed to be independent of velocity, both the selection of the time window for $t^{*}$ estimation and the choice of wave paths are dependent upon a good understanding of seismic propagation, which in turn requires a well-constrained velocity model. Two models of the cross-axis P-wave velocity structure have been obtained at $9^{\circ} 30^{\prime} \mathrm{N}$ on the EPR [Vera et al., 1990; Toomey et al., 1990a], but for the purpose of $\mathrm{Q}^{-1}$ tomography both have marked deficiencies. The three-dimensional delay time tomographic model [Toomey et al., 1990a] (Figure 1.2) was obtained using arrival times for about 4500 P-wave paths. However, since the longest paths of the tomography experiment were excluded from the inversion, the model does not image the velocity structure at depths greater than about $3 \mathrm{~km}$. While delay time tomographic techniques can successfully resolve the the location and relative magnitude of velocity anomalies, absolute velocity variations are not always well determined. Moreover, the 
resolution of delay time tomographic models is limited. The EPR velocity model is parameterized at nodes on a regular grid with a $1 \mathrm{~km}$ nodal separation in the vertical direction and perpendicular to the rise axis and a $2 \mathrm{~km}$ separation parallel to the rise axis. Such a grid is inadequate to parameterize fully many features of the velocity structure in oceanic crust.

Vera et al. [1990] present a two-dimensional model of velocities across the rise axis (Figures 1.3) which includes many fine-scale details and better estimates of the absolute velocities. However, since the model is obtained by interpolating between one-dimensional velocity profiles obtained from ESP data, parts of the model are subjective while other features have subsequently been shown to be inaccurate. The width of the magma lens is probably overestimated [Kent et al., 1990] while the precise thickness is not known [Vera et al., 1990; Kent et al., 1990]. The structure of the low-velocity zone which extends to the base of the crust beneath the magma body is poorly constrained [Vera et al., 1990], while we suggest that the model overestimates mantle velocities beneath the Moho transition zone at the rise axis (Chapter 2). Moreover, the delay time tomographic inversion apparently resolves significant along-axis variations in the magnitude of the axial low-velocity anomaly [Toomey et al., 1990a]. Inspection of delay times for long range arrivals suggests that significant along-axis variations are also present in the lower crustal and uppermost mantle velocity structure [Toomey et al., 1990b].

Even assuming adequate knowledge of the velocity structure, obtaining accurate three-dimensional wave paths for $\mathrm{P}$-wave phases is problematic. In Chapter 3 the finite-difference technique was used to model wave propagation through a twodimensional model similar to that of Vera et al. [1990]. The results provide important guidance on the length of the time window for $\mathrm{t}^{*}$ estimates and on the choice of wave paths. However, even with the aid of finite-difference 'snapshots', estimating wave paths for non ray-theoretical phases is difficult. The energy diffracted from below the magma chamber appears to propagate over a large range of depths beneath the rise axis, and the selection of a single wave path is subjective. Unfortunately, for computational reasons the finite-difference technique cannot be applied to a three-dimensional model of the axial velocity structure, and therefore our understanding of seismic propagation along paths forming high angles with the rise axis is more limited. To obtain a full set of three-dimensional wave paths the approximate ray-tracing algorithm of Thurber [1983] is used. This ray tracer searches for a minimum time path of constant curvature and then seeks to deform 
this into an approximately ray theoretical path using an iterative scheme. In Chapter 3 we demonstrated that the algorithm consistently approximates neither the shortest time path nor the path of the first arrival with significant amplitude. However, in the following sections we show how an understanding of seismic propagation across the rise axis can be used to limit the extent of the search for a minimum time travel path in the approximate ray-tracing algorithm, so that the final path much better approximates that of the phase which dominates $t^{*}$ estimates.

\section{RECEIVERS 20 KM OFF-AXIS}

Four AOBHs and 4 DOBHs were deployed at a distance of $20 \mathrm{~km}$ from the rise axis (Figure 1.1). In Chapter 2 high quality waveforms recorded by the DOBHs for rise-parallel paths were used to obtain one-dimensional models of the off-axis $Q$ structure. In comparison with the DOBHs the response of the AOBHs has large uncertainties and a smaller bandwidth and the instrument is frequently saturated by crustal phases at shorter ranges. Unfortunately, the experiment geometry is such that the AOBHs which were deployed near the center of the outer refraction lines record waveforms for a large number of source-receiver paths orientated approximately perpendicular to the rise axis, while nearly all the paths for the DOBHs, which were located near the ends of the outer refraction lines, make an angle of at least $20^{\circ}$ with the spreading direction. Therefore, the higher quality waveforms are obtained for paths along which the seismic propagation is not as well understood.

\section{Record Sections and Wave Paths}

Figure 4.1 shows record sections orientated perpendicular to the rise axis for two of the outer AOBHs. Although the source and receiver locations are reversed, there is a marked similarity between these sections and the finite-difference solution obtained for the $20 \mathrm{~km}$ source (Figure $3.4 \mathrm{~b}$ ). Arrival times predicted by an exact ray tracing algorithm using the velocity structure of Vera et al. [1990] and assuming a horizontal seafloor are also shown. In Chapter 3 such arrival times were shown to be in good agreement with full-waveform finite-difference solutions (Figure 3.4). However, in this case systematic offsets may result from errors in the large-scale features of the velocity model, from local variations in shallow structure immediately beneath receivers, and from the failure to include the effects of bathymetry in the predicted times. The direct comparison of synthetic times with the data is further 
complicated by the fairly large and somewhat irregular spacing of seismic records, and by the characteristics of the attenuated source-signature (Figure $1.5 \mathrm{~b}$ ), which is over $0.4 \mathrm{~s}$ long and which displays peak amplitudes $0.2 \mathrm{~s}$ and $0.4 \mathrm{~s}$ after the onset of the waveform.

In both sections the predicted travel times for the first arrival at ranges shorter than about $23 \mathrm{~km}$ are consistently about $0.05-0.1 \mathrm{~s}$ too small, although both the predicted and the observed reduced travel times decrease for sources on the rise axis ( $\pm 20 \mathrm{~km}$ range) due to the higher near-surface velocities on axis. At longer ranges the finite-difference results (Chapter 3) suggest that the amplitude of a minimum time arrival that diffracts above the magma chamber may be too small to observe. Indeed, at such ranges the times of the automatic picks [Toomey et al., 1990a] seem to be consistent with a diffraction from beneath the magma chamber. However, in contrast to the first arrival at shorter ranges, the predicted travel time is larger than observed. Such a discrepancy may result from the velocity model of Vera et al. [1990] incorporating velocities that are too low in the region directly beneath the magma body. Alternatively, the picked first arrival at such ranges may in some cases not be a diffraction from beneath the magma chamber since an early arriving PP phase may propagate above the magma chamber.

At ranges larger than about $23 \mathrm{~km}$ the most pronounced feature of the record sections is a high-amplitude secondary arrival which is identified as a Moho-turning phase and whose predicted arrival time is about $0.2 \mathrm{~s}$ delayed with respect to the diffraction below the magma chamber. The high amplitude of this arrival, which is qualitatively consistent with results of finite-difference modelling (Figure 3.4a), results from the large velocity increase at the Moho near the rise axis. At the longest ranges the ray tracing solutions suggest that this arrival may be preceded by a mantle arrival with a much smaller amplitude. Since the seismic records are complex at longer ranges and the velocity structure beneath the axial magma chamber is poorly constrained, it is not possible to exclude the possibility that energy turning at depths intermediate between the region directly beneath the magma chamber and the Moho transition zone may also contribute to the seismic records. However, since the Moho arrivals have such large amplitudes it seems fairly clear that they will dominate the spectral content of time windows that extend much more than about $0.2 \mathrm{~s}$ beyond the picked first arrivals.

In Figure 4.2a-c examples of wave paths determined for rise-perpendicular paths to $\mathrm{OBH} 1$ are compared. Figure $4.2 \mathrm{a}$ shows ray-theoretical paths for the velocity 
model of Vera et al. [1990]; these paths correspond to the predicted travel times shown in Figure 4.1. Figure 4.2b shows the approximate paths [Thurber, 1983] obtained from the delay time tomographic inversion [Toomey et al., 1990a]. The two sets of paths differ significantly. As was the case for the inversion of finitedifference travel times presented in Chapter 3, the approximate ray paths are placed above the magma chamber at ranges exceeding those at which such arrivals have significant amplitudes. Moreover, the turning depth of these phases is generally larger than those of the ray-theoretical paths, a result primarily of the coarse parameterization of the delay time tomographic model. At longer ranges the approximate paths propagate beneath the axial magma chamber, but turn at a range of depths intermediate between that of energy diffracted beneath the magma chamber and the Moho-turning phase.

In Figure 4.2c constrained approximate paths obtained from the delay time tomographic model are shown. Such paths are obtained by adjusting the velocity at strategic nodes to very low non-zero values to exclude paths that do not conform to that of the desired phase and by restricting the range of constant curvature paths used in the initial search for a minimum time path in the algorithm of Thurber [1983]. Such paths can be seen to be a much better approximation to the ray-theoretical paths shown in Figure 4.2a. The shorter range paths and the paths located above the magma chamber are obtained by placing low velocities beneath the rise axis at depths $\geq 3 \mathrm{~km}$ and by limiting the initial paths to depths no greater than $3 \mathrm{~km}$. Conversely, the diffractions below the magma chamber are calculated by placing very low velocities at axial nodes with 0 and $1 \mathrm{~km}$ depths and by requiring initial paths with a turning depth of $2.5 \mathrm{~km}$. The Moho-turning arrivals are approximated by choosing an initial path that lies in a vertical plane and has a turning depth of $7 \mathrm{~km}$, and by placing near-zero velocities at axial nodes with depths $\geq 2 \mathrm{~km}$.

Since the approximate ray tracing algorithm assumes initial paths of constant curvature and the velocity model is necessarily smoothed by the $1 \mathrm{~km}$ nodal separation, the algorithm does not generally successfully approximate paths in regions where ray-theoretical paths have a high curvature. The deepest portions of the Moho-turning phase do not reproduce the high curvature of the ray theoretical paths even though the nodal velocity model in this region is derived directly from the two-dimensional model [Vera et al., 1990]. However, since the velocity structure is not well known in this region both sets of paths may be poor approximations to the true paths. In the case of shots close to the rise axis, the location of the high 
curvature portions of the paths which pass beneath the magma chamber is determined by the location of nodes with near-zero velocities. Therefore, while such paths may approximate the ray-theoretical paths obtained from the symmetric twodimensional model of Vera et al. [1990] they are unlikely to show the effects of along-axis variability and cross-axis asymmetry resolved by the delay time tomographic inversion [Toomey et al., 1990a].

Figure 4.3 shows two record sections for profiles to the outer DOBHs which are orientated at about $50^{\circ}$ to the rise axis. The principal features are very similar to the axis-perpendicular sections of Figure 4.1. At shorter ranges the first arrival has a phase velocity close to $7 \mathrm{~km} / \mathrm{s}$, while at longer ranges the record sections are dominated by a high-amplitude Moho-turning phase. The Moho arrival for the western receiver (DOBH 12) has markedly higher amplitudes than for the eastern receiver (DOBH 14), a characteristic that is also apparent in Figure 4.1. Both the delay time tomography [Toomey et al., 1990a] and multichannel reflection profiles [Mutter et al., 1988] suggest that the magma chamber is offset to the west of the rise axis in the southern portion of the experiment area. While upper crustal asymmetry may affect the amplitude of the Moho-turning phase to some extent, the large difference in amplitudes between east- and west- propagating arrivals strongly suggests that the structure at the Moho is also asymmetric across the rise axis. While such asymmetry may involve changes in the location of the Moho transition zone, there seems a strong possibility that the largest velocity gradients within the Moho transition zone are also offset to the west of the rise axis. However, careful modelling would be required to to obtain a full understanding of the Moho velocity structure in this region.

In comparison with Figure 4.1 the waveforms at longer ranges are markedly simpler and seem qualitatively consistent with a single Moho arrival. The diffraction from beneath the magma chamber may be masked by the Moho phase since extrapolating the predicted travel times of Figure 4.1 suggests that the arrival times of the two phases may be fairly similar. Indeed, the apparently high phase velocities required by the three largest range picks on DOBH 12 may be an indication that the first arrival is a mantle phase. However, in the absence of quantitative predictions obtained from a well-constrained velocity model, it is not possible to specify with certainty the nature of the picked first arrival, although the Moho phase will probably dominate the spectral content of all but the shortest time window. Constrained approximate wave paths appropriate to the oblique record section for DOBH 12 
(Figure 4.3a) are shown in Figure 4.2d after projection onto a vertical plane orientated perpendicular to the rise axis. The paths are constrained using the criteria described earlier and have characteristics that are very similar to the riseperpendicular paths.

Figure 4.4 shows a rise-parallel record section for the longest-range axiscrossing shots recorded by DOBH 12 and 14. The source-receiver separation varies between about $55 \mathrm{~km}$ at the north of the sections to $40 \mathrm{~km}$ near the south. The firstarriving mantle phase is followed by a complex, higher amplitude crustal phase at a delay of about $0.2 \mathrm{~s}$, an observation in good agreement with the predictions obtained from the velocity model of Vera et al. [1990]. The amplitude of the crustal phase varies considerably with path azimuth and is always high for approximately riseperpendicular paths, suggesting that such arrivals may be triplicated. Indeed, the length of the rise-perpendicular paths is close to the typical range of the highamplitude Moho triplication observed in older oceanic crust. However, the Mohoturning arrivals predicted by the model of Vera et al. [1990] are confined to considerably shorter ranges, and the ray-theoretical path for this phase (Figure 4.2a) is a low-amplitude crustal phase that turns at about $5 \mathrm{~km}$ depth, well above the Moho transition zone. Therefore, the precise propagation depth of this phase beneath the rise axis is poorly constrained. However, since the shorter-range Moho-turning paths apparently turn several kilometers from the rise axis and propagate across the rise axis at mid to lower crustal depths, the longer-range arrivals almost certainly propagate below this phase beneath the rise axis. This is manifested in the choice of a $7 \mathrm{~km}$ turning depth for this phase in the calculations of the constrained approximate ray paths (Figure $4.2 \mathrm{c}$ ).

\section{t* Estimates}

It is qualitatively apparent from Figures 4.1 and 4.3 that the waveforms for paths that propagate more than a few kilometers across the rise axis are characterized by a marked decrease in high frequency content. Figure 4.5 shows examples of source- and receiver-corrected power spectra and $t^{*}$ estimates for DOBH 12 obtained from a window that includes $0.6 \mathrm{~s}$ of the waveform immediately following the automatic pick of the first arrival. At larger ranges, the upper frequency limit of the estimate, which is determined by the signal-to-noise ratio, is markedly lower and the $t^{*}$ estimates are nearly twice those at shorter ranges. In Figure 4.6, $\mathrm{t}^{*}$ estimates obtained from $0.3 \mathrm{~s}$ and $0.6 \mathrm{~s}$ of data for centrally located shots recorded 
by DOBH 14 are plotted at the shot locations. For both choices of window length there is a marked increase in $t^{*}$ values at ranges corresponding to those at which the amplitude of the phase that propagates above the magma chamber becomes insignificant. In the case of the 0.6 -s estimate this change is particularly abrupt.

A careful comparison of $t^{*}$ values at shorter ranges shows that the 0.6-s estimates generally show smaller variations between adjacent $t^{*}$ values. This characteristic was also observed for the outer refraction lines (Chapter 2) and is a result of the larger uncertainties associated with spectral estimates obtained from shorter time windows. Since the 0.6-s estimates show no large systematic offset from the 0.3-s estimates for any instrument we choose to use the 0.6-s estimates for waveforms whenever estimates from the longer window are not compromised by high-amplitude secondary arrivals. In Figure $4.7, \mathrm{t}^{*}$ estimates for such waveforms are presented for several instruments located $20 \mathrm{~km}$ off axis.

At longer ranges the 0.3-s and 0.6-s t* estimates for the same shot (Figure 4.6) frequently differ considerably. While such differences may in part be due to the increased uncertainties resulting from the decreased frequency interval available for spectral estimates, they presumably also result from the greater contribution of secondary arrivals to the power spectra obtained from the longer window. Indeed this effect is immediately apparent from an inspection of the two waveforms at 27 and $29 \mathrm{~km}$ range in Figure 4.5. The power spectra of the 0.6-s estimates are dominated by high amplitudes in the second half of the window that would be excluded from an 0.3-s estimate. Moreover, since the automatic picks which determine the position of the data window are not always consistent (e.g., Figure 4.2), such an effect may also contribute to variations between $t^{*}$ values for adjacent shots that are calculated using the same window length.

The $t^{*}$ values obtained from the earliest portion of the waveform suggest that both the diffraction below the magma chamber and the Moho-turning phase are characterized by high levels of attenuation. However, $t^{*}$ estimates with significant contributions from more than one phase are of little use in a quantitative inversion based on the linear relationship of equation (1.6). Not only do such values invalidate the assumption of a single wave path, but the combined $t^{*}$ value depends on both the attenuated amplitude of each phase and the individual $t^{*}$ values. Therefore, even if weighted multi-paths are included in the inversion, the problem becomes very nonlinear. The analysis of the record sections presented in the last section suggests that to obtain $t^{*}$ estimates for the diffraction below the magma chamber requires a 
window no longer than about $0.2 \mathrm{~s}$ for rise-perpendicular paths and an even shorter window for oblique paths. Moreover, since the velocity structure is not very well known and the earliest portion of the attenuated source signature has relatively low amplitudes (Figure 1.5b), it is difficult to maximize the length of a window which includes the diffracted phase while simultaneously ensuring that the Moho-turning phase is entirely excluded from the window. Unfortunately, $t^{*}$ estimates obtained from windows shorter than $0.3 \mathrm{~s}$ are unsatisfactory. Not only are spectral estimates obtained from very short windows subject to large uncertainties, but in Chapter 1 it was shown that the resulting $\mathrm{t}^{*}$ estimates may be severely biased toward lower values (Figure 1.9).

Therefore, our approach is to seek $t^{*}$ estimates for 0.6 -s-long windows aligned with a conservatively early estimate of the onset time of the Moho-turning phase. Examples of $t^{*}$ values for this phase are shown in Figure 4.8. The estimates are generally considerably higher than those obtained for shorter range paths (Figure 4.7). If a time window is dominated by a single high-amplitude phase then the $t^{*}$ estimate obtained from the window will closely approximate that of this phase provided lower amplitude phases within the window do not have significantly lower levels of attenuation. If low-amplitude, low-t* phases are included they may contribute significantly to the power spectrum at higher frequencies, thus biasing $\mathrm{t}^{*}$ values to lower values. The 0.6 -s window used to estimate $t *$ for the Moho-turning phase will in all cases contain at least a portion of the diffraction from beneath the magma chamber. However, in the next section it will be shown that the diffracted phase has $t^{*}$ values that are comparable to those of the Moho phase and so is unlikely to bias $t^{*}$ estimates significantly. At shorter ranges, phases propagating above the magma chamber may have significant amplitudes and since such paths are apparently associated with lower $t^{*}$, a bias may result. Indeed, some of the shorter range $t^{*}$ estimates in Figure 4.8 do have significantly smaller values than the estimates at longer ranges.

Inspection of Figure 4.8 shows that, even after ignoring low values at shorter ranges, $t^{*}$ estimates for individual instruments do show significant variations and that these variations often appear related to the shot distance from the rise axis. These variations may be related to the axial attenuation structure since the depth of wave paths beneath the rise axis (Figure 4.2) apparently changes significantly with the source-receiver separation. In addition, frequency-dependent focusing may contribute to the observed variations. However it is difficult to demonstrate 
convincingly that the observed variations are not primarily due to the effects of secondary phases. For this reason and because of the large uncertainties accompanying the estimates of the wave paths, the fine structure of the lower crustal $\mathrm{Q}^{-1}$ structure beneath the rise-axis is unlikely to be fully resolved by inversions of these data.

Figure 4.9 shows all the $t^{*}$ estimates for two instruments together with uncertainties and the maximum frequency used to obtain the estimates. In both cases the uncertainties associated with the Moho phase are significantly greater than those for the shorter range crustal phase. While this is in part a reflection of the smaller frequency interval available to estimate $t^{*}$ values, such an effect does not account entirely for the difference. The straight line fit to the natural logarithm of the power spectrum (equation 1.7) is markedly poorer for the Moho-turning phase, a further indication that the $t^{*}$ estimates may be affected by other phases.

In Figure 4.10, we present $t^{*}$ estimates for paths that propagate between the two outer refraction lines. Since, the first arriving mantle phase is followed by a much higher amplitude crustal phase at $\leq 0.2 \mathrm{~s}$ delay, a reliable $\mathrm{t}^{*}$ estimate cannot be obtained for the former phase. Therefore $t^{*}$ estimates presented are for a $0.6-\mathrm{s}$ window aligned with the onset of the higher amplitude phase. Since this arrival is apparently triplicated for rise-perpendicular paths but not for longer-range oblique paths, the path of the dominant arrival may well vary. Moreover the $t^{*}$ estimates may include significant contributions from more than one path, particularly in the case of rise-perpendicular paths where the waveforms appear complex (Figure 4.4). While the $t^{*}$ estimates for these arrivals are noticeably larger than those for short range upper crustal paths, they appear to be significantly smaller than those for the Moho-turning phase (Figure 4.11). Since these arrivals probably cross the rise axis at larger depths than shorter range Moho-turning phases, this difference may reflect vertical variations in the $\mathrm{Q}^{-1}$ structure within the lower crust and uppermost mantle.

\section{RECEIVERS 9 KM OFF-AXIS}

Four receivers, comprising an OBS, two AOBHs, and a DOBH, were deployed near the corners of the central area of dense shooting, at a distance of $9 \mathrm{~km}$ from the rise axis (Figure 1.1). Unfortunately, OBS 3 was poorly coupled and the resulting seismograms are dominated by an azimuthally dependent resonance which prohibits spectral $t^{*}$ estimates. 


\section{Record Sections and Wave Paths}

Figure 4.12 shows rise-perpendicular record sections for AOBH 4 and DOBH 15 together with ray-theoretical arrival times obtained from the velocity model of Vera et al. [1990]. The record section shows many similarities with the finite difference solution for a source at $8 \mathrm{~km}$ range (Figure 3.4a). At all but the largest ranges the picked first arrival propagates above the magma chamber. However, at ranges greater than about $12 \mathrm{~km}$ the amplitude of this phase is small and its identification is strongly dependent upon the signal-to-noise ratio, an observation in excellent agreement with finite-difference solution obtained for an 8-km source (Figure 3.5). In the case of $\mathrm{AOBH} 4$ the predicted travel times of this phase are almost $0.1 \mathrm{~s}$ too small.

For paths that cross the rise axis, both the finite-difference solutions (Figure 3.4a) and the exact ray tracing algorithm predict a number of secondary arrivals within the first $0.5 \mathrm{~s}$ of the waveform. Unfortunately because of the large and irregular trace separations, the complexity of the source signature, and the possible systematic errors in predicted times, this portion of the record sections is difficult to interpret unambiguously. In the case of DOBH 15 the predicted time of the diffraction below the magma chamber coincides with the onset of higher amplitudes. Comparison of the waveforms with the attenuated source signature (Figure 1.5b) suggest that at the largest ranges this phase may account for the high amplitude portion of the waveform. A diffracted arrival can also be identified on AOBH 4, but as is the case for the first arrival the predicted time is about $0.1 \mathrm{~s}$ too small. Unlike DOBH 15, the amplitudes of the records suggest the presence of an additional high amplitude phase with a larger travel time.

After correcting amplitudes for realistic crustal velocities at the seafloor, the finite-difference solutions (Figures 3.4a and 3.11b) suggest that a PP phase with a downward reflection from the seafloor near the rise axis may be an important arrival. Indeed the relative amplitude of such an arrival may be enhanced by the high nearsurface $Q$ observed on the rise axis (Chapter 2). The high amplitude of the PP phase is, however, dependent upon the presence of a small upper crustal triplication which for the off-axis velocity structure of Vera et al. [1990] is located at about 7-8 km range. In addition the low velocities within and below the axial magma body inhibit propagation across the rise axis of PP phases whose bounce points are not immediately above the magma chamber. The distance of the receivers from the rise axis $(9 \mathrm{~km})$ is slightly greater than the range of the shallow crustal triplication $(7$ 
$\mathrm{km}$ ) obtained from the two-dimensional velocity model of Vera et al. [1990], and as a result ray-theoretical PP arrivals of significant amplitude are not predicted at ranges greater than about $12 \mathrm{~km}$. Indeed no PP arrival is apparent at large ranges on DOBH 15. However, in the case of AOBH 4 a high amplitude phase is observed at 14-16 $\mathrm{km}$ range whose arrival time is consistent with the predicted arrival time of a very low-amplitude ray-theoretical PP phase after accounting for the relative delay observed for other phases. As noted above, the results of travel time tomography [Toomey et al., 1990a] and of mutli-channel seismic observations [Mutter et al., 1988] suggest that the axial magma chamber is offset to the west in the southern portion of the experiment. Such asymmetry may enhance the propagation of the PP phase by reducing the distance from $\mathrm{AOBH} 4$ to the magma chamber to a value similar to the range of the shallow crustal triplication, thus facilitating the propagation of a high amplitude PP phase at 14-16 km range.

Alternatively the high amplitude arrival observed on AOBH 4 might be a Mohoturning phase. The velocity model of Vera et al. [1990] predicts the presence of raytheoretical Moho arrivals at ranges greater than $14 \mathrm{~km}$. However, the existence of Moho arrivals at such short ranges is dependent upon the velocity within the uppermost mantle near the the rise axis. The model of Vera et al. [1990] includes values close to $8 \mathrm{~km} / \mathrm{s}$ which in Chapter 2 we suggest may be significantly too high. Reducing uppermost mantle velocities to $7.25 \mathrm{~km} / \mathrm{s}$, the value suggested in Chapter 2 , increases the minimum range of ray-theoretical Moho arrivals to about $20 \mathrm{~km}$. For computational reasons the finite-difference results (Chapter 3) include maximum velocities of only $7 \mathrm{~km} / \mathrm{s}$ and elevate the Moho $1 \mathrm{~km}$ beneath the rise axis, and therefore cannot be compared directly with the experimental data. However, the finite-difference solutions do include Moho arrivals with significant amplitude at ranges that are significantly shorter than predicted by ray theory. Thus, the high amplitude arrival observed on $\mathrm{AOBH} 4$ might be a Moho arrival though if this phase is present on DOBH 15, the amplitude must be markedly lower. Indeed, this discrepancy is consistent with the higher amplitude Moho arrivals observed on western receivers at $20 \mathrm{~km}$ range from the rise axis, which where interpreted in terms of an asymmetric Moho velocity structure.

Figure 4.13 shows record sections for the same instruments as Figure 4.12 but orientated at about $50^{\circ}$ to the rise axis. At ranges much greater than the rise axis the seismic records appear to be dominated by a single phase with relatively high amplitudes. Such an arrival is most straightforwardly interpreted as a diffraction 
from beneath the magma chamber. However, since the maximum ranges are significantly greater in the oblique section, this arrival might also be a Moho-turning phase. The two dashed lines show estimates of the minimum and maximum arrival times for the Moho phase. For the minimum time a velocity structure is obtained by projecting the velocity model of Vera et al. [1990] parallel to the rise axis onto the plane of the record section. For the maximum time the velocity structure of Vera et al. [1990] is rotated about the rise axis into the plane of the obliquely orientated section. The results suggest that the highest amplitude portion of the waveform has too small a travel time to be a Moho arrival. This conclusion seems to be supported by the observation that the amplitude of the arrival is much smaller than the amplitude of the Moho arrival recorded by the receivers $20 \mathrm{~km}$ off-axis (Figures 4.1 and 4.3). However, the apparent absence of a Moho phase with significant amplitudes on this section is slightly puzzling. Clearly, the explanation requires a better knowledge of the velocity structure and the ability to model seismic arrival times and amplitudes in a three-dimensional medium.

Figure 4.14 shows exact and approximate direct crustal ray paths for AOBH 4. As is the case for the receivers $20 \mathrm{~km}$ off-axis, the approximate ray paths obtained from the delay time tomographic inversion (Figure 4.14b) do not always match the ray-theoretical path of the first arrival with significant amplitude (Figure 4.14a). However, the constrained approximate paths (Figure 4.14c) which are obtained using the criteria outlined in the last section, match the ray-theoretical paths fairly well and can be obtained for paths orientated obliquely to the rise axis (Figure 4.14d).

\section{$\mathbf{t}^{*}$ Estimates}

The analysis of the record sections suggests that $\mathrm{t}^{*}$ estimates for the 9-km receivers may be obtained for both the direct upper crustal arrivals at shorter ranges (Figure 4.15) and the diffraction beneath the magma chamber at longer ranges (Figure 4.16). However, for diffractions below the magma chamber an 0.6-s-long data window is clearly too long to ensure that later arrivals are excluded from $t^{*}$ estimates. Therefore $t^{*}$ estimates for this phase are obtained from an 0.3-s-long window, the shortest window for which $t^{*}$ biases arising from $4 \pi$-prolate MWSA (Figure 1.9) are acceptable. The window is aligned with a manual pick of the diffracted phase, and estimates are confined to ranges at which the amplitude of the first arrival propagating above the magma chamber is negligible. Using an 0.3-s-long 
window to obtain $\mathrm{t}^{*}$ estimates for the arrival that propagates above the magma chamber would extend the maximum range of such estimates by $1-2 \mathrm{~km}$ by excluding the diffraction from beneath the magma chamber from the later part of the window. However, we chose to use an 0.6-s-long window since the uncertainty of the resulting $\mathrm{t}^{*}$ estimates is smaller. Figure 4.17 shows all $\mathrm{t}^{*}$ estimates together with uncertainties and the maximum frequency used for AOBH 6. As was the case for the receivers $20 \mathrm{~km}$ off-axis, the higher uncertainties of the $\mathrm{t}^{*}$ estimates for the diffractions beneath the magma chamber results from a combination of the decreased frequency interval available for $\mathrm{t}^{*}$ estimates and a generally poorer straight line fit to the logarithm of the power spectrum. In this case the poorer fit is at least in part a reflection of the higher uncertainties accompanying spectral estimates from the 0.3-slong window.

Estimates of $t^{*}$ for a Moho-turning phase that propagates across the rise axis from shots on the outer refraction lines may also be obtained for these instruments. Figure 4.18 show examples of such measurements for AOBH 4 and DOBH 15. The $t^{*}$ values for this phase, whose paths (dot-dashed line in Figure 4.2a, c) cross the rise axis at substantially greater depths than the diffraction beneath the magma chamber, are generally lower than the $t^{*}$ values for the diffraction below the magma chamber, indicating that $Q$ values may be lower immediately beneath the axial magma chamber than in the lowermost crust and upper mantle beneath the rise axis.

\section{RECEIVERS ON THE RISE AXIS}

Two AOBHs and an OBS were deployed on the rise axis. In Chapter $2 t^{*}$ estimates for rise-parallel paths were used to resolve relatively high $Q$ in the uppermost crust and very low $Q$ values within and beneath the axial magma chamber. In Figure 4.19, $t^{*}$ estimates and uncertainties obtained from an 0.6-s-long window immediately following the automatic pick are presented for AOBH 5 and OBS 1 . In both cases $\mathrm{t}^{*}$ estimates are markedly lower than those obtained for deeper rise-crossing paths to other instruments, a reflection of the absence of $t^{*}$ estimates for paths passing through the low-velocity region within and beneath the axial magma lens. $t^{*}$ values for the OBS have appreciably higher uncertainties, a reflection of the narrower bandwidth of these instruments. The highest $t^{*}$ values for AOBH 5 are located slightly to the west of the rise axis at the northern limit of the central region of dense shooting. Such $t^{*}$ values are probably representative of paths that have been confined to depths shallower than the depth of the axial magma 
chamber, which is also offset slightly to the west in the southern half of the experiment region [Mutter et al., 1988; Toomey et al., 1990a]. Because of the difficulty of approximating paths for phases that propagate at low angles to the rise axis, we generally exclude $t^{*}$ estimates for shots within $2 \mathrm{~km}$ of the rise axis, although the $t^{*}$ values for the rise-parallel paths which were presented in Chapter 2 are included in an inversion of all the data.

Figure 4.20 compares examples of rise perpendicular ray paths for AOBH 5 . Since no paths cross the rise axis no constraints are necessary to obtain approximate paths that match the ray-theoretical paths, though the approximate paths do have appreciably greater turning depths than the ray-theoretical paths, which as noted earlier is a consequence of the coarse parameterization of the delay time tomographic model.

\section{BIASES TO AOBH $t^{*}$ DATA}

Table 4.1 lists average $t^{*}$ values recorded by each instrument for different categories of wave paths. It is immediately apparent both from Table 4.1 and from inspection of the plots of $t^{*}$ values that the estimates for the AOBHs are generally markedly lower than those obtained for the DOBHs. The small systematic differences observed between values for the outer DOBHs (e.g., DOBHs 10 and 13 in Figures 4.7 and 4.8) may be accounted for by slight variations in the distribution of source-receiver paths, by variations in local $\mathrm{Q}$ structure beneath each instrument, and by small variations in instrument response, as well as by three-dimensional variations in the $\mathrm{Q}^{-1}$ structure. However, such explanations cannot account for the large systematic offset of AOBH t* values. The discrepancy must almost certainly be the result of a systematic error in correcting power spectra for instrument responses. Moreover, since the theoretical response derived for the DOBHs agrees very well with the results of on-bottom calibration tests, the error must be attributed to the AOBHs. There are three effects that might significantly affect response of the AOBHs.

First, in Appendix A we show that AOBH $t^{*}$ estimates may be biased by an effect that we term spectral leakage. The direct recording system of the AOBHs introduces signal distortion and signal generated noise which result in recorded power spectra that have a significant non-monochromatic component when the input signal is a sine wave (Figures A6 and A7). Extrapolating such results to predict the recorded spectra of seismic waveforms suggests that such a process can 
significantly bias $t^{*}$ estimates for highly attenuated waveforms toward lower values (Figure A8). In such cases, the input signal is dominated by low frequencies, and the spectral power at higher frequencies may be determined by spectral leakage. If $t^{*}$ estimates are limited to a bandwidth that includes uncorrected spectral powers no larger than $1 / 1000$ the maximum recorded power, a criterion that is satisfied by the $t^{*}$ estimates presented for the AOBHs, the tests suggest that $t^{*}$ values will be limited to a maximum value of about $0.06 \mathrm{~s}$. Such an effect might account for the offset to lower estimates of $t^{*}$ for more highly attenuated waveforms (path types 3-5 in Table 4.1). However, the results also suggest that $t^{*}$ values below about $0.04 \mathrm{~s}$ will be unaffected. Thus, spectral leakage cannot explain the offset of lower $t^{*}$ values for paths that do not cross the rise axis or which pass above the axial magma chamber (path types 1 and 2 in Table 4.1).

Second, the $t^{*}$ estimates may be affected by saturation of the direct recording system in AOBHs. In Appendix A the effects of saturating the AOBH are discussed in detail for both sine and square wave inputs. In both cases saturation occurs fairly abruptly. Below a certain input amplitude threshold the response of the instrument is approximately linear, while above the threshold the recorded amplitudes rapidly achieve a fixed value that is independent of the input amplitude. In the case of the response tests the maximum recorded amplitude is about 25 times the calibration pulse amplitude or $200 \mu \mathrm{bar}$ (peak to peak) (Figure A5). Figure 4.21 shows examples of the maximum recorded amplitude of the first 0.6-s of the waveform plotted against range for similarly configured AOBHs and DOBHs. The effects of saturation are immediately apparent. At ranges below about $10 \mathrm{~km}$ the AOBH amplitudes achieve a fixed value while the DOBH amplitudes increase rapidly with decreasing range and achieve values which lie well above the plot bounds. However, for an unknown reason the maximum recorded amplitudes for each AOBH are consistently 2-3 times higher than the tests predict.

To account for the effects of saturation, AOBH waveforms are excluded which achieve a maximum amplitude more than about two-thirds the maximum recorded amplitude for each instrument (Figure 4.21). This threshold, which is chosen assuming that the shape of the saturation curve measured for the AOBH is correct, must be accompanied by some doubt because of the discrepancy between the measured and the observed saturation amplitudes. The effect of saturation upon the power spectra for both sine wave (Figure A4) and square wave (Figure A11) inputs is to decrease the relative amplitudes of high frequencies, an effect that would tend 
to increase $\mathrm{t}^{*}$ estimates and which is opposite to the observed offset of $\mathrm{t}^{*}$ values. However, the magnitude of the $t^{*}$ bias that might result from saturation is small $(<0.005 \mathrm{~s}$ for $\mathrm{AOBH} 1)$, and since saturation can also increase the levels of spectral leakage (Figure A7) the overall effect of saturation on $t^{*}$ values for highly attenuated waveforms is unclear. A careful inspection of $t^{*}$ estimates obtained for all the AOBHs shows that $t^{*}$ values for waveforms which approach the saturation threshold are not significantly different from $t^{*}$ values for similarly configured waveforms which fall well below the threshold. Therefore, we are confident that our saturation threshold is adequate and that saturation does not cause the offset of AOBH t* values.

Third, the measured AOBH responses might be wrong. The responses were measured over one year after the tomography experiment and several of the instruments were deployed elsewhere during the intervening period. The difference between the measured responses for AOBH 1 and AOBH 7 (Figure 1.4) is equivalent to a $t^{*}$ difference of $0.012 \mathrm{~s}$ over the frequency interval $10-40 \mathrm{~Hz}$. If such differences result from some aspect of the pre-deployment instrument preparation they might account for the offset of $t^{*}$ values. However, a careful evaluation of all the steps involved in the instrument preparation (Appendix A) suggests that while changes in the response of an instrument might be expected between deployments, the magnitudes of such changes are relatively small. Inspection of the measurements of the amplitude response made routinely prior to deployments confirms that the variations in the measured response cannot account for the offset of $t^{*}$ values.

Therefore, we are led to conclude that the response of the AOBHs is significantly different during deployments than in the laboratory, showing a markedly better high frequency response on the seafloor. Indeed, a change in the response also seems necessary to explain the increased saturation threshold amplitude observed during the experiment. While the cause of such changes is unknown it is almost certainly related to the direct recording mechanism. Since the manufacturers specifications for $1 / 4^{\prime \prime}$ inch tape used in the $\mathrm{AOBH}$ do not extend below $10^{\circ} \mathrm{C}$, the change is response may well be a result of the low temperatures at the seafloor. 


\section{INVERSIONS FOR $Q^{-1}$ \\ Station Corrections}

Inversions of seismic travel time data are frequently accompanied by the application of fixed station corrections to account for local structure beneath seismic receivers. Indeed, if the scale of the local structure beneath receivers is much smaller than the typical separation of instruments or if the receivers are not located within the study region, the failure to apply station corrections may introduce substantial artifacts into velocity models obtained. The observed offset of the AOBH $t^{*}$ data clearly necessitate a similar station correction for $t^{*}$. Indeed as noted in the last section $\mathrm{t}^{*}$ values for DOBHs show smaller systematic differences which presumably also warrant the application of a station correction.

However, it is not clear whether the optimal station correction is a fixed correction at each instrument. Local variations in upper crustal $\mathrm{Q}$ structure [Jacobson and Lewis, 1990] (Chapter 2) may well occur on a small enough scale to warrant azimuthally dependent station corrections. However, for most receivers in this study such corrections are probably not warranted because of the limited azimuthal distribution of source-receiver paths. Moreover, since paths crossing the rise axis are generally characterized by high $t *$ values, it would be difficult to estimate azimuthally dependent corrections without incorporating signal arising from lateral variations in the attenuation structure. The other primary component of the station corrections accounts for errors in the instrument response. In the case of the AOBHs this component will dominate the effects of local structure. Assuming that the instrument correction is made according to equation (1.4) and that $t^{*}$ estimates are obtained from a least squares straight line fit to the logarithm of the power spectra, errors in the instrument response will result in a fixed $t^{*}$ error for estimates obtained over a specific frequency interval. However, the $t^{*}$ error may be dependent upon the choice of frequency limits. Unfortunately, since highly attenuated waveforms are characterized by adequate signal-to-noise ratios over small bandwidths, the spectral $t^{*}$ estimates are strongly correlated with the reciprocal of the maximum frequency used to obtain the estimate (Figures 4.9 and 4.17). In consequence it is not possible to determine a station correction which is dependent upon the upper frequency limit without including signal arising from the attenuation structure.

For these reasons fixed station corrections are used in the inversions presented in this chapter. The simplest method to obtain station corrections is to include them 
as a free parameter in all inversions. However, corrections are strongly weighted in the inversion since they contribute to each $\mathrm{t}^{*}$ estimate and it is difficult to constrain the values to fall within reasonable limits. To overcome this problem the sum of the corrections for the DOBHs is constrained to be zero. Thus the absolute values of the $\mathrm{Q}^{-1}$ models are referenced to $t^{*}$ estimates obtained for instruments for which the biases are likely to be small. It is clear from a cursory inspection of the data and from the results presented in Chapter 2 that a model which is laterally invariant across the rise axis will not fit the $t^{*}$ data. However, it is not immediately apparent that the data require any significant asymmetry or along-axis variations. To minimize the possibility of such variations being introduced as artifacts of the station corrections, the corrections are always derived assuming a two-dimensional axissymmetric model and are then held fixed at such values for inversions which permit asymmetry and along-axis variations. In the case of smoothest model inversions the optimal station corrections may not be independent of the smoothing weight. However, in the case of the inversions presented here, once the smoothing weight falls below a value which permits significant deviations from the a priori model, the station corrections derived for axis-symmetric two-dimensional models are virtually independent of the smoothing weight. Therefore, a single set of fixed station corrections can be obtained for each inversion. Station corrections are presented in Table 4.2 for several subsets of the $t^{*}$ data. The values are generally very similar between inversions, and the variations that do exist can be shown to have no significant effect upon the solutions.

\section{A Two-Dimensional Inversion for a Symmetric $\mathbf{Q}^{-1}$ Structure}

The first inversion we present is a simple smoothest model inversion for an axis-symmetric two-dimensional structure. The one-dimensional starting model (Figure 4.22) is based upon the inversions of the off-axis refraction line data (Chapter 2). At distances greater than $8 \mathrm{~km}$ from the rise axis the $\mathrm{Q}^{-1}$ structure is held fixed to starting model while for distances less than $8 \mathrm{~km}$, the model is parameterized on a $0.5-\mathrm{km}$ grid that is sheared vertically to conform with the seafloor. Uniform smoothing is applied throughout the model to minimize the first derivative of deviations from the starting model (equation 1.34). All the $t^{*}$ estimates presented in this chapter are included in the inversion (provided the ray paths pass within $8 \mathrm{~km}$ of the rise axis) as are the axial t* estimates presented in

Chapter 2. The total data set comprises about 3500 values. The wave paths used in 
this and other inversions are the constrained approximate paths (Figures $4.2 \mathrm{c}, \mathrm{d}$, $4.14 \mathrm{c}, \mathrm{d}$, and $4.20 \mathrm{~b}$ ) obtained from the delay time tomographic model of Toomey et al. [1990a]. Wave paths for the axial $t^{*}$ estimates of Chapter 2 are derived assuming initial constant curvature paths that lie within a vertical plane and which have turning depths of $\leq 1.5 \mathrm{~km}$ and $7 \mathrm{~km}$ for the upper crustal and Moho-turning phases, respectively. Figure 4.23 shows the distribution of wave paths used in the inversion.

Figure 4.24 shows the normalized squared data misfit (equation 1.40) as a function of smoothing weight, while Figure 4.25 shows the resulting model for seven choices of smoothing weight. The variance reduction is over $50 \%$ and is about $70 \%$ when only paths crossing the rise axis are considered. All the solutions show a pronounced decrease in $\mathrm{Q}^{-1}$ values within the upper crust close to the rise axis. Such a result is consistent with the one-dimensional axial $\mathrm{Q}^{-1}$ model derived in Chapter 2, the quantitative modelling of ESP seismic amplitudes [Vera et al., 1990], and a qualitative comparison of amplitudes recorded during on-bottom refraction experiments [Christeson et al., 1991a, b]. However, in this particular inversion such a feature might be an artifact of the station corrections. None of the axial instruments is a DOBH for which station $t^{*}$ correction can be assumed to be small, and thus the inversion results may be affected by a the trade-off between nearsurface axial $Q^{-1}$ values and the station correction for axial receivers.

As the smoothing weight is decreased the inversion resolves a region of low $Q$ values which in this symmetric inversion is centered below the rise axis. Initially this body appears at fairly large depths though as the smoothing weight decreases the minimum depth of the anomaly progressively decreases to about $2 \mathrm{~km}$ which is the minimum axial depth of wave paths assumed for the diffractions below the magma chamber (Figure 4.23). The initial appearance of the anomaly at larger depths can be explained by the combination of two factors. First, the number of Moho-turning paths significantly exceeds the number of paths for diffractions beneath the magma chamber. Since the Moho-turning paths cross the rise axis at larger depths the low $\mathrm{Q}$ anomaly at such depths is more strongly required by the data. Second, the minimization of the model roughness (equation 1.34) is better satisfied by maintaining a large separation between the negative axial near-surface $\mathrm{Q}^{-1}$ anomaly and the positive anomaly beneath. Therefore, the inversions with larger smoothing weight place the positive anomaly at greater depths.

The solution with the second smallest smoothing weight (Figure 4.25f) includes 
a number of fine scale structures in the lower part of the model. While such features are probably not fully resolved because of the uncertainty accompanying deeper wave paths, their existence can be explained in terms of the distribution of ray paths used in the inversion (Figure 4.23). At $2-3 \mathrm{~km}$ depth below the rise axis a low- $Q$ region with $\mathrm{Q}^{-1}>0.03$ is required to model the $\mathrm{t}^{*}$ values for the diffractions below the magma chamber. The Moho-turning phase for off-axis instruments also requires a deeper region of substantially increased $Q^{-1}$ values. However since the $t^{*}$ estimates for the Moho-turning phase recorded on the axial instruments require an average $Q^{-1}$ beneath the rise axis of about $\sim 0.02$ (Chapter 2 ), the maximum value of $\mathrm{Q}^{-1}$ directly beneath the rise axis is limited. The relatively low $\mathrm{t}^{*}$ values for the longest paths which propagate between the two outer refraction lines result in relatively low $\mathrm{Q}^{-1}$ values at $7 \mathrm{~km}$ depth below the rise axis. Moreover, these paths coincide closely with the deepest part of the paths for the Moho-turning phase (Figures 4.2c, d, and 4.23), preventing very high $\mathrm{Q}^{-1}$ values at large depths off-axis. As a result the anomaly required to match $t^{*}$ estimates for the off-axis Moho-turning phase is placed $2 \mathrm{~km}$ off the rise axis at about $5 \mathrm{~km}$ depth, a location that does not coincide with the wave paths for other phases. The solution with the smallest smoothing weight (Figure $4.25 \mathrm{~g}$ ) is characterized by very rapid variations in lower crustal $\mathrm{Q}^{-1}$ values and is clearly undersmoothed.

Figure 4.26 shows the formal linearized uncertainty factors (equation 1.38) for three of the solutions shown in Figure 4.25. The presence of a large number of ray paths in the upper crust is reflected by the relatively low uncertainties in such regions, while the largest uncertainties are associated with the deepest parts of the model off-axis where there is a sparse distribution of wave paths. The effect of decreasing the smoothing weight is to increase the uncertainty of $\mathrm{Q}^{-1}$ estimates. However, the formal uncertainties for the second roughest model (Figure 4.26c) are still small enough to suggest that many of the features within the deeper parts of model are resolved. However, the detailed structure in the lower part of the model depends on the relative location of ray paths, and the uncertainties that accompany the location of the paths are not included in the formal uncertainties.

Figure 4.27 shows the square root of the spread fuction (equation 1.42) calculated for the three solutions. This quantity, calculated from the resolution matrix is a measure of the spatial resolution of individual model parameters. Indeed the formal uncertainties presented in Figure 4.26 are of little meaning without some estimate of the area over which such estimates are obtained. The very low 
uncertainties of the smoothest models are generally associated with large spread values since such estimates are spatial averages over a large area. The low spread values observed for near-surface nodes indicates that this region of the model is well resolved, but the absolute values should be treated with caution for two reasons. First, in a continuous model a spread of zero indicates that the model value at a point is perfectly resolved. However, in a discrete model a spread which approaches zero indicates only that the model parameter is formally resolved on a scale similar to the grid spacing. Second, the values may be significantly affected by the nonlinear parameterization. A t* value is much more sensitive to a small change in $\ln \left(\mathrm{Q}^{-1}\right)$ in high- $\mathrm{Q}^{-1}$ regions than in low $\mathrm{Q}^{-1}$ regions along the wave path. Therefore, surface nodes which have high $\mathrm{Q}^{-1}$ values both in the a priori model and in all the solutions will be strongly weighted in the logarithmically parameterized matrix of partial derivatives ( $\mathrm{A}$ in equation 1.29). As a result the linearized estimates of resolution suggest that high- $\mathrm{Q}^{-1}$ regions including the uppermost crust are better resolved than would be the case for a linear parameterization in which $t^{*}$ values would be equally sensitive to changes in the $\mathrm{Q}^{-1}$ structure at all locations along the ray path.

Indeed, for the same reason estimates of uncertainty and resolution for localized low-Q regions in models with low smoothing weights should be treated with caution. One common effect of progressively reducing the smoothing weight in the $\mathrm{Q}^{-1}$ inversions is that $\mathrm{Q}^{-1}$ values at individual nodes progressively grow to very large values which, because of the logarithmic parameterization, may have linearized uncertainties significantly smaller than the actual value. While such an effect will eventually produce models that are clearly unrealistic (e.g., Figure $4.25 \mathrm{~g}$ ), the effects at slightly higher levels of smoothing might be more subtle. Thus, even if the deeper wave paths in the inversion can be considered correct many of the localized lower crustal high $\mathrm{Q}^{-1}$ features that are apparently resolved in Figure $4.25 \mathrm{f}$ might in fact have significantly larger uncertainties in a solution which uses a linear parameterization.

\section{Inversions for Upper Crustal Structure}

The second roughest inversion presented in the last section (Figure 4.25f) apparently resolves a low $\mathrm{Q}$ body centered at $2.5 \mathrm{~km}$ depth beneath the rise axis, a feature that can be attributed to the diffractions beneath the magma chamber. However, since some of the paths for the Moho-turning phase cross the rise axis at 
depths only slightly greater than $3 \mathrm{~km}$ (Figures $4.14 \mathrm{c}, \mathrm{d}$, and 4.23 ), it is not clear what affect $t^{*}$ estimates for these paths have on the model in this region. Moreover, since we argue in the last section that fine-scale features in the lower-crustal low-Q anomaly may be artifacts resulting from the relative distribution of poorly known wave paths, it is difficult to argue convincingly that shallowest portion of the low- $Q$ anomaly is well resolved. Therefore, in this section we present inversions for a data set that excludes all phases turning at depths significantly greater than $3 \mathrm{~km}$.

Figure 4.28 shows the results of inverting this subset of the data using a parameterization identical to that used for the solution presented in the last section except that the smoothing weight in the upper $1 \mathrm{~km}$ is quadruple that at larger depths. The solutions for three choices of smoothing parameters (Figures $4.28 \mathrm{c}-\mathrm{e}$ ) resolve high $\mathrm{Q}^{-1}$ values beneath the rise axis at depths greater than $2 \mathrm{~km}$. Indeed the minimum depth of this feature is controlled by the minimum depths of the paths ascribed to the diffractions beneath the magma chamber. However, the finitedifference solutions obtained in Chapter 3 suggest that this diffraction propagates over a fairly broad range of depths within and below the axial magma body and may not be well approximated by a single path. Therefore, the details of this high $\mathrm{Q}^{-1}$ anomaly such as those which are apparent in Figure 4.28e are certainly not well resolved.

To search for along-axis variations in the $Q$ structure within this portion of the model the solution presented in Figure 4.28d was used as the starting model for a three-dimensional inversion. To minimize the number of free parameters the horizontal spacing of nodes across the rise axis (the $\mathrm{x}$ direction) was increased to 1 $\mathrm{km}$ at distances greater than $2 \mathrm{~km}$ from the rise axis while the separation of nodes parallel to the rise axis (the y direction) was $2 \mathrm{~km}$. To stabilize the solutions further and to prevent unwarranted deviations from the starting structure the starting model was assigned a logarithmic uncertainty of 0.5 (equivalent to an uncertainty factor of 1.6) and the smoothing weight was doubled parallel to the rise axis. Figure 4.29 shows two sections through a solution which achieves a $15 \%$ variance reduction over the starting model. Both the horizontal section at $2.5 \mathrm{~km}$ depth (Figure $4.29 \mathrm{a}$ ) and the vertical section which runs along the rise axis (Figure 4.29b) show an apparent increase in the magnitude of the low- $Q$ anomaly in the northern half of the model.

Figure 4.30a shows the maximum $\mathrm{Q}^{-1}$ value obtained within the axial anomaly at different locations along the rise axis. While a northward increase in values is apparent, the formal uncertainties are large and are compatible with a constant 
value. However, $\mathrm{Q}^{-1}$ values averaged within 2- and 4-km-wide regions that are centered about the rise axis and which extend over a 2-3 km depth range show a similar trend, and the formal uncertainties suggest that the northward increase in $\mathrm{Q}^{-1}$ values is resolved. However, the most convincing evidence for along-axis variations comes from inspection of the $t^{*}$ data. $t^{*}$ values of the diffracted phase obtained at the three OBHs located $9 \mathrm{~km}$ off-axis (Figure 4.16 and 4.17) are markedly higher for northern shots.

The structure of the upper crustal axial low $Q$ anomaly does not correlate very well with the low velocity volume imaged using delay time tomography (Figure 1.2) [Toomey et al., 1990a]. The lowest velocities in the delay time tomographic model are offset to the west of the rise axis in the southern half of the model. However, the $\mathrm{Q}^{-1}$ inversions are unlikely to resolve such asymmetry. Paths for the diffraction below the magma chamber are constrained to depths greater than $2 \mathrm{~km}$ by placing near zero velocities at axial nodes with depths of 0 and $1 \mathrm{~km}$. Since these nodes largely determine the location of wave paths near the rise axis, the paths for eastand west-propagating phases in this region are to first order mirror images of each other and asymmetry in the velocity model is not reflected in the paths.

A more important difference between the velocity and $\mathrm{Q}^{-1}$ models is that the along-axis variations in the magnitude of the anomaly are not well correlated. The largest negative velocity perturbations in the delay time model (Figure $1.2 \mathrm{~b}$ and Figure 4.30c) are observed $2 \mathrm{~km}$ to the south of the experiment center in a region where the $\mathrm{Q}^{-1}$ anomaly has relatively small values. However, the anomaly does show a secondary maximum near the northern limit of the model. There are several explanations of the apparent differences between the velocity and $\mathrm{Q}^{-1}$ models. First, the models are measures of different quantities, and while a positive correlation between $\mathrm{Q}$ and velocity might be expected due to thermal effects it is not clear how good the correlation should be. Second, the travel time delay and $t^{*}$ values are dependent upon spatial integrals of functionals of seismic parameters but the spatial weighting within the two integrals is not the same. As noted earlier, inspection of the finite-difference models suggests that the propagation of the diffracted phase beneath the magma chamber is complex, and the energy arriving within the first few tenths of a second after the onset of the waveform may not be well approximated by a single path. In contrast algorithms that calculate travel times and ray paths using minimum time criteria [Vidale 1988, 1990; Moser, 1991; Toomey et al., 1991] can successfully approximate the arrival time of non-ray-theoretical arrivals from a single 
path. Delay time tomographic models can image low-velocity bodies even though no first arrivals propagate through such regions because of the effect such bodies have in perturbing ray paths. Clearly $t^{*}$ estimates are sensitive only to regions through which the waves propagate. Third, in Chapter 3 we showed that $t^{*}$ estimates may be sensitive to the effects of scattering. While scattering is unlikely to account for the bulk of the $\mathrm{Q}^{-1}$ anomaly it might contribute significantly to the highest $\mathrm{Q}^{-1}$ values observed (Figure 3.17). Along-axis variations in scattering resulting from changes in the velocity structure might introduce trends in the apparent $\mathrm{Q}^{-1}$ structure that are unrelated to intrinsic attenuation.

A fourth possibility is that the lowest velocities in the delay time tomographic models are not well resolved. The finite-difference solutions show that many of the approximate ray paths used in the inversion are misplaced with respect to the axial magma chamber. Moreover because the amplitude of the diffraction above the magma chamber is very low the identification of this phase is strongly dependent upon the signal-to-noise ratio. Consider solely picks obtained for the instruments 9 $\mathrm{km}$ off-axis. It is conceivable that a centrally located region of anomalously low axial velocities might be an artifact of inconsistencies in the automatic picks. The amplitude of the diffraction above the magma chamber is strongly dependent upon the distance the phase propagates after crossing the rise axis (Figure 3.5). Thus, in the case of rise-perpendicular paths (Figure 4.12) the diffractions above the magma chamber may be consistently picked for shots at greater perpendicular distances from the rise axis than in the case of oblique paths (Figure 4.13). Since the perpendicular paths from the $9-\mathrm{km}$ receivers cross the rise axis near the limits of the central region of the experiment while many of the oblique paths cross the rise axis near the center of the experiment the model may be biased. The approximately relative delay of the diffraction below the magma chamber with respect to the diffractions above is of the order of $0.1 \mathrm{~s}$, a value that may be large enough to account for the lower velocities seen in the center of the experiment. However, no such bias should exist for the receivers $20 \mathrm{~km}$ off axis which also contribute to the velocity model in this region. Moreover, picks for arrivals identified as diffractions were excluded from the delay time tomographic inversions. Therefore, it is not clear whether inconsistencies in the picks significantly affect the along-axis structure.

In Figure 4.30d we present spatial averages of the low-velocity anomaly obtained over the same regions used to obtain the spatial averages of $\mathrm{Q}^{-1}$ shown in Figure $4.30 \mathrm{~b}$. These averages do not show the same trends as the maximum 
negative velocity perturbations. Indeed, the average obtained over a 4-km-wide area correlates very well with the $\mathrm{Q}^{-1}$ model showing a general increase in magnitude in the northern half of the experiment.

\section{Inversions for Shallow Structure}

Previous inversions consistently resolve a region of relatively high near-surface $\mathrm{Q}$ centered on the rise axis. However, it was noted above that this might be an artifact of the station corrections for the axial instruments. To investigate this possibility we performed two sets of inversions using $t^{*}$ data sets comprised solely of paths that do not pass below the axial magma chamber. The first data set includes such $t^{*}$ values for all instruments while the second set excludes values for axial instruments. In both cases the data were inverted for axis-symmetric variations in $\mathrm{Q}^{-1}$ in the upper $1 \mathrm{~km}$ across the rise axis assuming a laterally invariant model at greater depths. The results (Figure 4.31) are almost identical and show conclusively that the values of high near-surface axial $Q$ are not an artifact of the axial station corrections.

Inspection of $t^{*}$ values for shots located near the rise axis shows that while such values are noticeably lower for some instruments (e.g., DOBH 14 in Figure 4.6, AOBH 1 and DOBH 10 in Figure 4.7, and DOBH 15 in Figure 4.15), in other cases axial values show no systematic difference from values for similar paths located well off axis (e.g., DOBH 13 and AOBH 7 in Figure 4.7, AOBH 4 in Figure 4.15, AOBH 6 in Figure 4.16). Indeed, a large component of the decreased axial $\mathrm{Q}^{-1}$ values results not from changes in the $t^{*}$ values themselves but from the characteristics of the paths. Paths which propagate above the magma chamber include longer segments within the upper 1-1.5 km near the rise axis (Figures 4.2 and 4.14).

The width of the near-surface axial high- $Q$ zone is not very well resolved. In the inversions presented in this Chapter the anomalous region extends $2-3 \mathrm{~km}$ off-axis. Since the smoothing constraints act to broaden the anomaly this value should be considered a maximum. The data are also compatible with a much narrower high- $\mathrm{Q}$ zone with proportionately lower $\mathrm{Q}^{-1}$ values. Indeed the only constraint on the

minimum width of the high- $\mathrm{Q}$ region comes from the total magnitude of the anomaly. Halving the width of the anomalous region in Figure 4.31 would require $\mathrm{Q}^{-1}$ values close to zero and therefore a half width of $1-1.5 \mathrm{~km}$ probably represents a lower limit to the width of the anomaly.

Another feature of the inversions which is evident in Figure $4.31 \mathrm{~b}$ as well as in 
other inversions (Figures 4.25 and 4.28 ) is that $\mathrm{Q}^{-1}$ values for surface nodes well away from the rise axis attain values that are slightly higher than the a priori model which is based on the structure $20 \mathrm{~km}$ off-axis. Such a result suggests that as the upper crust moves off axis, near surface $Q$ values must initially decrease rapidly and may at larger distances show a slight increase with distance. Similar trends have been suggested for the evolution of upper crustal velocities [McClain et al., 1985; Burnett et al., 1989; Caress et al., 1992]. The most straightforward method to determine whether such a characteristic is required by the data is to examine the $t^{*}$ values. In Figure $4.32 \mathrm{t}^{*}$ values obtained for the outer DOBHs using $4 \pi$-prolate MWSA and an 0.6-s-long window are plotted against range both for the shots on the outer refraction lines (Chapter 2) and for shots located at distances of 3-6 km and 6$9 \mathrm{~km}$ off-axis and on the same side of the axis as the instrument. The differences are small, but in all cases the average $t^{*}$ value for the outer refraction lines are 5-15\% smaller than average values for shots located 3-9 $\mathrm{km}$ off-axis. While this observation lends support to models which show a small decrease in near-surface $Q$ between distances of about 10 and $20 \mathrm{~km}$ off axis, two alternative explanations can account for this observation. First, the increase in $Q$ with distance from the rise axis need not be placed in the uppermost crust and may equally well be located at $2-3 \mathrm{~km}$ depth, the predominant depth at which these arrivals propagate. Second, the data may be equally well explained by a small component $(<10 \%)$ of anisotropy in nearsurface $Q$, with lower $Q$ values for propagation perpendicular to the rise axis. Unfortunately, the azimuthal distribution of paths is not adequate to distinguish between the anisotropic model and laterally varying models.

Figure 4.33 shows the results of inverting the off-axis and shallow rise-crossing estimates of $t^{*}$ for a two-dimensional model of surface attenuation using a uniform smoothing weight in the $\mathrm{x}$ and $\mathrm{y}$ directions. The variance reduction achieved by such an inversion (Figure 32a) is $25 \%$ with respect to a laterally invariant $Q$ model but is only about $10 \%$ when compared with the axis-symmetric models of $Q$ structure. Indeed, while the primary feature of the model is still a general decrease in $Q^{-1}$ values near the rise axis, a large number of other poorly resolved complexities are also present in the solutions with smaller smoothing weights (Figures $4.33 \mathrm{c}$ and d). The spacing of shots is almost certainly too small to resolve adequately along-axis variations in near-surface $Q$. Many features in the $t^{*}$ data such as the general northto-south increase in $\mathrm{t}^{*}$ values for DOBH 12 (Figure $4.9 \mathrm{~b}$ ) affect the solutions but are not consistent with observations obtained from other instruments and thus 
presumably originate from regions not included in the inversions.

\section{An Inversion of $t^{*}$ Values for Paths Between the Two Outer Refraction Lines}

Since many of the paths between the two outer refraction lines cross the rise axis outside the central area of the experiment we choose to invert these data separately for a simple $\mathrm{Q}^{-1}$ model. Away from the rise axis the model is held fixed to the laterally invariant model of Figure 4.22 , while $\mathrm{Q}^{-1}$ values within an $8-\mathrm{km}$-wide region centered on the rise axis are allowed to vary parallel to the rise axis. The width of this region is chosen arbitrarily to be the maximum width of the low- $Q$ anomaly in the two-dimensional inversion of all the data (Figure 4.25). The inversion (Figure 4.34) achieves a $40 \%$ variance reduction over the best fitting model without along-axis variations. Since the width of the axial low-Q body is not well known the absolute $\mathrm{Q}^{-1}$ values may be incorrectly scaled. In contrast to the earlier inversion for upper crustal structure which shows increased levels of axial attenuation towards the north, the primary feature resolved by the inversion is an apparent doubling of $\mathrm{Q}^{-1}$ values in the southern half of the experimental region. Most of the change occurs more than $5 \mathrm{~km}$ to the south of the experiment center; the highest model values are determined entirely in this region by $t^{*}$ measurements for the two most southerly DOBHs (DOBH 12 and 14). A comparison of the data and the model predictions for these two instruments (Figure 4.35) shows that the increase in $\mathrm{Q}^{-1}$ values is primarily a result of trying to fit the data for DOBH 14. Since $t^{*}$ values may also be influenced by local structure near the receiver, any result that relies upon data from a single instrument should be treated with caution. However, this result is consistent with the travel times for these paths which for all instruments consistently show increased delay times toward the south of the experiment [Toomey et al., 1990b].

\section{An Inversion of the Moho-Turning Phase}

The precise location of wave paths for the Moho-turning phase that propagate across the rise axis are not well known and any model obtained from such paths is likely to have limited resolution. Therefore, we chose to invert paths for this phase using a data set that includes paths that do not cross the rise axis or that cross the rise axis above the magma chamber but excludes all other phases that propagate below the axial magma chamber (Figure 4.36a). The lower crustal structure will thus depend entirely on the $t^{*}$ estimates for the Moho-turning path and will not 
include features that result from other phases (e.g., Figure 4.25f).

The starting model used for a three-dimensional inversion of the data set is shown in Figure 4.36b. The model is obtained from a two-dimensional axissymmetric inversion of this data set with the added constraint that below $4 \mathrm{~km}$ depth the model is vertically invariant. For the three-dimensional inversion, the model is parameterized with 1-km grid spacings in the vertical direction and perpendicular to the rise axis and with a 2-km spacing parallel to the rise axis. The smoothing weight is quadrupled in the upper $1 \mathrm{~km}$ and doubled parallel to the rise axis. To further limit unwarranted deviations from the starting model a logarithmic uncertainty of 0.5 (equivalent to an factor of 1.6) is assigned to the a priori model value at each node. Figure 4.37 shows sections through a solution which achieves a variance reduction of about $20 \%$. The vertical sections (Figure 4.37a-c) all show a region of very low $Q$ centered at $4 \mathrm{~km}$ depth below the rise axis. The magnitude of highest $\mathrm{Q}^{-1}$ values are almost certainly not resolved and may well be a result of a combination of the a priori model chosen, relative locations of ray paths, and biases in the $t^{*}$ estimates.

Both the vertical sections and a horizontal section through the model (Figure 4.37c) show a relatively small variation along axis in the magnitude of the low- $Q$ anomaly. Figure 4.38 shows the along-axis variations of the minimum $\mathrm{Q}^{-1}$ values, which have large uncertainties, and of the average $Q^{-1}$ values, which have uncertainties small enough to suggest that along-axis variation might be resolved. Average $\mathrm{Q}^{-1}$ values near the center of the experiment are relatively small and increase near the edges of the model particularly toward the south. While average $\mathrm{Q}^{-1}$ values show a maximum along-axis variation of less than $20 \%$, inspection of the $t^{*}$ data (Figures 4.8 and 4.9) suggests that this feature is resolved. Many of the instruments show markedly lower $t^{*}$ values for paths that cross the rise axis near the center of the experiment (e.g., DOBH 10 and DOBH 13 in Figure 4.8 and AOBH 2 in Figure 4.9) and higher $t^{*}$ values near both the southern (AOBH 7 and DOBH 10 in Figure 4.8 and AOBH 2 in Figure 4.9) and northern (e.g. DOBH 13 in Figure 4.8) limits of the central region.

In Figure $4.38 \mathrm{c}$ average $\mathrm{Q}^{-1}$ values over an area that extends $4 \mathrm{~km}$ to either side of the rise axis are compared for this inversion and the inversions of the paths between the two outer refraction lines (Figure 4.34). The two models show similar trends although the $\mathrm{Q}^{-1}$ values for the shorter range Moho-turning phase are a factor of about two higher. This difference presumably reflects a decrease in axial $\mathrm{Q}^{-1}$ values with depth since the longer range phase almost certainly traverses the rise 
axis at a larger depth.

\section{SUMMARY}

In this Chapter we have presented a series of inversions for $\mathrm{Q}^{-1}$ based upon the full $t^{*}$ data set which comprises over 3500 measurements. By comparing seismic record sections with the finite difference solutions presented in Chapter 3 and with the travel times predicted by an exact two-dimensional ray-tracing algorithm, we are able to select objectively the positions and lengths of time windows used for spectral calculations, so that $t^{*}$ estimates are predominantly the result of a single phase. By limiting the position of initial trial paths and by preventing paths from entering certain regions of the model, the three-dimensional approximate ray tracing algorithm of Thurber [1983] is constrained to calculate paths from the delay-time tomographic velocity model [Toomey et al., 1990a] that are reasonable approximations to the true paths of the correct phases. The data set includes $t^{*}$ estimates for direct crustal phases, diffractions from above and below the magma chamber, and Moho-turning phases.

The starting model for the inversions is the one-dimensional off-axis structure presented in the Chapter 2. A two-dimensional, axis-symmetric model obtained by a smoothest model inversion is able to fit the data quite well, although we note that the symmetric nature of the wave paths may mask cross-axis asymmetry such as that resolved in the delay time tomographic models [Toomey et al., 1990a]. Within the upper $1 \mathrm{~km}$ the inversions resolve an axial high- $\mathrm{Q}$ anomaly which is constrained to extend no more than $2-4 \mathrm{~km}$ off axis and in which $\mathrm{Q}$ values averaged over the upper $1 \mathrm{~km}$ decrease from about 30 off-axis to $>50$ on axis. High $\mathrm{Q}$ values are also resolved beneath the axial magma chamber. The diffractions beneath the magma chamber also resolve a 2-4 km-wide region of low $\mathrm{Q}$, with the lowest well-resolved $\mathrm{Q}$ values about 30. The principal depth of propagation of this phase beneath the magma chamber is poorly known, but inspection of the finite difference solutions suggests that it probably samples the axial Q structure no more than $1 \mathrm{~km}$ below the roof of the magma chamber. The detailed structure in the lower crust is not well resolved. However, the models suggest that the width of the anomaly increases in this region and that the minimum $\mathrm{Q}$ values are similar to those determined at shallower depths.

Although two-dimensional models fit the data fairly well, three-dimensional inversions do resolve significant along-axis variations in the axial low- $Q$ anomaly. 
The diffractions below the magma chamber show a northward increase in near-axial attenuation of $\sim 25 \%$. This along-axis variation does not correlate with trends in the maximum low-velocity anomaly imaged by travel time tomography [Toomey et al., 1990a]. However, when the velocity anomaly is averaged over $4 \mathrm{~km}$ width between 2 and $3 \mathrm{~km}$ depth the trends in both models are fairly similar. The lower crustal $\mathrm{Q}$ structure is fairly invariant along axis, but the magnitude of the anomaly shows a small minimum near the center of the experiment and, in contrast to observations at shallower depths, the highest values are observed toward the south of the experiment. Indeed, inversions of the longest-range paths which turn in the Moho velocity gradient beneath the rise axis also show a large increase in attenuation values to the south, most of which occurs outside the central portion of the experiment. While such an increase should be considered poorly resolved since it is primarily the result of $t^{*}$ values from one instrument, it does correlate with a northto-south increase in delay times observed for the same phase [Toomey et al., 1990b]. 
Table 4.1. Summary of the $t^{*}$ data.

\begin{tabular}{|c|c|c|c|c|c|c|c|c|c|c|c|c|c|c|c|}
\hline \multirow[b]{3}{*}{ Instrument } & \multirow{3}{*}{$\begin{array}{c}\text { Distance } \\
\text { Off-Axis, } \\
\quad \text { km }\end{array}$} & \multicolumn{14}{|c|}{ Path Type } \\
\hline & & \multicolumn{3}{|c|}{1} & \multicolumn{3}{|c|}{2} & \multicolumn{3}{|c|}{3} & \multicolumn{2}{|r|}{4} & \multicolumn{3}{|c|}{5} \\
\hline & & $\mathbf{N}$ & $\begin{array}{l}\overline{\mathrm{t}^{*}} \\
\mathrm{~ms}\end{array}$ & $\begin{array}{l}\overline{\Delta \mathrm{t}^{*}} \\
\mathrm{~ms}\end{array}$ & $\mathbf{N}$ & $\begin{array}{l}\overline{\mathrm{t}^{*}} \\
\mathrm{~ms}\end{array}$ & $\begin{array}{l}\overline{\Delta \mathrm{t}^{*}} \\
\mathrm{~ms}\end{array}$ & $\mathbf{N}$ & $\begin{array}{l}\overline{\mathrm{t}^{*}} \\
\mathrm{~ms}\end{array}$ & $\begin{array}{l}\Delta \mathrm{t}^{*} \\
\mathrm{~ms}\end{array}$ & $\mathrm{~N}$ & $\begin{array}{c}\overline{\mathrm{t}^{*}} \overline{\Delta \mathrm{t}^{*}} \\
\mathrm{~ms} \\
\mathrm{~ms}\end{array}$ & $\mathrm{~N}$ & $\begin{array}{l}\overline{t^{*}} \\
\mathrm{~ms}\end{array}$ & $\begin{array}{l}\overline{\Delta \mathrm{t}^{*}} \\
\mathrm{~ms}\end{array}$ \\
\hline $\begin{array}{r}\text { A1 } \\
\text { A2 } \\
\text { A7 } \\
\text { D10 } \\
\text { D12 } \\
\text { D13 } \\
\text { D14 }\end{array}$ & 20 & $\begin{array}{r}151 \\
90 \\
155 \\
201 \\
201 \\
205 \\
208\end{array}$ & $\begin{array}{l}18 \\
17 \\
11 \\
24 \\
26 \\
30 \\
23\end{array}$ & $\begin{array}{r}-4 \\
-4 \\
-10 \\
+3 \\
+5 \\
+8 \\
+2\end{array}$ & $\begin{array}{r}14 \\
4 \\
18 \\
34 \\
37 \\
34 \\
34\end{array}$ & $\begin{array}{l}20 \\
15 \\
16 \\
24 \\
23 \\
24 \\
17\end{array}$ & $\begin{array}{r}0 \\
-5 \\
-4 \\
+4 \\
+3 \\
+4 \\
-3\end{array}$ & & & & $\begin{array}{r}111 \\
107 \\
113 \\
88 \\
101 \\
109 \\
97\end{array}$ & $\begin{array}{rr}46 & -3 \\
40 & -10 \\
43 & -6 \\
44 & -5 \\
54 & +4 \\
61 & +12 \\
57 & +7\end{array}$ & $\begin{array}{l}40 \\
30 \\
44 \\
37 \\
39 \\
42 \\
44\end{array}$ & $\begin{array}{l}32 \\
29 \\
34 \\
34 \\
43 \\
47 \\
46\end{array}$ & $\begin{array}{l}-6 \\
-9 \\
-4 \\
-4 \\
+5 \\
+9 \\
+9\end{array}$ \\
\hline $\begin{array}{r}\text { A4 } \\
\text { A6 } \\
\text { D15 }\end{array}$ & 9 & $\begin{array}{l}124 \\
102 \\
182\end{array}$ & $\begin{array}{l}24 \\
26 \\
27\end{array}$ & $\begin{array}{r}-1 \\
0 \\
+1\end{array}$ & $\begin{array}{l}21 \\
20 \\
43\end{array}$ & $\begin{array}{l}38 \\
40 \\
29\end{array}$ & $\begin{array}{l}+2 \\
+5 \\
-7\end{array}$ & $\begin{array}{l}73 \\
84 \\
88\end{array}$ & $\begin{array}{l}47 \\
46 \\
54\end{array}$ & $\begin{array}{r}-3 \\
-3 \\
+5\end{array}$ & $\begin{array}{l}33 \\
37 \\
42\end{array}$ & $\begin{array}{rr}37 & -1 \\
38 & 0 \\
39 & +1\end{array}$ & & & \\
\hline $\begin{array}{l}\text { A3 } \\
\text { A5 } \\
\text { O1 }\end{array}$ & 0 & $\begin{array}{r}34 \\
145 \\
117\end{array}$ & $\begin{array}{l}18 \\
15 \\
25\end{array}$ & $\begin{array}{r}-1 \\
-5 \\
+6\end{array}$ & $\begin{array}{l}81 \\
87 \\
44\end{array}$ & $\begin{array}{l}18 \\
15 \\
23\end{array}$ & $\begin{array}{l}-1 \\
-4 \\
+4\end{array}$ & & & & $\begin{array}{l}6 \\
5\end{array}$ & $\begin{array}{r}72-14 \\
100+14\end{array}$ & & & \\
\hline
\end{tabular}

Path type definitions are as follows: 1, Arrivals with paths that do not cross the rise axis or which pass above the axial magma chamber for shots in the central area of dense shooting; 2 , as for type 1 except shots are on the outer refraction lines; 3 , diffractions from beneath the axial magma chamber; 4 , Moho turning phases for ranges $<40 \mathrm{~km} ; 5$, arrivals propagating between the two outer refraction experiments. For each path type and instrument three quantities are listed; the number of paths $\mathrm{N}$, the mean $\mathrm{t}^{*}$ value $\overline{\mathrm{t}^{*}}$, and the deviation from the average $\overline{\mathrm{t}^{*}}$ value $\overline{\Delta \mathrm{t}^{*}}$ for similarly configured instruments. $t^{*}$ values are determined using $4 \pi$-prolate MWSA and $0.6 \mathrm{~s}$ of the waveform except for path type 3 where $0.3 \mathrm{~s}$ of the waveform is used. 
Table 4.2. Station $\mathrm{t}^{*}$ Corrections.

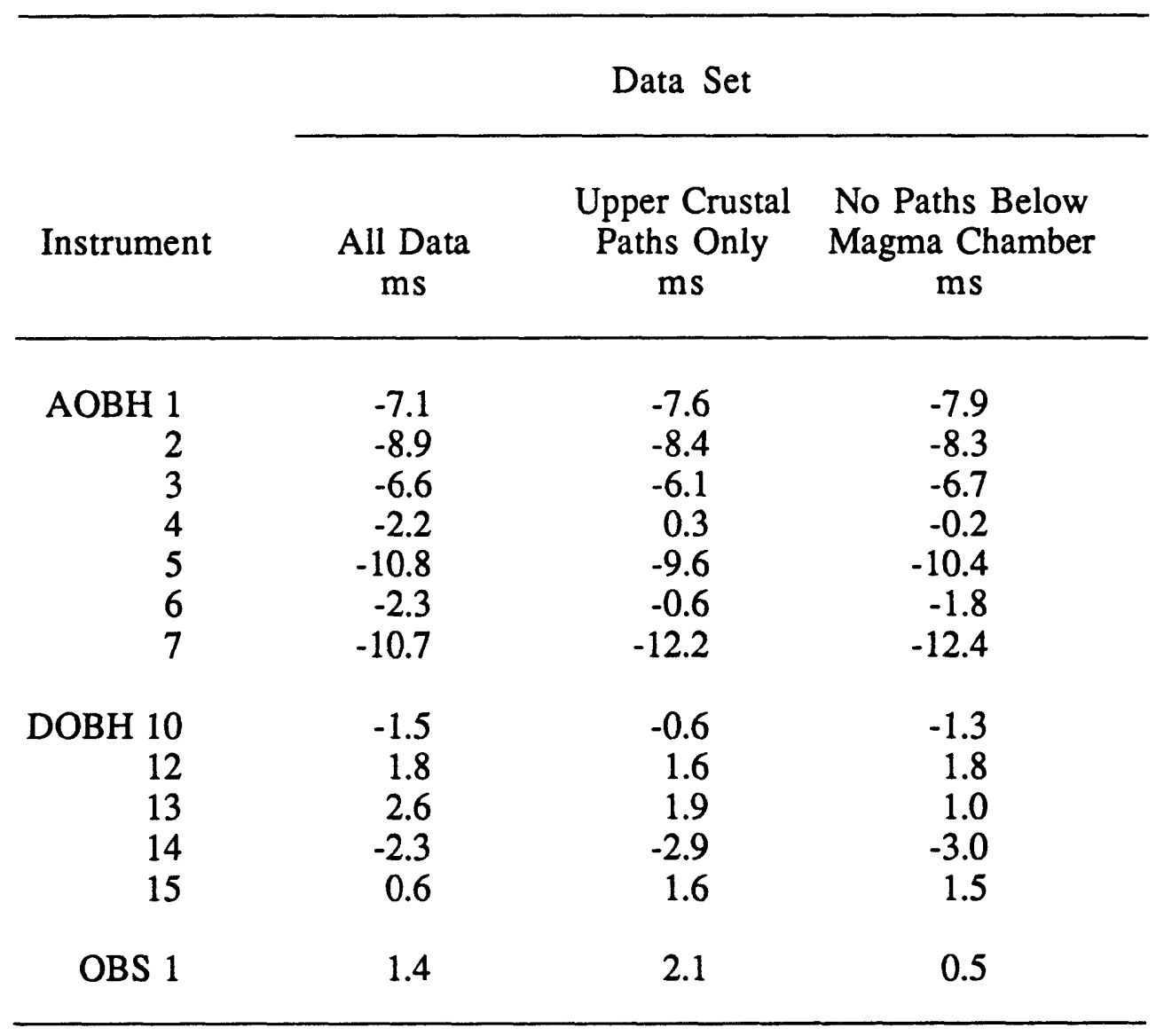

The sign convention is that the station corrections are added to the t* values predicted by the models. Station corrections are calculated using two-dimensional inversions for an axis-symmetric $\mathrm{Q}^{-1}$ structure with the constraint that the sum of the DOBH corrections is equal to zero. "Upper Crustal Paths" include no paths turning at depths significantly greater than $3 \mathrm{~km}$. 


\section{FIGURE CAPTIONS}

Figure 4.1. Record sections for (a) AOBH 1 and (b) AOBH 7 for profiles orientated perpendicular to the rise axis. A water path correction has been applied to the ranges and travel times, and amplitudes are scaled linearly with range to correct for wave divergence. Dashed lines show predicted travel times obtained from ray-theoretical paths through the unmodified velocity model proposed by Vera et al. [1990] assuming a horizontal seafloor. The location of the rise axis is shown as a dotted line.

Figure 4.2. (a) Ray-theoretical crustal wave paths, corresponding to the AOBH 1 records shown in Figure 4.1a, calculated assuming the velocity model of Vera et al. [1990] and a horizontal seafloor. Paths are shown for direct upper-crustal arrivals and diffractions above the axial magma chamber (solid), diffractions below the magma chamber (dotted), and a Moho-turning phase (dashed). (A dot-dashed line also shows a single ray path for a Moho-turning phase recorded by an instrument 9 km off-axis) (b) Approximate crustal ray paths [Thurber, 1983] for the same source-receiver combinations as (a) determined for the velocity model obtained from delay time tomography [Toomey et al., 1990a]. Since the delay time tomographic model only extends to $5 \mathrm{~km}$ depth, the deeper structure is based upon a $1-\mathrm{km}$ nodal parameterization of the model of Vera et al. [1991]. The paths are plotted with respect to a datum $2880 \mathrm{~m}$ below sealevel. (c) Wave paths corresponding to those shown in (a) obtained from the delay time tomographic velocity model by introducing constraints into the approximate ray tracing algorithm of Thurber [1983] (see text). (d) As for (c) except paths correspond to the oblique record section for DOBH 12 shown in Figure 4.3a and are shown after projection onto a plane that is perpendicular to the rise axis.

Figure 4.3. Record sections plotted as for Figure 4.1 for (a) DOBH 12 and (b) DOBH 14 . The profiles are orientated at $42^{\circ}$ and $302^{\circ}$ respectively, azimuths that make an angle of about $50^{\circ}$ with the rise axis. 
Figure 4.4. Record sections for (a) DOBH 12 and (b) DOBH 14 showing waveforms for paths that propagate between the two outer refraction lines (Figures 1.1 and 2.1). The plotting conventions are as for Figure 4.1 except that the origin of the plots is located at the most northerly shot location (Shots 1 and 221 for the western and eastern lines, respectively) and the profiles are orientated parallel to rise axis.

Figure 4.5. Examples of $t^{*}$ estimates obtained from a 0.6-s-long data window for DOBH 12 waveforms from the obliquely-oriented record section shown in Figure 4.3a. (a) The windowed waveforms are plotted against source-receiver range. The waveforms are scaled to show equal maximum amplitudes and are aligned with respect to the automatic pick. The ranges of several waveforms have been adjusted slightly so that the traces do not overlap. (b) Portions of the sourceand receiver-corrected (equation 1.24) $4 \pi$-prolate MWSA spectra with signal-to-noise ratios greater than 2 (solid) and least-squares straight line fits used to estimate $t^{*}$ (equation 1.7) (dashed).

Figure 4.6. $t^{*}$ estimates for DOBH 14 obtained using $4 \pi$-prolate MWSA from windows that include (a) $0.3 \mathrm{~s}$ and (b) $0.6 \mathrm{~s}$ of the waveform immediately following the automatic pick. Estimates are shown for all shots within the central region of the experiment and are plotted at the shot location. $t^{*}$ values $<0.03 \mathrm{~s}$ are shown as pluses which decrease linearly in size as the $t^{*}$ value increase while $t^{*}$ values $>0.03 \mathrm{~s}$ are shown as crosses whose size increases linearly with $t^{*}$. Thus, $t^{*}$ values close to 0.03 are represented by very small symbols. The locations of instruments are shown as open squares. DOBH 14 lies to the southeast of the plotted region.

Figure 4.7. $t^{*}$ estimates for paths that do not cross the rise axis or pass above the axial magma chamber for (a) AOBH 1 (located to the west of the plotted area), (b) AOBH 7 (located to the east), (c) DOBH 10 (located to the northwest), and (d) DOBH 13 (located to the northeast). Estimates are obtained using $4 \pi$-prolate MWSA and 0.6 
$\mathrm{s}$ of the waveform immediately following the automatic pick. The plotting conventions are the same as for Figure 4.6.

Figure 4.8. As for Figure 4.7 but showing $\mathrm{t}^{*}$ estimates from an 0.6-s window aligned with the onset of the Moho-turning phase.

Figure 4.9. (a) (i) $t^{*}$ estimates, (ii) relative uncertainties, and (iii) the maximum frequency used to obtain the $t^{*}$ estimates for both direct crustal and Moho-turning phases recorded by AOBH 2 (located to the west). $t^{*}$ values are plotted using the same conventions as Figure 4.6. The uncertainties are shown as crosses whose size increases with uncertainty using the same scaling factor used to plot the $t^{*}$ estimates. The maximum frequency is also shown using crosses whose size is proportional to the frequency interval used to estimate $t^{*}$ value (the lower frequency is $10 \mathrm{~Hz}$ ). (b) As for (a) except for DOBH 12 (located to the southwest). The maximum frequency is shown using a smaller scale.

Figure 4.10. $t^{*}$ estimates and uncertainties, obtained for seismic waveforms which propagate west to east between the two outer refraction lines, for (a) DOBH 13, (b) AOBH 7, and (c) DOBH 14 plotted against the shot distance from the most northerly shot of the western refraction profile (Shot 1 ). $t^{*}$ estimates are obtained using $4 \pi$-prolate MWSA and an 0.6-s data window positioned with respect to a manual pick of the higher amplitude secondary.

Figure 4.11. $\mathrm{t}^{*}$ estimates for paths that pass below the axial magma chamber for (a) AOBH 1 (filled square) and (b) DOBH 12. The plot convention is the same as for Figure 4.6 .

Figure 4.12. Record sections and predicted arrival times for (a) AOBH 4 and (b) DOBH 15 for profiles orientated perpendicular to the rise axis. The plotting conventions are the same as for Figure 4.1.

Figure 4.13. Record sections plotted as for Figure 4.1 for (a) AOBH 4 and (b) 
DOBH 15 for profiles orientated at about $50^{\circ}$ to the rise axis. The dashed lines show minimum and maximum travel times for a Mohoturning phase (see text).

Figure 4.14. (a) Ray-theoretical crustal wave paths, corresponding to the AOBH 4 records shown in Figure 4.12a, calculated assuming the velocity model of Vera et al. [1990] and a horizontal seafloor. Paths are shown for direct upper-crustal arrivals and diffractions above the axial magma chamber (solid or dotted) and diffractions below the magma chamber (dashed or dotted). The dotted lines denote paths for phases for which a reliable $t^{*}$ value cannot be determined for either path. (b) Approximate crustal ray paths [Thurber, 1983] for the same sourcereceiver combinations as (a) determined for the velocity model obtained from delay time tomography [Toomey et al., 1990a] and plotted with respect to a datum $2880 \mathrm{~m}$ below sealevel. (c) Wave paths corresponding to those shown in (a) obtained from the delay time tomographic velocity model by introducing constraints into the approximate ray tracing algorithm of Thurber [1983] (see text). (d) As for (c) except wave paths are for the oblique paths to AOBH 4 shown in Figure 4.13a and have been projected onto a plane that is perpendicular to the rise axis.

Figure 4.15. $\mathrm{t}^{*}$ estimates for arrivals with paths that do not cross the rise axis or which pass above the axial magma chamber for (a) AOBH 4 and (b) DOBH 15. Estimates are obtained using $4 \pi$-prolate MWSA and $0.6 \mathrm{~s}$ of the waveform immediately following the automatic pick The plotting conventions are the same as for Figure 4.11

Figure 4.16. As for Figure 4.15 but showing $t^{*}$ estimates from an 0.3-s-long data window aligned with the onset of the diffraction below the magma chamber.

Figure 4.17. (a) $t^{*}$ estimates, (b) relative uncertainties, and (c) the maximum frequency used to obtain the $t^{*}$ estimates for non-rise-crossing phases and diffractions above and below the magma chamber for AOBH 6. 
The plotting conventions are the same as for Figure 4.9.

Figure 4.18. (a) $t^{*}$ estimates and uncertainties for shots on the eastern refraction line recorded by AOBH 4 (located to the west of the axis). $t^{*}$ values are plotted against the distance parallel to the rise axis from the most northerly shot (Shot 221). $t^{*}$ estimates are obtained using $4 \pi$-prolate MWSA and an 0.6-s-long data window aligned with the automatic pick. (b) As for (a) except for shots on the western line (Shot 1 is the most northerly shot) recorded by DOBH 15 (located to the east of the axis).

Figure 4.19. (a) (i) $t^{*}$ estimates and (ii) uncertainties for AOBH 5 obtained using $4 \pi$-prolate MWSA and an 0.6-s-long data window aligned with the automatic pick. (b) As for (a) except for OBS 1.

Figure 4.20. (a) Ray-theoretical crustal wave paths to AOBH 5 for approximately rise-perpendicular paths from shots to the east of the rise axis calculated assuming the velocity model of Vera et al. [1990] and a horizontal seafloor. (b) Approximate paths [Thurber, 1983] for the same source-receiver combinations as (a) determined for the velocity model obtained from delay time tomography [Toomey et al., 1990a] and plotted with respect to a datum $2880 \mathrm{~m}$ below sealevel.

Figure 4.21. Maximum absolute amplitudes within the first $0.6 \mathrm{~s}$ of the waveform for (a) AOBH 1, (b) AOBH 6, (c) DOBH 10, and (d) DOBH 15 plotted against source-receiver range. In the case of the AOBHs amplitudes have be normalized to the amplitude of the calibration pulse. At short ranges values for the DOBHs fall well above the plot limits. The cutoff amplitude used to exclude saturated waveforms on the AOBHs is shown as a dashed line.

Figure 4.22. The off-axis $\mathrm{Q}^{-1}$ profile that is assumed in areas of the experiment not included in the inversions and which is used as a starting model in the axis-symmetric two-dimensional inversions. The profile is shown for $0.5-\mathrm{km}$ (solid) and $1.0-\mathrm{km}$ (dashed) nodal spacings. The model is 
based on the $\mathrm{Q}^{-1}$ structures obtained from smoothest model inversions of the the outer refraction lines presented in Chapter 2; profiles with a smoothing weight $b=10$ in Figure 2.13 are averaged and a minimum value of 0.002 is assigned at large depths).

Figure 4.23. Approximate wave paths used in the inversion of all the data for a two-dimensional symmetric structure. Selected paths are shown to illustrate the full spatial distribution paths used in the inversion after projection onto a rise-perpendicular plane. With the exception of a few rise parallel paths for a Moho-turning phase for which $t^{*}$ estimates have a large uncertainty (Chapter 2), there is a marked absence of paths at depths between about 1.5 and $2.0 \mathrm{~km}$ depth beneath the rise axis.

Figure 4.24. Normalized squared data misfit (equation 1.40) plotted against smoothing weight for an inversion for a two-dimensional axissymmetric $Q^{-1}$ structure using the full $t^{*}$ data set. Vertical dotted and dashed lines show the smoothing weights for which solutions are presented in Figure 4.25. Dashed vertical lines delineate smoothing weights for which contour plots of the uncertainty and spread are also presented in Figures 4.26 and 4.27, respectively.

Figure 4.25. Smoothest model solutions showing the two-dimensional axissymmetric $\mathrm{Q}^{-1}$ structure obtained from an inversion of all the data for smoothing weights of (a) $b=180$, (b) $b=56$, (c) $b=18$, (d) $b=5.6$, (e) $b=1.8$, (f) $b=0.56,(\mathrm{~g}) b=0.18$. The contour interval is 0.01 .

Figure 4.26. Formal linearized uncertainty factors (equation 1.38) for the $\mathrm{Q}^{-1}$ models with (a) $b=56$ (Figure 4.25b), (b) $b=5.6$ (Figure 4.25d), and (c) $b=0.56$ (Figure 4.25f). The contour interval is 0.025 for dashed contours, 0.1 for solid contours, and 0.5 for bold contours.

Figure 4.27. Square root of the spread function (equation 1.42) calculated for the $Q^{-}$ 1 models with (a) $b=56$ (Figure 4.25b), (b) $b=5.6$ (Figure 4.25d), and 
(c) $b=0.56$ (Figure $4.25 f$ ). The spread is normalized to yield a value of unity for a two-dimensional boxcar measuring $1 \mathrm{~km}$ on each side. Contours are separated by factors of two.

Figure 4.28. Results of inversions for two-dimensional axis-symmetric $\mathrm{Q}^{-1}$ structure using paths confined to depths above $3 \mathrm{~km}$. (a) The normalized squared data misfit plotted against smoothing parameter. Vertical dashed lines show smoothing weights for which $\mathrm{Q}^{-1}$ models are shown. (b) The distribution of wave paths used in the inversion plotted as for Figure 4.23. (c) The $Q^{-1}$ model for a smoothing weight $b=10$. (d) (i) The $Q^{-1}$ model for a smoothing weight $b=1$. (ii) The formal uncertainty factors for the model shown in (i). (e) The $Q^{-1}$ model for a smoothing weight $b=10$.

Figure 4.29. (a) Horizontal section at a depth of $2.5 \mathrm{~km}$ below the seafloor through a three-dimensional $\mathrm{Q}^{-1}$ model obtained from a constrained inversion for upper crustal structure (see text). (b) A vertical section along the rise axis through the same model.

Figure 4.30. (a) Maximum $\mathrm{Q}^{-1}$ values and formal uncertainties for the 2-3-km-deep axial low- $Q$ anomaly in the three-dimensional inversions presented in Figure 4.29 plotted against location along the rise axis. (b) As for (a) except $\mathrm{Q}^{-1}$ values and uncertainties are averages obtained within areas centered on the rise axis. Averages are obtained between 2 and $3 \mathrm{~km}$ depth and over a half width of $2 \mathrm{~km}$ (solid) and $4 \mathrm{~km}$ (dashed). (c) The maximum negative velocity perturbation in the delay time tomographic model [Toomey et al., 1990a] plotted against position along the rise axis. (d) Averages of the velocity anomaly obtained over the same areas as (b).

Figure 4.31. Results of inversions for axis-symmetric surface $\mathrm{Q}^{-1}$ models which are invariant along the rise axis. Solutions are shown for inversions obtained from a data set that includes $t^{*}$ values from non-rise-crossing and shallow rise-crossing paths. (a) The normalized squared data misfit plotted against smoothing weight $b$ for the inversion that 
includes data for all the instruments. Dashed lines show smoothing weights for which solutions are presented. (b)-(d) A comparison of models obtained from a data set that that includes values for all instruments (solid lines with error bars) and a data set that excludes values for instruments located on the rise axis (dashed lines). The smoothing weights are (b) $b=100$, (c) $b=10$, (d) $b=1$.

Figure 4.32. $t^{*}$ values for non-rise crossing paths to (a) DOBH 10, (b) DOBH 12, (c) DOBH 13, and (d) DOBH 14. Values are shown for shots located on the outer refraction lines approximately $20 \mathrm{~km}$ off-axis (open circles), 6-9 km off axis (pluses), and 3-6 km off axis (crosses). All $\mathrm{t}^{*}$ values are calculated from an 0.6 -s-long window using $4 \pi$-prolate MWSA.

Figure 4.33. Results of inversions for two-dimensional near-surface $\mathrm{Q}^{-1}$ structure (see text). (a) The normalized squared data misfit plotted against smoothing parameter. Vertical dashed lines show smoothing weights for which $Q^{-1}$ models are shown. (b-d) Surface $Q^{-1}$ models for smoothing weights (a) $b=10$, (b) $b=1$, and (c) $b=0.1$.

Figure 4.34. The results of an inversion of $t^{*}$ values obtained for paths between the two outer refraction lines. The inversion determines along axis variations in an 8-km-wide region of constant $\mathrm{Q}^{-1}$ centered on the rise axis; the $\mathrm{Q}^{-1}$ model of Figure 4.22 is adopted elsewhere. (a) The normalized squared data misfit plotted against the smoothing parameter $b$. Vertical dashed lines delineate smoothing weights for which $\mathrm{Q}^{-1}$ models are shown. (b) The $\mathrm{Q}^{-1}$ models for smoothing weights $b=3000$ (dot-dashed), $b=300$ (solid with formal error bars), and $b=30$ (dashed). (c) The square root of the spread for a smoothing weight $b=300$. The values are normalized to yield a value of unity for a 1-km-wide boxcar

Figure 4.35. $t^{*}$ values (error bars) and model predictions (asterisks) for the solution presented in Figure 4.34 with a smoothing weight $b=300$ for (a) DOBH 12 and (b) DOBH 14. 
Figure 4.36. (a) The distribution of wave paths plotted as for Figure 4.23 for the inversion of a data set comprising $t^{*}$ estimates for non-rise crossing paths, shallow rise-crossing paths, and the Moho-turning phase (excluding paths between the two outer refraction lines) for non-rise axis instruments. (b) The starting model which is obtained from a two-dimensional axis-symmetric inversion of the data set with the constraint that the model is vertically invariant below $4 \mathrm{~km}$ depth.

Figure 4.37. Sections through the three dimensional $\mathrm{Q}^{-1}$ model obtained by a constrained inversion (see text) of a data set comprising $t^{*}$ estimates for non-rise crossing paths, shallow rise crossing paths, and the Moho-turning phase (excluding paths between the two outer refraction lines) for non-rise axis instruments. (a)-(c) Vertical sections orientated perpendicular to the rise axis at $y=-4 \mathrm{~km}, y=0$ $\mathrm{km}$, and $\mathrm{y}=4 \mathrm{~km}$. (d) A horizontal section at a depth of $4 \mathrm{~km}$ below the seafloor.

Figure 4.38. (a) Maximum $\mathrm{Q}^{-1}$ value and formal uncertainties for the axial low- $\mathrm{Q}$ anomaly in the model shown in Figure 4.37 plotted against position on the rise axis. (b) As for (a) except the average $Q^{-1}$ value below $4 \mathrm{~km}$ depth and within $2 \mathrm{~km}$ of the rise axis is shown. (c) The average $\mathrm{Q}^{-1}$ value below $4 \mathrm{~km}$ depth and within $4 \mathrm{~km}$ of the rise axis (solid) is compared with the along axis $\mathrm{Q}^{-1}$ model $(\mathrm{b}=300)$ for paths that propagate between the two outer refraction lines (Figure 4.34b, $b=300$ ). 
(a)

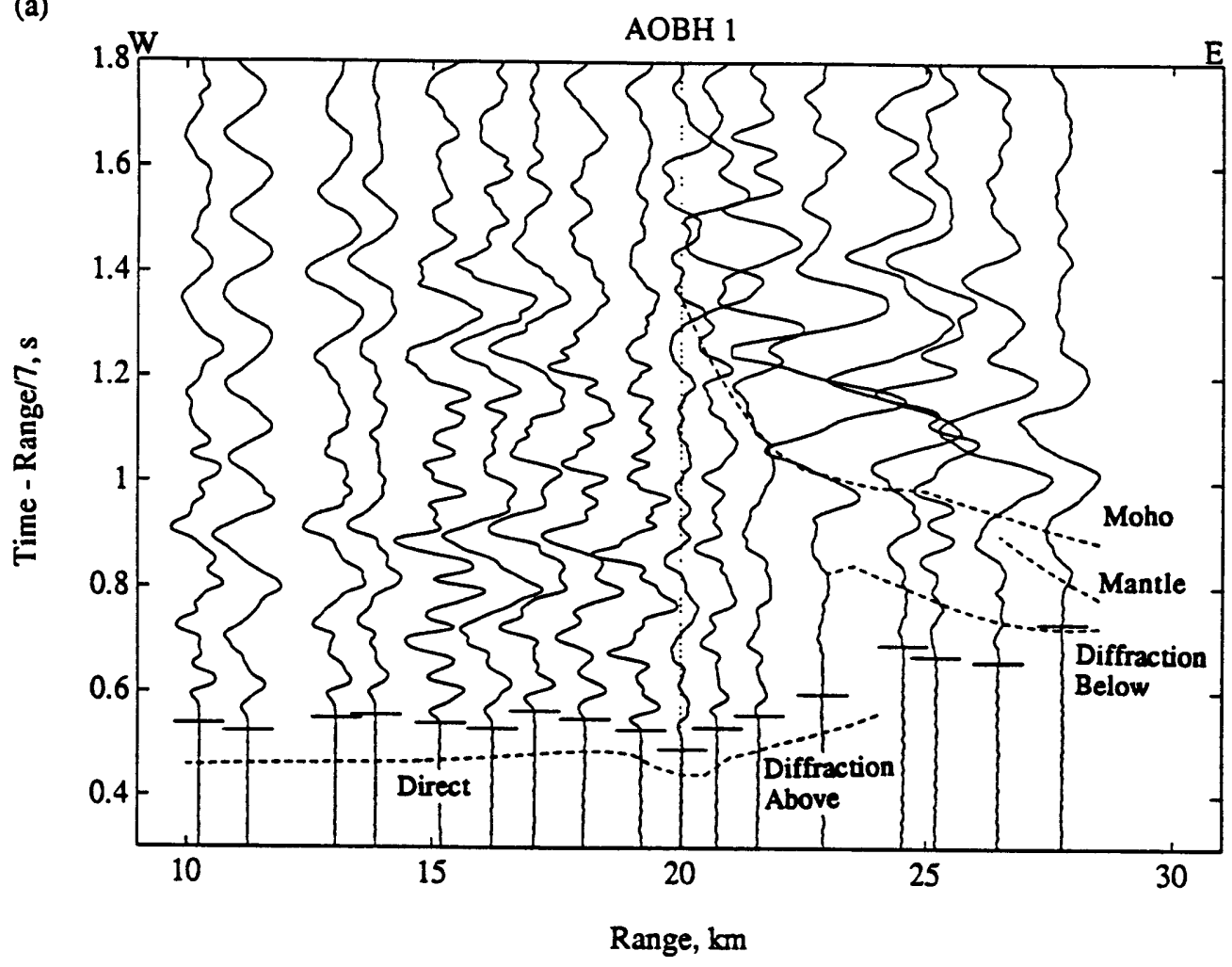

(b)

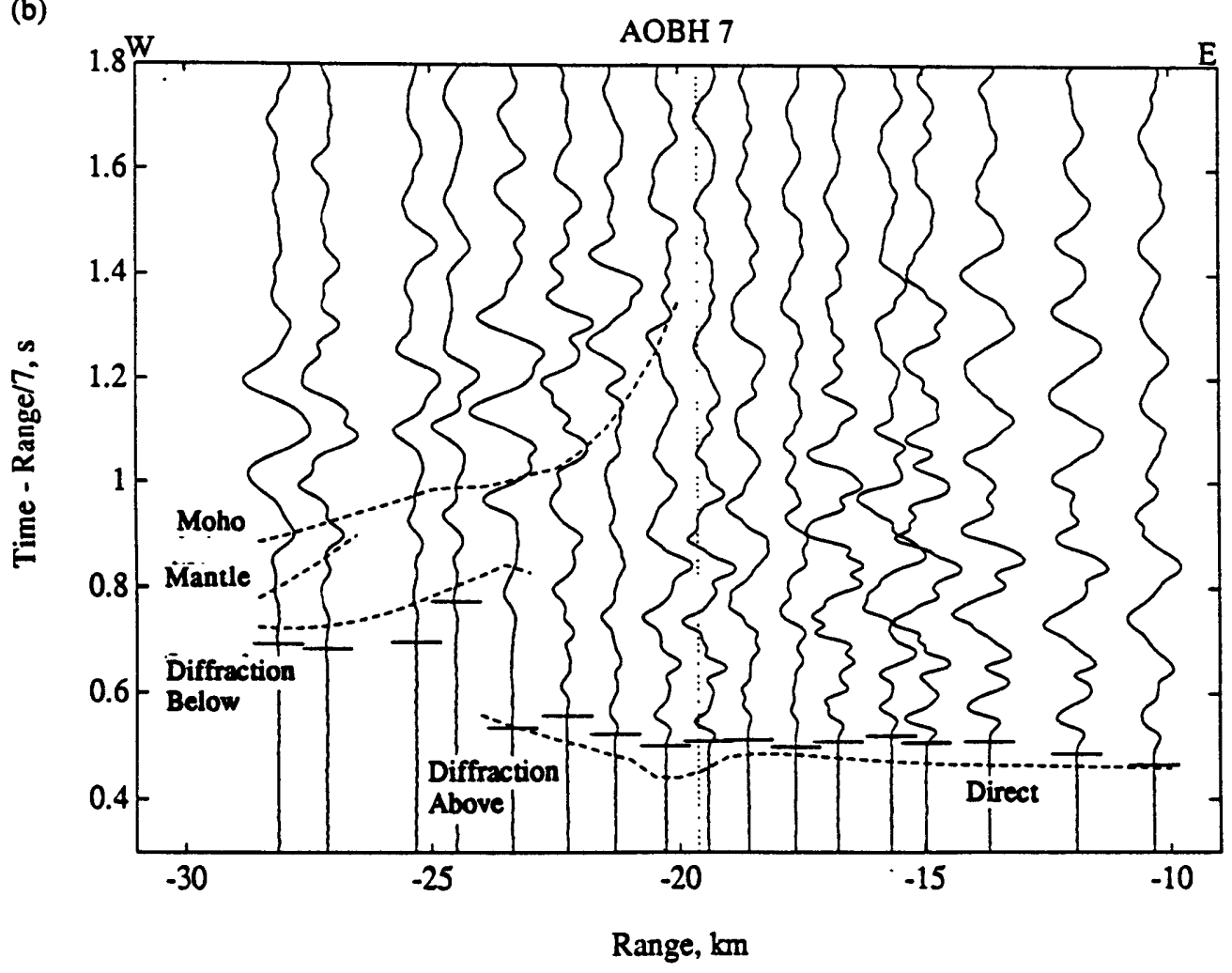

Figure 4.1 
(a)

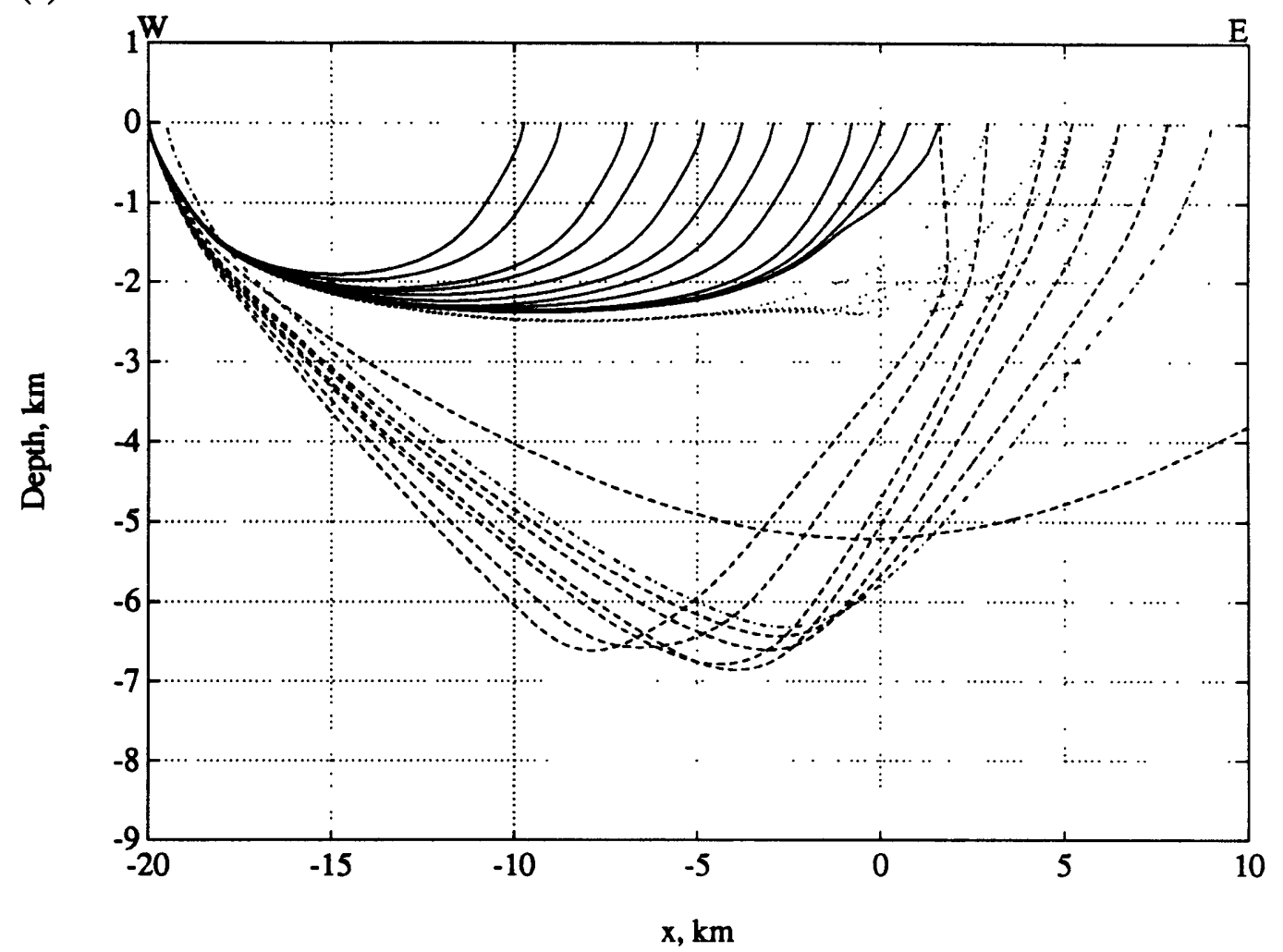

(b)

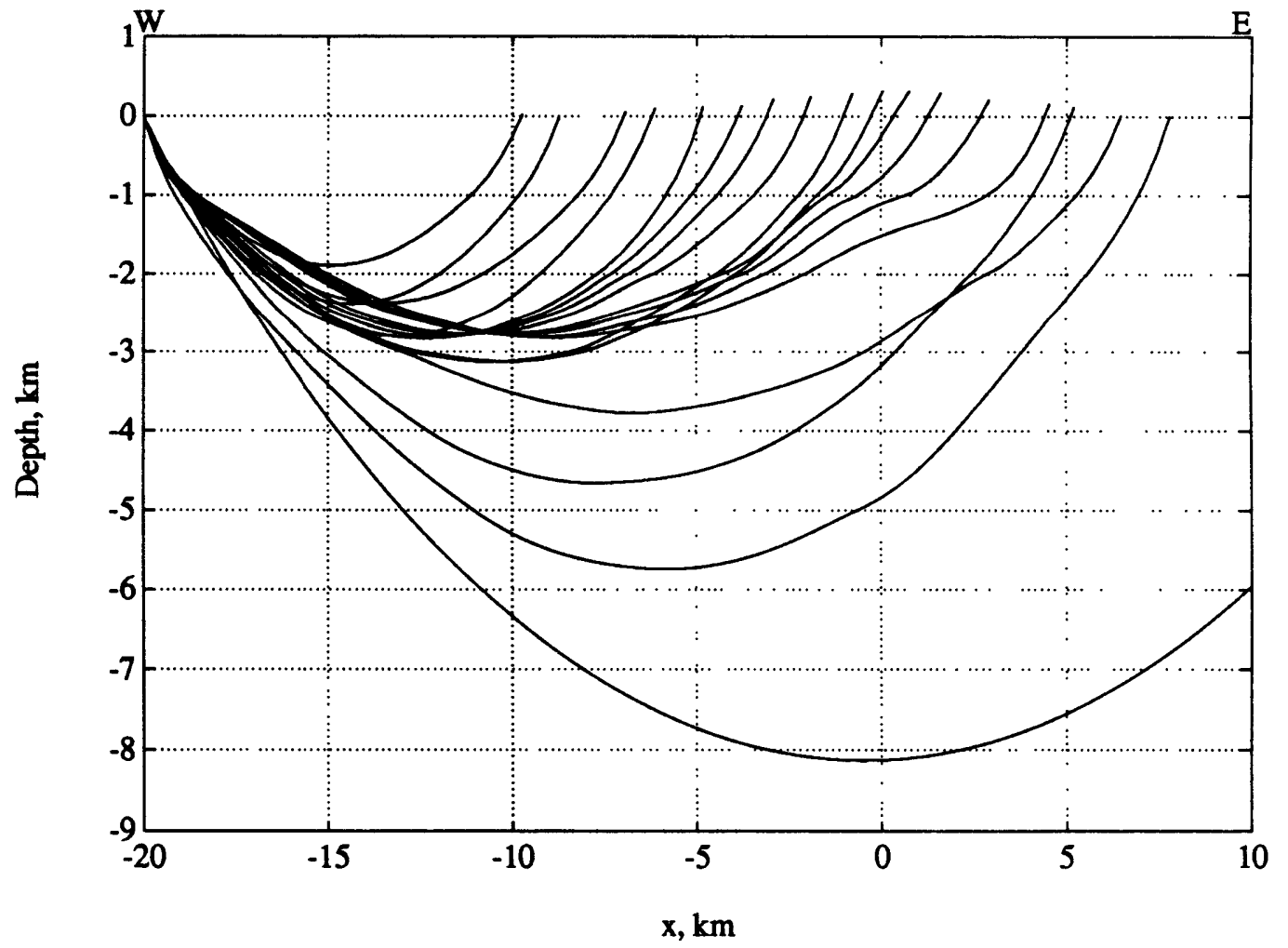

Figure 4.2 
(c)

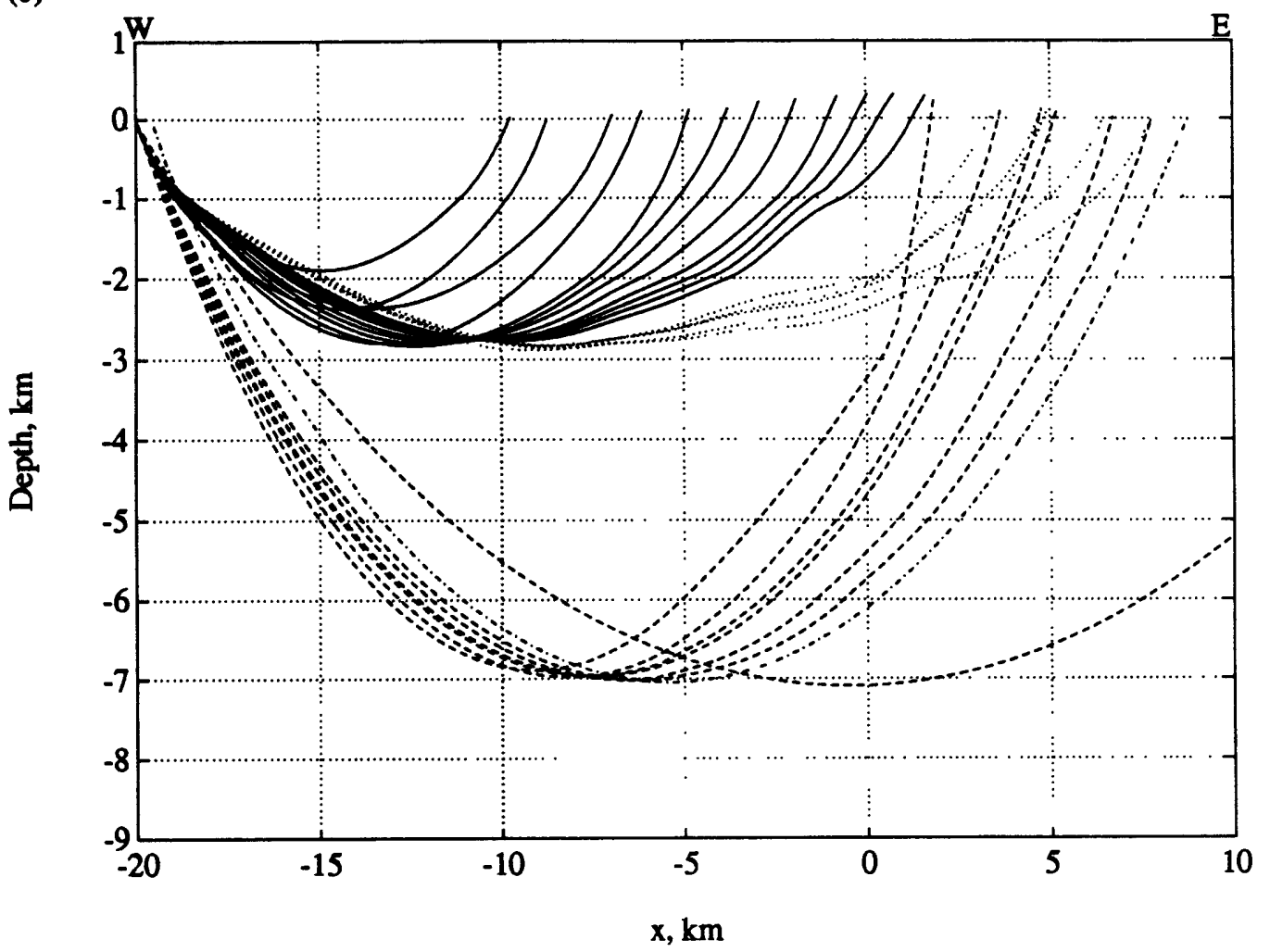

(d)

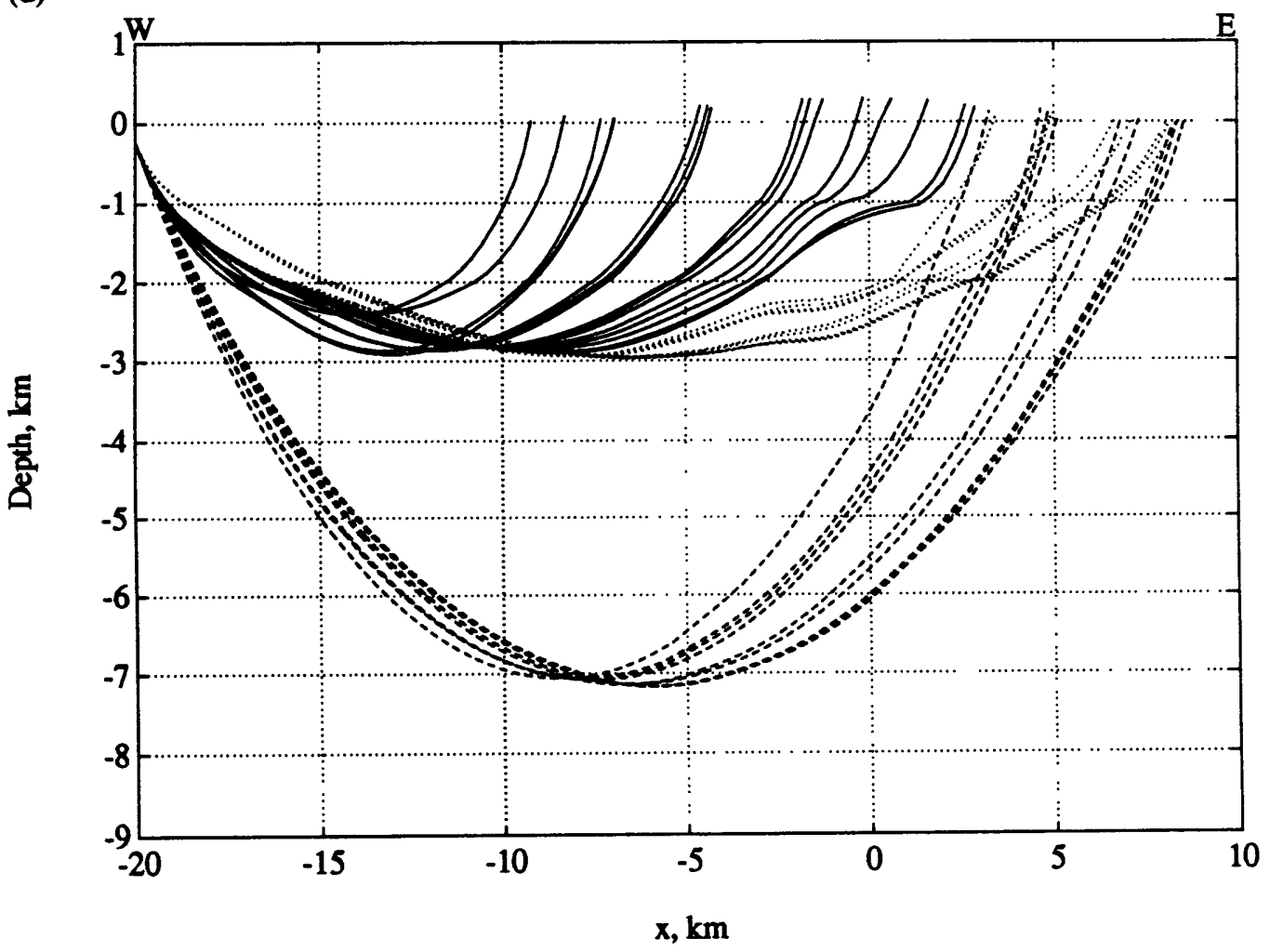

Figure 4.2 cont. 
(a)

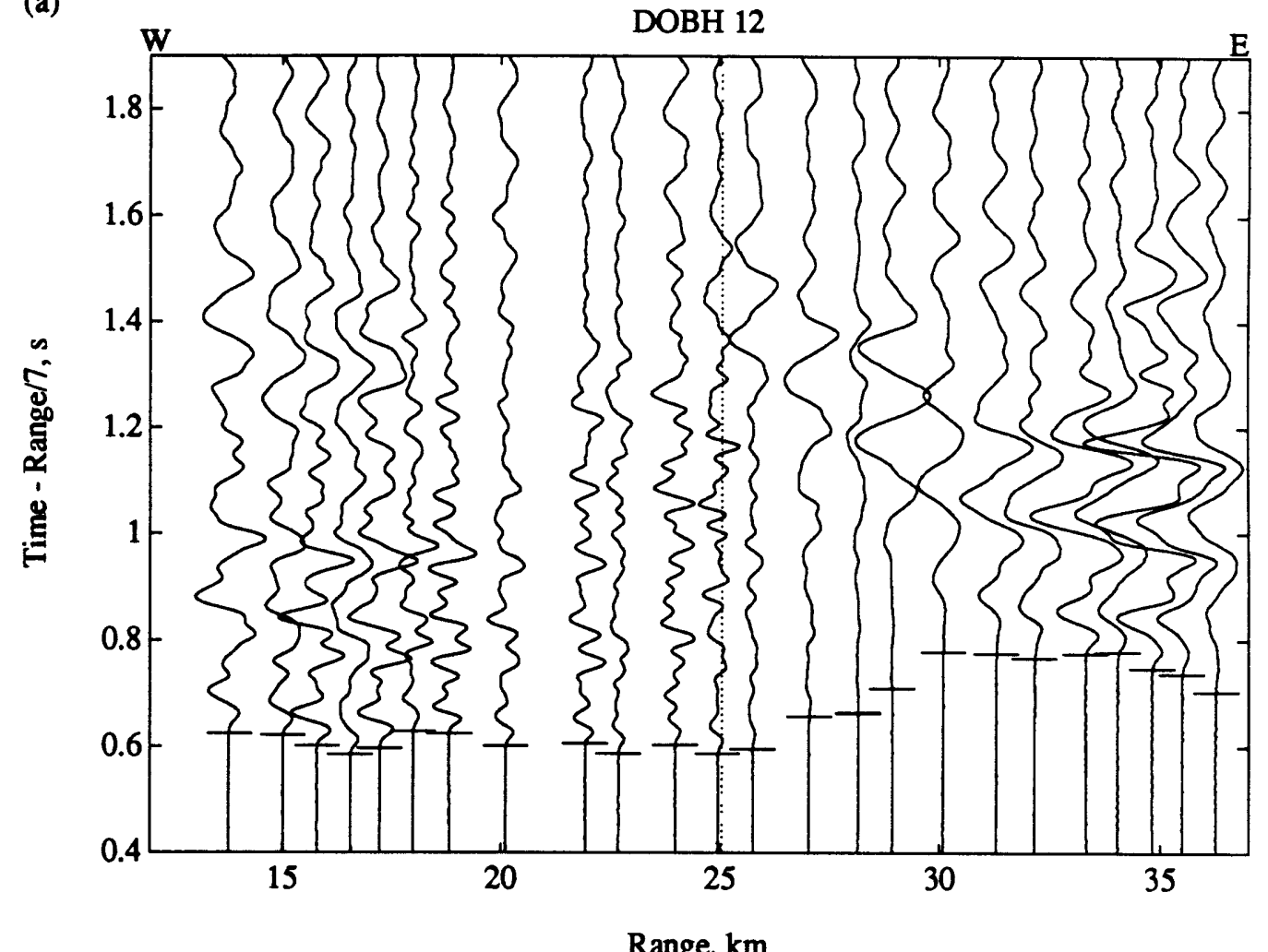

(b)

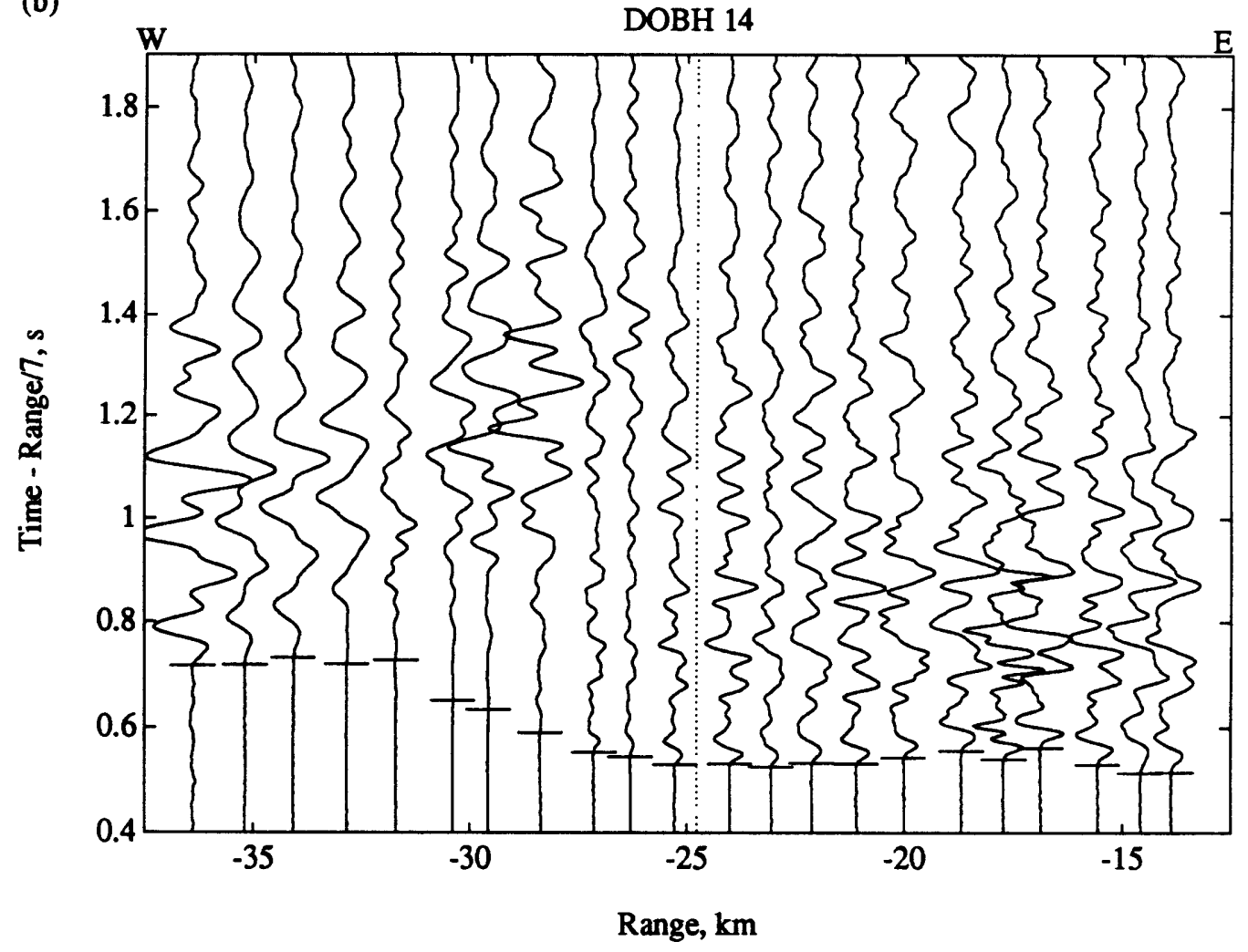

Figure 4.3 
(a)

DOBH 12

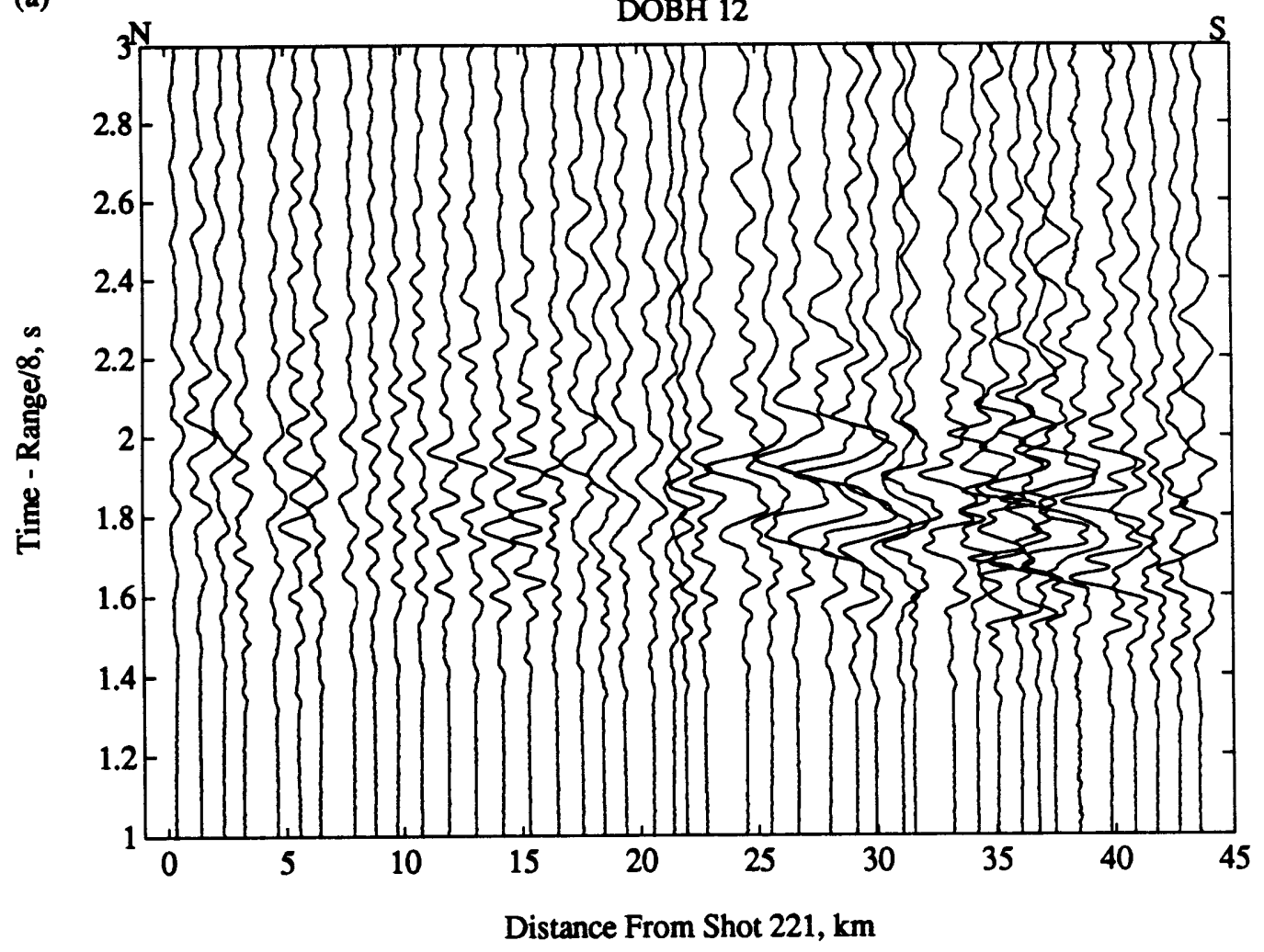

(b) DOBH 14

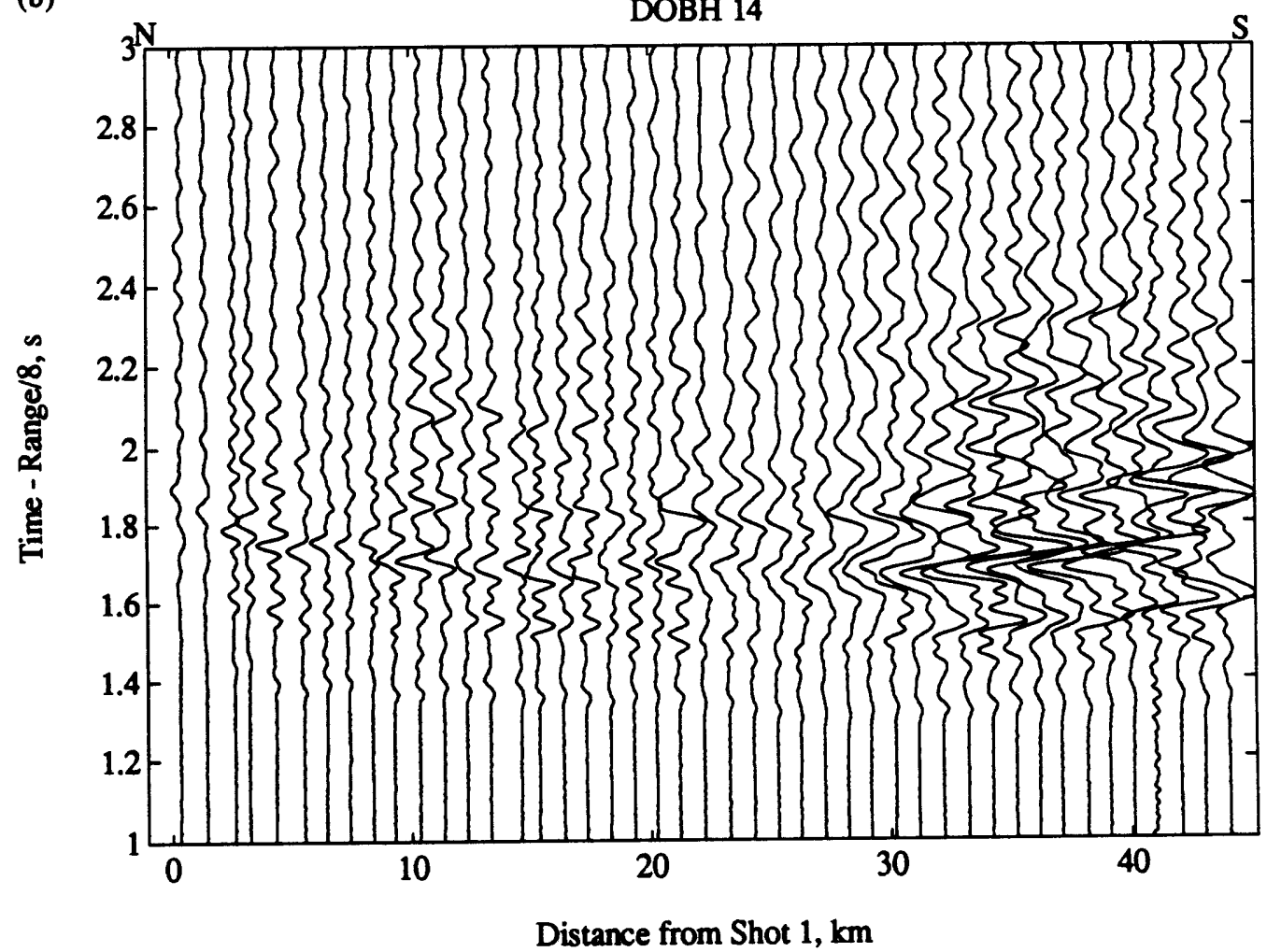

Figure 4.4 
(a)

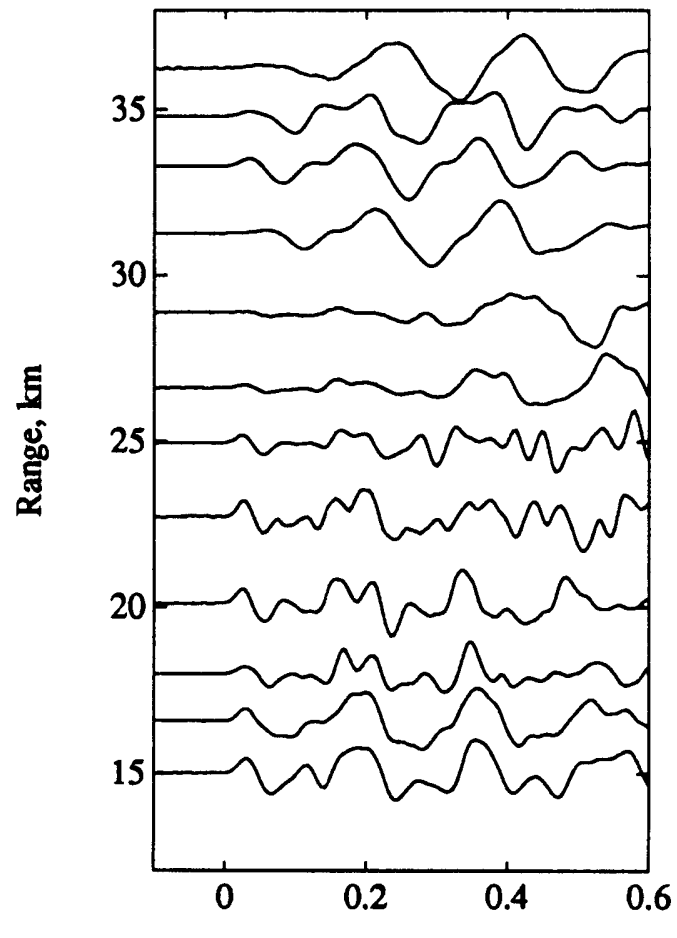

Time, $s$ (b)

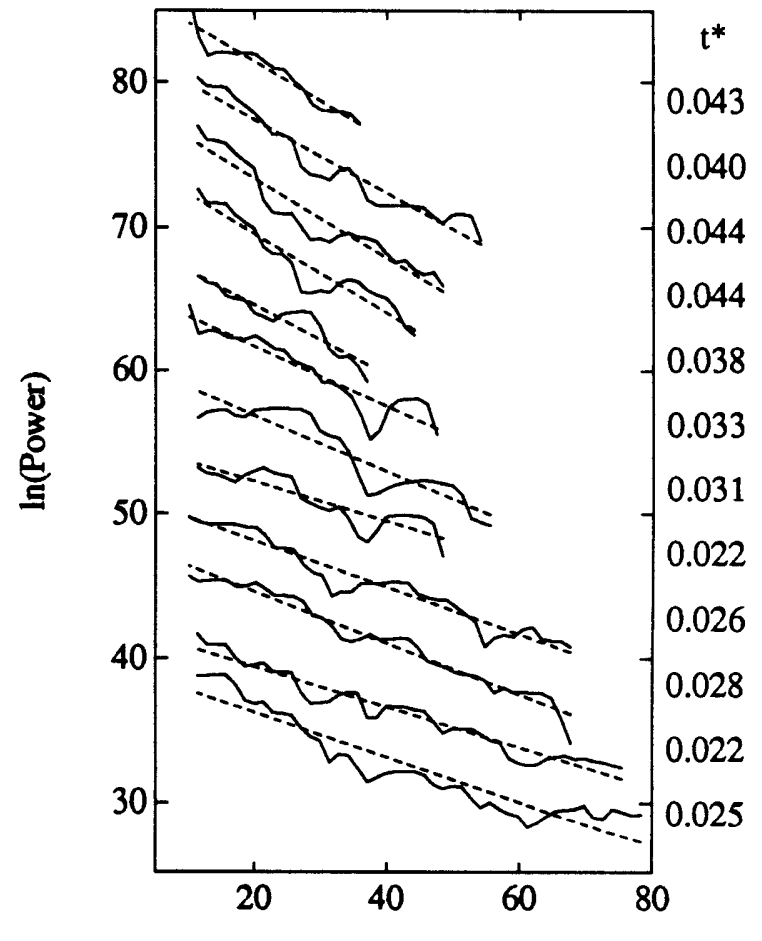

Frequency, $\mathrm{Hz}$ 
(a)

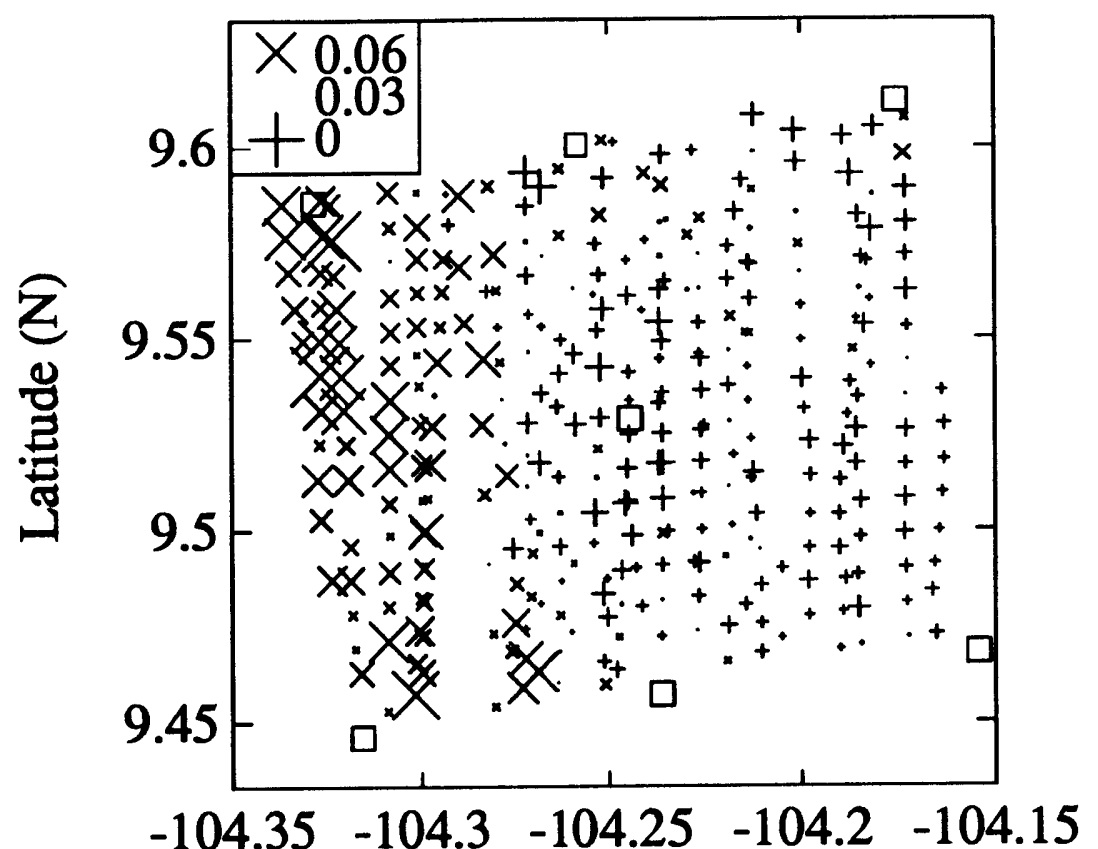

(b)

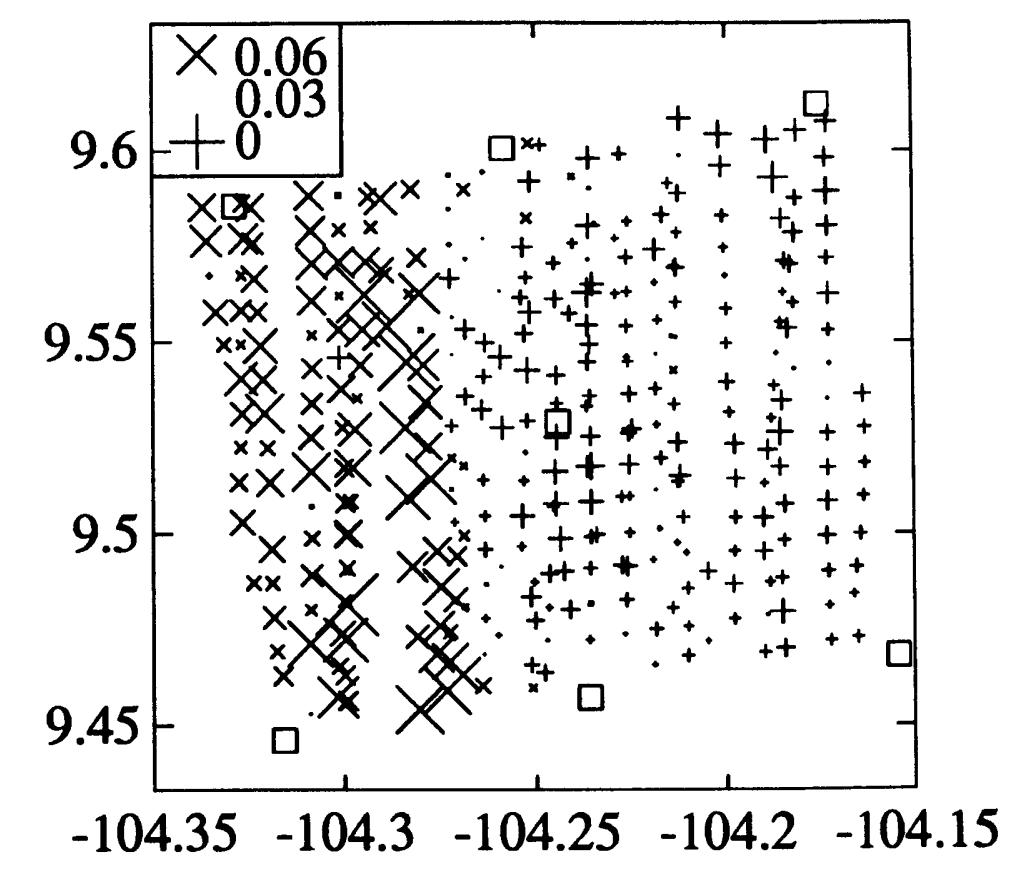

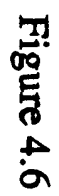

Longitude (W) 


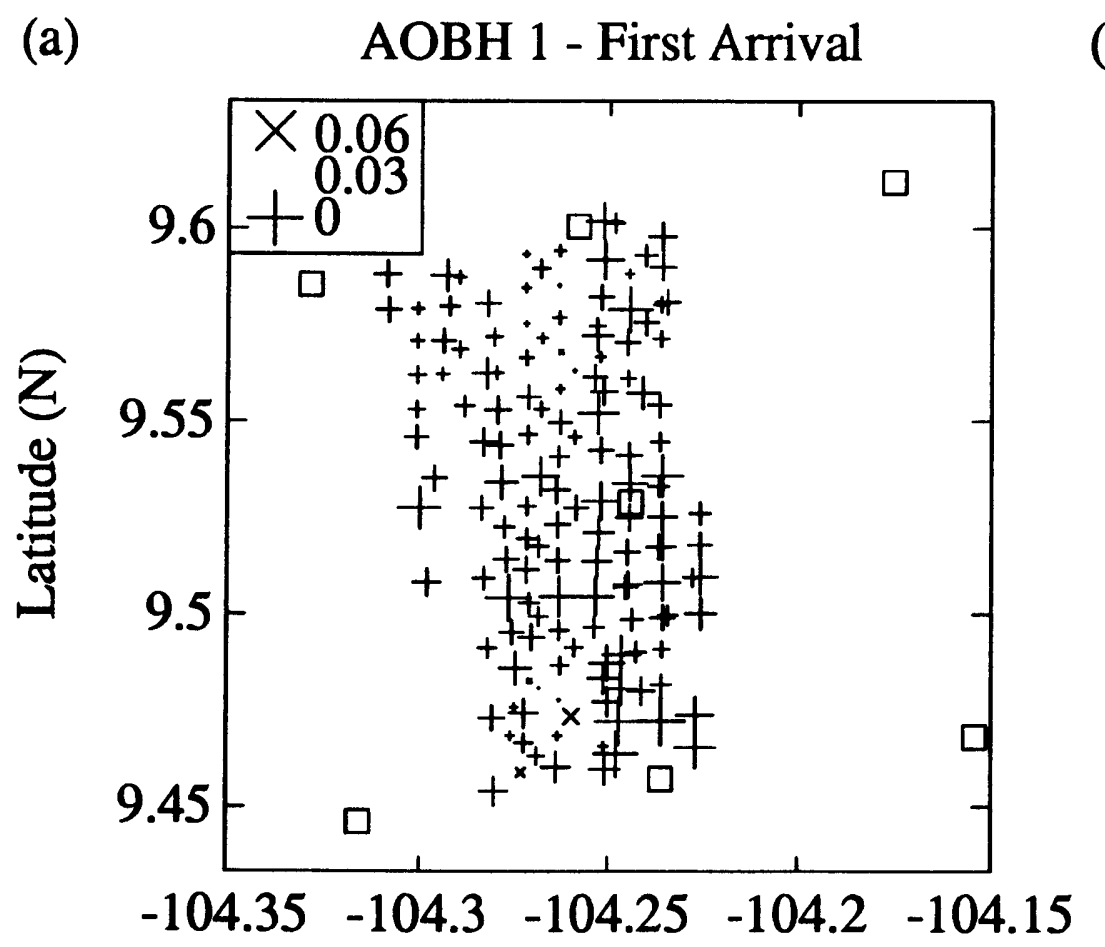

(b)

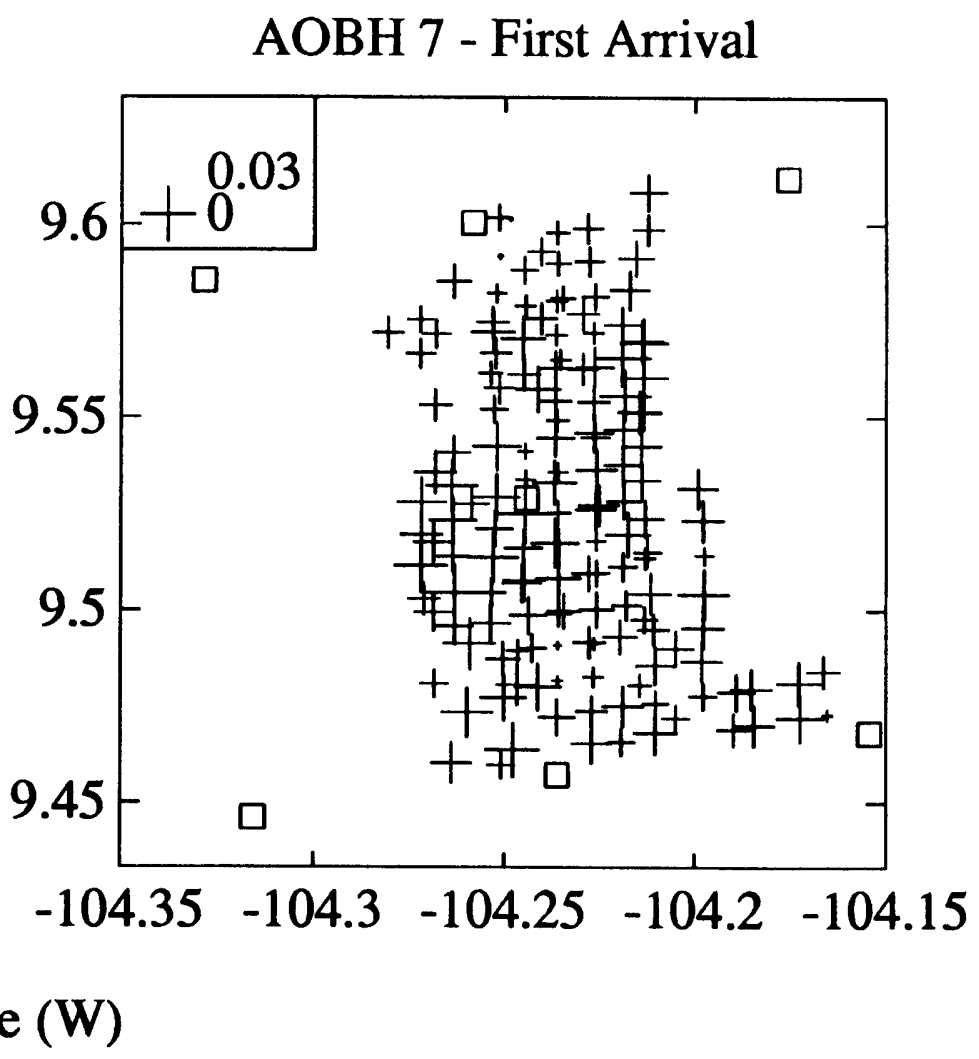

\section{Longitude (W)}


(c)

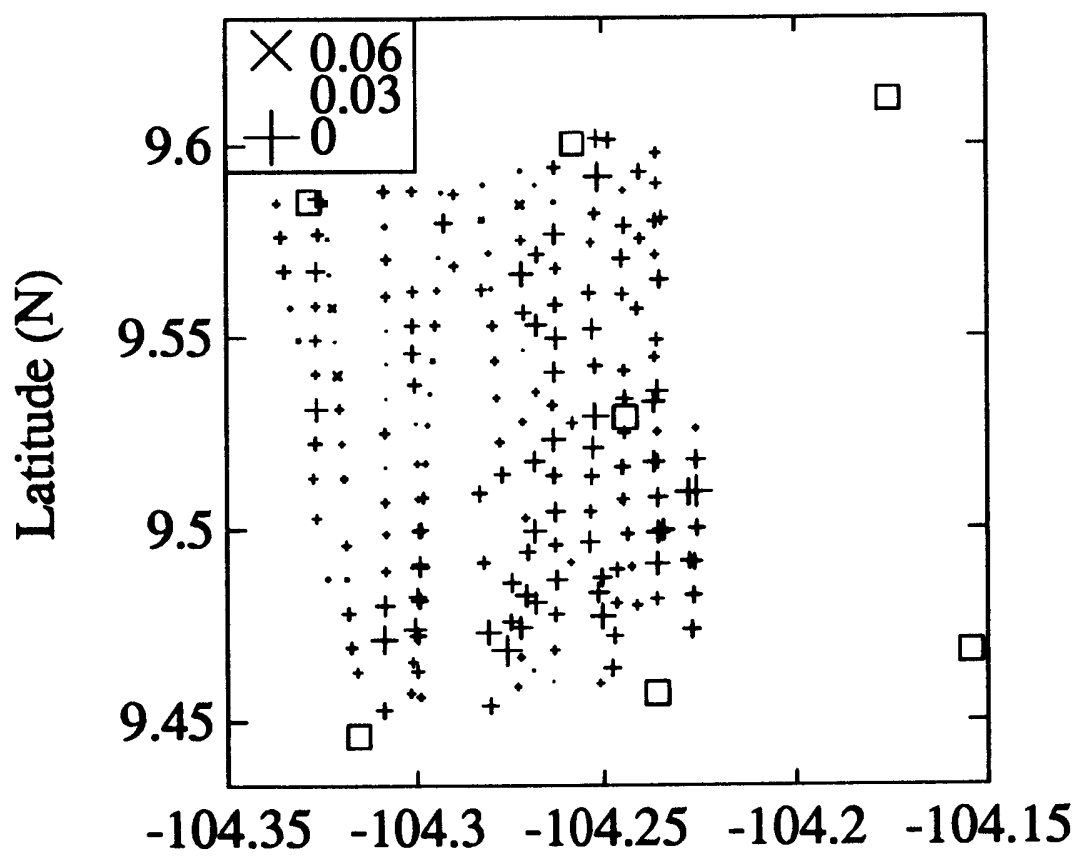

(d)

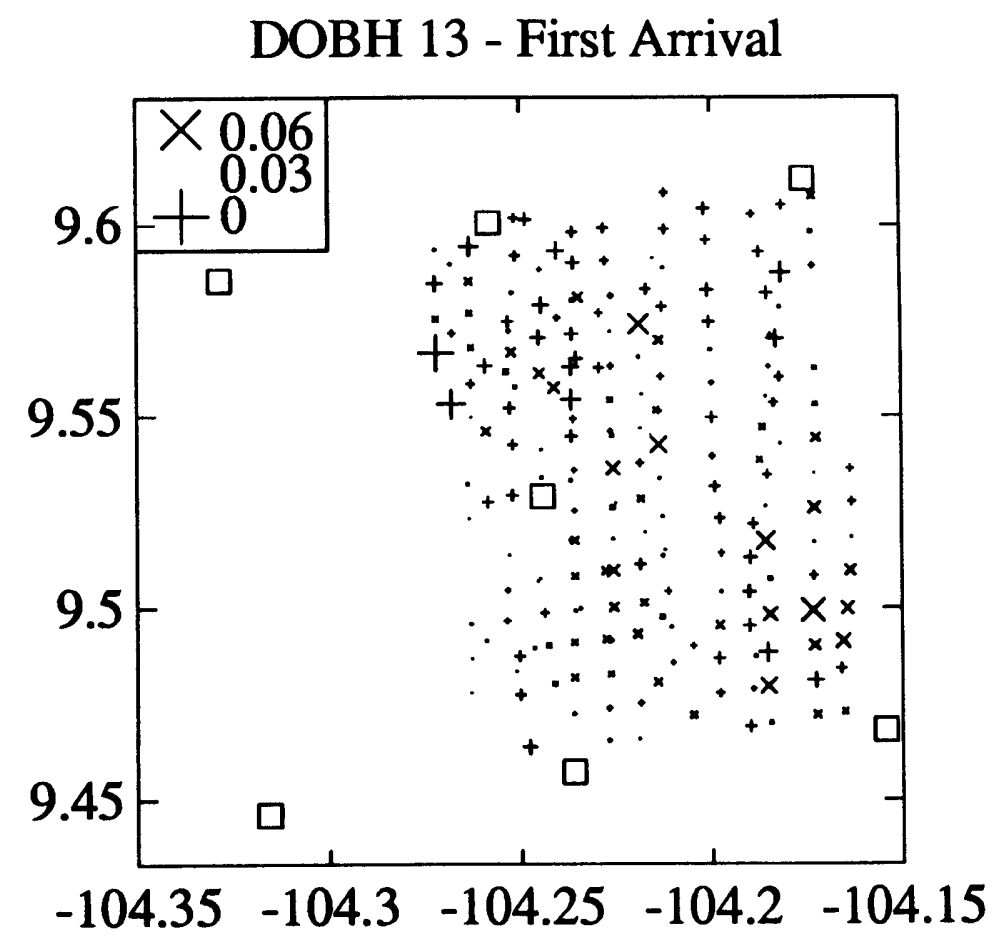


(a)

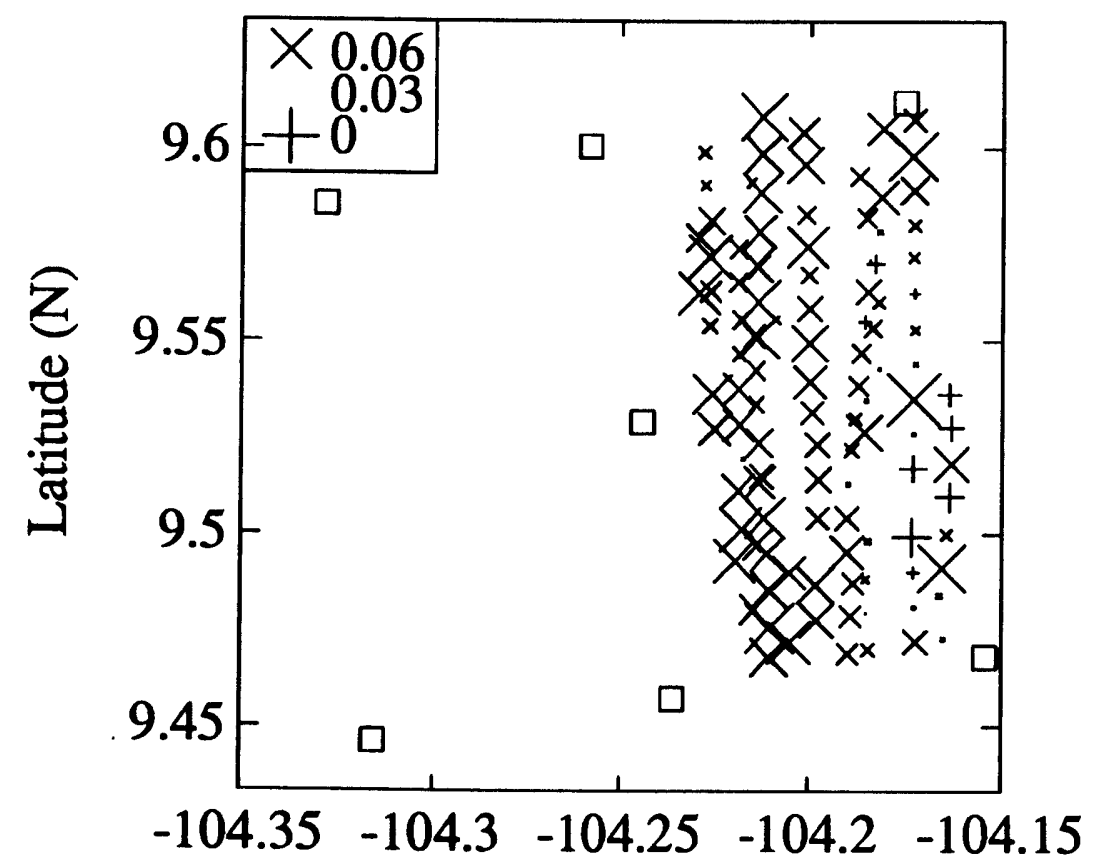

(b)

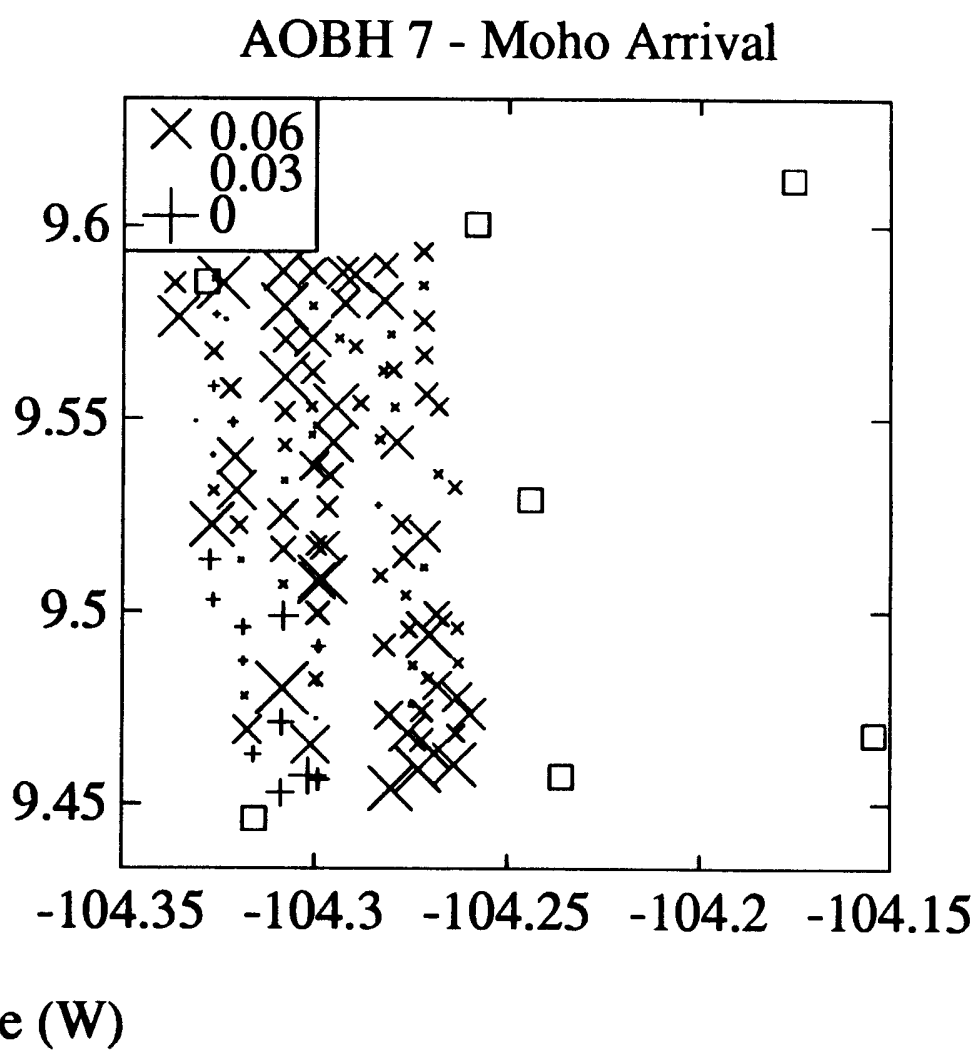

Longitude (W) 
(c)

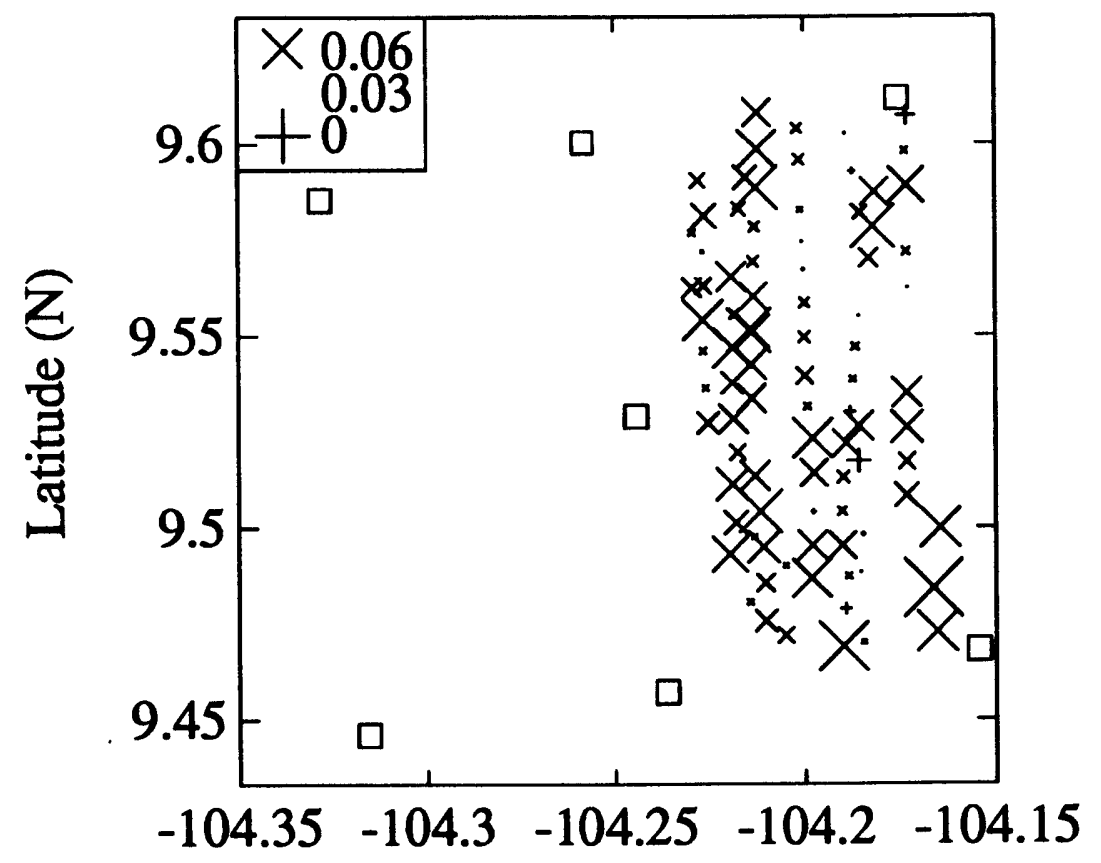

(d)

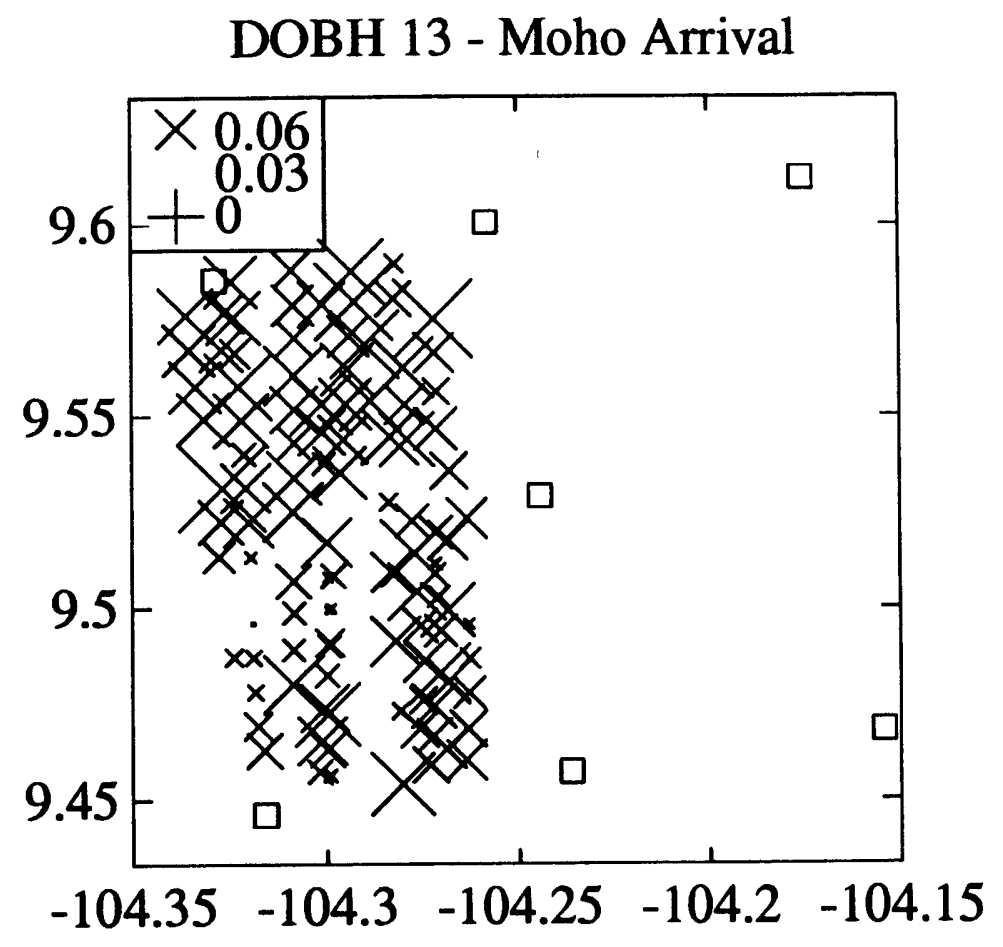

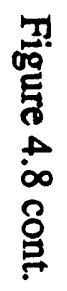
Longitude (W) 
(a) (i)

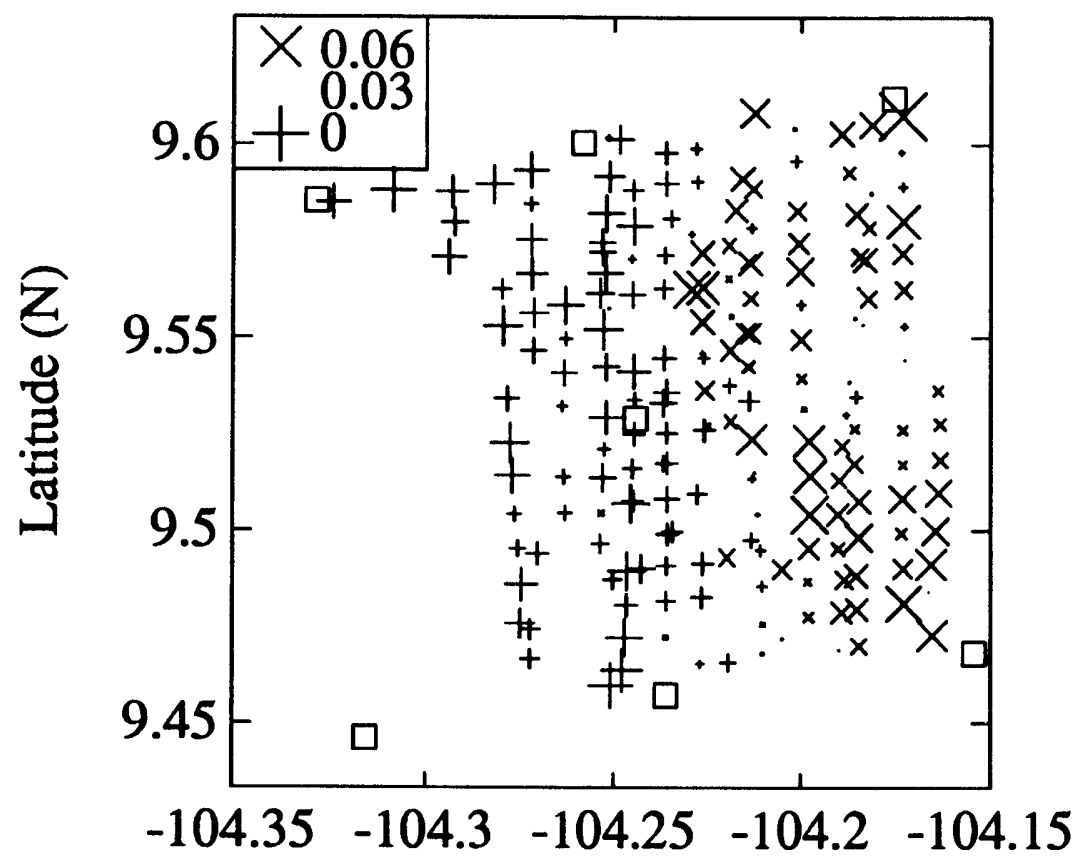

(ii)

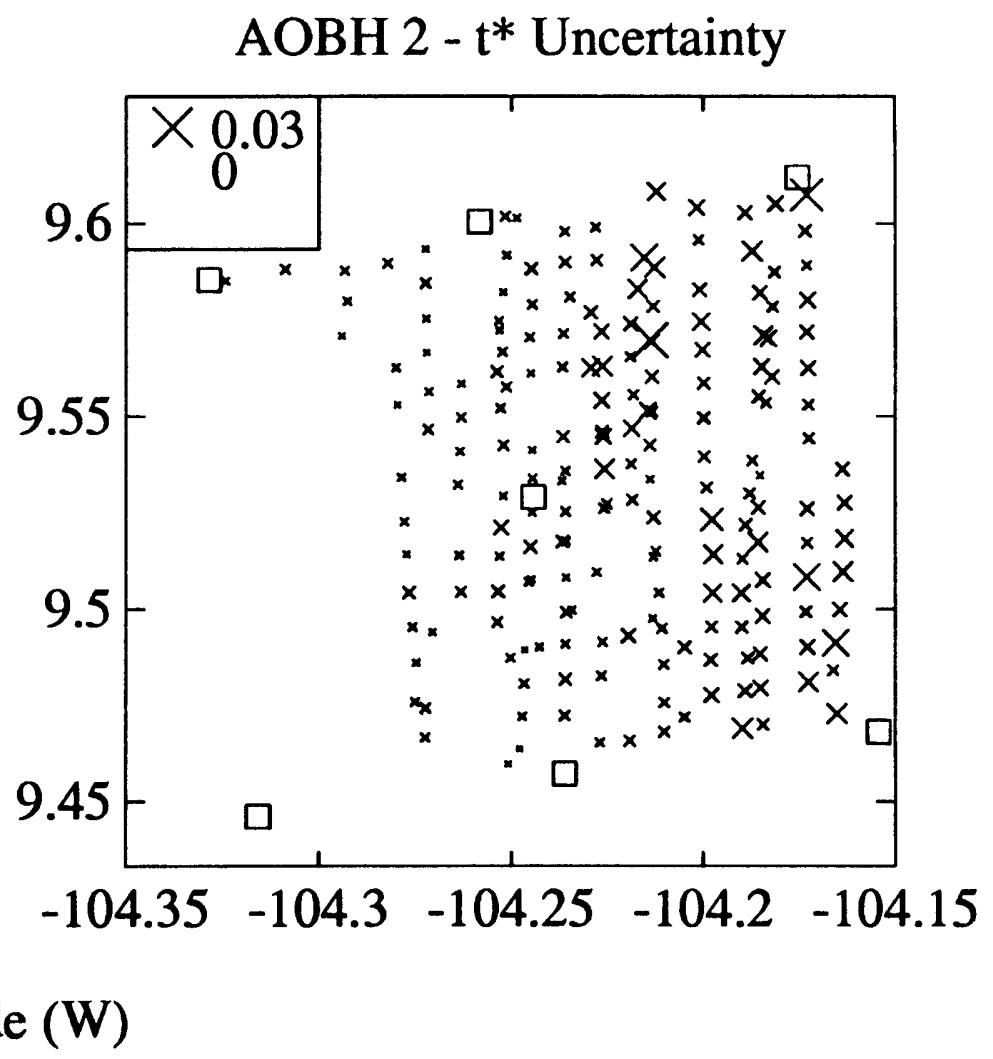

\section{Longitude (W)}


(a) (iii) AOBH 2 - Upper Frequency Limit

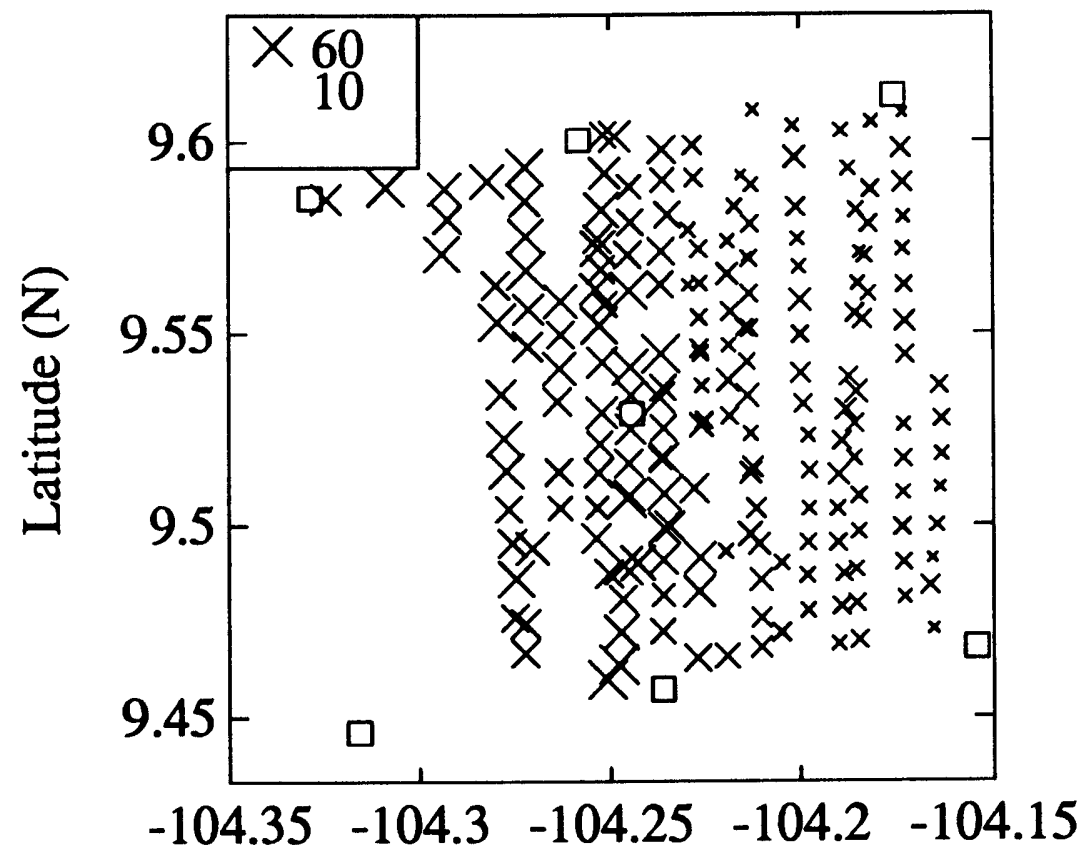

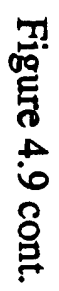

\section{Longitude (W)}




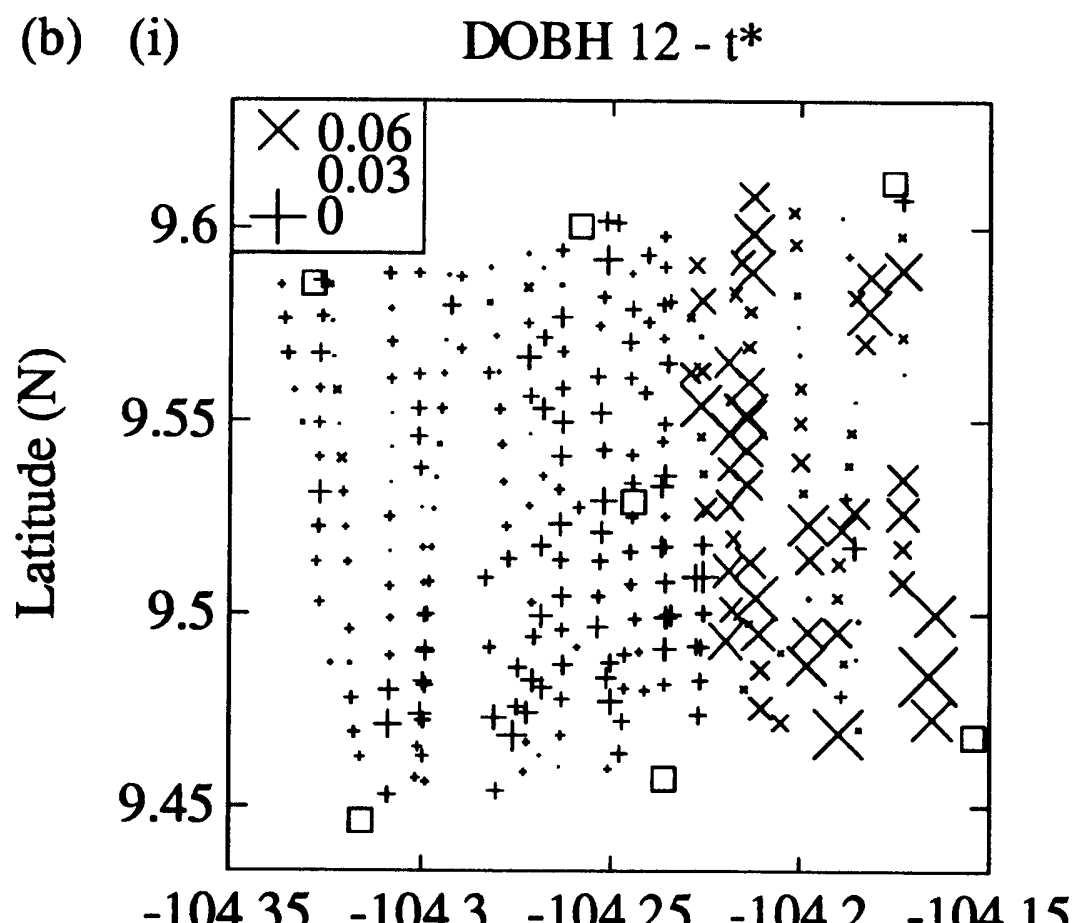

(ii)

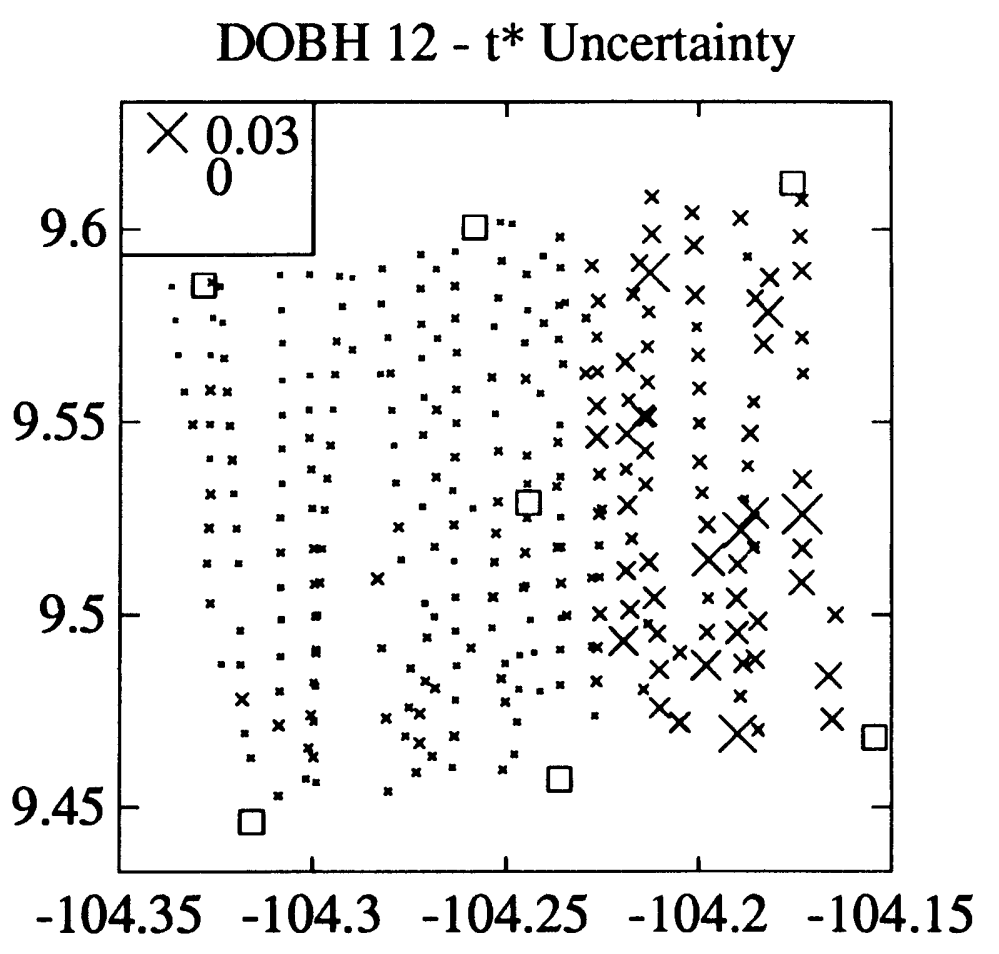

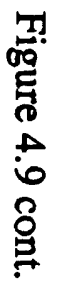

Longitude (W) 
(b) (iii) DOBH 12 - Upper Frequency Limit

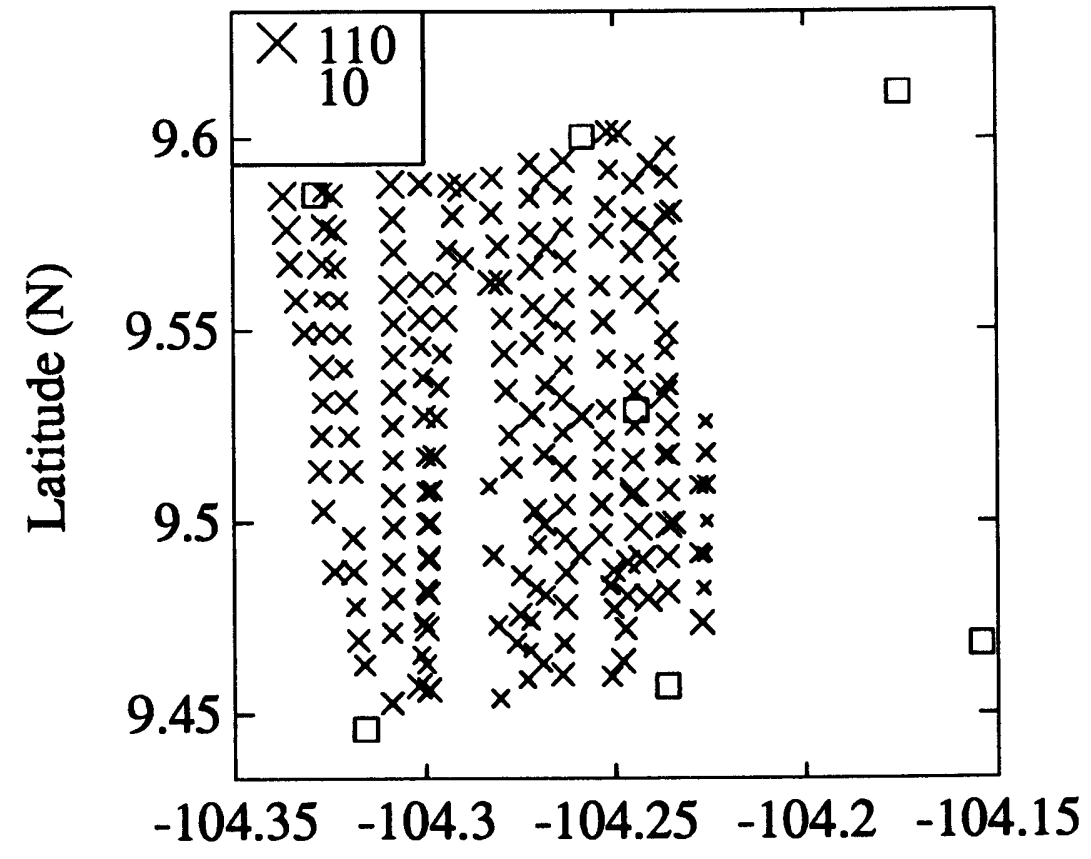

\section{Longitude (W)}



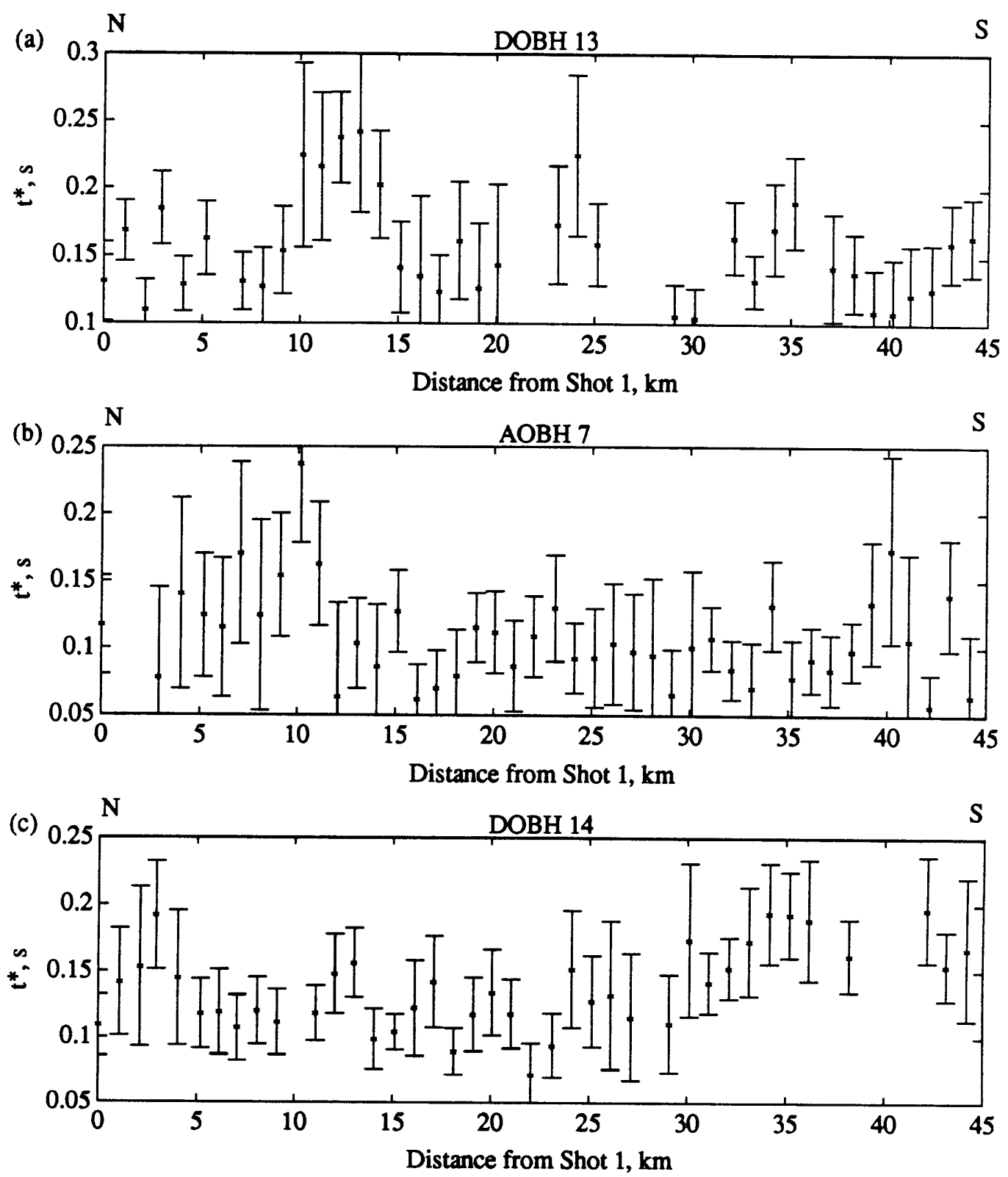

Figure 4.10 


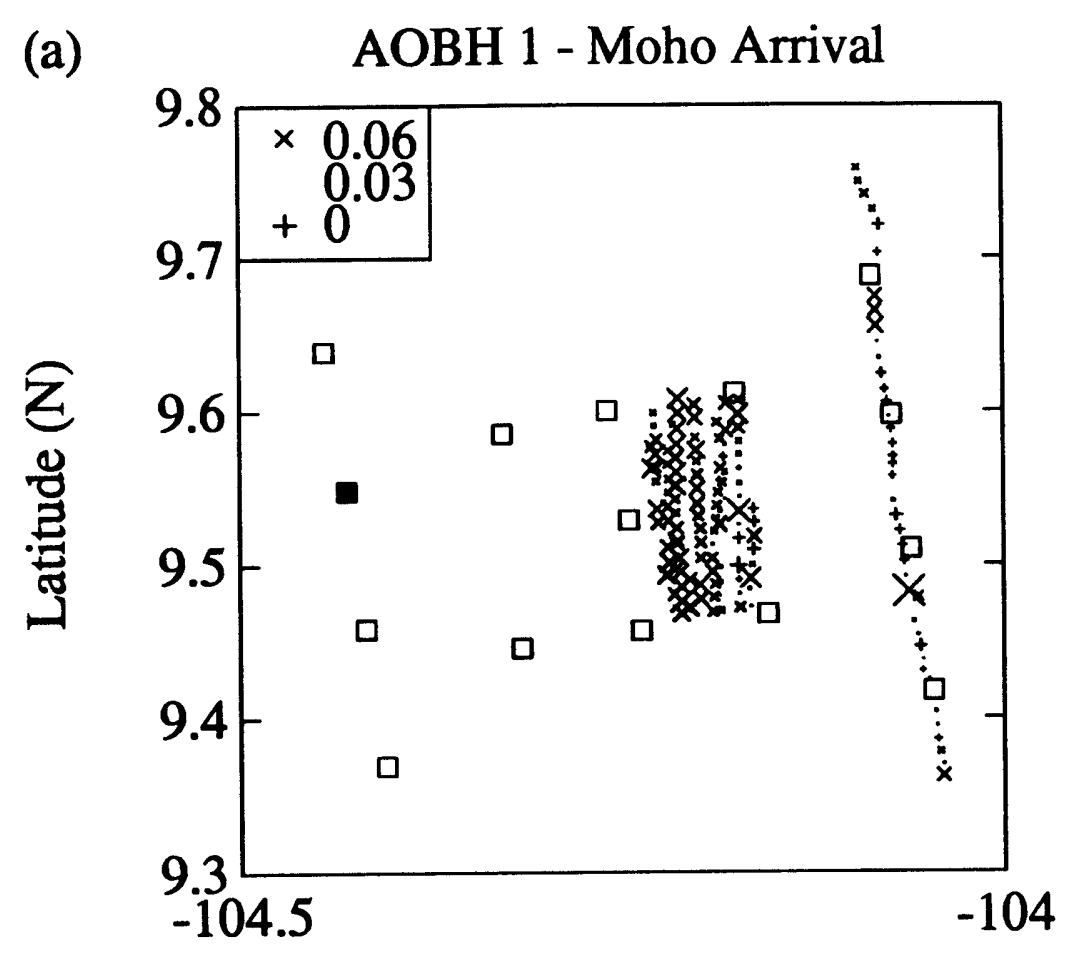

(b) DOBH 12 - Moho Arrival

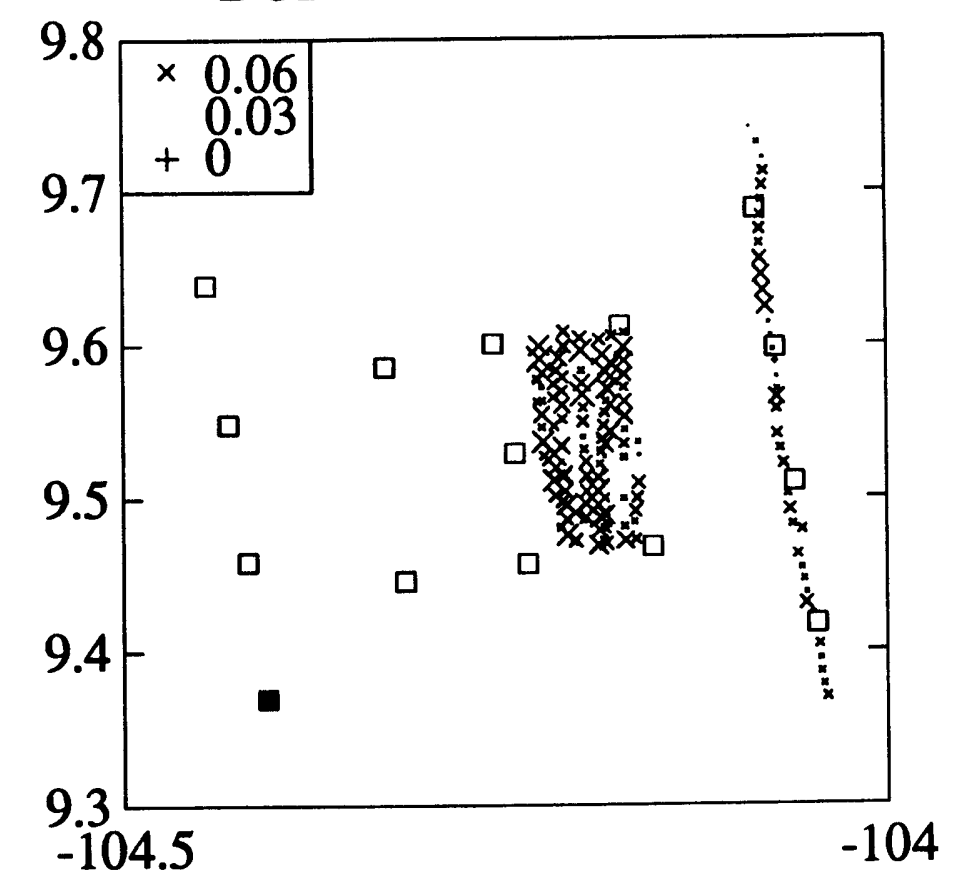

Longitude (W) 
(a)
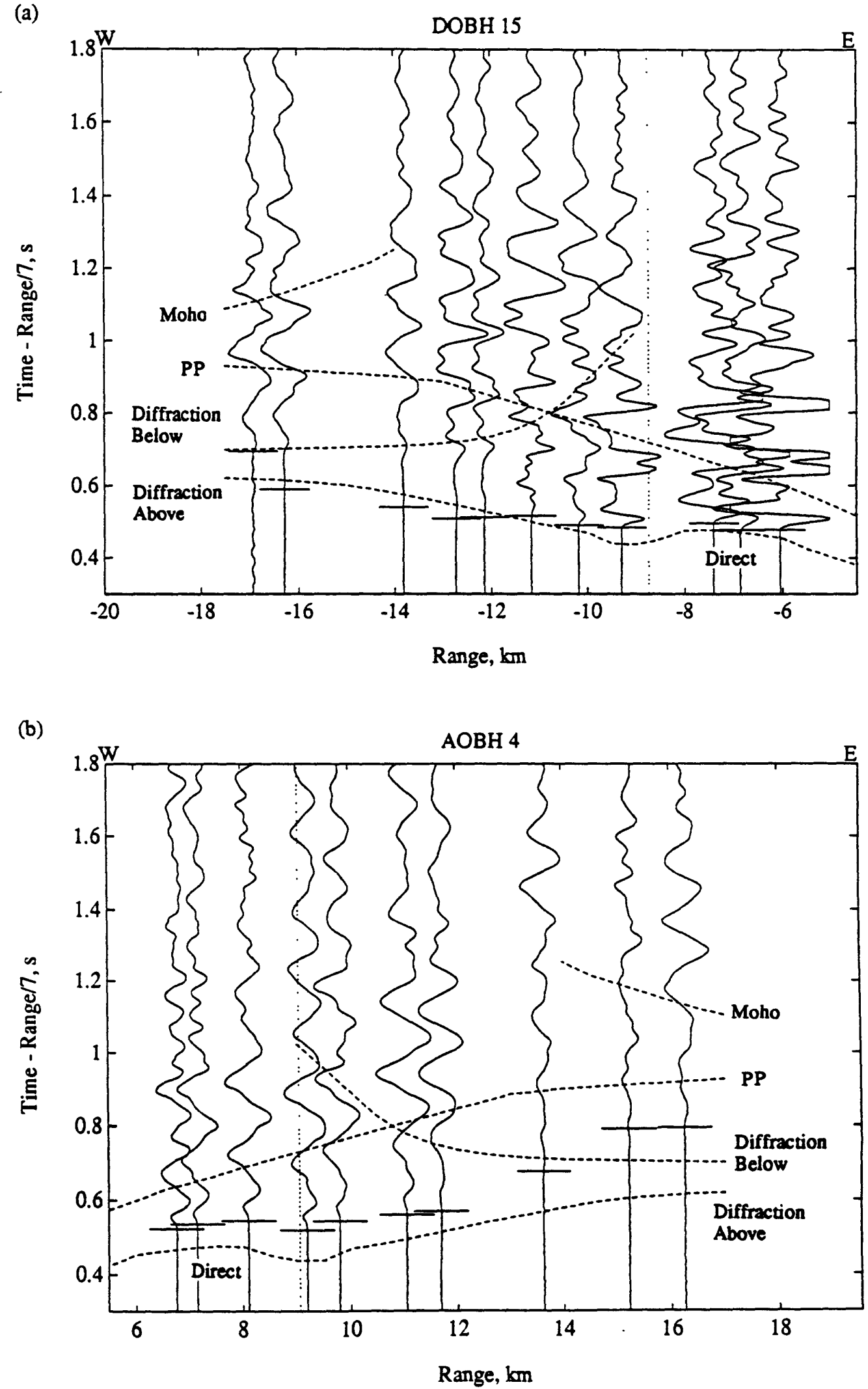

Figure 4.12 
(a)

DOBH 15

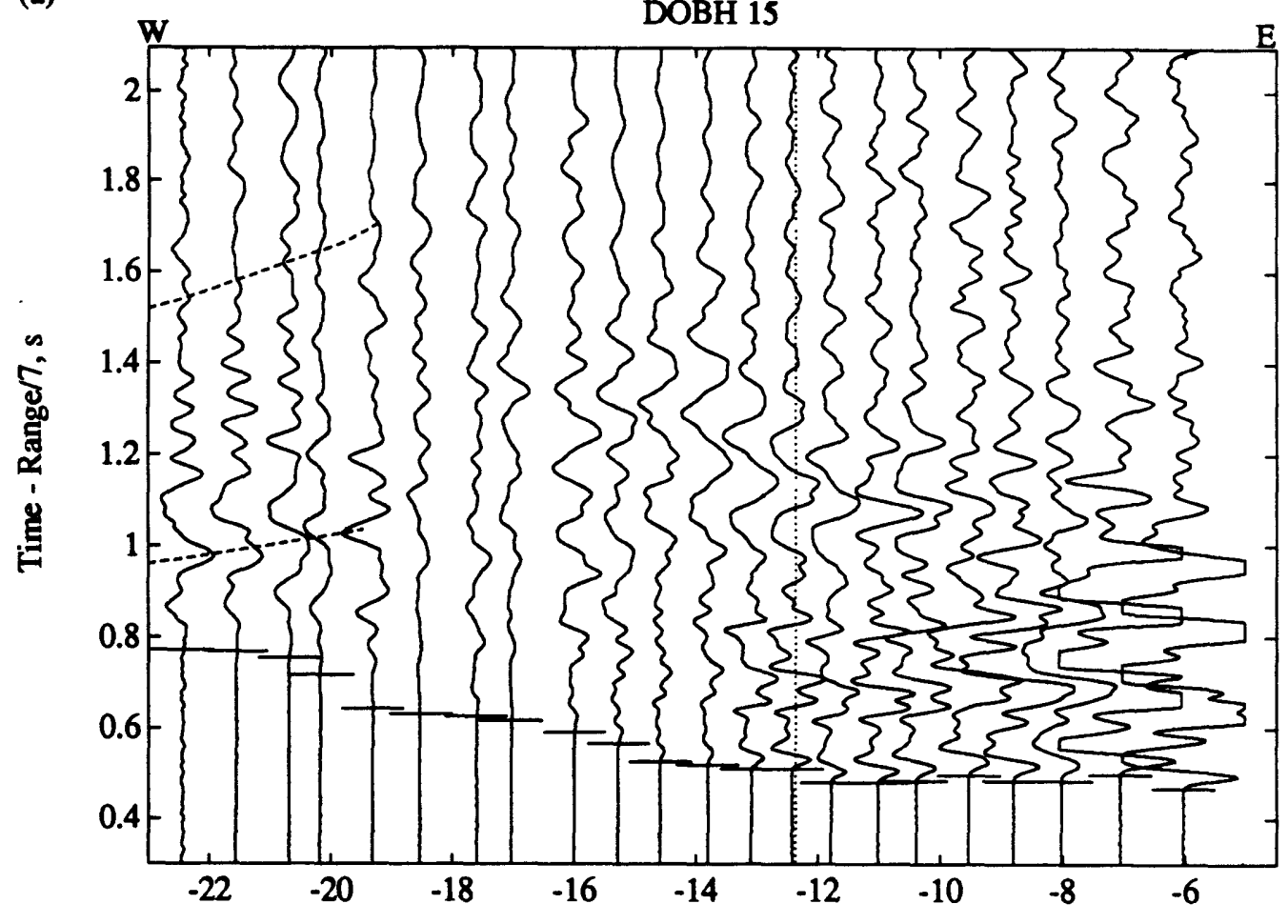

Range, km

(b)

AOBH 4

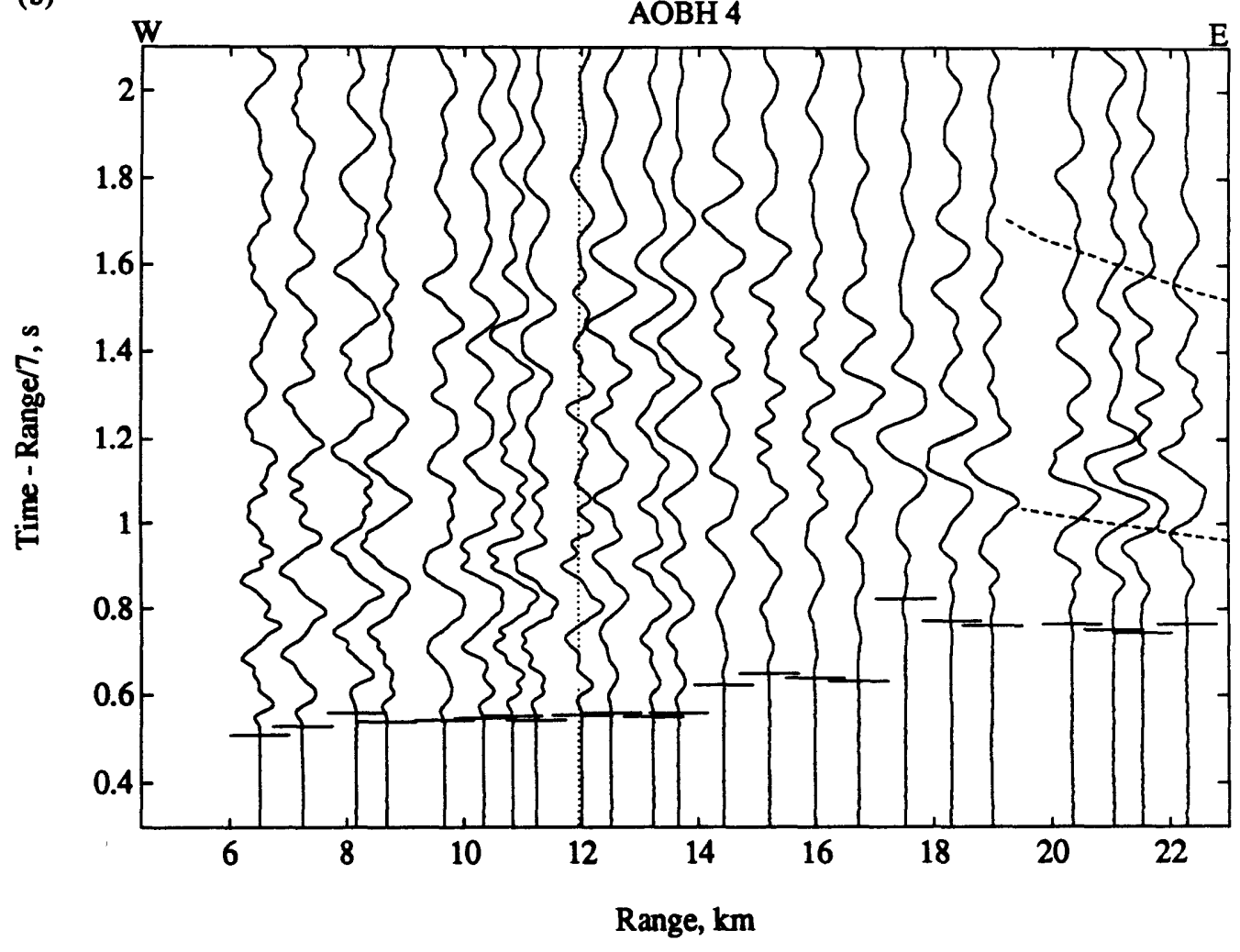

Figure 4.13 

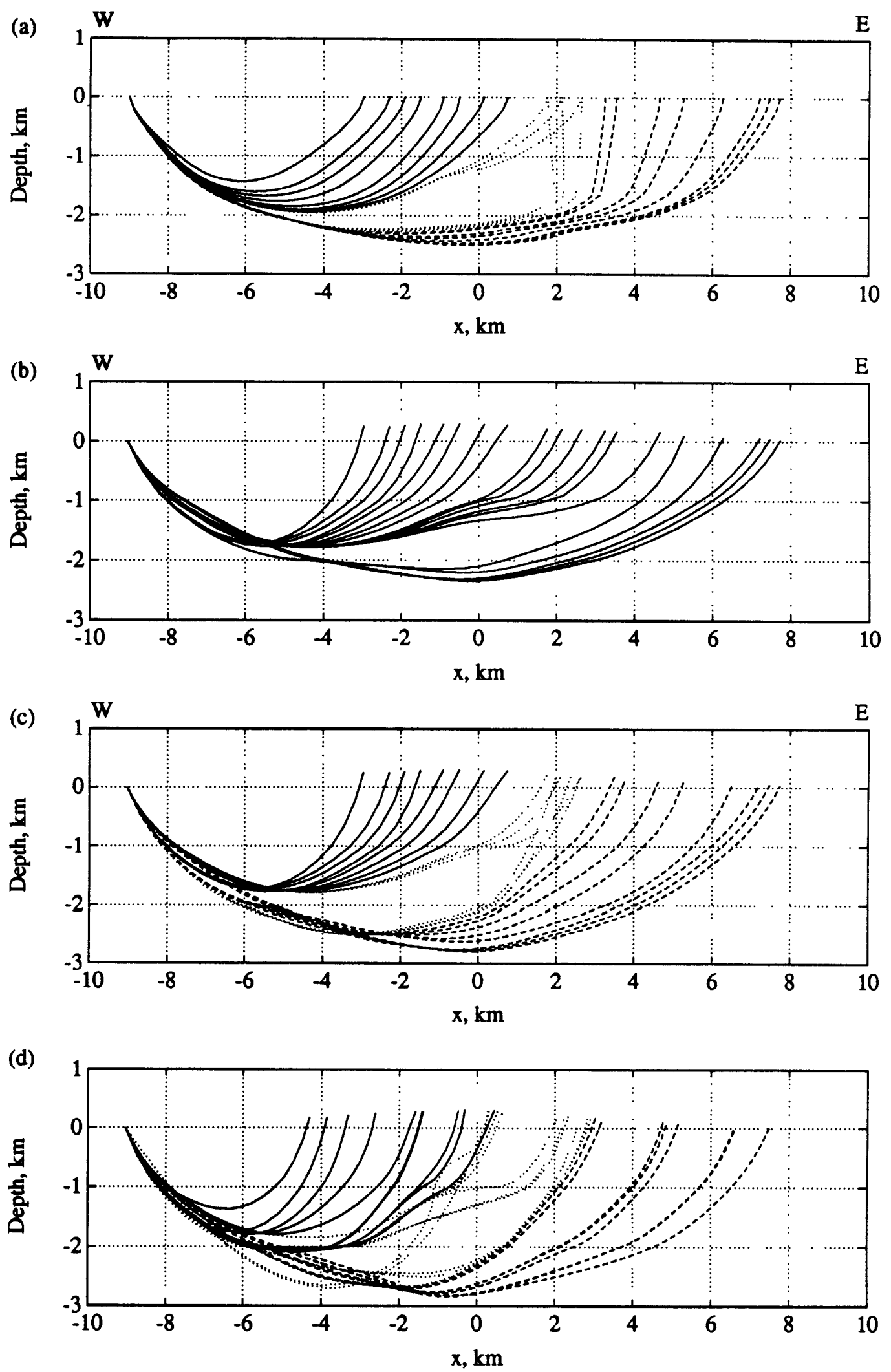

Figure 4.14 


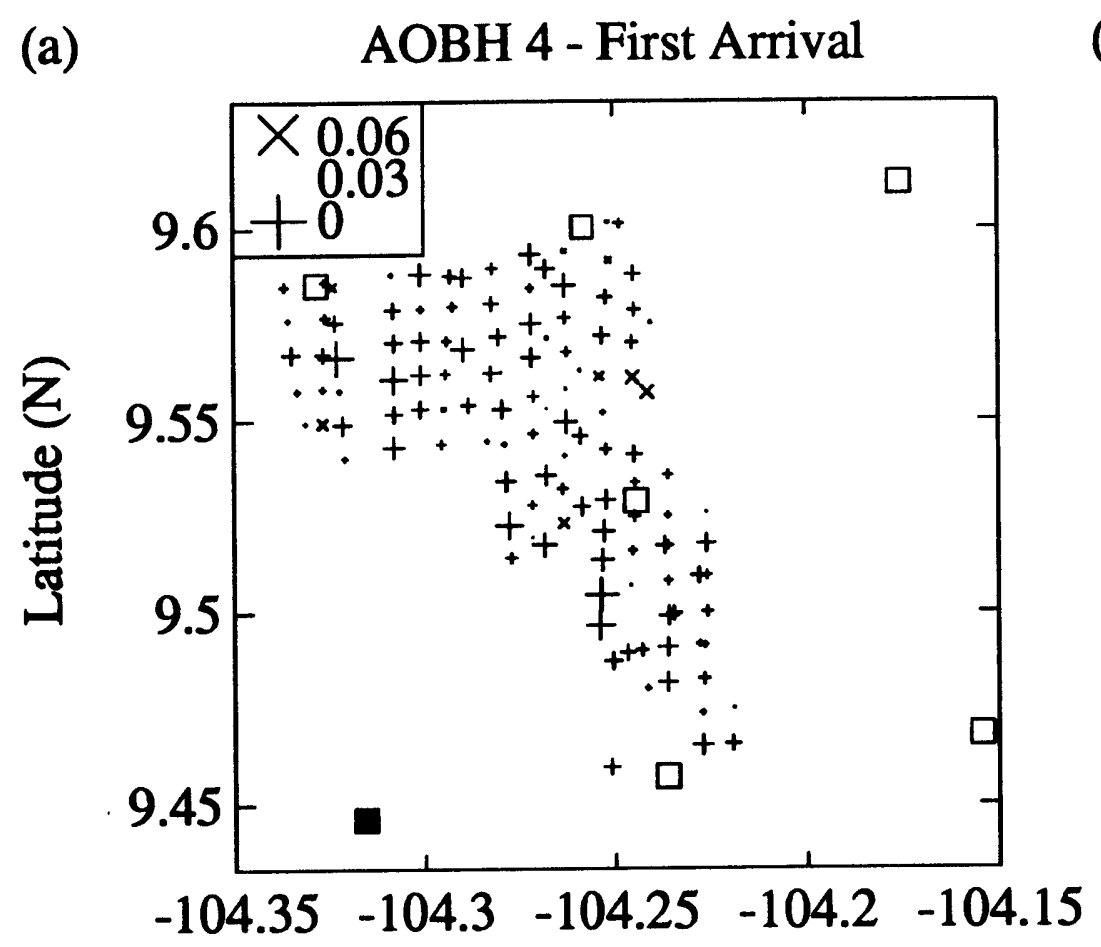

(b)

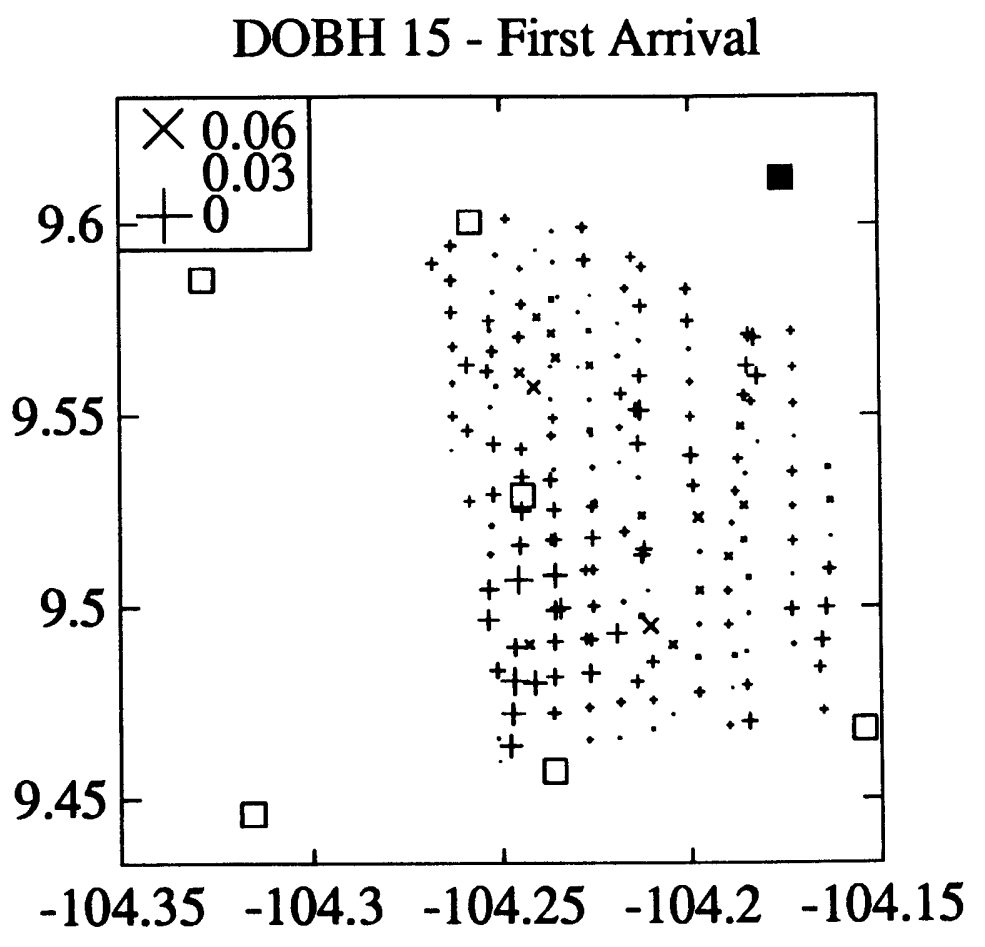

\section{Longitude (W)}


(a) AOBH 4 - Magama Chamber Diffraction (b) DOBH 15 - Magma Chamber Diffraction
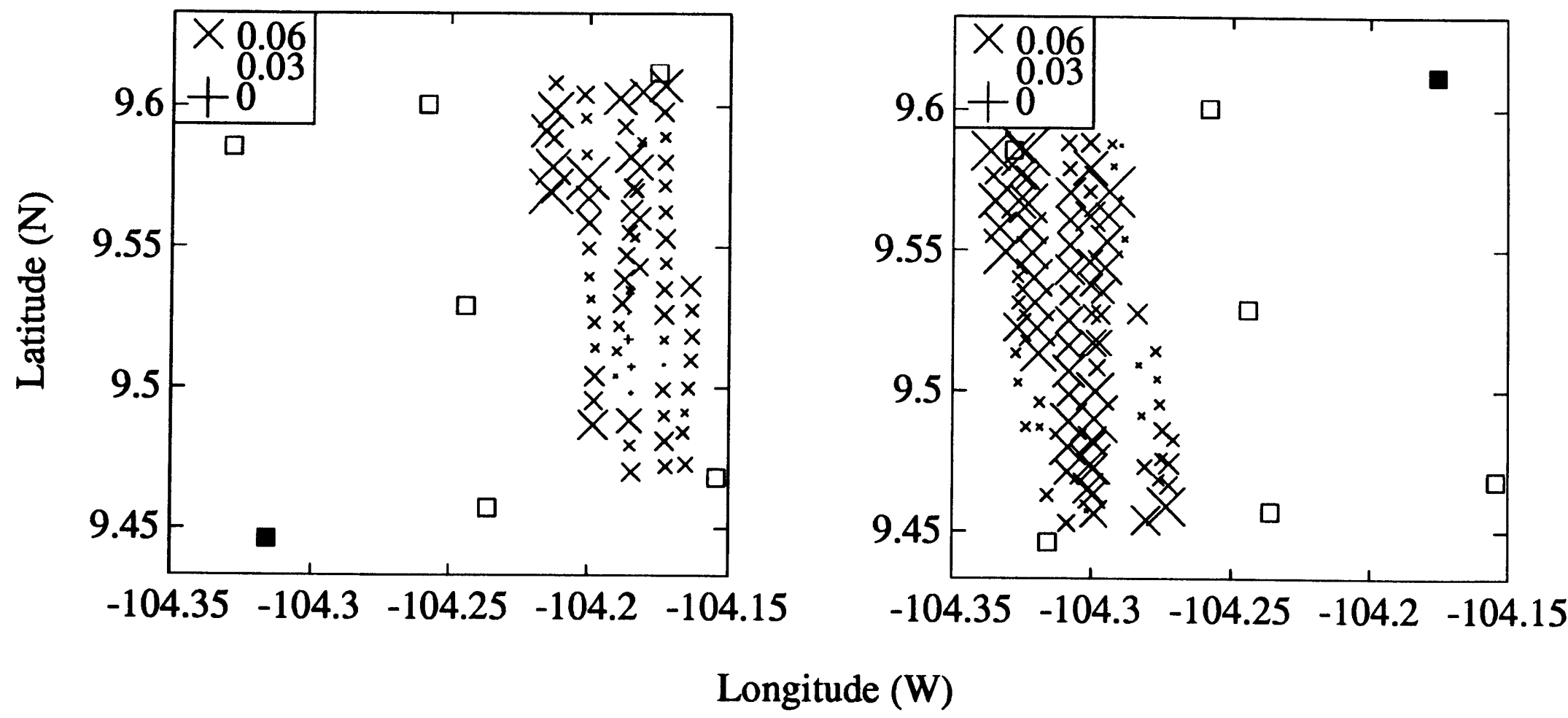
(a)

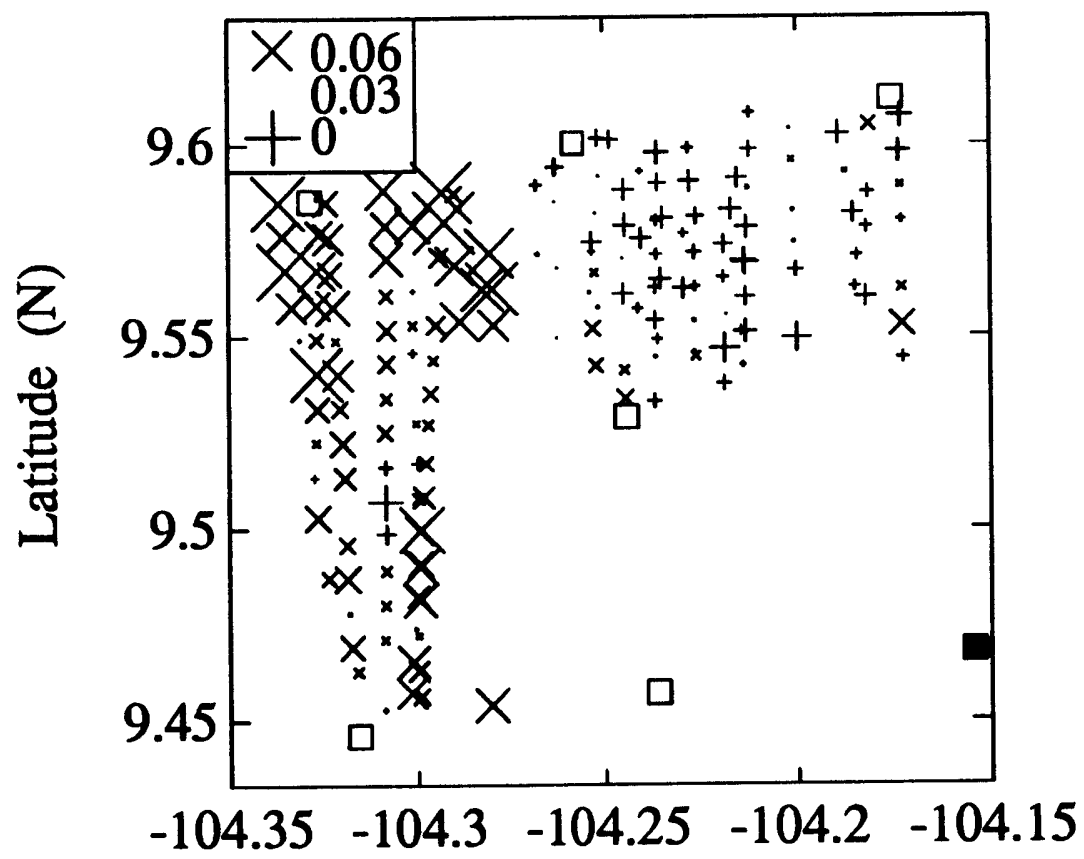

(b)

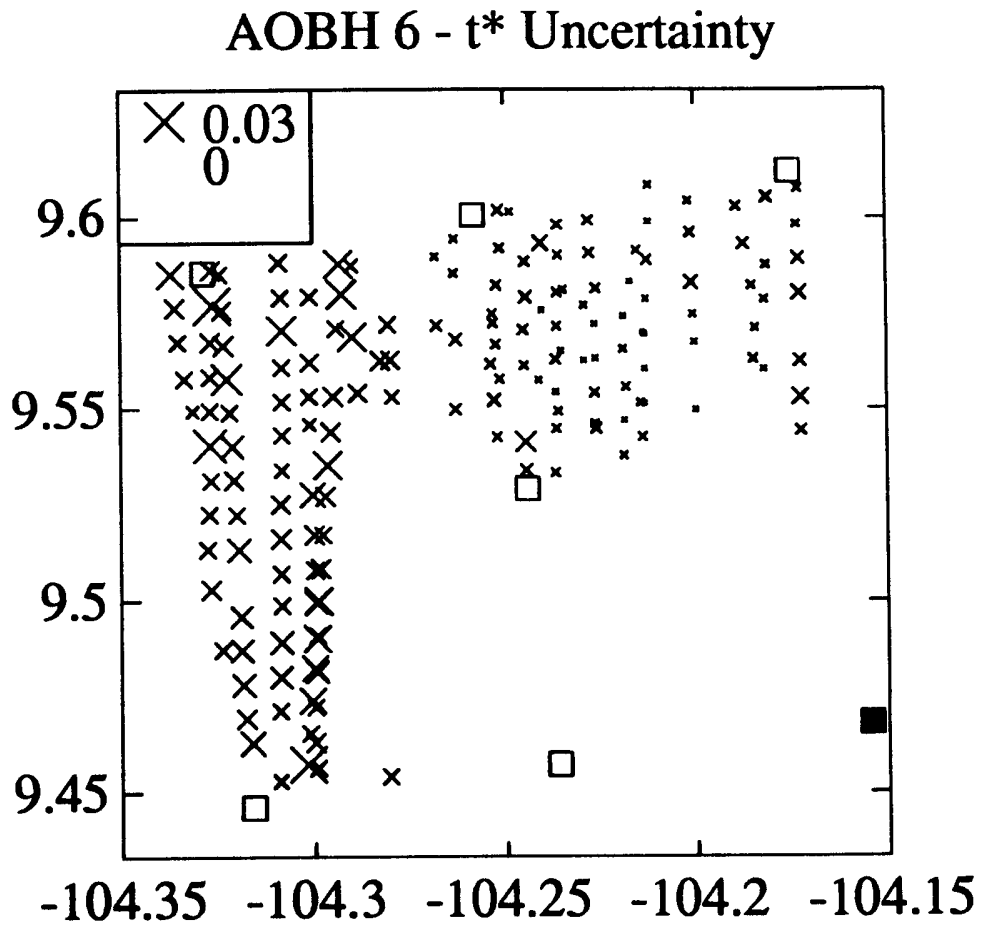


(c) $\quad \mathrm{AOBH} 6$ - Upper Frequency Limit

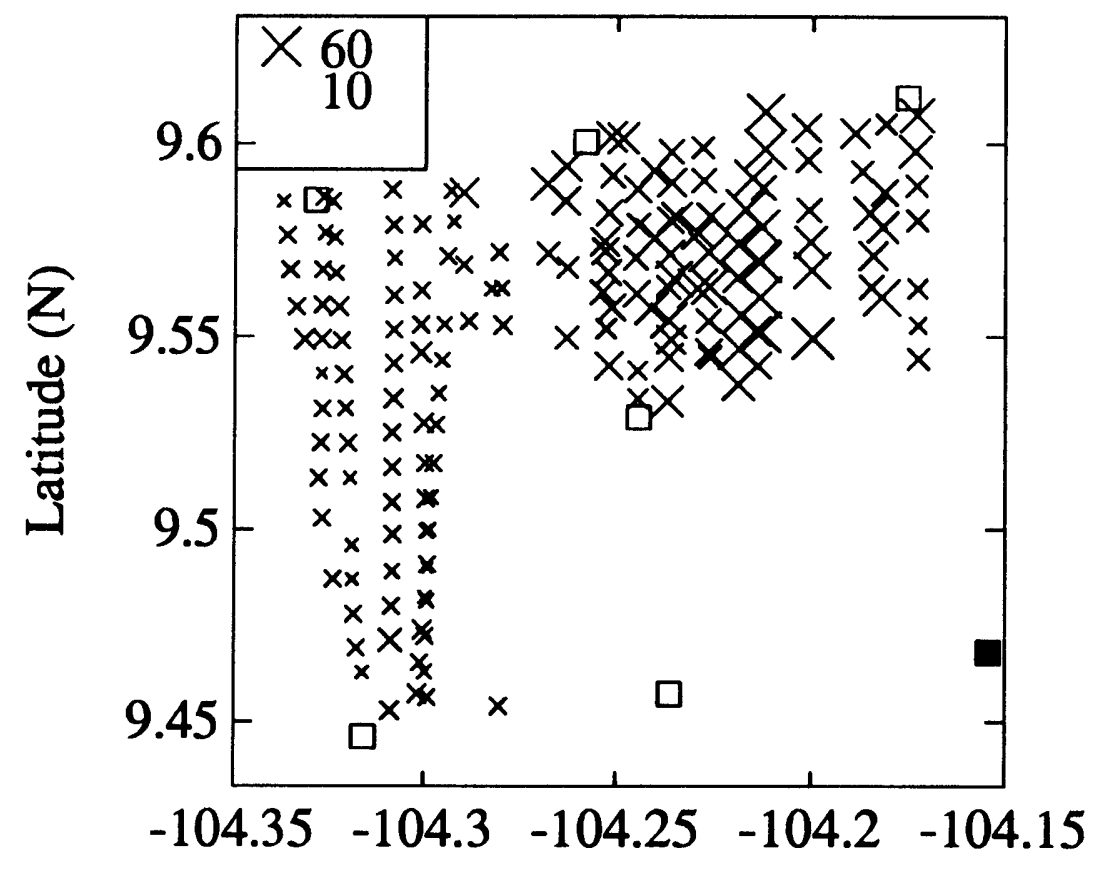

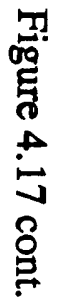

\section{Longitude (W)}



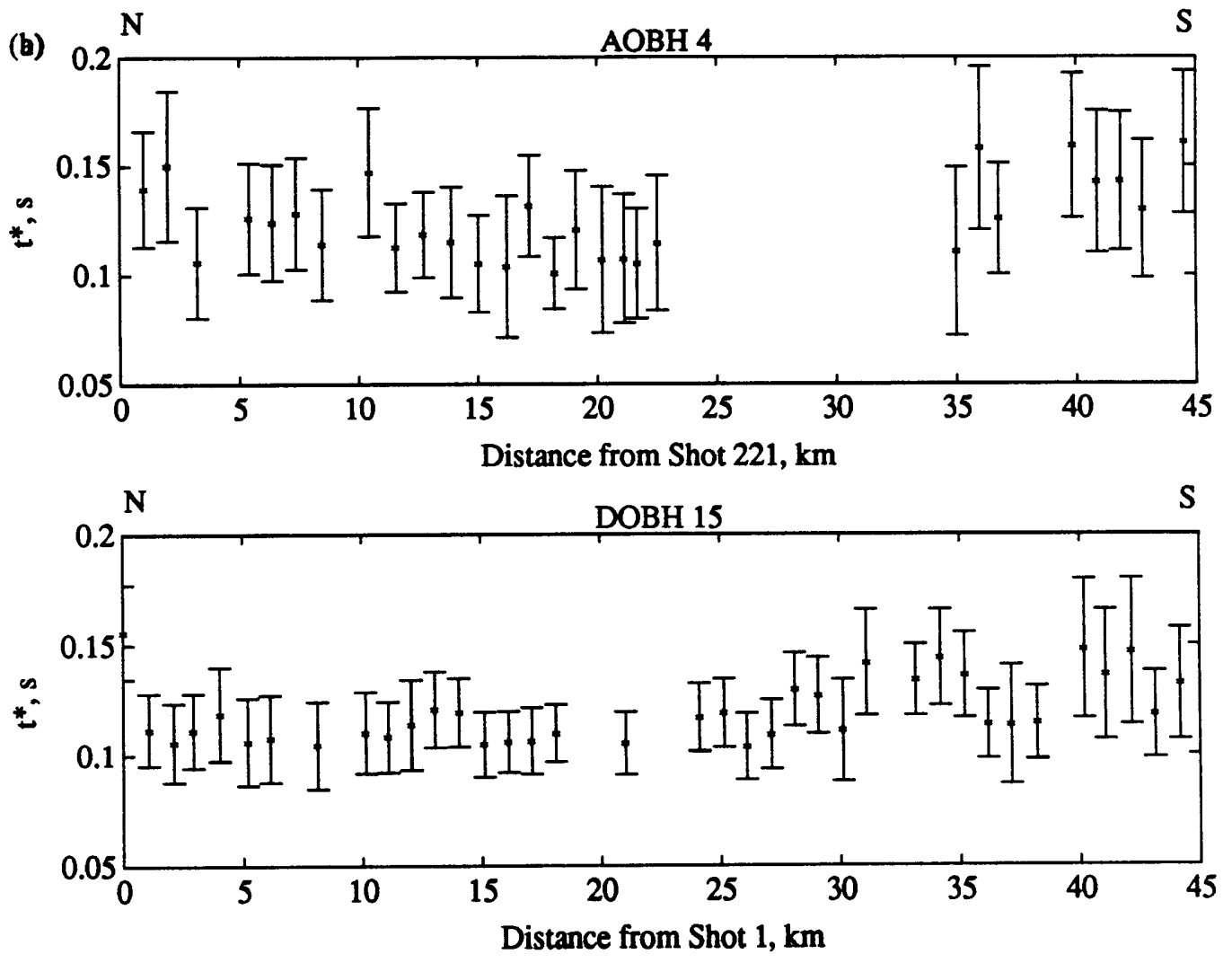

Figure 4.18 

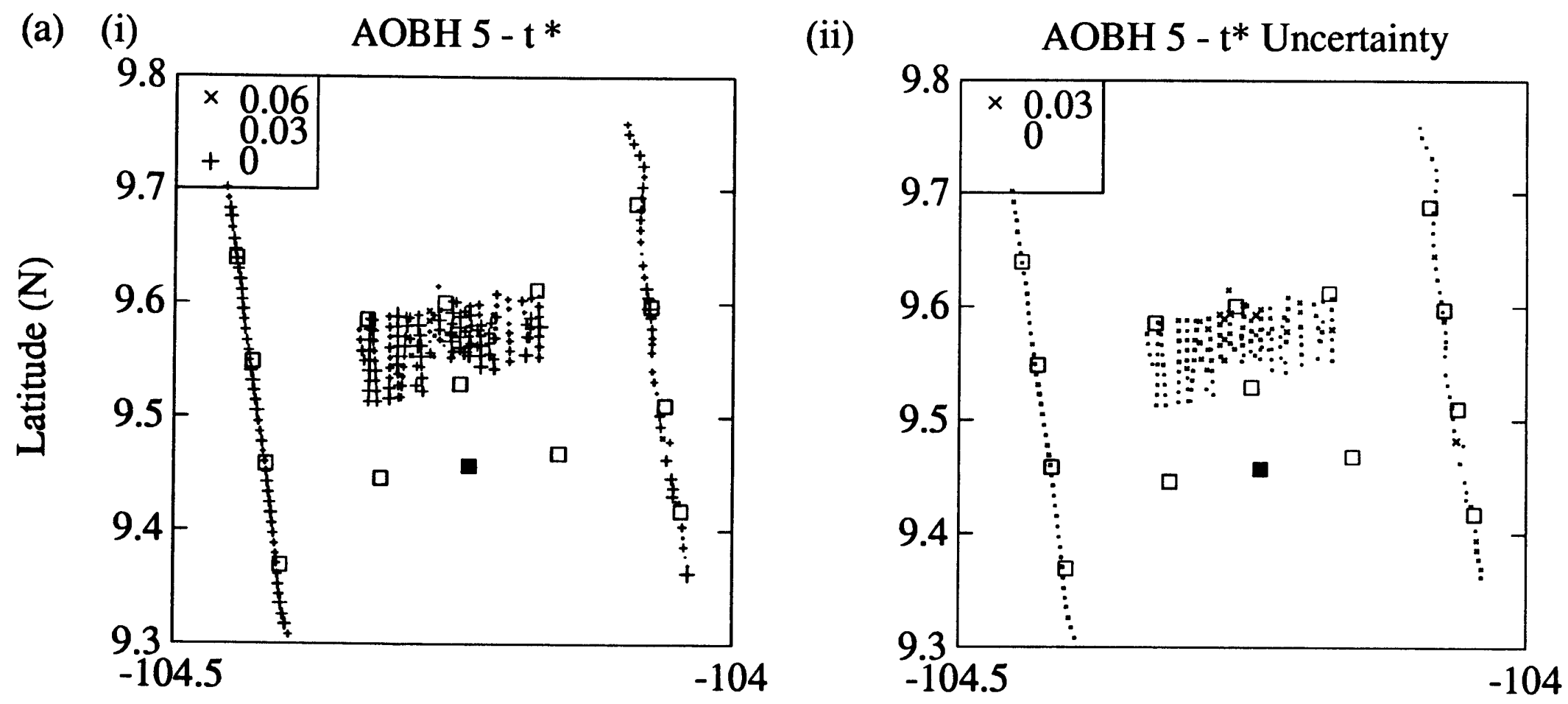

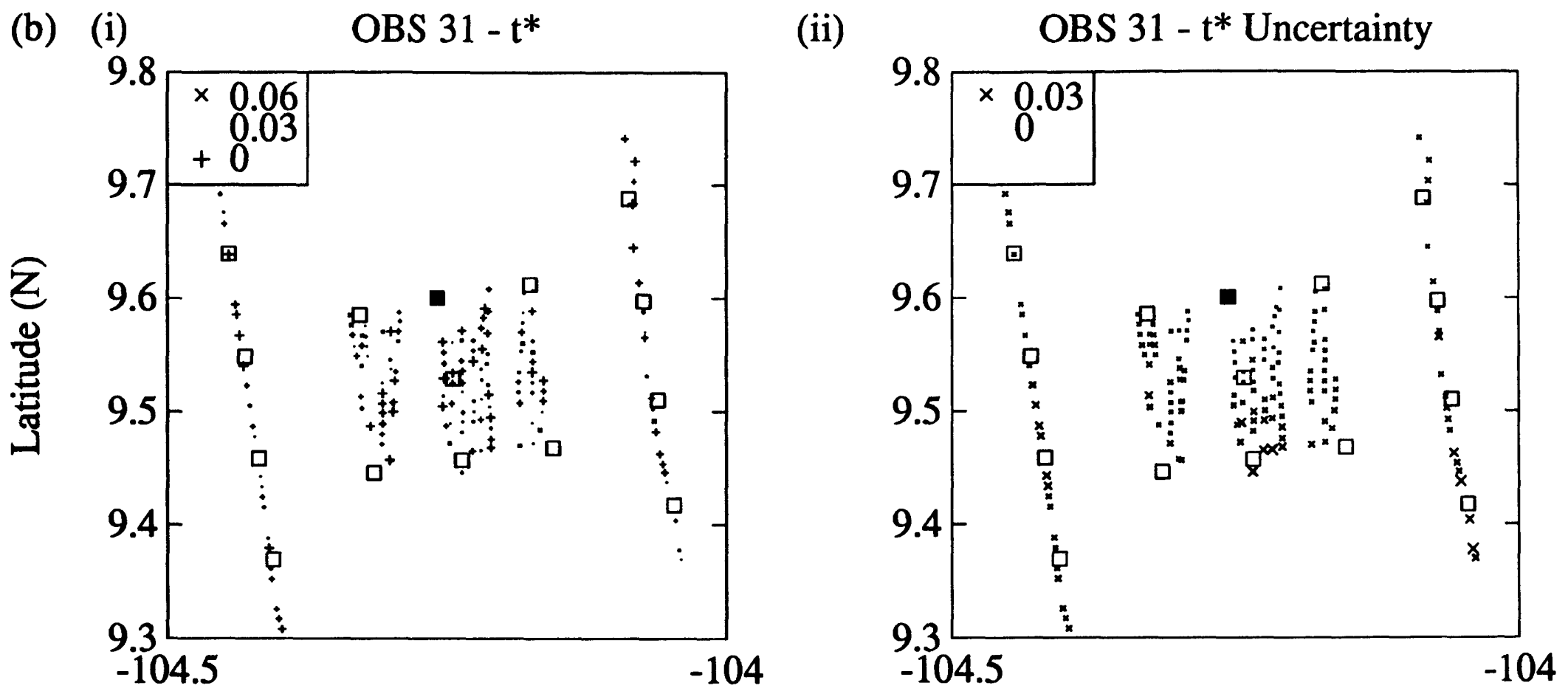

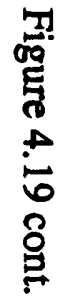

\section{Longitude (W)}



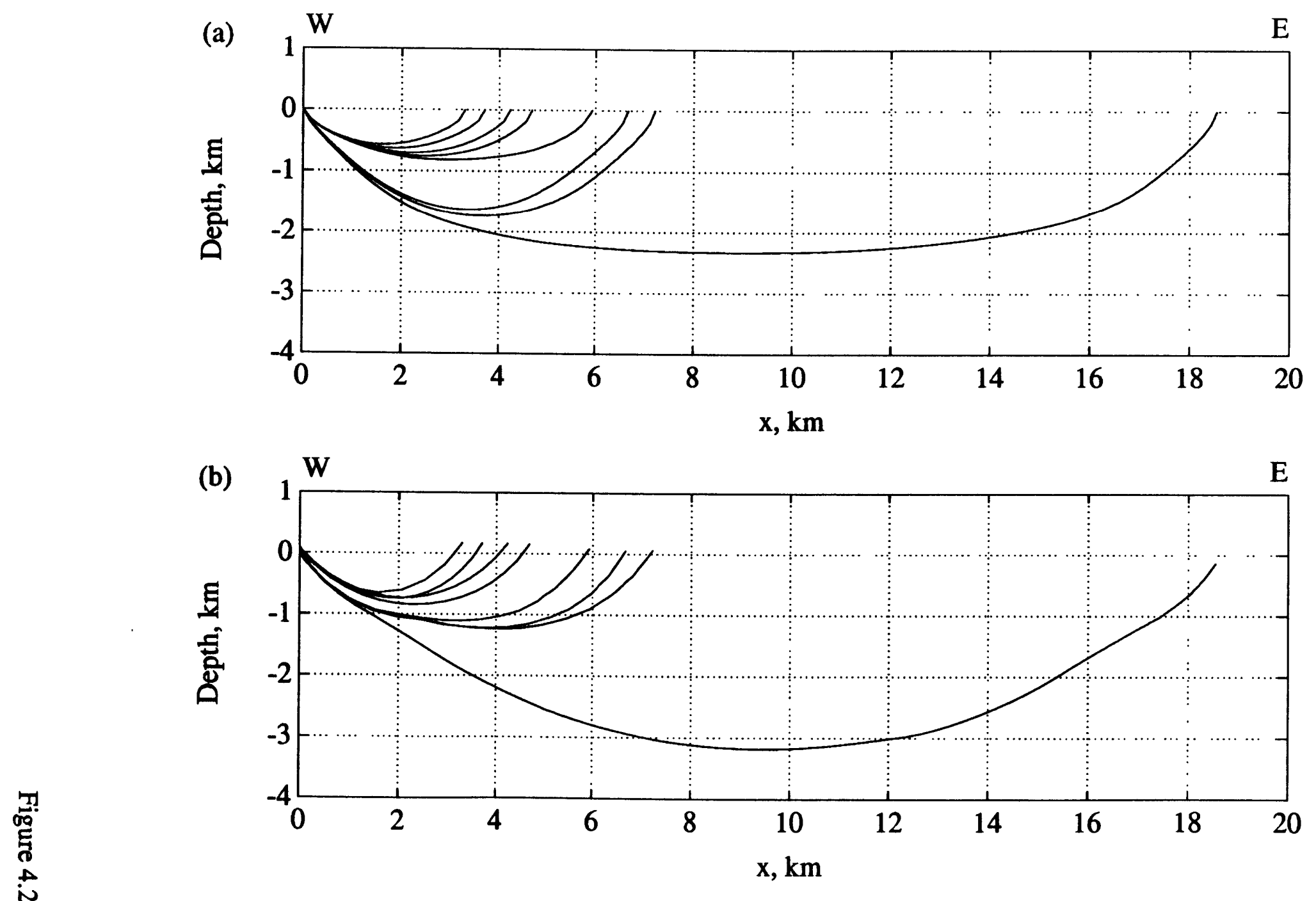
(a)

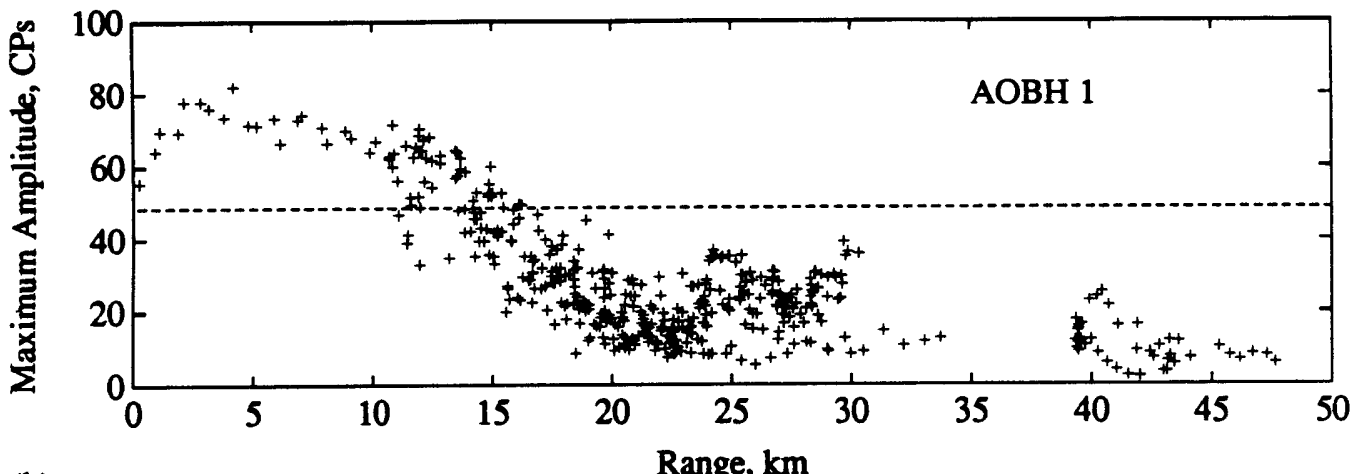

(b)

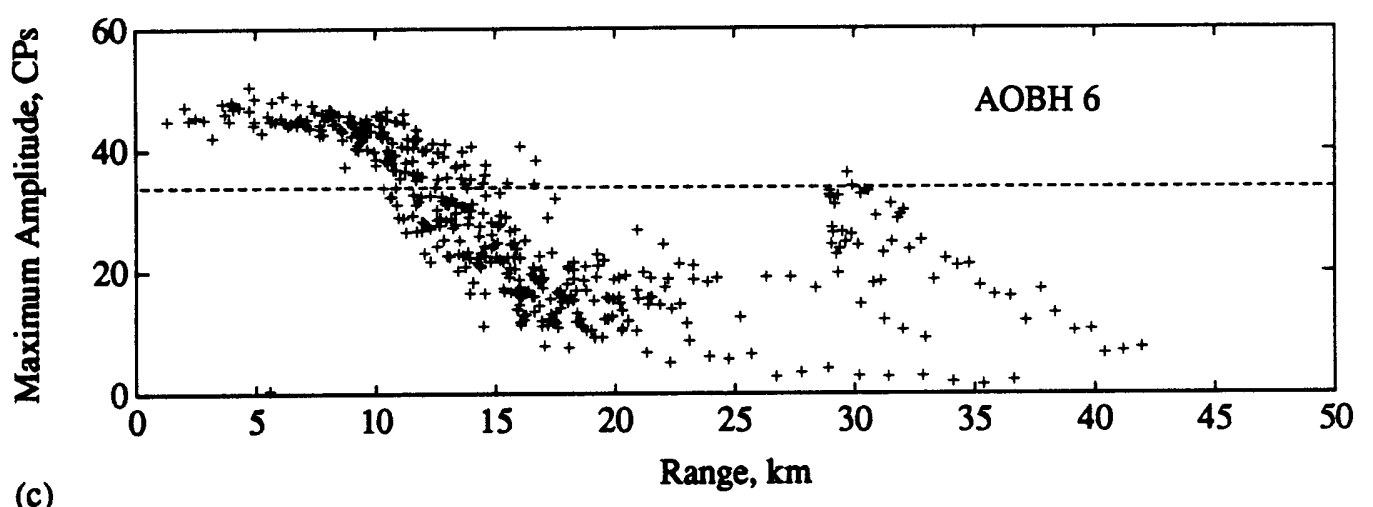

(c)

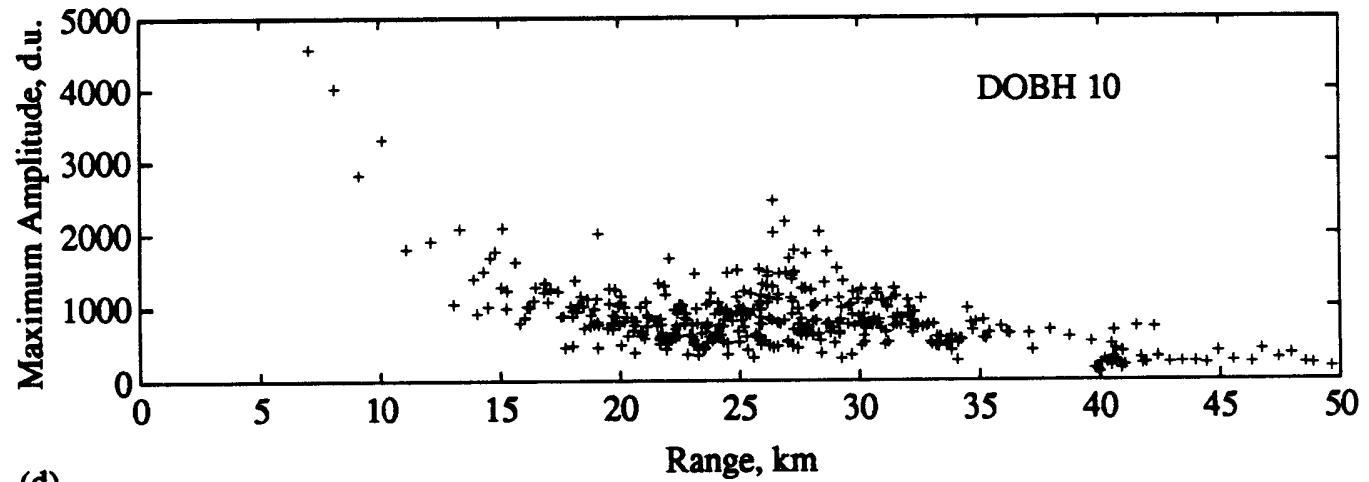

(d)

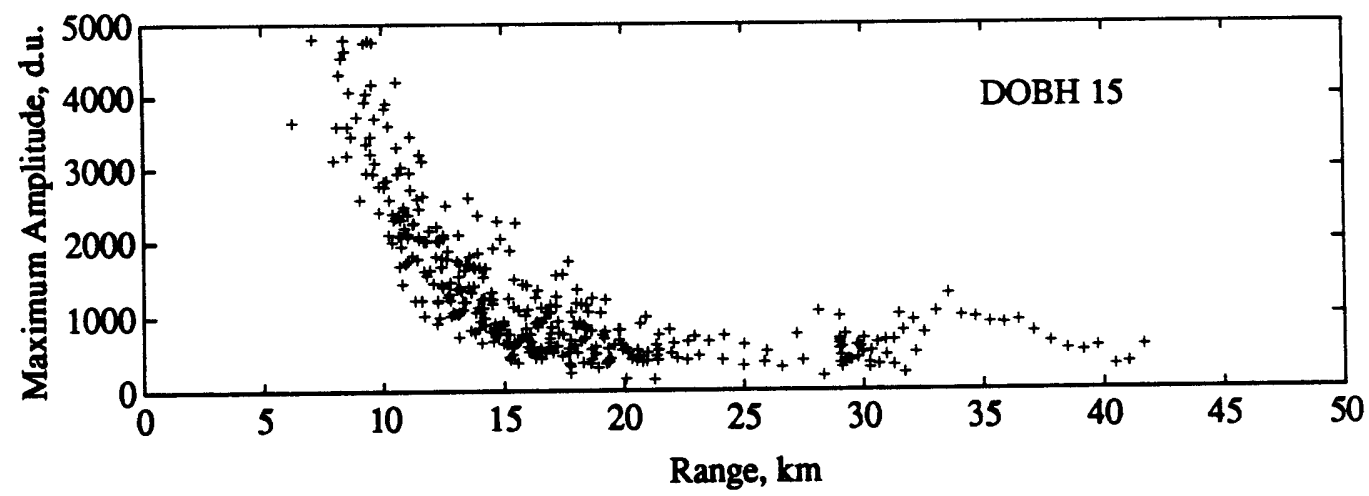

Figure 4.21 


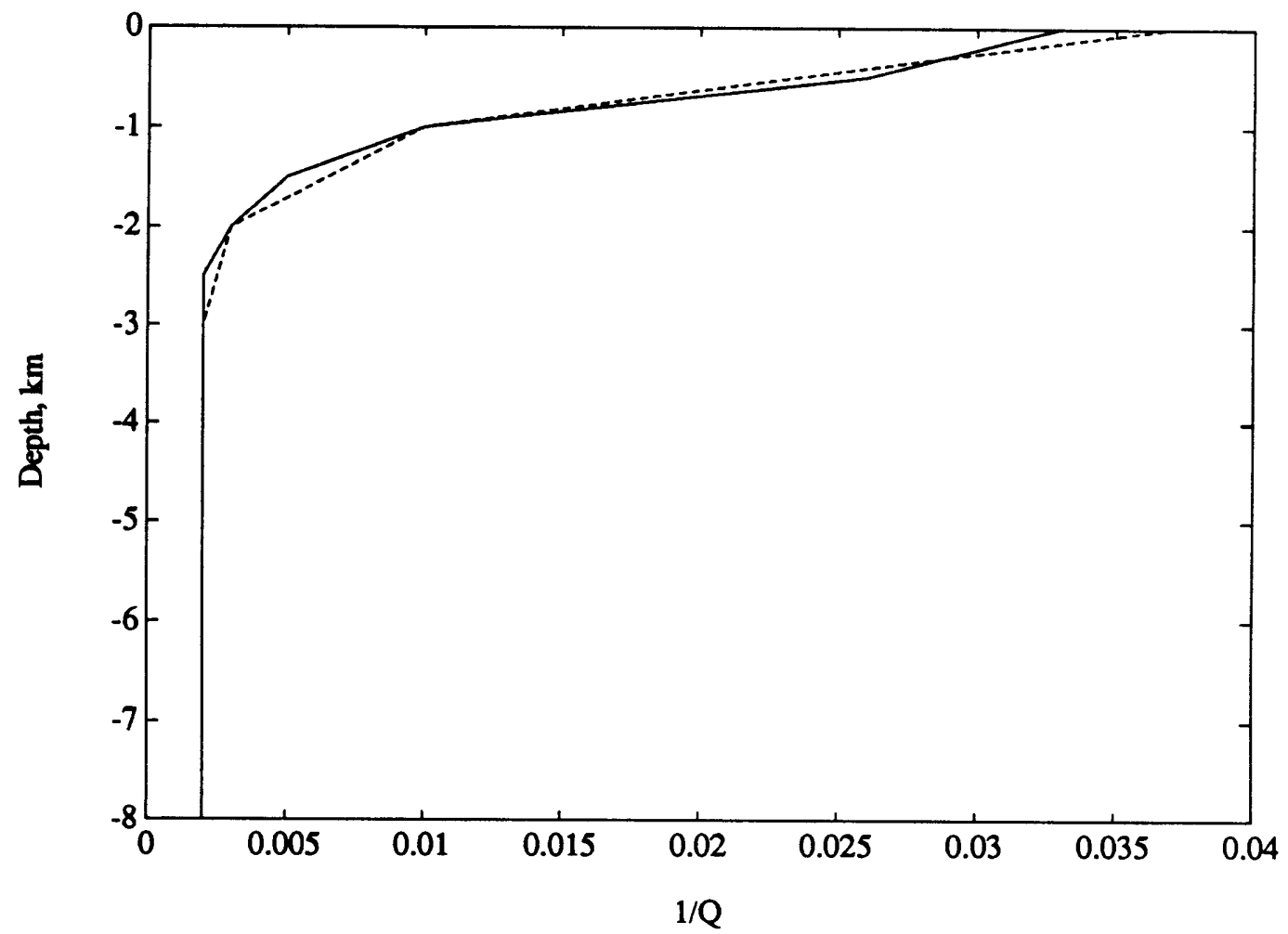

Figure 4.22 


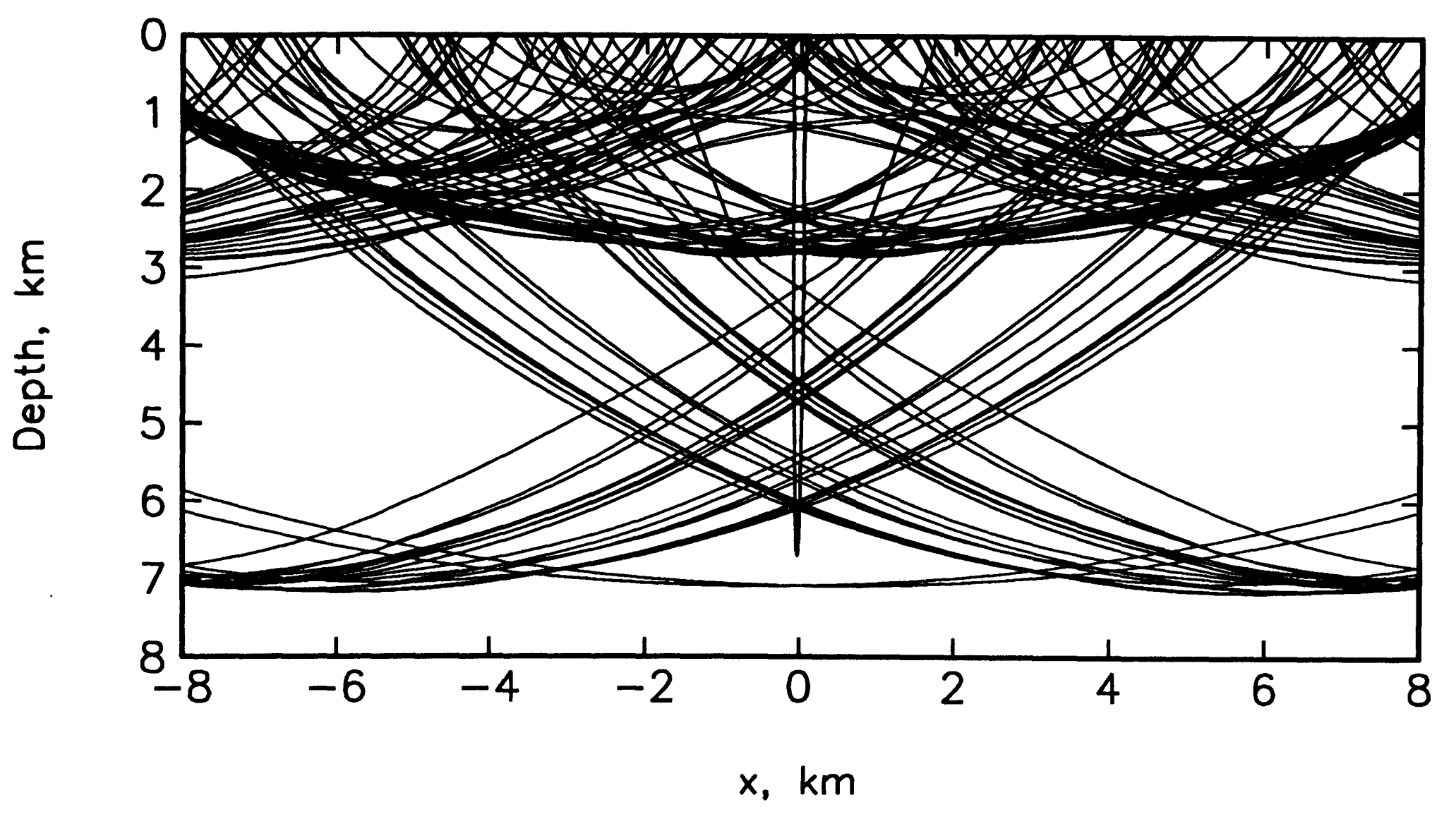




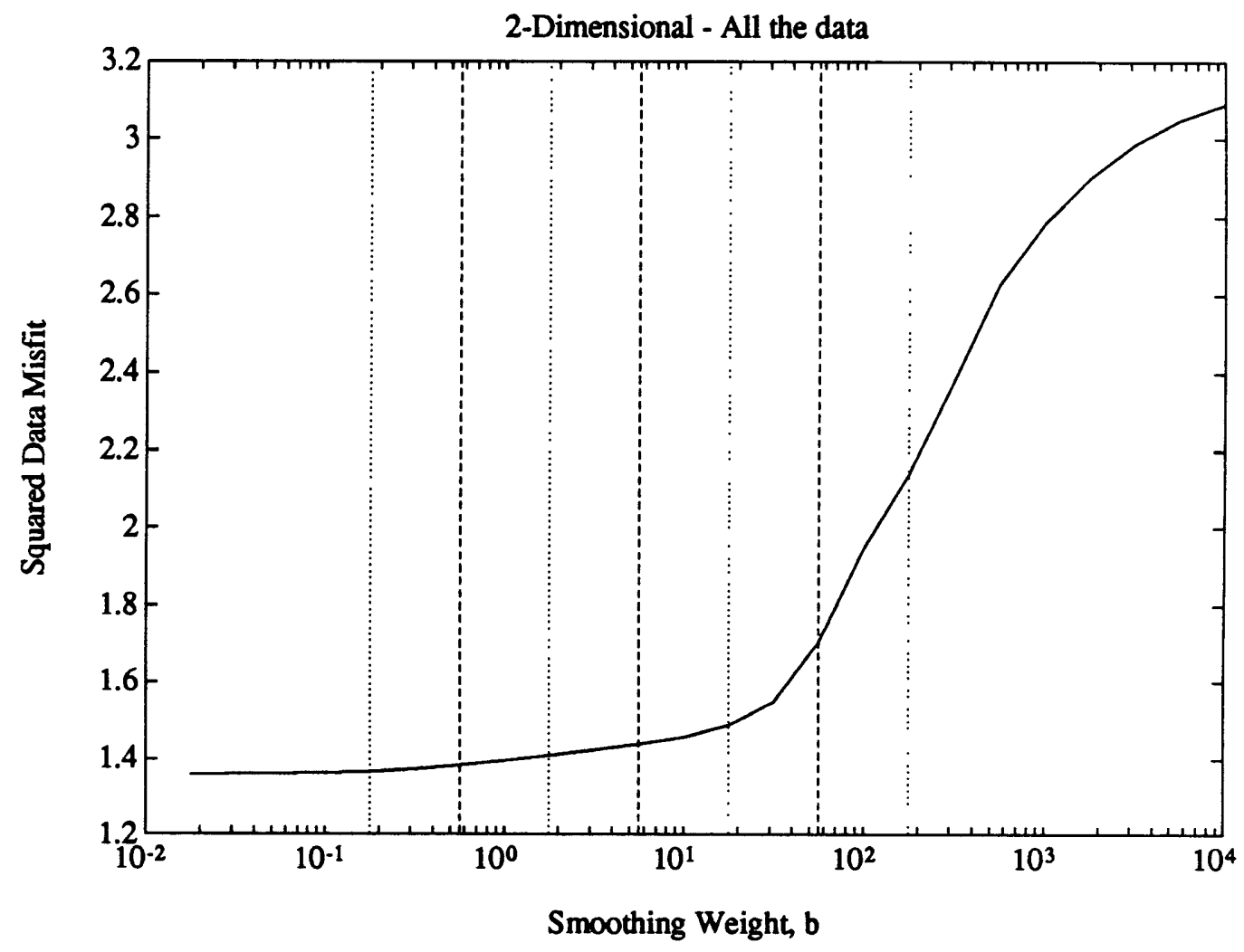

Figure 4.24 


$$
1 / 0 \quad b=180
$$
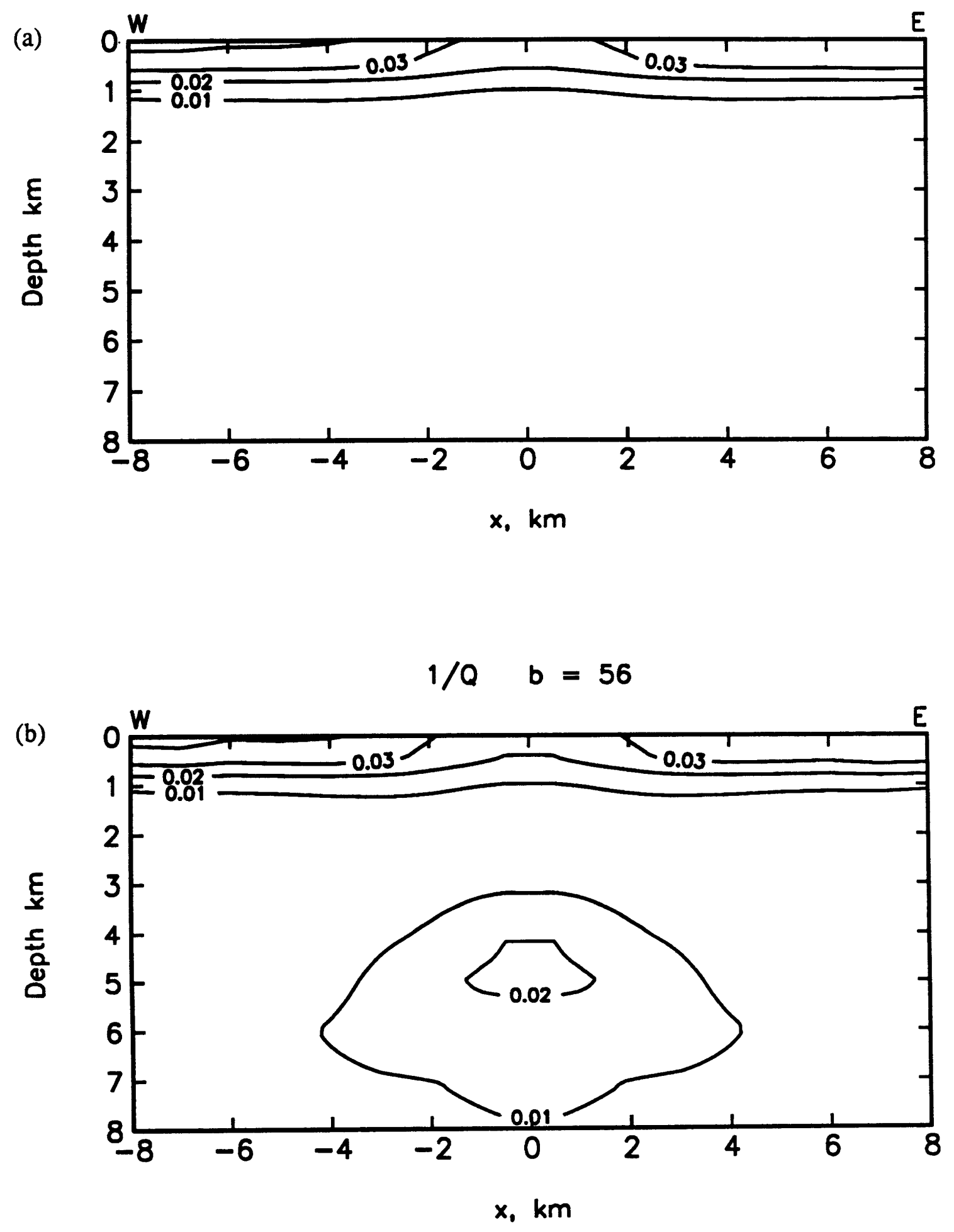

Figure 4.25 

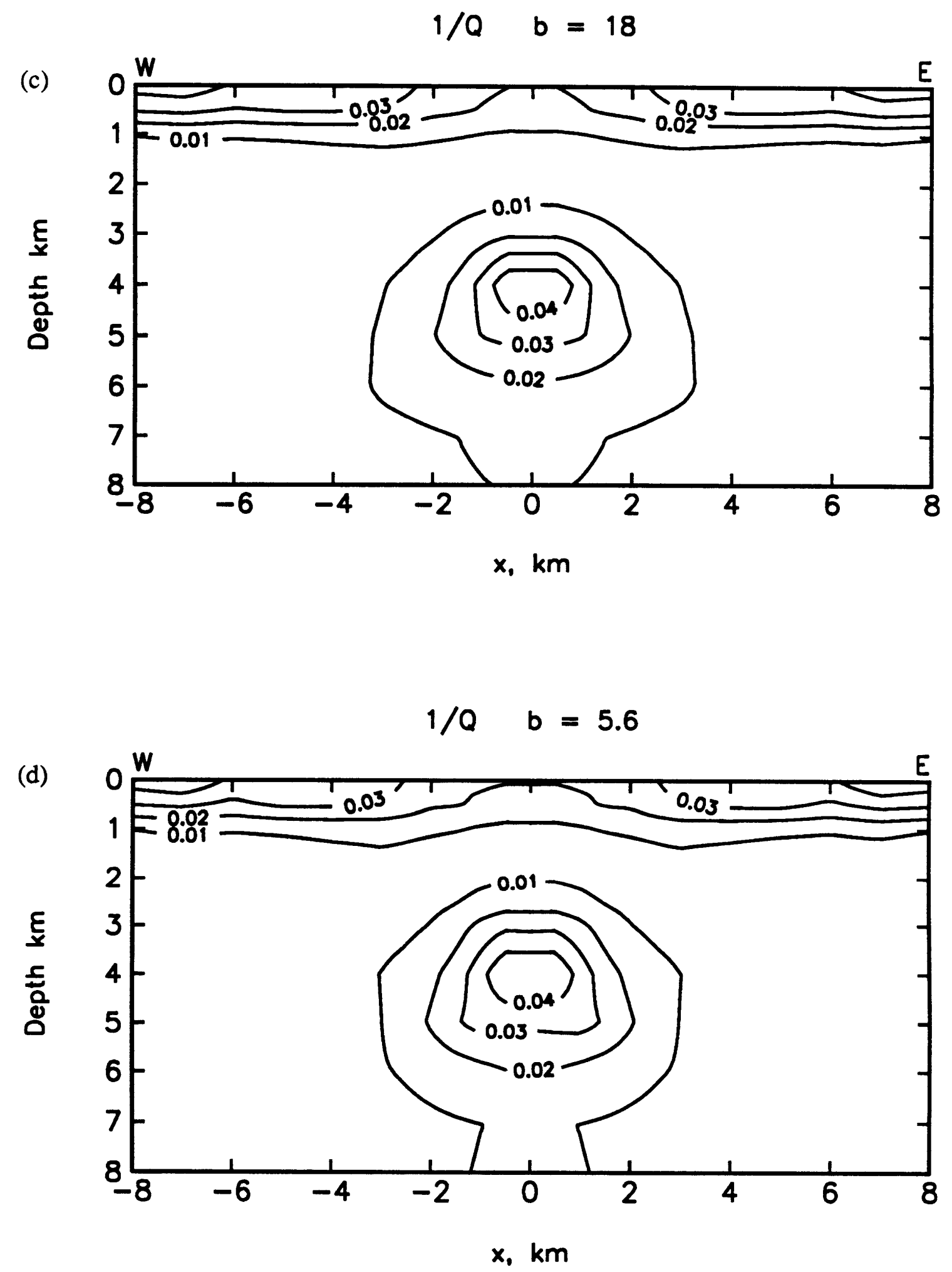

Figure 4.25 cont. 

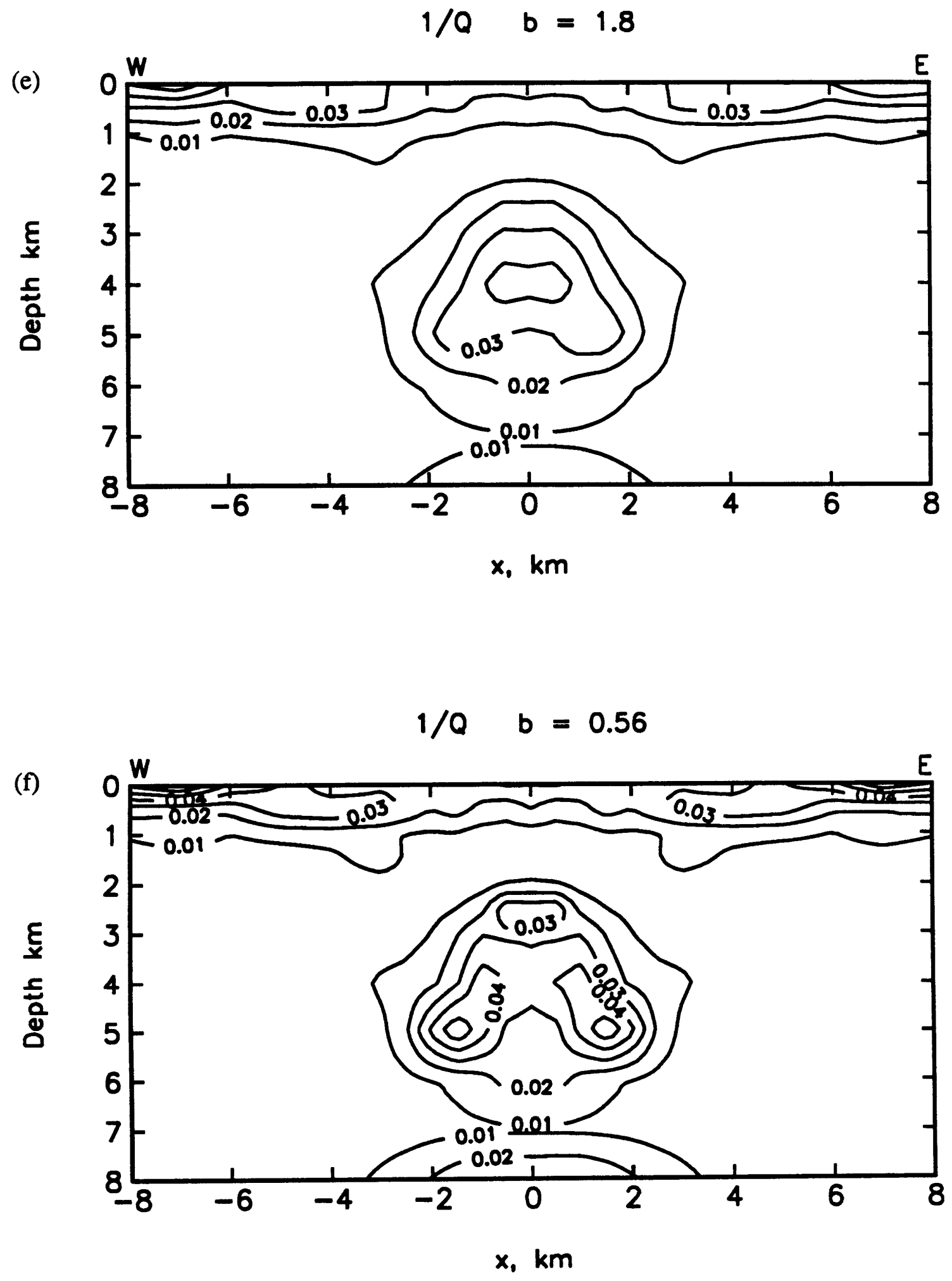

Figure 4.25 cont. 


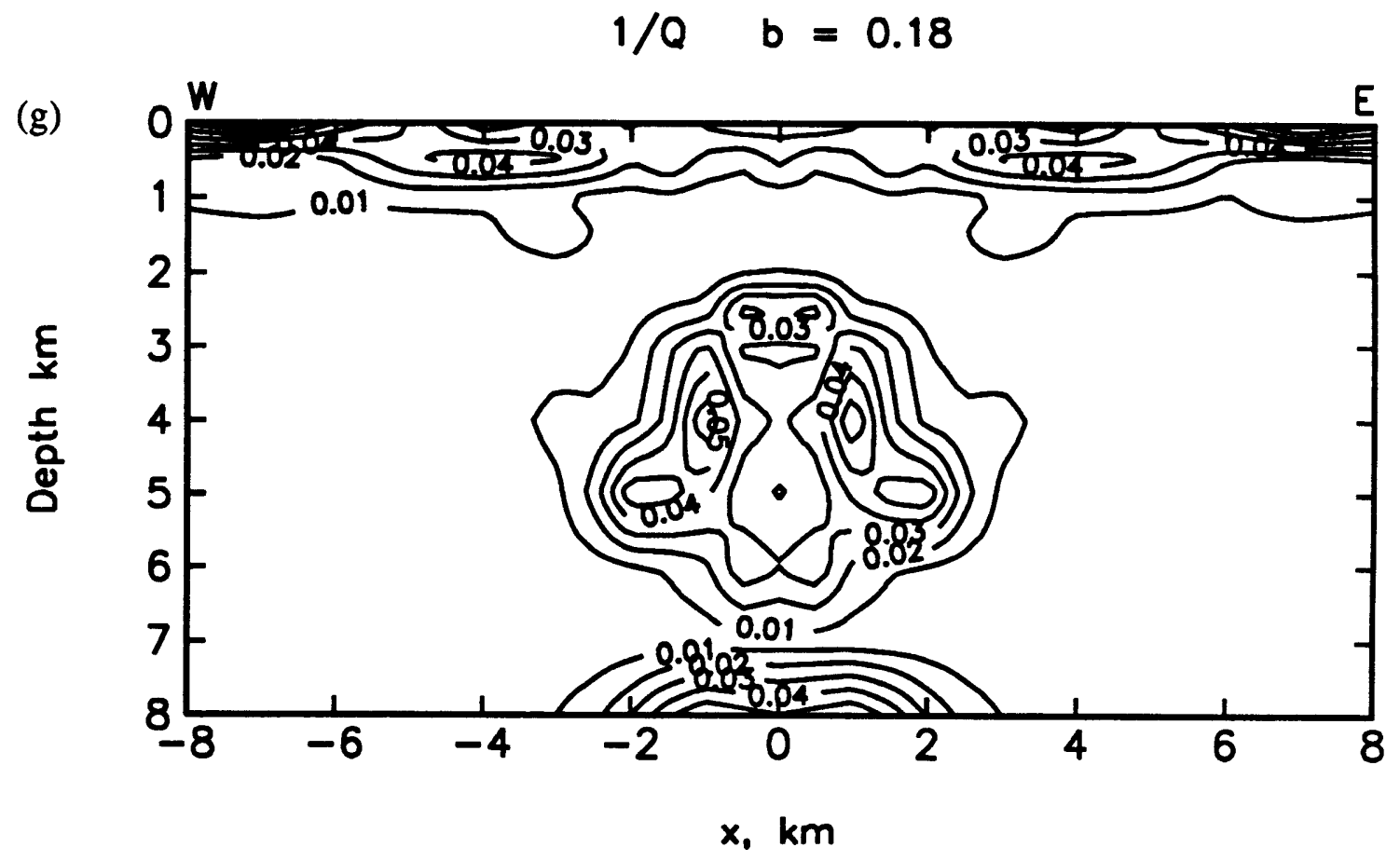

Figure 4.25 cont. 
$1 / Q$ Uncertainty $b=56$

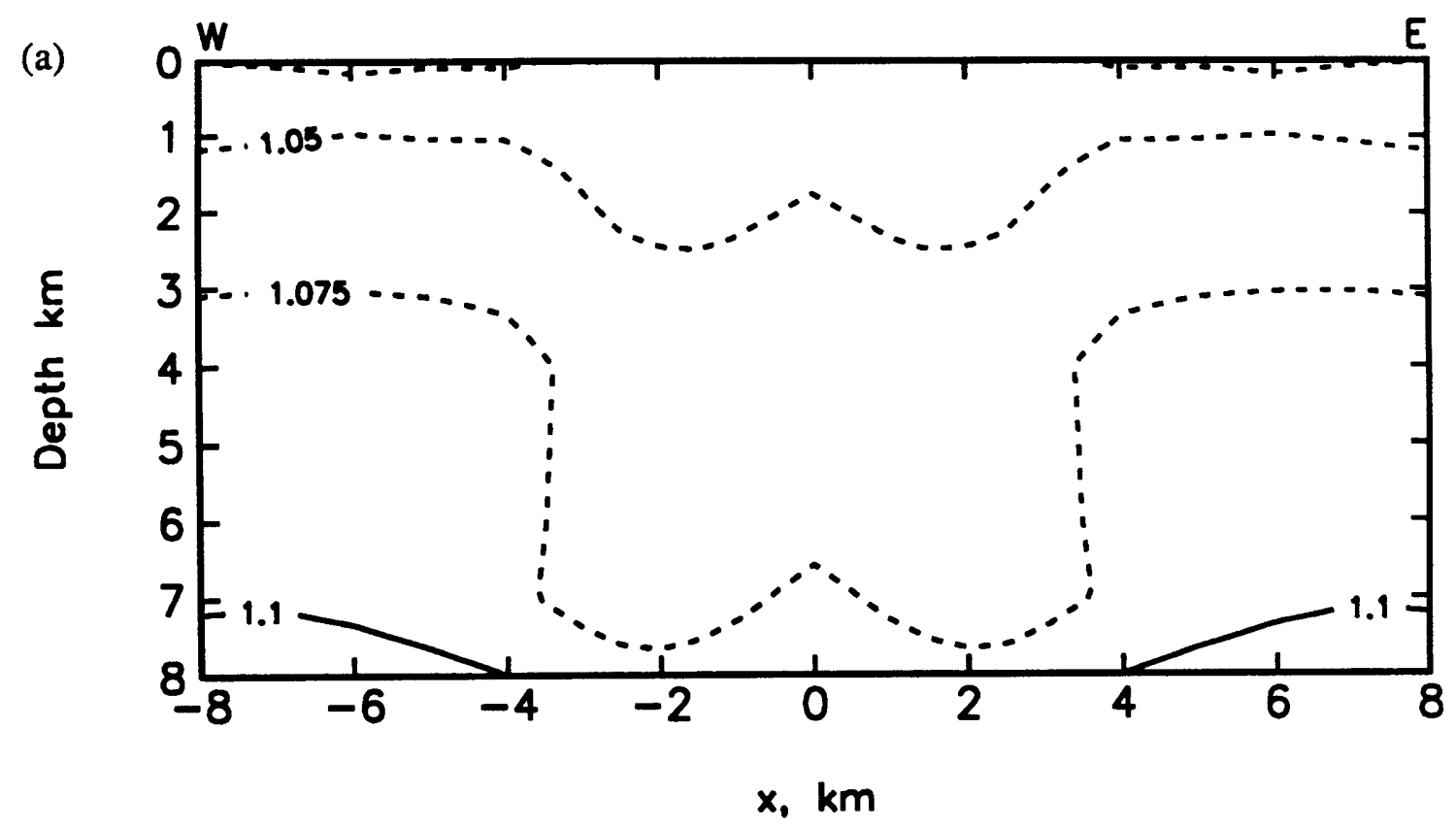

$1 / Q$ Uncertainty $b=5.6$

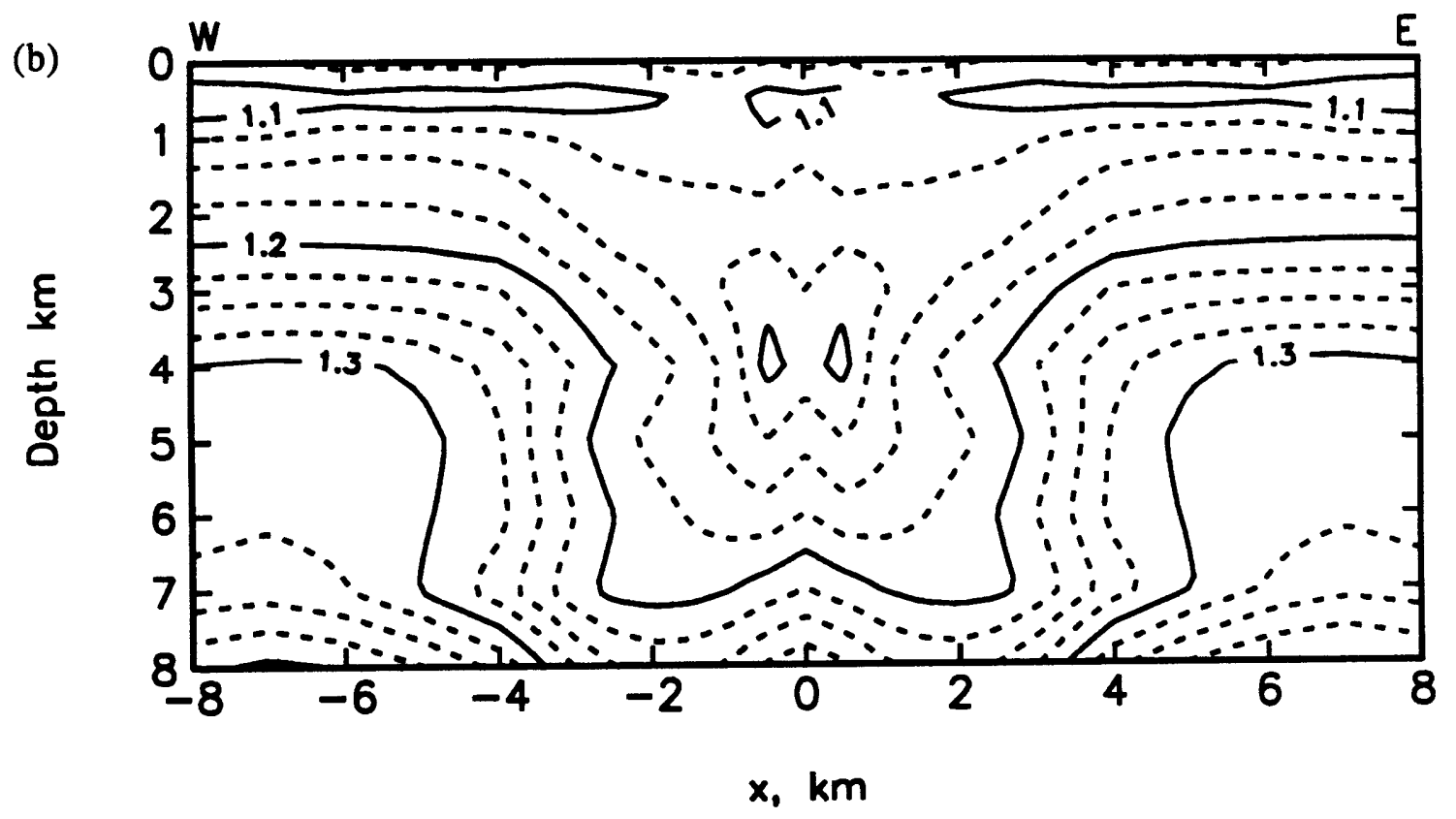

Figure 4.26 


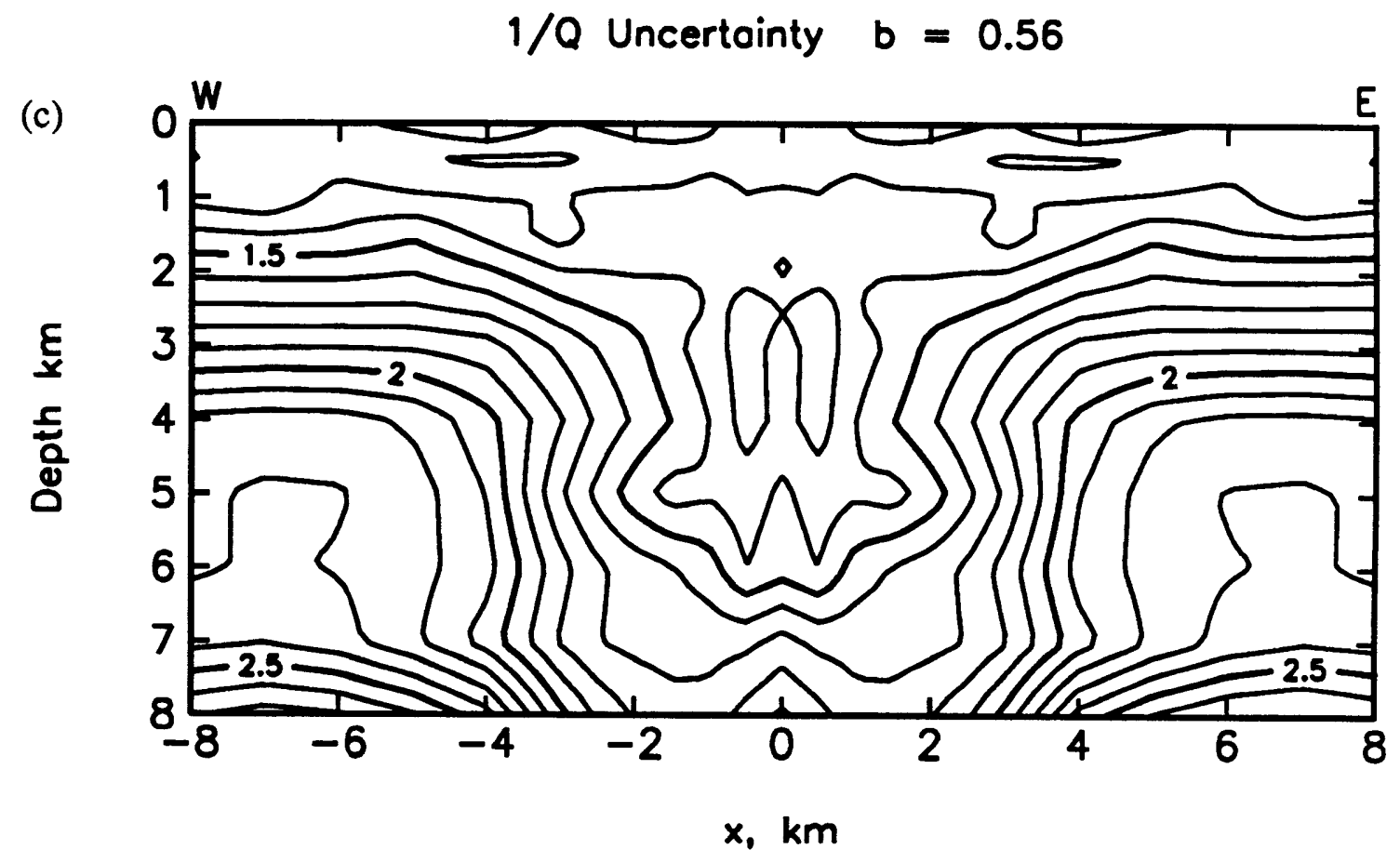

Figure 4.26 cont. 
$1 / Q$ Spread $b=56$

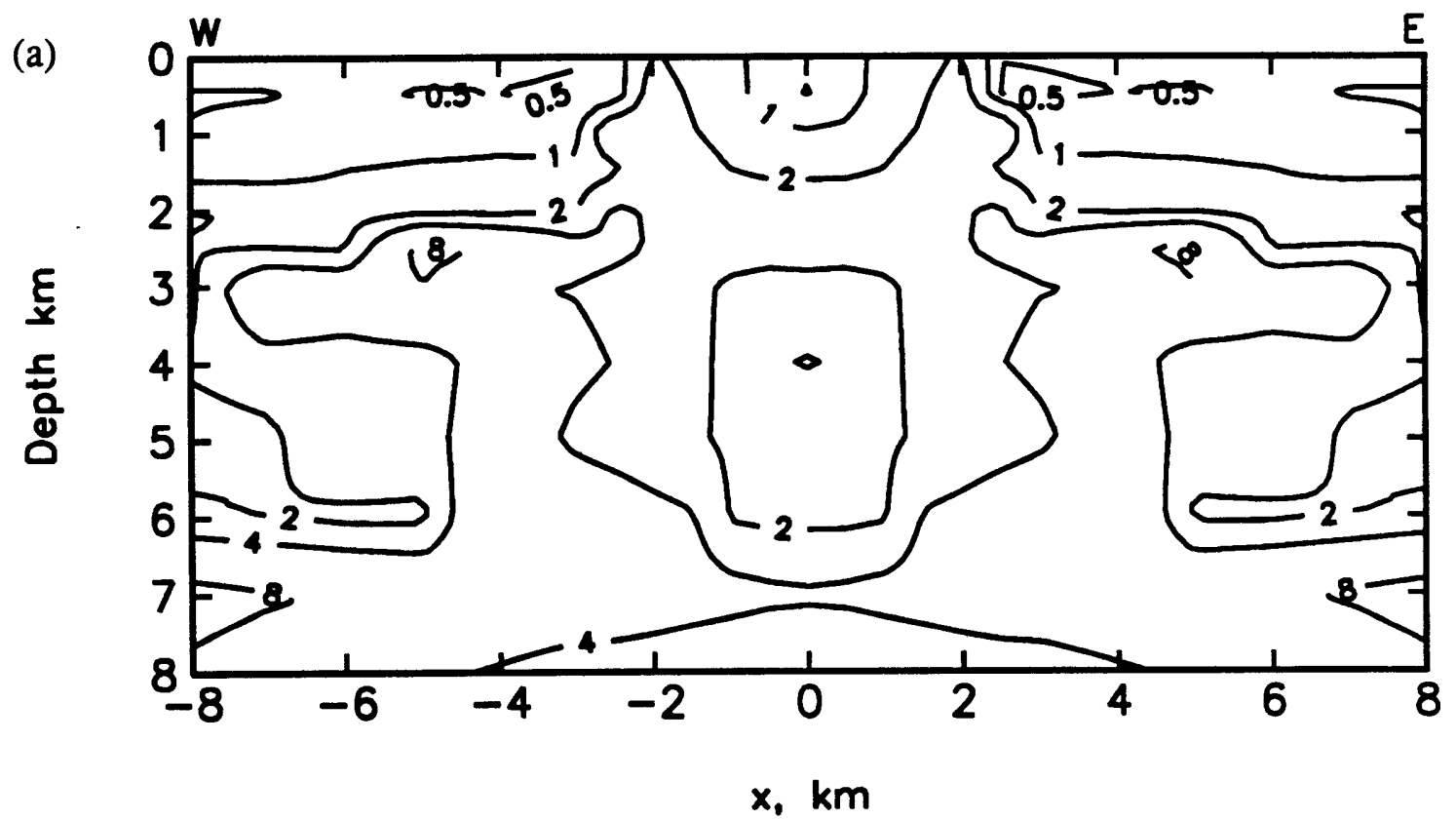

$1 / Q$ Spread $b=5.6$

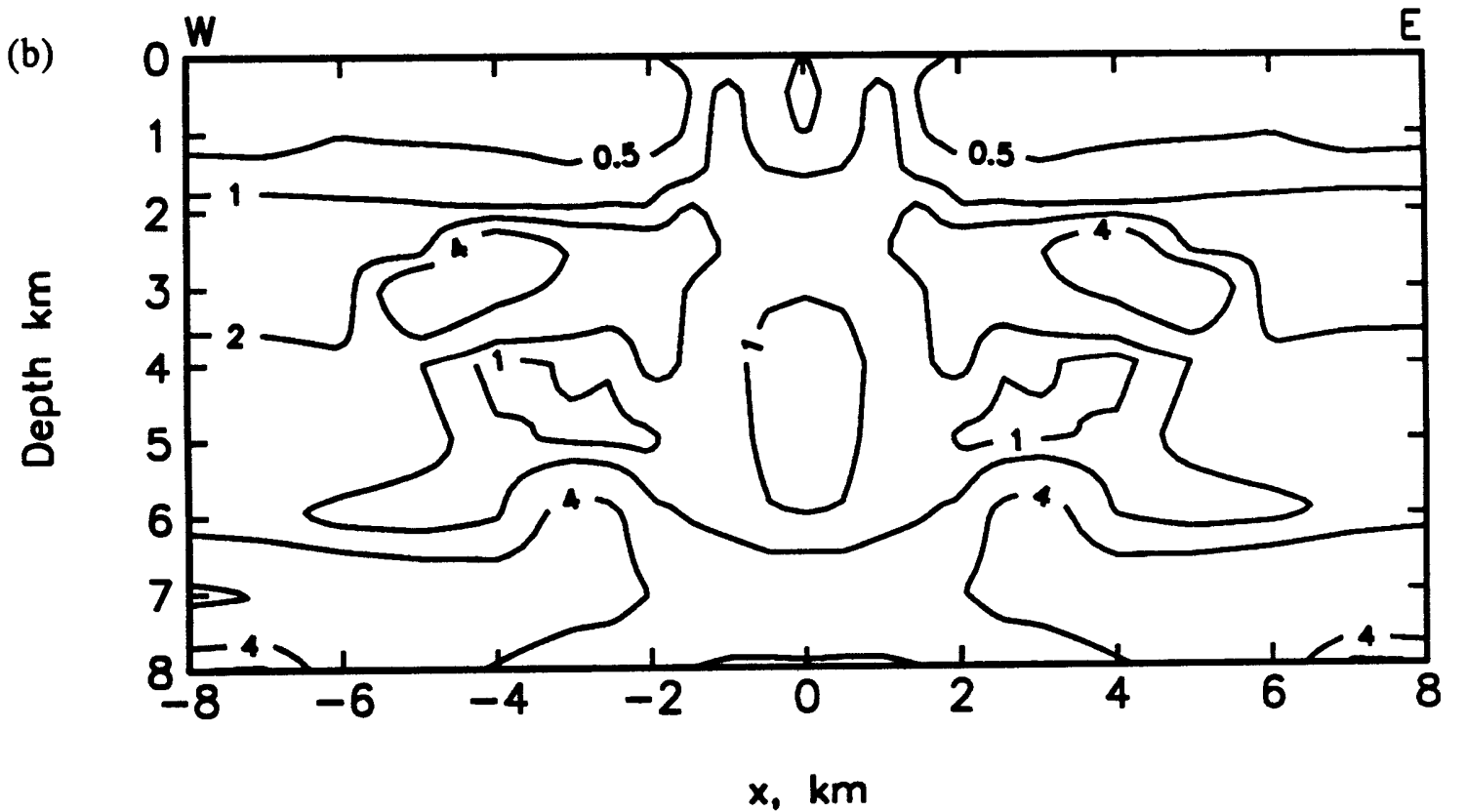

Figure 4.27 


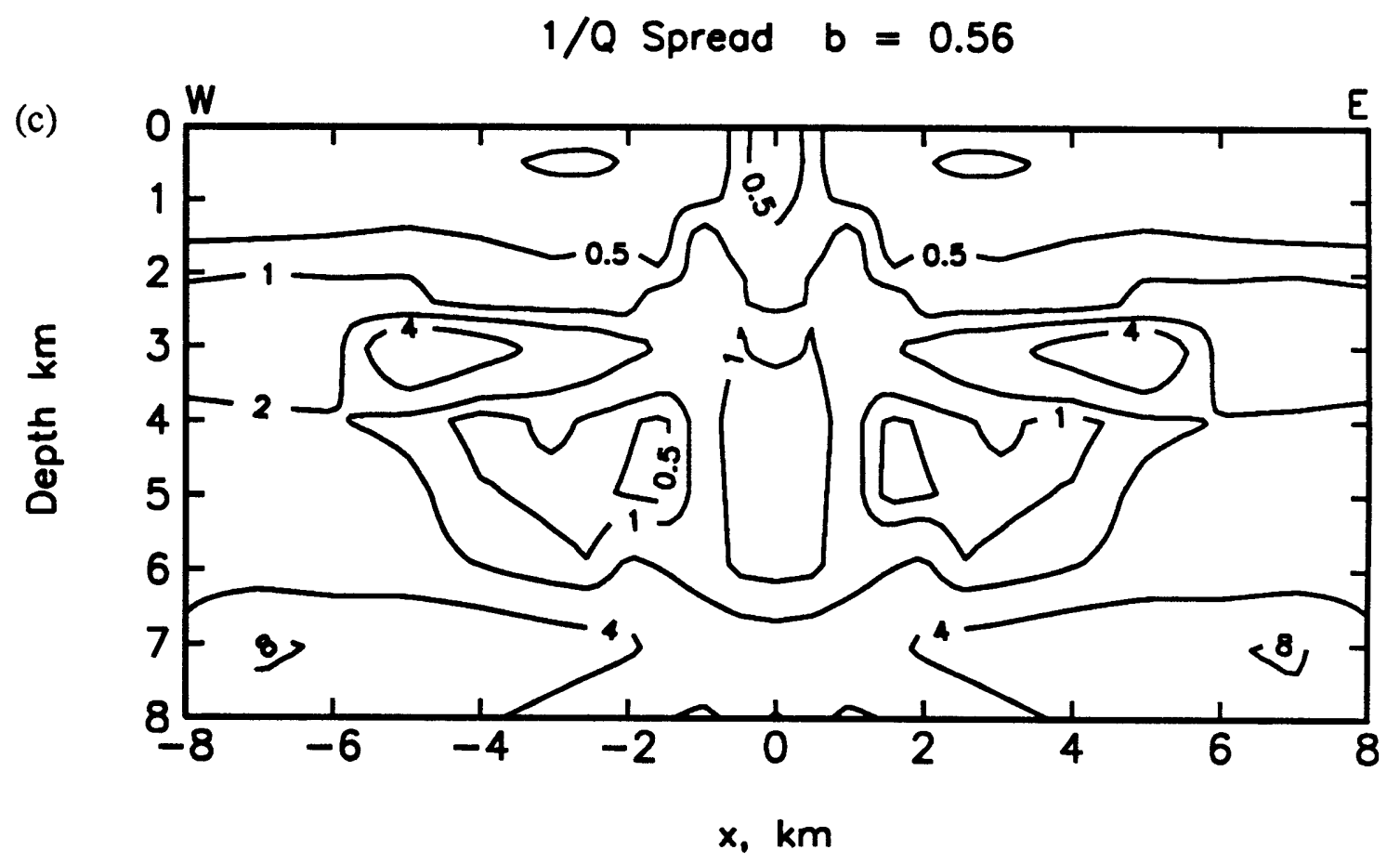

Figure 4.27 cont. 


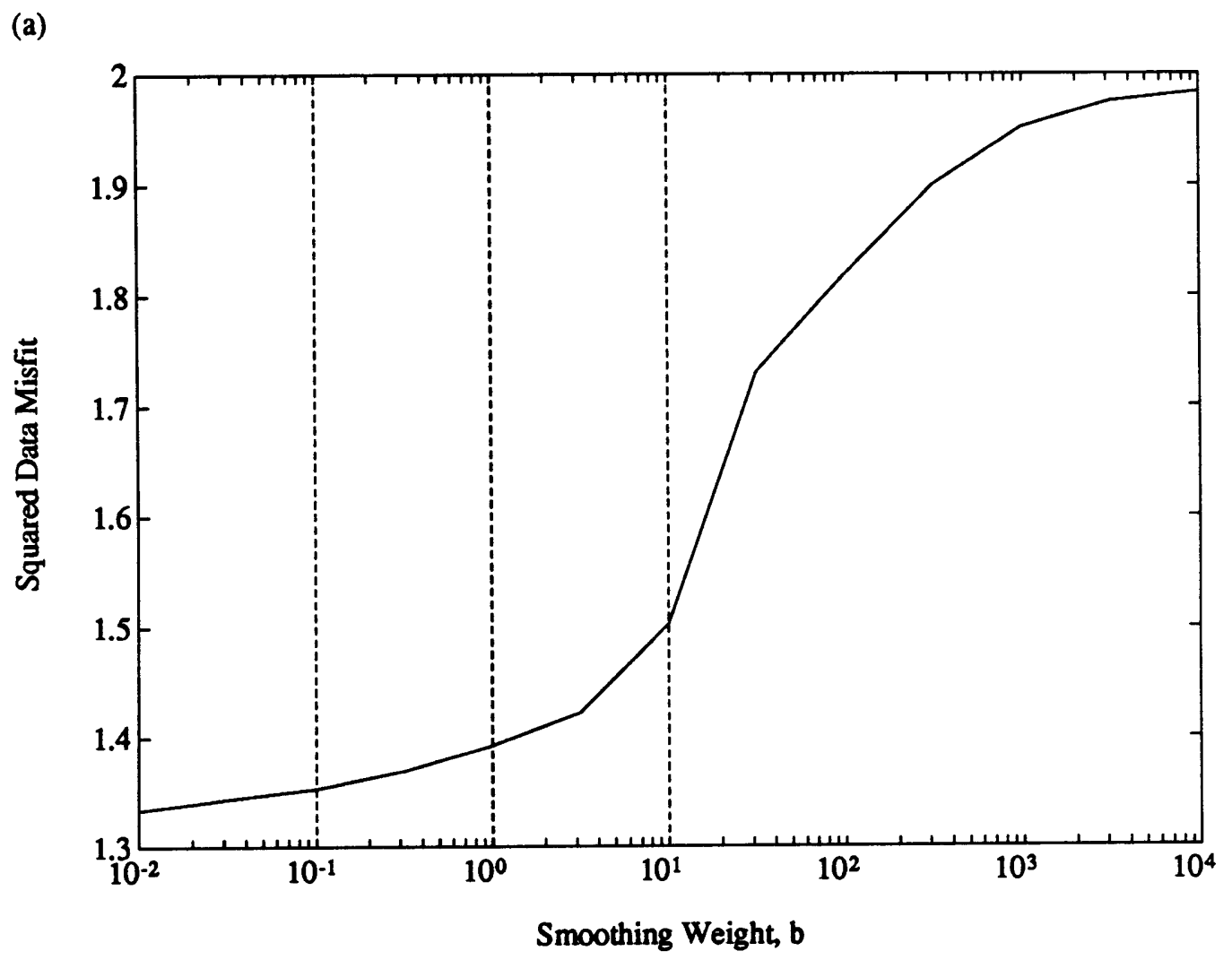

Figure 4.28 
(b)

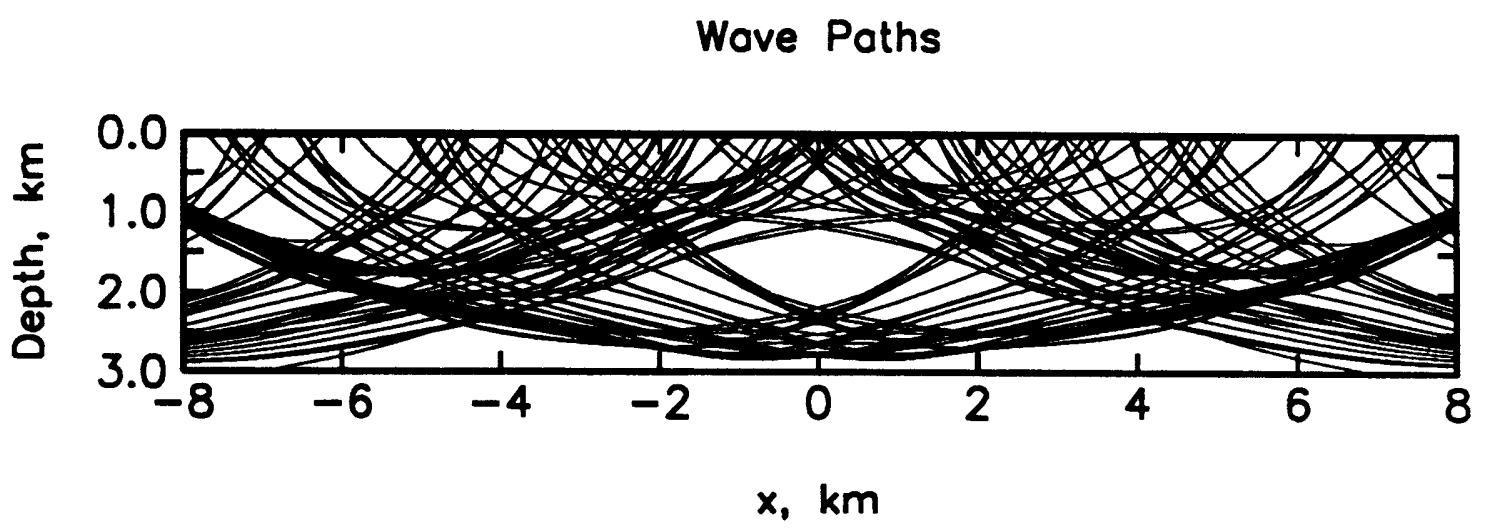

(c)

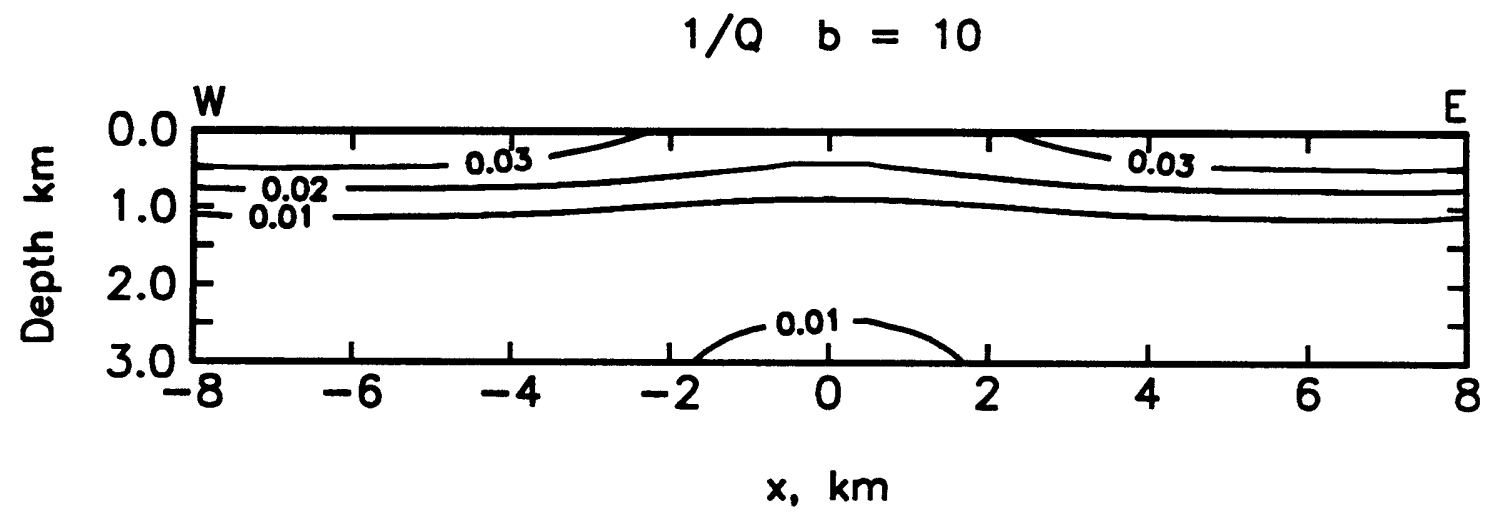

Figure 4.28 cont. 
(d) (i)

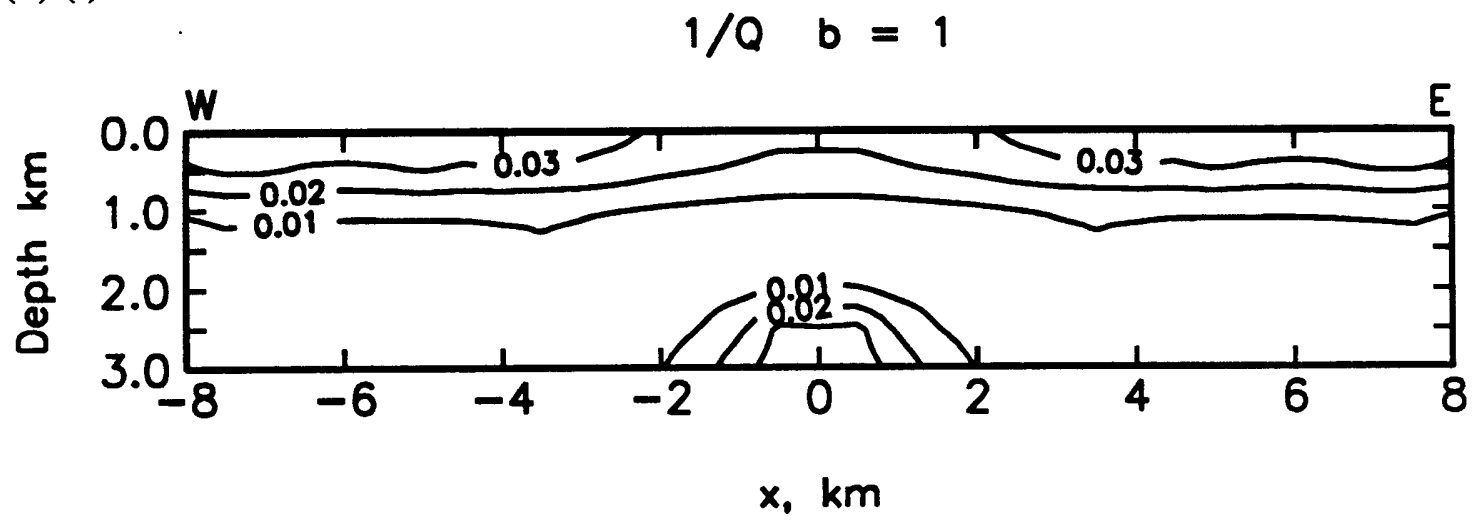

(d) (ii)

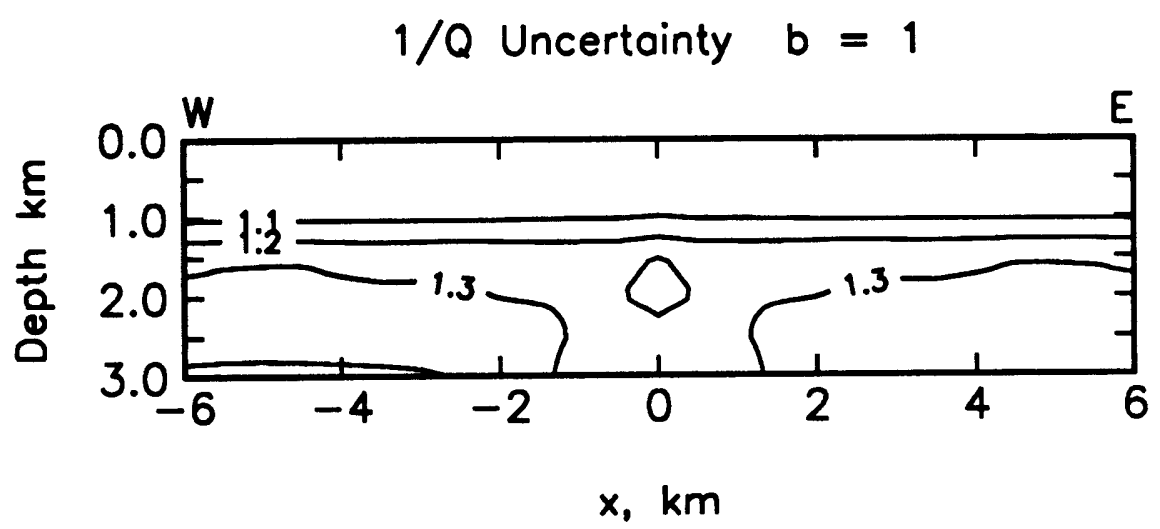

(e)

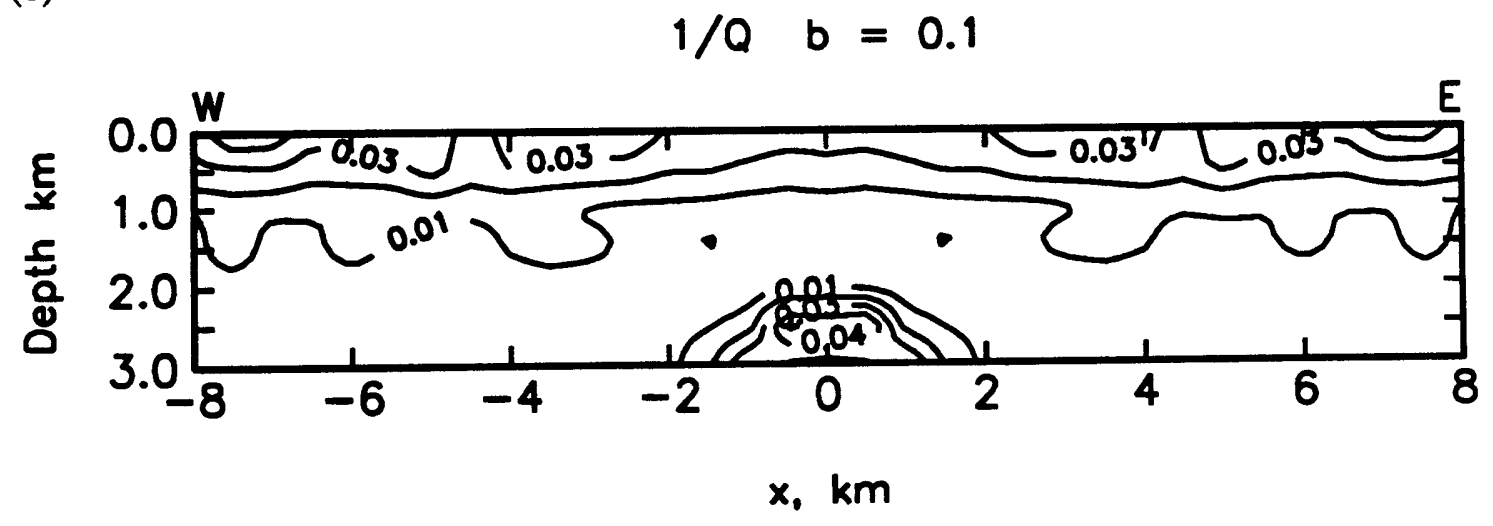

Figure 4.28 cont. 
(a)

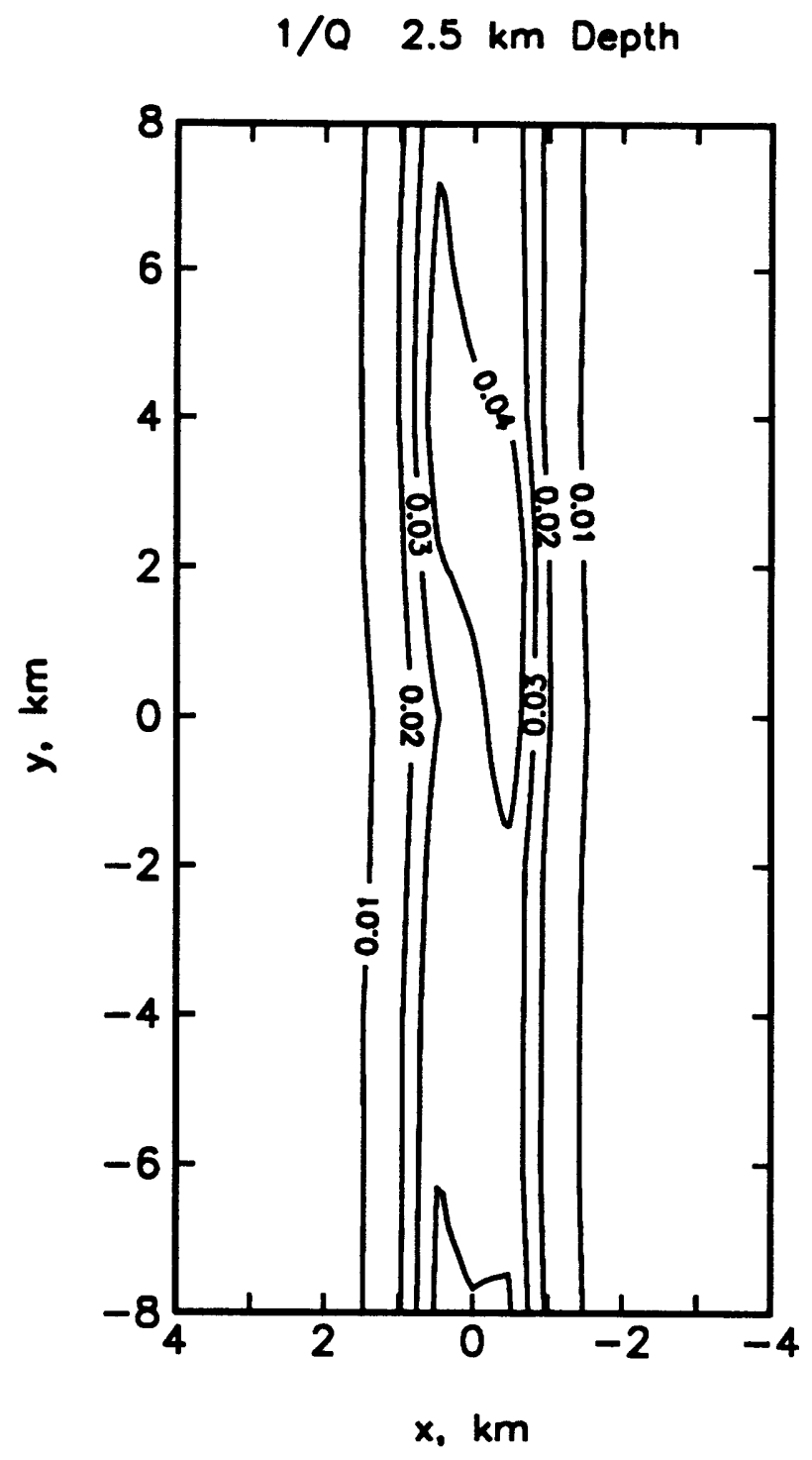

(b)

$1 / 0 \times=0 \mathrm{~km}$

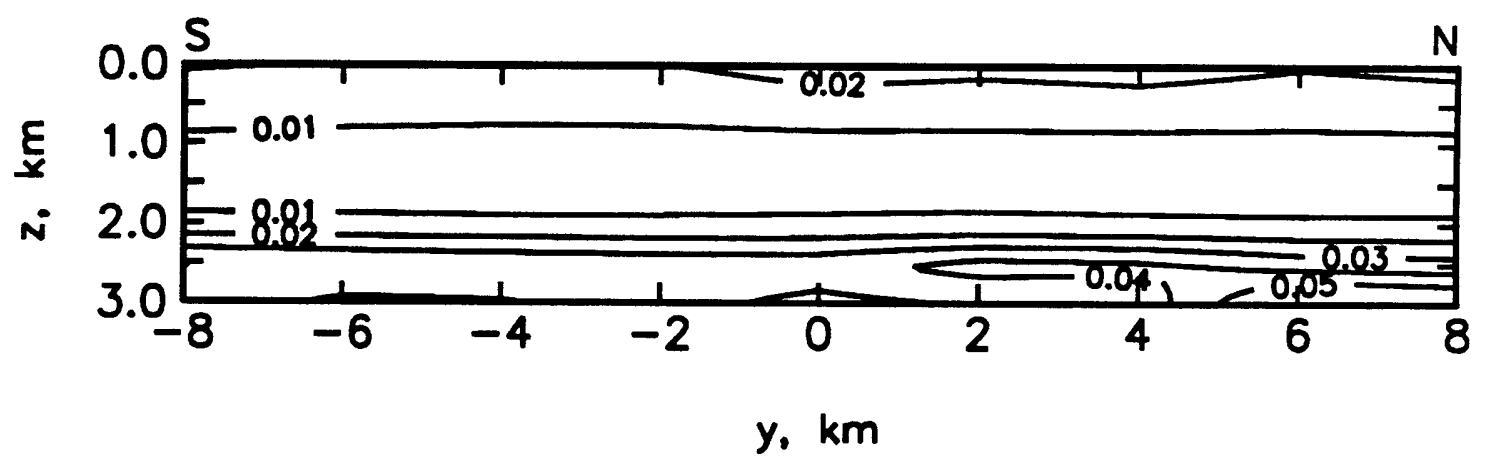

Figure 4.29 

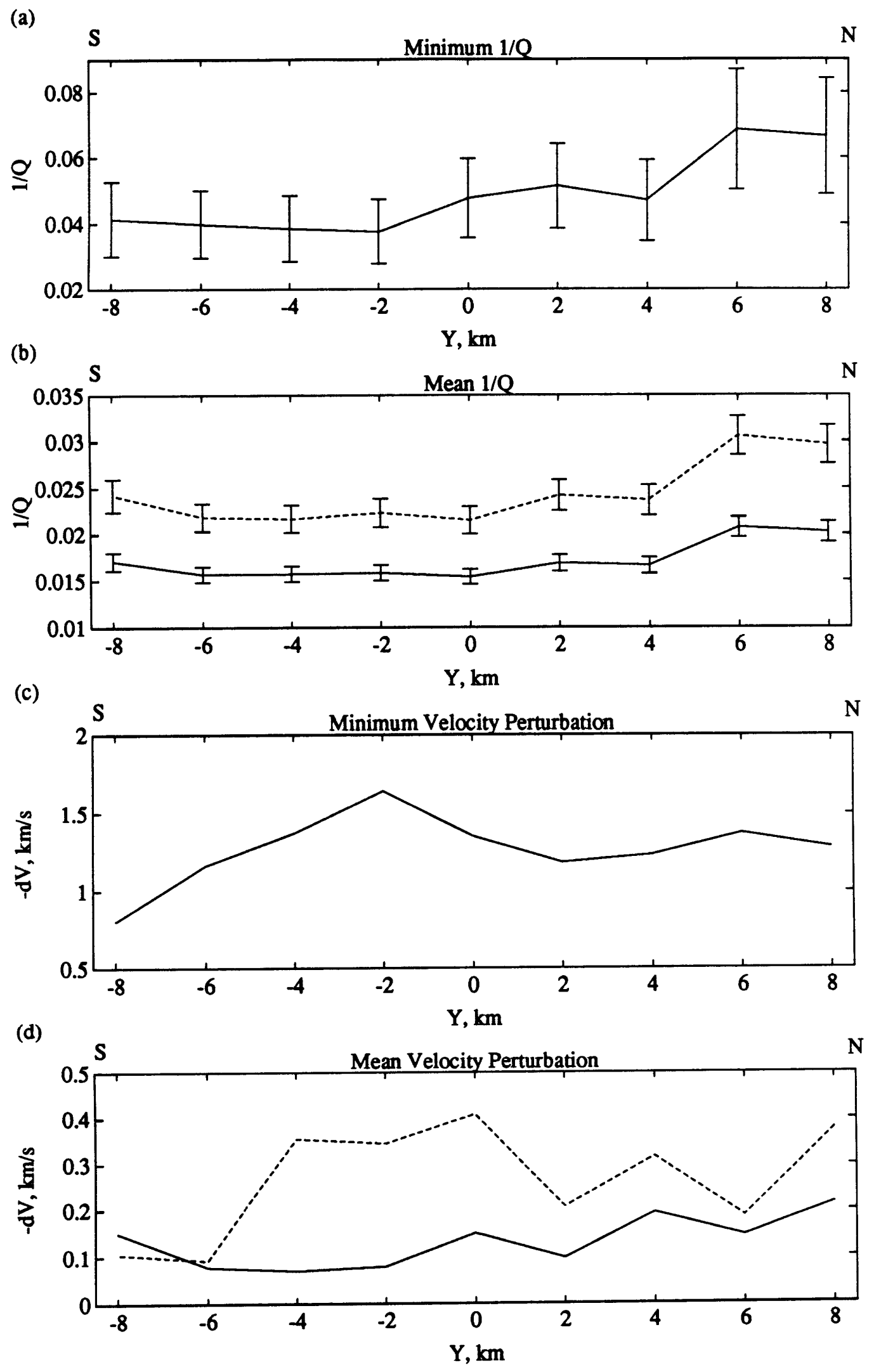

Figure 4.30 
(a)

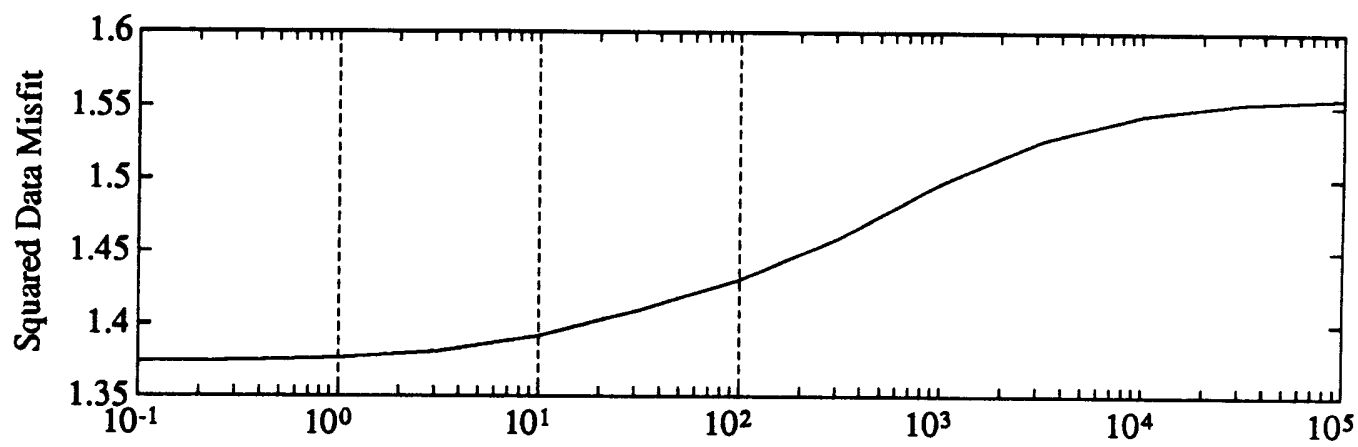

(b)

Smoothing Weight, b
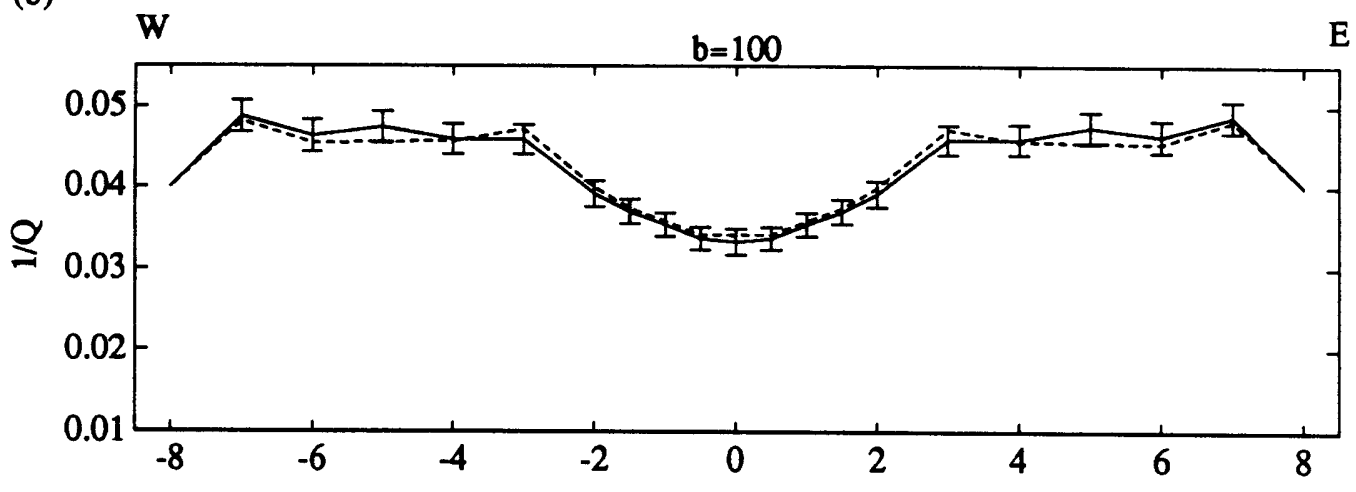

(c)
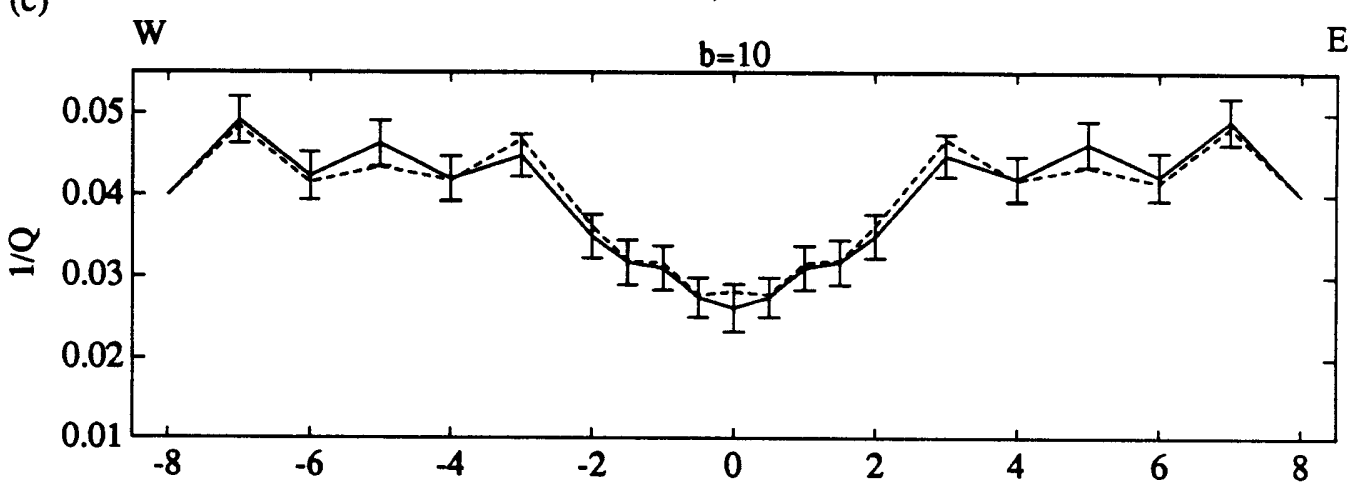

(d)

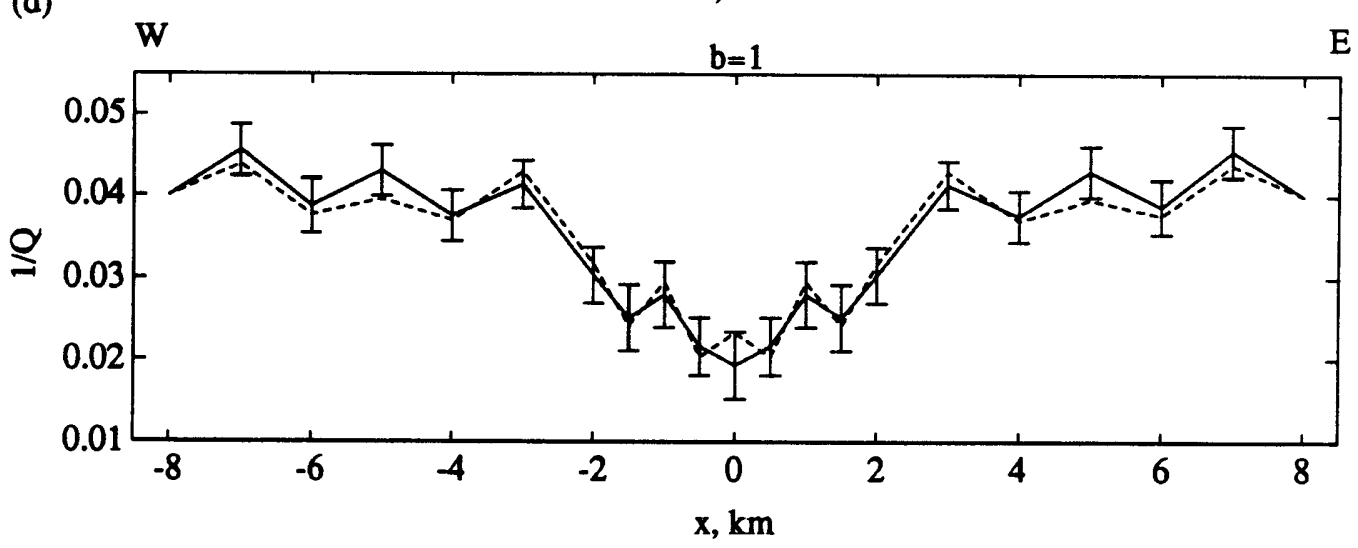

Figure 4.31 

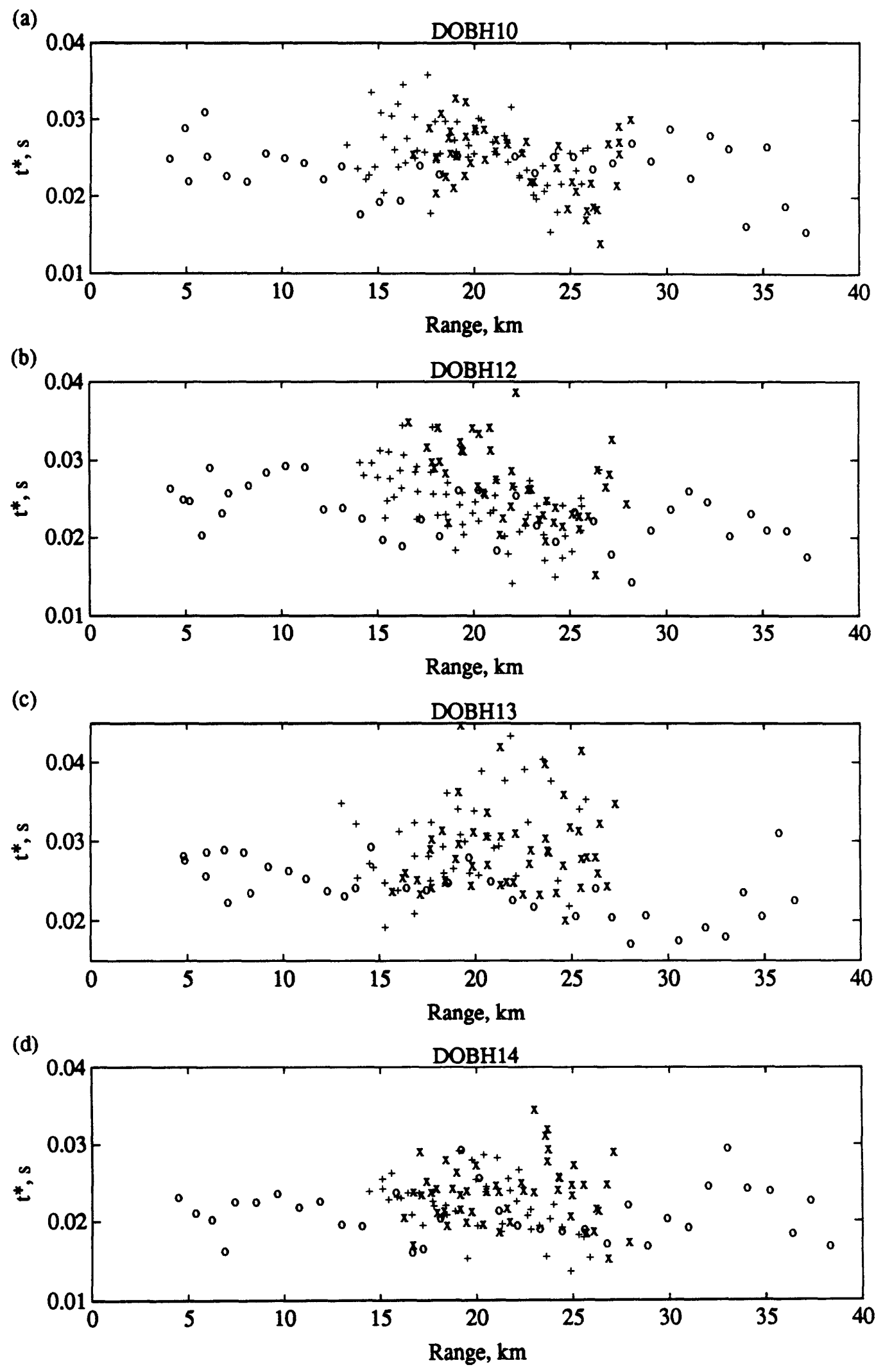

Figure 4.32 
(a)

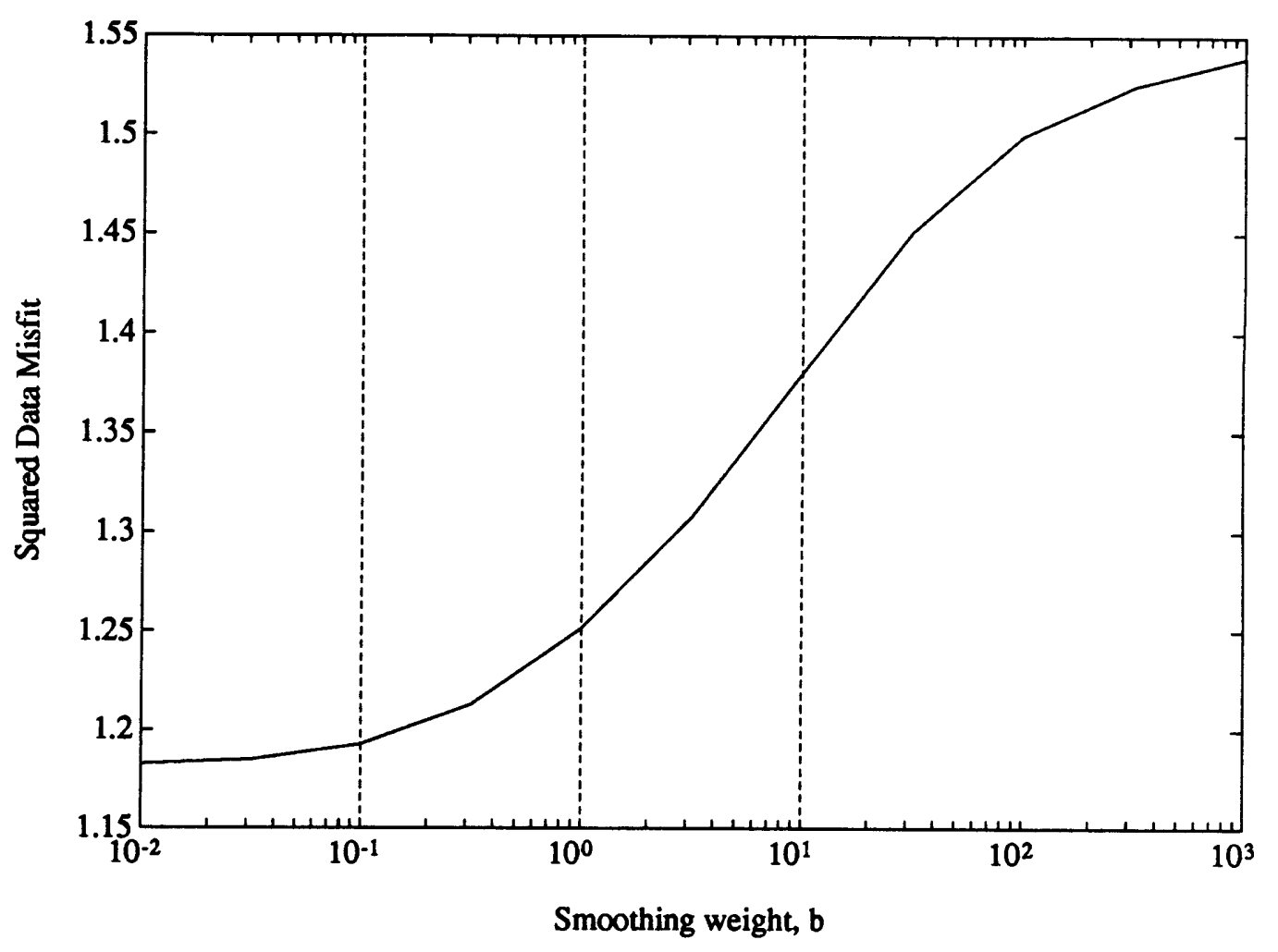

(b)

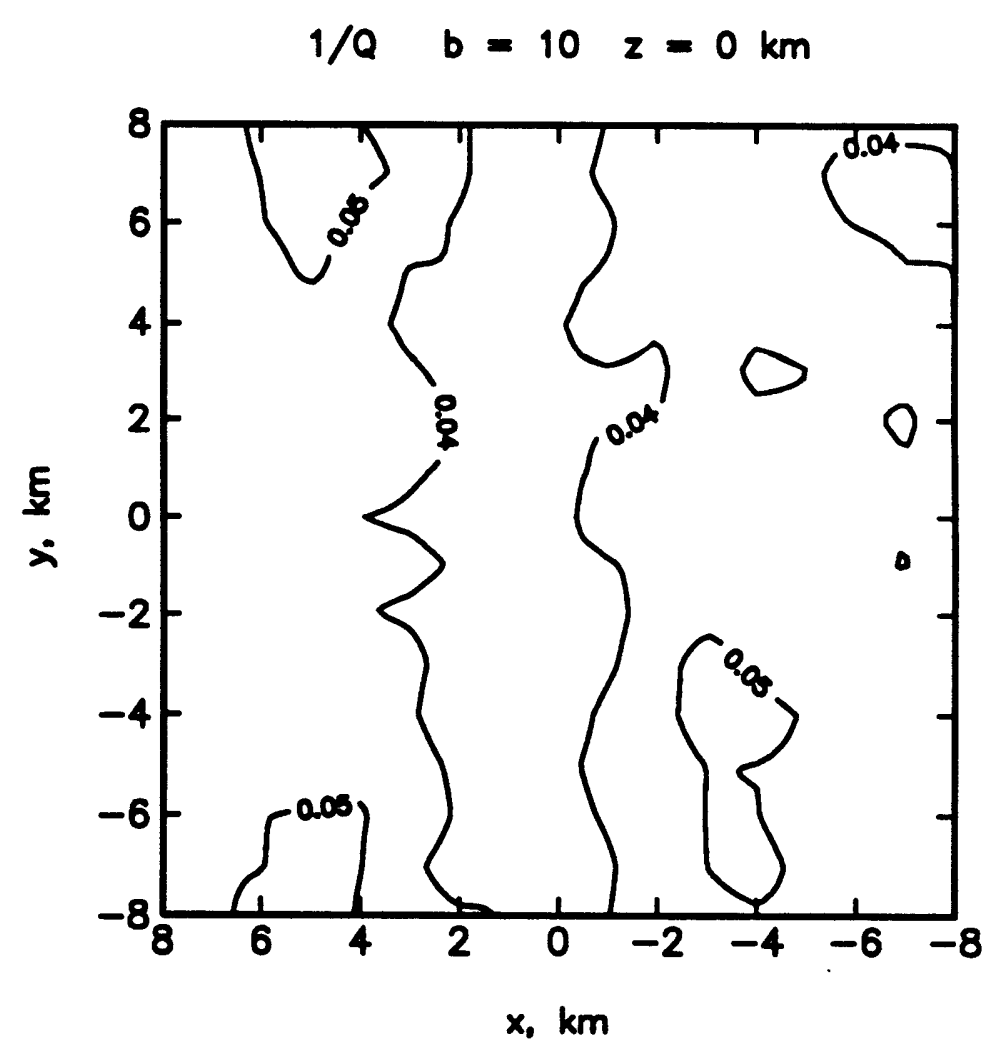

Figure 4.33 
(c)

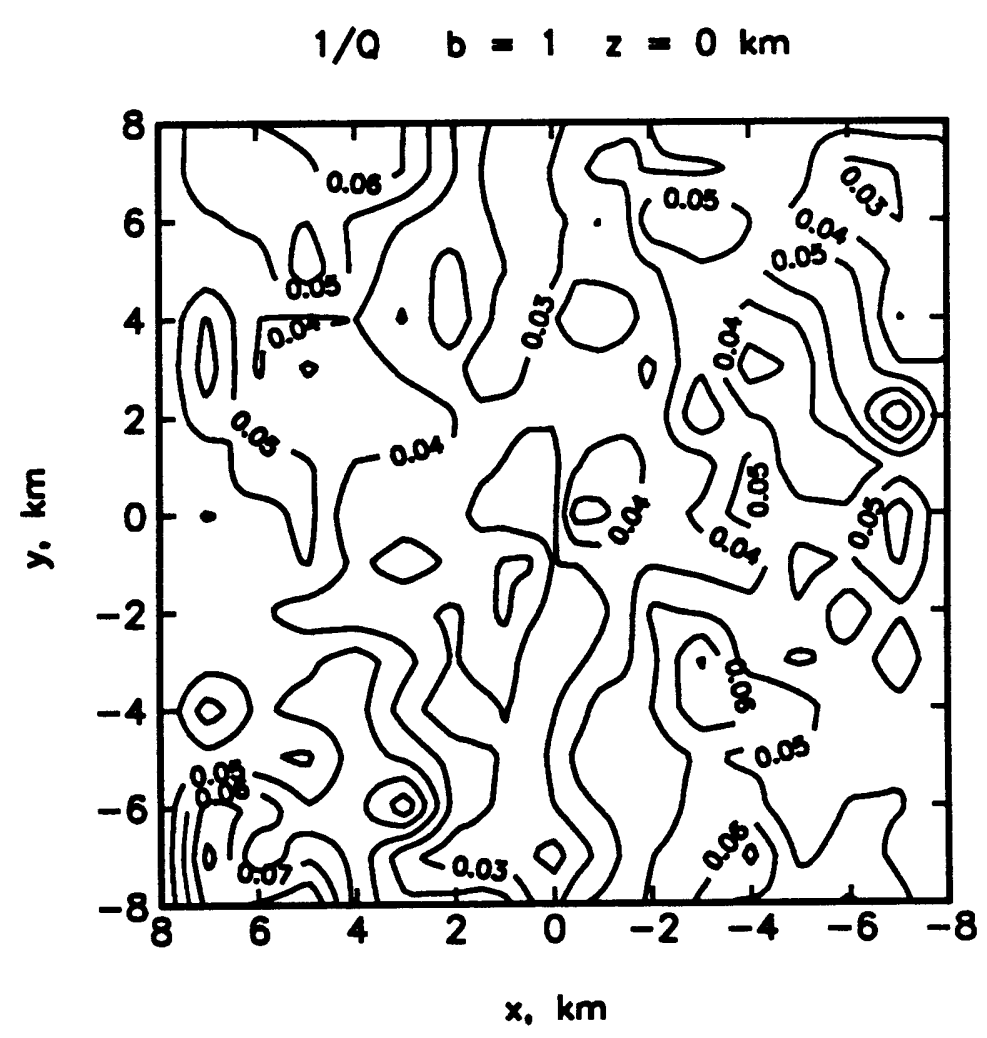

(d)

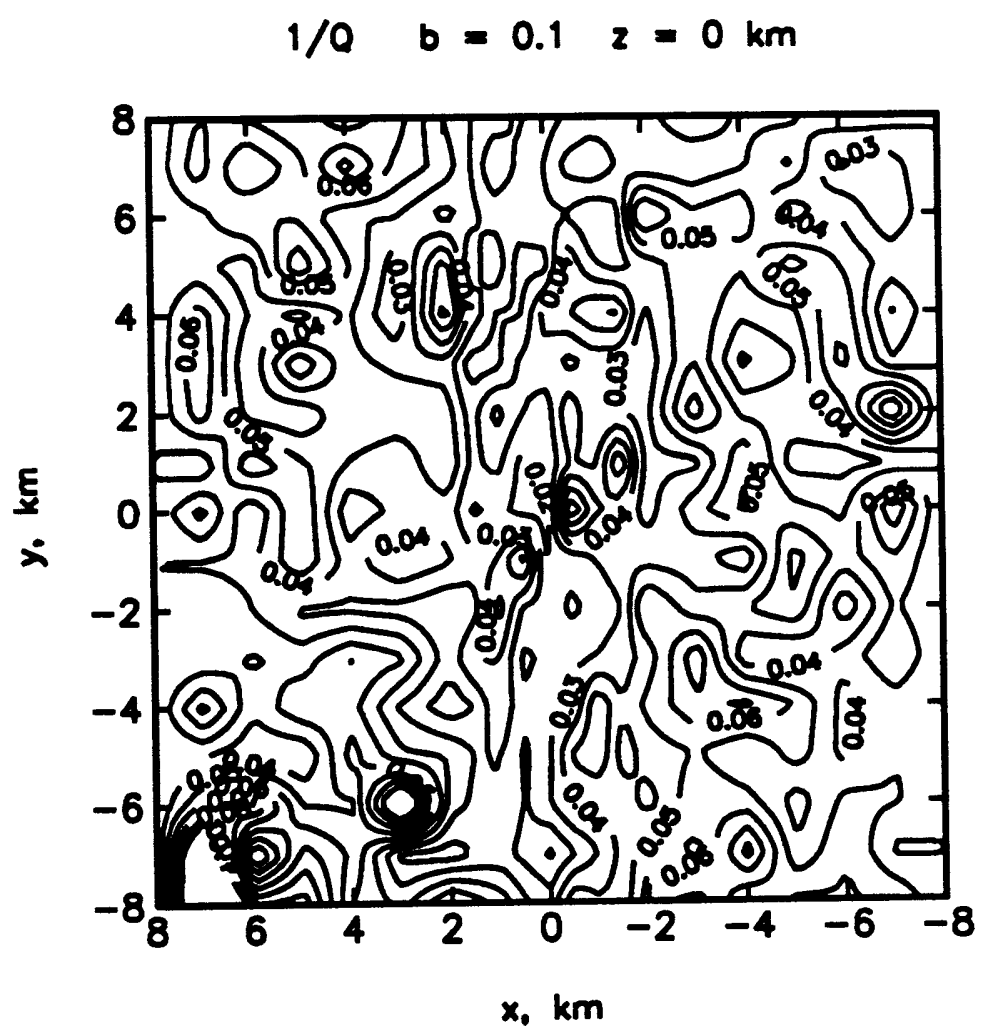

Figure 4.33 cont. 

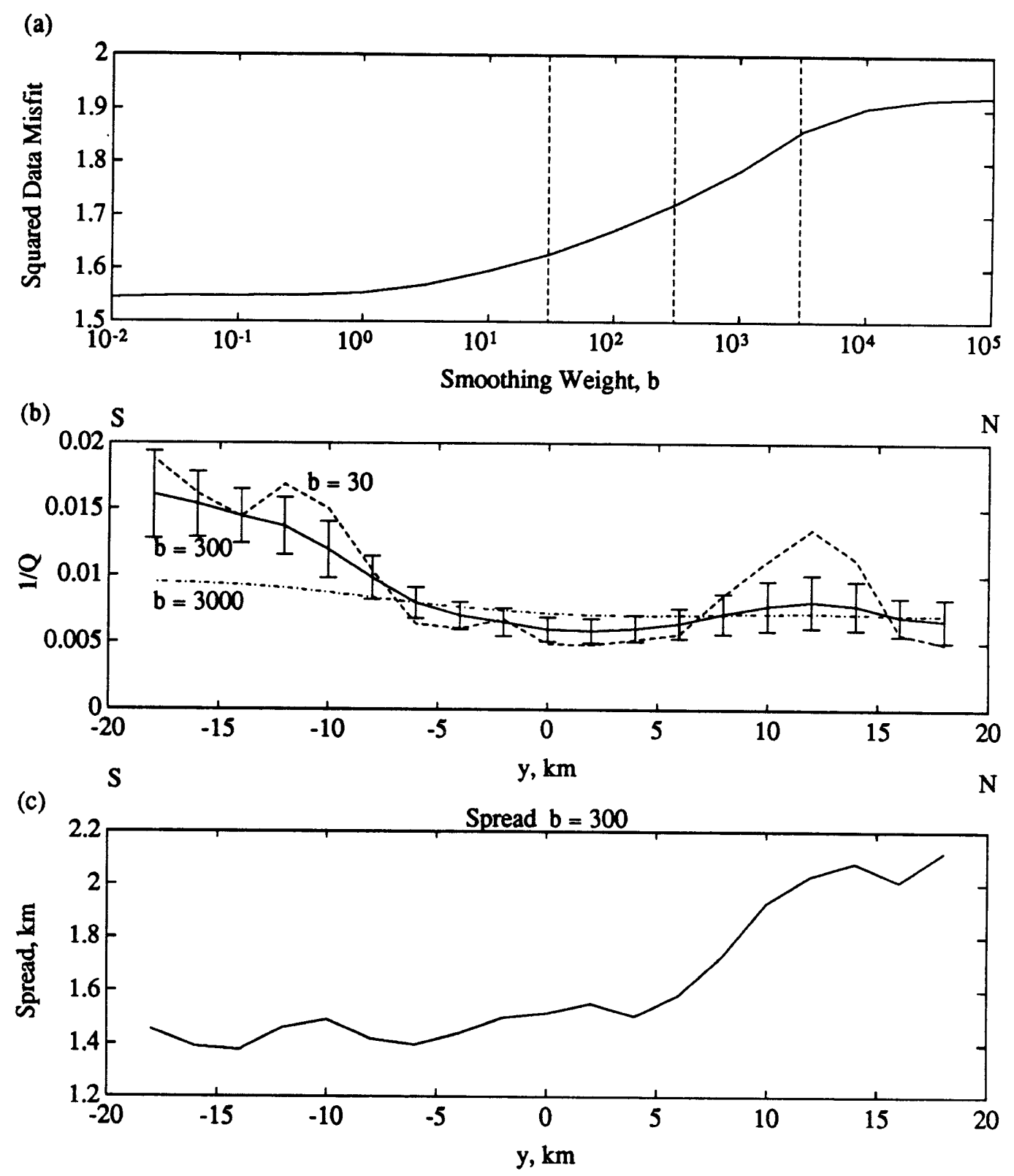

Figure 4.34 

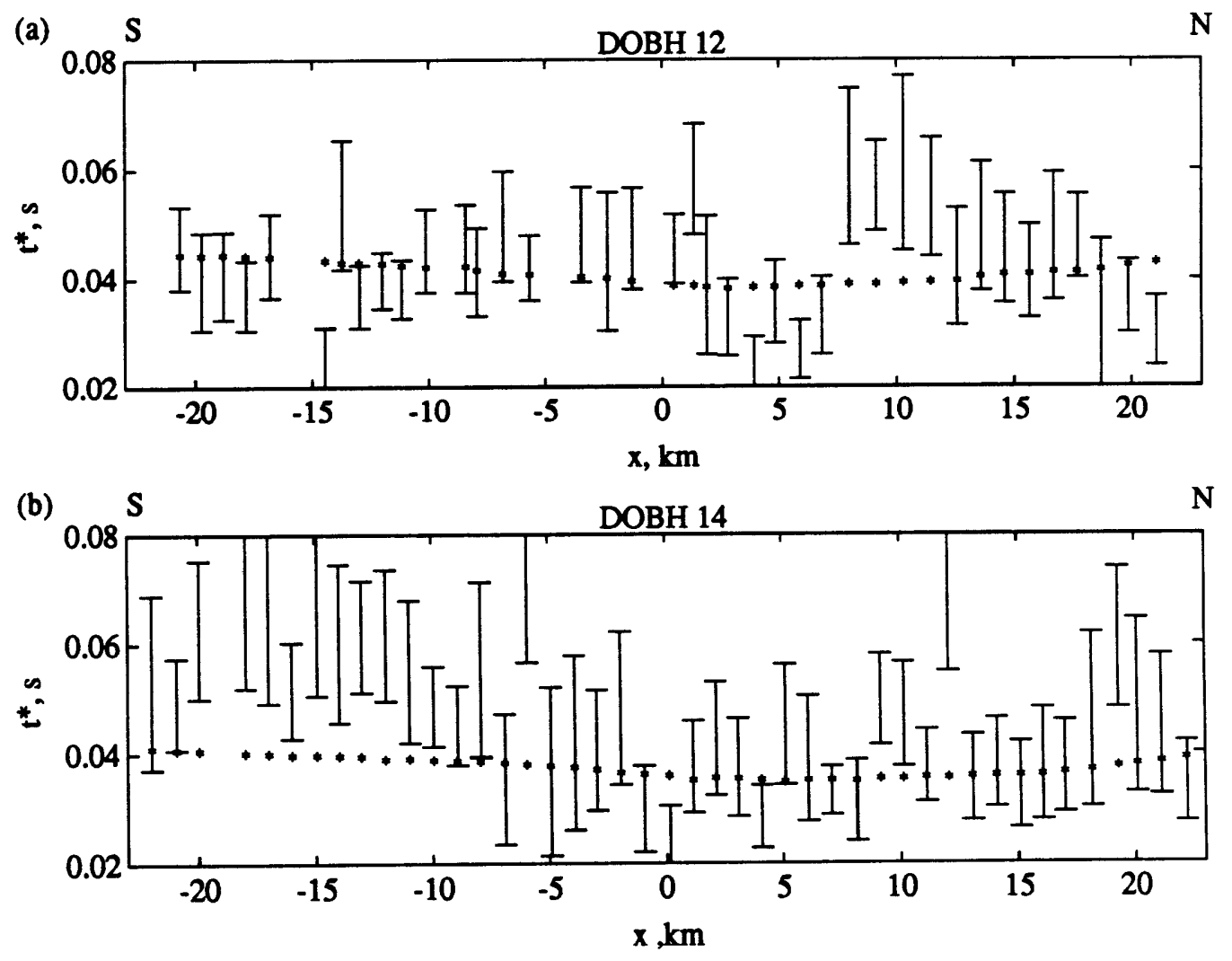

Figure 4.35 
(a)

Apriori Model

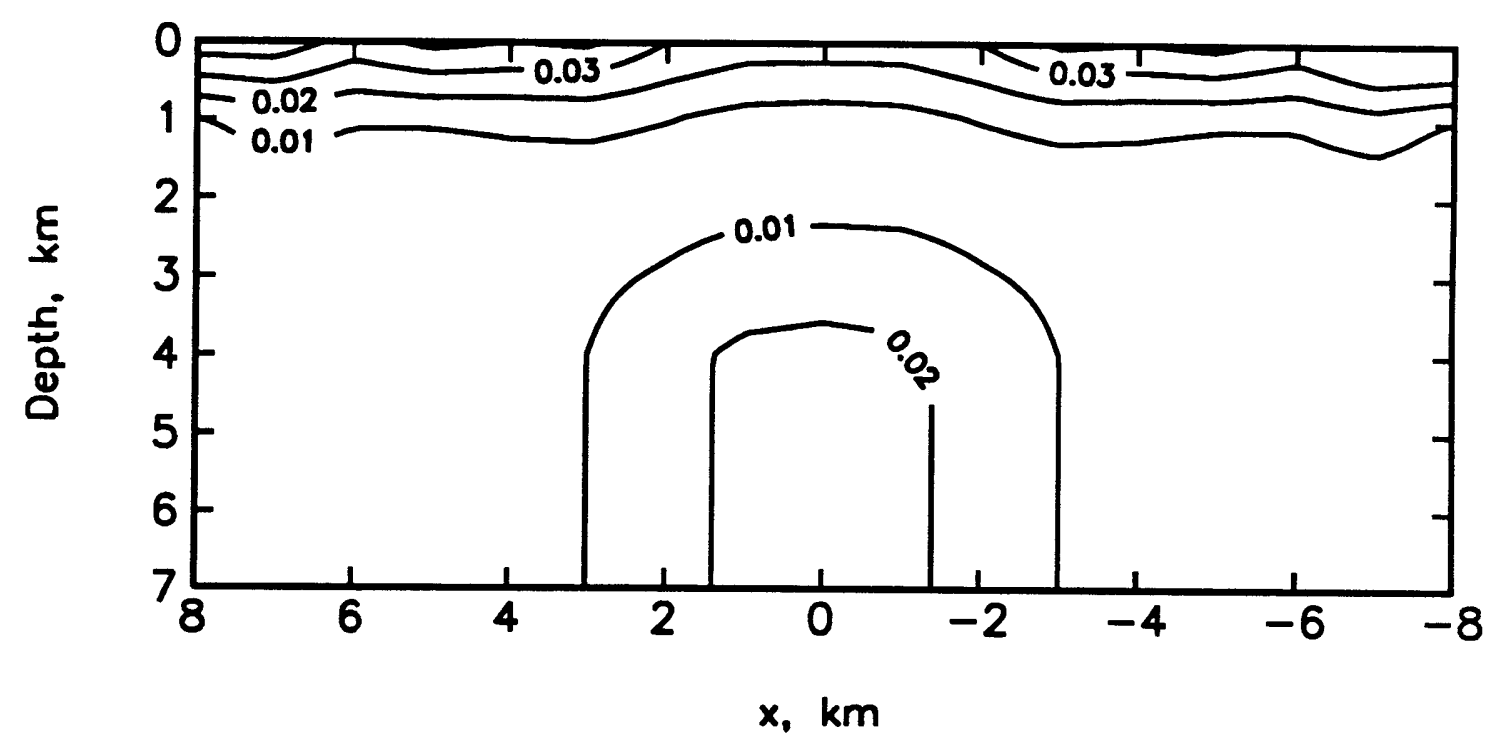

(b) Wove Paths

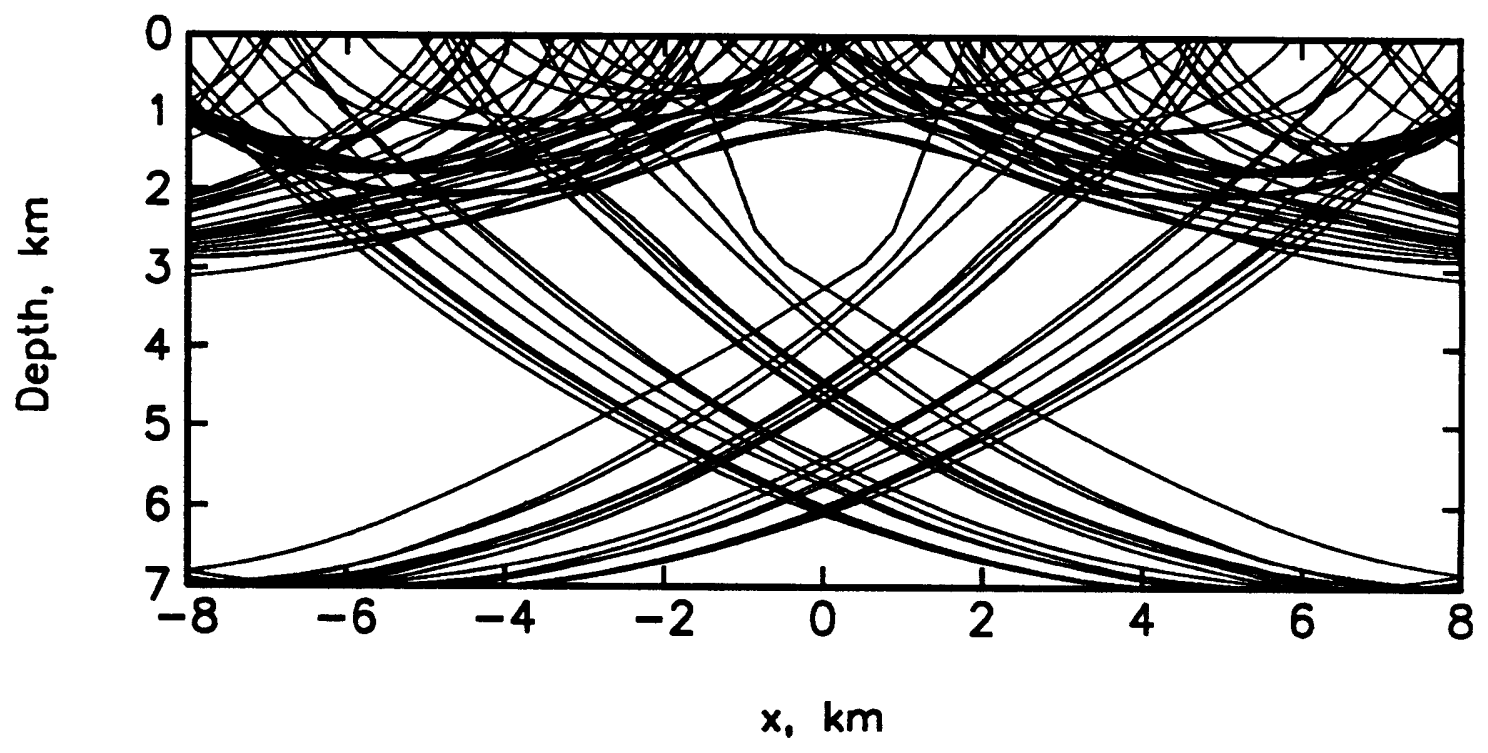

Figure 4.36 

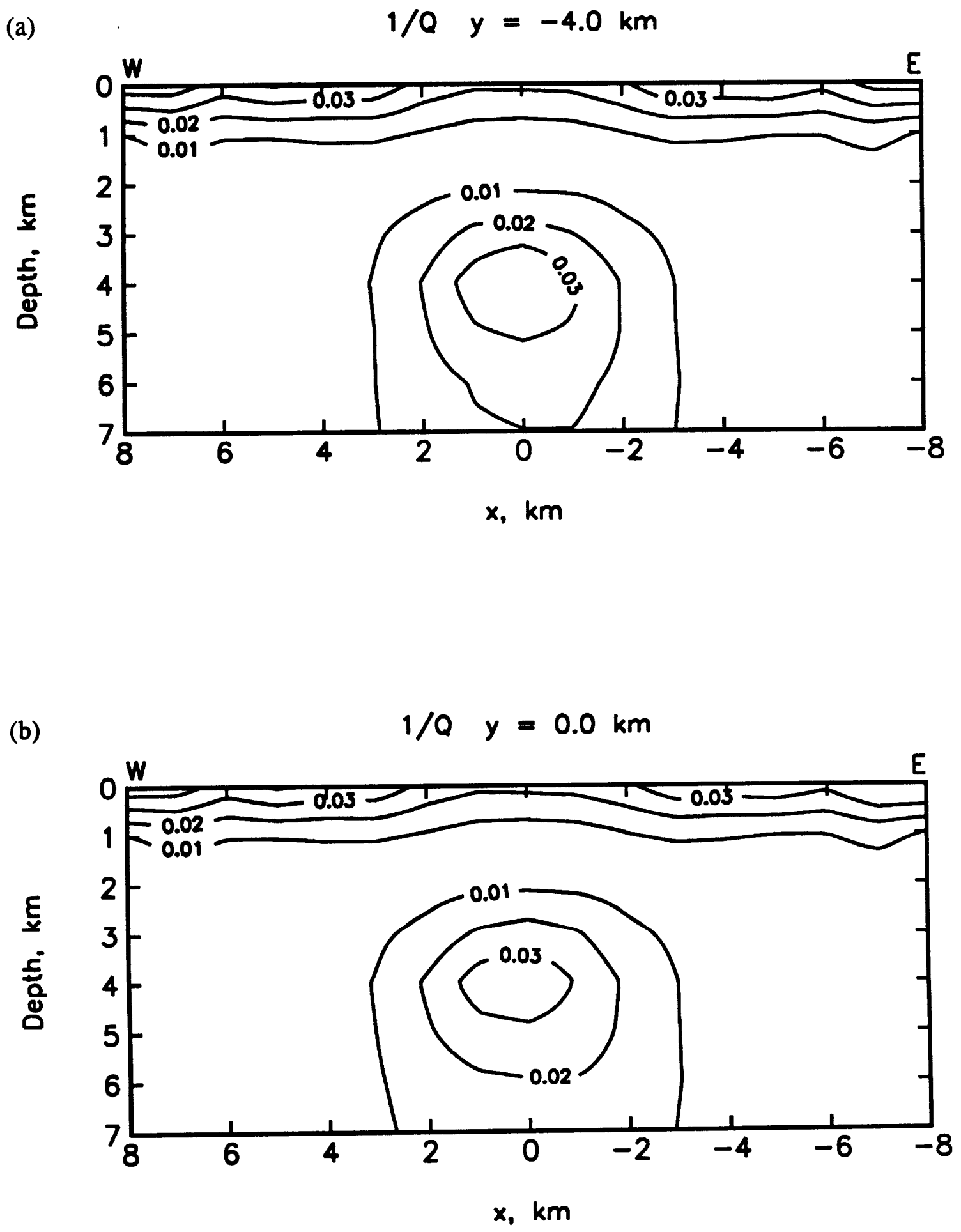

Figure 4.37 
(c)

$1 / Q \quad y=4.0 \mathrm{~km}$

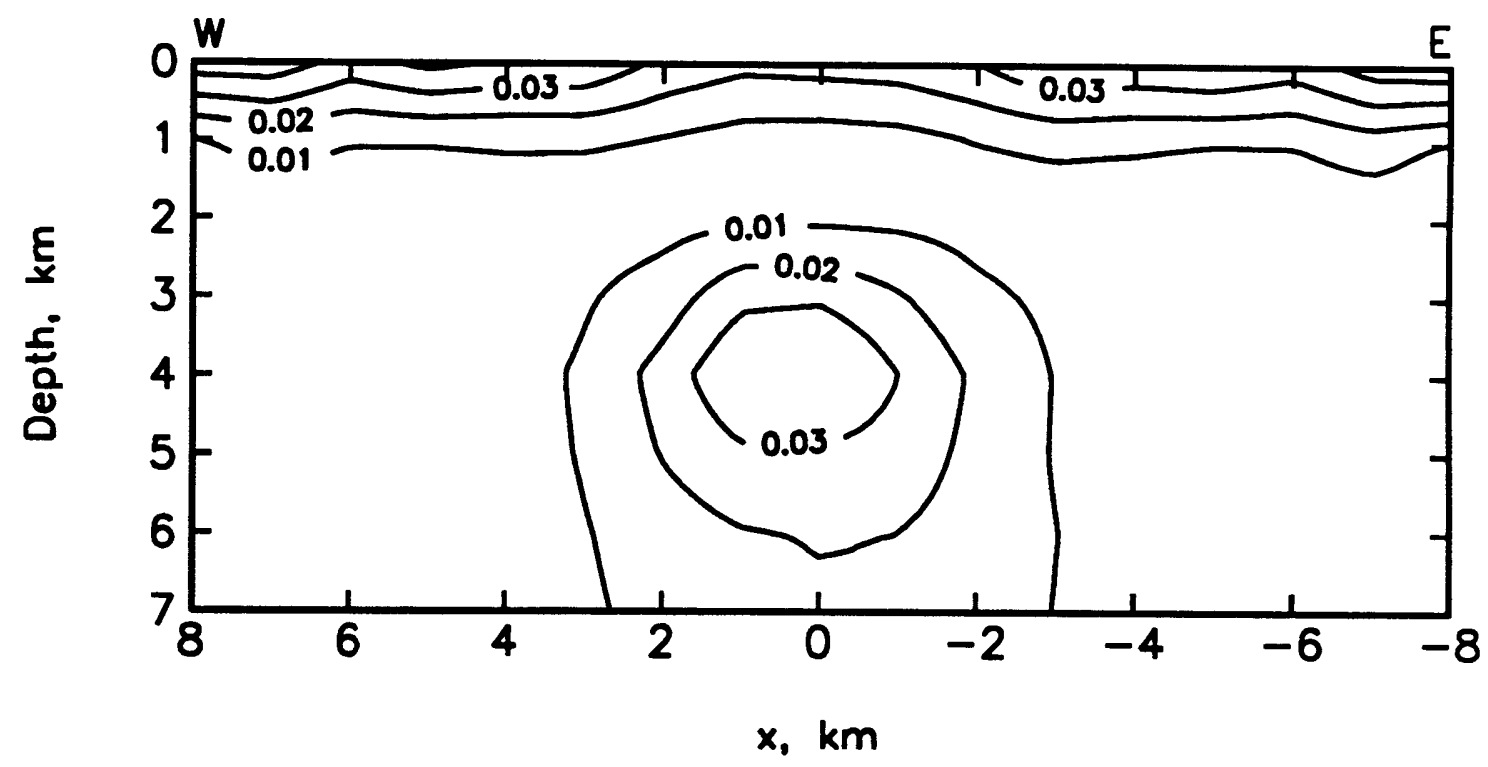

(d)

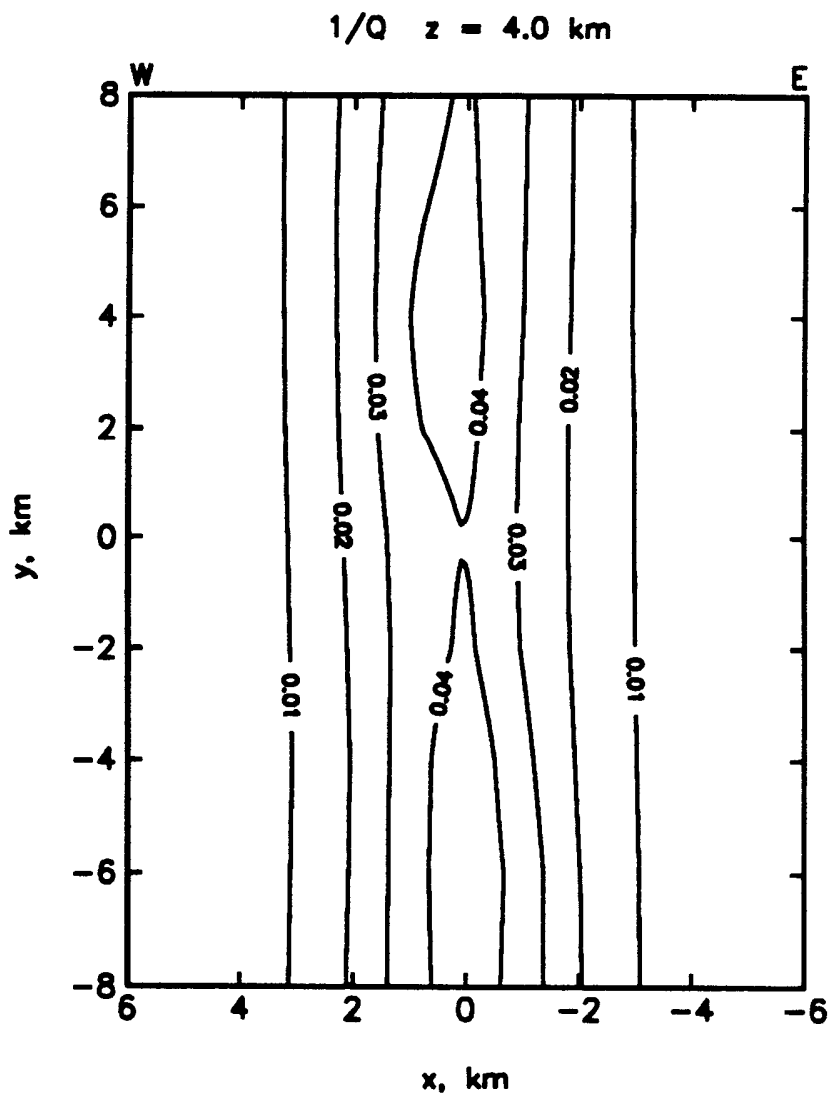

Figure 4.37 cont. 

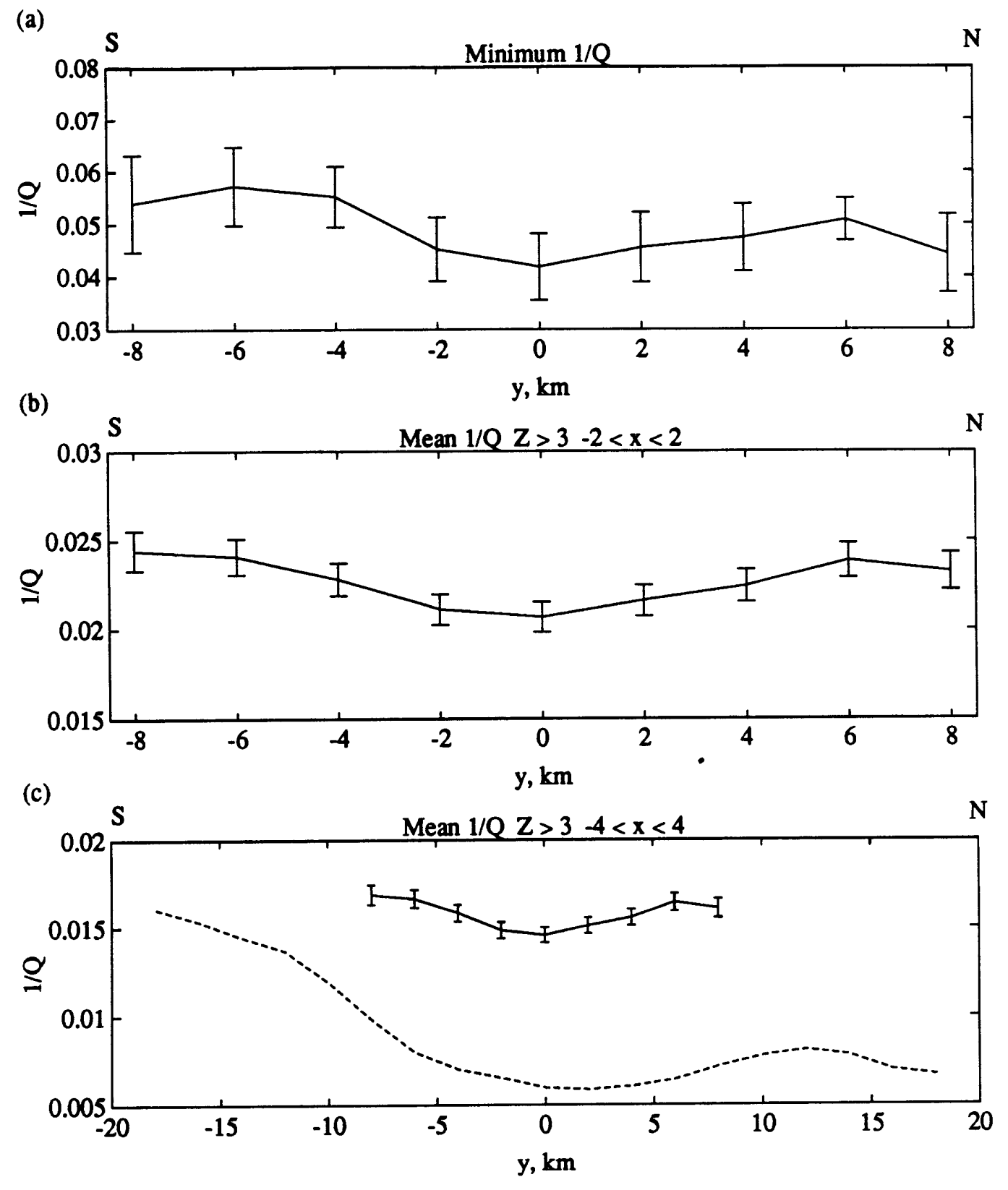

Figure 4.38 


\section{ChaPTer 5}

\section{THE INTERPRETATION OF THE ATTENUATION STRUCTURE OF THE EAST PACIFIC RISE}

The East Pacific Rise at $9^{\circ} 30^{\prime} \mathrm{N}$ has been the site of many targeted studies of in seismology [Orcutt et al., 1976; Herron et al., 1978; Detrick et al., 1987; Vera et al., 1990; Toomey et al., 1990a; Christeson et al., 1991a, b], bathymetry [Macdonald et al., 1984, 1992; Wilcock et al., 1992], petrology [Langmuir et al., 1986], gravity [Madsen et al., 1990], high-resolution imaging [Haymon et al., 1991], and magnetics [Carbotte and Macdonald, 1992]. Indeed, the large number of observational constraints led Sinton and Detrick [1992] to base their model for magma chambers on fast-spreading ridges on this locality. In this chapter we attempt to incorporate the knowledge of the attenuation structure presented in earlier chapters into geological models developed from previous work.

The $\mathrm{Q}^{-1}$ models show that $\mathrm{P}$-wave attenuation in the crust on the East Pacific Rise is dominated by two regions of low- $Q$. High levels of attenuation in the uppermost crust are presumably the result of high porosity and possibly alteration. The observation that near-surface $Q$ values decrease rapidly as crust moves off axis, a change that correlates with a decrease in seismic velocities [e.g., Vera et al., 1990; Toomey et al., 1990a; Christeson et al., 1991a, b], suggests that studies of attenuation can contribute to a better understanding of the processes which control the evolution of the shallow crust. A low-Q region beneath the rise axis is related to the high temperatures and the presence of partial melt within the crustal injection zone. If the $\mathrm{Q}$ structure within this region can be used to obtain constraints upon the distribution of temperature and partial melt, then such information can provide a basis for refining existing models of axial crustal structure [e.g., Sinton and Detrick, 1992].

\section{THE NEAR-SURFACE Q STRUCTURE}

The inversions presented in both Chapter 2 and Chapter 4 show a substantial evolution in the near-surface attenuation structure of oceanic crust with age. In Chapter 2 one-dimensional $Q$ profiles were derived from rise-parallel refraction lines both on the rise axis and $20 \mathrm{~km}$ off axis. The off-axis $\mathrm{t}^{*}$ values, which correspond to 
0.35-My-old crust, show little variation with range and are dominated by a highly attenuative upper layer for which the minimum thickness is poorly constrained but for which the maximum thickness is no greater than about $1.5 \mathrm{~km}$. The $\mathrm{t}^{*}$ data are not compatible with the model of oceanic $\mathrm{Q}$ structure obtained from laboratory measurements of ophiolite samples at ultrasonic frequencies $(1 \mathrm{MHz})$, room temperature, and the appropriate pressure [Wepfer and Christensen, 1991]. Their model includes $Q$ values of about 50 both in the uppermost crust and in the gabbros which form the lower crust. Since the frequencies at which the laboratory measurements were made are separated from those of our seismic observations by about five orders of magnitude, the discrepancy presumably results from a frequency dependence of $\mathrm{Q}$ in gabbro. One-dimensional models derived from inversions of our data require an average $Q \sim 30$ in the upper $1 \mathrm{~km}$, while values in the lower crust lie in the range 500-1000.

In contrast, upper crustal $Q$ values on the rise axis are markedly higher than those observed off-axis although the measurements have a higher degree of uncertainty due to the poorer responses of the instruments deployed on the axis. The data for OBS 1 suggest an average $Q$ value in the crust above the magma chamber of $90 \pm 10$ in good agreement with the results of Vera et al. [1990], while the inversion for AOBH 5 yields a substantially larger $Q$ of $200 \pm 40$. However, while repeating the inversions with the station corrections derived from inversions of all the data in Chapter 4 (Table 4.2) produces little change in the results for OBS 1 , the value for $A O B H 5$ is reduced to $120 \pm 15$.

Inversions utilizing the entire $t^{*}$ data set that are presented in Chapter 4 resolve a fairly narrow region of relatively high $\mathrm{Q}$ centered on the rise axis. This high-Q region appears to extend to distances of about 2-4 km off axis, but the width of the high-Q zone is not well-resolved, and the smoothing constraint will act to broaden the anomaly. The on-axis surface $\mathrm{Q}^{-1}$ values obtained in an inversion for an axis-symmetric, two-dimensional model of near surface $Q$ (Figure 4.31) are about $0.02-0.03$. Such values correspond to average $Q$ values over the upper $1 \mathrm{~km}$ of about 50-70, values appreciably less than those obtained in Chapter 2. Thus, the bulk of the high-Q anomaly probably extends only $1-2 \mathrm{~km}$ from the rise axis.

These observations correlate strongly with the observed changes in the velocity structure. Both the ESP data [Vera et al., 1990; Vera and Diebold, 1991] and delay time tomographic inversion [Toomey et al., 1991a] (Figure 1.2a) resolve high nearsurface velocities along the rise axis at $9^{\circ} 30^{\prime} \mathrm{N}$. Such a feature is also indicated by 
similar studies at $13^{\circ} \mathrm{N}$ [McClain et al., 1985; Burnett et al., 1989; Harding et al., 1989; Caress et al., 1992]. On-bottom seismic experiments [Christeson et al., 1991a, b] resolve axial velocities of $5.0-5.5 \mathrm{~km} / \mathrm{s}$ at depths of $100-200 \mathrm{~m}$ below the seafloor, values which contrast with maximum velocities of about $4.5 \mathrm{~km} / \mathrm{s}$ observed in the uppermost $300 \mathrm{~m}$ of crust off axis. Harding et al. [1990, 1991] trace a reflector across the rise axis which they correlate with the base of layer $2 \mathrm{~A}$. The depth of the reflector increases from about $200 \mathrm{~m}$ on-axis to $400-600$ within 1-2 km of the rise axis, in good agreement with a value of $400 \mathrm{~m}$ off-axis obtained from an earlier multichannel experiment in the region [Herron, 1982] and with the average layer $2 \mathrm{~A}$ thickness of $0.7 \pm 0.2 \mathrm{~km}$ determined for young Pacific crust from a compilation of sonobuoy refraction data [Houtz and Ewing, 1976].

Two explanations have been advanced to explain the changes in shallow velocity structure. First, the decrease in velocities may result from an increase in porosity due to faulting [McClain et al., 1985; Burnett et al., 1989; Caress et al., 1992]. Second, the thickness of the extrusive layer may increase off-axis due to blanketing by volcanic flows that erupt from a narrow injection zone [Toomey et al., 1990a]. Two observations lend strong support for the second explanation. First, the narrow axial summit graben observed in this area [Haymon et al., 1990], coupled with models of mid-ocean ridge magmatism [Cann, 1974; Kidd, 1977], requires that dikes must penetrate close to the surface at the rise axis. Second, the travel time between the reflection from the base of layer $2 \mathrm{~A}$ and the magma chamber remains constant irrespective of the layer 2A thickness [Harding et al., 1990, 1991], suggesting that the thickness of layer $2 \mathrm{~A}$ increases by the addition of new material from above. However, the processes are not mutually exclusive, and both extrusive thickening and in situ porosity changes may contribute to the change in upper crustal properties.

The attenuation observations of this study provide new constraints on the nature of the shallow age-dependent structure in very young oceanic crust. A comparison of $t^{*}$ values between the axial and off-axis data presented in Chapter 2 suggests that two-way propagation through the upper crust must contribute an additional $\sim 0.015 \mathrm{~s}$ to the off-axis observations. The addition of a 400 -m layer of low- $Q$ layer $2 \mathrm{~A}$ yields a $\mathrm{t}^{*}$ increase of about 0.012 and $0.005 \mathrm{~s}$ for $\mathrm{Q}$ values of 20 and 50 , respectively. Thus, the maximum observed thickening of layer $2 \mathrm{~A}$ [Harding et al., 1990, 1991] combined with the minimum shallow crustal $Q$ value observed on the Juan de Fuca Ridge by Jacobson and Lewis [1990] can barely account for the entire 
increase in shallow crustal contributions to $t^{*}$. Assuming an average value for layer $2 \mathrm{~A}$ thickening $(300 \mathrm{~m})$ yields a near surface $\mathrm{Q} \sim 12$ if thickening is to account for the entire $t^{*}$ dicrepancy. Thus, the average near-surface $\mathrm{Q}(30)$ of Jacobson and Lewis [1990] suggests that crustal thickening and in situ evolutionary processes contribute about equally to changes in the upper crustal attenuation structure. Moreover, the increase in layer $2 \mathrm{~A}$ thickness obtained from the reflection data is larger than other estimates. The on-bottom experiment of Christeson et al. [1991a, b] shows that the thickness of the very-low-velocity surficial layer $\left(V_{P}<3 \mathrm{~km} / \mathrm{s}\right)$, which they suggest is composed of highly fractured and porous basalts, increases from about $50-100 \mathrm{~m}$ on-axis to only $130-180 \mathrm{~m}$ off axis, though an underlying layer with significantly higher velocities $\left(\mathrm{V}_{\mathrm{P}}>4\right)$ does extend to a depth of at least $300 \mathrm{~m}$ off axis. On the basis of the analysis of wide-aperture profile data, Vera and Diebold [1991] suggest that the thickness of layer $2 \mathrm{~A}$ increases from about $100-150 \mathrm{~m}$ on axis to $200-300 \mathrm{~m}$ off axis. Such thickness increases require that near-surface $Q$ be less than 10 . Thus, if the near-surface observations of Jacobson and Lewis [1990] are applicable to the East Pacific Rise, crustal thickening may account for only a small fraction of the increase in attenuation observed off-axis.

ESP data [Vera et al., 1990; Vera and Diebold, 1991], travel time tomography [Toomey et al., 1990a], mutli-channel seismic data [Harding et al., 1990, 1991], and on-bottom refraction experiments [Christeson et al., 1991a, b] all suggest that the evolution of upper crustal velocities at $9^{\circ} 30^{\prime} \mathrm{N}$ primarily occurs within less than $2 \mathrm{~km}$ $(0.035 \mathrm{My})$ of the rise axis. Similarly, the inferred width of the high-Q near-surface anomaly is $1-2 \mathrm{~km}$. Significant large scale tectonic faulting, in contrast is not observed inward of the flanks of the volcanic high [Macdonald, 1982], which at this location are located about $2 \mathrm{~km}$ off axis [Wilcock et al., 1992]. On-bottom observations suggest that tectonic extension also results in abundant faulting and fissuring [e.g., Macdonald, 1982]. Indeed, at this location fine scale fissuring is observed in on the crest of the rise axis [Haymon et al., 1990; Wright and Haymon, 1991]. However, such features are probably confined to incompetent surficial pillow basalts since at larger depths extension is likely to be accommodated by magmatic injection. Since the uppermost layer is presumably highly porous on eruption, it is unclear if tectonic processes will significantly affect the velocities and attenuation in this layer. Indeed, the on-bottom experiments of Christeson et al., [1991a, b] show a small increase in near-surface velocities off-axis. The spacing of fractures that develop in deeper, more competent units is likely to be related to that of major faults. 
Since the horst and graben structures [Lonsdale, 1977] and inward facing faults $[$ Rea, 1975$]$ that characterize the flanks of the EPR are spaced at intervals of several kilometers [Wilcock et al., 1992], it is unclear whether tectonic activity alone can significantly increase the average porosity of oceanic crust on the scales sampled in this experiment. These results suggest that tectonically-induced increases in porosity do not contribute significantly to rapid changes in crustal velocities and $\mathrm{Q}$, although the seismic observations cannot entirely rule out a more gradual, smaller scale evolution of seismic properties by such a mechanism.

An alternative explanation of near-axis variations in porosity is hydrothermal activity. The EPR at $9^{\circ} 30^{\prime} \mathrm{N}$ is hydrothermally active [Haymon et al., 1991], with the high-temperature venting confined to the flanks of the axial summit graben [Haymon et al., 1991]. High-temperature seawater may produce rapid increases in porosity [Lister, 1974] and alteration [Humphris and Thompson, 1978; Alt et al., 1986], both of which act to decrease Q [Wepfer and Christensen, 1990] at ultrasonic frequencies, though changes in porosity accompanying alteration may be of more importance than alteration itself. The maximum thickness of the low- $Q$ layer off-axis is constrained to be about $1.5 \mathrm{~km}$, a value that coincides with the thickness of the lid of the axial magma chamber, which in turn must be an upper bound to the depth of axial hydrothermal circulation.

Within downflow zones and at the base of the hydrothermal cell, the hydrothermal fluids will cool the host rock and induce thermal contraction, cracking [Lister, 1974], and an increase in porosity. In contrast, upflow zones will be characterized by hydrothermal fluids whose temperatures exceed those of the host rock and will therefore not be sites of additional thermal cracking. Moreover, the deposition of hydrothermal minerals within such regions [e.g., Delaney et al., 1987] will tend to reduce pre-existing porosity. While high-temperature vents are known to be confined to the rise axis the patterns of hydrothermal circulation are poorly known [e.g., Goldfarb and Delaney, 1988]. On the basis of a preferred rise-parallel orientation for hydrothermal veins in the Oman ophiolite Nehlig and Juteau [1988] argue that hydrothermal circulation is primarily confined to planes orientated parallel to the rise axis. However, the orientation of hydrothermal veins may reflect the configuration of the upflow zone rather than that of the whole hydrothermal system. At $9^{\circ} 30^{\prime} \mathrm{N}$ the gaps between high temperature vents are compatible with a pattern of circulation in which downflow zones are also located on axis [Haymon et al., 1991]. However, the along-axis continuity of low-temperature venting inferred from the the 
distribution of fauna suggests that the downflow is located off axis [Haymon et al., 1991]. If a significant proportion of the downflow for the large scale hydrothermal circulation occurs away from the rise crest, then increases in porosity occurring as crust moves off-axis from the vicinity of upflow to downflow zones might explain the observed increase in upper crustal attenuation.

McClain et al. [1985] suggest that near-surface velocities may increase slightly between crustal ages of 0.1 and $0.3 \mathrm{My}$ at $12^{\circ} 50^{\prime} \mathrm{N}$, an observation that is apparently supported by the delay-time tomographic inversions [Burnett et al., 1989; Caress et al., 1992]. McClain et al. [1985] suggest that such a change may be a consequence of a reduction in near-surface porosity resulting from the filling of voids by the alteration products of passive off-axis hydrothermal circulation [e.g., Lister, 1981; Fisher et al., 1990]. Indeed, such a mechanism is generally accepted as the explanation for a large increase in layer $2 \mathrm{~A}$ velocities observed in much older crust [Houtz and Ewing, 1976; Jacobson, 1992]. The inversions presented in Chapter 4 and a careful analysis of the $t^{*}$ data set suggest that a similar trend in attenuation may occur at this site. The data are compatible with a small 5-10\% reduction in near-surface $Q$ between 0.15 and 0.35 My-old crust. However, the effect is fairly subtle and might be explained equally well by $Q$ anisotropy. Since near surface cracks and fissures are preferentially orientated parallel to the rise axis [Macdonald, 1982; Wright and Haymon, 1991], attenuation may be higher for waves propagating perpendicular to the rise axis than for the rise-parallel paths used to constrain the 0.35-My-old structure. To distinguish between these two mechanisms would require a better azimuthal distribution of ray paths.

\section{THE AXIAL LOW-Q REgION}

Several early models of mid-ocean ridges [e.g., Cann, 1974; Dewey and Kidd, 1977; Palister and Hobson, 1981], which were constructed with few geophysical constraints, call for large molten magma chambers extending from upper crustal depths to the base of the crust. However, even on the East Pacific Rise, which has a high magmatic budget such models have been known for some while to be inconsistent with seismological observations. Early refraction experiments on the EPR [Orcutt et al., 1975, 1976; Rosendahl et al., 1976] detected a low-velocity zone whose top coincided with an upper crustal reflector [Herron et al., 1978, 1980; Hale et al., 1982] which was interpreted to be the roof of a magma chamber. However, the vertical extent and velocities of the low-velocity anomaly detected by the 
refraction experiments were not consistent with a very magma body. Indeed later refraction experiments which included rise-crossing paths [Lewis and Garmany, 1982; Bratt and Solomon, 1984; McClain et al., 1985] placed strong limits on the maximum dimensions of axial magma bodies. More recently, a large body of seismological data including extensive multi-channel reflection profiles [Detrick et al., 1987; Mutter et al., 1988; Kent et al., 1990; Vera et al., 1990], expanding spread profiles [Harding et al., 1989; Vera et al., 1990], and two delay-time tomographic experiments [Burnett et al., 1989; Toomey et al., 1990a; Caress et al., 1992] has resulted in a much better understanding of the upper crustal structure along the axis of the East Pacific Rise between $9^{\circ} \mathrm{N}$ and $13^{\circ} \mathrm{N}$. A thin, continuous, 1-2 km-deep magma lens is present along large segments of the rise axis [Detrick et al., 1987]. The width of the magma lens at $9^{\circ} 30^{\prime} \mathrm{N}$ is about $1.2 \mathrm{~km}$ [Kent et al., 1990], and the maximum thickness is much less than $1 \mathrm{~km}$ [Toomey et al., 1990a] and may be as small as a few tens of meters [Kent et al., 1990]. The magma lens is underlain by a more extensive region of lowered seismic velocities extending to the base of the crust. Along-axis discontinuities in the magma chamber reflections [Mutter et al., 1988] and variations in the low-velocity zone [Toomey et al., 1990a] are correlated with morphological discontinuities evident in bathymetric data. Thus, the seismic data are more compatible with models of mid-ocean ridges which include only a small sill-like magma body underlain by a more extensive region of partial melt [Sleep, 1975, 1978; Nicolas et al., 1988; Sinton and Detrick, 1992].

None of the more recent seismological studies however, places very strong constraints upon the structure of the lower crust. The multi-channel seismic reflection data do not image the Moho reflection immediately beneath the rise rise axis [e.g., Detrick et al., 1987], while the ESP data only constrains the average crustal velocity beneath the magma body [Harding et al., 1989; Vera et al., 1990]. Delay time tomographic inversions could, in principle, resolve the lower crustal structure. However, the travel time inversion at $9^{\circ} 30^{\prime} \mathrm{N}$ [Toomey et al., 1990a] does not include waves propagating at depths significantly deeper than $3 \mathrm{~km}$ beneath the rise axis. The inversion at $12^{\circ} 50^{\prime} \mathrm{N}$ [Burnett et al., 1989; Caress et al., 1992] does include a significant number of $\mathrm{PmP}$ (Moho turning) phases propagating at lower crustal depths, but the solution is based on a single iteration using ray-paths derived from a one-dimensional velocity model and it is not clear how well the resulting model resolves details of the sub-axial lower crustal structure. 
Obtaining good constraints on the structure beneath the magma body is essential if models of fast spreading mid-ocean ridges are to be further refined. Both the magma chamber model of Sleep [1975, 1978], which is based on thermal considerations, and the model of Nicolas et al. [1988] include only a small completely molten region. Indeed, the sill-like shape of the magma body in model proposed by Sleep [1975] is in excellent agreement with the seismic reflection data. However, both the models include large cumulate mush zones extending to the base of the crust and several kilometers to either side of the rise axis. Although the melt fraction is not explicitly stated, the implication of the models is that throughout much of this region this fraction is relatively high $(\geq 10 \%)$. In contrast, a more recent model proposed by Sinton and Detrick [1992] for the EPR at $9^{\circ} 30^{\prime} \mathrm{N}$ includes a smaller upper crystal mush zone lying immediately beneath the magma body in which the melt fraction exceeds about $40 \%$ and underlain by a more extensive region containing considerably smaller melt fractions. Since the attenuation measurements presented in this thesis include a large number of observations for paths crossing the rise axis at a range of depths, the data may help to distinguish between such models.

\section{Laboratory Determinations of $\mathbf{Q}$ at High Temperatures in Mafic Rocks}

Ideally, the $\mathrm{Q}^{-1}$ models obtained in this study should be combined with laboratory measurements of $\mathrm{Q}$ to obtain tight constraints on temperatures and the spatial distribution of partial melt beneath the EPR. However, the validity of such a procedure depends upon the accuracy and applicability of laboratory studies. Obtaining measurements that are valid for both the compositional, pressure, and temperature conditions in the Earth and for the frequency, scale, and strain rates of seismic observations is a formidable task [e.g., Jackson, 1986; Christensen and Wepfer, 1989]. A number of measurements have been carried out on mafic and ultramafic samples at elevated temperatures [Woirguard and Gueguen, 1978; Berckhemer et al., 1979; 1982; Sacks and Murase, 1983; Kampfmann and Berckhemer, 1985; Sato and Manghnani, 1985; Manghnani et al., 1986; Sato et al., 1988, 1989; Gueguen et al., 1989; Jackson et al., 1992]. The majority of these works were motivated by a desire to understand the properties of the asthenosphere and are thus primarily devoted to ultramafic samples.

The earlier measurements were obtained at room pressure. Woirguard and Gueguen [1978] obtained a limited number of $\mathrm{Q}_{S}$ measurements at seismic 
frequencies by studying the forced torsional oscillations of natural peridotite and polycrystalline forsterite and enstatite samples. Berckhemer et al. [1979] deduced Q spectra of peridotite up to $1 \mathrm{~Hz}$ from measurements of the creep response to the sudden changes in a uniaxial load. Sacks and Murase [1983] used a pulse transmission technique to measure $\mathrm{Q}_{\mathrm{P}}$ in peridotites at frequencies of $40-300 \mathrm{kHz}$ and temperatures up to $1180^{\circ} \mathrm{C}$. However, the most comprehensive set of measurements were obtained by Berckhemer et al. [1982] and Kampfmann and Berckhemer [1985] for a variety of mafic and ultramafic materials. Using a forced torsional oscillation method, Berckhemer et al. [1982] systematically measured $\mathrm{Q}^{-1}$ in a dunite and a synthetic forsterite over the frequency band $0.003-30 \mathrm{~Hz}$ and at temperatures ranging to values above the solidus. Kampfmann and Berckhemer [1985] repeated such measurements for a number of rock samples including basalts, gabbros, and a peridotite. Figure 5.1 shows results for peridotite and gabbro. Qualitatively, the form of the variations of $Q^{-1}$ with temperature and frequency is similar for all samples studied. A quantitative description of the attenuation spectra [Kampfmann and Berckhemer, 1985] suggests that three mechanisms are operating. The general increase with temperature results from high-temperature background, a mechanism that is poorly understood in rocks [e.g., Jackson and Anderson, 1970]. At lower temperatures an absorption peak which shifts to higher temperatures with increasing frequency is probably the result of a dislocation mechanism [Woirguard and Gueguen, 1978; Gueguen et al., 1981]. Above the solidus, whose precise temperature is not presented for these samples, a third frequency-independent mechanism is also operative. For these experiments in which the melt sample cannot be more than a few percent, the contribution from this latter mechanism is significant at the highest temperatures but never exceeds $50 \%$ of the total attenuation.

A significant problem with high-temperature measurements conducted at ambient pressures is that absorption in microcracks which result from thermal stresses may contribute to the measurements [e.g., Jackson et al., 1984]. Moreover, since the minimum depth of our observations of the axial low- $\mathrm{Q}$ zone is about $2 \mathrm{~km}$, it is not clear how applicable room pressure measurements are to our study. Sato et al. $[1988,1989]$ present $Q_{P}$ measurements for peridotite as a function of temperature obtained at pressures of $0.20,0.48$ and $0.72 \mathrm{GPa}$. The values were obtained by applying a spectral ratio method to pulse transmission measurements at $60-880 \mathrm{kHz}$, frequencies that are several orders of magnitude above the seismic 
band. The results show no resolvable frequency dependence and once $Q$ and the temperature are normalized to the values at the solidus, the trends in $Q$ are independent of pressure. The $Q$ values extend to melt concentrations of about $5 \%$, and show no pronounced contribution from the small melt component, increasing smoothly with temperature through the solidus. On the basis of calculations of the activation volume, Sato et al. [1989] argue that the attenuation mechanism is grainboundary damping, which they equate to the high temperature background. Sato and Sacks [1990] suggest that since the grain boundary mechanism is dependent upon the grain size, laboratory measurements at high frequencies and small grain sizes may scale to the lower frequencies and larger grain sizes typical of seismic observations. On the basis of the absence of a strong frequency dependence in their results they argue that their measurements fall within a regime that is equivalent to the region of weak frequency dependence in the absorption band model of Anderson and Given [1982]. By comparing oceanic geotherms derived from heat flow data in older oceanic crust with the temperatures their results predict for models of seismic Q, Sato and Sacks [1989] proceed to derive a small correction term to account for the differences between the laboratory and upper mantle conditions.

However, the extrapolation of their results to seismic frequencies, which is based on the assumption of a common grain boundary mechanism, may be without foundation [Jackson et al., 1992]. Jackson and Anderson [1970] suggest that the high-temperature background almost certainly results from a poorly understood mechanism involving dislocations and not grain-boundaries, though attenuation may be enhanced near grain boundaries due to the high concentrations of dislocations in such regions. A dislocation mechanism is also favoured by Gueguen et al. [1981] and Kampfmann and Berckhemer [1985]. Gueguen et al. [1989] present Q values obtained for single forsterite crystals at seismic frequencies. The measurements show that attenuation increases with temperature in a manner similar to that observed in polycrystaline samples. Moreover, $\mathrm{Q}^{-1}$ values increase by about a factor of 2 after the crystal is deformed. Such observations rule out a grain boundary mechanism as the cause of high-temperature background and lend strong support to a dislocation mechanism.

However, determining the appropriate mechanism for seismic observations requires measurements obtained simultaneously at high pressure and seismic frequencies. A forced torsional oscillation apparatus to obtain such measurements has recently been developed [Jackson et al., 1984, Jackson and Paterson, 1987]. The 
first measurements obtained at high temperatures are presented by Jackson et al. [1992] for a dunite sample from the same formation as that used by Berckhemer et al. [1982]. The results cannot be directly applied to the tomography experiment since the maximum temperatures studied were only $1000^{\circ} \mathrm{C}$. However, the $\mathrm{Q}_{\mathrm{S}}$ values obtained at $0.3 \mathrm{GPa}$ are not grossly inconsistent with the dunite measurements of Berckhemer et al. [1982] and the single forsterite crystal results of Gueugen et al. [1989], lending support to a dislocation mechanism at higher pressures. The results also suggest that the lower temperature measurements in the studies of Berckhemer et al. [1982] and Kampfmann and Berckhemer [1985] may be influenced by microcracking.

Measurements using forced torsional oscillations of rock cylinders are limited to small melt concentrations since they require a competent sample. While measurements at all melt concentrations are feasible using pulse transmission techniques, the only direct measurements available for basaltic magmas [Sato and Manghnani, 1985; Manghnani et al., 1986] were obtained at temperatures above the liquidus and frequencies of about $1 \mathrm{MHz}$. Measured levels of attenuation are high with $\mathrm{Q}$ values less than 10 at temperatures just above the liquidus and show a strong frequency dependence that is fit very well with a Gaussian distribution of the logarithm of the relaxation times. However, the relaxation times are very short, and extrapolating the results to seismic frequencies yields negligible levels of attenuation. Thus, while other mechanisms may be important at lower frequencies it is possible that the the level of attenuation in basaltic melts at seismic frequencies may be very low.

\section{Constraints on the Temperature and Melt Concentration Beneath the Axial Magma Body}

It is fairly clear from the discussion above that the experimental data obtained to date are not adequate to allow a quantitative conversion of the models of $\mathrm{Q}^{-1}$ presented in this thesis to meaningful models of the temperature and partial melt distribution beneath the rise axis. However, more qualitative comparisons are still worthwhile. As noted above the bulk of laboratory studies have been confined to ultramafic materials that are representative of mantle compositions. None of the $t^{*}$ estimates presented in this thesis are for phases that propagate within the mantle. For the $\mathrm{Q}^{-1}$ inversions, the deepest wave paths beneath the rise axis belong to a secondary arrival follows the mantle phase propagating between the two outer 
refraction lines. Indeed, the high amplitudes observed for rise perpendicular paths strongly suggest that this phase turns within the Moho transition zone beneath the rise axis, a region which seismic models of ophiolites suggest is composed of layered mafic and ultramafic cumulates [e.g., Karson et al., 1984; Collins et al., 1986]. Assuming that the axial low- $\mathrm{Q}$ zone is $8 \mathrm{~km}$ wide, the $\mathrm{Q}$ values obtained for this phase range from 70-140 (Figure 4.35), with the better constrained values above 100. Halving the width of the region to $4 \mathrm{~km}$ similarly halves the $Q$ values.

These values seem incompatible with the results of Sato et al. [1988, 1989]. The results obtained for peridotite at $0.2 \mathrm{GPa}$, a pressure that corresponds very closely to that at the Moho, predict $Q_{P} \sim 6$ at the solidus and suggest that the values obtained for the longest range paths require temperatures $200-300^{\circ}$ below the solidus. Applying the correction derived from comparisons with heat flow data [Sato and Sacks, 1989] decreases the predicted temperature further by about $50^{\circ}$. Simple models of the axial thermal structure preclude temperatures at the base of the crust below about $1200^{\circ} \mathrm{C}$ [Sinton and Detrick, 1992]. Experimental measurements [e.g., Ito and Kennedy, 1967] show that the solidus of a lherzolite at this depth is below $1200^{\circ} \mathrm{C}$ while that of a fully depleted harzburgite is about $1400^{\circ} \mathrm{C}$. While some ultramafic cumulate layers may have similarly high solidus temperatures, the average solidus temperature within the Moho transition zone will probably be significantly lower than $1400^{\circ} \mathrm{C}$. Thus, the predictions of Sato et al. $[1988,1989]$ seem implausible.

In contrast, the Qs values measured by Berckhemer et al. [1982] for dunite at room pressure are entirely compatible with our observations. Assuming $Q_{\mathrm{P}} / \mathrm{Q}_{\mathrm{S}}=2.25$ and using laboratory values obtained at $10 \mathrm{~Hz}$, the $\mathrm{Q}^{-1}$ values modelled in the Moho transition zone correspond to temperatures of $\sim 1200-1260^{\circ} \mathrm{C}$ for an $8-\mathrm{km}$-wide anomaly and $\sim 1260-1320^{\circ} \mathrm{C}$ for a $4-\mathrm{km}$ wide anomaly, values that are in excellent agreement with the thermal constraints of Sinton and Detrick [1992]. The $Q_{S}$ measurements for peridotite [Kampfmann and Berckhemer, 1985] require temperatures of $900-1250^{\circ} \mathrm{C}$. The large range of possible temperatures for this sample results from the presence of a prominent low-temperature absorption peak (Figure 5.1). However, as noted above, the experimental measurements of attenuation at the lower temperatures may be augmented by microfracturing.

Figure 5.2 shows $Q_{P}$ values at $10 \mathrm{~Hz}$ for the basalt and gabbro obtained by Kampfmann and Berckhemer [1985]. At low temperatures all the models show similar trends with $Q$ increasing exponentially with the reciprocal of the temperature. 
At temperatures close to the solidus, the affect of the absorption peak merging with the high temperature background (Figure 5.1) is to decrease the sensitivity of $Q$ to changes in temperature. Models derived from inversions of $t^{*}$ estimates for diffractions beneath the magma chamber (Figure 4.28) include well-resolved $Q$ values as low as 30 directly below the axial magma body. Inspection of Figure 5.2 suggests that such values might be compatible with very low melt fractions or even with no melt at all. However, the errors accompanying a quantitative comparison of the two sets of measurements may be large. Moreover, in the absence of experimental measurements it is not entirely clear how $Q$ will vary in rocks containing a larger melt fraction.

The frequency independent contribution to $\mathrm{Q}^{-1}$ at supersolidus temperatures inferred from the laboratory measurements [Kampfmann and Berckhemer, 1985] suggests that the contribution to $\mathrm{Q}^{-1}$ arising from melt increases rapidly with temperature. Extrapolating such results to higher melt fractions would predict $Q$ values much smaller than our results even at moderate $(\sim 10 \%)$ melt fractions. A frequently cited mechanism for melt-related anelasticity at low melt concentrations is melt squirt [Mavko and Nur, 1975], a process which involves local transient flow between cracks at different orientations in response to shear stresses. Quantitative models of melt squirt for various melt configurations [O'Connell and Budiansky, 1977; Mavko, 1980; Schmeling, 1985] suggest that the melt squirt contribution to $\mathrm{Q}^{-1}$ is strongly dependent upon the distribution of melt. The attenuation resulting from $<1$ $\%$ melt distributed in thin sheets along grain boundaries may be equivalent to that resulting from $5 \%$ melt in tubules along grain edges [Mavko, 1980]. The equilibrium distribution of melt is almost certainly a network of tubules along triple junctions [Cooper and Kohlstedt, 1986]. However as Kampfmann and Berckhemer [1985] point out an equilibrium melt distribution may not be achieved in their experiments and so it is not clear if the levels of melt-generated attenuation they obtain are applicable.

A feature of melt squirt mechanisms is that the levels of attenuation for a given melt configuration increase rapidly with the melt concentration. An alternative mechanism which has been invoked to explain the transient creep response of olivine-basalt partial melts and which involves changes in the size of triple junctions in response to isotropic stresses [Green et al., 1990] also shows substantial increases in attenuation with temperature and melt fraction. However, both mechanisms are based upon melt distributed in thin sheets or tubules along grain 
boundaries within a competent matrix, and it is not clear what mechanisms will be operative in a rock containing much higher melt concentration, particularly once melt fractions reach values close to $50 \%$, at which point the solid aggregate is no longer bonded [Marsh, 1989]. Indeed if there is no significant attenuation mechanism in basaltic melts other than that detected at ultrasonic frequencies [Sato and Manghnani, 1985; Manghnani et al., 1986], seismic Q may be relatively high in predominantly molten regions.

The finite difference models suggest that the diffraction beneath the magma chamber propagates over a large range of depths beneath the rise axis. While the depth of propagation of this phase will depend upon the velocity structure which is poorly known, a qualitative inspection of the finite difference results suggests that for the velocity model of Vera et al. [1990] significant energy may propagate at depths extending from the magma lens at $1.5 \mathrm{~km}$ depth to about $3.0 \mathrm{~km}$ depth. There are two possible explanations for the attenuation of this phase. The first is that the recorded signal propagates through a region with a melt fraction much less than about $10 \%$. Such an interpretation, which arises directly from the extrapolation of the laboratory measurements and theoretical models of attenuation, places strong limits upon the depth extent of the crystal mush zone beneath the magma lens. Conversely, it is conceivable that the melt fractions and consequently $\mathrm{Q}^{-1}$ at $2-3 \mathrm{~km}$ depth are much higher than the $t^{*}$ measurements suggest. In such a case the $Q$ values of the models might be representative of propagation both within a relatively high-Q magma body and a crystal mush region with very high melt fractions directly below. This second explanation is, however, highly speculative. Not only does it rely on unsupported assumptions about $\mathrm{Q}$ at high melt concentrations but it is probably incompatible with the seismic data. If the magma body and crystal mush zone represent a relatively high- $\mathrm{Q}$ path for propagation of the diffracted phase then either the diffracted arrival should be compatible with velocities within a largely molten region or the arrival should be emergent since energy propagating in higher velocity regions at larger depths would have higher levels of attenuation. Neither of these effects is observed. The diffracted arrival does not appear to be emergent, and the arrival times seem consistent with ray-theoretical paths crossing the rise axis at about $2.5 \mathrm{~km}$ depth. The lowest velocities imaged in the tomographic inversion [Toomey et al., 1990a] are about $5 \mathrm{~km} / \mathrm{s}$, well above the values of $<3 \mathrm{~km} / \mathrm{s}$ predicted for molten basalt [Murase and McBirney, 1973; Sato and Manghnani, 1985; Manghnani et al., 1986]. Therefore, it seems reasonable to conclude that the 
diffraction below the magma chamber propagates in a region of containing a low melt fraction and that the thickness of the crystal mush zone is fairly small.

The $\mathrm{Q}^{-1}$ models for the lower crust (Figures 4.25 and 4.37) suggest that axial $\mathrm{Q}^{-1}$ values in this region may range from 25-50, with average values close to 30 . Such results are lower than the average $Q$ value of 50 in the crust beneath the rise axis determined from $t^{*}$ values for rise parallel paths in Chapter 2 (see the discussion of Figure 4.25 in Chapter 4). However, the value of $60 \pm 10$ determined for AOBH 5 may be biased by the effects of signal distortion and signal-generated noise in the direct recording system of the AOBH (Appendix A). The value of $40 \pm$ 10 for OBS 1 , which may also be biased because of the very small frequency interval used to obtain $t^{*}$ estimates, is compatible with the $Q$ values derived from inversions of rise crossing paths. Moreover, the paths for these phases (Figure 2.19) propagate significant distances in the Moho transition zone which has a higher $\mathrm{Q}$ than the lower crust (Figures 4.34 and $4.38 \mathrm{c}$ ).

The axial $Q$ values are dependent upon the width of the low- $Q$ anomaly, which is not very well constrained by the data. Indeed, because of the uncertainties in the paths and in the $t^{*}$ estimates, the detailed $Q$ structure in the lower crust is not well resolved. However, the similarity of $Q$ values with those determined for the diffraction below the magma chamber suggests that the homologous temperature and the melt fraction may be fairly similar to the values at shallower depths.

Although the precise location of wave paths in the lower crust is not well constrained the relative depths of paths beneath the rise axis is probably correct. Figure 5.3 shows average $t^{*}$ values for all phases passing below the magma chamber plotted against the depth of the path beneath the rise axis for both the DOBH and AOBH data (the diffraction below the magma chamber is placed at $2 \mathrm{~km}$ depth). Variations in $t^{*}$ values with path depth may reflect changes in $Q$ values but will also be affected by structure off axis, particularly the width of the anomalous zone. Thus, the lower values for the diffraction below the magma chamber and the Moho turning phase at $3 \mathrm{~km}$ depth probably result from the smaller width of the anomaly at shallower depths. For both $\mathrm{AOBH}$ and DOBH data the maximum averaged $t^{*}$ values are for paths crossing the rise axis at $4 \mathrm{~km}$ depth, although at greater depths the trends are noticeably different. The DOBH $t^{*}$ values are generally relatively constant for paths crossing the rise axis between 3 and $6 \mathrm{~km}$ depth suggesting that the homologous temperature and melt fraction may also be relatively invariant. The average $t^{*}$ for paths between the outer refraction lines is 
placed at $7 \mathrm{~km}$ depth and is markedly lower. Such a difference is most simply explained in terms of a decrease in the homologous temperature resulting from the more mafic composition of the Moho transition zone. In contrast to the DOBHs, the AOBH $t *$ values which are subject to larger uncertainties but for which the wave paths are probably better known, show a progressive decrease for paths crossing the rise axis between 4 and $7 \mathrm{~km}$ depth. Such values are compatible with an appreciable decrease either in the homologous temperature or the melt fraction through the lower crustal section.

Clearly, if the wave paths are correct, the $t^{*}$ values for the AOBHs and DOBHs are incompatible. The effect of including data from both instruments in the inversion is to produce a small decrease in $t^{*}$ values for paths in the lower crust (Figures 4.25 and 4.37). The discrepancy may result from systematic errors in $t^{*}$ estimates arising from corruption from other phases or from non-attenuative frequency-dependent components of propagation. However the possibility exists that changes in the wave path locations resulting from a better understanding of the velocity structure in this region might account for the apparent discrepancy between AOBH and DOBH $t^{*}$ estimates and significantly improve the resolution of the $Q^{-1}$ inversions in the lower crust.

The primary result of this section is that axial $Q$ values at depths extending from no more than $1 \mathrm{~km}$ below the roof of the axial magma chamber to the base of the crust are consistent with relatively small $(\ll 10 \%)$ melt fractions that show only subtle variations with depth. The models of Sleep $[1975,1978]$ and Nicolas et al. [1988], which include a melt-rich cumulate mush extending throughout the crust. If such models are correct then the width of such a region within the lower crust must be small. The model of Sinton and Detrick [1992] includes a smaller mush zone with high melt fractions extending only to mid-crustal depths and underlain by a more extensive region with smaller melt fractions. Our results are compatible with this model and strongly suggest then the dimensions of the crystal mush zone may be fairly small extending less than $1 \mathrm{~km}$ below the magma lens. Following Ryan [1987], Sinton and Detrick [1992] suggest that the depth of the magma lens might be controlled by the depth of neutral buoyancy of the basaltic magma. If this is the case, compaction theory [McKenzie, 1984] would suggest that even in the presence of a small buoyancy force magmas would be delivered to such depths fairly efficiently. Such considerations seem to be consistent with models which include very small melt fractions at depth and a relatively thin crystal mush zone. On the 
other hand, the densities of magmas erupted on mid-ocean ridges are smaller than those for more basic magmas [e.g., Stolper, 1980; Sparks et al., 1980] and so the melt concentration in the crustal column may not be controlled by compaction theory but rather by changes in the depth of neutral buoyancy with crystal fractionation.

\section{Along-Axis Variations}

Although the inversions show apparent along-axis variations in the axial low-Q anomaly, when compared to the magnitude of the axial anomaly resolved in inversions for two-dimensional structure across the rise axis, such variations are fairly small. The variance reduction achieved between one-dimensional models and the axis-symmetric two-dimensional model is over 50\% (Figure 4.24) and is about $70 \%$ when only paths crossing the rise axis are considered. In contrast the decrease in variance between two- and three-dimensional models is no more than $20 \%$. However, while two-dimensional models fit the data fairly well, the inversions presented in the last chapter suggest that some along-axis variations are resolved by the data.

The diffractions below the magma chamber require about a $25 \%$ increase in $Q^{-1}$ values in the shallowest portions of the low-Q anomaly toward the north of the experiment (Figure 4.38). As discussed in the last section such results do not correlate with the largest negative velocity perturbations in the delay time tomographic models [Toomey et al., 1990a] which show a pronounced minimum $2 \mathrm{~km}$ south of the center of the experiment. Toomey et al. [1990a] argue that the largest low-velocity anomaly which falls between two devals (deviations from axial linearity) at $9^{\circ} 28^{\prime} \mathrm{N}$ and $9^{\circ} 35^{\prime} \mathrm{N}$, represents a region of elevated temperatures along a thermally segmented rise axis. To evaluate the plausibility of such an explanation it is necessary to consider the expected characteristics of a high-temperature portion of a thermally segmented rise axis. Axial temperatures below the magma body are presumably limited to values close to the liquidus temperature of the magma. Although erupted lavas do show compositional variations along this portion of the EPR [e.g., Langmuir et al., 1986], these will produce negligible changes in the liquidus temperature within the experimental area. A thermally robust portion of the rise axis is thus more likely to be characterized by higher melt fractions close to the rise axis and a broader thermal anomaly. Inspection of the velocity structure of Toomey et al. [1990a] (Figure 1.2) shows that the largest negative velocity anomaly coincides with the narrowest width of the axial low velocity zone. Indeed, the width 
of the anomaly progressively increases toward the north of the experiment, and values averaged over $4 \mathrm{~km}$ width show a trend that is very similar to the $\mathrm{Q}^{-1}$ models (Figure 4.38). Because of the limited resolution of the $\mathrm{Q}^{-1}$ inversions, the increase in $\mathrm{Q}^{-1}$ values we observe to the north might also be explained by an increase in width of the anomaly. Thus, both the velocity data and $\mathrm{Q}^{-1}$ data in the northern portion of the experiment are compatible with either an increase in width of the thermal anomaly underlying the magma chamber or an increase in the partial melt content over a fairly broad region.

If largest axial velocity anomalies in the model of Toomey et al. [1990a] are well resolved, they are presumably the manifestation of along-axis variations in the size of the melt lens and the crystal mush zone, a region that may not be sampled by the $t^{*}$ measurements. Since this feature does not correlate with the broader anomaly observed in both the $\mathrm{Q}^{-1}$ and velocity models it is not compatible with a long-term segmented structure. Indeed simple arguments can be presented to argue against long-lived segmentation. The depth of the magma lens [Harding et al., 1990, 1991] is relatively constant along this portion of the East Pacific Rise. The multi-channel seismic reflection data [Barth and Mutter, 1991] suggests a progressive increase in crustal thickness of $\sim 700 \mathrm{~m}$ per $10 \mathrm{~km}$ from north to south across the experiment area. However, such an observation does not appear compatible with gravity measurements [Madsen et al., 1990] which suggest a relatively uniform crustal thickness. Moreover, a cursory inspection of the record sections for shots between the two outer refraction lines (e.g., Figure 4.4) shows that the arrival times of the first-arriving mantle phase and the delay of higher amplitude secondary crustal phases with respect to the mantle phase do not show the large systematic variations that would be expected for a change of more than $2 \mathrm{~km}$ in crustal thickness. Thus, the crustal thickness may be relatively constant within the experiment area. Since the EPR extends primarily by constructional volcanism, in the absence of lateral motions of solid material and crustal thickness variations the time averaged heat budget must be constant along the rise axis throughout this region. Along-axis variations in the magnitude of the thermal anomaly and partial melt fraction must be short lived. Indeed the structure within the magma body and upper crustal portion of the thermal anomaly may be primarily controlled by the episodicity of eruptions and the efficiency of hydrothermal cooling, processes that have time scales of the order of $10^{2}-10^{3}$ years in this region [Haymon et al., 1991]. 
The deeper $Q$ structure also shows along-axis variations. The lower crustal model (Figures 4.37 and 4.38 ) shows small but possibly significant increases in $Q^{-1}$ toward the south and north of the experiment. The $\mathrm{Q}$ structure determined for the longest wave paths (Figures 4.37 and 4.38), which is probably representative of the structure near the axial Moho transition zone, also shows a minimum near the center of the experiment. $\mathrm{Q}^{-1}$ values in this region show a very large apparent increase to the south which is not well resolved since it is primarily the result of data from one instrument. However, this increase correlates with a general north-to-south increase in delay times observed on all instruments [Toomey et al., 1990b], although changes in delay times could also result from a proposed southward increase in crustal thickness [Barth and Mutter, 1991] rather than from an increase in the axial thermal anomaly. Although the minimum amplitudes of the along-axis variations in the magnitude of the low- $\mathrm{Q}$ anomaly coincide fairly closely at all depths, the models do not extend for sufficient distance along the rise axis to determine if the upper and lower crustal structures are well correlated.

Large scale mid-ocean ridge segmentation resulting from transform faults and large overlapping spreading centers is commonly explained in terms of gravitational instabilities within the upper mantle [Whitehead et al., 1984]. The length scale of such instabilities $(\sim 100 \mathrm{~km})$ [Whitehead et al., 1984, Crane, 1985; Schouten et al., 1985 ] is too large to be resolved by this experiment, though such processes may contribute to the trends in $\mathrm{Q}^{-1}$ structure observed across the experiment region. Toomey et al. [1990a] propose that along-axis variations in the magnitude of the axial thermal anomaly may correlate with smaller scale segmentation on a scale of 10-15 km which on the EPR results is manifested by small offsets of the axial summit caldera and changes in orientation of the rise axis [e.g., Macdonald et al., 1992]. To the extent that such segmentation is correlated with the distribution of eruptions and hydrothermal activity [Haymon et al., 1991] this seems plausible. Indeed, the axial chamber chamber has a segmented structure [Mutter et al., 1988] with the width of the magma lens varying systematically between segments that are bounded by devals [Kent et al., 1991a, b]. However, small scale segmentation may also be determined by the brittle response of the uppermost crust to spreading forces and it is not clear if such a mechanism requires a strong correlation with variations in the underlying thermal structure. 


\section{CONCLUSIONS}

In this thesis we have demonstrated the feasibility of using simple assumptions about the attenuation of seismic waves to obtain models of apparent $\mathrm{Q}^{-1}$ in the crust and Moho transition zone of the East Pacific Rise at $9^{\circ} 30^{\prime} \mathrm{N}$. Many features of the $Q$ structure appear very similar to the velocity models obtained at $9^{\circ} 30^{\prime} \mathrm{N}$ [Toomey et al., 1990a; Vera et al., 1990], with low $Q$ values corresponding to regions of low velocities. However, since well-resolved $Q$ values vary by over an order of magnitude (from about 25-30 to 500-1000), while compressional wave velocities lie between 2 and $8 \mathrm{~km} / \mathrm{s}$, the t* measurements are particularly sensitive to regions of anomalously low $\mathrm{Q}$. The $\mathrm{Q}^{-1}$ models resolve low-Q regions in the uppermost crust and beneath the rise axis at depths greater than $2 \mathrm{~km}$. Figure 5.4 is a schematic section across the East Pacific Rise summarizing how such observations can be incorporated into a geological model of crustal structure.

In the shallow crust both seismic velocities and $\mathrm{Q}$ are observed to decrease rapidly with seafloor age within 1-2 km of the rise axis. Following Toomey et al. [1990a] it is now generally accepted that the decrease in near-surface velocities at this site results primarily from an increase in the thickness of surficial high-porosity basalts due to blanketing by flows erupted along the rise axis [Harding et al., 1990, 1991; Christeson et al., 1990a, b; Vera and Diebold, 1991]. However, the increase in layer 2A thickness is not sufficient to account for the changes in $Q$ values averaged over the upper $1 \mathrm{~km}$ unless near-surface $Q$ values are much lower than observed elsewhere [Jacobson and Lewis, 1990]. Thus, in situ changes in porosity probably also contribute to decreases in $Q$ offaxis. Since tectonic fissuring and fracturing is primarily observed at distances greater than $2 \mathrm{~km}$ from the rise axis, we suggest that hydrothermal processes may contribute substantially to the rapid evolution of crust off-axis. Within the upflow zones which are located on-axis hydrothermal deposits may reduce preexisting porosity, while in downflow zones located off-axis porosity will increase as a result of thermally induced cracking (Figure 5.4).

The uppermost portion of the low-Q anomaly resolved in the inversions presented in this thesis coincides closely with the low-velocity anomaly imaged by delay time tomography. However, the $t^{*}$ data set also includes a substantial number of highamplitude Moho-turning phases which cross the rise axis at lower crustal depths and whose travel times are not included in the inversion for velocity conducted to data [Toomey et al., 1990a]. The $\mathrm{Q}^{-1}$ models resolve a fairly constant axial $\mathrm{Q}$ of about 25-50 extending from about $2.5 \mathrm{~km}$ depth to the base of the crust. Laboratory studies suggest 
that such values require at most small melt fractions. Our preferred model of the axial structure (Figure 5.4) includes a small crystal mush zone whose thickness is constrained to be no more than $1 \mathrm{~km}$, underlain by a much larger region of low melt concentrations. Such a model suggests that magma is transported efficiently through the lower crust to the axial magma chamber.

There are several ways in which future work could improve the resolution of attenuation studies. The minimum source-receiver separation was limited to $4 \mathrm{~km}$ in this study due to the presence of the water wave within the spectral-estimation window at shorter ranges. As a result we are unable to resolve vertical variations in the attenuation structure at crustal depths less than $1 \mathrm{~km}$. Studies employing near-bottom sources and receivers can overcome this problem. The resolution of $\mathrm{Q}^{-1}$ models within the axial low$\mathrm{Q}$ zone is limited by the large uncertainties in wave paths and might be substantially improved by a better knowledge of the velocity structure. An important requirement of a model of the lower crustal velocity structure is that it predicts the high amplitudes of the Moho-turning phase. However, calculations of seismic amplitudes require that the effects of attenuation be taken into account. Such considerations suggest that seismic $Q$ and velocity should be determined by simultaneous inversions. Indeed, high-resolution models of both $Q$ and velocity might be able to constrain variations in both homologous temperature and melt fraction, properties that cannot be independently determined using a single seismic parameter. The analysis of rise-perpendicular record sections in Chapter 4 was rendered particularly difficult by the large separation of traces. Refraction experiments utilizing rise-perpendicular lines of closely spaced shots could significantly improve our understanding of axial structure and might be suitable for forward modelling by the full-waveform finite difference technique. Additional constraints upon rise axis structure might be obtained by constraining seismic models to show variations compatible with simple models of thermal structure across the rise axis. In particular, the width of the axial low-Q anomaly should be compatible with thermal models. Finally, while the formidable difficulties associated with experimental measurements of seismic properties at high temperatures and frequencies and appropriate frequencies must be recognized, there is clearly a need for more laboratory measurements obtained under conditions more closely approximating those of seismic propagation within the oceanic crust and uppermost mantle. 


\section{FIGURE CAPTIONS}

Figure 5.1 Experimentally determined $\mathrm{Q}_{\mathrm{S}}{ }^{-1}$ of (a) peridotite and (b) gabbro at ambient pressure as a function of frequency and temperature [from Kampfmann and Berckhemer, 1985].

Figure 5.2 $\mathrm{Q}_{\mathrm{P}}$ values for a plagioclase basalt (solid), an olivine-plagioclase basalt (dashed), and gabbros with mean grain diameters of $0.25 \mathrm{~mm}$ (dot-dashed) and $0.5 \mathrm{~mm}$ (dotted) plotted against homologous temperature. The results are obtained from the experimental determinations of $\mathrm{Q}_{S}$ by Kampfmann and Berckhemer [1985] assuming $\mathrm{Q}_{\mathrm{S}} / \mathrm{Q}_{\mathrm{P}}=2.25$ and solidus temperatures for the basalt and gabbros of $990^{\circ} \mathrm{C}$ and $1040^{\circ} \mathrm{C}$, respectively.

Figure 5.3 (a) Averaged DOBH $t *$ values for paths passing beneath the magma body plotted against the depth of the approximate wave path beneath the rise axis (the diffracted phase is plotted at $2 \mathrm{~km}$ depth). Station corrections are applied to the $t^{*}$ data before averaging. The solid line and error bars do not include measurements for DOBH 10 which has anomalously low values for these paths even after application of the station correction. The dashed line shows the effect of including DOBH 10. (b) As for (a) except data are for the AOBHs.

Figure 5.4. Schematic cross-section of the East Pacific Rise with a geological interpretation of the low- $Q$ anomalies (speckled). The increase in near-surface attenuation off-axis results from an increase in the thickness of pillow basalts and from thermally induced fracturing in hydrothermal downflow zones. The axial low- $Q$ anomaly includes a small upper crustal magma chamber comprising a magma lens and a crystal mush zone underlain by a more extensive region containing at most a small fraction of partial melt. 

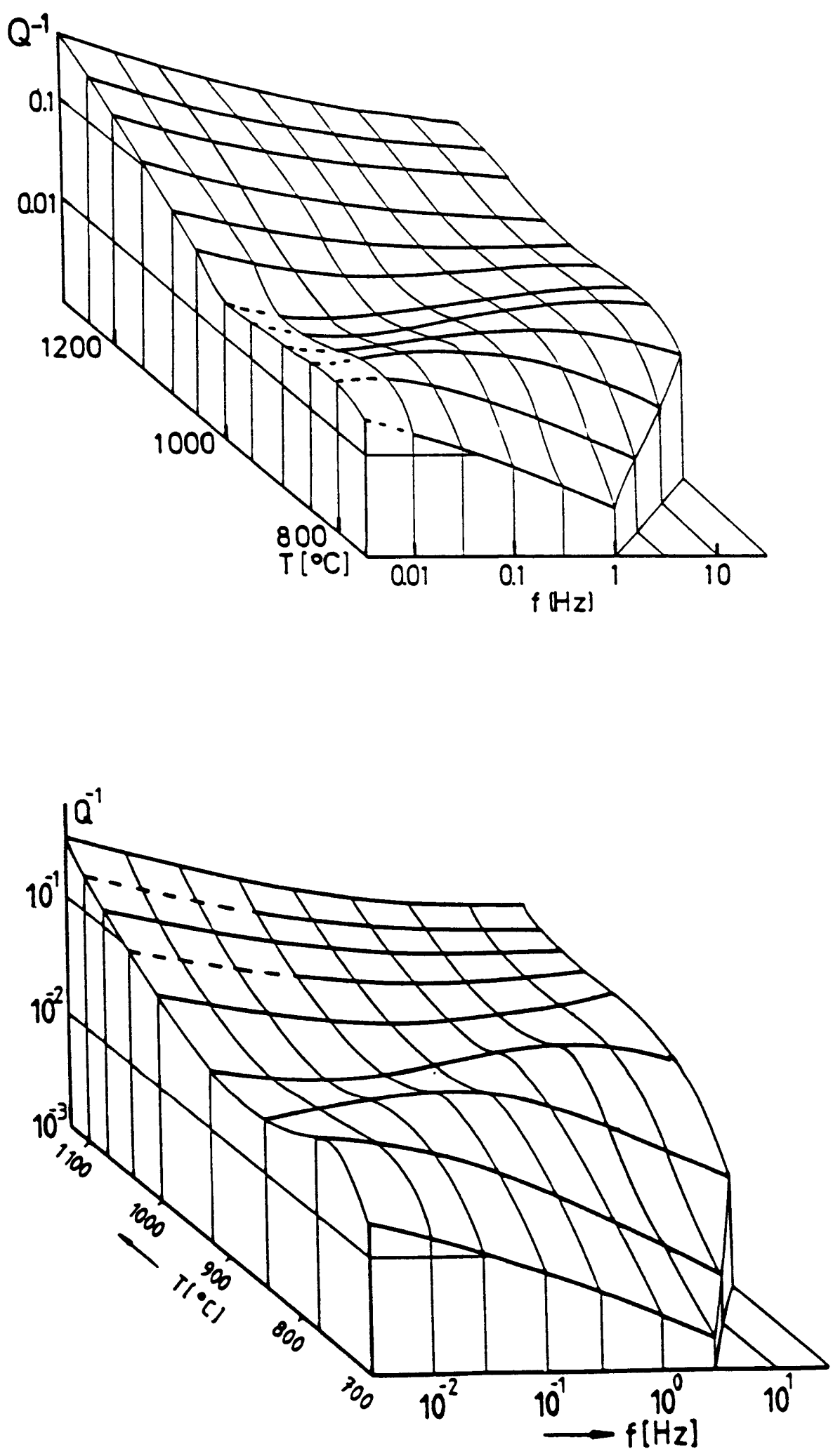

Figure 5.1 


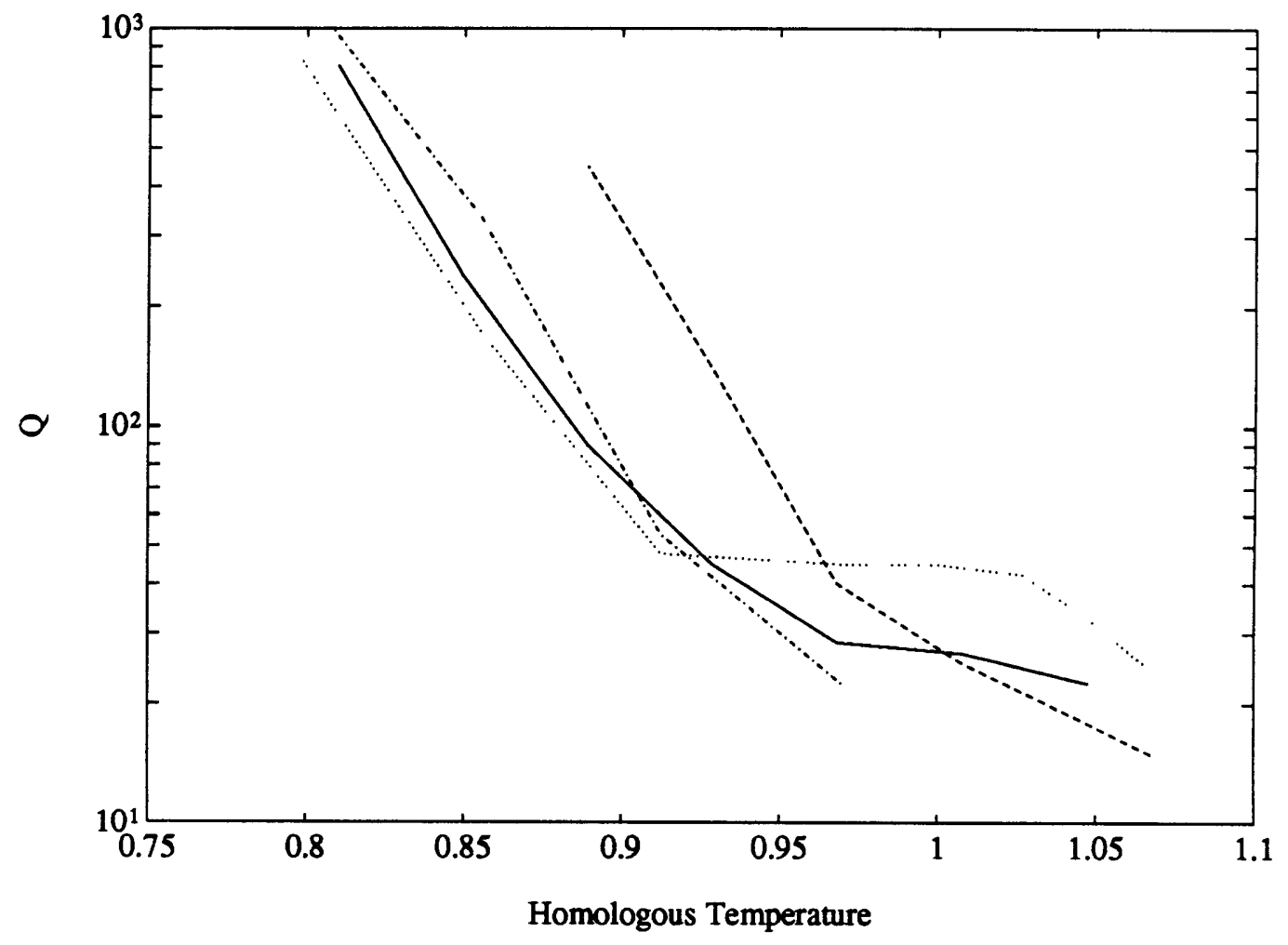

Figure 5.2 

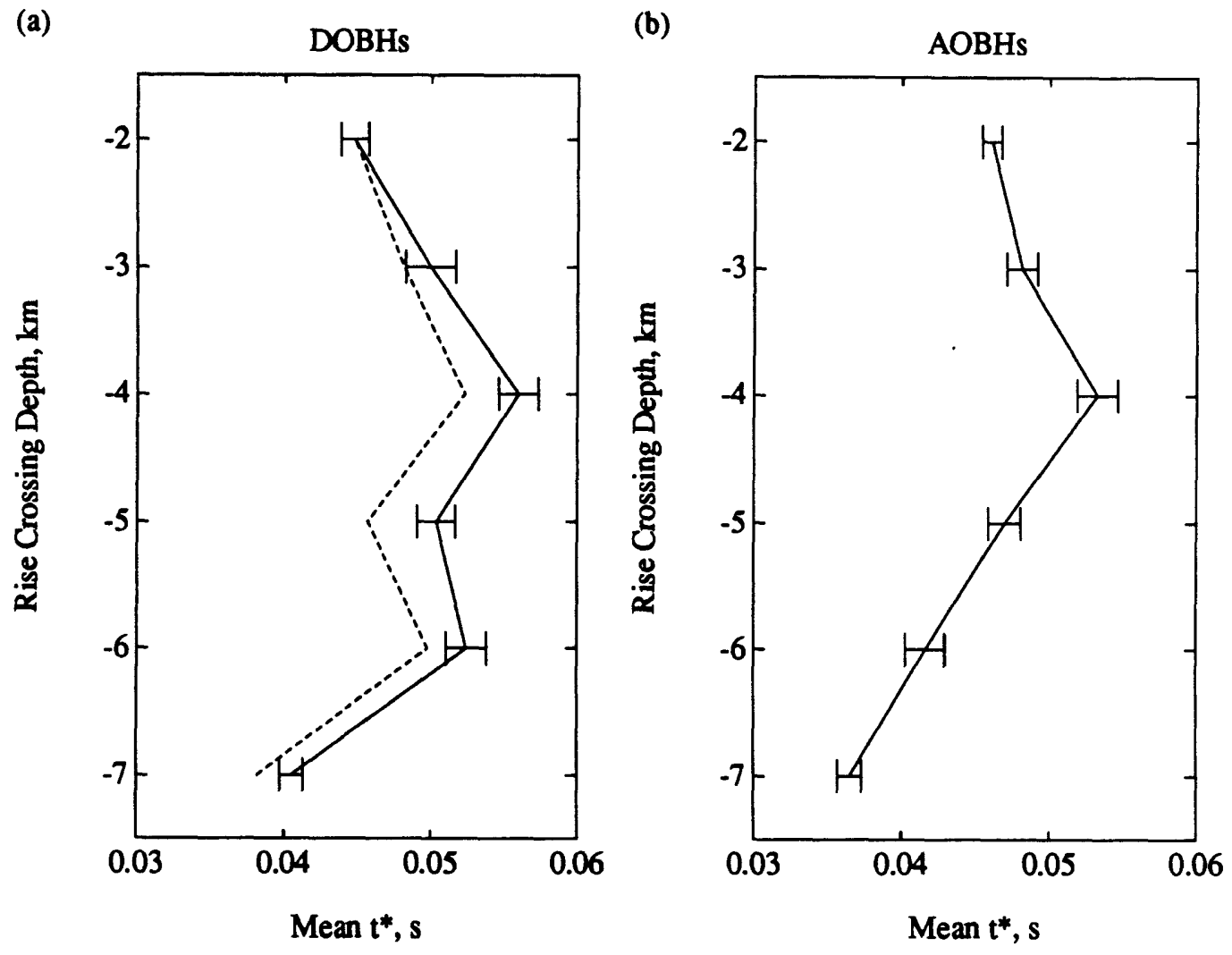

Figure 5.3 


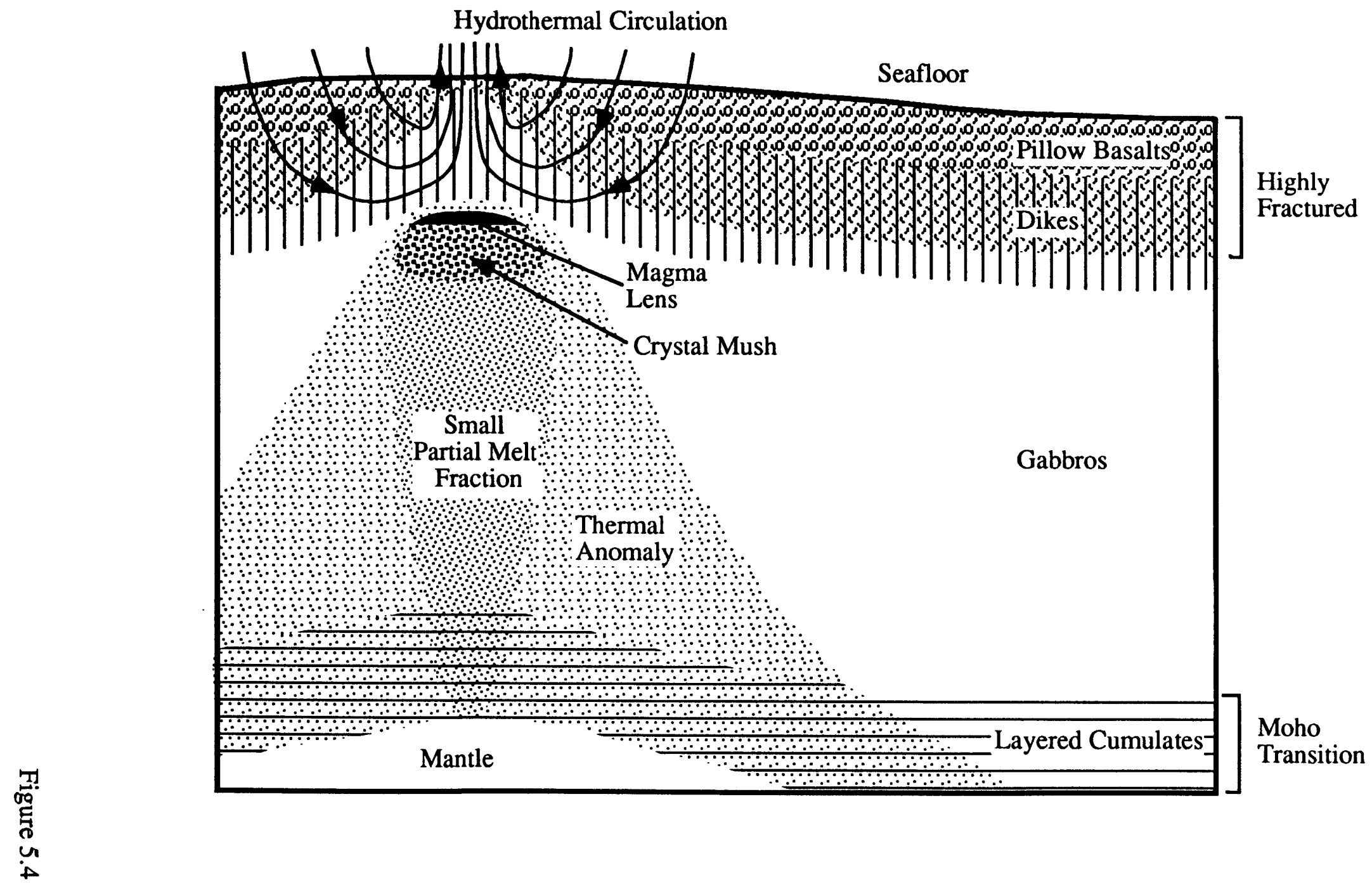




\section{APPENDIX A}

\section{THE INSTRUMENT RESPONSES}

\section{INTRODUCTION}

The EPR tomography experiment utilized three different seismic recorders, the WHOI analog ocean bottom hydrophone (AOBH), the WHOI digital ocean bottom hydrophone (DOBH) and the MIT ocean bottom seismometer (OBS). The attenuation studies presented in this work require a good knowledge of the amplitude (or power) response of each instrument. In this Appendix the response of each instrument in discussed in turn. The AOBH incorporates a direct analog tape recorder, and its response must be measured by a series of tests. The DOBH is a sophisticated instrument for which a theoretical transfer function can be derived. An on-bottom self-calibration test provides a check on these results. The response of the OBS has been described in detail by Tréhu [1982] and is discussed only briefly here.

\section{ANALOG OCEAN BOTTOM HYDROPHONE}

\section{Introduction}

From the first deployments in 1976 to the last in 1988, the WHOI AOBH was the workhorse of the Woods Hole seismology group. About 150 deployments were successfully made without loss of an instrument and with a data recovery rate well in excess of $90 \%$. The design and construction of the AOBH are described in detail by Koelsch and Purdy [1978]. The instrument package, comprising an Ocean \& Atmospheric Science (OAS) model E-2SD pressure-compensated hydrophone, a recording package, an AMF model 325 acoustic-release transponder, strobe lights, a radio beacon, and 4 glass flotation spheres, is generally deployed 3-5 $\mathrm{m}$ above the seafloor. There are four channels, namely two low-frequency channels with gains separated by $20 \mathrm{~dB}$ which record the seismic data, a modulated envelope-detected high-frequency channel which allows precise identification of water wave arrival times, and IRIG-B time code carried on a $25 \mathrm{HZ}$ signal. These are recorded continuously using a slow-speed, direct-recording analog tape recorder which has a dynamic range of about $30 \mathrm{~dB}$. 
To permit use of waveform amplitude data and to detect instrument malfunctions, two calibration tests are routinely carried out. First, prior to each cruise the amplitude response of the low-frequency channels is measured by recording a sinusoidal signal generated by an oscillator connected in parallel with the hydrophone. Second, during each deployment 2-s-long 10-Hz, 200- $\mu \mathrm{V}$ (peak-topeak) calibration signals are introduced in series with the hydrophone four times near the end of each hour. However, while some use has been made of waveform amplitude ratios [Fischer and Purdy, 1986] and of the principal features of amplitude spectra of microearthquake recordings [Toomey et al., 1988; Wilcock et al., 1990; Kong et al., 1992], the majority of work utilizing AOBH data has been confined to the use of $\mathbf{P}$ wave arrival times and to qualitative comparisons of amplitudes. Since no previous work had required accurate corrections for instrument response, it was necessary in this study to determine the frequency response of the low-gain, lowfrequency channels which were used for all the spectral measurements, the reliability and uncertainty in the estimated responses, and the characteristics of tape saturation and other non-linear effects. The results are presented in detail below.

\section{The Low-Frequency Low-Gain Channel}

The principal objective in designing the AOBH was to enable the determination of water wave and $\mathrm{P}$ wave arrival times on the high- and low-gain channels, respectively, with a timing uncertainty of $10 \mathrm{~ms}$ or better. To this end the gains on the low-frequency channels were set conservatively high so that the ratio of signal to ambient noise and not the instrument gain would control the amplitude threshold for phase identification. As a consequence, the explosive source deployed in the tomography experiment generates $\mathrm{P}$-waves that saturate the low-frequency, highgain channel at all but the largest ranges, while the low-gain channel is frequently saturated at ranges below $10-15 \mathrm{~km}$. For this reason, the waveforms used in this work are obtained exclusively from the low-gain channel.

Figure A1 is a flow chart showing the recording system for the low-frequency low-gain channel. The hydrophone output passes through a preamplifier and a lowpass ( $\sim 100 \mathrm{~Hz}$ cutoff) filter at which point the low- and high- gain channels diverge. The appropriate low-gain amplification is applied to the signal which is added to a 5 $\mathrm{kHz}$ bias current and passed through the head driver (voltage to current) amplifier which feeds directly into the tape head. The data are recorded on $1 / 4$ " $(0.6 \mathrm{~cm}) 3 \mathrm{M}$ Scotch magnetic tape at a speed of $1 / 40 \mathrm{in} / \mathrm{s}(0.064 \mathrm{~cm} / \mathrm{s})$. The tape recorder is 
designed to ensure a smooth, constant-speed motion of tape over the tape heads with flutter and wow never exceeding 10\% [Koelsch and Purdy, 1978].

Figure A2 is a flow chart showing the playback and analog-to-digital conversion scheme. Immediately after deployment the $1 / 4$ " data tape is transcribed onto $1 / 2$ " $(1.3 \mathrm{~cm})$ Ampex 787 precision magnetic tape for archiving. The data tape is played back at $17 / 8 \mathrm{in} / \mathrm{s}(4.7 \mathrm{~cm} / \mathrm{s}, 75$ times real time) on a Crown 700 Series tape recorder, and the output, after attenuation to $1 \mathrm{~V}$ (peak to peak), is recorded on a Honeywell $5600 \mathrm{C}$ FM tape recorder at a tape speed of $15 \mathrm{in} / \mathrm{s}(38 \mathrm{~cm} / \mathrm{s})$. The $1 / 2$ " tape is subsequently used for playback and analog-to-digital conversion. For this step the output channels of the Honeywell recorder are amplified to $10 \mathrm{~V}$ (peak to peak), and the low-frequency channels are bandpass-filtered using Krohn-Hite 3500 filters. Analog-to-digital conversion is performed by a Digital LPA11-K 12-bit converter attached to a Digital Vax 11-780 computer. A short term/long-term event detection algorithm allows retention of selected portions of the digital data.

To accurately determine arrival times on the data channels requires that the channels are correctly aligned with the time code channel. Misalignments arise from relative skew of the tape record and reproduce heads. Skew can be measured straightforwardly for both the AOBH/Crown record/reproduce tape head and for the Honeywell tape heads by generating a test tape for which the input to all channels is the same. Unfortunately, due to an initial oversight and a subsequent hardware failure, no skew test tape was available for the particular Honeywell recorder used for the tomography experiment transcription. To overcome this problem an alternative playback scheme involving analog-to-digital conversion directly from the original 1/4" tape was devised (Figure A2). However, since the quality of the signal on the $1 / 4$ " tape degrades rapidly after several playbacks, the waveform data used for the attenuation studies were obtained using the conventional playback scheme. The skew introduced by the Honeywell was shown subsequently to be negligible.

While a theoretical response may straightforwardly be calculated for most components of the AOBH instrument and playback system, this is not feasible for the 1/4" tape recorder [D. E. Koelsch, pers. comm., 1989]. Moreover, the fidelity of low speed analog direct recording systems is notoriously unreliable and is the major source of variability and uncertainty in the $\mathrm{AOBH}$ response. Therefore, to determine the instrument response of the $\mathrm{AOBH}$, an extensive series of tests was conducted. By carefully calibrating the results using the $10-\mathrm{Hz}$ calibration pulses recorded 
during the tomography experiment an estimate of the absolute amplitude response of the AOBH during the deployment can be recovered.

\section{Response Tests}

\section{Testing Configuration}

The instrument response test comprised recording the attenuated output of a signal generator and analyzing the results, after playback and analog-to-digital conversion. To overcome the problems associated with reproducing the hydrophone input impedance, the preamplifier was bypassed during these tests (Figure A1). To facilitate more efficient data reduction, the alternative playback scheme (Figure A2), which eliminates the Honeywell tape recorder, was used. The transfer function $f_{T}$ determined from the tests can be related straightforwardly to the full instrument response (including playback) i by

$$
i(s)=f_{1}(s) f_{2}(s) f_{3}(s) f_{4}(s) f_{T}(s)
$$

where $s$ is the complex frequency and the transfer functions are defined as follows:

$$
\begin{aligned}
f_{1}(s)= & \text { Hydrophone response. } \\
f_{2}(s)= & \text { Hydrophone capacitance and preamplifier input network. } \\
f_{3}(s)= & \text { Honeywell record-reproduce, input attenuator, and output amplifier } \\
& \text { response. }
\end{aligned}
$$

$\mathrm{f}_{4}(\mathrm{~s})=$ Correction for changes in the settings of the anti-alias bandpass filters Before discussing the test results these four components of the full response not incorporated in the tests will be presented in turn

\section{Hydrophone response}

The hydrophone sensor used in the AOBHs is a high-quality pressurecompensated hydrophone designed to operate at all ocean depths. The manufacturers specifications require a flat response in the interval $0-500 \mathrm{~Hz}$ with a tolerance of $\pm 10 \%(1 \mathrm{~dB})$ and a sensitivity of $-87 \mathrm{~dB}$ with respect to $1 \mathrm{~V} / \mu \mathrm{bar}$. Thus hydrophone response may be simply written

$$
f_{1}(s)=44.6 \mathrm{~V} / \mathrm{bar}
$$

Hydrophone capacitance and preamplifier input network

The schematic for the preamplifier used in the AOBHs is shown in Figure A3. At frequencies above $1 \mathrm{~Hz}$ the capacitance $\mathrm{C}_{2}$ can be ignored. The hydrophone capacitance and preamplifier input form a simple RC high-pass filter for which the transfer function is 


$$
f_{2}(s)=G_{1} G_{2} \frac{s / \omega}{1+s / \bar{\omega}}
$$

where the two gains $G_{1}$ and $G_{2}$ are defined in terms of the components shown in Figure A3

$$
\begin{aligned}
G_{1} & =\frac{R_{2}}{R_{1}+R_{2}+R_{3}} \\
G_{2} & =\frac{R_{4}+R_{5}}{R_{4}}
\end{aligned}
$$

and the characteristic frequency is

$$
\sigma=\frac{C_{1}+C_{H}}{\left(R_{1}+R_{2}+R_{3}\right) C_{1} C_{H}}
$$

Component values, which in some cases differ slightly between instruments, are presented in Table A1 along with the resulting amplifier parameters. In all cases the total gain is approximately 10 and the characteristic frequency is less than $1 \mathrm{~Hz}$. Honeywell Record-Reproduce (with attenuation and amplification)

The Honeywell $5600 \mathrm{C}$ is a high quality FM tape recorder. A simple calibration using a monochromatic source shows that the record-reproduce amplitude response, including the required attenuation and amplification, is flat to $\pm 0.5 \%$ within the frequency band of interest. However, since the Honeywell underwent hardware repairs and a fresh calibration between the times of transcription and analog to digital conversion, the transfer function for the tomography experiment may have a higher uncertainty, although it is almost certainly insignificant when compared with other contributions. The absolute gain of record-reproduce system is unity with an uncertainty of $\pm 10 \%(1 \mathrm{~dB})$. The gain of the attenuators and amplifiers is adjustable with increments of $3 \mathrm{~dB}$ and $2 \mathrm{~dB}$, respectively. However, since absolute amplitude measurements are always referenced to the $10 \mathrm{~Hz}$ calibration pulses, the gains are of no importance provided the tape recorder is not overloaded. Thus, the transfer function can be simply written

$$
f_{3}(s)=1
$$

\section{Anti-alias Filter}

The anti-alias filter is a Krohn-Hite Model 3500C bandpass filter with low and high cutoff frequencies that are independently adjustable between $2 \mathrm{~Hz}$ and $200 \mathrm{kHz}$. The response in the "'Max Flat" setting approximates a 4-pole Butterworth, a filter which has a transfer function that is optimally flat within the passband and which can be written 


$$
f_{4}(s)=\frac{1}{\prod_{i=1}^{4}\left(\frac{s}{\omega_{H C}}-(-1)_{i}^{1 / 8}\right) \prod_{i=1}^{4}\left(\frac{s}{\omega_{L C}}-(-1)_{i}^{1 / 8}\right)}
$$

where $\omega_{\mathrm{HC}}$ and $\omega_{\mathrm{LC}}$ are the low and high cutoff angular frequencies and $(-1)^{1 / 8_{i}}$ are chosen to be the four roots with negative real components. A filter calibration was conducted. Within the passband, the response lies within $2 \%$ of the theoretical response. However, the cutoff frequencies can only be set to an accuracy of $10 \%$. Thus filter corrections are only reliable within the passband. For this reason the passband used for the instrument tests $(0.4-80 \mathrm{~Hz})$ was chosen to be larger than that typically used for data reduction $(3-40 \mathrm{~Hz}$ or $2-67 \mathrm{~Hz})$.

\section{Sine Wave Tests}

Tests conducted using a sine wave signal provide the most straightforward method to measure the amplitude response and saturation characteristics of the AOBH.

\section{Amplitude}

For each instrument one minute of data was recorded at input voltages of 2,10 , and $50 \mathrm{mV}$ (peak to peak), for about 10 frequencies logarithmically spaced between 1 and $60 \mathrm{~Hz}$. After playback and analog-to-digital conversion, a simple least squares inversion was used to determine the amplitude of the best fitting sine wave. Such a method is less efficient than a calculation of the root mean squared (rms) amplitude but avoids biases that might result from signal distortion or signal-generated noise. The method gives almost identical results to those obtained using a fast Fourier transform but is computationally more efficient for long time series, and the misfit can be used to obtain an estimate of the uncertainty.

Figure A4 shows examples of the normalized amplitude responses for AOBHs 1 and 7 for three different input voltages. For each instrument the responses estimated from the 2 and $10 \mathrm{mV}$ input signals are almost identical. However, the 50 $\mathrm{mV}$ test consistently yields lower normalized amplitudes than the 2 and $10 \mathrm{mV}$ tests, indicating that at this input voltage the $\mathrm{AOBH}$ tape recorder is at least partially saturated. A comparison between the two instruments shows that while the responses at lower frequencies are very similar, the upper frequency limit differs significantly between the two instruments. At $40 \mathrm{~Hz}$ the response of AOBH 7 is 16 $\mathrm{dB}$ below the peak response while for AOBH 1 it is only $4 \mathrm{~dB}$ lower. 
In Table A2 the amplitude responses derived from these tests are presented for all instruments. The results are normalized to $10 \mathrm{~Hz}$, the frequency of the calibration pulse which can be used to determine absolute amplitudes. The values for the 2 and $10 \mathrm{mV}$ tests represent a good estimate of the amplitude response below saturation levels. In terms of bandwidth, the responses of AOBH 1 and 7 (Figure A4) represent extremes of the range of measured $\mathrm{AOBH}$ responses.

\section{Saturation}

To analyze the saturation characteristics of the AOBH the response at selected frequencies was determined for a larger set of input voltages. Figure A5 shows the results for AOBHs 1 at $5,10,20$ and $40 \mathrm{~Hz}$, while Table A3 lists results at $10 \mathrm{~Hz}$ for all instruments. The tests show that saturation occurs fairly abruptly. Below $20 \mathrm{mV}$ the response is nearly linear with a less than $20 \%$ decrease in sensitivity between 2 and $20 \mathrm{mV}$ at all frequencies, while at $50 \mathrm{mV}$ the instrument is almost completely saturated and shows an almost constant recorded amplitude at higher voltages. Indeed, at input voltages above $100 \mathrm{mV}$ the recorded amplitudes actually decrease slightly. The saturation voltage decrease slightly with increasing frequency. Signal Distortion and Signal Generated Noise

Measurements of spectral slopes from highly attenuated waveforms require good estimates of higher frequency spectral amplitudes in the presence of a predominantly low-frequency signal. Such estimates will be severely compromised if the instrument-playback response includes significant levels of signal distortion and signal-generated noise, since the spectral leakage from low-frequency signals may swamp the low-amplitude, high-frequency component of the input signal.

The majority of response tests were conducted using a Heath SG-1271 signal generator which has poor specifications for harmonic distortion. To assess the importance of spectral leakage additional tests were conducted on AOBH 1 using an accurate signal generator which has a harmonic distortion $60 \mathrm{~dB}$ below the input signal. The harmonic distortion $D_{H}$ in units of $d B$ is defined by

$$
D_{H}=-10 \log _{10}\left(\frac{P(F)}{P(n F)}\right)
$$

where $P(f)$ is the spectral power density at frequency $f, F$ the input frequency, and $n$ is the order of the harmonic of interest. Alternatively, distortion $\mathrm{D}$ may be quantified by measuring the spectral power outside a small bandwidth $\Delta(2 \mathrm{~Hz})$ centered upon the input frequency $F$ 


$$
D=-10 \log _{10}\left(\sum_{1-\frac{f=F-\Delta / 2}{f=F_{\text {Nyquist }}} P(f)}^{\sum_{f=F_{\min }}^{F+\Delta / 2} P(f)}\right)
$$

A choice of $F_{\min }=0$ results in a measure of spectral leakage to all frequencies while a value $F_{\min }=f-\Delta / 2$ yields estimates of spectral leakage to higher frequencies, a more meaningful quantity when considering the biases distortion might introduce into spectral measurements of $t^{*}$.

Figure A6 shows examples of power spectra recorded by AOBH 1 for 10 and 20 $\mathrm{Hz}$ input signals. In both cases spectral amplitudes away from the input frequency are markedly higher than ambient noise levels recorded with no input signal. The levels of distortion for AOBH 1 measured according to equation (A7) are shown in Figure $A 7$ for input frequencies of $5,10,20$, and $40 \mathrm{~Hz}$ together with ambient noise levels. The results show that signal distortion on the AOBH may be significant. At very low input amplitudes distortion is not resolved above ambient noise levels, while at higher amplitudes values range between about -30 and $-20 \mathrm{~dB}$. For the 5 and $10 \mathrm{~Hz}$ tests, the lowest levels of distortion $(\sim-30 \mathrm{~dB})$ are observed for input voltages which are below saturation levels. One result of saturating the instrument is to increase the levels of distortion by up to $10 \mathrm{~dB}$ for lower frequency input signals.

The effect of spectral leakage upon the expected values of $t^{*}$ estimates can be predicted from these tests if it is assumed that the process is linear. Equation (1.7) can be used to estimate a theoretical power spectrum for an incoming signal assuming an impulsive source. In the frequency domain this input is multiplied by the power response, cross-correlated with the $10 \mathrm{~Hz}$ power spectrum shown in Figure A6a, and then divided by the power response. Equation (1.7) is then used to obtain the expected value of the $t^{*}$ estimate. The results are shown in Figure A8. Estimates for low $t^{*}$ signals are little affected by spectral leakage but $t^{*}$ estimates obtained for higher actual $t^{*}$ values achieve a maximum value that may actually decrease with further inputs in $t^{*}$. The $t^{*}$ value at which such biases become apparent is dependent upon the frequency. Choosing a frequency band whose minimum recorded powers are $1 / 1000$ the maximum recorded amplitude results in $t^{*}$ estimates that are little effected below $t^{*}=0.04 \mathrm{~s}$ but which achieve a maximum value of about $0.06 \mathrm{~s}$. While decreasing the frequency interval further would increase 
the maximum resolvable $t^{*}$ values slightly, it will also increase the uncertainties accompanying the straight line fit to the logarithm of the power spectrum. In this thesis $t^{*}$ values are estimated over frequency intervals which are constrained to have power ratios of no more than 1000 . Indeed, many such intervals are similar to those that would be obtained from inspection of the signal to ambient noise ratios. Two Frequency Tests

A small number of tests were conducted on AOBH 1 to measure the response of the AOBH to a signal comprised of two sine waves of different frequencies. The relative signal amplitude was held constant while the absolute amplitude was varied. Figure A9 shows the results for 5 and $20 \mathrm{~Hz}$ signals with an amplitude ratio of 20:1. Over a $40 \mathrm{~dB}$ range of input voltages, the response is approximately linear, while the relative amplitude of the higher frequency decreases markedly once the saturation voltage is achieved.

\section{Square Wave Tests}

Sine wave tests provide an accurate method of measuring the amplitude response of a linear system. However, they provide no direct estimate of the phase response, nor do they provide an adequate method to detect non-linear response characteristics for broadband signals. To remedy these deficiencies the response of the AOBHs was also measured using a low-frequency $(\sim 0.3 \mathrm{~Hz})$ square wave with a range of input amplitudes.

Considering a square wave of period $\mathrm{T}$ and unit amplitude

$$
\begin{array}{ll}
\mathrm{f}(\mathrm{t})=1 & (0<\mathrm{t}<\mathrm{T} / 2) \\
\mathrm{f}(\mathrm{t})=0 & (\mathrm{~T} / 2<\mathrm{t}<\mathrm{T})
\end{array}
$$

the Fourier transform of one cycle is

$$
F(\omega)=-\frac{2 i}{\omega}\left[\cos \left(\frac{\omega t}{2}\right)-1\right]
$$

This is a notched response with maximum amplitudes proportional to the reciprocal of the frequency. While the decrease in spectral amplitudes with frequency does not follow the relationship predicted for attenuated seismic waveforms, the general decline in spectral amplitudes can be considered analogous to an attenuated wave when considering a relatively small frequency interval. Assuming an impulsive source the decrease in spectral amplitudes between 10 and $40 \mathrm{~Hz}$ is equivalent to an intrinsic attenuation of $t^{*}=0.015 \mathrm{~s}$ while the amplitude decrease over smaller 
frequency intervals is representative of higher degrees of attenuation. The discrete Fourier transform corresponding to equation (A8) is

$$
\begin{array}{ll}
\mathrm{F}(\mathrm{n})=\frac{2 \mathrm{i} N}{\pi \mathrm{n}} & \mathrm{n}=1,3, \ldots \mathrm{N} \\
\mathrm{F}(\mathrm{n})=0 & \mathrm{n}=2,4, \ldots \mathrm{N}
\end{array}
$$

where $\mathrm{N}$ is the number of samples. A smoothed discrete Fourier transform obtained from the square wave response will thus be

$$
\mathrm{F}_{\mathrm{S}}(\mathrm{n}) \sim-\frac{\mathrm{iN}}{\pi \mathrm{n}}
$$

This can be converted to the Fourier transform of a delta function $F_{D}(\omega)=N / T$ straightforwardly

$$
\mathrm{F}_{\mathrm{D}}(\mathrm{n}) \sim \frac{\mathrm{ipN}}{\mathrm{T}} \mathrm{F}_{\mathrm{S}}(\mathrm{n})
$$

Such an operation, which is the equivalent of differentiation, is unstable at high frequencies since noise levels are amplified. However, by taking the Fourier transform of several cycles $(\sim 10)$ and smoothing the result using a running mean over $9 \mathrm{M}$ samples where $\mathrm{M}$ is the number of cycles, a reasonably stable estimate of the the impulse response over the frequency band of the AOBH can be obtained. The procedure is illustrated in Figure A10.

\section{Amplitude}

Figure A11 shows the square wave amplitude response measured at various input amplitudes for $\mathrm{AOBHs} 1$ and 7 together with the sine wave results presented earlier. At lower input voltages the shapes of the amplitude responses are in good agreement, the response of AOBH 7 being markedly lower than AOBH 1 at higher frequencies. The apparently lower amplitudes observed for the square wave tests below about $4 \mathrm{~Hz}$ are an artifact of smoothing the spectral estimates. As was the case for the sine wave tests the effects of saturation is to decrease the relative amplitudes of high frequencies. However, in comparison with the sine wave tests, saturation appears to occur at markedly lower input voltages, an effect which produces the large misalignment of the $50-\mathrm{mV}$ sine and square wave responses. This apparent discrepancy can be explained by considering the mechanism of saturation. Saturation occurs when the head current in the tape recorder reaches a certain threshold. To compare the sine and square wave results it is necessary to compare not the input amplitude levels but the maximum head current amplitudes. For tests which do not saturate the instrument, a square wave results in a maximum recorded amplitude about twice that of a sine wave test with the same rms 
amplitude. Thus, one might expect saturation for square waves to occur at half the input voltage of sine waves. Indeed comparing the $25-\mathrm{mV}$ square wave and $50-\mathrm{mV}$ sine wave tests the discrepancy is small. The offset between the sine wave and square wave responses at 2 and $10 \mathrm{mV}$ is due either to the small amount of nonlinearity seen below saturation voltages (e.g., Figure A5) or to variations with time of the absolute sensitivity, a topic discussed in detail later in this section.

Phase

The square wave tests also provide good estimates of the phase response of the AOBHs (Figure A10) at frequencies below about $50 \mathrm{~Hz}$. Indeed, attempts to obtain the phase response solely from the amplitude response using the method of Bode diagrams [e.g., Close, 1966] were unsuccessful, since this method requires accurate measurements of the amplitude response at frequencies well outside the instrument passband.

\section{Uncertainties}

Prior to each deployment the AOBH undergoes an extensive series of checks and adjustments, the procedures for which are well established and documented. Indeed, the thoroughness with which they have been routinely performed, together with the quality of the instrument design, accounts for the consistently high data return rates achieved for these instruments. Since the response tests were performed nearly eighteen months after the tomography experiment, and several instruments were deployed elsewhere in the intervening period, those steps in the instrument preparation pertinent to the response were also performed prior to the tests. In particular, there are three dashpot adjustments, controlling the tape speed, the bias current and the head current, which affect the response of the instrument. We discuss below the uncertainties introduced by these adjustments.

\section{Bias Current}

Direct current tape recorders require the addition of a high frequency bias current to the signal prior to recording (Figure A1). The AOBHs use a bias current at 5 $\mathrm{kHz}$, a frequency that is readily available from the chronometer and which is well above the data frequency band. The bias current levels are set prior to each deployment by attaching a current meter to the output of each head driver amplifier and adjusting a dashpot until the peak to peak amplitude viewed on an oscilloscope is $4 \mathrm{ma}$. This adjustment is relatively straightforward to make accurately and is probably good to within better than $\pm 10 \%$. Systematic errors might arise from 
inaccuracies in the sensitivity of the current meter, but since the same current meter was used for all deployments they are presumably be invariant between cruises. In Figure A12 the effect upon the amplitude responses of AOBHs 3 and 6 of reducing the bias current amplitude by $10 \%$ is shown. In both cases the recorded amplitude increases by about $10 \%$ at all frequencies. There is no resolvable change in the shape of the amplitude response.

\section{Head Current}

The amplitude levels of the data signal output from the head driver amplifier (Figure A1) are adjusted prior to each cruise using a $25 \mathrm{~Hz}$ test signal in parallel with the hydrophone. For the low-frequency, low-gain channel a 4-mV input signal is used, and the dashpot adjusted until the head driver current amplitude is $0.5 \mathrm{~mA}$. This is a very difficult adjustment to make accurately. First, the head current is added to the higher amplitude bias current, so the amplitude level must be read from variations in the bias current envelope. Second, with the current meter and oscilloscope both set to maximum gain, the current meter sensitivity is only $1 \mathrm{~mA}$ per division $(\sim 1 \mathrm{~cm})$. In consequence this adjustment is probably accurate to no better than $\pm 20 \%$. As is the case for the bias current adjustment, additional systematic errors are presumably largely invariant between deployments. The effect upon the amplitude response of reducing the head current $20 \%$ is also shown in Figure A12. The amplitudes recorded are similarly reduced by about $20 \%$ with no resolvable change in the shape of the amplitude response.

\section{Tape Speed}

Prior to each cruise the tape recorder undergoes a complete overhaul. Just before deployment the tape speed is adjusted so that the tape capstan flywheel revolves once every $16 \mathrm{~s}$. Figure A12 shows the change in amplitude response resulting from a $10 \%$ reduction in the tape speed. At lower frequencies the effect is small, but at higher frequencies there is a marked decrease in amplitudes by up to $25 \%$. Thus, large variations in tape speed would significantly increase the uncertainties accompanying corrections for the instrument response. While the initial tape speed can easily be set to within $\pm 1 \%$, it is possible that the tape speed may vary significantly during a deployment, due the varying amount of tape on supply and take-up reels, the lower temperatures at the seafloor, and the reduction in battery voltages during the deployment. The effect of changing the tape-driving d.c. voltage is shown in Figure A13. Provided the voltage is higher than about $13 \mathrm{~V}$ the tape speed is not sensitive to the driving voltage. For most deployments, including 
all those in the tomography cruise, the battery voltages are above this threshold immediately before a deployment but upon recovery are at values between 12 and 13 V. Such a drop in voltage should result in a tape speed reduction of a few percent. However, the effect of the progressive redistribution of tape and of the lower temperatures found on the seafloor are harder to estimate.

Fortunately, a precise measure of the tape speed may be made using the time code channel. Since the Crown and Honeywell tape speeds and the digitization rate are constant, all variations in the number of digitized samples per second of time code can be directly related to tape speed variations. Moreover, since the instrument is recording time code within minutes of the final tape check, the absolute tape speed can also be estimated. Figure A14 shows tape speeds during the tomography cruise for $\mathrm{AOBH} 1$ and $\mathrm{AOBH} 4$. Both instruments show tape speed variations of only a few percent. Indeed, the $7.5 \%$ speed variation observed for AOBH 4 is the larger than all the other instruments. AOBH 4 shows a smooth decrease in tape speeds, a trend that is characteristic of most other instruments and is presumably a result of the decrease in tape driving voltage. AOBH 1 has a nearly constant tape speed, except for six hours of increased speeds near the start of the deployment and a steady increase in speed over the last two days, an increase that is observed on several other instruments. While the observed tape speed changes should produce perceptible changes in instrument response the resulting errors in $t^{*}$ measurements should be small ( $\leq 0.002 \mathrm{~s})$.

\section{Reproducibility}

To test the reproducibility of the measured responses the full set of sine- and square-wave calibration tests were repeated for AOBH 1 and AOBH 3 without recalibrating the instruments. The $10-\mathrm{mV}$ amplitude responses are shown in Figure A15. In both cases there is good agreement (within about $10 \%$ ) in the shape of the response, with the largest discrepancies occurring at higher frequencies. However, there are discrepancies of up to $50 \%$ in the overall amplitude levels.

The cause of this large effect is a change in the relative alignment of the AOBH record and Crown playback heads, since for a direct-recording tape recorder a relative misalignment of the heads will result in a decrease in the output amplitudes. As will be shown in the next section, the calibration pulse amplitudes and time code amplitude can be used to correct for this effect. 


\section{Calibration Pulses and Absolute Amplitudes}

All measurements of absolute amplitudes using the AOBH must be referenced to the calibration pulses. While accurate measurements of absolute amplitude are not explicitly used in this thesis, approximate values are required to determine whether individual waveforms saturated the instrument. Moreover, absolute amplitudes may be required for future work. Therefore, a method by which precise estimates of absolute amplitude may be obtained using the calibration pulses is presented in some detail below.

During each deployment, four 2-s-long calibration pulses are recorded towards the end of each hour, at times 58:45, 58:55, 59:45, 59:55 (hours:minutes). Figure A16 shows the circuit used to generate the calibration pulse from a 0 to $5 \mathrm{~V}$ (peak to peak) $10 \mathrm{~Hz}$ square wave. The output is introduced across resistor $R_{3}$, which is in series with the hydrophone (see $\mathrm{R}_{3}$ in Figure A3). Ignoring the initial d.c. offset of the calibration pulse the output of the calibration circuit is

$$
V_{\text {out }}=\frac{R_{3} V_{\text {in }}}{\left[\left(\frac{1}{s C}+R_{1}+R_{2}\right)\left(\frac{R_{2}+R_{3}+R_{4}}{R_{2}}\right)-R_{2}\right]}=\frac{3.7 V_{\text {in }}}{\left(\frac{2.1 \times 10^{-6}}{s}+10^{5}\right)}
$$

At $10 \mathrm{~Hz}$, the principal frequency of the calibration pulse, the amplitude is $220 \mu \mathrm{V}$ (peak to peak). Figure A17a shows the predicted form of the recorded calibration pulse.

To check the theoretical amplitude, the calibration pulse was recorded during the response tests for each instrument. This was achieved by reconnecting the preamplifier and placing a shielded $0.012-\mu \mathrm{F}$ capacitor across the hydrophone input sockets to mimic the impedance of the hydrophone. Figure A17b shows the result for $\mathrm{AOBH}$ 4. The predicted and the observed form of the pulse are in excellent agreement. An estimate of the calibration pulse amplitude can be obtained by comparing the amplitude at $10 \mathrm{~Hz}$ with the result of the $10-\mathrm{Hz} 2-\mathrm{mV}$ sine wave test amplitude, after correction for the preamplifier gain. Measured amplitudes, which range between 190 and $230 \mu \mathrm{V}$, are listed in Table A4. Considering the uncertainties arising from the direct recording system and the tolerances of electronic components $( \pm 5 \%)$, these amplitudes are in good agreement with the theoretical predictions.

The calibration pulse amplitudes were also monitored throughout the tomography experiment for each instrument. Figure A17c shows a typical example. The signal-to-noise ratio is only about 2. A least squares inversion algorithm was 
used to fit a $10-\mathrm{Hz}$ sine wave to each pulse. The method has the advantage that it is robust in the presence of noise, and the rms least squares misfit can be used to obtain a formal linearized uncertainty. The uncertainties for individual measurements are generally of the order of $5 \%$. In Figure A18, calibration pulse amplitudes are plotted against time for $\mathrm{AOBH} 4$. Although the specific form of the plot varies between instruments, several features are common to all instruments. Calibration pulse amplitudes show very large variations during the experiment, particularly during the first few days. Amplitude levels show gradual trends punctuated by large, rapid (perhaps instantaneous) changes. As noted above, such variations result from changes in relative alignment of the instrument record and Crown playback heads. Sudden amplitude jumps sometimes coincide with changes in the Honeywell transcription tape, a time when Crown tape-head alignments are likely to change because the $1 / 4$ " tape is manually rewound a few turns. In other cases sudden changes may originate during acquisition. Contributions to progressive changes may arise from changes in tape tension, from varying amounts of tape on the reels, and from slack in the tape guiding mechanism.

The most obvious method to interpolate between calibration pulse amplitudes is to fit a smooth curve to the data. However, such methods ignore short-term variations in the data. To measure the magnitude of such variations the mean squared amplitude change $S^{2}$ (or variance) over a time interval $\Delta T$ can be defined

$$
\left(S_{\Delta T}\right)^{2}=\frac{\sum_{i=1}^{N}\left[a_{i}(t)-a_{i}(t+\Delta T)\right]^{2}}{N}
$$

where $a(t)$ is the calibration pulse amplitude at time $t$, and $N$ is the number of pairs of calibration pulses separated by $\Delta \mathrm{T}$. Because of the regular spacing of calibration pulses this quantity can only be evaluated for a limited number of values $\Delta T$. In Table A5 values of $S$ are presented for time intervals of $10 \mathrm{~s}, 1$ minute, and 1 hour together with the rms uncertainty of measurements of calibration pulse amplitudes. In the absence of any variation of amplitude with time all these quantities should have the same value. However, in all cases values of $S$ obtained for a 10-s interval are larger than the measurement uncertainty. Moreover, in most cases $S$ values are significantly greater for a $\Delta \mathrm{T}=1$ hour than for $\Delta \mathrm{T}=10 \mathrm{~s}$ or 1 minute. These results suggest that appreciable variations in absolute amplitude scales occur over time intervals less than 1 hour, variations that cannot be modelled by fitting a smooth curve to the calibration pulse data. 
Fortunately, the time code amplitude can be used to interpolate between calibration pulses. Since tape head misalignments are presumably largely invariant between channels, the resulting amplitude fluctuations should be the same on all channels. The time code comprises a $25 \mathrm{~Hz}$ signal that modulates between high and low amplitudes twice a second (Figure A17d). The amplitude of the high amplitude portions of the time code can be measured precisely over a small time period enclosing the calibration pulse. A linear relationship relating time code amplitudes to calibration pulse amplitudes may be determined and used to obtain estimates of calibration pulse amplitudes at the desired time.

For the tomography experiment, the mean of the four calibration pulse measurements at the end of each hour was fitted to the mean high-amplitude time code amplitude (taken over $5 \mathrm{~s}$ ) by minimizing the rms misfit obtained from

$$
a(t)=b(t)(C+D t)
$$

where $\mathrm{b}$ is the time code amplitude and $\mathrm{C}$ and $\mathrm{D}$ are the fitting parameters. The quality of the fit can be assessed by evaluating the normalized value $\chi^{2}$

$$
\chi^{2}=\frac{\left(\sum_{i=1}^{N} \frac{\left[a_{i}(t)-b_{i}(t)(C+D t)\right]^{2}}{\sigma_{i}}\right)}{N-2}
$$

where $\mathrm{N}$ is the number of data points used. In most cases $\chi^{2}$ values close to unity are obtained from a single fit to each day of data. Large values of $\chi^{2}$ can invariably be reduced significantly by using two fits on either side of an obvious discontinuity. Mean values of $\chi^{2}$ obtained for each instrument are presented in Table A5.

The process is illustrated for AOBH 4 in Figure A19. The calibration amplitudes predicted for shot times are also shown. It is immediately apparent that there are rapid fluctuations in the predicted calibration pulse amplitudes, variations that are severely aliased by the available calibration pulse amplitudes. The quality of the fit leads to the conclusion that such variations are real and not an artifact of unrelated fluctuations in the time code amplitude. Assuming that equation (A15) correctly models all variations in time code amplitude fluctuations, this relationship would imply an error in time code derived estimates of calibration pulse amplitude of less than $1 \%$. Since mean values of $\chi^{2}$ do exceed unity slightly, this assumption is not completely justified. However, the uncertainties are clearly considerably smaller than values of $5-10 \%$ obtainable from a smooth fit to the data. 


\section{Conclusions}

The preceding section has detailed how the amplitude response and absolute amplitude levels have been determined for the AOBH. The procedure is too complex to permit the calculation of a precise uncertainty in the amplitude response. However, the tests suggest that the relative amplitudes at widely spaced frequencies should be reproducible to an accuracy of a few tens of percent. Thus, the correction for the instrument power response should be accurate to within a factor of two which for a frequency range $10-40 \mathrm{~Hz}$ suggests a maximum systematic error in $\mathrm{t}^{*}$ of $0.004 \mathrm{~s}$. The results also suggest that instrument saturation and spectral leakage may further bias $t^{*}$ for high-amplitude and highly attenuated waveforms, respectively.

\section{DIGITAL OCEAN BOTTOM HYDROPHONE}

\section{Introduction}

The WHOI DOBH [Koelsch et al., 1982] is similar to the AOBHs in its mechanical configuration, employing an identical hydrophone sensor and recovery and release system. The recording electronics, however, are radically different. the DOBH is microprocessor controlled, recording digitally the outputs of one high frequency and up to four low-frequency channels on a commercially available 16.7Mbyte cartridge tape. Both hardware and software are designed to permit operational flexibility. A modular design allows sensors, amplifiers and antialiasing filters to be changed with little effort prior to each deployment. Within a single deployment the instrument may be programmed to record in a variety of modes. Start and end times delimit tasks, during which a number of parameters control the mode of acquisition (event detect or preset), the record length and sample rate of each channel, and the nature of self-calibration tests.

For the tomography experiment all instruments operated in an event-detect mode. However, it was necessary to specify four tasks per instrument to allow for dormant periods in the later part of the experiment during which airgun refraction data could be recorded by the AOBHs without filling the DOBH cartridge tapes. The high-frequency channel was bandpassed filtered from 300 to $500 \mathrm{~Hz}$, and an envelope threshold algorithm was used to detect events, triggered by the arrival of the water wave. A short 2-s record of the modulated, integrated signal was recorded to allow for subsequent determination of the water wave arrival time. Simultaneously a longer 30-s record of low-frequency data commencing $28 \mathrm{~s}$ before the water wave 
arrival time was recorded. A sample rate of $250 \mathrm{~Hz}$ was used together with a $80-\mathrm{Hz}$ low-pass filter. A system self-calibration test, comprising $121-\mathrm{s}, 0.9-\mathrm{mV}$ (peak to peak) sine waves logarithmically spaced between 1 and $2000 \mathrm{~Hz}$, was performed at the start of each task and subsequently after every 100 events.

Unlike the AOBH the frequency response of the low-frequency channel of the DOBH may be predicted by a series of theoretically derived equations $[D . E$.

Koelsch, pers. comm., 1989]. The self-calibration tests serve both as an independent check on the validity of these equations and a precautionary test to detect instrument malfunctions.

\section{Theoretical Response}

Figure A20 is a schematic flow chart showing the major components of the lowfrequency channel. The instrument response I(s) may be written as the response of a series of reactive networks

$$
I(s)=f_{1}(s) \cdot f_{2}(s) \cdot f_{3}(s) \cdot f_{4}(s) \cdot f_{5}(s) \cdot f_{6}(s)
$$

where $s$ is the complex frequency and transfer functions $f_{i}(s)$ are defined as follows:

$$
\begin{aligned}
& f_{1}(s)=\text { Hydrophone response } \\
& f_{2}(s)=\text { Hydrophone capacitance and preamplifier input network } \\
& f_{3}(s)=\text { Preamplifier output and antialias filter input } \\
& f_{4}(s)=\text { Antialias filter } \\
& f_{5}(s)=\text { Gain ranging input } \\
& f_{6}(s)=\text { Gain ranging amplifier }
\end{aligned}
$$

The response of each component [D. E. Koelsch, pers. comm., 1989] is described below.

\section{Hydrophone Response}

The hydrophone sensor used in the DOBH is the same as that used in the $\mathrm{AOBH}$. The response is given by equation (A2).

\section{Hydrophone Capacitance and Preamplifier Input}

Three different preamplifiers were used in DOBH units during the tomography cruise. In each case the hydrophone capacitance and preamplifier input form a highpass filter with a configuration identical to that used in the AOBH (Figure A3) except that the capacitor $C_{2}$ is absent. The response is given by equation (A3). Component values and filter parameters for each DOBH are presented in Table A6. 
The preamplifier unit in DOBH 11 was designed for the source monitoring experiment and has a gain about $1 / 100$ that of the other units. The preamplifier in DOBH 10 has a characteristic frequency of $1 \mathrm{~Hz}$ while for other units it is about 5 $\mathrm{Hz}$.

\section{Preamplifier Output and Filter Input}

The output of the preamplifier and input into the filter section (Figure A21) forms a simple RC network whose response is

$$
f_{3}(s)=G \frac{s / \Phi}{1+s / \Phi}
$$

where the gain is given by

$$
G=\frac{R_{1}}{R_{1}+R_{2}}=0.989
$$

and the characteristic frequency is

$$
\bar{\sigma}=\frac{1}{\left(R_{1}+R_{2}\right) C}=11.0 \mathrm{~s}^{-1}
$$

\section{Filter Section}

The design of the DOBH allows the use of a variety of low-pass and band-pass antialias filters. For all deployments during the tomography cruise a 6-pole, 2-dBripple, low-pass Chebyshev filter with a cut-off frequency of $80 \mathrm{~Hz}$ was used. This filter is approximated by three cascaded, 2-pole Sallen and Key filters whose network is shown in Figure A22. The response of each two-pole filter may be written as

$$
f_{S K}=\frac{1}{\left(1+\frac{2 \zeta s}{\omega}+\frac{s^{2}}{\Phi^{2}}\right)}
$$

where the characteristic filter frequency is given by

$$
\varpi=\frac{1}{\left(R_{1} R_{2} C_{1} C_{2}\right)^{1 / 2}}
$$

and the damping factor by

$$
\zeta=\frac{\left(R_{1}+R_{2}\right) C_{2}^{1 / 2}}{\left(4 R_{1} R_{2} C_{1}\right)^{1 / 2}}
$$

The component values and corresponding filter parameters for the three filters used to construct the low-pass filter are listed in Table A7. The response of the whole filter is simply 


$$
f_{4}(s)=\prod_{i=1}^{3} \frac{1}{\left(1+\frac{2 \zeta_{i} s}{\varpi_{i}}+\frac{s^{2}}{\sigma_{i}^{2}}\right)}
$$

where $i$ is the index of the Sallen and Key filter.

\section{Gain-Ranging Amplifier Input}

The input to the gain ranging amplifier (Figure A21) is a simple RC network whose response is

$$
f_{5}(s)=\frac{s / \Phi}{1+s / \Phi}
$$

where

$$
\varpi=\frac{1}{\mathrm{RC}}=10.0 \mathrm{~s}^{-1}
$$

\section{Gain-Ranging Amplifier and Analog-to-Digital Converter}

The gain-ranging amplifier chooses between the output of four simple transistor amplifiers separated by $12 \mathrm{~dB}$ and the unamplified signal. The precise gains calculated from the resistor values are 4.007, 16.07, 64.37, $256.2( \pm 1 \%)$. After gain ranging the signal passes through a sample-and-hold circuit into a 12 -bit $\pm 10 \mathrm{~V}$ analog-to-digital converter. During data reduction the output of the analog-to-digital converter is normalized with respect to the maximum gain of 256. Thus, the response of this section may be written in terms of digital units (du) per volt

$$
\mathrm{f}_{6}(\mathrm{~s})=\frac{256 \times 4096}{20}=5.243 \times 10^{4} \mathrm{~d} \text {.u. } / \mathrm{V}
$$

To prevent overloading of the gain-ranging amplifiers diodes are included on the inputs. As a result, the maximum amplitude on the lowest gain setting is clipped at about one half the saturation amplitude of the digitizer.

Figure A23 shows the total theoretical amplitude and phase responses of DOBH 15 derived from equations (A2), (A3), (A16), (A17), (A21), (A22), and (A23). The low-frequency cutoff results from the preamplifier input and output circuits and the gain- ranging amplifier input while the response at higher frequencies is controlled by the Chebyshev low-pass filter.

\section{Calibration Tests}

The calibration signal is generated using a 4410 chip and consists of twelve 1-slong sine waves with frequencies spaced at factors of 2 between 0.997 and $2041 \mathrm{~Hz}$ and a nominal peak-to-peak amplitude of $1 \mathrm{~V}$. This signal is attenuated to $0.894 \mathrm{mV}$ (peak to peak) using the circuit shown in Figure A24 and is introduced in series with 
the hydrophone. Due to a software oversight, calibration tests recorded within a task were conducted immediately after the preceeding event. As a result, these tests are swamped by seismic reverberations within the water column. Thus, for each instrument only the four calibration tests recorded at the start of each task may be used to estimate the instrument response. These were recorded at 0:600 GMT on 24 January, 30 January, 31 January and 1 February (the explosive shoots were deployed in eight sessions between 24 and 31 January).

Figure A23 shows the results along with theoretical predictions for DOBH 14. The primary features are common to all instruments. The amplitudes of the all but the first calibration test always agree within a few percent or better. The first test, however, recorded consistently lower amplitudes than succeeding tests; discrepancies listed in Table A8 vary between 10 and 30\% (1-3 dB). The cause of this is unknown. While some components of the DOBH may be temperaturesensitive, the first calibration test always occurs over 40 hours after deployment, a period that should easily allow the instrument to reach thermal equilibrium. Since most explosive shots occurred between the first and second tests which are separated by 6 days, there is a dilemma as to which better represents the instrument response at the time of the shots. Without an understanding of the cause of the effect this question cannot be answered unambiguously. However, the lower amplitude levels are a transient feature and there is no resolvable increase in amplitude levels after the second test. Thus, it is reasonable to infer that the time constant for the persistence of lower amplitude levels is much less than six days and that the later tests better represent the instrument response for the majority of the tomography experiment.

A comparison of the calibration test results with the theoretical predictions (Figure A23) shows that there is excellent agreement between the shapes of the responses. Within the instrument pass band the measured and predicted ratios of the responses at two frequencies never differ by more than about $10 \%(1 \mathrm{~dB})$. However, there is a discrepancy in the absolute amplitude levels. The later calibration tests yield sensitivities (Table A8) between $70 \%$ and $85 \%$ of the predicted value, while for the first tests the discrepancy is even larger. There are a number of possible effects that together may account for this discrepancy. First, the 4410 chip used to generate the calibration pulse has specifications which permit a tolerance in output voltage at $25^{\circ} \mathrm{C}$ of $\pm 12 \%$. At $4^{\circ} \mathrm{C}$ there may also be a systematic offset from the $25^{\circ} \mathrm{C}$ levels though the temperature specifications suggest it will be 
small. The theoretical response may be in error due to uncertainties in component values. However, since all critical resistors have tolerances of $\pm 1 \%$, and gain primarily comes from two components, the preamplifier and the gain-ranging amplifier, component value errors should not alter the output amplitude by more than a few percent. A significant systematic bias towards lower measured amplitudes may be introduced during multiplexing and analog-to-digital conversion. Sample-andhold circuits are used to pass samples from each channel to the digitizer in turn. If the RC time constant of this circuit does not greatly exceed the sample interval then a significant but constant fractional loss of signal amplitude will occur prior to digitization. There is no straightforward method to measure the magnitude of this effect.

\section{Conclusions}

The DOBH is a sophisticated digital instrument for which a theoretical approximation to the response may be calculated. The shape of this response agrees very well with the results of self-calibration tests though the measured amplitude levels are about $25 \%$ lower than those predicted. By using the theoretical response shape and calibration test amplitude levels, the absolute amplitude response can be obtained to the accuracy of about $\pm 10 \%$.

\section{Digital OCEAN BotTOM SEISMOMETER}

The MIT OBS [Mattaboni and Solomon, 1977; Duschenes et al., 1981] is an event-triggered digital instrument that records the output of three orthogonal $4.5-\mathrm{Hz}$ seismometers deployed in an external geophone package. The response has been described in detail by Tréhu [1982]. Since that time the instrument has undergone only minor modifications comprising the replacement of the geophones and a doubling of the digital unit voltage.

Following equation (3.6) of Tréhu [1982] the complex spectrum of geophone displacement $M$ in $\mathrm{cm} / \mathrm{s}$ may be related to the recorded seismogram $D$ by

$$
\mathrm{M}(\mathrm{s})=\frac{0.0024 \mathrm{D}(\mathrm{s})}{103 \frac{\mathrm{g}}{256} \mathrm{~s} \mathrm{~V}(\mathrm{~s}) \mathrm{F}(\mathrm{s})}
$$

where $\mathrm{g}$ is the automatically controlled gain (which for all explosive shots recorded by the tomography experiment was 256 ), $\mathrm{V}$ is the geophone response, and $\mathrm{F}$ the filter board response. 
The geophone currently in use in the MIT OBS is a Geo Space HS-1. The transfer function for the geophone may be written

$$
V(s)=\frac{A s^{2}}{\omega_{\mathrm{r}}^{2}+s^{2}+2 b_{t} s \omega_{\mathrm{r}}}
$$

where $\omega_{\mathrm{r}}=28.3$ radians/s is the natural resonant frequency of the geophone and $\mathrm{A}$ and $b_{t}$ are dependent upon the geophone properties and are given by

$$
\begin{aligned}
& b_{t}=b_{o}+\frac{G^{2}}{2 \omega_{r} m\left(R+R_{c}\right)} \\
& A=\frac{G R}{R+R_{c}}
\end{aligned}
$$

where $b_{0}=0.27$ is the open circuit damping, $G=0.41 \mathrm{~V} / \mathrm{m} / \mathrm{s}$ is the intrinsic sensitivity, $\mathrm{m}=28.5 \mathrm{~g}$ the moving mass, $R_{c}=900 \Omega$ the coil resistance, and $R=$ $1800 \Omega$ the shunt resistance. These values yield $b_{t}=0.656$ and $A=0.273 \mathrm{~V} / \mathrm{cm} / \mathrm{sec}$.

The low pass filter used in the OBS is an approximation to a 6-pole Chebyshev filter with a cut off frequency of $30 \mathrm{~Hz}$ and a passband ripple amplitude of $2 \mathrm{~dB}$. The response is

$$
\mathrm{F}(\mathrm{s})=\left(\frac{1.8776}{\left(\mathrm{~s} / \omega_{\mathrm{c}}\right)^{2}+1.1722 \mathrm{~s} / \omega_{\mathrm{c}}+1}\right)^{3}
$$

where the cut off frequency $\omega_{c}=193.4$ radians/sec. The amplitude response (Figure A25) is characterized by a rapid drop in amplitudes above $30 \mathrm{~Hz}$. 
Table A1. Component values and filter parameters for the AOBH preamplifiers

\begin{tabular}{|c|c|c|c|c|c|c|c|c|c|c|}
\hline \multirow[b]{2}{*}{ Instrument } & \multicolumn{7}{|c|}{ Nominal component values } & \multicolumn{3}{|c|}{ Filter parameters } \\
\hline & $\begin{array}{r}\mathrm{R}_{1} \\
\mathrm{M} \Omega\end{array}$ & $\begin{array}{r}\mathrm{R}_{2} \\
\mathrm{M} \Omega\end{array}$ & $\begin{array}{l}\mathrm{R}_{3} \\
\boldsymbol{\Omega}\end{array}$ & $\begin{array}{l}\mathrm{R}_{4} \\
\mathrm{k} \Omega\end{array}$ & $\begin{array}{l}R_{5} \\
\mathrm{k} \Omega\end{array}$ & $\begin{array}{l}\mathrm{C}_{\mathrm{H}}, \\
\mu \mathrm{F}\end{array}$ & $\begin{array}{l}C_{1}, \\
\mu F\end{array}$ & $\mathrm{G}_{1}$ & $\mathrm{G}_{2}$ & $\begin{array}{l}\bar{\sigma} \\
\mathrm{Hz}\end{array}$ \\
\hline 1 & 1.50 & 10.2 & 100 & 11.0 & 100 & 1.0 & 0.012 & 0.87 & 9.9 & 5.6 \\
\hline 2 & 1.50 & 10.2 & 100 & 11.0 & 100 & 1.0 & 0.012 & 0.87 & 10.4 & 5.6 \\
\hline 3 & 1.50 & 40.0 & 100 & 11.0 & 100 & 1.0 & 0.012 & 0.96 & 10.2 & 1.6 \\
\hline 4 & 1.00 & 10.2 & 100 & 11.0 & 100 & 1.0 & 0.012 & 0.91 & 9.9 & 5.7 \\
\hline 5 & 1.50 & 10.2 & 100 & 11.0 & 100 & 1.0 & 0.012 & 0.87 & 9.9 & 5.6 \\
\hline 6 & 1.50 & 10.2 & 100 & 11.0 & 100 & 1.0 & 0.012 & 0.87 & 10.1 & 5.6 \\
\hline 7 & 1.50 & 10.2 & 100 & 11.0 & 100 & 1.0 & 0.012 & 0.88 & 10.3 & 5.4 \\
\hline
\end{tabular}

Components symbols correspond to the circuit diagram shown in Figure A3, while the filter parameters are those of equation (A3). The component values presented are the nominal values ( $\pm 5 \%$ for resistors and $\pm 10 \%$ for capacitors). The filter parameters are calculated using accurate measurements of all resistor values $( \pm 1 \%)$. 
Table A2. Amplitude responses of the AOBHs determined from sine wave tests

\begin{tabular}{|c|c|c|c|c|c|c|c|c|c|c|c|c|c|c|}
\hline \multirow[b]{2}{*}{ Instrument } & \multirow{2}{*}{$\begin{array}{c}\text { Input } \\
\text { Voltage, } \\
\text { mV }\end{array}$} & \multicolumn{13}{|c|}{ Normalized Amplitude at Frequency, $\mathrm{Hz}$} \\
\hline & & 1 & 2 & 3 & 4 & 5 & 7 & 10 & 15 & 20 & 30 & 40 & 50 & 60 \\
\hline \multirow[t]{3}{*}{1} & 2 & 0.39 & 0.88 & 1.05 & - & 1.07 & 1.08 & 1.00 & 0.98 & 0.89 & 0.79 & 0.64 & 0.41 & 0.21 \\
\hline & 10 & 0.39 & 0.85 & 1.00 & - & 1.05 & 1.04 & 1.00 & 0.92 & 0.88 & 0.73 & 0.61 & 0.44 & 0.22 \\
\hline & 50 & 0.50 & 0.89 & 1.04 & - & 1.09 & - & - & 0.78 & 0.69 & 0.57 & 0.43 & 0.29 & - \\
\hline \multirow[t]{3}{*}{2} & 2 & 0.41 & 0.86 & 1.04 & 1.08 & 1.10 & 1.04 & 1.00 & 0.89 & 0.78 & 0.51 & 0.19 & - & - \\
\hline & 10 & 0.41 & 0.90 & 1.05 & 1.09 & 1.08 & 1.06 & 1.00 & 0.89 & 0.76 & 0.47 & 0.24 & - & - \\
\hline & 50 & 0.52 & 1.00 & 1.15 & - & 1.18 & 1.12 & 1.00 & 0.80 & 0.63 & 0.40 & 0.21 & - & - \\
\hline \multirow[t]{3}{*}{3} & 2 & 0.46 & 0.89 & 1.04 & 1.05 & 1.08 & 0.96 & 1.00 & 0.85 & 0.79 & 0.61 & 0.43 & 0.21 & - \\
\hline & 10 & 0.45 & 0.85 & 1.00 & 1.07 & 1.08 & 0.99 & 1.00 & 0.88 & 0.82 & 0.58 & 0.40 & 0.14 & - \\
\hline & 50 & 0.52 & 0.97 & 1.12 & 1.14 & 1.06 & 1.07 & 1.00 & 0.84 & 0.66 & 0.53 & 0.40 & 0.22 & - \\
\hline \multirow[t]{3}{*}{4} & 2 & 0.46 & 0.92 & 1.05 & 1.10 & 1.10 & 1.10 & 1.00 & 0.93 & 0.87 & 0.65 & 0.51 & 0.34 & - \\
\hline & 10 & 0.46 & 0.93 & 1.06 & 1.10 & 1.10 & 1.10 & 1.00 & 0.93 & 0.86 & 0.71 & 0.52 & 0.34 & 0.18 \\
\hline & 50 & 0.53 & 1.03 & 1.21 & 1.23 & 1.19 & 1.12 & 1.00 & 0.88 & 0.75 & 0.63 & 0.51 & 0.36 & 0.17 \\
\hline
\end{tabular}


Table A2. continued

\begin{tabular}{|c|c|c|c|c|c|c|c|c|c|c|c|c|c|c|}
\hline \multirow{2}{*}{ Instrument } & \multirow{2}{*}{$\begin{array}{l}\text { Input } \\
\text { Voltage, } \\
\text { mV }\end{array}$} & \multicolumn{13}{|c|}{ Normalized Amplitude at Frequency, $\mathrm{Hz}$} \\
\hline & & 1 & 2 & 3 & 4 & 5 & 7 & 10 & 15 & 20 & 30 & 40 & 50 & 60 \\
\hline \multirow[t]{3}{*}{5} & 2 & 0.46 & 0.97 & 1.11 & 1.14 & 1.14 & 1.11 & 1.00 & 0.91 & 0.80 & 0.58 & 0.36 & - & - \\
\hline & 10 & 0.49 & 0.96 & 1.10 & 1.15 & 1.13 & 1.08 & 1.00 & 0.91 & 0.81 & 0.56 & 0.38 & 0.18 & - \\
\hline & 50 & 0.58 & 1.06 & 1.18 & 1.21 & 1.20 & 1.14 & 1.00 & 0.81 & 0.69 & 0.50 & 0.32 & 0.19 & \\
\hline \multirow[t]{3}{*}{6} & 2 & 0.39 & 0.80 & 0.95 & 0.99 & 1.01 & 1.06 & 1.00 & 0.93 & 0.85 & 0.74 & 0.62 & 0.42 & - \\
\hline & 10 & 0.44 & 0.86 & 1.01 & 1.06 & 1.09 & 1.06 & 1.00 & 0.96 & 0.91 & 0.78 & 0.60 & 0.45 & 0.21 \\
\hline & 50 & 0.49 & 0.95 & 1.07 & 1.12 & 1.11 & 1.06 & 1.00 & 0.80 & 0.73 & 0.61 & 0.49 & 0.28 & - \\
\hline \multirow[t]{3}{*}{7} & 2 & 0.56 & 1.15 & 1.25 & 1.33 & 1.27 & 1.19 & 1.00 & 0.80 & 0.65 & 0.38 & - & - & - \\
\hline & 10 & 0.46 & 1.10 & 1.27 & 1.28 & 1.25 & 1.17 & 1.00 & 0.79 & 0.64 & 0.38 & 0.20 & - & - \\
\hline & 50 & 0.58 & 1.14 & 1.26 & 1.28 & 1.27 & 1.16 & 1.00 & 0.77 & 0.63 & 0.38 & 0.18 & - & - \\
\hline
\end{tabular}

These results are normalized to $10 \mathrm{~Hz}$ and do not include the preamplifier response. The signal generator used for the tests is adjustable to within $<10 \%$ of the nominal frequencies given above. The amplitudes above have been corrected to the nominal frequencies using the slope of a fourth order polynomial fit to the logarithms of the data. 
Table A3. Saturation characteristics of the AOBHs at $10 \mathrm{~Hz}$ determined from the sine wave tests

\begin{tabular}{|c|c|c|c|c|c|c|c|c|c|c|c|}
\hline \multirow[b]{2}{*}{ Instrument } & \multirow[b]{2}{*}{$\begin{array}{c}\text { Frequency, } \\
\mathrm{Hz}\end{array}$} & \multicolumn{10}{|c|}{ Normalized amplitude at input voltage, $\mathrm{mV}$} \\
\hline & & 2 & 5 & 10 & 25 & 40 & 50 & 70 & 100 & 150 & 300 \\
\hline 1 & 10.2 & 2.00 & 5.08 & 10.0 & 23.2 & 33.2 & - & 41.9 & 42.0 & 42.0 & - \\
\hline 2 & 10.2 & 2.00 & 4.81 & 9.52 & 21.9 & - & 35.7 & - & 39.9 & 40.2 & 39.0 \\
\hline 3 & 10.0 & 2.00 & - & 9.7 & 22.9 & - & 36.1 & 39.3 & 40.4 & 41.2 & 40.0 \\
\hline 4 & 10.2 & 2.00 & 4.92 & 9.54 & 22.3 & 34.3 & 37.9 & 44.5 & 45.9 & 44.5 & 43.4 \\
\hline 5 & 10.2 & 2.00 & 5.05 & 9.79 & 22.3 & 33.9 & 38.0 & 43.2 & 44.5 & 44.6 & 44.0 \\
\hline 6 & 10.2 & 2.00 & 4.60 & 9.06 & 22.0 & 30.7 & 34.9 & 38.5 & 38.9 & 38.2 & 37.2 \\
\hline 7 & 10.2 & 2.00 & 4.85 & 9.76 & 23.8 & 35.7 & 41.5 & 50.3 & 52.2 & 52.8 & 53.9 \\
\hline
\end{tabular}

Recorded amplitudes are normalized to a value of 2.00 for an input voltage of $2 \mathrm{mV}$. 
Table A4. Measured AOBH calibration pulse amplitudes

\begin{tabular}{ccc}
\hline Instrument & $\begin{array}{c}\text { Amplitude, } \\
\mu \mathrm{V}\end{array}$ & $\begin{array}{c}\text { Formal Uncertainty, } \\
\mu \mathrm{V}\end{array}$ \\
\hline 1 & 196 & 4 \\
2 & 205 & 4 \\
3 & 204 & 4 \\
4 & 230 & 4 \\
5 & 192 & 4 \\
6 & - & - \\
7 & 201 & 4 \\
\hline
\end{tabular}

Due to an experimental oversight no value was obtained for AOBH 6. The amplitudes and formal uncertainties are obtained by fitting a $10-\mathrm{Hz}$ sine wave to a recorded calibration pulse using a least squares inversion algorithm. 
Table A5. Fractional rms uncertainty of calibration pulse amplitude measurements, the fractional rms time variation of calibration pulse amplitude measurements, and the misfit $\chi^{2}$ for linear relationships between calibration pulse and time code amplitudes for the AOBHs

\begin{tabular}{cccccc}
\hline & \multirow{2}{*}{$\begin{array}{c}\text { Measurement } \\
\text { Uncertainty }\end{array}$} & \multicolumn{2}{c}{ rms amplitude variation over } & Time code fit \\
\cline { 3 - 5 } Instrument & $\mathrm{rms}$ & $10 \mathrm{~s}$ & 1 minute & 1 hour & $\chi^{2}$ \\
\hline 1 & 0.037 & 0.052 & 0.070 & 0.093 & 2.11 \\
2 & 0.027 & 0.035 & 0.046 & 0.065 & 1.16 \\
3 & 0.057 & 0.086 & 0.089 & 0.088 & 0.86 \\
4 & 0.037 & 0.058 & 0.065 & 0.096 & 2.08 \\
5 & 0.031 & 0.038 & 0.070 & 0.115 & 1.40 \\
6 & 0.039 & 0.052 & 0.078 & 0.107 & 0.69 \\
7 & 0.028 & 0.039 & 0.051 & 0.062 & 1.57 \\
\hline
\end{tabular}

The rms measurement uncertainty and rms variation of calibration pulse amplitudes with time are normalized to the calibration pulse amplitudes. The rms variation of calibration pulse amplitudes with time is calculated according to equation (A13). The misfit $\chi^{2}$ of the time code and the calibration pulse amplitudes is calculated for the whole deployment using equation (A15) with parameters determined separately for each day. 
Table A6. Component values and filter parameters for DOBH preamplifiers

Component values

\begin{tabular}{|c|c|c|c|c|c|c|c|c|c|c|}
\hline \multirow[b]{2}{*}{ Instrument } & & \\
\hline & $\begin{array}{l}\mathrm{R}_{1}, \\
\mathrm{M} \Omega\end{array}$ & $\begin{array}{r}\mathrm{R}_{2} \\
\mathrm{M} \Omega\end{array}$ & $\begin{array}{l}\mathrm{R}_{3}, \\
\Omega\end{array}$ & $\begin{array}{l}\mathrm{R}_{4} \\
\mathrm{k} \Omega\end{array}$ & $\begin{array}{l}\mathrm{R}_{5} \\
\mathrm{k} \Omega\end{array}$ & $\begin{array}{l}C_{H}, \\
\mu F\end{array}$ & $\begin{array}{l}C_{1,} \\
\mu F\end{array}$ & $\mathrm{G}_{1}$ & $\mathrm{G}_{2}$ & $\begin{array}{r}\overline{\boldsymbol{\Phi}} \\
\mathrm{Hz}\end{array}$ \\
\hline 10 & 0.1 & 10.2 & 100 & 11.0 & 100 & 1.0 & 0.012 & 0.990 & 10.09 & 6.56 \\
\hline $11^{*}$ & 2.1 & 0.02 & 100 & 11.0 & 100 & 1.0 & 0.012 & 0.00943 & 10.09 & 31.87 \\
\hline $12,13,14,15$ & 0.1 & 2.0 & 100 & 11.0 & 100 & 1.0 & 0.012 & 0.952 & 10.09 & 32.22 \\
\hline
\end{tabular}

* This preamplifier was used on DOBH 14 for the source monitoring experiment. Component specifications are $\pm 1 \%$ for resistors and $\pm 2 \%$ for capacitors. 
Table A7. Component values and filter parameters for three 2-pole Sallen and Key filters used to approximate the 6-pole Chebyshev filter in the DOBH

\begin{tabular}{|c|c|c|c|c|c|c|c|}
\hline \multirow[b]{2}{*}{$\begin{array}{c}\text { 2-pole } \\
\text { filter number }\end{array}$} & \multicolumn{5}{|c|}{ Component values } & \multicolumn{2}{|c|}{ Filter parameters } \\
\hline & $\begin{array}{l}R_{1}, \\
k \Omega\end{array}$ & $\begin{array}{c}\mathrm{R}_{2} \\
\mathrm{k} \Omega\end{array}$ & $\begin{array}{l}R_{3}, \\
k \Omega\end{array}$ & $\begin{array}{l}C_{1}, \\
\mu F\end{array}$ & $\begin{array}{l}C_{2}, \\
\mu F\end{array}$ & $\begin{array}{l}\varpi \\
\mathrm{Hz}\end{array}$ & $\xi$ \\
\hline 1 & 56.2 & 56.2 & 10.0 & 0.2 & 0.0628 & 159 & 0.560 \\
\hline 2 & 56.2 & 56.2 & 10.0 & 0.276 & 0.0083 & 371 & 0.173 \\
\hline 3 & 42.2 & 42.2 & 10.0 & 1.0 & 0.0024 & 484 & 0.049 \\
\hline
\end{tabular}

Component specifications are $\pm 1 \%$ for resistors and $\pm 2 \%$ for capacitors. 
Table A8. Absolute amplitude levels obtained from the DOBH self-calibration test

\begin{tabular}{ccc}
\hline & \multicolumn{2}{c}{ Sensitivity as a fraction of the theoretical value } \\
\cline { 2 - 3 } Instrument & 1st test & 2nd, 3rd, and 4th tests \\
\hline 10 & - & $0.71( \pm 0.01)$ \\
12 & 0.77 & $0.86( \pm 0.06)$ \\
13 & 0.63 & $0.77( \pm 0.01)$ \\
14 & 0.56 & $0.79( \pm 0.01)$ \\
15 & 0.62 & 0.72 \\
\hline
\end{tabular}




\section{Figure Captions}

Figure A1. Flow chart showing the main components of the recording system for the low-frequency, low-gain channel on the $\mathrm{AOBH}$.

Figure A2. Flow chart showing the analog-to-digital conversion scheme used to obtain seismic records from the AOBH. An alternative scheme that was used to obtain $P$ wave arrival times and for calibration tests is also shown (see text).

Figure A3. Circuit diagram for the AOBH preamplifier. The calibration pulse is introduced across resistor $R_{3}$. Component values are listed in Table A1.

Figure A4. Amplitude responses for (a) AOBH 1 and (b) AOBH 7 obtained from $2 \mathrm{mV}$ (pluses and solid), $10 \mathrm{mV}$ (crosses and dashed), and $50 \mathrm{mV}$ (circles and dot-dashed) sine wave tests. The curves are obtained using a fourth order polynomial fit to the data. The results are divided by the input voltage and normalized with respect to the $2 \mathrm{mV}, 10 \mathrm{~Hz}$ result.

Figure A5. Sine wave saturation of characteristics of AOBH 1 at $5 \mathrm{~Hz}$ (pluses and solid), $10 \mathrm{~Hz}$ (crosses and dashed), $20 \mathrm{~Hz}$ (circles and dotdashed) and $40 \mathrm{~Hz}$ (asterisks and dotted). (a) The recorded amplitude as a function of input voltage. (b) The sensitivity, obtained by dividing the recorded amplitude by the input voltage, as a function of input voltage. Both plots are normalized to an input voltage of 2 $\mathrm{mV}$.

Figure A6. Examples of recorded power spectra (solid) for high-quality 20-mV sine wave inputs with frequencies of (a) $10 \mathrm{~Hz}$ and (b) $20 \mathrm{~Hz}$. Ambient noise levels are also shown (dashed).

Figure A7. Distortion levels D determined using equation (A7) for AOBH 1 at (a) $5 \mathrm{~Hz}$, (b) $10 \mathrm{~Hz}$, (c) $20 \mathrm{~Hz}$, and (d) $40 \mathrm{~Hz}$. The solid lines show 
the spectral leakage to frequencies above a $2-\mathrm{Hz}$-wide band centered on the input frequency, while the dashed lines show spectral leakage to all frequencies outside the frequency band. Ambient noise levels are also shown (dotted lines).

Figure A8. Predicted effects of the measured spectral leakage upon estimates of $t^{*}$ under the assumption that spectral leakage is a linear process. The expected values of $t^{*}$ are plotted against the input $t^{*}$ value for estimates obtained over the frequency intervals 10-20, 10-30, 10-40, and $10-50 \mathrm{~Hz}$ (solid). The expected $\mathrm{t}^{*}$ estimate is also shown for a frequency interval constrained to include recorded powers no less than $1 / 1000$ the largest recorded spectral power (dashed).

Figure A9. Recorded amplitude ratio of $5 \mathrm{~Hz}$ and $20 \mathrm{~Hz}$ signals plotted against the input amplitude of the $5 \mathrm{~Hz}$ signal, for an input signal comprising 5 and $20 \mathrm{~Hz}$ sine waves with an amplitude ratio of 20.

Figure A10. Series of plots illustrating the technique used to obtain the impulse response of the $\mathrm{AOBH}$ from the response to a low-frequency square wave. (a) A portion to the times series recorded by AOBH 1 for a 0.3 $\mathrm{Hz}$ square wave input with a $12.6 \mathrm{mV}$ peak-to-peak amplitude. (b) Smoothed amplitude and (c) phase spectra obtained from 10 cycles of the square wave response. (d) Time series obtained from an inverse fast Fourier transform of (b) and (c). (e) Time series, (f) amplitude spectrum, and $(\mathrm{g})$ phase spectrum for the impulse response of $\mathrm{AOBH}$ 1 obtained using equation (A11).

Figure A11. Amplitude responses estimated for (a) AOBH 1, and (b) AOBH 7 using both square wave (solid) and sine wave (dashed) tests. The sine wave results are shown for peak-to-peak input amplitudes of 2, 10 and $50 \mathrm{mV}$. The square wave results are shown for peak-to-peak input amplitudes of 2, 5, 10, 25, 50, and $100 \mathrm{mV}$ (AOBH 1 only) and are obtained by multiplying the responses obtained from square waves with 1.26 times the stated amplitude. 
Figure A12. The effect on the 10-mV sine wave amplitude response of errors in bias current, head current, and tape speed adjustments for (a) AOBH 3 and (b) AOBH 6. The effect of reducing the bias current by $10 \%$ (circles and dot-dashed), the head current by $20 \%$ (asterisks and dotted), and the tape speed by $10 \%$ (crosses and dashed) are shown together with the initial amplitude response (pluses and solid).

Figure A13. Tape speed as a function of the tape driving d.c. voltage normalized to the value at $13 \mathrm{~V}$.

Figure A14. Tape speed normalized to the speed at the time of deployment for (a) AOBH 1 and (b) AOBH 4 plotted against time (Julian days) for the tomography experiment. Values are obtained from digitization rate observed during analog-to-digital conversion.

Figure A15. Repeated measurements of 10-mV amplitude response for (a) AOBH 1 and (b) AOBH 3 derived from sine wave tests (pluses and solid) and square wave tests (dashed). Tests were repeated after reloading the $1 / 4$ " tape but without recalibrating the instrument.

Figure A16. Circuit used to generate the AOBH calibration pulse; the circuit is introduced in series with the hydrophone. The resistor $R_{3}$ is the same as $R_{3}$ of Figure $A 3$.

Figure A17. Calibration pulses for AOBH 4: (a) The theoretical recorded calibration pulse. The calibration pulse predicted by the circuit in Figure A16 has been convolved with the instrument response normalized to unit amplitude at $10 \mathrm{~Hz}$. (b) A calibration pulse recorded during the instrument response tests. (c) A typical calibration pulse recorded during the tomographic experiment. (d) $25-$ $\mathrm{Hz}$ time code recorded simultaneously with the calibration pulse shown in (c).

Figure A18. Calibration pulse amplitudes at $10 \mathrm{~Hz}$ plotted against Julian day for AOBH 4. (a) All measured amplitudes are shown as pluses. (b) 
Uncertainties obtained for groups of four calibration pulses at the end of each hour are shown as error bars. Dashed lines show the times the Honeywell tape was changed during transcription.

Figure A19. Calibration pulse amplitudes predicted from time code amplitude variations for $\mathrm{AOBH} 4$. For each day one set of parameters (except for Julian day 26 where two sets are calculated) is determined for the linear relationship between calibration pulse and time code amplitudes expressed in equation (A14). The mean amplitudes and uncertainties for the four calibration pulses at the end of each hour are shown as error bars connected by a dotted line. The calibration pulse amplitudes predicted from the time code amplitude using equation (A14) are shown both for the times of calibration pulses (open circles) and the times of the explosive shots (pluses).

Figure A20. Flow chart showing the reactive components of the recording system for the low-frequency channel on the DOBH.

Figure A21. Circuit diagram for the preamplifier and filter section. Component values are: $R_{1}=10^{3} \Omega, R_{2}=9.0 .9 \mathrm{k} \Omega, C=1 \mu \mathrm{F}$. The portion of the circuit outside the dashed box has the same configuration as the input to the gain-ranging amplifier, which has component values: $R_{2}=10^{5}$ $\Omega, \mathrm{C}=1 \mu \mathrm{F}$.

Figure A22. Circuit diagram the two-pole Sallen and Key filter used to construct the 6 pole, 2-dB-ripple, low-pass Chebyshev filter used in the DOBH during the tomography experiment.

Figure A23. Theoretical response and calibration test responses for DOBH 14 using (a) logarithmic and (b) linear axes. The theoretical response is shown in both plots as a solid line. In (a) the first test (06:00 GMT 24 January) is shown as a dot-dashed line while the three later tests (06:00 GMT 30, 31 January, and 1 February) are shown as dashed lines. In (b) the third test is plotted with error bars after normalization to the theoretical amplitude levels. 
Figure A24. Circuit used to generate the DOBH calibration test signal; the circuit is introduced in series with the hydrophone. The resistor $R_{3}$ is the same as $R_{3}$ of Figure 3.

Figure A25. Amplitude response of the MIT digital OBS. 


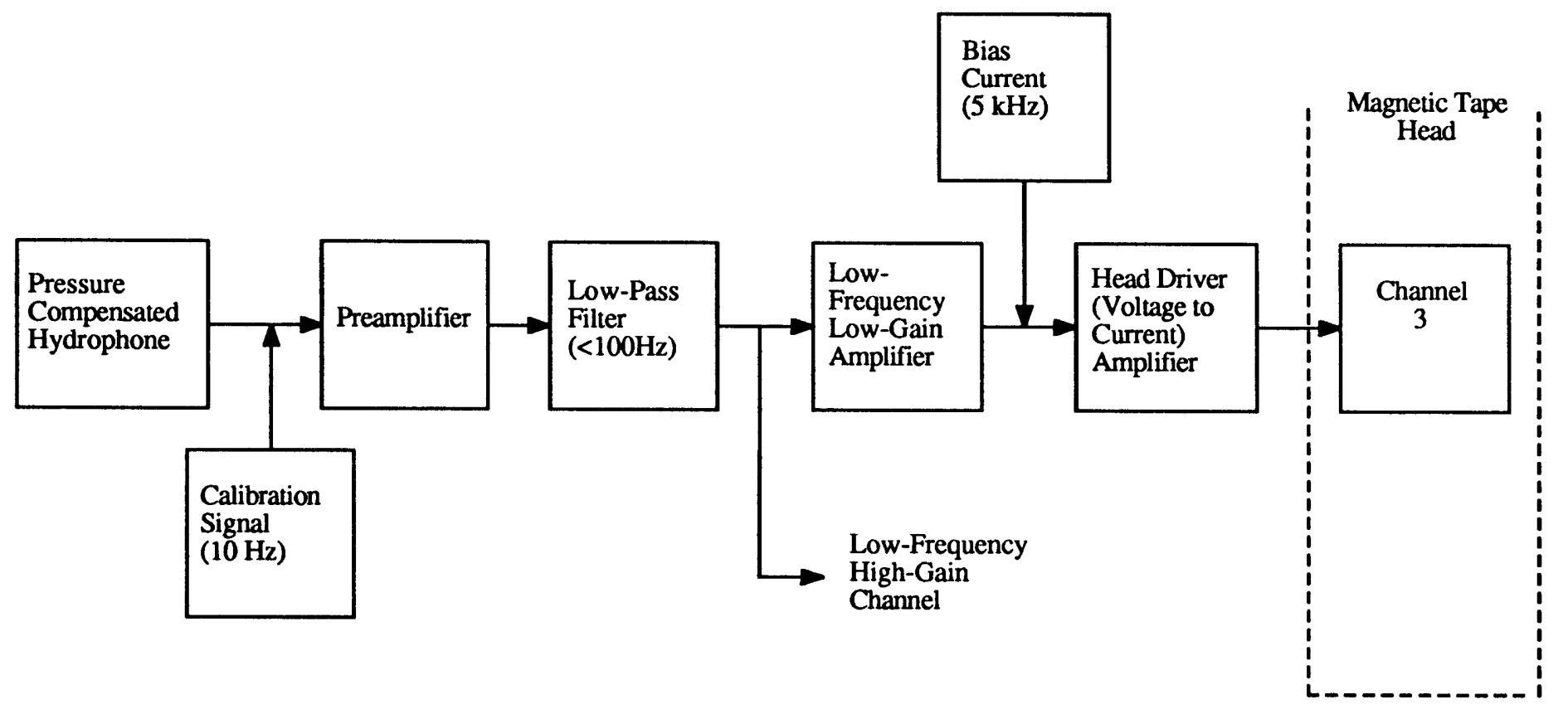

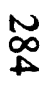


Conventional Analog to Digital Conversion After Transcription

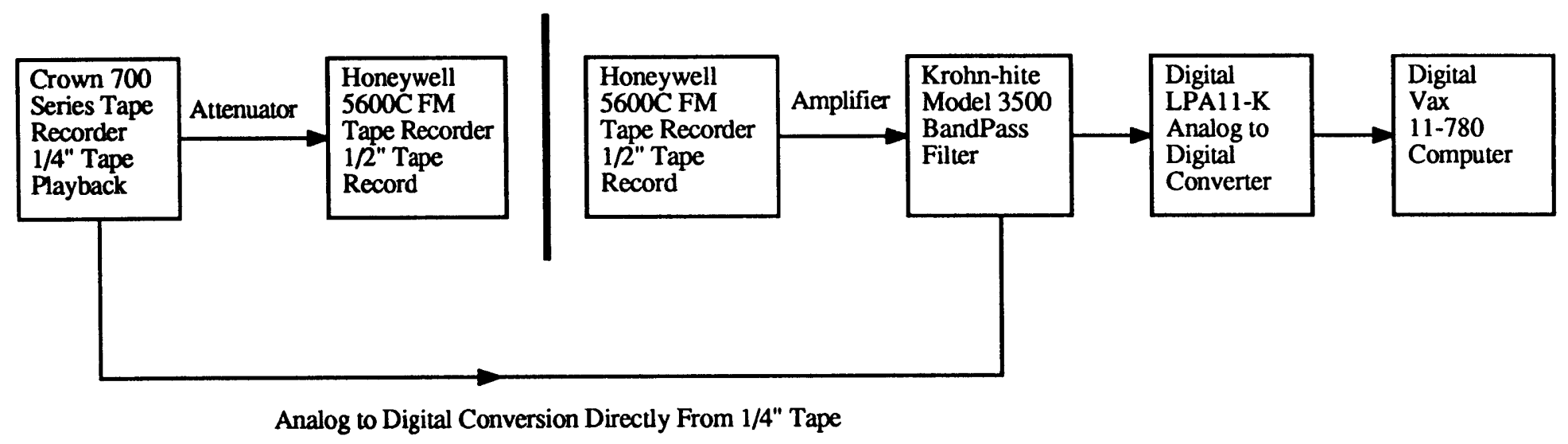




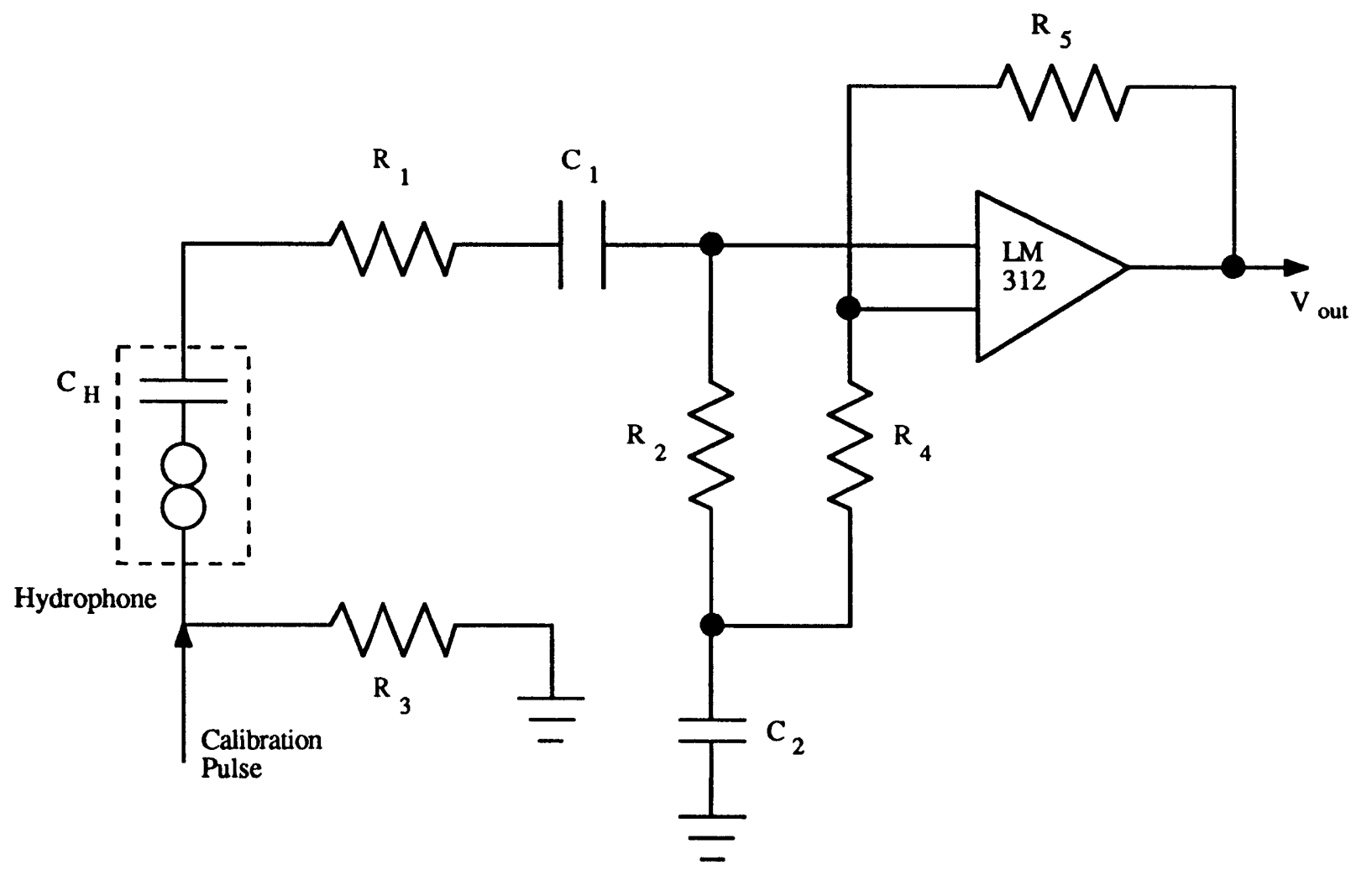

变 

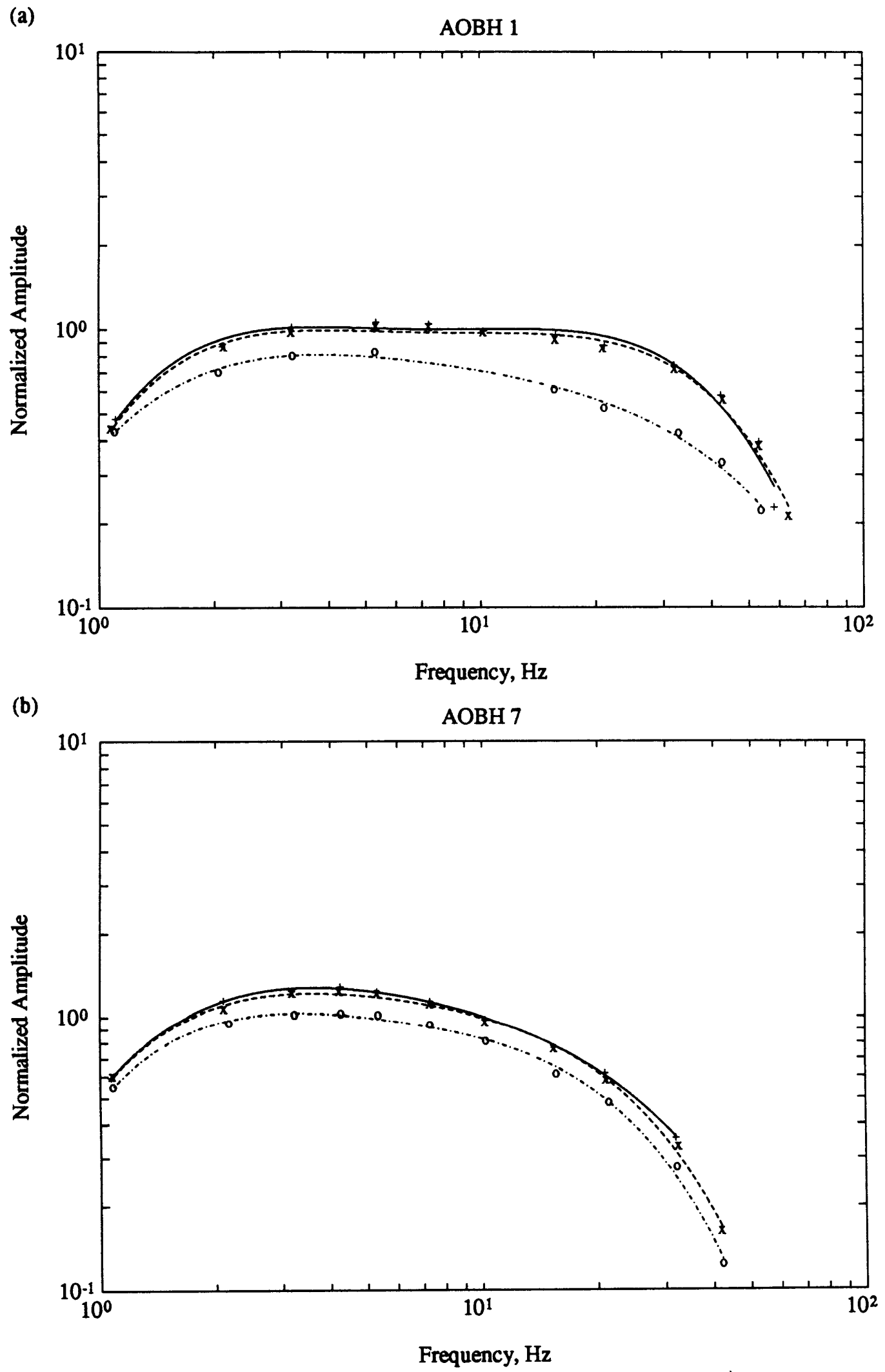

Figure A4 
(a)

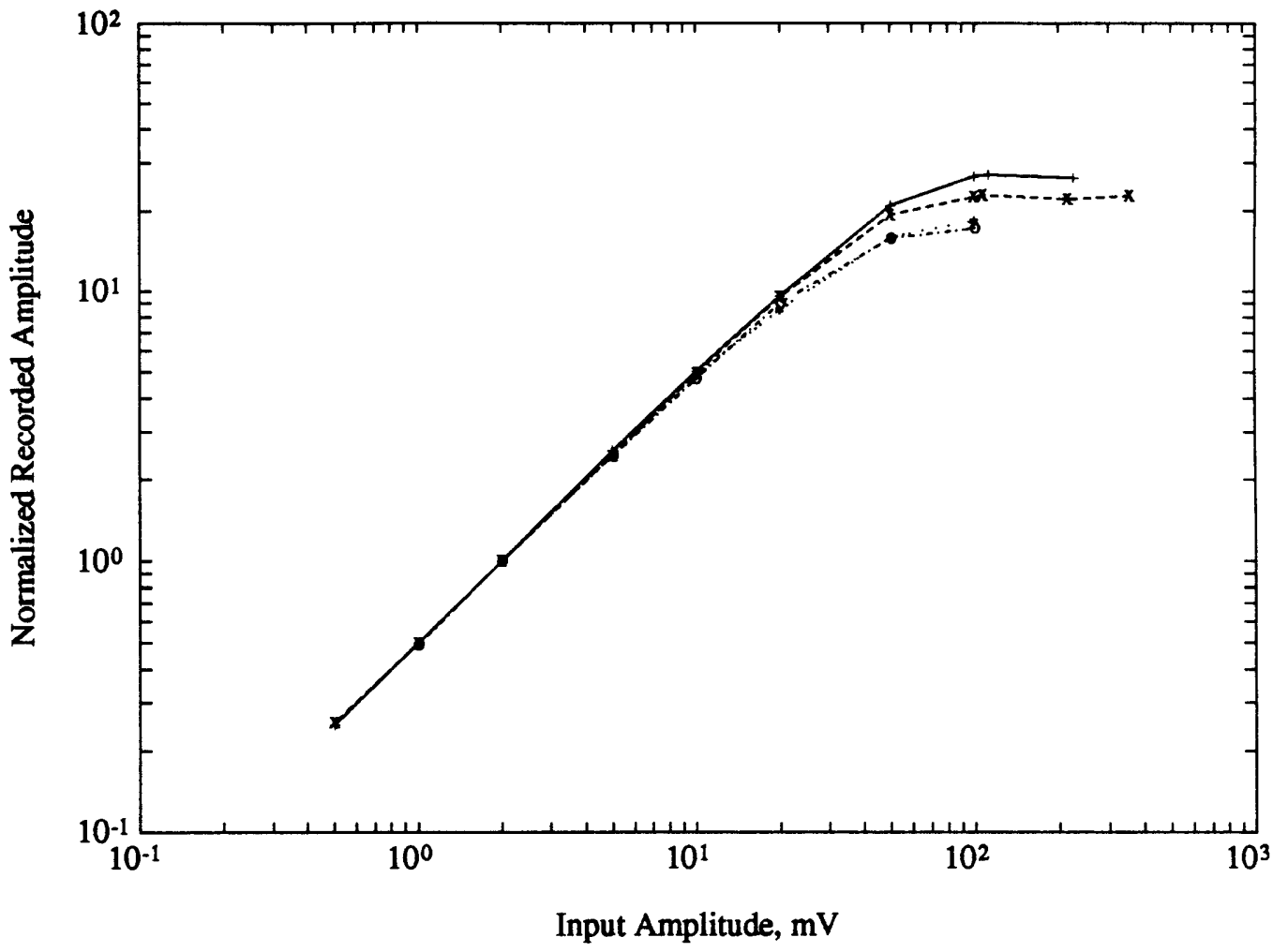

(b)

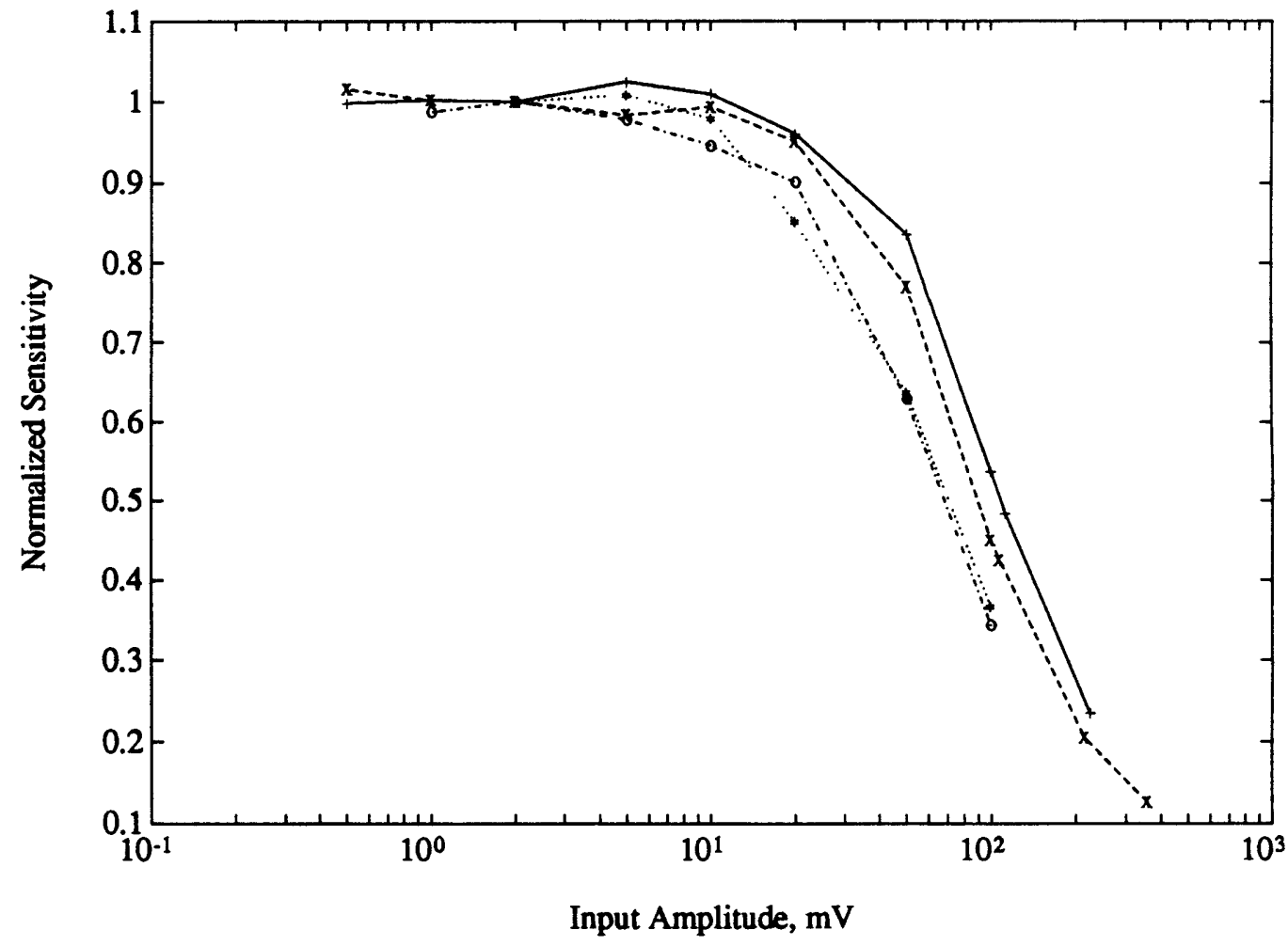

Figure A5 

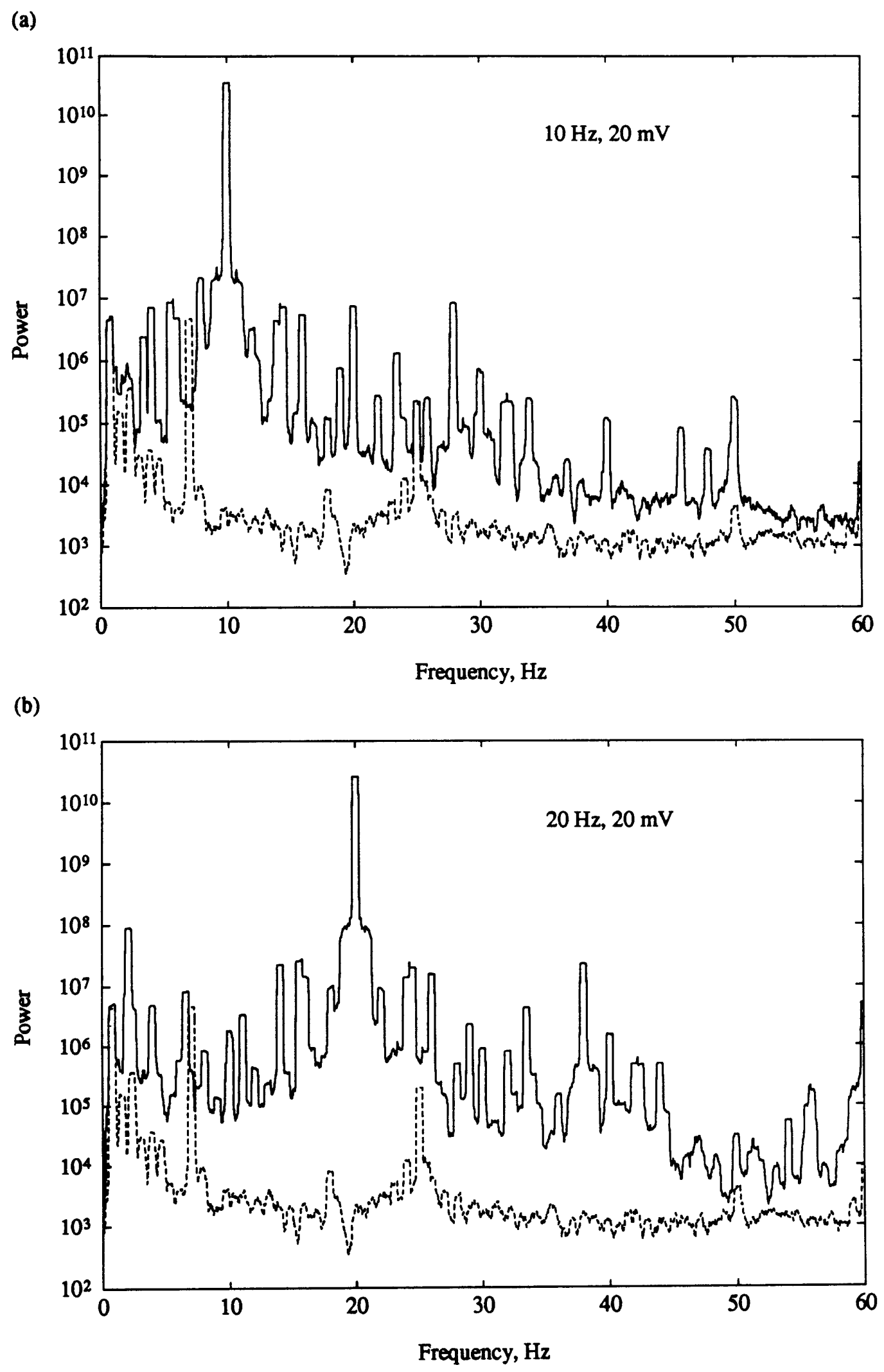

Figure A6 

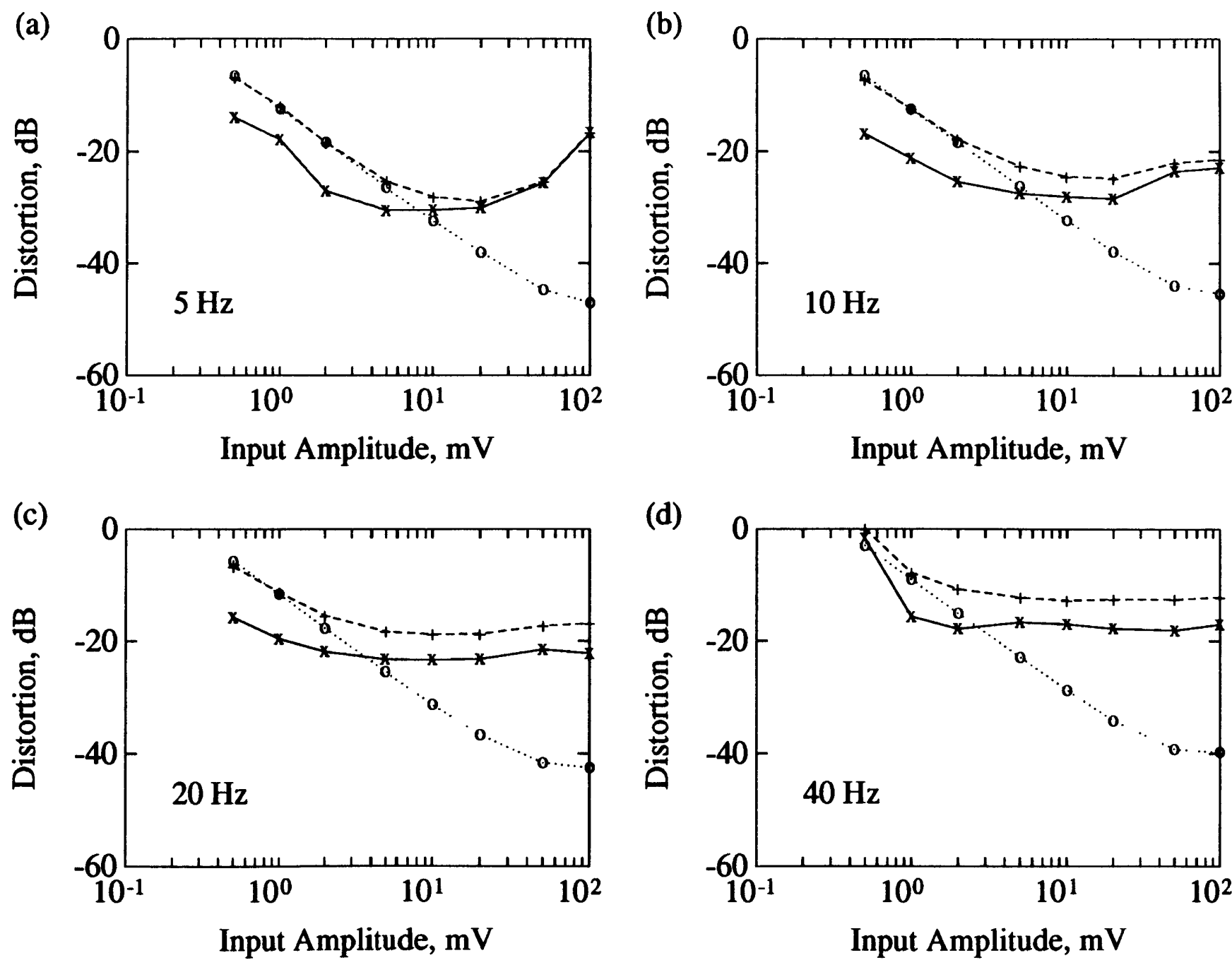


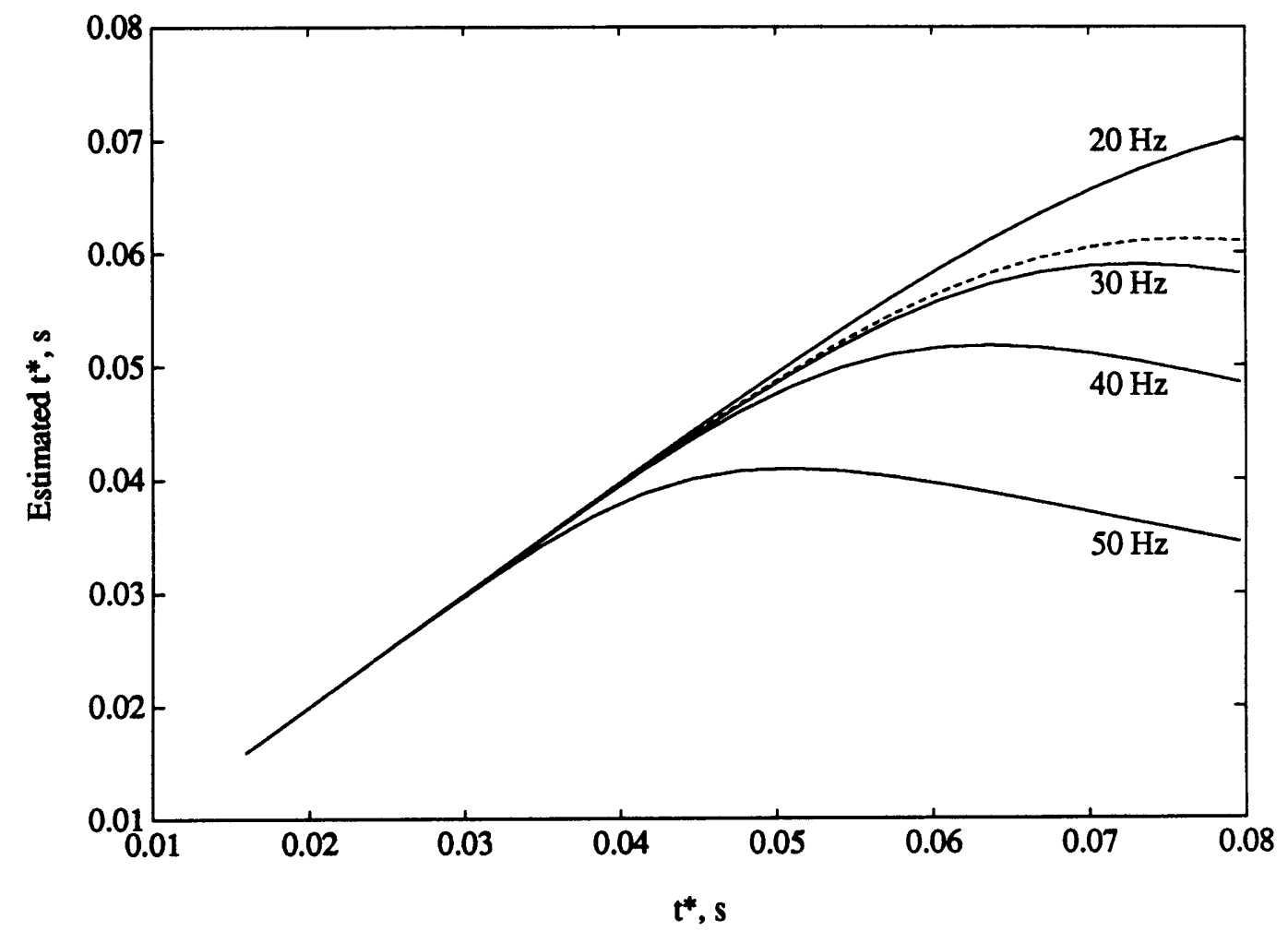

Figure A8 


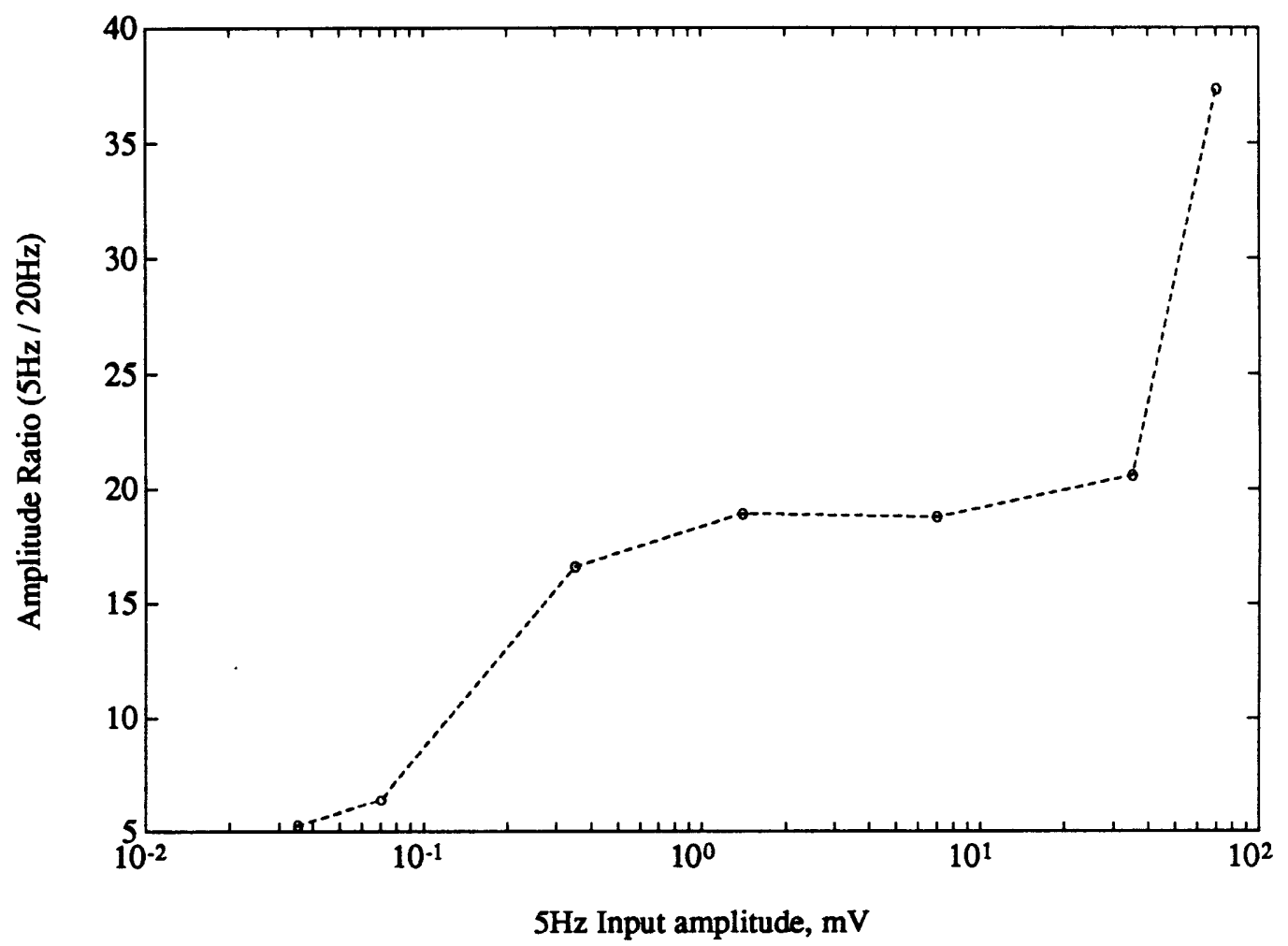

Figure A9 

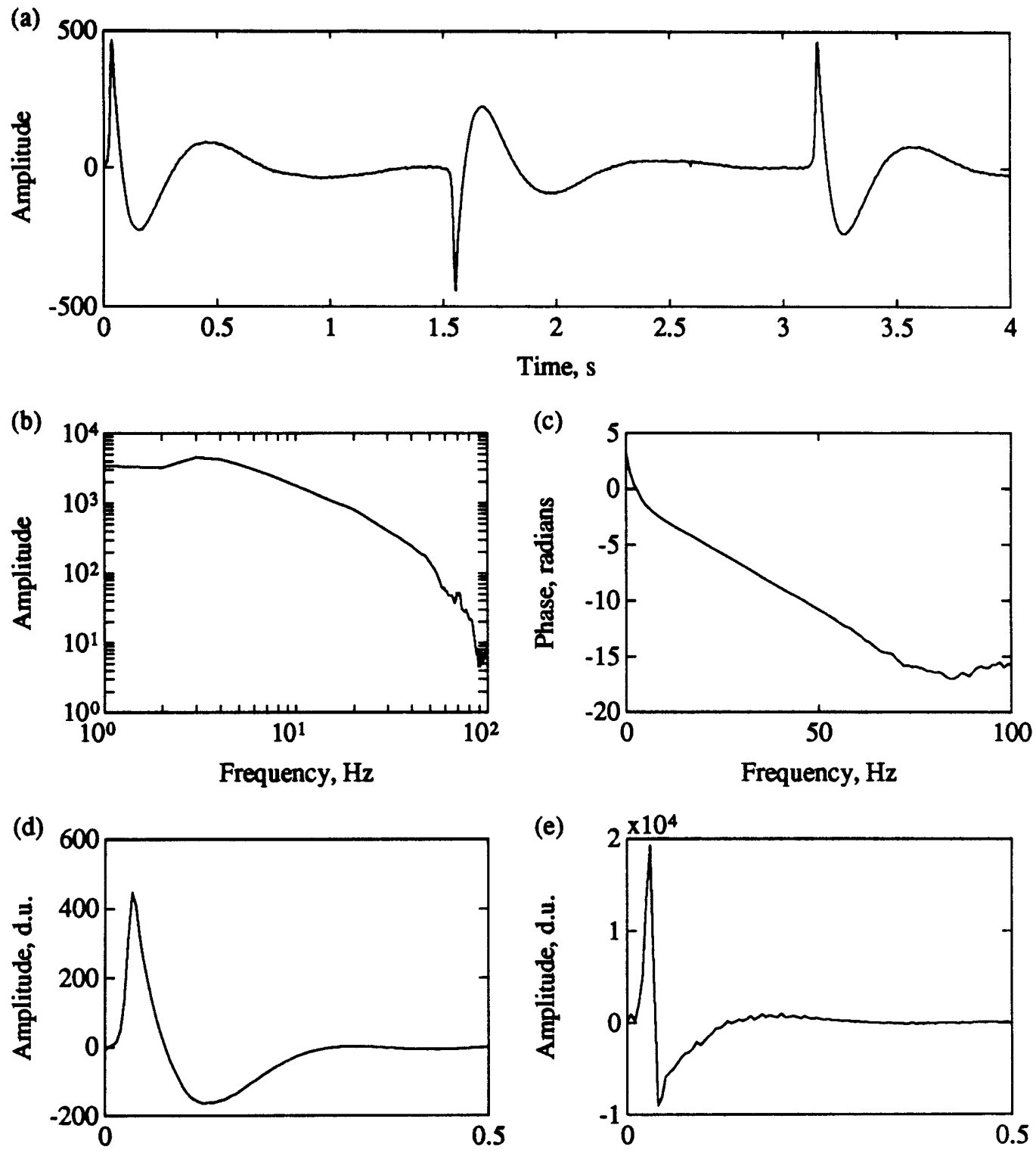

Time, $s$

Time, $\mathrm{s}$
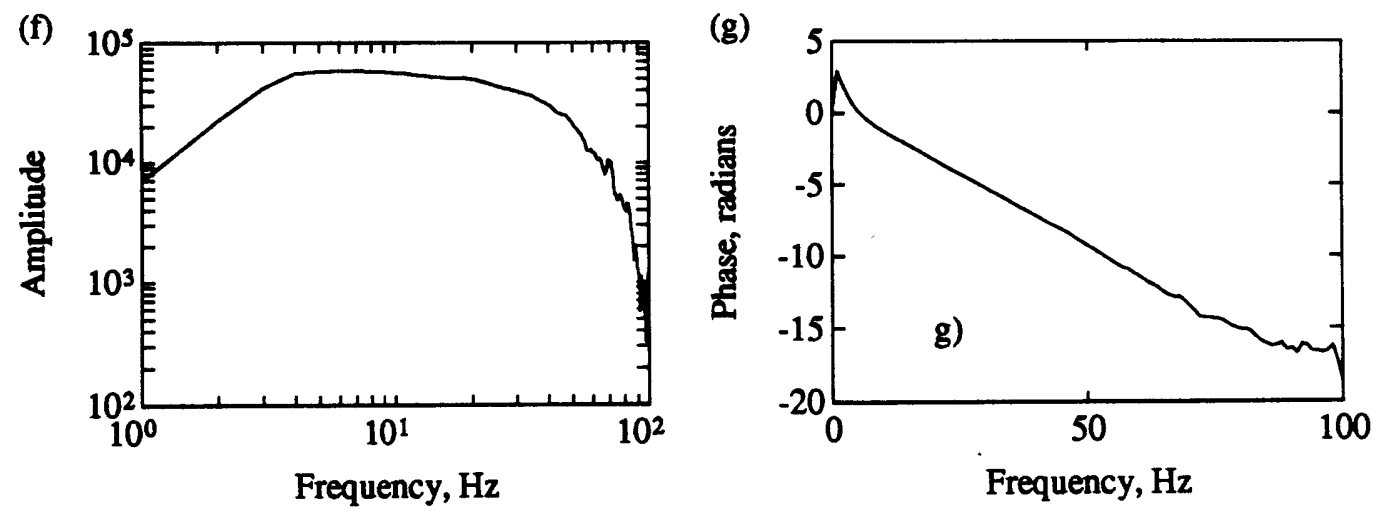

Figure A10 
(a)

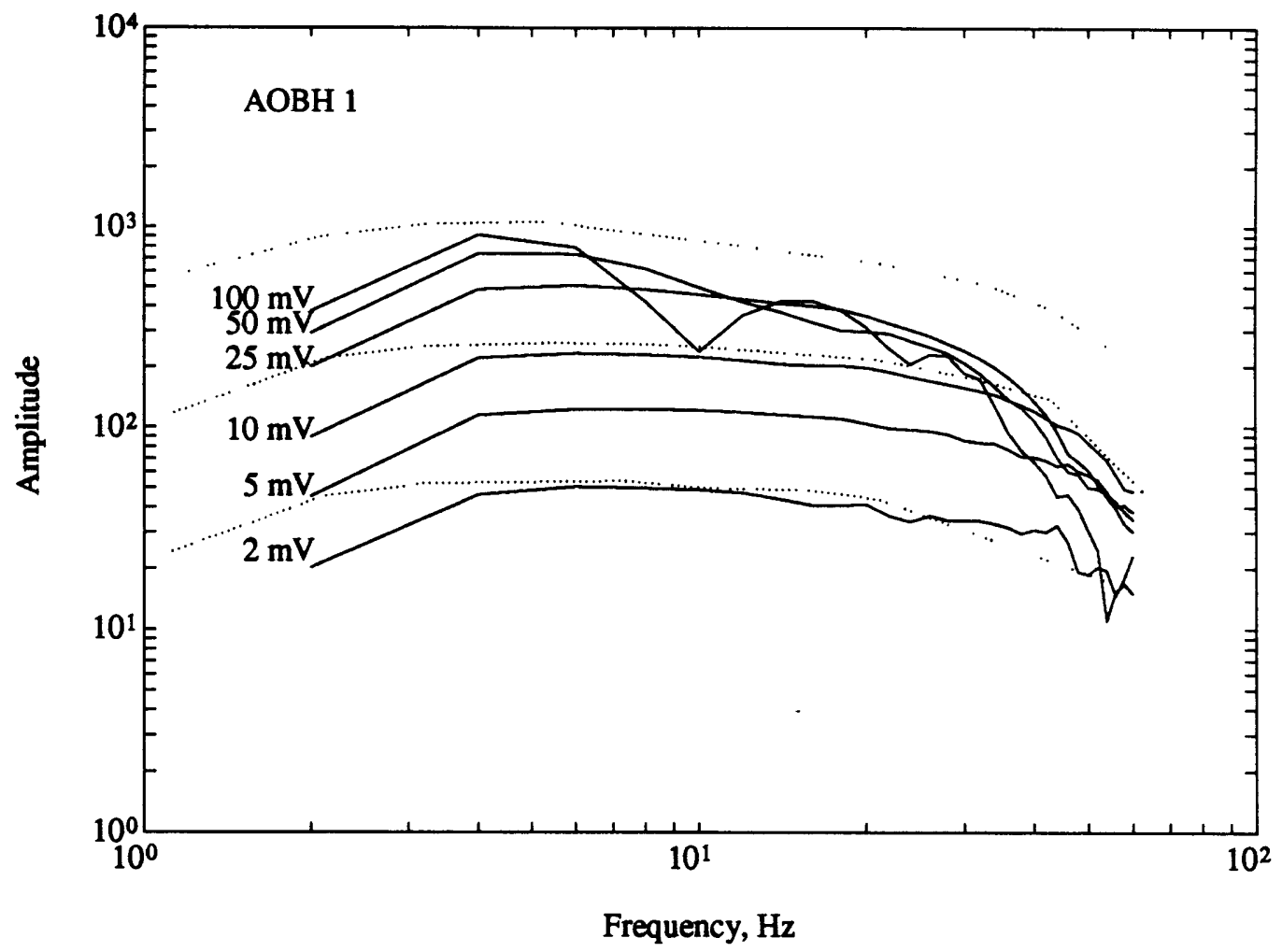

(b)

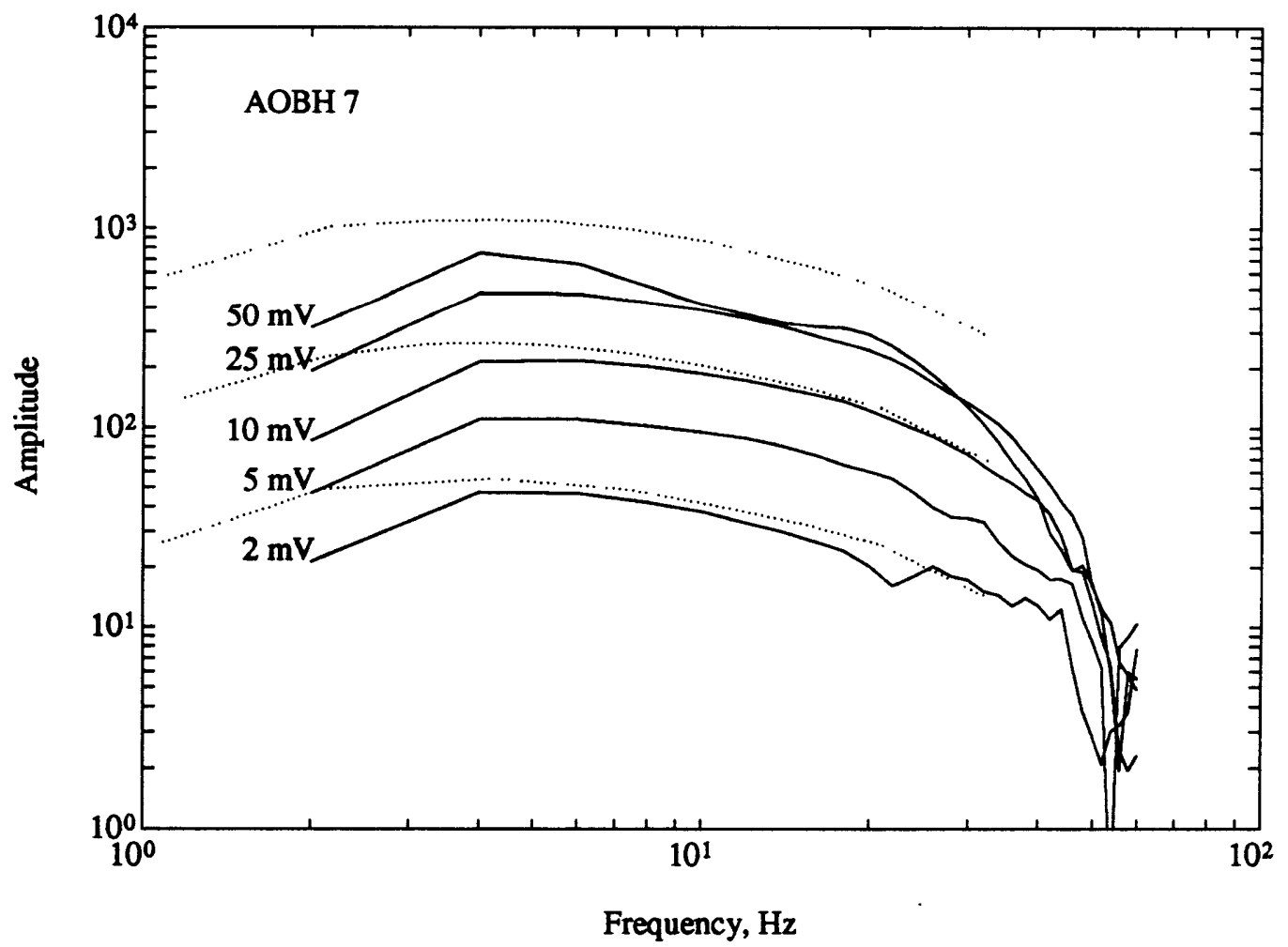

Figure A11 
(a)

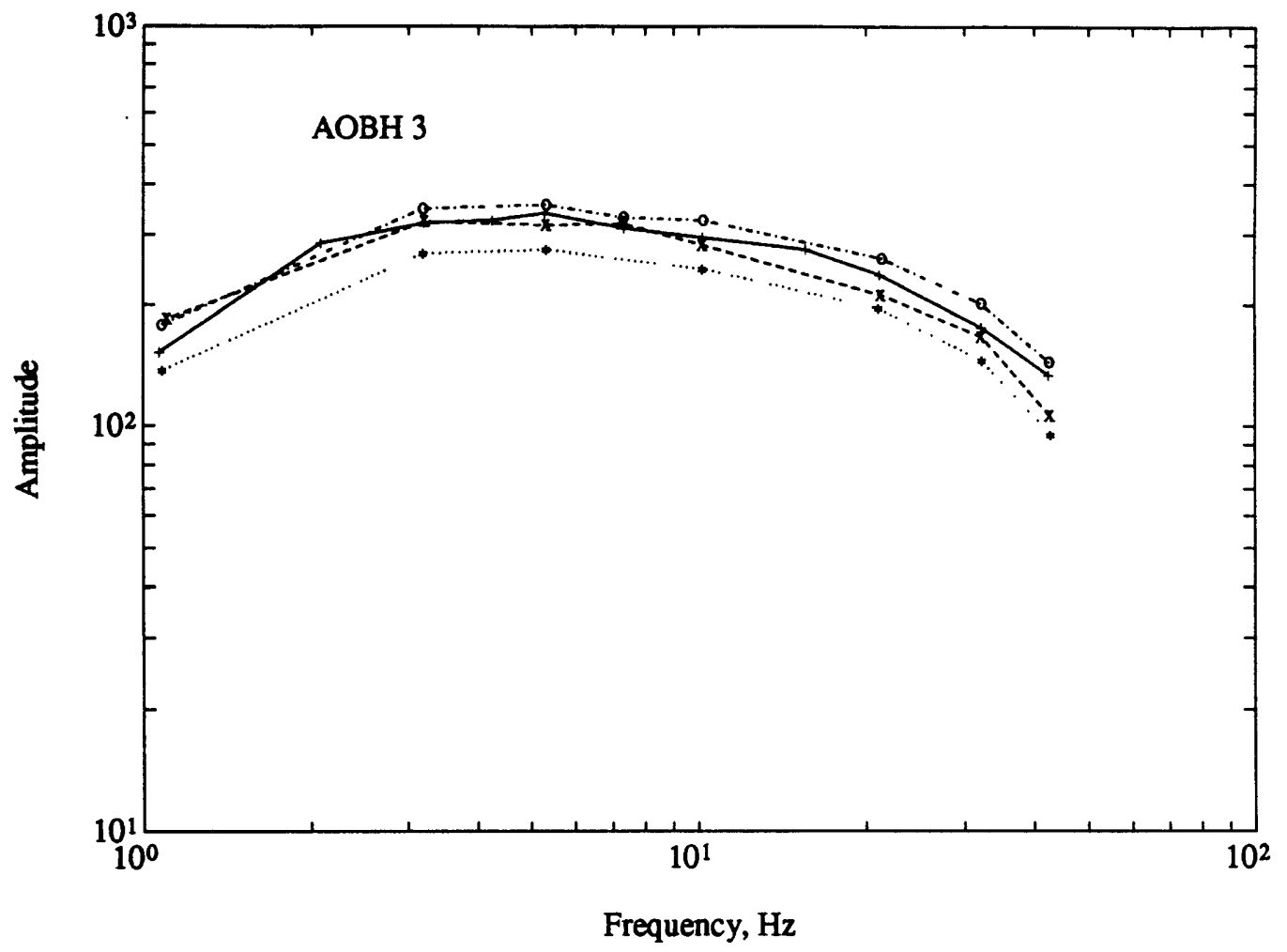

(b)

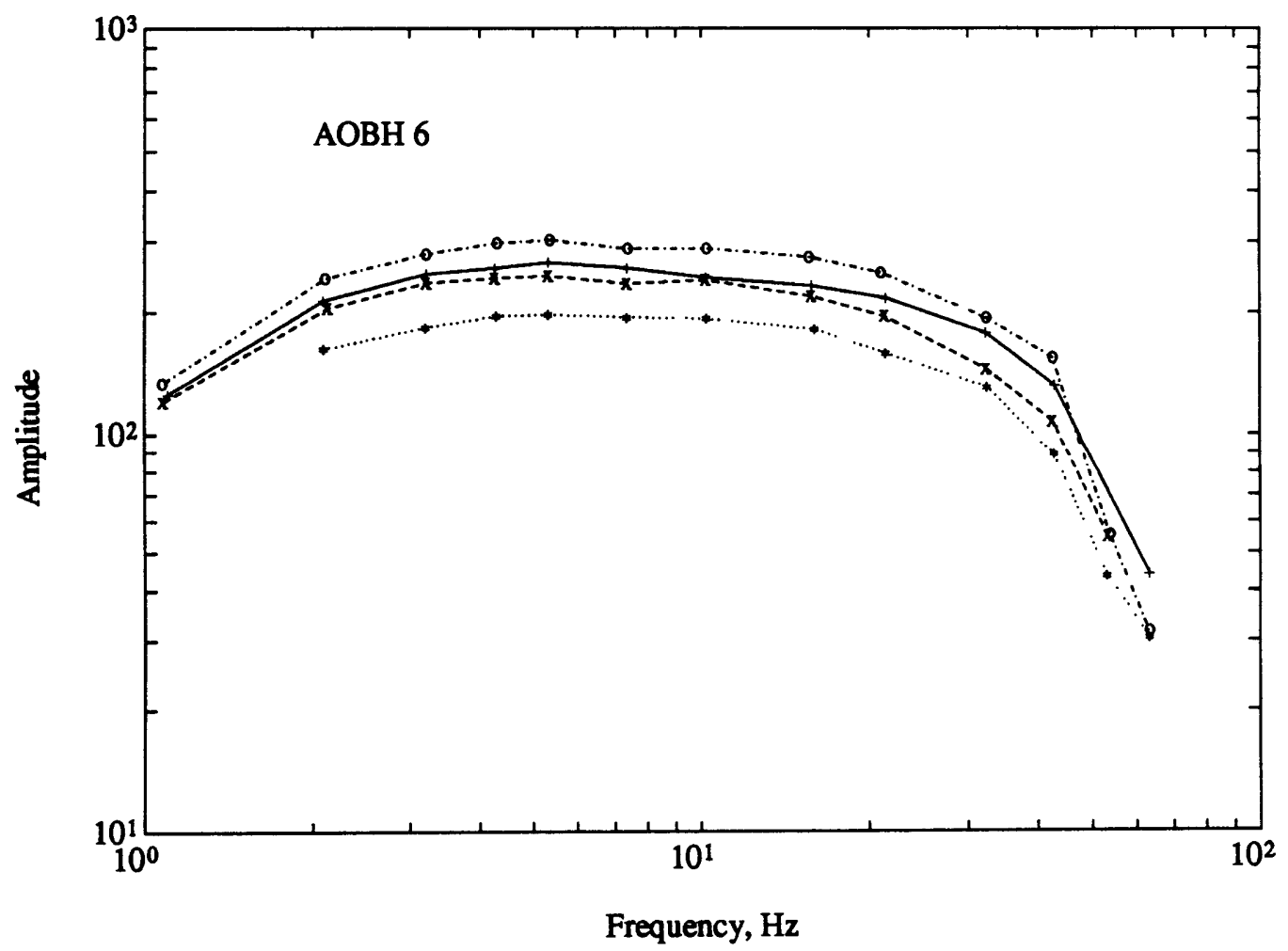

Figure A12 


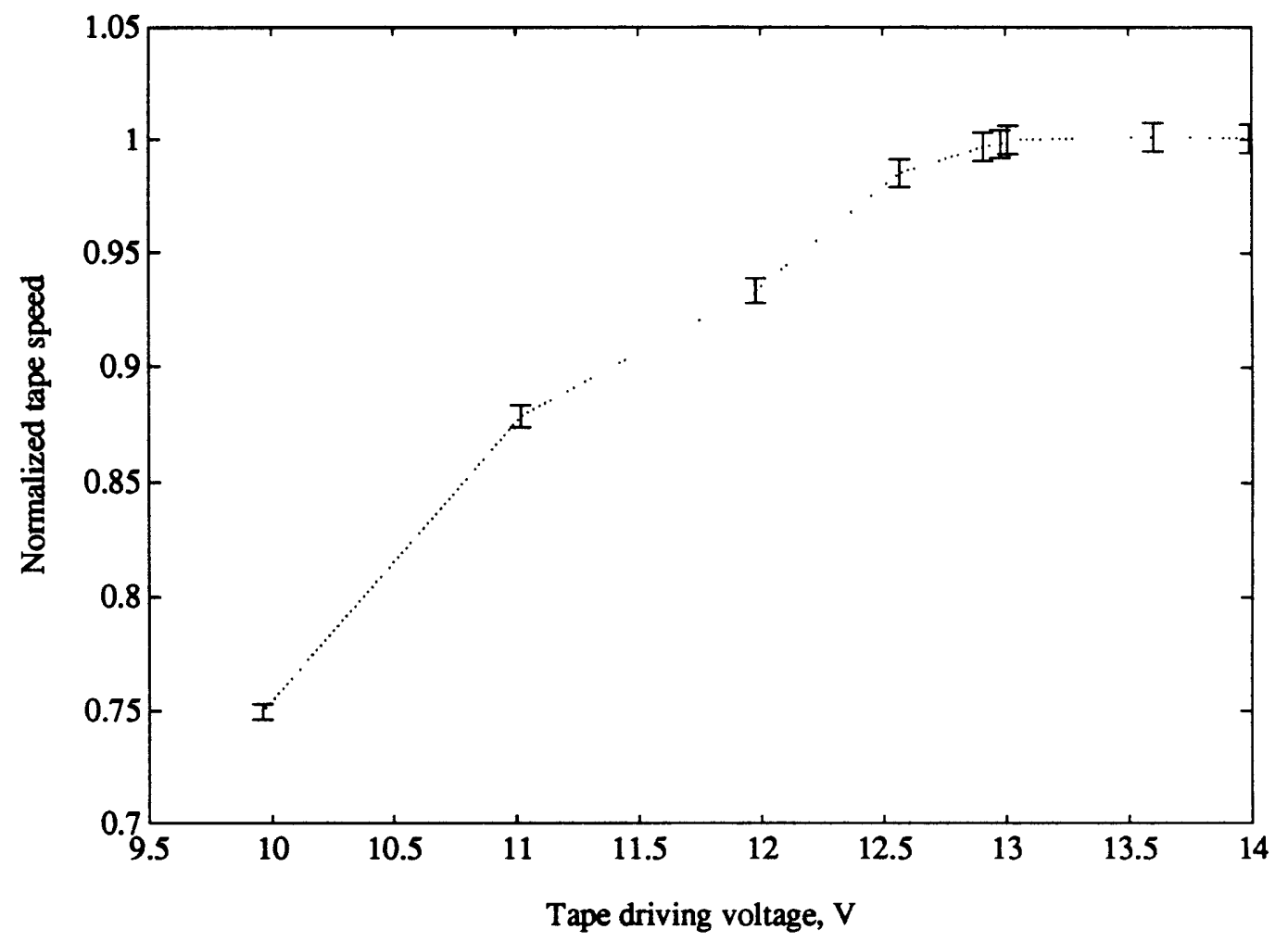

Figure A13 


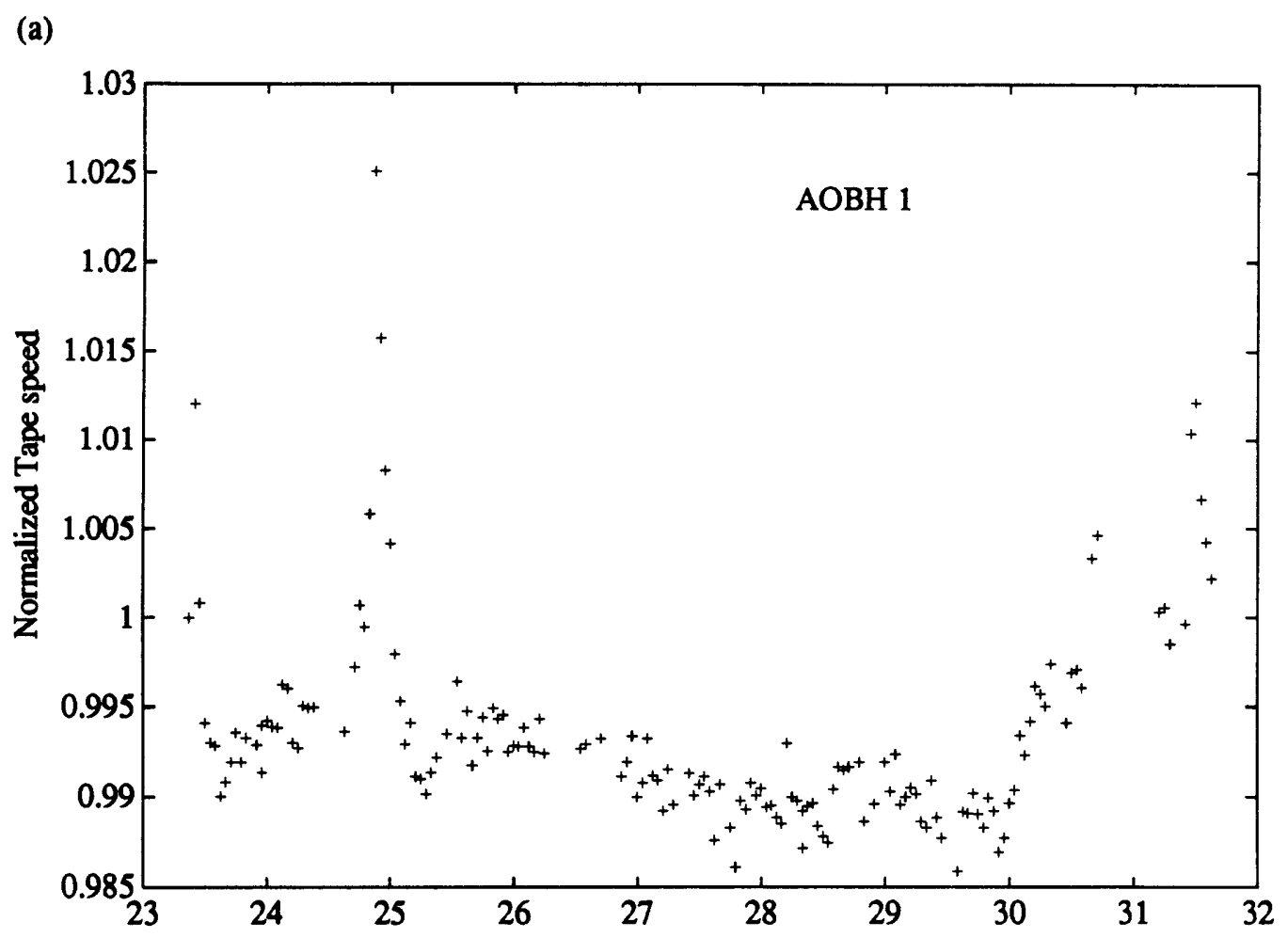

Time, Julian Days

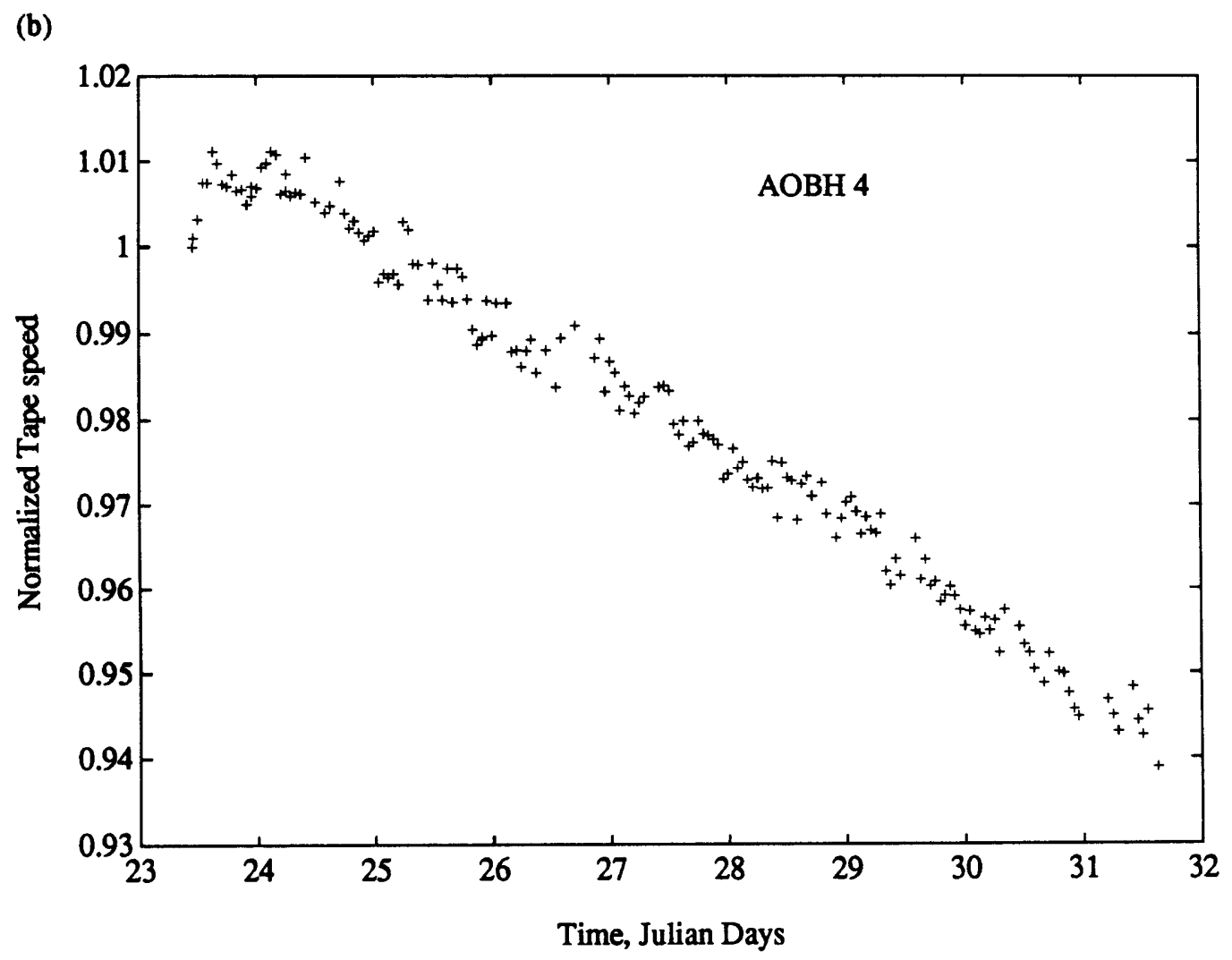

Figure A14 
(a)

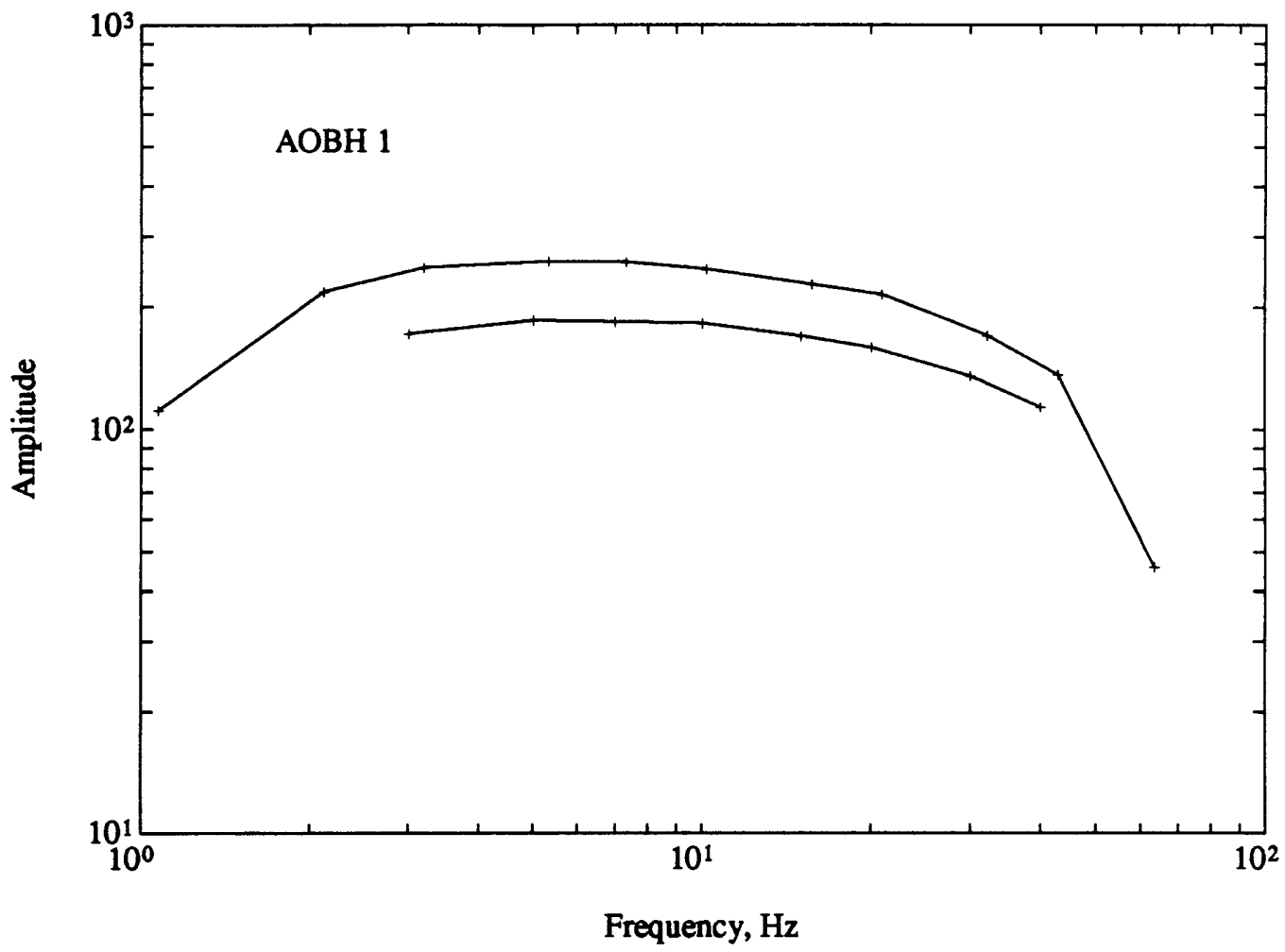

(b)

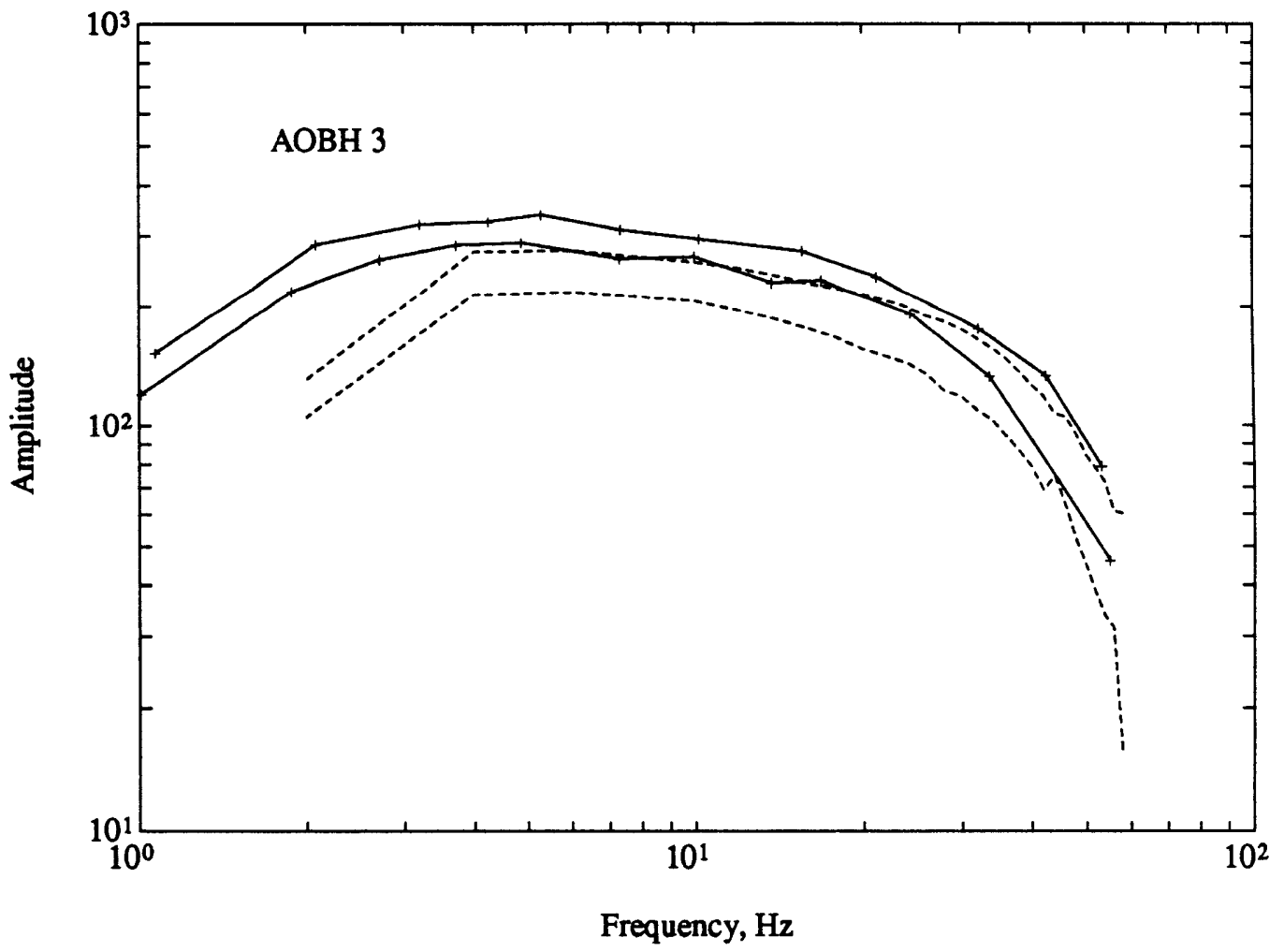

Figure A15 


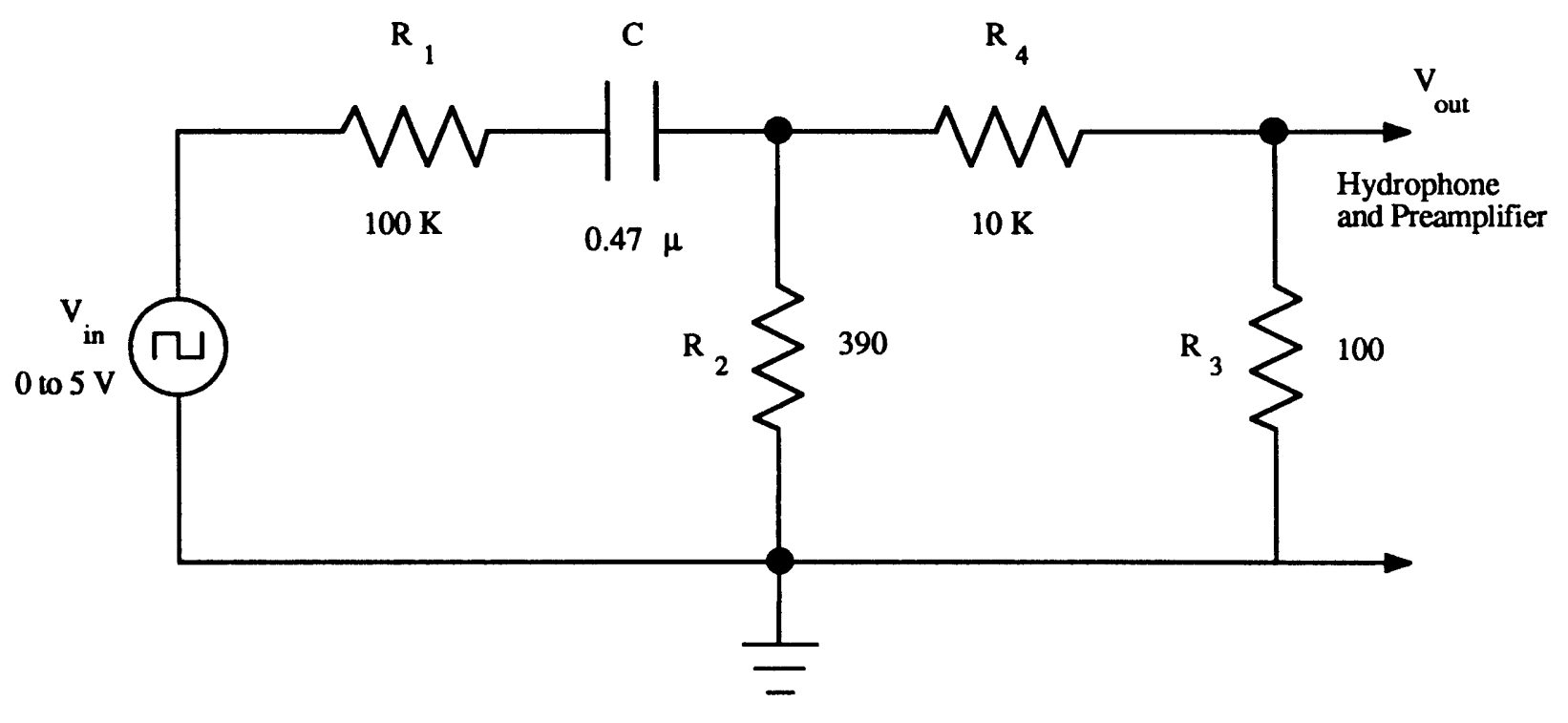

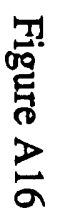



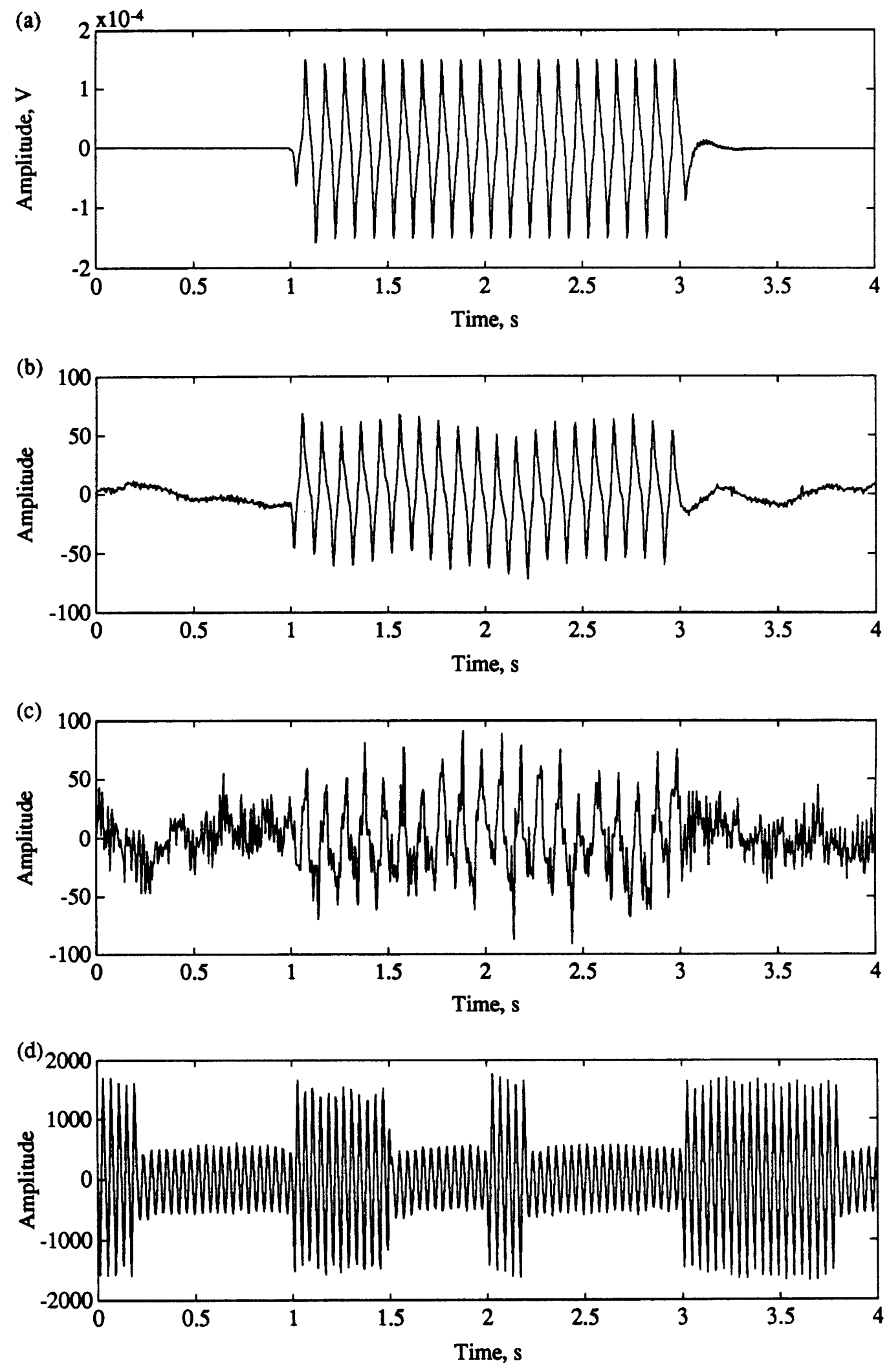

Figure A17 
(a)

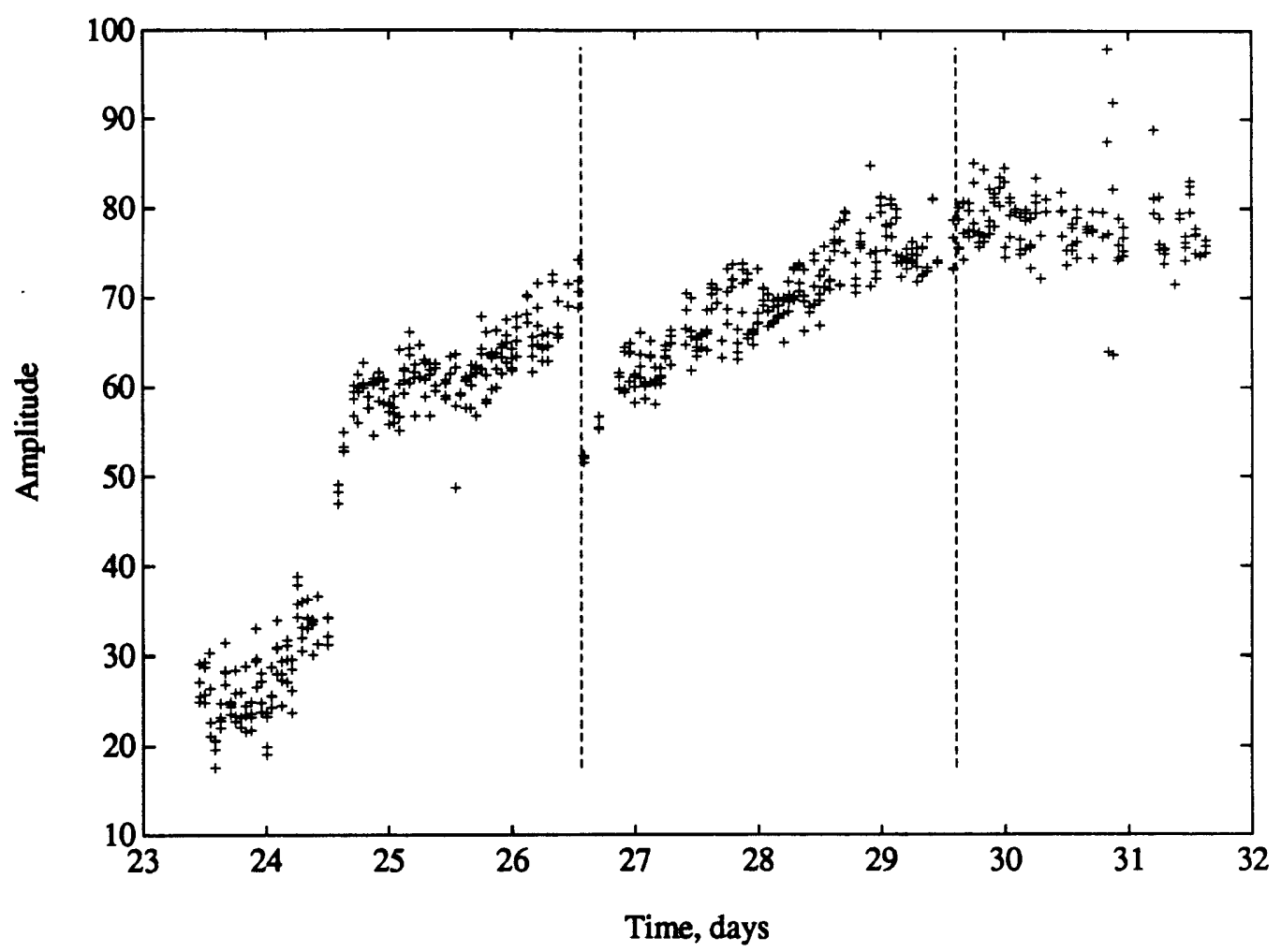

(b)

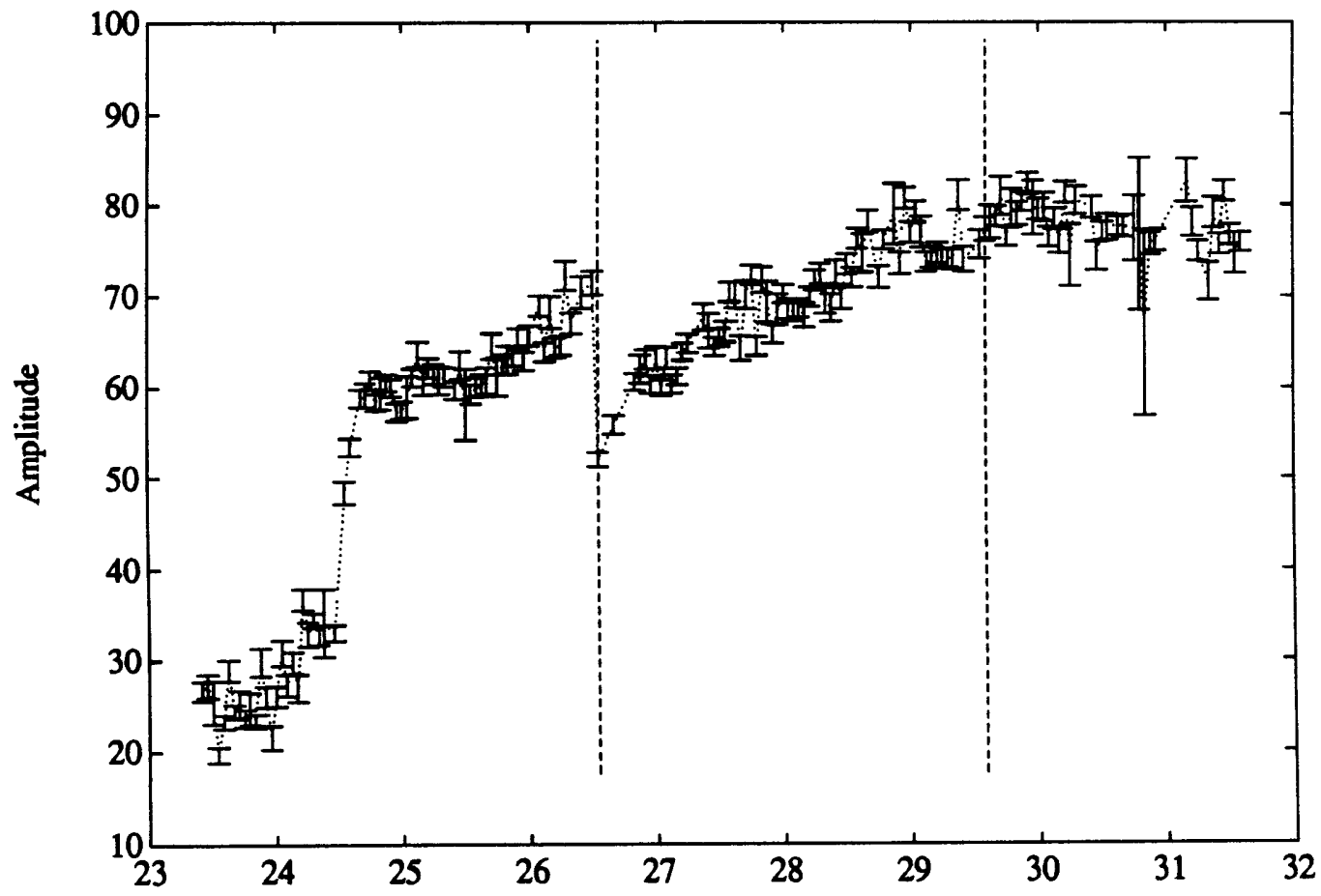

Time, days

Figure A18 

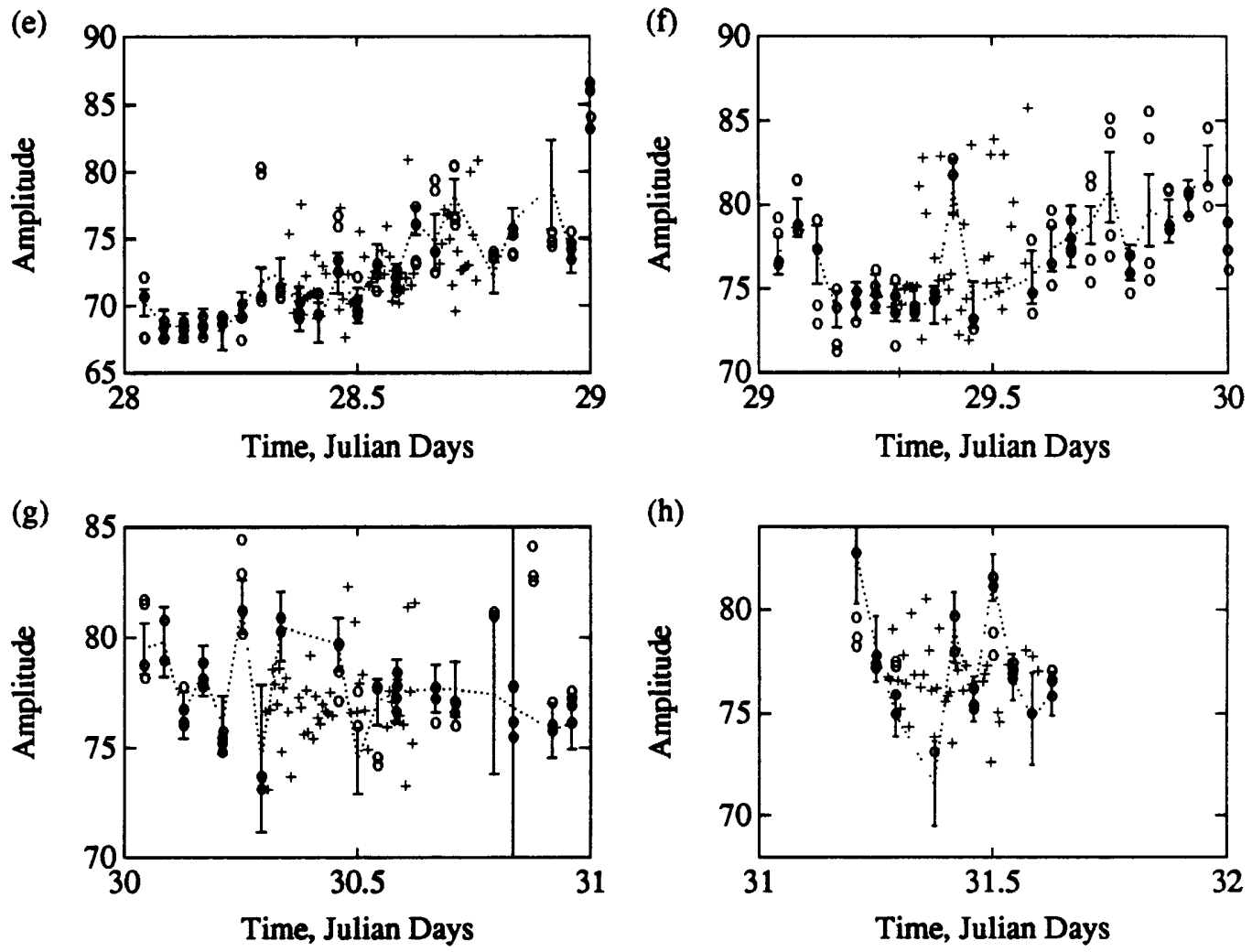

Figure A19 

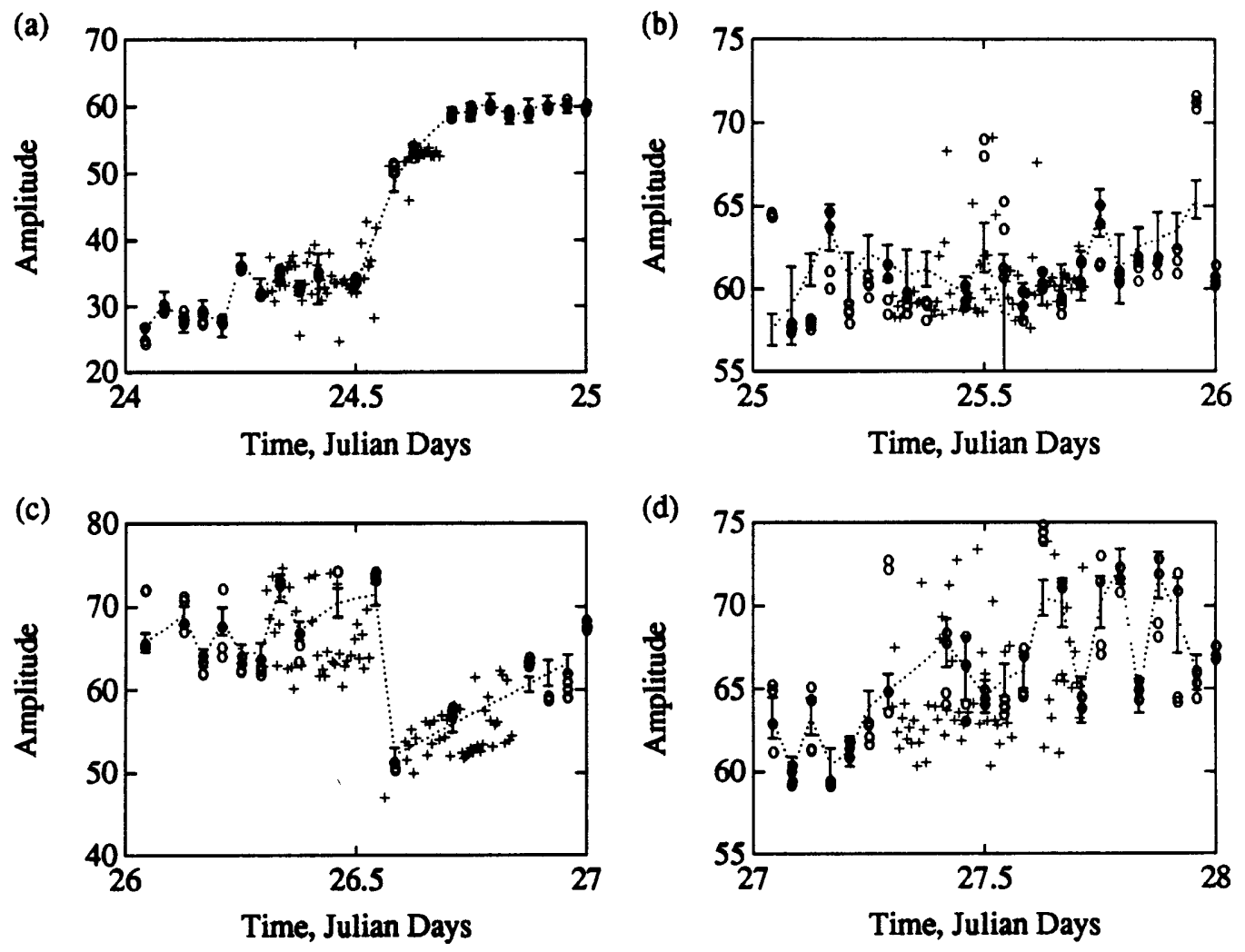

Figure A19 cont. 


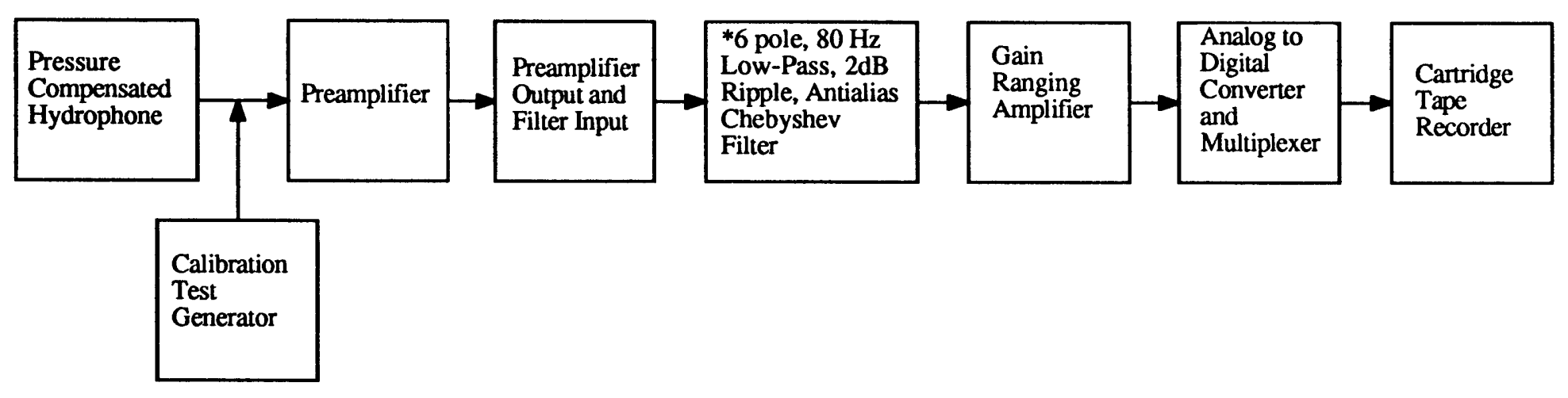




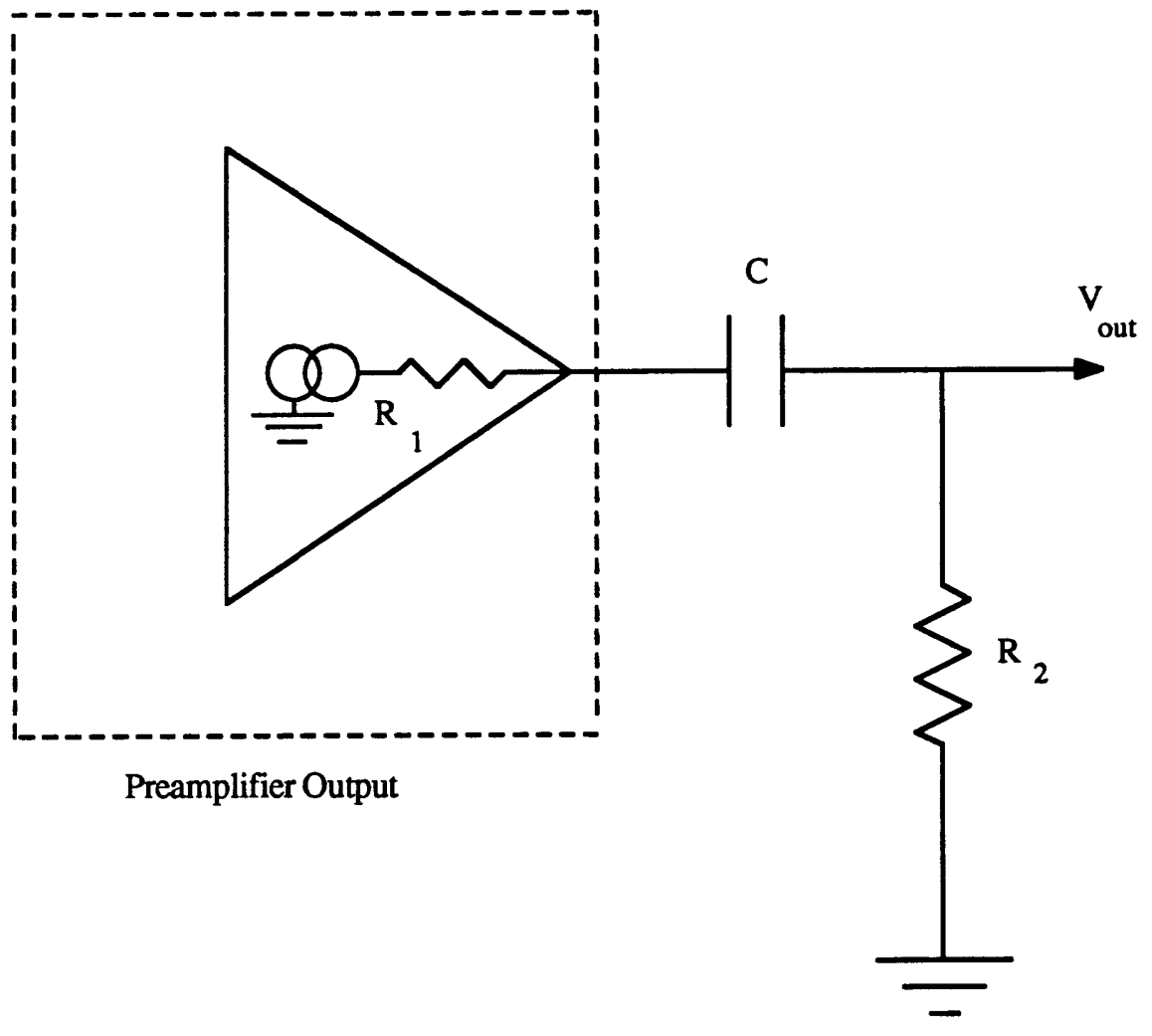

Figure A21 


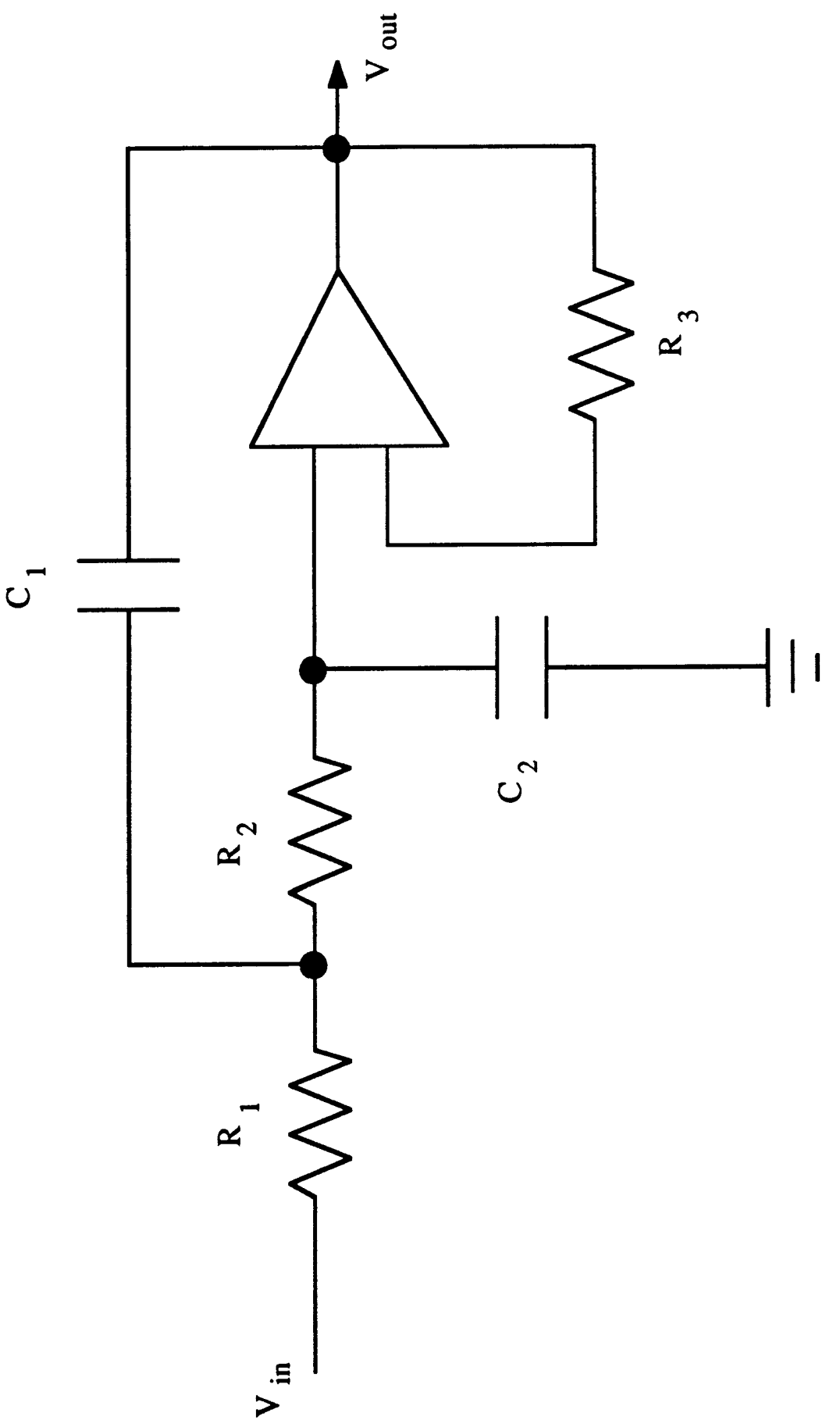

Figure A22 
(a)

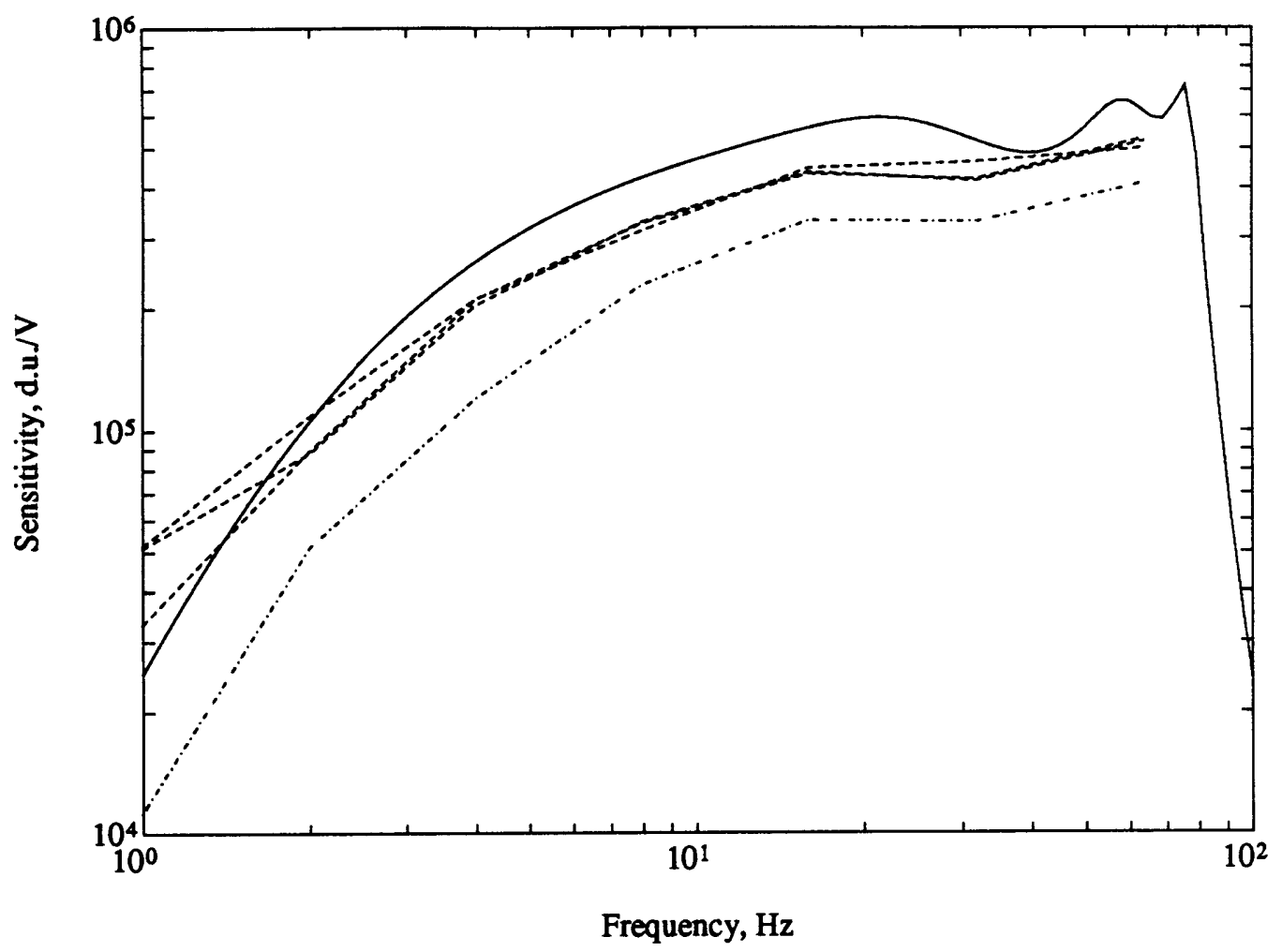

(b) $\times 10^{5}$

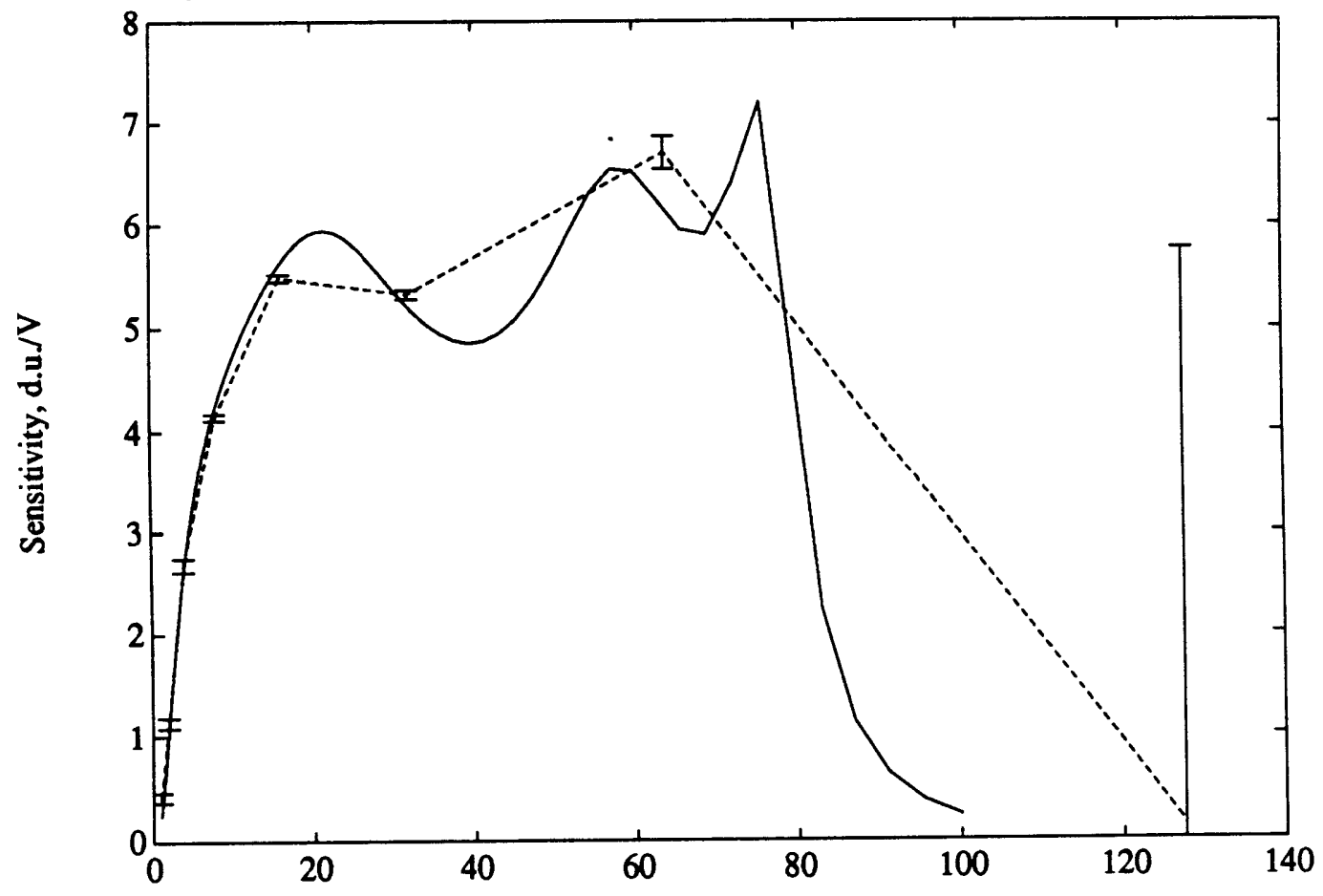

Frequency, $\mathrm{Hz}$

Figure A23 


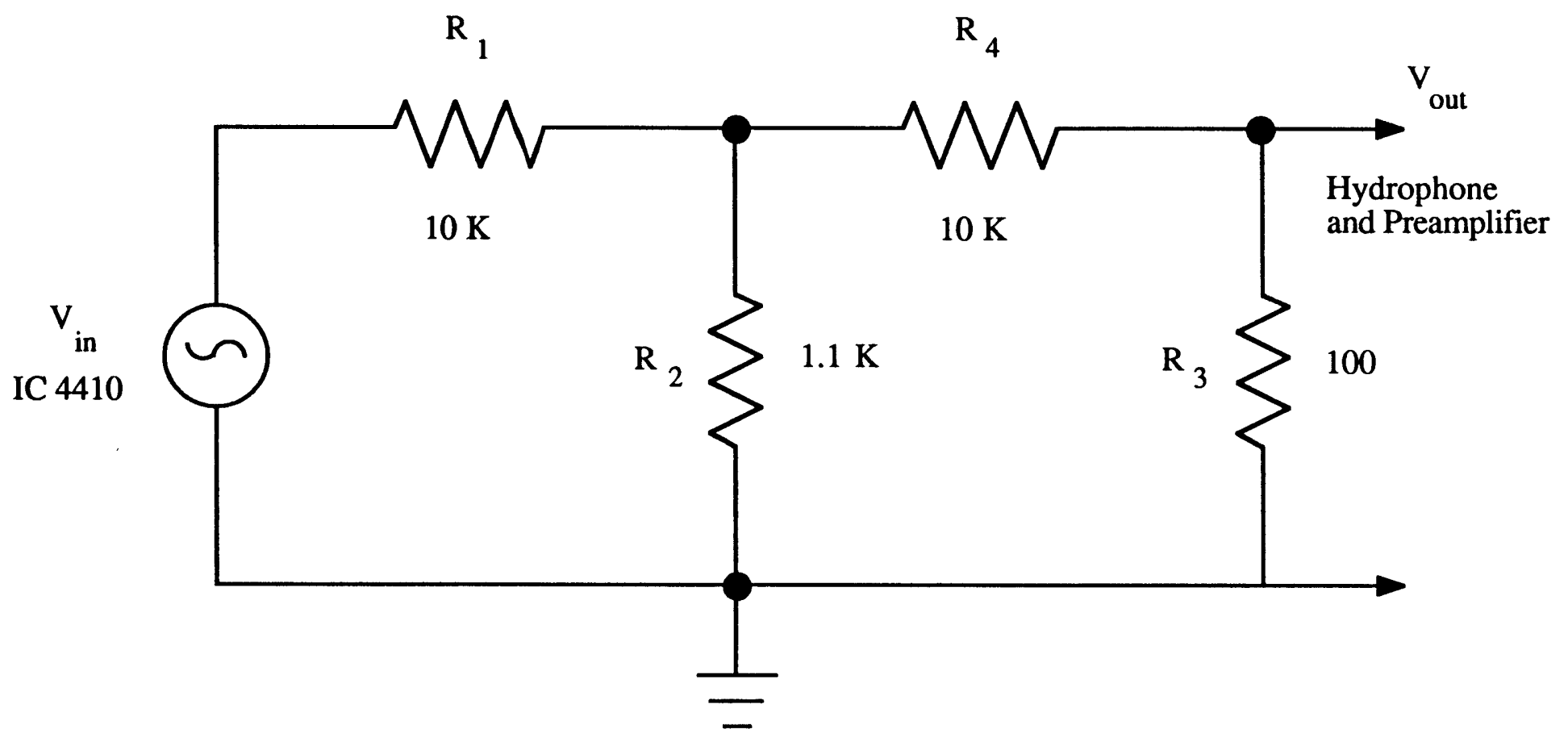




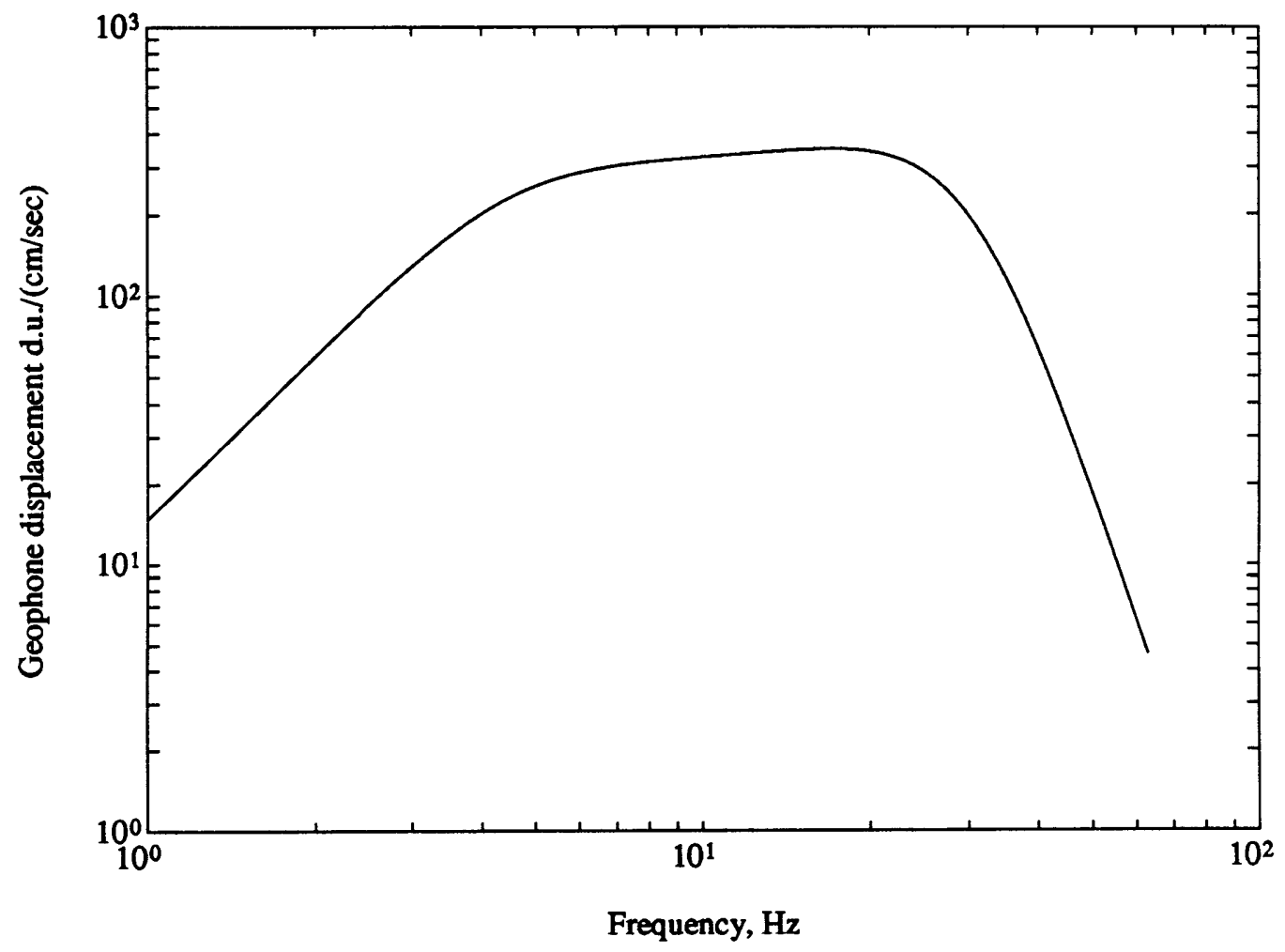

Figure A25 


\section{APPENDIX B}

\section{The SPECTRAL ACCURACY OF THE FinIte-DifferenCE SOLUTIONS}

\section{NUMERICAL STABILITY}

Evaluation of the accuracy of finite-difference seismograms is an important problem that has received considerable attention [e.g., Alford et al., 1974; Kelly et al., 1976; Stephen, 1983, 1988]. The formulation used in this study [Virieux, 1986] has been tested for several types of models, none of which closely corresponds to the magma chamber model. Virieux [1986] considered a corner-edge model and a water-solid step discontinuity. Dougherty [1989] compared analytical and finite difference solutions for acoustic scattering from an infinite elastic cylinder. Stephen [1990] examined acoustic benchmark solutions for a perfect wedge, a lossless penetrable wedge, and a plane-parallel waveguide. Finite-difference studies of complex media have dealt extensively with the scattering from random media [Frankel and Clayton, 1984, 1986; McLaughlin et al., 1986; McLaughlin and Anderson, 1987; Dougherty and Stephen, 1988; Charrette, 1991; Toksöz et al., 1991]. However, such studies have generally been limited to mean velocity perturbations of $5-20 \%$, values significantly smaller than the variations in the magma chamber model (Figure 3.3). Moreover, few studies have explicitly considered the broadband spectral content of finite difference waveforms [Frankel and Clayton, 1984; Charrette, 1991]. By far the most frequently used source wavelets are derivatives of a Gaussian function [Kelly et al., 1976; Stephen et al., 1985], for which the half power bandwidth is limited to about one octave. For these reasons, it is important to verify the stability and broadband spectral accuracy of the finite difference waveforms generated for this study.

The von Neumann numerical stability requirement for this formulation [Virieux, 1986] requires that the quantity $\gamma$, defined by

$$
\gamma \equiv \sqrt{2} \mathrm{~V}_{\mathrm{P}} \frac{\Delta \mathrm{t}}{\Delta \mathrm{x}}
$$

where $\Delta t$ is the sample interval and $\Delta \mathrm{x}$ is the grid spacing, have a value of less than or equal to unity. For a maximum $P$-wave velocity of $7 \mathrm{~km} / \mathrm{s}$, a value $\Delta t / \Delta x \leq 0.1$ is required. A more limiting constraint arises from grid dispersion, a process whereby high frequencies travel more slowly than low frequencies. This effect may alter the 
characteristics of the waveform, broadening the initial pulse and producing a characteristic oscillatory tail, while possibly altering the spectral content of the waveform. To avoid this problem it is generally recommended that the quantity $\mathrm{H}$, defined by

$$
\mathrm{H}=\frac{\Delta \mathrm{x}}{\lambda}
$$

where $\lambda$ is the wavelength, should have a maximum value of about 0.1 [e.g., Alford et al., 1974; Virieux, 1986], though Stephen [1988] recommends a more stringent limit of 0.05 . In practice the maximum acceptable value for $\mathrm{H}$ may vary between studies since the amount of dispersion increases with the propagation distance as well as the grid spacing and the acceptable level of dispersion may depend on the application of the finite-difference solutions.

Virieux [1986] presents a quantitative expression for the grid dispersion in his formulation. The ratio $\mathrm{q}_{\mathrm{P}}$ of the finite-difference $\mathrm{P}$-wave velocity to the true velocity is always less than unity and can be written

$$
\mathrm{q}_{\mathrm{P}}=\frac{\sqrt{2}}{\pi \gamma \mathrm{H}} \sin ^{-1}\left[\frac{\gamma}{\sqrt{2}} \sqrt{\sin ^{2}(\pi \mathrm{H} \cos \theta)+\sin ^{2}(\pi \mathrm{H} \sin \theta)}\right]
$$

where $\theta$ is the angle of propagation with respect to a grid axis. By substituting $\mathrm{V}_{\mathbf{S}}$ for $V_{P}$ in equation (B1), equation (B3) can be used to calculate a value for the corresponding $S$-wave ratio $\mathrm{q}_{\mathrm{S}}$. For all the models in this work the value of $\mathrm{H}$ at the upper half-power source frequency is

$$
\mathrm{H}_{\mathrm{U}}=\frac{0.1875}{\mathrm{~V}}
$$

Within the water column $\mathrm{H}_{U}=0.125$, a value slightly greater than the generally recommended value. Corresponding $q_{P}$ values range from 0.975 to 0.987 for propagation parallel and at $45^{\circ}$ to the grid. Figure B1 shows waveforms for a test model comprised solely of a homogeneous water layer. At a depth of 160 grid points (Figure B1b), which is equivalent to 20 wavelengths at the upper half-power frequency, the effects of dispersion are very apparent, particularly for propagation parallel to the grid. However at a depth of 40 grid points (Figure B1a), which is the thickness of the water layer in the magma chamber models, the effect on waveforms is slight. Indeed, a visual comparison of the water waves with the source signature (Figure 3.2) shows no additional oscillations on the tail of the waveform. Spectral analysis shows that even at the larger ranges the changes in spectral content are negligible. 
Within the crustal component of the model, propagation distances are much larger and the heterogeneous velocity structure makes the cumulative effects of dispersion more difficult to estimate. Therefore, more stringent limitations on the maximum size of $\mathrm{H}$ are necessary. The maximum crustal $\mathrm{P}$-wave wave velocity is limited to $4 \mathrm{~km} / \mathrm{s}\left(\mathrm{H}_{U}=0.047\right)$ except in a small region corresponding to the location of an axial magma chamber where the minimum value is $3 \mathrm{~km} / \mathrm{s}\left(\mathrm{H}_{U}=0.063\right)$. Such values yield maximum $\mathrm{q}_{\mathrm{P}}$ values of 0.997 and 0.994 , respectively, and none of the $\mathrm{P}$ wave phases within the models show considerable dispersion. The minimum nonzero shear wave velocity is $2 \mathrm{~km} / \mathrm{s}\left(\mathrm{H}_{U}=0.096\right)$ which yields a maximum $\mathrm{q}_{S}=0.985$. Some of the $\mathrm{S}$-wave phases do show appreciable dispersion (e.g., Figure 3.4a), but since spectral analysis is confined to P-waves this is considered acceptable.

\section{A COMPaRison OF FinITE-DifFERENCE AND REFLECTIVITY SOLUTIONS}

A complete test of the finite difference solutions for the magma chamber model is not possible since no other full-waveform technique exists to model adequately the waveforms for such a complex structure. However, as a partial test of the finite difference method, solutions for laterally-invariant models are compared with those obtained by the reflectivity method [Fuchs and Müller, 1971], a comparison that has been previously used to evaluate a different finite-difference formulation [Stephen, 1983]. Two velocity profiles (Figure B2), representative of off-axis and axial structure in the magma chamber model, were used in this comparison. Finite difference solutions were calculated with a $7.5-\mathrm{m}$ grid spacing and a $4-25 \mathrm{~Hz}$ source for both profiles and with a $3.75-\mathrm{m}$ grid and a $8-50 \mathrm{~Hz}$ source for the off-axis profiles. The velocity profiles contain smooth velocity gradients which must be approximated by a layered model in the reflectivity method. White and Stephen [1980] and Stephen [1983] recommend a minimum layer spacing of one fifth of the compressional wavelength. However, Chapman and Orcutt [1985] suggest that such a fine spacing is not necessary in most cases. In the reflectivity solutions presented here, the layer thickness is constrained to be no more than one half the minimum compressional wavelength and to incorporate a velocity jump of no more than 0.1 $\mathrm{km} / \mathrm{s}$. Tests using the upper portion of the models suggest that further reduction in the layer thickness has little effect on the waveforms. Since no attenuation is incorporated in the models, very long time series (15-45 s) are calculated to minimize wraparound in the time domain [e.g., Mallick and Frazer, 1987]. To minimize computation time reflectivity solutions are calculated for ray parameters 
ranging between $1 / 15$ and $1 / 3.2 \mathrm{~s} / \mathrm{km}$ and for angle of incidence increments of $0.03^{\circ}$.

Record sections calculated by both techniques are shown in Figure B3 for the off-axis profile and the $4-25 \mathrm{~Hz}$ source. A range-dependent amplitude correction has been applied to account for divergence, which differs between the two solutions, since the reflectivity algorithm includes a point source while the finite-difference algorithm utilizes a line source. The reflectivity solutions to not include the water wave because of the angle limits of integration. The shapes and amplitudes of the $P$ arrivals are in reasonable agreement at all ranges though small phase shifts between the two solutions are apparent. The PP ampitudes correspond well at ranges greater than about $10 \mathrm{~km}$ but show appreciable phase shifts, while at shorter ranges the finite difference solutions have noticeably higher amplitudes. The finite difference record section also includes appreciably higher amplitudes immediately following the PP arrival. At larger ranges this energy clearly separates into coherent arrivals whose paths include more than one downward surface reflection (PPP, PPPP, etc.), an observation which discounts a dispersive origin for the difference. In contrast, the PPP phase in the reflectivity solutions has a very low amplitude, and higher order phases are barely visible. The reflectivity solution also shows a high amplitude headwave at ranges above $8 \mathrm{~km}$, propagating at $4 \mathrm{~km} / \mathrm{s}$, an arrival that is not apparent in the finite difference solutions. The results of ray-theoretical amplitude calculations which include the correct amplitude coefficients for planewave water-solid reflections [Ergin, 1952] correspond closely to the finite-difference amplitudes, suggesting that the finite difference solution is correct. The discrepancy in the reflectivity solution might result from the limited range of angle integration, the layered approximation of the velocity model, or the failure of the particular reflectivity algorithm used in this study to model correctly the water-solid interface at low angles of incidence [J. A. Collins, pers. comm., 1991].

To compare the spectral content of the waveforms, apparent $t^{*}$ values were estimated from the spectral slope (equation (1.7)) and are shown in Figure B4. The results show fairly good agreement at all but the shortest ranges. For the off-axis model (Figures B4a and b) there is good agreement at ranges greater than $6 \mathrm{~km}$. At ranges less than $6 \mathrm{~km}$ the $4-25 \mathrm{~Hz}$ reflectivity solution yields markedly higher t* values, although the discrepancy is smaller for the $8-50 \mathrm{~Hz}$ source. For the on-axis model (Figure B4c) there is also good agreement at ranges greater than $6 \mathrm{~km}$. At short ranges the rapid fluctuations in apparent $t^{*}$ values observed in the finite difference solution cannot be reproduced using the reflectivity technique. 
We can advance no satisfactory explanation for the discrepancy between reflectivity and finite difference solutions at short ranges. However, two observations suggest that the finite-difference solutions may be incorrect. First, in Figure B3a the water wave at $3 \mathrm{~km}$ range has a highly oscillatory tail while at shorter and longer ranges this is not the case. Comparison with Figure B1 and the observation that this effect is not apparent at shorter ranges discounts the possibility that this is a straightforward consequence of grid dispersion. Second, a series of test models incorporating a constant gradient beneath the water-solid interface frequently resulted in an unrealistic loss of high frequency P-wave energy content at ranges just above the critical range even after $\mathrm{H}_{U}$ was decreased significantly. Such results, while not fully quantifying the problem, suggest that the water-solid interface in the Virieux [1986] formulation may be unstable for certain choices of model parameters. While this discrepancy deserves further study, we emphasize that the spectral analysis of finite difference waveforms presented in Chapter 3 is not critically dependent on short-range paths. 


\section{Figure CaPTIONS}

Figure B1. Finite-difference record sections showing the a water wave recorded by a horizontal lines of receivers located (a) 40 grid points and (b) 160 grid points below the upper surface of the finite difference grid.

Figure B2. Layered velocity profiles used in the comparison of finite-difference and reflectivity solutions. The layered approximations used in the reflectivity solutions are shown for the axial (dashed line) and off-axis (solid line) profiles. The smooth profiles used in the finite difference models are shown as dotted lines.

Figure B3. A comparison of (a) finite-difference and (b) reflectivity record sections for the off-axis velocity profile calculated using the $4-25 \mathrm{~Hz}$ source. To correct for divergence, the finite-difference seismograms have been scaled by the square root of the range while the reflectivity seismograms have been scaled by the range.

Figure B4. (a) A comparison of apparent $t^{*}$ values estimated for finite-difference (circles) and reflectivity (crosses) solutions for the off-axis velocity structure. $t^{*}$ has been estimated in the frequency band $4-25 \mathrm{~Hz}$ using $4 \pi$-prolate MWSA and a 0.4 -s-long window which includes $0.3 \mathrm{~s}$ of the waveform. (b) As for (a) except that the source waveforms and the $t^{*}$ estimates are for a frequency interval of $8-50 \mathrm{~Hz}$. (c) As for (a) except that the axial velocity structure is used. 
(a)

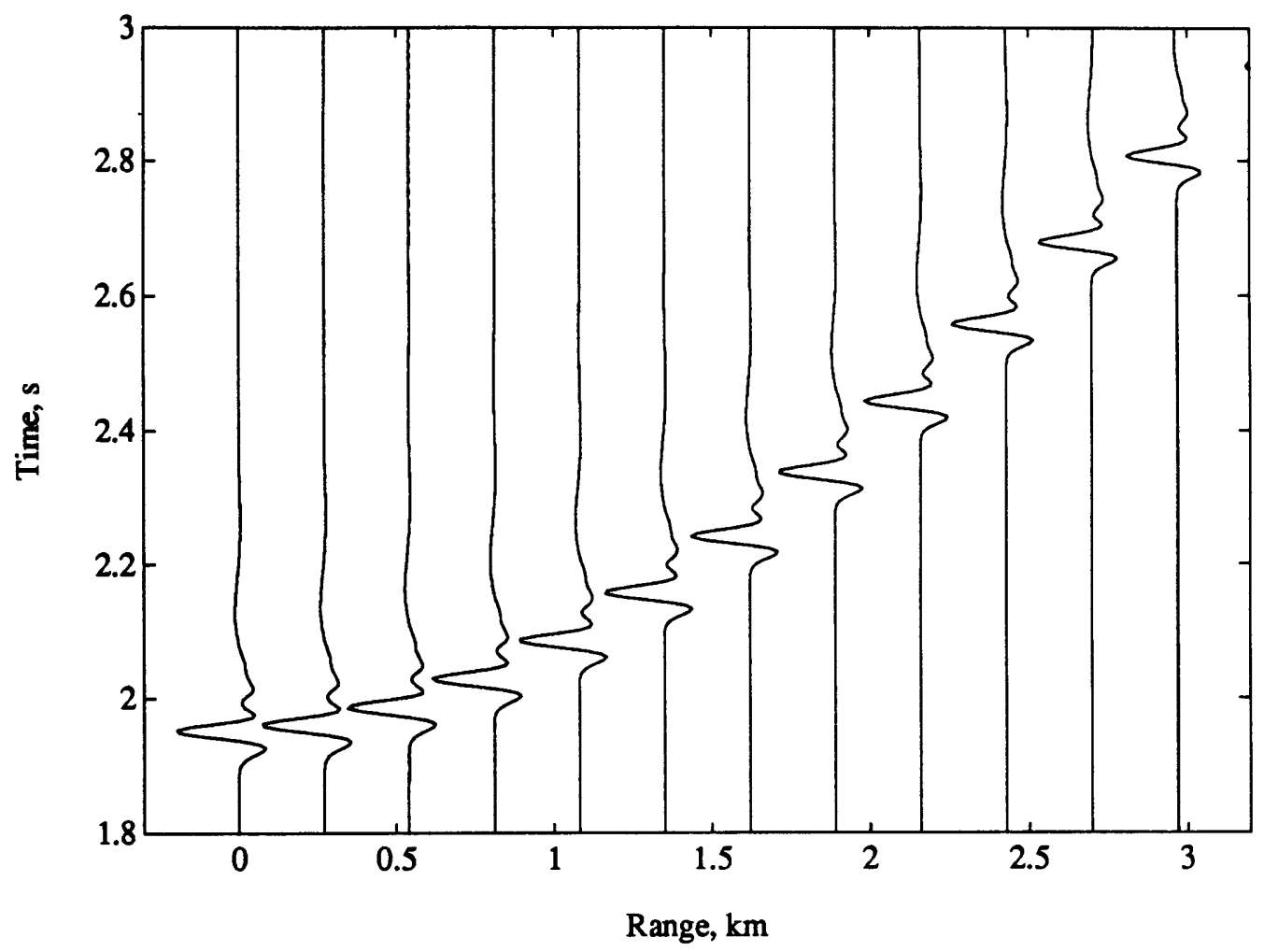

(b)

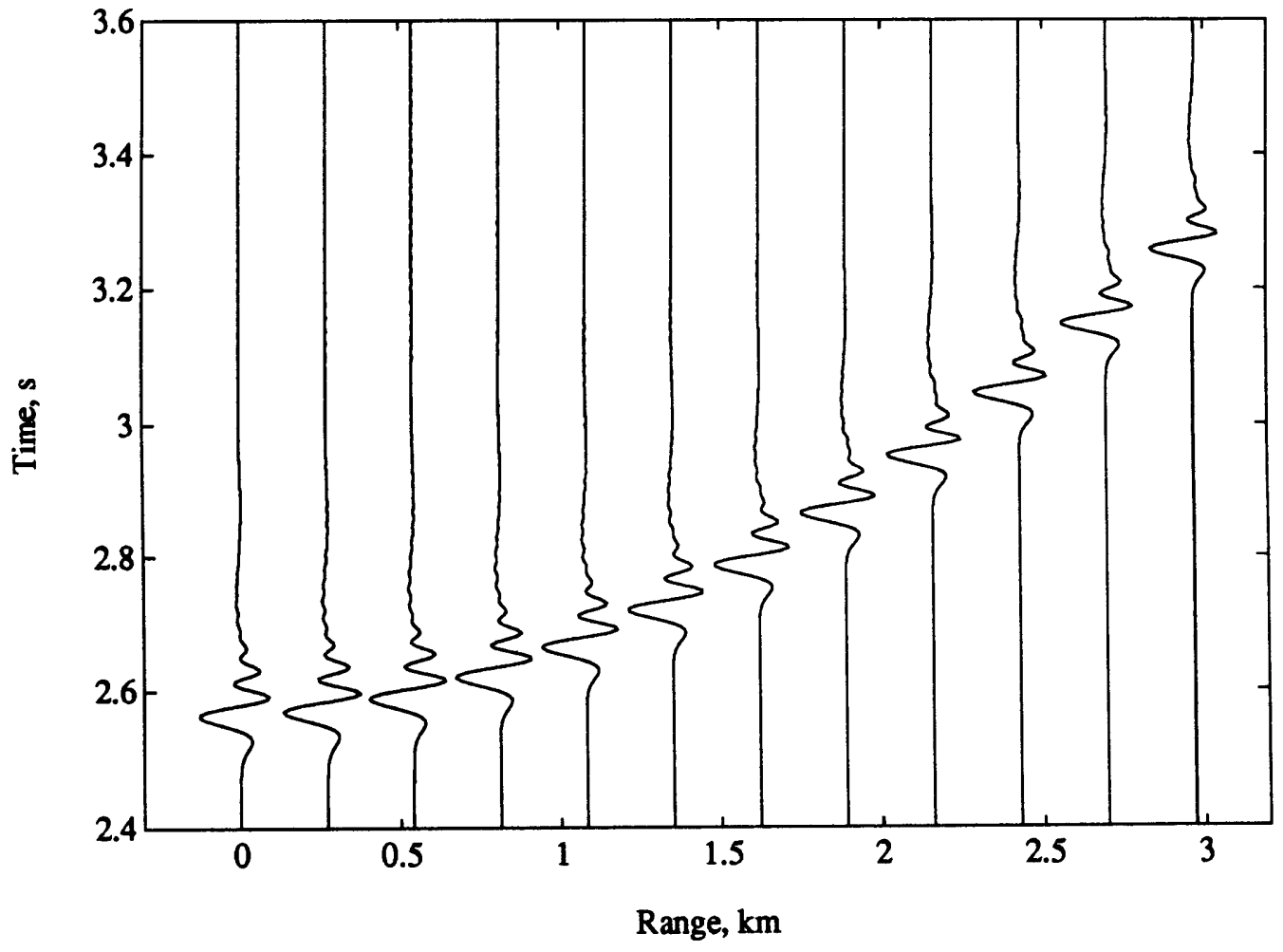

Figure B1 


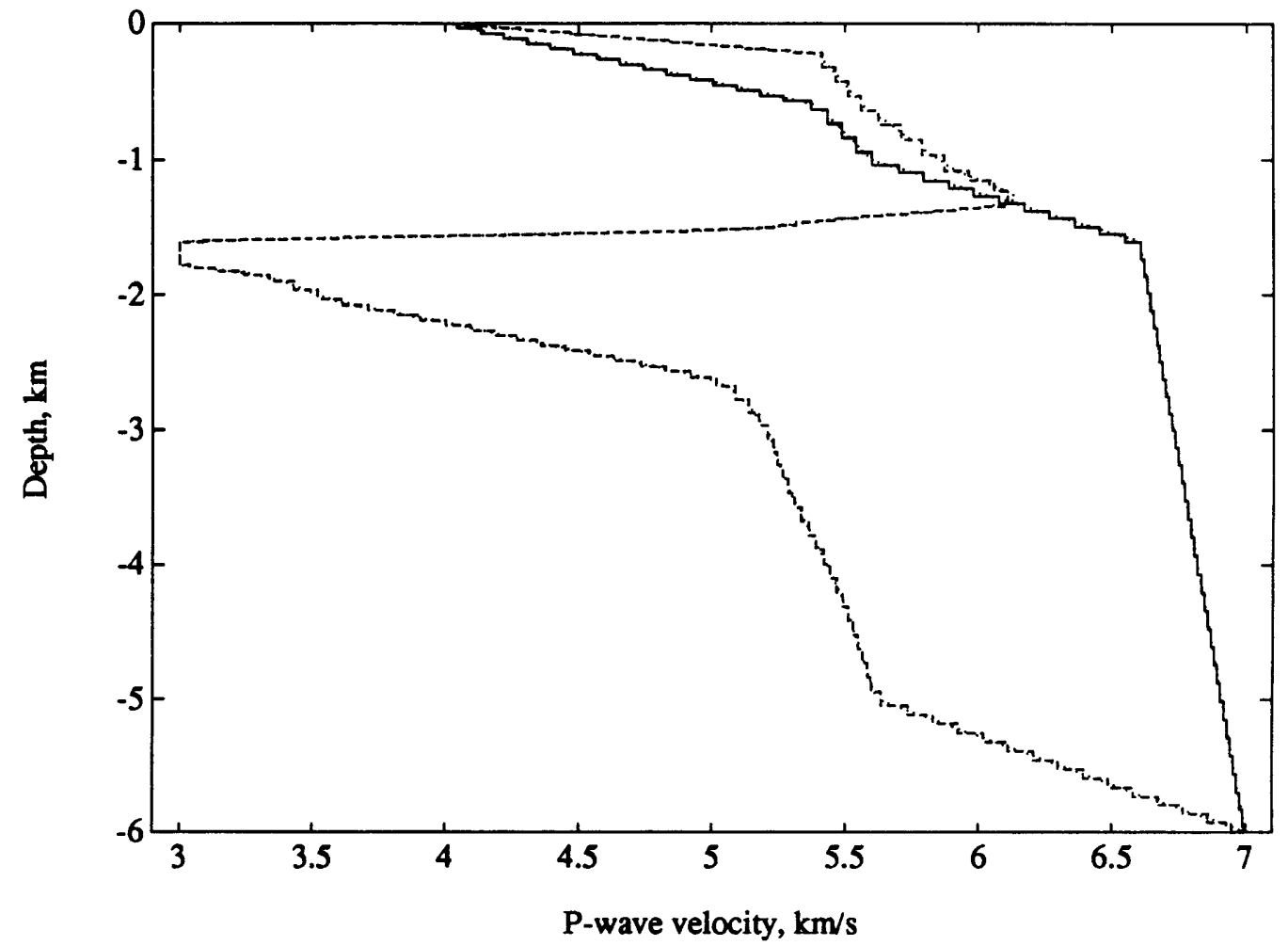

Figure B2 
(a)

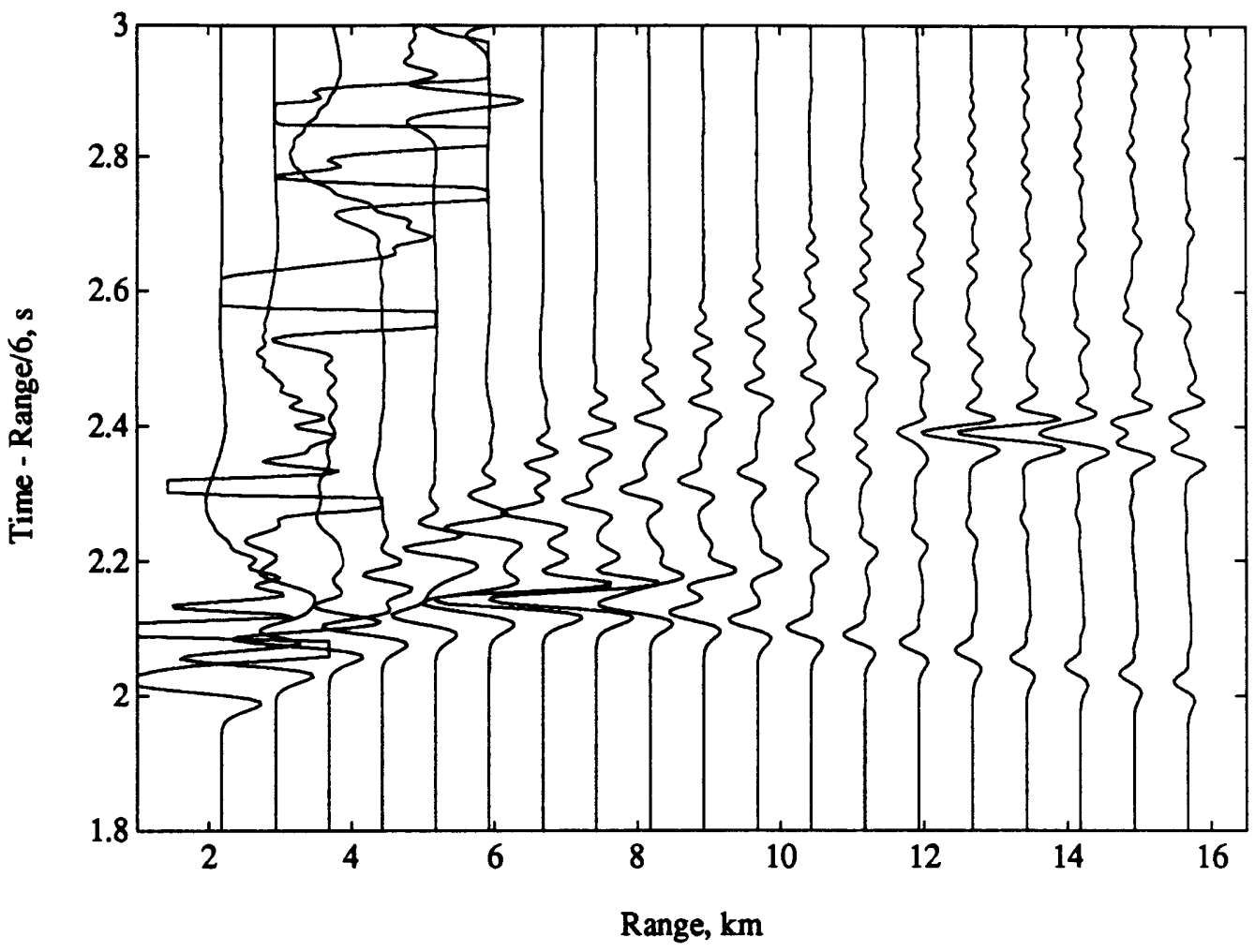

(b)

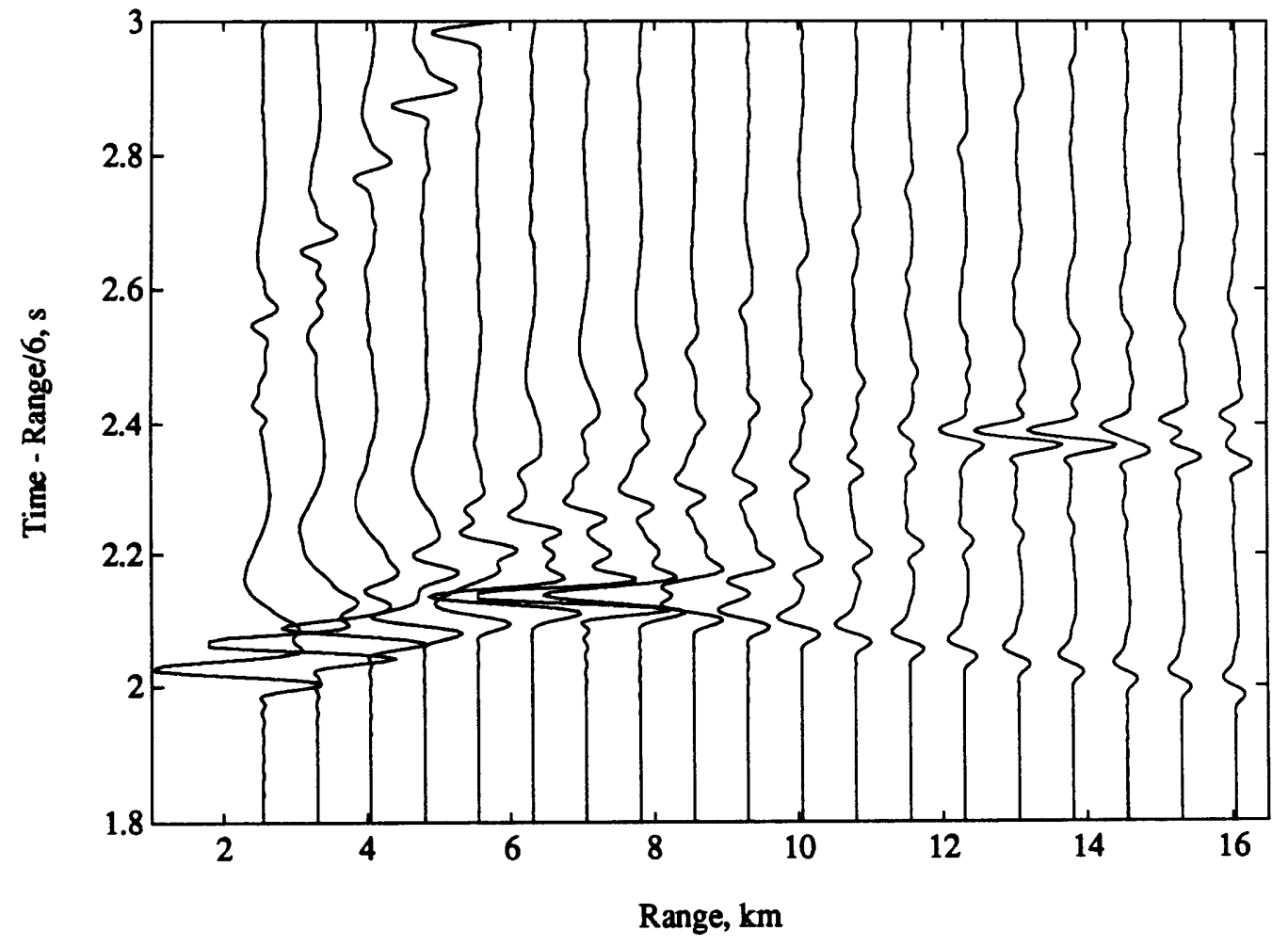

Figure B3 

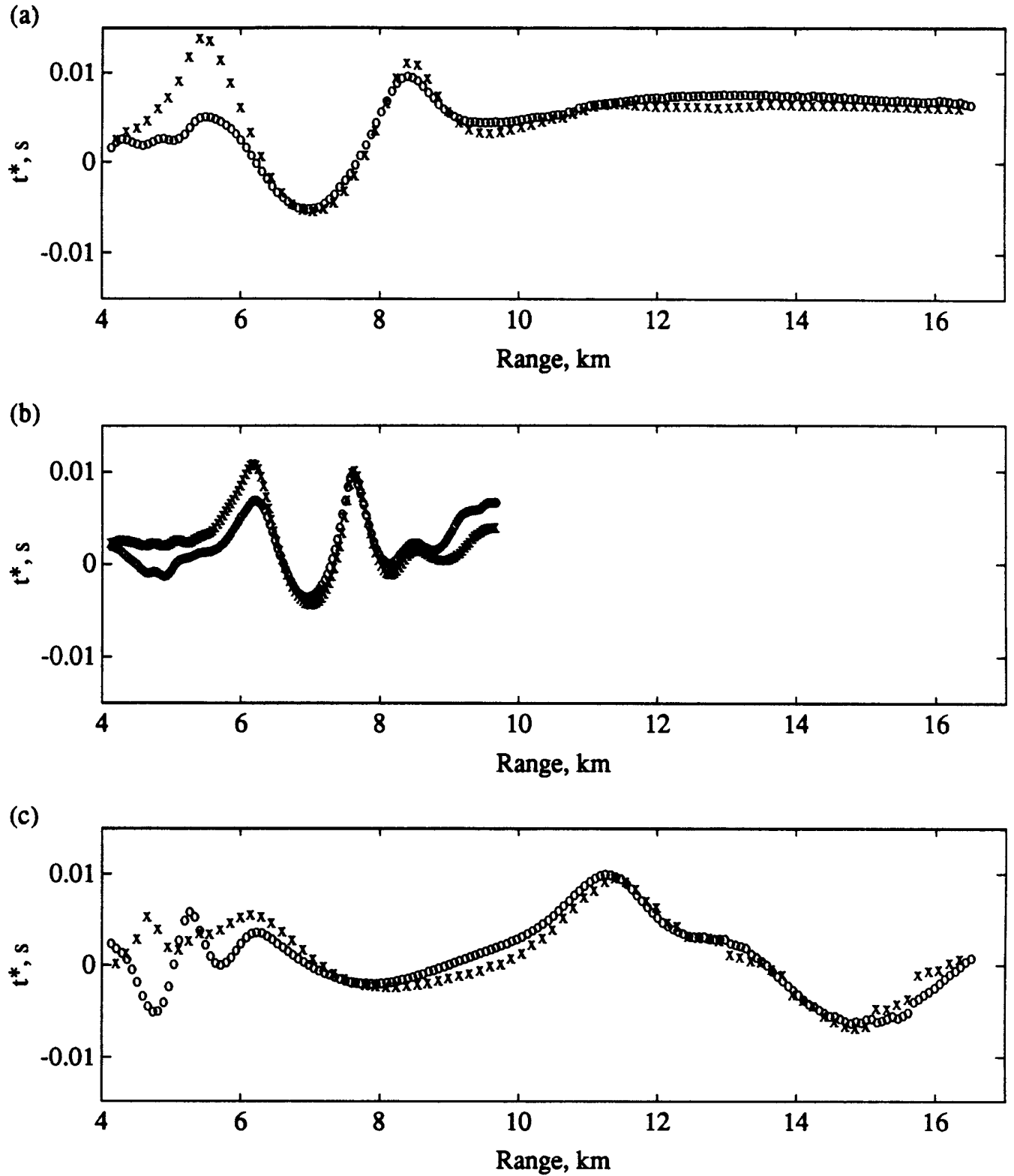

Figure B4 


\section{REFERENCES}

Aki, K., Attenuation of shear-waves in the lithosphere for frequencies from 0.05 to 25 Hz, Phys. Earth Planet. Inter., 21, 50-60, 1980.

Aki, K., and B. Chouet, Origin of coda waves: Source, attenuation, and scattering effects, J. Geophys. Res., 80, 3322-3342, 1975.

Aki, K., and P. G. Richards, Quantitative Seismology, Theory and Methods, pp. 167185, W. H. Freeman, New York, 1980.

Alford, R. M., K. R. Kelly, and D. M. Boore, Accuracy of finite-difference modeling of the acoustic wave equation, Geophysics, 39, 834-842, 1974.

Allen, R. Aoutomatic phase pickers: Their present use and future prospects, Bull. Seismol. Soc. Am., 72, S225-242, 1982.

Alt, J. C., J. Honnorez, C. Laverne, and R. Emmerman, Hydrothermal alteration of a 1 km section through the upper oceanic crust, Deep Sea Drilling Project hole 504B: Mineralogy, chemistry, and evolution of seawater-basalt interactions, J. Geophys. Res., 91, 10309-10335, 1986.

Anderson, D. L., The anelasticity of the mantle, Geophys. J. R. Astron. Soc., 14, 135164, 1967.

Anderson, D. L., and J. W. Given, Absorption band Q model for the Earth, $J$. Geophys. Res., 87, 3893-3904, 1982.

Archambeau, C. B., E. A. Flinn, and D. G. Lambert, Fine structure of the upper mantle, J. Geophys. Res., 74, 5825-5865, 1969.

Asada, T., and K. Takano, Attenuation of short period $\mathrm{P}$ waves in the mantle, J. Phys. Earth, 11, 25-34, 1963.

Azimi, Sh. A., A. V. Kalinin, and B. L. Pivovarov, Impulse and transient characteristics of media with linear and quadratic absorption laws, Izv. Earth Phys., 2, 42-54, 1968.

Backus, G., and F. Gilbert, The resolving power of gross Earth data, Geophys. J. R. Astron. Soc., 16, 169-205, 1968.

Backus, G., and F. Gilbert, Uniqueness in the inversion of inaccurate gross Earth data, Phil. Trans. R. Soc. Lond., Ser. A, 266, 123-192, 1970.

Barth, G. A., and J. C. Mutter, Patterns of crustal thickness produced along the East Pacific Rise, $8^{\circ} 50^{\prime} \mathrm{N}$ to $9^{\circ} 50^{\prime} \mathrm{N}$ (abstract), Eos Trans. AGU, 72, Fall meeting suppl. 490, 1991.

Berckhemer, H., F. Auer, and J. Drisler, High-temperature anelasticity and elasticity of mantle peridotite, Phys. Earth Planet. Inter., 20, 48-59, 1979. 
Berckhemer, H., W. Kampfmann, E. Aulbach, and H. Schmeling, Shear modulus and Q of forsterite and dunite near partial melting from forced-oscillation experiments, Phys. Earth Planet. Inter., 29, 30-41, 1982.

Bratt, S. R., and S. C. Solomon, Compressional and shear wave structure of the East Pacific Rise at $11^{\circ} 20 \mathrm{~N}$ : Constraints from three-component ocean bottom seismometer data, J. Geophys Res., 89, 6095-6110, 1984.

Briggs, I. C., Machine contouring using minimum curvature, Geophysics, 39, 39-48, 1974.

Burnett, M. S., J. A. Orcutt, and A. H. Olson, A finite element computation of sesimic diffraction about the rise axis magma chamber, Geophys. Res. Lett., 15, 1487-1490, 1988.

Burnett, M. S., D. W. Caress, and J. A. Orcutt, Tomographic image of the magma chamber at $12^{\circ} 50^{\prime} \mathrm{N}$ on the East Pacific Rise, Nature, 339, 206-208, 1989.

Cann, J. R., A model for oceanic crustal structure developed, Geophys. J. R. Astron. Soc., 39, 169-187, 1974.

Carbotte, S., and K. Macdonald, East Pacific Rise $8^{\circ}-10^{\circ} 30^{\prime} \mathrm{N}$ : Evolution of ridge segments and discontinuities from SeaMARC II and three-dimensional magnetic studies, J. Geophys. Res., in press, 1992.

Caress, D. W., M. S. Burnett, and J. A. Orcutt, Tomographic image of the axial low velocity zone at $12^{\circ} 50^{\prime} \mathrm{N}$ on East Pacific Rise, J. Geophys. Res., in press, 1992.

Carter, D. J. T., Echo-Sounding Correction Tables (formerly Matthews' Tables), Hydrographic Department, Ministry of Defence, Taunton, Somerset, U. K., 150 pp., 1980.

Cerjan, C., D. Kosloff, R. Kosloff, and M. Reshef, A nonreflecting boundary condition for discrete acoustic and elastic wave equations, Geophysics, 50, 705-708, 1985.

Chapman, C. H., and J. A. Orcutt, The computation of body wave synthetic seismograms in laterally homogeneous media, Rev. Geophys., 23, 105-163, 1985.

Charrette, E. E., Elastic wave scattering in laterally inhomogeneous media, Ph.D. thesis, Massachusetts Institute of Technology, Cambridge, 222 pp., 1991.

Christensen, N. I., and W. W. Wepfer, Laboratory techniques for determining seismic velocities and attenuations, with applications to the continental lithosphere, Geophysical framework of the continental United States, Geol. Soc. Am. Memoir., 172, pp. 91-102, edited by L. C. Pakiser and W. D. Mooney, Boulder, Colorado, 1989. 
Christeson, G. L., and G. M. Purdy, and G. J. Fryer, Upper crustal structure of the East Pacific Rise from ocean bottom refraction experiments: The velocity structure (abstract), Eos Trans. AGU, 72, Spring meeting suppl. 262, 1991a.

Christeson, G. L., G. M. Purdy, and G. J. Fryer, A dramatic change in the upper crustal structure of the East-Pacific Rise from ocean bottom refraction experiments (abstract), Eos Trans. AGU, 72, Fall meeting suppl. 480, 1991b.

Clawson, S. R., R. B. Smith, and H. M. Benz, P wave attenuation of the Yellowstone caldera from three-dimensional inversion of spectral decay using explosion source seismic data, J. Geophys. Res., 94, 7205-7222, 1989.

Close, M., The Analysis of Linear Circuits, Harcourt Brace and World, New York, 210pp, 1966.

Collins, J. A., T. M. Brocher, and J. A. Karson, Two-dimensional seismic reflection modeling of the inferred fossil oceanic crust/mantle transition in the Bay of Islands ophiolite, J. Geophys. Res., 91, 12520-12538, 1986.

Constable, S. C., R. L. Parker, C. G. Constable, Occam's inversion: A practical algorithm for generating smooth models of electromagnetic sounding data, Geophysics, 52, 289-300, 1987.

Cooper, R. F., and D. L. Kohlstedt, Rheology and structure of olivine-basalt partial melts, J. Geophys Res, 91, 9315-9323, 1986.

Cormier, V. F., The effect of attenuation on seismic body waves, Bull. Seismol. Soc. Am., 72, S169-S200, 1982.

Crane, K., The spacing of rift axis highs: Dependence upon diapiric processes in the underlying asthenosphere?, Earth Planet. Sci. Lett., 72, 405-414, 1985.

Creager, K. C., and L. M. Dorman, Location of instruments on the seafloor by joint adjustment of instrument and ship positions, J. Geophys. Res., 87, 8379-8388, 1982.

Delaney, J. R., D. W. Mogk, and M. J. Mottl, Quartz-cemented breccias from the MidAtlantic Ridge: Samples of a high-salinity hydrothermal upflow zone, J. Geophys. Res., 92, 9175-9192, 1987.

Detrick, R. S., P. Buhl, E. Vera, J. Mutter, J. Orcutt, J. Madsen, and T. Brocher, Multichannel seismic imaging of a crustal magma chamber along the East Pacific Rise, Nature, 326, 35-41, 1987.

Dewey, J. F., and W. S. F. Kidd, Geometry of plate accretion, Geol. Soc. Am. Bull., 88, 960-968, 1977. 
Dougherty, M. E., Ocean bottom seismic scattering, Ph.D. thesis, MIT/WHOI Joint Program in Oceanography, Woods Hole, Mass., 310 pp., 1989.

Dougherty, M. E., and R. A. Stephen, Seismic energy partitioning and scattering in laterally heterogeneous ocean crust, Pure Appl. Geophys, 128, 195-229, 1988.

Dougherty, M. E., and R. A. Stephen, Seismo/acoustic propagation through rough seafloors, J. Acoust. Soc. Am., 90, 2637-2651, 1991.

Duschenes, J. D., T. W. Barash, P. J. Mattaboni, and S. C. Solomon, On the use of an externally deployed geophone package on an ocean bottom seismometer, Mar. Geophys. Res., 4, 437-450, 1981.

Einarsson, P., S-wave shadows in the Krafla caldera in NE-Iceland, evidence for a magma chamber in the crust, Bull. Volcanol., 41, 187-195, 1978.

Ergin, K., Energy ratio of seismic waves reflected and refracted at a rock-water boundary, Bull. Seismol. Soc. Am., 42, 349-372, 1952.

Evans, J. R, and J. J. Zucca, Active high-resolution seismic tomography of compressional wave velocity and attenuation at Medicine Lake volcano, northern California Cascade Range, J. Geophys. Res., 93, 15016-15036, 1988.

Fischer, K. M., and G. M. Purdy, Seismic amplitude modeling and the shallow crustal structure of the East Pacific Rise at $12^{\circ} \mathrm{N}$, J. Geophys. Res., 91, 14006-14014, 1986.

Fisher, A. T., K. Becker, T. N. Narasimhan, M. G. Langseth, and M. J. Mottl, Passive, off-axis convection through the southern flank of the Costa Rica rift, $J$. Geophys. Res., 95, 9343-9370, 1990.

Frankel, A., and R. W. Clayton, A finite-difference simulation of wave propagation in two-dimensional random media, Bull. Seismol. Soc. Am., 74, 2167-2186, 1984.

Frankel, A., and R. W. Clayton, Finite difference simulations of seismic scattering: Implications for propagation of short-period seismic waves in the crust and models of crustal heterogeneity, J. Geophys. Res., 91, 6465-6489, 1986.

Frantti, G. E., Attenuation of $\mathrm{P}_{\mathrm{n}}$ from offshore Maine explosions, Bull. Seismol. Soc. Am., 55, 417-423, 1965.

Fuchs, K., and G. Müller, Computation of synthetic seismograms with the reflectivity method and comparison with observations, Geophys. J. R. Astron. Soc., 23, 417433, 1971.

Futterman, W. I., Dispersive body waves, J. Geophys. Res., 67, 5279-5291, 1962.

Gladwin, M. T., and F. D. Stacey, Anelastic degradation of acoustic pulses in rock, Phys. Earth Planet. Inter., 8, 332-336, 1974. 
Goldfarb, M. S., and J. R. Delaney, Response of two-phase fluids to fracture configurations within submarine hydrothermal systems, J. Geophys. Res., 93, 45854594, 1988.

Green, D. H., R. F. Cooper, and S. Zhang, Attenuation spectra of olivine/basalt partial melts: Transformation of newtonian creep response, Geophys. Res. Lett., 17, 2097 2100, 1990.

Gueguen, Y,. J. Woirgard, and M. Darot, Attenuation mechanisms and analasticity in the upper mantle, Analasticity in the Earth, Geodynamics Series, vol 4, edited by F. D. Stacey, M. S. Paterson, and A. Nicholas, pp. 86-94, AGU, Washington, D.C., 1981.

Gueguen, Y., M. Darot, P. Mazot, and J. Woirgard, $Q^{-1}$ of fosterite single crystals, Phys. Earth Planet. Inter., 55, 254-258, 1989.

Gutenberg, B., Attenuation of seismic waves in the Earth's mantle, Bull. Seismol. Soc. Am., 48, 269-282, 1958.

Hale, L. D., C. J. Morton, and N. H. Sleep, Reinterpretation of seismic reflection data over the East Pacific Rise, J. Geophys. Res., 87, 7707-7717, 1982.

Hamilton, E. L., Sound attenuation as a function of depth in the sea floor, J. Acoust. Soc. Am., 59, 528-535, 1976.

Harding, A. J., J. A. Orcutt, M. E. Kappus, E. E. Vera, J. C. Mutter, P. Buhl, R. S. Detrick, and T. M. Brocher, Structure of young oceanic crust at $13^{\circ} \mathrm{N}$ on the East Pacific Rise from expanding spread profiles, J. Geophys Res., 94, 12163-12196, 1989.

Harding, A. J., G. M. Kent, M. E. Kappus, and J. A. Orcutt, Examination of layer 2A between $8^{\circ} 500^{\prime} \mathrm{N} \& 9^{\circ} 50^{\prime} \mathrm{N}$ on the East Pacific Rise using CDP data (abstract), Eos Trans. AGU, 72, Fall meeting suppl. 494, 1991.

Harding, A. J., G. M. Kent, and J. A. Orcutt, Forward modeling of CDP reflection profiles across the East Pacific Rise between $9^{\circ} 17 \mathrm{~N}$ and $9^{\circ} 49^{\prime} \mathrm{N}$, poster presentation at AGU Fall National Meeting, San Francisco, December 3-7, 1990.

Harris, F. J., On the use of windows for harmonic analysis with the discrete Fourier transform, Proc. I.E.E.E., 66, 51-83, 1978.

Hashida, T., and K. Shimazaki, Determination of seismic attenuation structure and source strength by inversion of seismic intensity data: Tohoku district, northeastern Japan arc, J. Phys. Earth, 35, 67-92, 1987. 
Hashida, T., G. Stavrakakis, and K. Shimazaki, Three-dimensional seismic attenuation structure beneath the Aegean region and its tectonic implication, Tectonophysics, 145 , 43-54, 1988.

Haymon, R. M., D. J. Fornari, M. H. Edwards, S. Carbotte, D. Wright, and K. C. Macdonald, Hydrothermal vent distribution along the East pacific Rise crest $\left(9^{\circ} 09^{\prime}\right.$ $54^{\prime} \mathrm{N}$ ) and its relationship to magmatic and tectonic processes on fast-spreading midocean ridges, Earth. Planet. Sci. Lett., 104, 513-534, 1991.

Helmberger, D. V., The crust-mantle transition in the Bering Sea, Bull. Seismol. Soc. Am., 58, 179-214, 1968.

Herron, T. J., Lava flow layer - East Pacific Rise, Geophys. Res. Lett., 9, 17-20, 1982.

Herron, T. J., W. J. Ludwig, P. L. Stoffa, T. K. Kan, and P. Buhl, Structure of the East Pacific Rise from multichannel seismic reflection data, J. Geophys Res., 83, 798-804, 1978.

Herron, T. J., P. L. Stoffa, and P. Buhl, Magma chamber and mantle reflections - East Pacific Rise, Geophys. Res. Lett., 7, 989-992, 1980.

Ho-Liu, P., H. Kanamori, and R. W. Clayton, Applications of attenuation tomography to Imperial Valley and Coso-Indian Wells region, southern California, J. Geophys. Res., 93, 10501-10520, 1988.

Ho-Liu, P., J.-P. Montagner, and H. Kanamori, Comparison of iterative back-projection inversion and generalized inversion without blocks; case studies in attenuation tomography, Geophys. J. R. Astron. Soc., 97, 19-29, 1989.

Ho-Liu, P., C. Johnson, J.-P. Montagner, H. Kanamori, R. W. Clayton, Threedimensional attenuation structure of Kilauea-East rift zone, Hawaii, J. Geophys. Res., submitted, 1990.

Holt, M., Underwater explosions, Ann. Rev. Fluid. Mech., 9, 187-214, 1977.

Houtz, R., and J. Ewing, Upper crustal structure as a function of plate age, J. Geophys. Res., 81, 2490-2498, 1976.

Humphris, S. E., and G. Thompson, Hydrothermal alteration of oceanic basalts by seawater, Geochim. Cosmochim. Acta, 42, 107-125, 1978.

Ito, K., and G. C. Kennedy, Melting and phase relations in a natural peridotite to 40 kilobars, Amer J. Sci., 265, 519-538, 1967.

Jackson, D. D., and D. L. Anderson, Physical mechanisms of seismic-wave attenuation, Rev. Geophys. Space Phys., 8, 1-63, 1970. 
Jackson, I., The laboratory study of seismic wave attenuation, in Mineral and rock deformation: Laboratory studies, AGU Geophys. Monogr. Ser., 36, pp. 11-23, edited by B. E. Hobbs and H. C. Heard, AGU, Washington , D.C., 1986.

Jackson, I., and M. S. Paterson, Shear modulus and internal friction of calcite rocks at seismic frequencies: pressure, frequency and grain size dependence, Phys. Earth, Planet. Inter., 45, 349-367, 1987.

Jackson, I., M. S. Paterson, H. Niesler, and R. M. Waterford, Rock analasticity measurements at high pressure, low strain amplitude and seismic frequency, Geophys. Res. Lett., 11, 1235-1238, 1984.

Jackson, I., M. S. Paterson, and J. D. Fitz Gerald, Seismic wave dispersion and attenuation in Åheim dunite: An experimental study, Geophys. J. Int., in press, 1992.

JJacobson, R. S., An investigation into the fundamental relationships between attenuation, phase dispersion, and frequency using seismic refraction profiles over sedimentary structures, Geophysics, 52, 72-87, 1987.

acobson, R. S., The impact of crustal evolution on changes of the seismic properties of the uppermost ocean crust, Rev. Geophys., in press, 1992.

Jacobson, R. S., and B. T. R. Lewis, The first direct measurements of upper oceanic crustal compressional wave attenuation, J. Geophys. Res., 95, 17417-17429, 1990.

Jacobson, R. S., G. G. Shor, and L. M. Dorman, Linear inversion of body wave datapart II: Attenuation versus depth using spectral ratios, Geophysics, 46, 152-162, 1981.

Jacobson, R. S., G. G. Shor, and M. Beé, A comparison of velocity and attenuation between the Nicobar and Bengal deep sea fans, J. Geophys. Res., 89, 6181-6196, 1984.

Kampfmann, W., and H. Berckhemer, High temperature experiments on the elastic and anelastic behaviour of magmatic rocks, Phys. Earth Planet. Inter., 40, 223-247, 1985.

Kanamori, H., and D. L. Anderson, Importance of physical dispersion in surface wave and free oscillation problems: Review, Rev. Geophys. Space Phys., 15, 105-112, 1977.

Karson, J. A., J. A. Collins, and J. F. Casey, Geologic and seismic velocity structure of the crust/mantle transition in the Bay of Islands ophiolite complex, J. Geophys. Res., 89, 6126-6138, 1984.

Kelly, K. R., R. W. Ward, S. Treitel, and R. M. Alford, Synthetic seismograms: A finite-difference approach, Geophysics, 41, 2-27, 1976. 
Kent, G. M., A. J. Harding, and J. A. Orcutt, Evidence for a smaller magma chamber beneath the East Pacific Rise at 9³0'N, Nature, 344, 650-653, 1990.

Kent, G. M., A. J. Harding, and J. A. Orcutt, Reprocessed CDP lines between $8^{\circ} 50^{\prime} \mathrm{N}$ and $9^{\circ} 50^{\prime} \mathrm{N}$ on the East Pacific Rise: Implications for layer 2A thickening, segmentation of the axial magma chamber, and decoupling of the melt source region from the neovolcanic zone,(abstract), Eos Trans. AGU, 72, Fall meeting suppl. 490491, 1991a.

Kent, G. M., A. J. Harding, and J. A. Orcutt, Constraints on the along-axis variation of magma chamber width between $8^{\circ} 50 \mathrm{~N}$ and $9^{\circ} 50^{\circ} \mathrm{N}$ on the East Pacific Rise from the forward modeling of the CDP data (abstract), Eos Trans. AGU, 72, Fall meeting suppl. 494, $1991 \mathrm{~b}$.

Kidd, R. G. W., A model for the process of formation of the upper oceanic crust, Geophys. J. R. Astron. Soc., 51, 149-183, 1977.

Knopoff, L., Q, Rev. Geophys., 2, 625-660, 1964.

Kobayashi, A., I. Furuya, and K. Uhira, Re-examination of magma reservoirs by spectral analysis of $\mathrm{P}$ and $\mathrm{S}$ waves, Geophys. Mag., 41, 173-187, 1986.

Koelsch, D. E., and G. M. Purdy, An ocean bottom hydrophone instrument for seismic refraction experiments in the deep ocean, Mar. Geophys. Res., 4, 115-125, 1979.

Koelsch, D. E., K. R. Peal, and G. M. Purdy, The Woods Hole Oceanographic Institution digital ocean bottom hydrophone instrument, WHOI Tech. Rep., 82-30, 15 pp., Woods Hole Oceanogr. Inst., Woods Hole, Mass., 1982.

Kong, L. S. L., S. C. Solomon, and G. M. Purdy, Microearthquake characterization of a mid-ocean ridge along-axis high, J. Geophys. Res., in press, 1992

Kurita, T., Attenuation of short-period P-waves and Q in the mantle, J. Phys. Earth, 16, 61-78, 1968.

Langmuir, C. H., J. F. Bender, and R. Batiza, Petrological and tectonic segmentation of the East pacific Rise, $5^{\circ} 30^{\prime}-14^{\circ} 30^{\prime} \mathrm{N}$, Nature, 322, 422-429, 1986.

Latter, J. H., Location of zones of anomalously high S-wave attenuation in the upper crust near Ruapehu and Ngauruhoe volcanoes, New Zealand, J. Volcanol. Geotherm. Res., 10, 125-156, 1981.

Lawson, C. L., and R. J. Hanson, Solving Least Squares Problems, Prentice-Hall, Englewood Cliffs, New Jersey, 340 pp., 1974.

Levander, A. R., Use of the telegraphy equation to improve absorbing boundary efficiency for fourth-order acoustic wave finite difference schemes, Bull. Seismol. Soc. Am., 75, 1847-1852, 1985. 
Lewis, B. T. R., and J. D. Garmany, Constraints on the structure of the East Pacific Rise from seismic refraction data, J. Geophys. Res., 87, 8417-8425, 1982.

Lewis, B. T. R., and H. Jung, Attenuation of refracted seismic waves in young oceanic crust, Bull. Seismol. Soc. Am., 79, 1070-1088, 1989.

Liu, H.-P., D. L. Anderson, and H. Kanamori, Velocity dispersion due to anelasticity; implications for seismology and mantle composition, Geophys. J. R. Astron. Soc., 47, 41-58, 1976.

Lister, C. R. B., On the penetration of water into hot rock, Geophys. J. R. Astron. Soc., 39, 465-509, 1974.

Lister, C. R. B., "Active" and "passive" hydrothermal systems in the oceanic crust: Predicted physical conditions, The dynamic environment of the ocean floor, pp. 441470, edited by K. E. Fanning and F. T. Manheim, , D. C. Heath, Lexington, Mass.., 1981.

Lonsdale, P., Structural geomorphology of a fast-spreading rise crest: The East Pacific Rise near 3²5'S, Mar. Geophys. Res., 3, 251-293, 1977.

Luetgert, J. H., User's manual for RAY84/R83PLT interactive two-dimensional ray tracing/synthetic seismogram package, U. S. Geol. Surv. Open File Rep., 88-238, 1988.

Lundquist, G. M., and V. C. Cormier, Constraints on the absorption band model of Q, J. Geophys. Res., 85, 5244-5256, 1980.

Macdonald, K. C., Mid-ocean ridges: Fine scale tectonic, volcanic and hydrothermal processes within the plate boundary zone, Ann. Rev. Earth Planet. Sci., 10, 155$190,1982$.

Macdonald, K., J. C. Sempere, and P. J. Fox, East Pacific Rise from Siqueiros to Orozco fracture zones: Along strike continuity of axial neovolcanic zone and structure and evolution of overlapping spreading centers, J. Geophys. Res., 89, 6049-6069, 1984.

Macdonald, K. C., P. J. Fox, S. Carbotte, M. Eisen, S. Miller, L. Perram, D. Scheirer, S. Tighe, and C. Weiland, The East Pacific Rise and its flanks $8^{\circ}-17^{\circ} \mathrm{N}$ : History of segmentation, propagation and spreading direction based on SeaMARC II and Sea Beam studies, Marine Geophys. Res., in press, 1992.

Madsen, J. A., R. S. Detrick, J. C. Mutter, P. Buhl, J. A. Orcutt, A two- and threedimensional analysis of gravity anomalies associated with the East Pacific Rise at $9^{\circ} \mathrm{N}$ and $13^{\circ}$ N., J. Geophys. Res., 95, 4967-4987, 1990. 
Mallick, S., and L. N. Frazer, Practical aspects of reflectivity modeling, Geophysics, 52, 1355-1364, 1987.

Manghnani, M. H., H. Sato, and C. S. Rai, Ultrasonic velocity and attenuation measurements on basalt melts to $1500^{\circ} \mathrm{C}$ : Role of composition and structure in the viscoelastic properties, J. Geophys. Res., 91, 9333-9342, 1986.

Marsh, B. D., Magma chambers, Ann. Rev. Earth Planet. Sci., 17, 439-474, 1989.

Mattaboni, P. J., and S. C. Solomon, MITOBS: A seismometer system for ocean bottom earthquake studies, Mar. Geophys. Res., 3, 87-102, 1977.

Matumoto, T., Seismic body waves observed in the vicinity of the Mount Katmai, Alaska, and evidence for the existence of molten chambers, Geol. Soc. Am. Bull., 82, 2905-2920, 1971.

Mavko, G. M., Velocity and attenuation in partially molten rocks, J. Geophys. Res., 85, 5173-5189, 1980.

Mavko, G., and A. Nur, Melt squirt in the asthenosphere, J. Geophys. Res., 80, 14441448, 1975.

McClain, J. S., J. A. Orcutt, and M. Burnett, The East Pacific Rise in cross section: A seismic model, J. Geophys. Res., 90, 8627-8639, 1985.

McKenzie, D. P., The generation and compaction of partially molten rock, J. Petrol., 25, 713-765, 1984.

McLaughlin, K. L., and L. M. Anderson, Stochastic dispersion of short period P-waves due to scattering and multipathing, Geophys. J. R. Astron. Soc., 89, 933-963, 1987.

McLaughlin, K. L., L. M. Anderson, and Z. A. Der, Investigations of seismic waves using 2-dimensional finite difference calculations, presented at Symposium on scattering of waves in random media and random rough surfaces, The Pennsylvania State University, 1985.

Menke, W., Geophysical Data Analysis: Discrete Inverse Theory, 2nd edition, Academic Press, San Diego, California, 289 pp., 1989.

Mitchell, S. K., and K. C. Focke, New measurements of compressional wave velocity in deep sea sediments, J. Acoust. Soc. Am., 67, 1582-1589, 1980.

Molnar, P., and J. Oliver, Lateral variations of attenuation in the upper mantle and discontinuities in the lithosphere, J. Geophys. Res., 74, 2648-2682, 1969.

Moser, T. J., Shortest path calculation of seismic rays, Geophysics, 56, 59-67, 1991.

Murase, T., and A. R. McBirney, Properties of some common igneous rocks and their melts at high temperatures, Geol. Soc. Am. Bull., 84, 3563-3592, 1973. 
Mutter, J. C., G. A. Barth, P. Buhl, R. S. Detrick, J. Orcutt, and A. Harding, Magma distribution across ridge-axis discontinuities on the East Pacific Rise from multichannel seismic images, Nature, 336, 156-158, 1988.

Nafe, J. E., and C. L. Drake, Variation with depth in shallow and deep water marine sediments of porosity, density, and the velocities of compressional and shear waves, Geophysics, 22, 523-552, 1957.

Nehlig, P., and T. Juteau, Deep crustal seawater penetration and circulation at oceanic ridges: Evidence from the Oman ophiolite, Mar. Geol., 84, 209-228, 1988.

Nicolas, A., I. Reuber, and K. Benn, A new magma chamber model based on structural studies in the Oman ophiolite, Tectonophysics, 151, 87-105, 1988.

O'Connell, R. J., and B. Budiansky, Viscoelastic properties of fluid-saturated cracked solids, J. Geophys. Res., 82, 5719-5735, 1977.

O'Connell, R. J., and B. Budiansky, Measures of dissipation in viscoelastic media, Geophys. Res. Lett., 5, 5-8, 1978.

Orcutt, J., B. Kennett, L. Dorman., and W. Prothero, A low velocity zone underlying a fast-spreading rise crest, Nature, 256, 475-476, 1975.

Orcutt, J. A., B. L. N. Kennett, and L. M. Dorman, Structure of the East Pacific Rise from an ocean bottom seismometer survey, Geophys. J. R. Astron. Soc., 45, 305$320,1976$.

Pallister, J. S., and C. A. Hopson, Samail ophiolite plutonic suite: Field relations, phase variation, and cryptic variation and layering, and a model of a spreading ridge magma chamber, J. Geophys. Res., 86, 2593-2644, 1981.

Park, J., C. R. Lindberg, and D. J. Thomson, Multiple-taper spectral analysis of terrestrial free oscillations: Part I, Geophys. J. R. Astron. Soc., 91, 755-794, 1987a.

Park, J., C. R. Lindberg, and F. L. Vernon, III, Multitaper spectral analysis of highfrequency seismograms, J. Geophys. Res., 92, 12675-12684, $1987 \mathrm{~b}$.

Purdy, G. M., The correction for the travel time effects of seafloor topography in the interpretation of marine seismic data, J. Geophys. Res., 87, 8389-8396, 1982.

Rea, D. K., Model for the formation of topographic features of the East Pacific Rise crest, Geology, 3, 77-80, 1975.

Reid, I., J. A. Orcutt, and W. A. Prothero, Seismic evidence for a narrow zone of partial melt underlying the East Pacific Rise at $21^{\circ}$ N., Geol. Soc. Am. Bull., 88, 678-682, 1977. 
Rodi, W. L., Regularization and Backus-Gilbert estimation in nonlinear inverse problems: Application to magnetotellurics and surface waves, Ph.D. thesis, The Pennsylvania State University, 297 pp., 1989.

Rosendahl, B. R., R. W. Raitt, L. M. Dorman, L. D. Bibee, D. W. Hussong, and G. H. Sutton, J. Geophys. Res., 81, 5294-5304, 1976.

Rothman, D. H., Automatic estimation of large residual statics corrections, Geophysics, 51, 332-346, 1986.

Ryall, F., and A. Ryall, Attenuation of $P$ and $S$ waves in a magma chamber in Long Valley caldera, California, Geophys. Res. Lett., 8, 557-560, 1981.

Sacks, I. S., Distribution of absorption of shear waves in South America and its tectonic significance, Year Book Carnegie Inst. Washington, 67, 339-344, 1969.

Sacks, I. S., and T. Murase, The anelasticity of peridotite and partial melt in the asthenosphere, Year Book Carnegie Inst. Washington, 82, 509-512, 1983.

Sanders, C. O., Location and configuration of magma bodies beneath Long Valley, California determined from anomalous earthquake signals, J. Geophys. Res., 89, 8287-8302, 1984.

Sanders, C., P. Ho-Liu, D. Rinn, H. Kanamori, Anomalous shear wave attenuation in the shallow crust beneath the Coso volcanic region, California, J. Geophys. Res., 93, 3321-3338, 1988.

Sato, H., and M. H. Manghnani, Ultrasonic measurements of $\mathrm{V}_{\mathrm{P}}$ and $\mathrm{Q}_{\mathrm{P}}$ : Relaxation spectrum of complex modulus on basalt melts, Phys. Earth Planet. Inter., 41, 18-33, 1985.

Sato, H., and I. S. Sacks, Analasticity and thermal structure of the oceanic upper mantle: Temperature calibration with heat flow data, J. Geophys. Res., 94, 5705-5715, 1989.

Sato, H., I. S. Sacks, T. Murase, G. Muncill, and H. Fukuyama, Attenuation of compressional waves in peridotite measured as a function of temperature at $200 \mathrm{MPa}$, Pure Appl. Geophys., 128, 433-447, 1988.

Sato, H., I. S. Sacks, T. Murase, G. Muncill, and H. Fukuyama, Qp-melting temperature relation in peridotite at high pressure and temperature: Attenuation mechanism and implications for the mechanical properties of the upper mantle, $J$. Geophys. Res., 94, 10647-10661, 1989.

Scherbaum, F., and M. Wyss, Distribution of attenuation in the Kaoiki, Hawaii, source volume estimation by inversion of P wave spectra, J. Geophys. Res., 95, 12439$12448,1990$. 
Schmeling, H., Numerical models on the influence of partial melt on elastic, anelastic and electric properties of rocks, part I: Elasticity and anelasticity, Phys. Earth Planet. Inter., 41, 34-57, 1985.

Schouten, H., K. D. Klitgord, and J. A. Whitehead, Segmentation of mid-ocean ridges, Nature, 317, 225-229, 1985.

Sheehan, A. F., and S. C. Solomon, Differential shear wave attenuation and its lateral variation in the North Atlantic region, J. Geophys. Res., in press, 1992.

Sinton, J. M., and R. S. Detrick, Mid-ocean ridge magma chambers, J. Geophys. Res., 97, 197-216, 1992.

Sipkin, S. S., and T. H. Jordan, Frequency dependence of $\mathrm{Q}_{\mathrm{scS}}$, Bull. Seismol. Soc. Am., 69, 1055-1079, 1979.

Sleep, N. H., Formation of oceanic crust: Some thermal constraints, J. Geophys Res., 80, 4037-4042, 1975.

Sleep, N. H., Thermal structure and kinematics of mid-ocean ridge axis, some implications to basaltic volcanism, Geophys. Res. Lett., 5, 426-428, 1978.

Solomon, S. C., Seismic-wave attenuation and partial melting in the upper mantle of North America, J. Geophys. Res., 77, 1483-1502, 1972.

Solomon, S. C., Shear wave attenuation and melting beneath the Mid-Atlantic Ridge, $J$. Geophys. Res., 78, 6044-6059, 1973.

Solomon, S. C., and M. N. Toksöz, Lateral variation of attenuation of $P$ and $S$ waves beneath the United States, Bull. Seismol. Soc. Am., 60, 819-838, 1970.

Sparks, R. S. J., Meyer, P., and H. Sigurdsson, Density variation amongst mid-ocean ridge basalts: Implications for magma mixing and the scarcity of primitive lavas, Earth Planet. Sci. Lett., 46, 419-430, 1980.

Spudich, P., and J. Orcutt, Petrology and porosity of an oceanic crustal site: Results from wave form modeling of seismic refraction data, J. Geophys. Res., 85, 14091433, 1980a.

Spudich, P., and J. Orcutt, A new look at the seismic velocity structure of the oceanic crust, Rev. Geophys. Space Phys., 18, 627-645, $1980 \mathrm{~b}$.

Stephen, R. A., A comparison of finite difference and reflectivity seismograms for marine models, Geophys. J. R. Astron. Soc., 72, 39-58, 1983.

Stephen, R. A., A review of finite difference methods for seismo-acoustic problems at the seafloor, Rev. Geophys., 26, 445-458, 1988.

Stephen, R. A., Solutions to range-dependent benchmark problems by the finite difference method, J. Acoust. Soc. Am., 87, 1527-1534, 1990. 
Stephen, R. A., F. Cardo-Casas, and C. H. Cheng, Finite-difference synthetic acoustic logs, Geophysics, 50, 1588-1609, 1985.

Stoll, R. D., and R. E Houtz, Attenuation measurement with sonobuoys, J. Acoust. Soc. Am., 73, 163-172, 1983.

Stolper, E., and D. Walker, Melt density and the average composition of basalt, Contrib. Mineral. Petrol., 74, 7-12, 1980.

Sutton, G. H., W. Mitronovas, and P. W. Pomeroy, Short-period seismic energy radiation patterns from underground nuclear explosions and small-magnitude earthquakes, Bull. Seismol. Soc. Am., 57, 249-267, 1967.

Sutton, G. H., F. K. Duennebier, and B. Iwatake, Coupling of ocean bottom seismometers to soft bottom, Mar. Geophys. Res., 5, 35-51, 1981.

Swain, C. J., A Fortran IV program from interpolating irregularly spaced data using the difference equations for minimum curvature, Computers and Geoscience, 1, 231240, 1976.

Swift, S. A., M. E. Dougherty, and R. A. Stephen, Finite difference seismic modelling of axial magma chambers, Geophys. Res. Lett., 17, 2105-2108, 1990.

Tarantola, A., Inverse problem theory: Methods for Data Fitting and Model Parameter Estimation, Elsevier, New York, 613 pp., 1987.

Tarantola, A., and Valette, B., Inverse problems = quest for information, J. Geophys., $50,159-170,1982 \mathrm{a}$.

Tarantola, A., and Valette, B., Generalized nonlinear inverse problems solved using the least squares criterion, Rev. Geophys. Space Phys., 20, 219-232, 1982b.

Teng, T.-L., Attenuation of body waves and the Q structure of the mantle, J. Geophys. Res., 73, 2195-2208, 1968.

Thomson, D. J., Spectrum estimation techniques for characterization and development of WT4 waveguide - I, Bell System Tech. J., 56, 1769-1815, 1977.

Thomson, D. J., Spectrum estimation and harmonic analysis, Proc. I.E.E.E., 70, 10551096, 1982.

Thurber, C. H., Earthquake locations and three-dimensional crustal structure in the Coyote Lake area, central California, J. Geophys. Res., 88, 8226-8236, 1983.

Tikhonov, A. N., and V. Y. Arsenin, Solutions of Ill-Posed Problems, 258 pp., Wiley, New York, 1977.

Toksöz, M. N., A. M. Dainty, E. Reiter, R.-S. Wu, A model for attenuation and scattering in the Earth's crust, Pure Appl. Geophys., 128, 81-100, 1988. 
Toksöz, M. N., A.. M. Dainty, and E. E. Charrette, Coherency of ground motion at regional distances and scattering, Phys. Earth Planet. Inter., 67, 162-179, 1991.

Tonn, R., Comparison of seven methods for the computation of Q, Phys. Earth Planet. Inter., 55, 259-268, 1989.

Toomey, D. R., The tectonics and three-dimensional crustal structure of spreading centers: Microearthquake studies and tomographic inversions, Ph.D. thesis, MIT/WHOI Joint Program in Oceanography, Woods Hole, Mass., 210 pp., 1987.

Toomey, D. R., S. C. Solomon, and G. M. Purdy, Microearthquakes beneath the median valley of the Mid-Atlantic Ridge near $23^{\circ} \mathrm{N}$ : Tomography and tectonics, $J$. Geophys Res., 93, 9093-9112, 1988.

Toomey, D. R., G. M. Purdy, S. C. Solomon, and W. S. D. Wilcock, The threedimensional sesimic velocity structure of the East Pacific Rise near latitude $9^{\circ} 30^{\circ} \mathrm{N}$, Nature, 347, 639-645, 1990a.

Toomey, D. R., G. M. Purdy, and S. C. Solomon, Deep crustal seismic structure beneath the East Pacific Rise near 9०30'N (abstract), Eos Trans. AGU, 71, 1636, $1990 \mathrm{~b}$.

Toomey, D. R., S. C. Solomon, and G. M. Purdy, Application of new tomographic methods to data from the East Pacific Rise near $9^{\circ} 30^{\prime} \mathrm{N}$ (abstract), Eos Trans. AGU, 72, Fall meeting suppl. 494, $1990 \mathrm{~b}$.

Trampert, J., J.-P. Montagner, and P. Ho-Liu, and H. Kanamori, Comparison of iterative back-projection inversion and generalized inversion without blocks; case studies in attenuation tomography, comment and reply, Geophys. J. Int., 103, 755758, 1990.

Tréhu, D. R., Seismicity and structure of the Orozco transform fault from ocean bottom seismic observations, Ph.D. thesis, MIT/WHOI Joint Program in Oceanography, Woods Hole, Mass., 370 pp., 1982.

Vera, E. E., and J. B. Diebold, Seismic imaging of oceanic layer 2A between $9^{\circ} 30^{\prime} \mathrm{N}$ and $10^{\circ} \mathrm{N}$ on the East Pacific Rise (abstract), Eos Trans. AGU, 72, Fall meeting suppl. 495, 1991.

Vera, E. E., J. C. Mutter, P. Buhl, J. A. Orcutt, A. J. Harding, M. E. Kappus, R. S. Detrick, and T. M. Brocher, The structure of 0- to 0.2 -m.y.-old oceanic crust at $9^{\circ} \mathrm{N}$ on the East Pacific Rise from expanded spread profiles, J. Geophys. Res., 95, 15529-15556, 1990.

Vidale, J., Finite-difference calculation of travel times, Bull. Seismol. Soc. Am., 78, 2062-2076, 1988. 
Vidale, J. E., Finite-difference calculation of traveltimes in three dimensions, Geophysics, 55, 521-526, 1990.

Virieux, J., P-SV wave propagation in heterogeneous media: Velocity-stress finitedifference method, Geophysics, 51, 889-901, 1986.

Welch, P. D., The use of the fast Fourier transform for the estimation of power spectra: A method based on time averaging over short modified periodograms, I.E.E.E. Trans. Audio Electroacoust., AU-15, 70-74, 1967.

Wepfer, W. W., and N. I. Christensen, Compressional wave attenuation in oceanic basalts, J. Geophys. Res., 95, 17431-17439, 1990.

Wepfer, W. W., and N. I. Christensen, Q structure of the oceanic crust, Mar. Geophys. Res., 13, 227-237, 1991.

White, D. J., and R. M. Clowes, Shallow crustal structure beneath the Juan de Fuca Ridge from 2-D seismic refraction tomography, Geophys. J., 100, 349-367, 1990.

White, R. S., and R. A. Stephen, Compressional to shear wave conversion in oceanic crust, Geophys. J. R. Astron. Soc., 63, 547-565, 1980.

Whitehead, J. A., H. J. B. Dick, and H. Schouten, A mechanism for magmatic accretion under spreading centers, Nature, 312, 146-148, 1984.

Wilcock, W. S. D., G. M. Purdy, and S. C. Solomon, Microearthquake evidence for extension across the Kane transform fault, J. Geophys Res., 95, 15439-15462, 1990.

Wilcock, W. S. D., D. R. Toomey, G. M. Purdy, and S. C. Solomon, The renavigation of Sea Beam bathymetric data between $9^{\circ} \mathrm{N}$ and $10^{\circ} \mathrm{N}$ on the East Pacific Rise, Marine Geophys. Res., in press, 1992.

Wright D. J., and R. M. Haymon, Along-strike variations in fissure density and widths along the axial zone of the EPR $\left(9^{\circ} 12^{\prime}-54^{\prime} \mathrm{N}\right)$ (abstract), Eos Trans. AGU, 72, Fall meeting suppl. 491, 1991.

Woirgard, J., and Y. Gueguen, Elastic modulus and internal friction in enstatite, forsterite and peridotite at seismic frequencies and high temperatures, Phys. Earth Planet. Inter., 17, 140-146, 1978.

Yanovskaya, T. B., and P. G. Ditmar, Smoothness criteria in surface wave tomography, Geophys. J. Int., 102, 63-72, 1990.

Young, C.-Y., and R. W. Ward, Three-dimensional $\mathrm{Q}^{-1}$ model of the Coso Hot Springs known geothermal resource area, J. Geophys. Res., 85, 2459-2470, 1980.

Zener, C., Elasticity and Analasticity of Metals, University of Chicago Press, Chicago, Illinois, 170 pp., 1948. 
Zhu, T., K. Chun, and G. F. West, High-frequency P-wave attenuation determination using multiple-window spectral analysis method, Bull. Seismol. Soc. Am., 79, 1054-1069, 1989. 